\title{
Environmental Report 1999
}

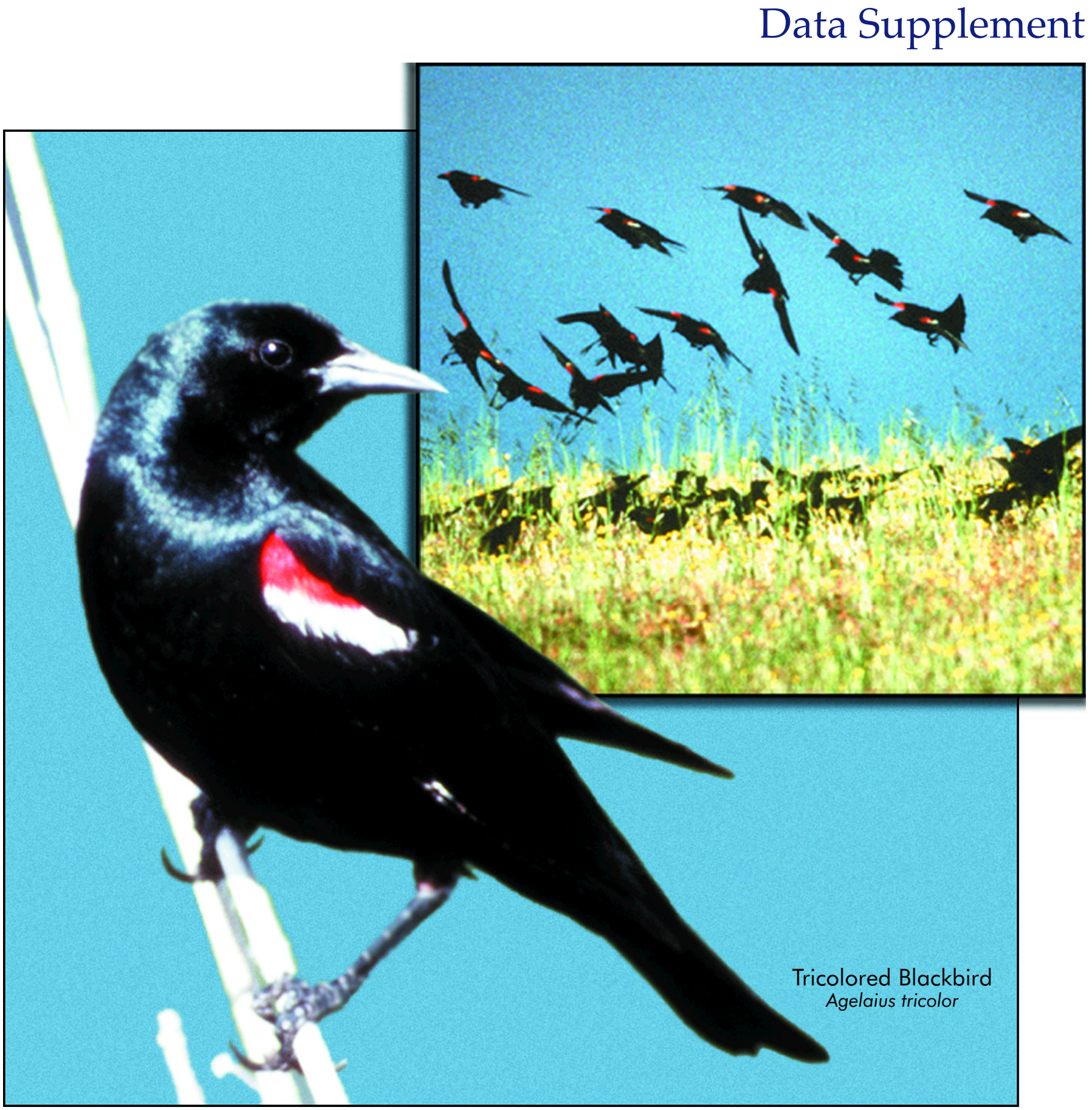

Lawrence Livermore National Laboratory 


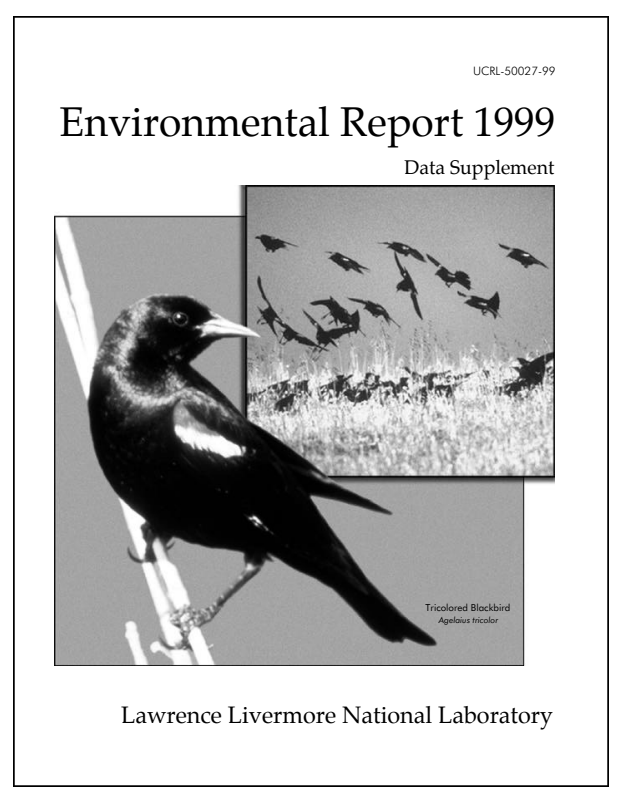

\section{Composition}

Beverly L. Chamberlain

Sherry A. Emmons

\section{Art and Design}

Lee A. Dravidzius

Brett S. Clark

\section{Cover}

The Tricolored Blackbird (Agelaius tricolor), which has state and federal status as a species of Special Concern, is endemic only to California and is largely restricted to the San Joaquin Valley where it is estimated that populations have declined by at least $50 \%$ in the last half-century. It roosts and nests in cattail or tule marshes in dense colonies that can number in the thousands of birds. The male Tricolored has a darker red patch than the Red-winged Blackbird and a conspicuous white margin that is identifiable even in flight.

Historically, Site 300 has had a small population nesting in the Elk Ravine wetland. This colony is considered unique because Tricoloreds typically do not inhabit foothill habitat areas like those at Site 300. When Tricoloreds are actively nesting, LLNL restricts traffic levels to protect the adult birds during their egg incubation period and rearing of young (roughly late March through May). LLNL wildlife biologists collect information on the density of nesting birds, productivity at the nest sites, and the areas used by the colony for foraging. This information is important to our understanding of the natural history and wildlife management aspects of the site as well as to state and federal resource agencies that track Tricolored population trends across California.

Cover photos provided by:

- flock-Jim S. Woollett, Jr., wildlife biologist, LLNL

- single bird-David Menke, outdoor recreation planner, Lower Klamath National Wildlife Refuge, U.S. Fish and Wildlife Service

For further information about this report contact: Bert Heffner, LLNL Public Affairs Department, P.O. Box 808, Livermore, CA 94550, (510) 424-4026. This report can be accessed on the Internet at http:/ / www.llnl.gov/ saer. It is also available to DOE employees and DOE contractors from: Office of Scientific and Technical Information, P.O. Box 62, Oak Ridge, TN 37831 and to the public from: National Technical Information Service, U.S. Department of Commerce, 5285 Port Royal Road, Springfield, VA 22161.

\section{DISCLAIMER}

This document was prepared as an account of work sponsored by an agency of the United States Government. Neither the United States Government nor the University of California nor any of their employees makes any warranty, express or implied, or assumes any legal liability or responsibility for the accuracy, completness, or usefulness of any information, apparatus, product, or process disclosed, or represents that its use would not infringe on privately owned rights. References herein to any specific commercial product, process, or service by trade name, trademark, manufacturer, or otherwise does not necessarily constitute or imply its endorsement, recommendation, or favoring by the United States Government or the University of California. The views and opinions of authors expressed herein do not necessarily state or reflect those of the United States Government or the University of California and shall not be used for advertising or product endorsement purposes. 


\section{Environmental Report 1999 Data Supplement}

\section{Authors}

Jennifer M. Larson Gretchen M. Gallegos

Lucinda M. Garcia

Arthur H. Biermann Ted A. Giesing

Robert J. Harrach

Allen R. Grayson

Paris E. Althouse

Linda C. Hall

Nicholas A. Bertoldo

Donald H. MacQueen

Richard G. Blake

Sandra Mathews

Erich R. Brandstetter

S. Ring Peterson

Shari L. Brigdon

Michael J. Taffet

Richard A. Brown

Paula J. Tate

Eric Christofferson

Robert J. Vellinger

Karen J. Folks

Rebecca J. Ward

Robert A. Williams

\section{Editors}

Karen S. Rath

Nancy J. Woods
Gloria J. Cannon

Nona M. Sanford 



\section{Preface}

This Data Supplement to the Lawrence Livermore National Laboratory's (LLNL's) annual Environmental Report 1999 was prepared for the U.S. Department of Energy. The main volume is intended to provide all information on LLNL's environmental impact and compliance activities that is of interest to most readers. The Data Supplement supports main volume summary data and is essentially a detailed data report that provides individual data points, where applicable. Some summary data are also included in the Data Supplement, and more detailed accounts are given of sample collection and analytical methods.

The two volumes are organized in a parallel fashion to aid the reader in crossreferencing between them. This supplement includes more detailed information to support the nine chapters in the main volume that cover monitoring of air, air effluent, sewerable water, surface water, ground water, soil and sediment, vegetation and foodstuff, environmental radiation, and quality assurance. The other five chapters in the main volume have no supporting information in the Data Supplement.

As in our previous annual reports, data are presented in Système International (SI) units. In particular, the primary units used for radiological results are becquerels and sieverts for activity and dose, with curies and rem used secondarily $\left(1 \mathrm{~Bq}=2.7 \times 10^{-11} \mathrm{Ci} ; 1 \mathrm{~Sv}=100 \mathrm{rem}\right)$. 



\section{Table of Contents}

List of Tables

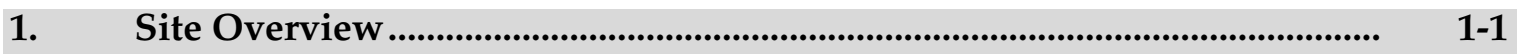

2. Compliance Summary …..................................................................................... 2-1

3. Environmental Program Information............................................................. 3-1

4. $\quad$ Air Effluent Monitoring ...................................................................................... 4-1

Air Effluent Sampling Methods .................................................................. $4-1$

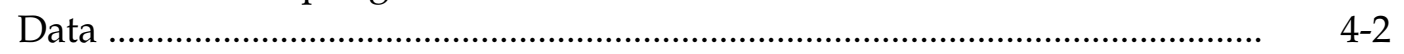

5. Air Surveillance Monitoring ..................................................................................... 5-1

Air Surveillance Sampling ............................................................................... 5-1

Air Particulate Networks....................................................................... $\quad 5-1$

Air Particulate Radiological Networks ......................................................... 5 5

Air Particulate Beryllium ........................................................................... $\quad 5-5$

Air Tritium …........................................................................................... $5-5$

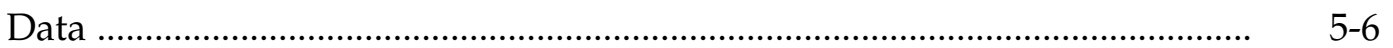

6. Sewerable Water Monitoring ........................................................................ 6-1

Discharges of Treated Ground Water .......................................................... 6 6 6

Flow Monitoring Methods ............................................................................. 6-1

Sewage Sampling Methods and Analytical Procedures................................. 6. 6-1

Quality Assurance Methods ............................................................................ $\quad$ 6-5

7. Surface Water Monitoring ................................................................................. 7-1

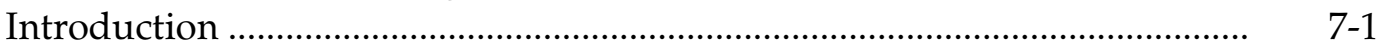

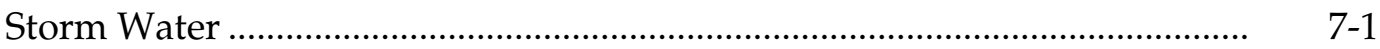

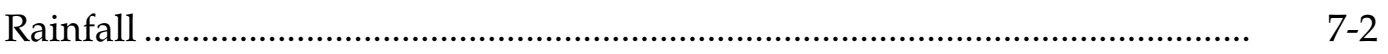

Drainage Retention Basin .......................................................................... $\quad$ 7-2

Other Waters ..............................................................................................

8. Ground Water Investigation and Remediation ................................................ 8-1

9. Ground Water Monitoring ........................................................................... 9-1

Methods .............................................................................................

Livermore Site ............................................................................ 9

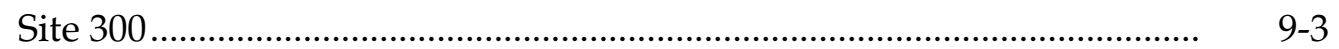

10. Soil and Sediment Monitoring ....................................................................... 10-1

Surface Soil Methods ........................................................................... 10...

Surface Sediment Methods ..................................................................... 10 10

Vadose Zone Soil Methods .............................................................................. 10-3

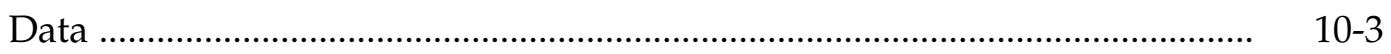

Indicates no supplemental data in this volume. Please see the main volume for detailed information on this subject. 
11. Vegetation and Foodstuff Monitoring …............................................................... 11-1

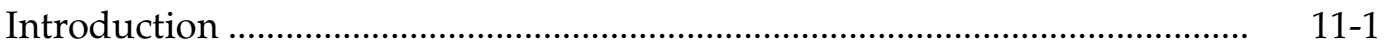

Vegetation Sampling Methods ........................................................................ 11-1

Wine Sampling Methods ............................................................................... 11-2

12. Environmental Radiation Monitoring …............................................................ 12-1

Methods of Gamma Radiation Monitoring ................................................ 12-1

Tables ................................................................................. $12-2$

13. Radiological Dose Assessment.......................................................................... 13-1

14. Quality Assurance ......................................................................................... 14-1

Participation in Laboratory Intercomparison Studies ................................. 14- 14

Indicates no supplemental data in this volume. Please see the main volume for detailed information on this subject. 


\section{List of Tables}

Table 4-1. Summary of gross alpha and gross beta in air effluent samples from monitored emission points at Building 175, 1999 .....................

Table 4-2. Summary of gross alpha and gross beta in air effluent samples from monitored emission points at Building 177, 1999 .....................

Table 4-3. Summary of gross alpha and gross beta in air effluent samples from monitored emission points at Building 251, 1999 ..................... $\quad 4-4$

Table 4-4. Summary of gross alpha and gross beta in air effluent samples from monitored emission points at Building 292, 1999

Table 4-5. Summary of tritium in air effluent samples from monitored emission points at Building 331, 1999

Table 4-6. Summary of gross alpha and gross beta in air effluent samples from monitored emission points at Building 332, 1999

Table 4-7. Summary of gross alpha and gross beta in air effluent samples from monitored emission points at Building 490, 1999

Table 4-8. Summary of gross alpha and gross beta in air effluent samples from monitored emission points at Building 491, 1999

Table 5-1. Monthly median activities for gross alpha and gross beta summarized from weekly data for the LLNL perimeter locations, 1999

Table 5-2. Monthly median activities for gross alpha and beta summarized from weekly data for the Livermore Valley upwind locations, 1999

Table 5-3. Monthly median activities for gross alpha and beta summarized from weekly data for Livermore Valley downwind and special interest locations, 1999.

Table 5-4. Gamma activity in particulate air samples, Livermore site perimeter, 1999

Table 5-5. Plutonium-239+240 activity in air particulate samples, Livermore site perimeter, 1999.

Table 5-6. Plutonium-239+240 activity in air particulate samples, Livermore Valley, 1999

Table 5-7. Plutonium-239+240 activity in air particulate samples, diffuse sources, 1999

Table 5-8. Uranium mass concentration in air particulate samples, Livermore site perimeter 1999 
Table 5-9. Monthly median activities for gross alpha summarized from weekly data from low-volume air samplers, 1999 ...

Table 5-10. Monthly median activities for gross beta summarized from weekly data from low-volume air samplers, 1999

Table 5-11. Tritium concentration in air, Livermore Valley, 1999.

Table 5-12. Tritium concentration in air, Livermore site perimeter, 1999.

Table 5-13. Tritium concentration in air at locations near diffuse sources, 1999

Table 5-14. Monthly beryllium in air particulate composites, Livermore site perimeter, 1999

Table 5-15. Gross alpha and gross beta activities summarized from weekly data for Site 300 perimeter and associated off-site locations, 1999 .

Table 5-16. Gamma activity in particulate air samples, Site 300, 1999

Table 5-17. Plutonium-239+240 activity in air particulate samples, Site 300, 1999.

Table 5-18. Uranium mass in air particulate samples for Site 300 composite and PRIM location, 1999

Table 5-19. Tritium concentration in air, Site 300, 1999

Table 5-20. Beryllium in air particulate samples, Site 300 network, 1999

Table 6-1. Laboratory analytical results for ground water discharges to the sanitary sewer, January 1 through December 31, 1999.

Table 6-2a. Daily flow totals for LLNL site sanitary sewer effluent (ML), 1999.

Table 6-2b. Monthly and annual flow summary statistics for LLNL site sanitary sewer effluent (ML), 1999

Table 6-3. Daily monitoring results for gross alpha, gross beta, and tritium in the sanitary sewer effluent, 1999

Table 6-4. Weekly composite results for tritium $(\mathrm{mBq} / \mathrm{mL})$ for the LWRP effluent, 1999

Table 6-5. Weekly composite results for metals in LLNL sanitary sewer effluent, 1999

Table 6-6. Monthly 24-hour composite results for metals in LLNL sanitary sewer effluent, 1999

Table 6-7. Monthly monitoring results for physical and chemical characteristics of the LLNL sanitary sewer effluent, 1999 
Table 7-1. Numerical comparison criteria for storm water constituents of concern

Table 7-2. Routine tritium, gross alpha, and gross beta sampling in storm water runoff at the Livermore site, 1999.

Table 7-3. Tritium source investigation sampling in storm water runoff $(\mathrm{Bq} / \mathrm{L})$ at the Livermore site, 1999

Table 7-4. Plutonium in storm water runoff, Livermore site, 1999 ..................... 7-9

Table 7-5. Metals detected in storm water runoff, Livermore site, 1999 ............ 7-10

Table 7-6. Nonradioactive constituents (other than metals) detected in storm water runoff, Livermore site, 1999

Table 7-7. Number of nondetects in storm water runoff, Livermore site, 1999.

Table 7-8. Radioactivity in storm water runoff, Site 300, 1999

Table 7-9. Nonradioactive constituents in storm water runoff, Site 300, 1999.

Table 7-10. Dioxins in storm water runoff, Site 300, 1999

Table 7-11. Pit 6 post-closure storm water monitoring, Site 300, 1999

Table 7-12. Tritium in rain (Bq/L), Livermore site and Livermore Valley, 1999

Table 7-13. Drainage Retention Basin discharge limits for CDBX, identified in CERCLA Record of Decision as amended, and sampling frequencies for CDBX and WPDC

Table 7-14. Routine water quality management action levels and monitoring plan for the Drainage Retention Basin

Table 7-15. Compliance monitoring data for releases from the Drainage Retention Basin, 1999.

Table 7-16. Monthly analyses of water samples collected from the Drainage Retention Basin location CDBE, 1999.

Table 7-17. Quarterly analyses of water samples collected from the Drainage Retention Basin location CDBE, 1999

Table 7-18 Semiannual/annual analyses of water samples collected from the Drainage Retention Basin location CDBE, 1999

Table 7-19. Field data collected from the Drainage Retention Basin at eight locations, 1999

Table 7-20. Seasonal inventory of plants and animals, Livermore site, 1999 ......

Table 7-21. Radioactivity in surface and drinking water $(\mathrm{Bq} / \mathrm{L})$ in the Livermore Valley, 1999. 
Table 9-1a. Analytical methods and reporting limits for inorganic constituents of concern in ground water

Table 9-1b. Analytical methods and reporting limits for organic constituents of concern in ground water

Table 9-1c. Radioisotopes and reporting limits for gamma spectroscopic analysis of constituents of concern in ground water

Table 9-2. Livermore site surveillance well W-008

Table 9-3. Livermore site surveillance well W-221

Table 9-4. Livermore site surveillance well W-017.

Table 9-5. Livermore off-site surveillance well 14B1

Table 9-6. Livermore off-site surveillance well W-121 .

Table 9-7. Livermore off-site surveillance well $W-151$.

Table 9-8. $\quad$ Livermore off-site surveillance well $\mathrm{W}-571$...................................... 9-23

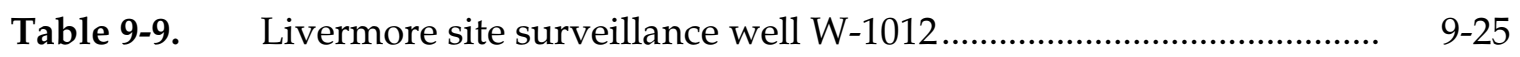

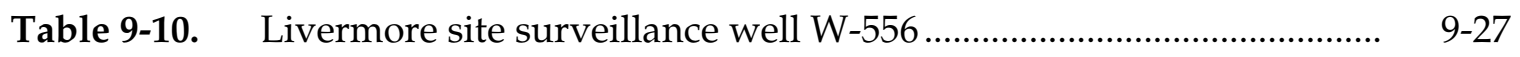

Table 9-11. Livermore site surveillance well $W-373$.............................................. 9-29

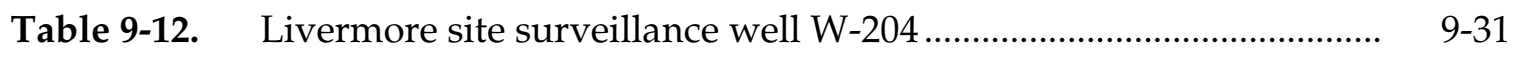

Table 9-13. Livermore site surveillance well $W-363$............................................. 9-33

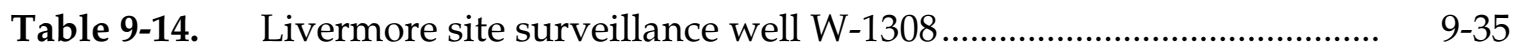

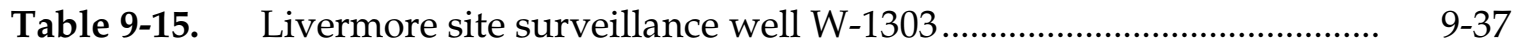

Table 9-16. Livermore site surveillance well $\mathrm{W}-119$................................................ 9-39

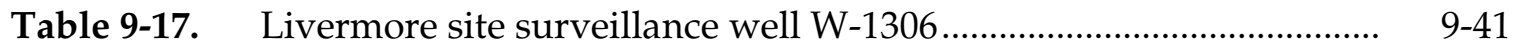

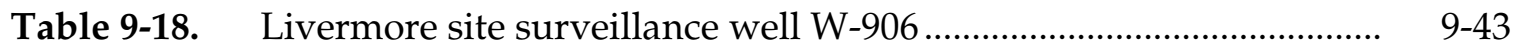

Table 9-19. Livermore site surveillance well $\mathrm{W}-593$............................................... 9-45

Table 9-20. Livermore site surveillance well $W-270$............................................. 9-47

Table 9-21. Livermore site surveillance well $\mathrm{W}-359$................................................ 9-49

Table 9-22. Livermore site surveillance well GSW-011 .......................................... 9-51

Table 9-23. Livermore site surveillance well $\mathrm{W}-307$............................................... 9-53

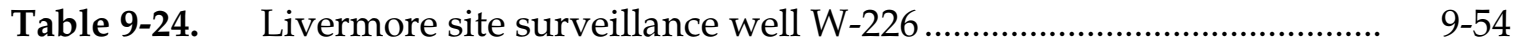

Table 9-25. Livermore site surveillance well $\mathrm{W}-306$............................................... 9-55

Table 9-26. Livermore site surveillance well W-305 ............................................. 9-56

Table 9-27. Livermore site surveillance well SIP-331-001 ...................................... 9-58

Table 9-28. Livermore site surveillance well $\mathrm{W}-148$................................................ 9-60

Table 9-29. Tritium activity in Livermore Valley wells, 1999 .............................. 9-62 
Table 9-30. Site 300, Elk Ravine surveillance wells ............................................... 9-63

Table 9-31. Site 300, Pit 2 surveillance wells ........................................................... 9-66

Table 9-32. Site 300, Pit 8 surveillance wells ........................................................ 9-68

Table 9-33. Site 300, Pit 9 surveillance wells .......................................................... 9-69

Table 9-34. 1999 analytical results for Site 300 Building 829 area deep monitoring wells

Table 9-35. 1999 analytical results for Site 300 Building 829 area shallow monitoring wells .......................................................................... $9-76$

Table 9-36. Site 300 potable standby supply well 18 ............................................. 9-78

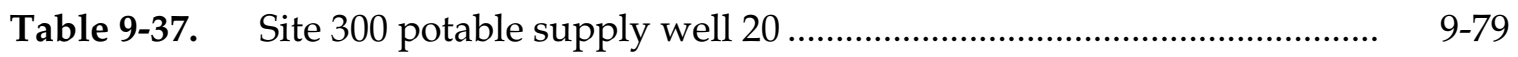

Table 9-38. Site 300 off-site well CARNRW1 ........................................................... 9-80

Table 9-39. Site 300 off-site well CDF1 ................................................................. 9-81

Table 9-40. Off-site surveillance well CON1 ........................................................ 9-82

Table 9-41. Site 300, off-site surveillance well GALLO1 ....................................... 9-83

Table 9-42. Site 300 off-site surveillance well CARNRW2 …................................. 9-84

Table 9-43. Site 300 off-site surveillance well CON2 …..................................... 9-85

Table 9-44. Annually monitored off-site surveillance wells ................................ 9-86

Table 10-1. Radionuclides in soils and sediments in the Livermore Valley, 1999.

Table 10-2. Radionuclides and beryllium in soils at Site 300, 1999 ...................... 10-6

Table 10-3. Background screening concentration values for metals in soils at the Livermore site

Table 10-4. De minimis concentration levels for soluble metals found in Livermore site soils

Table 10-5. De minimis concentration levels for organic and radioactive constituents of concern found in Livermore site soils and sediments

Table 10-6. Semi-volatile organic compounds measured by EPA Method 8240 in Livermore site vadose zone soil, 1999

Table 10-7. Total metals in Livemore site vadose zone soil, 1999 ........................ 10-11

Table 10-8. Soluble metals in Livermore site vadose zone soil, 1999 ................... 10 10-12

Table 11-1. Concentrations of tritium in plant water (Bq/L), 1999 ..................... 11-3

Table 11-2. Tritium in retail wine (Bq/L), 1999 .................................................... 11-4

Table 12-1. Calculated dose from TLD environmental radiation measurements, Livermore site perimeter, 1999. 


\section{List of Tables}

Table 12-2. Calculated dose from TLD environmental radiation measurements, Livermore Valley, 1999.

Table 12-3. Calculated dose from TLD environmental radiation measurements, Site 300 perimeter, 1999

Table 12-4. Calculated dose from TLD environmental radiation measurements, Tracy and other off-site locations in the vicinity of Site 300, 1999

Table 14-1. LLNL's CES EMRL results from the DOE EML Quality Assurance Program, 1999

Table 14-2. LLNL's HCAL results from the DOE EML Quality Assurance Program, 1999

Table 14-3. LLNL CES EMRL performance in the MAPEP-98-W6 Intercomparison Program for Water

Table 14-4. LLNL CES EMRL performance in the MAPEP-99-S6 Intercomparison Program for Soil 
There are no supplemental data in this chapter. Please see the main volume for details about Site Overview. 

There are no supplemental data in this chapter.

Please see the main volume for details about Compliance Summary. 

There are no supplemental data in this chapter.

Please see the main volume for details about Environmental Program Information. 



\section{Air Effluent Monitoring}

Arthur H. Biermann

Linda C. Hall

\section{Air Effluent Sampling Methods}

At the beginning of 1999, Lawrence Livermore National Laboratory used 101 continuously operating radiological sampling systems on air exhausts at eight facilities at the Livermore site (main volume, Table 4-1). These samplers were used to determine actual emissions from operations involving radioactive materials at the facilities and to verify the integrity of emission control systems. Some sampling systems at Buildings 251, 292, and 490 were either deactivated or removed in 1999. For a further discussion see Chapter 4 of the main volume.

Air samples for particulate emissions are extracted downstream of high-efficiency particulate air (HEPA) filters and prior to the discharge point to the atmosphere. In most cases, simple, filter-type aerosol collection systems are used. However, in some facilities (Buildings 251 and 332), continuous air monitors (CAMs) are used for sampling to check for alpha activity. In addition to collecting a sample of particles, the CAM units provide an alarm capability for the facility in the event of a release of particulates containing alpha activity. Both types of sampling systems, the simple filter type and alpha alarm monitors, are used to monitor discharge points from Building 332. In the event of a power outage, the air sampling systems in critical facilities are switched to auxiliary power and continue to operate.

The sample filters are 47-mm-diameter membrane filters and are changed weekly or biweekly, depending on the facility. After sample collection, filters are placed in glassine envelopes, and each envelope is tagged with a unique bar code label. Filter sample data-including location, equipment identification, bar code, sampling start date, sampling stop date, and flow rate-are entered into the Hazards Control Department (HCD) sample tracking and reporting (STAR) computer system. Sampling procedures are contained in the environmental section of the discipline action plan for a facility. Filters are analyzed at the HCD Radiological Measurements Laboratory (RML) for gross alpha and beta activity using gas proportional counters. Analysis is delayed for at least four days following sample termination to allow for the decay of naturally occurring radon daughters. To verify the operation of the counting system, calibration and background samples are intermixed with the sample filters for analysis. Analytical 


\section{Air Effluent Monitoring}

techniques are consistent with the Environmental Protection Agency (EPA) recommended procedures. Further details about sampling and analysis are discussed in the Environmental Monitoring Plan (Tate et al. 1999).

Each stack of the Tritium Facility (Building 331) is monitored for tritium release by both a continuous monitoring alarm system and continuous molecular sieve samplers. The alarmed samplers, Overhoff ionization chambers, provide real-time total tritium concentration release levels (tritiated hydrogen gas and tritiated water combined). The sieve samplers, which can discriminate between tritiated water vapor and tritiated hydrogen gas, provide the values used for environmental reporting. Each sieve sampler (not alarmed) runs in parallel with an alarmed monitor and consists of two molecular sieves. The first sieve collects tritiated water vapor; the second sieve contains a palladiumcoated catalyst that converts tritiated hydrogen to tritiated water and collects the tritiated water on the sieve. Sieves are changed weekly. The sieve samples are logged into the HCD STAR sample tracking system and submitted to the HCD Analytical Laboratory, where tritiated water is baked out and collected. RML analyzes the retrieved tritium for beta activity using scintillation counting techniques.

\section{Data}

Annual summaries of gross alpha, gross beta, and tritium data for samplers at each monitored facility are summarized in Tables 4-1 through 4-8. The tables present the ratio of the number of results that have activity concentration greater than the minimum detectable concentration (MDC) of the analysis to the total number of samples in the year, and the minimum, median, and maximum activity concentrations of the samples (in $\mathrm{Bq} / \mathrm{m}^{3}$ ). If the concentration is negative, the result is considered to be a nondetection. The MDC is defined as the smallest concentration of radioactive material that can be detected (distinguished from background) with some specified degree of confidence. Analytical results are reported as a measured concentration in $\mathrm{Bq}$ per volume of air. If the concentration reported is negative, the result is considered to be a nondetection. A detailed discussion of these results is provided in Chapter 14 of the main volume of this report. 
Table 4-1. Summary of gross alpha and gross beta in air effluent samples from monitored emission points at Building 175, 1999.

\begin{tabular}{|c|c|c|c|c|}
\hline $\begin{array}{c}\text { Emission } \\
\text { point }\end{array}$ & $\begin{array}{l}\text { No. >MDC(a)/ } \\
\text { total samples }\end{array}$ & $\begin{array}{c}\text { Minimum } \\
\left(\mathrm{Bq} / \mathrm{m}^{3}\right)\end{array}$ & $\begin{array}{l}\text { Median } \\
\left(\mathrm{Bq} / \mathrm{m}^{3}\right)\end{array}$ & $\begin{array}{c}\text { Maximum } \\
\left(\mathrm{Bq} / \mathrm{m}^{3}\right)\end{array}$ \\
\hline \multicolumn{5}{|l|}{ Gross alpha } \\
\hline 1 & $2 / 48$ & $-6.88 \times 10^{-5}$ & $7.46 \times 10^{-6}$ & $3.02 \times 10^{-4}$ \\
\hline 2 & $0 / 48$ & $-6.51 \times 10^{-5}$ & $3.81 \times 10^{-6}$ & $1.20 \times 10^{-4}$ \\
\hline 3 & $1 / 48$ & $-2.80 \times 10^{-5}$ & $1.03 \times 10^{-6}$ & $1.02 \times 10^{-4}$ \\
\hline 4 & $0 / 49$ & $-2.58 \times 10^{-5}$ & $-5.98 \times 10^{-7}$ & $5.74 \times 10^{-5}$ \\
\hline 5 & $0 / 47$ & $-6.33 \times 10^{-5}$ & $3.92 \times 10^{-6}$ & $1.43 \times 10^{-4}$ \\
\hline 6 & $0 / 47$ & $-6.59 \times 10^{-5}$ & $4.63 \times 10^{-6}$ & $2.59 \times 10^{-4}$ \\
\hline \multicolumn{5}{|l|}{ Gross beta } \\
\hline 1 & $8 / 48$ & $-1.76 \times 10^{-4}$ & $1.01 \times 10^{-4}$ & $4.70 \times 10^{-3}$ \\
\hline 2 & $5 / 48$ & $-1.11 \times 10^{-4}$ & $1.05 \times 10^{-4}$ & $1.08 \times 10^{-3}$ \\
\hline 3 & $10 / 48$ & $-7.73 \times 10^{-5}$ & $8.38 \times 10^{-5}$ & $6.11 \times 10^{-3}$ \\
\hline 4 & $0 / 49$ & $-8.47 \times 10^{-5}$ & $1.46 \times 10^{-5}$ & $1.32 \times 10^{-4}$ \\
\hline 5 & $2 / 47$ & $-1.38 \times 10^{-4}$ & $-1.33 \times 10^{-6}$ & $8.47 \times 10^{-4}$ \\
\hline 6 & $1 / 47$ & $-1.55 \times 10^{-4}$ & $4.37 \times 10^{-5}$ & $4.11 \times 10^{-4}$ \\
\hline
\end{tabular}

a $\mathrm{MDC}=$ Minimum detectable concentration. (See main volume, Chapter 14, Quality Assurance, for an explanation of MDC.)

Table 4-2. Summary of gross alpha and gross beta in air effluent samples from monitored emission points at Building 177, 1999.

\begin{tabular}{|c|c|c|c|c|}
\hline $\begin{array}{c}\text { Emission } \\
\text { point }\end{array}$ & $\begin{array}{c}\text { No. >MDC(a)/ } \\
\text { total samples }\end{array}$ & $\begin{array}{c}\text { Minimum } \\
\left(\mathrm{Bq} / \mathbf{m}^{\mathbf{3}}\right)\end{array}$ & $\begin{array}{c}\text { Median } \\
\left(\mathrm{Bq} / \mathbf{m}^{3}\right)\end{array}$ & $\begin{array}{c}\text { Maximum } \\
\left(\mathbf{B q} / \mathbf{m}^{\mathbf{3}}\right)\end{array}$ \\
\hline $\begin{array}{c}\text { Gross alpha } \\
1\end{array}$ & $0 / 50$ & $-2.58 \times 10^{-5}$ & $2.54 \times 10^{-6}$ & $6.36 \times 10^{-5}$ \\
\hline $\begin{array}{c}\text { Gross beta } \\
1\end{array}$ & $0 / 50$ & $-6.96 \times 10^{-5}$ & $2.52 \times 10^{-5}$ & $2.03 \times 10^{-4}$ \\
\hline
\end{tabular}

a $\mathrm{MDC}=$ Minimum detectable concentration. (See main volume, Chapter 14, Quality Assurance, for an explanation of MDC.) 


\section{Air Effluent Monitoring}

Table 4-3. Summary of gross alpha and gross beta in air effluent samples from monitored emission points at Building 251, 1999.

\begin{tabular}{|c|c|c|c|c|}
\hline $\begin{array}{c}\text { Emission } \\
\text { point }^{(a)}\end{array}$ & $\begin{array}{c}\text { No. }>\text { MDC }(b, c) / \\
\text { total samples }\end{array}$ & $\begin{array}{c}\text { Minimum } \\
\left(\mathrm{Bq} / \mathrm{m}^{3}\right)\end{array}$ & $\begin{array}{l}\text { Median } \\
\left(\mathrm{Bq} / \mathrm{m}^{3}\right) \\
\end{array}$ & $\begin{array}{c}\text { Maximum } \\
\left(\mathrm{Bq} / \mathrm{m}^{3}\right)\end{array}$ \\
\hline \multicolumn{5}{|l|}{ Gross Alpha } \\
\hline 1 & $0 / 26$ & $-6.36 \times 10^{-5}$ & $4.46 \times 10^{-5}$ & $1.84 \times 10^{-4}$ \\
\hline 2 & $0 / 1$ & $1.17 \times 10^{-4}$ & $1.17 \times 10^{-4}$ & $1.17 \times 10^{-4}$ \\
\hline 3 & $0 / 26$ & $-6.59 \times 10^{-5}$ & $2.41 \times 10^{-5}$ & $1.39 \times 10^{-4}$ \\
\hline 4 & $0 / 1$ & $4.85 \times 10^{-5}$ & $4.85 \times 10^{-5}$ & $4.85 \times 10^{-5}$ \\
\hline 5 & $1 / 26$ & $-2.58 \times 10^{-5}$ & $2.69 \times 10^{-5}$ & $1.60 \times 10^{-4}$ \\
\hline 6 & $1 / 25$ & $-6.18 \times 10^{-5}$ & $1.00 \times 10^{-4}$ & $5.70 \times 10^{-4}$ \\
\hline 7 & $0 / 26$ & $-1.51 \times 10^{-5}$ & $1.34 \times 10^{-5}$ & $8.55 \times 10^{-5}$ \\
\hline 8 & $0 / 2$ & $3.70 \times 10^{-6}$ & $1.40 \times 10^{-5}$ & $2.42 \times 10^{-5}$ \\
\hline 10 & $3 / 26$ & $-7.84 \times 10^{-5}$ & $9.62 \times 10^{-5}$ & $4.48 \times 10^{-4}$ \\
\hline 11 & $0 / 1$ & $1.84 \times 10^{-4}$ & $1.84 \times 10^{-4}$ & $1.84 \times 10^{-4}$ \\
\hline 12 & $0 / 1$ & $8.58 \times 10^{-7}$ & $8.58 \times 10^{-7}$ & $8.58 \times 10^{-7}$ \\
\hline 13 & $0 / 26$ & $-6.85 \times 10^{-5}$ & $2.39 \times 10^{-5}$ & $1.63 \times 10^{-4}$ \\
\hline 14 & $1 / 26$ & $-4.59 \times 10^{-5}$ & $4.44 \times 10^{-5}$ & $3.52 \times 10^{-4}$ \\
\hline 15 & $0 / 1$ & $3.92 \times 10^{-4}$ & $3.92 \times 10^{-4}$ & $3.92 \times 10^{-4}$ \\
\hline 16 & $1 / 26$ & $-2.69 \times 10^{-5}$ & $2.28 \times 10^{-5}$ & $1.35 \times 10^{-4}$ \\
\hline 17 & $2 / 26$ & $-2.38 \times 10^{-5}$ & $3.14 \times 10^{-5}$ & $1.74 \times 10^{-4}$ \\
\hline 18 & $1 / 26$ & $-3.96 \times 10^{-5}$ & $3.69 \times 10^{-5}$ & $1.96 \times 10^{-4}$ \\
\hline 19 & $1 / 26$ & $-2.89 \times 10^{-5}$ & $3.65 \times 10^{-5}$ & $1.74 \times 10^{-4}$ \\
\hline 20 & $1 / 26$ & $-1.89 \times 10^{-5}$ & $4.51 \times 10^{-5}$ & $1.41 \times 10^{-4}$ \\
\hline 21 & $3 / 26$ & $-9.36 \times 10^{-6}$ & $7.49 \times 10^{-5}$ & $1.96 \times 10^{-4}$ \\
\hline 22 & $0 / 1$ & $3.77 \times 10^{-5}$ & $3.77 \times 10^{-5}$ & $3.77 \times 10^{-5}$ \\
\hline 23 & $0 / 26$ & $-2.51 \times 10^{-5}$ & $2.85 \times 10^{-5}$ & $1.26 \times 10^{-4}$ \\
\hline 24 & $1 / 26$ & $-3.24 \times 10^{-5}$ & $8.45 \times 10^{-5}$ & $2.53 \times 10^{-4}$ \\
\hline 25 & $0 / 26$ & $-3.58 \times 10^{-5}$ & $2.69 \times 10^{-5}$ & $1.20 \times 10^{-4}$ \\
\hline 26 & $0 / 26$ & $-4.88 \times 10^{-5}$ & $4.24 \times 10^{-5}$ & $1.90 \times 10^{-4}$ \\
\hline 27 & $0 / 1$ & $-4.22 \times 10^{-6}$ & $-4.22 \times 10^{-6}$ & $-4.22 \times 10^{-6}$ \\
\hline 28 & $0 / 26$ & $-5.55 \times 10^{-5}$ & $6.96 \times 10^{-5}$ & $1.90 \times 10^{-4}$ \\
\hline 29 & $1 / 26$ & $-3.00 \times 10^{-5}$ & $4.18 \times 10^{-5}$ & $1.41 \times 10^{-4}$ \\
\hline 30 & $0 / 25$ & $-4.40 \times 10^{-5}$ & $6.01 \times 10^{-5}$ & $1.76 \times 10^{-4}$ \\
\hline 31 & $0 / 1$ & $9.92 \times 10^{-6}$ & $9.92 \times 10^{-6}$ & $9.92 \times 10^{-6}$ \\
\hline 32 & $0 / 1$ & $4.81 \times 10^{-5}$ & $4.81 \times 10^{-5}$ & $4.81 \times 10^{-5}$ \\
\hline 33 & $6 / 26$ & $-1.25 \times 10^{-5}$ & $6.42 \times 10^{-5}$ & $2.22 \times 10^{-4}$ \\
\hline 34 & $3 / 26$ & $-2.09 \times 10^{-5}$ & $5.70 \times 10^{-5}$ & $1.69 \times 10^{-4}$ \\
\hline 35 & $0 / 26$ & $-2.87 \times 10^{-5}$ & $2.29 \times 10^{-5}$ & $9.88 \times 10^{-5}$ \\
\hline
\end{tabular}


Table 4-3. Summary of gross alpha and gross beta in air effluent samples from monitored emission points at Building 251, 1999 (continued).

\begin{tabular}{|c|c|c|c|c|}
\hline $\begin{array}{c}\text { Emission } \\
\text { point }^{(a)}\end{array}$ & $\begin{array}{l}\text { No. >MDC(b,c)/ } \\
\text { total samples }\end{array}$ & $\begin{array}{l}\text { Minimum } \\
\left(\mathrm{Bq} / \mathrm{m}^{3}\right)\end{array}$ & $\begin{array}{l}\text { Median } \\
\left(\mathrm{Bq} / \mathrm{m}^{3}\right)\end{array}$ & $\begin{array}{c}\text { Maximum } \\
\left(\mathrm{Bq} / \mathrm{m}^{3}\right)\end{array}$ \\
\hline \multicolumn{5}{|l|}{ Gross alpha } \\
\hline 36 & $0 / 1$ & $3.37 \times 10^{-5}$ & $3.37 \times 10^{-5}$ & $3.37 \times 10^{-5}$ \\
\hline 37 & $0 / 1$ & $4.81 \times 10^{-5}$ & $4.81 \times 10^{-5}$ & $4.81 \times 10^{-5}$ \\
\hline 38 & $0 / 1$ & $2.52 \times 10^{-5}$ & $2.52 \times 10^{-5}$ & $2.52 \times 10^{-5}$ \\
\hline 39 & $0 / 1$ & $2.65 \times 10^{-5}$ & $2.65 \times 10^{-5}$ & $2.65 \times 10^{-5}$ \\
\hline 40 & $0 / 26$ & $-1.32 \times 10^{-5}$ & $2.24 \times 10^{-5}$ & $8.10 \times 10^{-5}$ \\
\hline 41 & $0 / 1$ & $5.62 \times 10^{-5}$ & $5.62 \times 10^{-5}$ & $5.62 \times 10^{-5}$ \\
\hline 42 & $0 / 1$ & $7.47 \times 10^{-6}$ & $7.47 \times 10^{-6}$ & $7.47 \times 10^{-6}$ \\
\hline 43 & $0 / 26$ & $-1.15 \times 10^{-5}$ & $1.26 \times 10^{-5}$ & $6.36 \times 10^{-5}$ \\
\hline 44 & $1 / 26$ & $-6.03 \times 10^{-5}$ & $8.10 \times 10^{-5}$ & $1.55 \times 10^{-4}$ \\
\hline 45 & $1 / 26$ & $-3.61 \times 10^{-5}$ & $4.13 \times 10^{-5}$ & $2.24 \times 10^{-4}$ \\
\hline 46 & $5 / 26$ & $-2.58 \times 10^{-5}$ & $3.10 \times 10^{-5}$ & $1.92 \times 10^{-4}$ \\
\hline 47 & $2 / 26$ & $-1.94 \times 10^{-5}$ & $5.40 \times 10^{-5}$ & $1.58 \times 10^{-4}$ \\
\hline 48 & $3 / 26$ & $-1.82 \times 10^{-5}$ & $5.18 \times 10^{-5}$ & $2.33 \times 10^{-4}$ \\
\hline 49 & $1 / 26$ & $-2.49 \times 10^{-5}$ & $3.85 \times 10^{-5}$ & $1.38 \times 10^{-4}$ \\
\hline \multicolumn{5}{|l|}{ Gross beta } \\
\hline 1 & $0 / 26$ & $-3.54 \times 10^{-5}$ & $2.23 \times 10^{-4}$ & $4.66 \times 10^{-4}$ \\
\hline 2 & $1 / 1$ & $5.81 \times 10^{-4}$ & $5.81 \times 10^{-4}$ & $5.81 \times 10^{-4}$ \\
\hline 3 & $4 / 26$ & $-6.18 \times 10^{-6}$ & $1.70 \times 10^{-4}$ & $4.59 \times 10^{-4}$ \\
\hline 4 & $1 / 1$ & $4.48 \times 10^{-4}$ & $4.48 \times 10^{-4}$ & $4.48 \times 10^{-4}$ \\
\hline 5 & $11 / 26$ & $4.81 \times 10^{-5}$ & $2.21 \times 10^{-4}$ & $7.40 \times 10^{-4}$ \\
\hline 6 & $1 / 25$ & $-1.14 \times 10^{-4}$ & $2.75 \times 10^{-4}$ & $6.36 \times 10^{-4}$ \\
\hline 7 & $7 / 26$ & $-1.07 \times 10^{-6}$ & $1.07 \times 10^{-4}$ & $2.55 \times 10^{-4}$ \\
\hline 8 & $0 / 2$ & $3.96 \times 10^{-7}$ & $1.25 \times 10^{-4}$ & $2.50 \times 10^{-4}$ \\
\hline 10 & $18 / 26$ & $2.54 \times 10^{-5}$ & $7.38 \times 10^{-4}$ & $1.88 \times 10^{-3}$ \\
\hline 11 & $0 / 1$ & $1.73 \times 10^{-3}$ & $1.73 \times 10^{-3}$ & $1.73 \times 10^{-3}$ \\
\hline 12 & $0 / 1$ & $1.45 \times 10^{-5}$ & $1.45 \times 10^{-5}$ & $1.45 \times 10^{-5}$ \\
\hline 13 & $2 / 26$ & $-4.29 \times 10^{-5}$ & $1.96 \times 10^{-4}$ & $4.14 \times 10^{-4}$ \\
\hline 14 & $13 / 26$ & $-5.11 \times 10^{-5}$ & $3.74 \times 10^{-4}$ & $9.25 \times 10^{-4}$ \\
\hline 15 & $0 / 1$ & $1.43 \times 10^{-3}$ & $1.43 \times 10^{-3}$ & $1.43 \times 10^{-3}$ \\
\hline 16 & $4 / 26$ & $-2.09 \times 10^{-5}$ & $1.19 \times 10^{-4}$ & $3.85 \times 10^{-4}$ \\
\hline 17 & $17 / 26$ & $8.55 \times 10^{-5}$ & $3.44 \times 10^{-4}$ & $1.26 \times 10^{-3}$ \\
\hline 18 & $2 / 26$ & $-5.14 \times 10^{-5}$ & $1.43 \times 10^{-4}$ & $3.48 \times 10^{-4}$ \\
\hline 19 & $3 / 26$ & $-1.27 \times 10^{-4}$ & $1.46 \times 10^{-4}$ & $2.96 \times 10^{-4}$ \\
\hline 20 & $2 / 26$ & $3.31 \times 10^{-5}$ & $1.70 \times 10^{-4}$ & $3.16 \times 10^{-4}$ \\
\hline
\end{tabular}




\section{Air Effluent Monitoring}

Table 4-3. Summary of gross alpha and gross beta in air effluent samples from monitored emission points at Building 251, 1999 (concluded).

\begin{tabular}{|c|c|c|c|c|}
\hline $\begin{array}{c}\text { Emission } \\
\text { point }^{(a)}\end{array}$ & $\begin{array}{c}\text { No. }>\mathrm{MDC}^{(\mathrm{b}, \mathrm{c}) /} \\
\text { total samples }\end{array}$ & $\begin{array}{c}\text { Minimum } \\
\left(\mathrm{Bq} / \mathrm{m}^{3}\right)\end{array}$ & $\begin{array}{l}\text { Median } \\
\left(\mathrm{Bq} / \mathrm{m}^{3}\right)\end{array}$ & $\begin{array}{c}\text { Maximum } \\
\left(\mathrm{Bq} / \mathrm{m}^{3}\right)\end{array}$ \\
\hline \multicolumn{5}{|l|}{ Gross beta } \\
\hline 21 & $19 / 26$ & $1.08 \times 10^{-4}$ & $3.64 \times 10^{-4}$ & $8.40 \times 10^{-4}$ \\
\hline 22 & $1 / 1$ & $1.04 \times 10^{-4}$ & $1.04 \times 10^{-4}$ & $1.04 \times 10^{-4}$ \\
\hline 23 & $5 / 26$ & $-3.01 \times 10^{-6}$ & $1.27 \times 10^{-4}$ & $3.15 \times 10^{-4}$ \\
\hline 24 & $1 / 26$ & $1.76 \times 10^{-5}$ & $2.04 \times 10^{-4}$ & $4.11 \times 10^{-4}$ \\
\hline 25 & $1 / 26$ & $-4.14 \times 10^{-5}$ & $1.29 \times 10^{-4}$ & $2.87 \times 10^{-4}$ \\
\hline 26 & $1 / 25$ & $5.48 \times 10^{-5}$ & $2.62 \times 10^{-4}$ & $5.66 \times 10^{-4}$ \\
\hline 27 & $0 / 1$ & $3.92 \times 10^{-4}$ & $3.92 \times 10^{-4}$ & $3.92 \times 10^{-4}$ \\
\hline 28 & $15 / 26$ & $-9.29 \times 10^{-5}$ & $4.42 \times 10^{-4}$ & $1.35 \times 10^{-3}$ \\
\hline 29 & $3 / 26$ & $4.40 \times 10^{-5}$ & $1.19 \times 10^{-4}$ & $2.94 \times 10^{-4}$ \\
\hline 30 & $12 / 26$ & $7.59 \times 10^{-5}$ & $3.29 \times 10^{-4}$ & $7.62 \times 10^{-4}$ \\
\hline 31 & $0 / 1$ & $5.77 \times 10^{-4}$ & $5.77 \times 10^{-4}$ & $5.77 \times 10^{-4}$ \\
\hline 32 & $0 / 1$ & $4.81 \times 10^{-5}$ & $4.81 \times 10^{-5}$ & $4.81 \times 10^{-5}$ \\
\hline 33 & $26 / 26$ & $2.56 \times 10^{-4}$ & $6.41 \times 10^{-4}$ & $1.82 \times 10^{-3}$ \\
\hline 34 & $14 / 26$ & $1.04 \times 10^{-4}$ & $2.87 \times 10^{-4}$ & $6.96 \times 10^{-4}$ \\
\hline 35 & $2 / 26$ & $8.84 \times 10^{-6}$ & $9.60 \times 10^{-5}$ & $2.35 \times 10^{-4}$ \\
\hline 36 & $1 / 1$ & $6.14 \times 10^{-4}$ & $6.14 \times 10^{-4}$ & $6.14 \times 10^{-4}$ \\
\hline 37 & $1 / 1$ & $4.11 \times 10^{-4}$ & $4.11 \times 10^{-4}$ & $4.11 \times 10^{-4}$ \\
\hline 38 & $0 / 1$ & $4.33 \times 10^{-5}$ & $4.33 \times 10^{-5}$ & $4.33 \times 10^{-5}$ \\
\hline 39 & $0 / 1$ & $-1.08 \times 10^{-6}$ & $-1.08 \times 10^{-6}$ & $-1.08 \times 10^{-6}$ \\
\hline 40 & $5 / 26$ & $1.45 \times 10^{-5}$ & $8.62 \times 10^{-5}$ & $2.16 \times 10^{-4}$ \\
\hline 41 & $0 / 1$ & $1.60 \times 10^{-4}$ & $1.60 \times 10^{-4}$ & $1.60 \times 10^{-4}$ \\
\hline 42 & $0 / 1$ & $7.77 \times 10^{-5}$ & $7.77 \times 10^{-5}$ & $7.77 \times 10^{-5}$ \\
\hline 43 & $3 / 26$ & $-1.97 \times 10^{-5}$ & $6.59 \times 10^{-5}$ & $2.98 \times 10^{-4}$ \\
\hline 44 & $12 / 26$ & $9.25 \times 10^{-5}$ & $2.61 \times 10^{-4}$ & $7.99 \times 10^{-4}$ \\
\hline 45 & $4 / 26$ & $1.95 \times 10^{-5}$ & $1.64 \times 10^{-4}$ & $5.48 \times 10^{-4}$ \\
\hline 46 & $25 / 26$ & $1.14 \times 10^{-4}$ & $3.38 \times 10^{-4}$ & $1.34 \times 10^{-3}$ \\
\hline 47 & $25 / 26$ & $1.21 \times 10^{-4}$ & $4.00 \times 10^{-4}$ & $1.51 \times 10^{-3}$ \\
\hline 48 & $15 / 26$ & $1.29 \times 10^{-5}$ & $3.11 \times 10^{-4}$ & $1.07 \times 10^{-3}$ \\
\hline 49 & $13 / 26$ & $2.41 \times 10^{-5}$ & $1.92 \times 10^{-4}$ & $1.87 \times 10^{-3}$ \\
\hline
\end{tabular}

a Results reported are from the simple, filter-type samplers (see text). The CAM samplers, located along with the simple filter samplers at stations $46-49$, are in place for facility alarm purposes rather than environmental reporting, so their results are not included here.

b $\mathrm{MDC}=$ Minimum detectable concentration. (See main volume, Chapter 14, Quality Assurance, for an explanation of MDC.)

c The sample numbers having only one or two samples were deactivated the beginning of 1999 (see main volume, Chapter 4). 
Table 4-4. Summary of gross alpha and gross beta in air effluent samples from monitored emission point at Building 292, 1999.

\begin{tabular}{|c|c|c|c|c|}
\hline $\begin{array}{c}\text { Emission } \\
\text { point }\end{array}$ & $\begin{array}{c}\text { No. }>\text { MDC(a,b)/ } \\
\text { total samples }\end{array}$ & $\begin{array}{c}\text { Minimum } \\
\mathbf{( B q / \mathbf { m } ^ { 3 } )}\end{array}$ & $\begin{array}{c}\text { Median } \\
\left(\mathbf{B q} / \mathbf{m}^{\mathbf{3}}\right)\end{array}$ & $\begin{array}{c}\text { Maximum } \\
\left(\mathbf{B q} / \mathbf{m}^{3}\right)\end{array}$ \\
\hline $\begin{array}{c}\text { Gross alpha } \\
1\end{array}$ & $1 / 14$ & $-8.99 \times 10^{-6}$ & $1.35 \times 10^{-6}$ & $7.10 \times 10^{-5}$ \\
\hline $\begin{array}{c}\text { Gross beta } \\
1\end{array}$ & $1 / 14$ & $-2.68 \times 10^{-5}$ & $2.28 \times 10^{-5}$ & $1.07 \times 10^{-4}$ \\
\hline
\end{tabular}

a $\mathrm{MDC}=$ Minimum detectable concentration. (See main volume, Chapter 14, Quality Assurance, for an explanation of MDC.)

b This sampler was removed in 1999 (see main volume, Chapter 4).

Table 4-5. Summary of tritium in air effluent samples from monitored emission points at Building 331, 1999.

\begin{tabular}{|c|c|c|c|c|}
\hline $\begin{array}{c}\text { Emission } \\
\text { point }^{(a)}\end{array}$ & $\begin{array}{l}\text { No. >MDC(b)/ } \\
\text { total samples }\end{array}$ & $\begin{array}{c}\text { Minimum } \\
\left(\mathrm{Bq} / \mathrm{m}^{3}\right)\end{array}$ & $\begin{array}{l}\text { Median } \\
\left(\mathrm{Bq} / \mathrm{m}^{3}\right)\end{array}$ & $\begin{array}{c}\text { Maximum } \\
\left(\mathrm{Bq} / \mathrm{m}^{3}\right)\end{array}$ \\
\hline \multicolumn{5}{|l|}{ HT } \\
\hline Stack 1 & $51 / 51$ & 6.77 & $1.40 \times 10^{1}$ & $2.96 \times 10^{2}$ \\
\hline Stack 2 & $51 / 51$ & $3.20 \times 10^{2}$ & $1.04 \times 10^{3}$ & $1.28 \times 10^{5}$ \\
\hline \multicolumn{5}{|l|}{ HTO } \\
\hline Stack 1 & $51 / 51$ & $2.58 \times 10^{1}$ & $9.51 \times 10^{2}$ & $2.33 \times 10^{3}$ \\
\hline Stack 2 & $51 / 51$ & $1.10 \times 10^{3}$ & $4.00 \times 10^{3}$ & $1.56 \times 10^{5}$ \\
\hline
\end{tabular}

a Results reported are from the molecular sieve samplers (see text). The ionization chamber samplers are in place for facility alarm purposes rather than environmental reporting, so their results are not included here.

b $\mathrm{MDC}=$ Minimum detectable concentration. (See main volume, Chapter 14, Quality Assurance, for an explanation of MDC.) 


\section{Air Effluent Monitoring}

Table 4-6. Summary of gross alpha and gross beta in air effluent samples from monitored emission points at Building 332, 1999.

\begin{tabular}{|c|c|c|c|c|}
\hline $\begin{array}{c}\text { Emission } \\
\text { point }^{(a)}\end{array}$ & $\begin{array}{l}\text { No. >MDC(b)/ } \\
\text { total samples }\end{array}$ & $\begin{array}{c}\text { Minimum } \\
\left(\mathrm{Bq} / \mathrm{m}^{3}\right)\end{array}$ & $\begin{array}{l}\text { Median } \\
\left(\mathrm{Bq} / \mathrm{m}^{3}\right)\end{array}$ & $\begin{array}{c}\text { Maximum } \\
\left(\mathrm{Bq} / \mathrm{m}^{3}\right)\end{array}$ \\
\hline \multicolumn{5}{|l|}{ Gross alpha } \\
\hline$S P-1 A$ & $0 / 52$ & $-3.45 \times 10^{-5}$ & $4.21 \times 10^{-6}$ & $1.52 \times 10^{-4}$ \\
\hline SP-1B & $0 / 52$ & $-5.14 \times 10^{-5}$ & $1.71 \times 10^{-6}$ & $8.07 \times 10^{-5}$ \\
\hline SP-2A & $0 / 52$ & $-3.81 \times 10^{-5}$ & $6.05 \times 10^{-6}$ & $9.32 \times 10^{-5}$ \\
\hline SP-2B & $0 / 52$ & $-3.77 \times 10^{-5}$ & $4.50 \times 10^{-6}$ & $1.20 \times 10^{-4}$ \\
\hline SP-3 & $0 / 52$ & $-5.33 \times 10^{-5}$ & $1.89 \times 10^{-6}$ & $1.14 \times 10^{-4}$ \\
\hline $\mathrm{SP}-4$ & $0 / 52$ & $-3.29 \times 10^{-5}$ & $-1.05 \times 10^{-6}$ & $8.84 \times 10^{-5}$ \\
\hline SP-5 & $0 / 52$ & $-3.47 \times 10^{-5}$ & $1.49 \times 10^{-6}$ & $1.04 \times 10^{-4}$ \\
\hline SP-6A & $0 / 52$ & $-5.07 \times 10^{-5}$ & $-1.05 \times 10^{-6}$ & $5.85 \times 10^{-5}$ \\
\hline SP-6B & $0 / 52$ & $-3.45 \times 10^{-5}$ & $1.31 \times 10^{-6}$ & $1.11 \times 10^{-4}$ \\
\hline SP-7A & $0 / 52$ & $-3.96 \times 10^{-5}$ & $2.01 \times 10^{-6}$ & $8.44 \times 10^{-5}$ \\
\hline SP-7B & $0 / 52$ & $-4.59 \times 10^{-5}$ & $3.87 \times 10^{-6}$ & $8.66 \times 10^{-5}$ \\
\hline SP-8 & $0 / 52$ & $-3.18 \times 10^{-5}$ & $2.34 \times 10^{-6}$ & $9.21 \times 10^{-5}$ \\
\hline SP-9 & $0 / 52$ & $-5.07 \times 10^{-5}$ & $3.26 \times 10^{-6}$ & $8.70 \times 10^{-5}$ \\
\hline SP-10 & $0 / 52$ & $-6.88 \times 10^{-5}$ & $2.44 \times 10^{-7}$ & $1.10 \times 10^{-4}$ \\
\hline SP-11 & $0 / 52$ & $-3.89 \times 10^{-5}$ & $-1.70 \times 10^{-5}$ & $1.04 \times 10^{-4}$ \\
\hline SP-12 & $0 / 52$ & $-1.03 \times 10^{-4}$ & $-2.90 \times 10^{-6}$ & $1.85 \times 10^{-4}$ \\
\hline \multicolumn{5}{|l|}{ Gross beta } \\
\hline SP-1A & $0 / 52$ & $-1.51 \times 10^{-4}$ & $3.50 \times 10^{-5}$ & $2.88 \times 10^{-4}$ \\
\hline SP-1B & $0 / 52$ & $-9.95 \times 10^{-5}$ & $1.04 \times 10^{-6}$ & $1.60 \times 10^{-4}$ \\
\hline SP-2A & $0 / 52$ & $-8.29 \times 10^{-5}$ & $2.80 \times 10^{-5}$ & $1.79 \times 10^{-4}$ \\
\hline SP-2B & $0 / 52$ & $-1.05 \times 10^{-4}$ & $3.00 \times 10^{-5}$ & $2.27 \times 10^{-4}$ \\
\hline SP-3 & $0 / 52$ & $-8.81 \times 10^{-5}$ & $1.38 \times 10^{-5}$ & $1.94 \times 10^{-4}$ \\
\hline $\mathrm{SP}-4$ & $0 / 52$ & $-1.33 \times 10^{-4}$ & $1.78 \times 10^{-5}$ & $2.67 \times 10^{-4}$ \\
\hline SP-5 & $0 / 52$ & $-1.16 \times 10^{-4}$ & $1.80 \times 10^{-5}$ & $2.33 \times 10^{-4}$ \\
\hline SP-6A & $2 / 52$ & $-1.38 \times 10^{-4}$ & $1.21 \times 10^{-5}$ & $1.59 \times 10^{-3}$ \\
\hline SP-6B & $0 / 52$ & $-1.38 \times 10^{-4}$ & $1.98 \times 10^{-5}$ & $1.73 \times 10^{-4}$ \\
\hline SP-7A & $0 / 52$ & $-1.39 \times 10^{-4}$ & $4.40 \times 10^{-6}$ & $2.20 \times 10^{-4}$ \\
\hline SP-7B & $0 / 52$ & $-1.15 \times 10^{-4}$ & $1.11 \times 10^{-5}$ & $1.38 \times 10^{-4}$ \\
\hline SP-8 & $0 / 52$ & $-1.71 \times 10^{-4}$ & $5.00 \times 10^{-7}$ & $1.50 \times 10^{-4}$ \\
\hline SP-9 & $0 / 52$ & $-1.52 \times 10^{-4}$ & $-3.35 \times 10^{-8}$ & $1.30 \times 10^{-4}$ \\
\hline SP-10 & $0 / 52$ & $-2.29 \times 10^{-4}$ & $-1.00 \times 10^{-6}$ & $3.01 \times 10^{-4}$ \\
\hline SP-11 & $0 / 52$ & $-1.55 \times 10^{-4}$ & $2.11 \times 10^{-5}$ & $1.81 \times 10^{-4}$ \\
\hline SP-12 & $0 / 52$ & $-3.25 \times 10^{-4}$ & $2.55 \times 10^{-6}$ & $3.10 \times 10^{-4}$ \\
\hline
\end{tabular}

a Results reported are from the simple, filter-type samplers (see text). The CAM samplers are in place for facility alarm purposes rather than environmental reporting, so their results are not included here.

b $\quad$ MDC = Minimum detectable concentration. (See main volume, Chapter 14, Quality Assurance, for an explanation of MDC.) 
Table 4-7. Summary of gross alpha and gross beta in air effluent samples from monitored emission points at Building 490, 1999.

\begin{tabular}{|c|c|c|c|c|}
\hline $\begin{array}{c}\text { Emission } \\
\text { point }\end{array}$ & $\begin{array}{c}\text { No. >MDC(a,b)/ } \\
\text { total samples }\end{array}$ & $\begin{array}{c}\text { Minimum } \\
\mathbf{( B q / \mathbf { m } ^ { 3 } )}\end{array}$ & $\begin{array}{c}\text { Median } \\
\left(\mathbf{B q} / \mathbf{m}^{3}\right)\end{array}$ & $\begin{array}{c}\text { Maximum } \\
\left(\mathbf{B q} / \mathbf{m}^{3}\right)\end{array}$ \\
\hline $\begin{array}{c}\text { Gross alpha } \\
1\end{array}$ & $0 / 15$ & $-1.51 \times 10^{-5}$ & $-1.12 \times 10^{-5}$ & $3.16 \times 10^{-5}$ \\
2 & $0 / 21$ & $-1.95 \times 10^{-4}$ & $-9.07 \times 10^{-6}$ & $3.85 \times 10^{-5}$ \\
3 & $0 / 15$ & $-1.38 \times 10^{-5}$ & $3.77 \times 10^{-6}$ & $2.16 \times 10^{-5}$ \\
4 & $0 / 20$ & $-9.25 \times 10^{-5}$ & $1.01 \times 10^{-6}$ & $4.63 \times 10^{-5}$ \\
\hline Gross beta & & & & $8.58 \times 10^{-5}$ \\
1 & $0 / 15$ & $-5.85 \times 10^{-5}$ & $3.49 \times 10^{-5}$ & $3.03 \times 10^{-4}$ \\
3 & $0 / 21$ & $-2.09 \times 10^{-5}$ & $2.78 \times 10^{-5}$ & $1.81 \times 10^{-4}$ \\
4 & $1 / 15$ & $-6.51 \times 10^{-5}$ & $2.74 \times 10^{-5}$ & $1.37 \times 10^{-4}$ \\
\hline
\end{tabular}

a $\mathrm{MDC}=$ Minimum detectable concentration. (See main volume, Chapter 14, Quality Assurance, for an explanation of MDC.)

b These sampling stations were deactivated in 1999 (see main volume, Chapter 4).

Table 4-8. Summary of gross alpha and gross beta in air effluent samples from monitored emission points at Building 491, 1999.

\begin{tabular}{|c|c|c|c|c|}
\hline $\begin{array}{c}\text { Emission } \\
\text { point }\end{array}$ & $\begin{array}{c}\text { No. >MDC(a)/ } \\
\text { total samples }\end{array}$ & $\begin{array}{c}\text { Minimum } \\
\mathbf{( B q / \mathbf { m } ^ { 3 } )}\end{array}$ & $\begin{array}{c}\text { Median } \\
\left(\mathbf{B q} / \mathbf{m}^{\mathbf{3}}\right)\end{array}$ & $\begin{array}{c}\text { Maximum } \\
\left(\mathbf{B q} / \mathbf{m}^{\mathbf{3}}\right)\end{array}$ \\
\hline $\begin{array}{c}\text { Gross alpha } \\
1\end{array}$ & $1 / 53$ & $-2.70 \times 10^{-5}$ & $1.76 \times 10^{-6}$ & $8.10 \times 10^{-5}$ \\
\hline $\begin{array}{c}\text { Gross beta } \\
1\end{array}$ & $3 / 53$ & $-3.03 \times 10^{-5}$ & $3.33 \times 10^{-5}$ & $9.10 \times 10^{-4}$ \\
\hline
\end{tabular}

a $\mathrm{MDC}=$ Minimum detectable concentration. (See main volume, Chapter 14, Quality Assurance, for an explanation of MDC.) 



\section{Air Surveillance Monitoring}

Paris E. Althouse

Paula J. Tate

\section{Air Surveillance Sampling}

Lawrence Livermore National Laboratory conducts air surveillance sampling using several different networks, each one representing a general location and type of analysis. There are separate networks for sampling radiological particulates and beryllium particulates at both the Livermore site and Site 300 as well as a low-volume radiological air surveillance sampling network and a tritium sampling network in Livermore. Four different collection media are employed: glass fibers for radiological particulates, cellulose for beryllium, Millipore AW-19 for low-volume radiological particulates, and silica gel for tritium. Table 5-1 in the main volume shows the organization of the networks; and sampling locations are shown in Figures 5-1, 5-2, and 5-3 in the main volume.

\section{Air Particulate Networks}

All particulate air samplers are positioned to ensure reasonable probability that any significant concentration of particulate effluents from LLNL operations will be detected. The air sampling locations are described in the locations database. Details for accessing the database are available in the Locations Database SOP Supplement EMP-QA-DM, Sample and Data Management.

The air particulate networks primarily use high-volume (hi-vol) air sampling units, which collect airborne particles on filters. These hi-vols use brushless motors and provide a readout of the total elapsed time and instantaneous and total flow rates.

Mass flow totalizers in the hi-vols are checked weekly using a portable field calibration unit. If a hi-vol stops running or the measured flow rate differs more than $10 \%$ from the expected flow rate, it is recalibrated using a calibration source traceable to the National Institute for Standards and Technology (NIST). During operation, the flow rate is maintained within 10\%, better than the Department of Energy (DOE) requirement of $\pm 20 \%$, of the nominal flow by using a mass flow controller that adjusts motor speed. All air particulate filters are changed each week at all locations. 


\section{Air Surveillance Monitoring}

After each particulate filter is removed from a sampler, it is identified by location, date on, date off, elapsed time, and flow rate; and it is given a sample identifier (a four-field code) that accompanies it throughout the analysis. Filters are then placed in glassine envelopes, and the sample information is recorded in a field tracking notebook. All air filters are processed at the end of each month according to their location and required analysis.

Radiological hi-vol samplers collect particulate at a continuous rate of $1 \mathrm{~m}^{3} / \mathrm{min}$ using glass-fiber filters. The low-volume samplers collect particulate at a continuous rate of $0.03 \mathrm{~m}^{3} / \mathrm{min}$ using Millipore AW-19 filters. Beryllium samplers collect particulate at a continuous rate of $0.43 \mathrm{~m}^{3} / \mathrm{min}$ using Whatman- 41 cellulose filters.

The details of air particulate sampling and sample change-out are described in Appendix B of the Environmental Monitoring Plan (Tate et al. 1999). Details of highvolume sampler flow calibration are also discussed in a procedure (ORAD EMP-AP-CA), and details of air sample analysis procedures are outlined in Hall and Edwards (1994a, b, and c).

\section{Air Particulate Radiological Networks}

The collection efficiency of particulate filters for radiological analysis should be greater than 95\% (Marshall and Stevens 1980). LLNL uses glass-fiber filters that have this level of efficiency and that maintain continuous flowrates. A total volume of approximately $10 \mathrm{ML}$ of air is sampled at each location each week for radiological analysis.

During 1999, two air particulate locations were moved due to nearby construction. Location COW was moved approximately 75 yards west, and location GOLF was moved approximately 60 yards east.

Data from each of the networks are grouped in categories representing the following areas: perimeter, upwind, downwind, diffuse source, and special interest locations.

The LLNL hi-vol radiological air particulate site perimeter network maintains six samplers at the perimeter (CAFE, COW, MESQ, MET, SALV, and VIS), two at diffuse source areas (B531 and CRED) shown the main volume Figure 5-1. The Livermore Valley network shown in the main volume Figure 5-2, consists of four locations in the least prevalent wind directions (CHUR, FCC, FIRE, and HOSP), considered to be upwind or background, and four samplers located in the most prevalent downwind 
directions (AMON, PATT, TANK, and ZON7). An additional sampler is located in an area of special interest at the Livermore Water Reclamation Plant (LWRP) because, in 1967 , there was a plutonium release to sewer that resulted in local soil contamination (see Results section in Chapter 5 of the main volume). The low-volume radiological air particulate network consists of two samplers located at HOSP and FCC.

The perimeter at Site 300 is monitored at seven locations (801E, ECP, EOBS, GOLF, NPS, WCP, and WOBS) as shown in the main volume Figure 5-3. Off-site monitoring at Site 300 occurs at two locations: PRIM (near the Site 300 boundary) and TFIR (in downtown City of Tracy).

Glass-fiber filters are collected from the field and placed in glassine bags. The glassine bags are gathered at the end of the month, and each filter is cut and separated to supply samples for the various analyses. Portions of all glass-fiber filters (except B531 and CRED) are sent in for gross alpha and gross beta analysis. These samples are sent to the analytical laboratory after a four-day delay to allow for decay of radon-thoron daughters. Gross alpha and gross beta activities are determined using a gas flow proportional counter.

The analytical laboratory uses thorium-230 and strontium-90 as calibration sources to determine alpha and beta counting efficiencies, respectively. Annual counting-efficiency measurements are made for each detector. Cross-checks using standards certified by the Environmental Protection Agency (EPA) are also completed periodically. Background and efficiency checks are completed daily, and a matrix and method blank are run with every batch of 20 samples. Records are kept of background and counting efficiency variations that occur in the counting equipment. The analytical laboratory reports the actual instrumentation values, including negative results, that arise when background measurements are higher than those for the filters.

As outlined in the Environmental Regulatory Guide for Radiological Effluent Monitoring and Environmental Surveillance (U.S. Department of Energy 1991), gross alpha and gross beta air-filter results are used only as trend indicators; specific radionuclide analysis is done for plutonium, uranium, and gamma emitters, depending on the location. All analytical results are reported as a measured concentration per volume of air, or at the minimum detection concentration (MDC) when no activity is detected. In all cases, the MDC is more than adequate for demonstrating compliance with the pertinent regulatory requirements for radionuclides that are present or may be present in the air sampled. Particle size distributions are not determined because the estimated effective dose 


\section{Air Surveillance Monitoring}

equivalent to the maximally exposed individual is well below the $0.01 \mathrm{mSv}$ ( $1 \mathrm{mrem}$ ) allowable limit (U.S. Department of Energy 1991).

For gamma scanning, one composite is created using another portion of all weekly glassfiber filters from each Livermore perimeter location. This composite, which represents filter portions from each of the Livermore perimeter locations (CAFE, COW, MESQ, MET, SALV, and VIS) for the month, is placed into a clear bag. This monthly composite is placed into a $214-\mathrm{cm}^{3}$ Teflon container and counted for gamma-emitting radionuclides using a low-background $\mathrm{Ge}(\mathrm{Li})$ detector.

Still other portions of the glass-fiber filters from each Livermore site perimeter location are analyzed for the presence of plutonium-239+240, uranium-235, and uranium-238 by LLNL's Chemistry and Materials Science Environmental Services Laboratory. The filters are placed in a muffled furnace to reduce organic content and then dissolved in a mixture of nitric acid and hydrochloric and/or hydrofluoric acids. Plutonium and uranium are separated by an ion-exchange process. Each separated element is purified further by ion exchange. Then the plutonium is electroplated onto a stainless steel disk and analyzed by alpha spectrometry, and the uranium solutions are analyzed by mass spectrometry.

The remaining glass fiber portions consisting of all Livermore Valley (CHUR, FCC, FIRE, HOSP, PATT, TANK, ZON7) and Site 300 off-site locations (PRIM and TFIR) are composited by location and analyzed for plutonium-239+240 as described above.

One composite is created using portions of all Site 300 perimeter locations (801E, ECP, EOBS, GOLF, NPS, WCP, and WOBS). This composite is gamma-scanned, and its entire contents analyzed for plutonium-239+240 by alpha spectrometry and for uranium-235 and uranium- 238 by mass spectrometry in the same manner as Livermore site perimeter locations.

Replicate radiological Quality Assurance (QA) samples are processed to confirm the precision of the analytical results obtained from the samplers. A duplicate QA sampler is operated for two months in parallel with the permanent sampler at a given site. In addition, a trip blank is collected during each route. The QA trip blanks and QA duplicates are processed in the same manner as the routine samples and analyzed for the same radiological parameters. 


\section{Air Particulate Beryllium}

Beryllium analysis requires an easily dissolvable filter with a low trace-metal background. Whatman-41 filters provide a balance between such requirements and particulate collection efficiency (Lindeken et al. 1963).

Beryllium is monitored at all Livermore perimeter locations (CAFE, COW, MESQ, MET, SALV, and VIS) as required by the Bay Area Air Quality Management District. Although there is no requirement to monitor beryllium at Site 300, as a best management practice, it is monitored at four locations (801E, EOBS, GOLF, and TFIR).

A total volume of approximately 4.3 ML of air is sampled at each location each week for beryllium analysis. The details of air particulate sampling and sample change-out are described in Appendix B of the Environmental Monitoring Plan (Tate et al. 1999). Details of high-volume sampler flow calibration are also discussed in a procedure (ORAD EMP-AP-CA).

The cellulose filters from each site are halved, with one portion saved on site for archiving, and the other composited into a monthly sample (one for each location) and sent out to the analytical laboratories for analysis. The off-site analytical digests the sample, using nitric acid, hydrochloric acid, and hydrogen perioxide during various heating and cooling phases. Care is taken to prevent the sample from boiling or baking dry. The resulting solution is diluted to $50 \mathrm{~mL}$ with blank water and the quantity of beryllium is determined by inductively coupled plasma-mass spectrometry.

Trip blanks are collected weekly from the Site 300 and Livermore networks, and split samples are chosen from the archived portions of the routine sample filters. LLNL sends them to the analytical laboratory as blind samples to help determine the accuracy of the analytical measurement.

\section{Air Tritium}

LLNL maintains 11 continuously operating, airborne tritium samplers on the Livermore site (main volume, Figure 5-1), six samplers in the Livermore Valley (main volume, Figure 5-2), and one near Site 300 (main volume, Figure 5-3). Four of the Livermore site locations (B292, B331, B514, and B624) monitor diffuse source emissions. The tritium sample locations are described in the locations database. The tritium samplers, operating at a flow rate of $700 \mathrm{~cm}^{3} / \mathrm{min}$, use a continuous vacuum pump to capture air moisture on silica gel contained in sampling flasks. These flasks are changed every two weeks, and the 


\section{Air Surveillance Monitoring}

samples are identified by location, date on, date off, elapsed sampling time, and flow rate. The flow rate is the average of the initial and final flow rates, which are measured biweekly with a rotameter that is calibrated once a year. LLNL is in the process of incorporating new flow meters to each of the sampling units. These flow meters provide the instantaneous flow and the minimum, maximum and total flow during the sample period.

Each sample is given a sample identifier that accompanies it through analysis. Two additional samplers are rotated among the locations at two-month intervals to provide duplicate QA samples. Details of the actual tritium sampling and a description of tritium sampler calibration can be found in Appendix B of the Environmental Monitoring Plan (Tate et al. 1999).

Once the samples are taken, water is separated from the silica gel by freeze-dried vacuum distillation, and the tritium concentration in the water is determined by liquidscintillation counting. Airborne tritium sample analysis is done by LLNL's Chemistry and Materials Science Environmental Services Laboratory. All analytical results are reported as a measured concentration per unit volume of air flow through the sampling medium. Details of the analytical procedure are described in Low-Level Tritium Analysis-Freeze Dry.

\section{Data}

Monthly summaries of gross alpha and gross beta data for the main site and Site 300 are presented in Tables 5-1, 5-2, 5-3, and 5-15. The activities shown in the tables displaying monthly medians are concentrations calculated from samples collected weekly.

Tables 5-4 and 5-16 present monthly gamma activity on air filters for the Livermore site perimeter and Site 300. Monthly plutonium data for each sampling location are shown in Tables 5-5, 5-6, 5-7, and 5-17. Monthly uranium data for the Livermore site perimeter and Site 300 are presented in Tables 5-8 and 5-18. The monthly low-volume gross alpha and gross beta data are presented in Tables 5-9 and 5-10. Biweekly tritium data for sampling locations in the Livermore Valley, Livermore site perimeter, and diffuse sources are shown in Tables 5-11, 5-12, and 5-13. Table 5-19 shows tritium-in-air data for Site 300. Tables 5-14 and 5-20 present monthly beryllium data for Livermore site perimeter and Site 300 sampling locations. The activities shown in the tables displaying monthly and biweekly data are measured concentrations and their associated $\pm 2 \sigma$ counting errors.

The data generally reflect historic data values for these analytes at these locations. A detailed discussion of these results is provided in the main volume of this report. 
Table 5-1. Monthly median activities for gross alpha and gross beta summarized from weekly data for the LLNL perimeter locations, 1999.

\begin{tabular}{|c|c|c|c|c|c|c|}
\hline \multirow{3}{*}{ Month } & \multicolumn{6}{|c|}{ Sampling location ${ }^{(a)}$} \\
\hline & CAFE & cow & MESQ & MET & SALV & VIS \\
\hline & \multicolumn{6}{|c|}{$\left(10^{-6} \mathrm{~Bq} / \mathrm{m}^{3}\right)$} \\
\hline \multicolumn{7}{|l|}{ Gross alpha } \\
\hline Jan & 4.01 & 32.9 & 39.0 & 19.7 & 35.2 & 28.2 \\
\hline Feb & 2.75 & 3.20 & 11.1 & 6.66 & 15.8 & 16.8 \\
\hline Mar & 40.2 & 42.0 & 45.0 & 46.6 & 50.9 & 42.0 \\
\hline Apr & 56.2 & 23.3 & 65.5 & 51.4 & 49.6 & 32.5 \\
\hline May & 18.7 & 9.42 & 21.1 & 23.3 & 16.1 & 24.8 \\
\hline Jun & 23.1 & 14.4 & 29.4 & 9.56 & 38.0 & 28.8 \\
\hline Jul & 28.2 & 43.7 & 43.3 & 50.3 & 45.1 & 29.1 \\
\hline Aug & 55.5 & 49.8 & 39.7 & 43.6 & 48.9 & 30.1 \\
\hline Sep & 78.6 & 60.5 & 88.8 & 61.1 & 55.1 & 72.5 \\
\hline Oct & 222 & 213 & 205 & 269 & 255 & 235 \\
\hline Nov & 112 & 105 & 106 & 121 & 147 & 126 \\
\hline Dec & 109 & 75.1 & 84.5 & 94.0 & 102 & 119 \\
\hline Annual median(b) & 48.5 & 43.7 & 53.5 & 50.7 & 50.7 & 41.2 \\
\hline$I_{Q} R^{(b, c)}$ & 65.7 & 60.1 & 60.4 & 52.6 & 59.6 & 61.2 \\
\hline Annual maximum ${ }^{(b)}$ & 328 & 321 & 321 & 385 & 364 & 377 \\
\hline \multicolumn{7}{|l|}{ Gross beta } \\
\hline Jan & 645 & 794 & 796 & 749 & 675 & 580 \\
\hline Feb & 238 & 198 & 202 & 214 & 168 & 189 \\
\hline Mar & 258 & 209 & 199 & 183 & 292 & 247 \\
\hline Apr & 257 & 327 & 238 & 269 & 396 & 237 \\
\hline May & 355 & 327 & 338 & 307 & 278 & 294 \\
\hline Jun & 232 & 148 & 228 & 150 & 188 & 223 \\
\hline Jul & 238 & 304 & 270 & 245 & 293 & 223 \\
\hline Aug & 391 & 318 & 364 & 298 & 358 & 311 \\
\hline Sep & 683 & 651 & 614 & 677 & 718 & 651 \\
\hline Oct & 1340 & 1230 & 1280 & 1270 & 1510 & 1430 \\
\hline Nov & 833 & 838 & 873 & 914 & 914 & 912 \\
\hline Dec & 623 & 668 & 688 & 696 & 653 & 694 \\
\hline Annual median(b) & 366 & 342 & 378 & 314 & 385 & 335 \\
\hline$I_{Q} R^{(b, c)}$ & 476 & 553 & 561 & 508 & 534 & 465 \\
\hline Annual maximum ${ }^{(b)}$ & 2140 & 2070 & 2220 & 2220 & 2660 & 2470 \\
\hline
\end{tabular}

a See Figure 5-1, main volume, for the description of sampling locations.

b Determined by data from the 52 -week period.

c $\quad \mathrm{IQR}=$ Interquartile range. 


\section{Air Surveillance Monitoring}

Table 5-2. Monthly median activities for gross alpha and beta summarized from weekly data for the Livermore Valley upwind locations, 1999.

\begin{tabular}{|c|c|c|c|c|}
\hline \multirow{3}{*}{ Month } & \multicolumn{4}{|c|}{ Sampling location ${ }^{(a)}$} \\
\hline & CHUR & FCC & FIRE & HOSP \\
\hline & \multicolumn{4}{|c|}{$\left(10^{-6} \mathrm{~Bq} / \mathrm{m}^{3}\right)$} \\
\hline \multicolumn{5}{|l|}{ Gross alpha } \\
\hline Jan & 54.0 & 7.61 & 10.4 & 33.2 \\
\hline Feb & 2.46 & -11.4 & 12.8 & 31.4 \\
\hline Mar & 47.2 & 47.5 & 24.3 & 41.8 \\
\hline Apr & 56.6 & 27.4 & 54.8 & 27.0 \\
\hline May & 36.7 & 26.7 & 16.3 & 11.7 \\
\hline Jun & 20.5 & 20.7 & 26.3 & 12.5 \\
\hline Jul & 50.0 & 18.1 & 33.3 & 35.7 \\
\hline Aug & 34.1 & 30.7 & 42.0 & 27.4 \\
\hline Sep & 62.9 & 73.6 & 68.1 & 63.1 \\
\hline Oct & 253 & 277 & 251 & 211 \\
\hline Nov & 125 & 132 & 79.6 & 106 \\
\hline Dec & 117 & 122 & 74.9 & 91.9 \\
\hline Annual median ${ }^{(b)}$ & 52.9 & 37.6 & 35.6 & 39.2 \\
\hline$I_{Q} R^{(b, c)}$ & 59.5 & 62.4 & 47.2 & 59.4 \\
\hline Annual maximum ${ }^{(\mathbf{b})}$ & 309 & 414 & 299 & 385 \\
\hline \multicolumn{5}{|l|}{ Gross beta } \\
\hline Jan & 657 & 679 & 637 & 646 \\
\hline Feb & 188 & 256 & 187 & 174 \\
\hline Mar & 227 & 234 & 223 & 260 \\
\hline Apr & 238 & 267 & 251 & 307 \\
\hline May & 310 & 275 & 224 & 268 \\
\hline Jun & 228 & 190 & 187 & 216 \\
\hline Jul & 260 & 239 & 238 & 257 \\
\hline Aug & 382 & 314 & 292 & 367 \\
\hline Sep & 718 & 670 & 646 & 660 \\
\hline Oct & 1260 & 1390 & 1290 & 1240 \\
\hline Nov & 890 & 971 & 788 & 759 \\
\hline Dec & 775 & 831 & 635 & 484 \\
\hline Annual median(b) & 270 & 275 & 253 & 289 \\
\hline $\operatorname{IQR}^{(\mathrm{b}, \mathrm{c})}$ & 478 & 485 & 472 & 447 \\
\hline Annual maximum ${ }^{(\mathbf{b})}$ & 2270 & 2500 & 1990 & 2110 \\
\hline
\end{tabular}

a See Figure 5-1, main volume, for the description of sampling locations.

b Determined by data from the 52 -week period.

c $\quad \mathrm{IQR}=$ Interquartile range. 
Table 5-3. Monthly median activities for gross alpha and beta summarized from weekly data for Livermore Valley downwind and special interest locations, 1999.

\begin{tabular}{|c|c|c|c|c|c|}
\hline \multirow{4}{*}{ Month } & \multicolumn{5}{|c|}{ Sampling location(a) } \\
\hline & \multicolumn{4}{|c|}{ Livermore Valley downwind } & \multirow{2}{*}{$\begin{array}{c}\text { Special interes } \\
\text { LWRP }\end{array}$} \\
\hline & AMON & PATT & TANK & ZON7 & \\
\hline & \multicolumn{5}{|c|}{$\left(10^{-6} \mathrm{~Bq} / \mathrm{m}^{3}\right)$} \\
\hline \multicolumn{6}{|l|}{ Gross alpha } \\
\hline Jan & 31.5 & 18.1 & 27.4 & 0.774 & 8.93 \\
\hline Feb & 3.08 & 3.91 & 25.6 & 23.5 & 20.7 \\
\hline Mar & 48.1 & 70.5 & 41.5 & 54.8 & 52.0 \\
\hline Apr & 53.7 & 41.4 & 30.1 & 59.2 & 51.4 \\
\hline May & 18.3 & 5.5 & 31.7 & 23.0 & 24.2 \\
\hline Jun & 21.6 & 25.6 & 28.7 & 37.2 & 28.2 \\
\hline Jul & 24.7 & 23.6 & 21.3 & 64.8 & 15.4 \\
\hline Aug & 49.0 & 54.8 & 36.2 & 48.1 & 49.4 \\
\hline Sep & 58.3 & 72.7 & 91.8 & 93.1 & 49.2 \\
\hline Oct & 138 & 231 & 238 & 275 & 235 \\
\hline Nov & 95.5 & 137 & 93.2 & 145 & 80.5 \\
\hline Dec & 88.4 & 97.3 & 80.7 & 101 & 133 \\
\hline Annual median (b) & 41 & 45 & 46 & 59 & 53 \\
\hline$I_{Q} R^{(b, c)}$ & 59 & 63 & 59 & 55 & 58 \\
\hline Annual maximum(b) & 298 & 492 & 366 & 370 & 353 \\
\hline \multicolumn{6}{|l|}{ Gross beta } \\
\hline Jan & 682 & 431 & 717 & 740 & 775 \\
\hline Feb & 136 & 199 & 200 & 218 & 167 \\
\hline Mar & 239 & 272 & 251 & 229 & 295 \\
\hline Apr & 316 & 266 & 265 & 336 & 318 \\
\hline May & 242 & 279 & 281 & 257 & 261 \\
\hline Jun & 208 & 205 & 217 & 178 & 203 \\
\hline Jul & 253 & 250 & 241 & 280 & 250 \\
\hline Aug & 323 & 414 & 414 & 361 & 330 \\
\hline Sep & 692 & 742 & 699 & 751 & 677 \\
\hline Oct & 1050 & 1280 & 1140 & 1260 & 1350 \\
\hline Nov & 699 & 823 & 821 & 931 & 905 \\
\hline Dec & 629 & 612 & 592 & 836 & 783 \\
\hline Annual median ${ }^{(b)}$ & 344 & 309 & 300 & 362 & 361 \\
\hline$I R^{(b, c)}$ & 466 & 520 & 484 & 522 & 544 \\
\hline Annual maximum(b) & 1870 & 2120 & 2120 & 2680 & 2450 \\
\hline
\end{tabular}

a See Figure 5-2, main volume for sampling locations.

b Determined by data from the 52-week period.

c $\quad I Q R=$ Interquartile range. 


\section{Air Surveillance Monitoring}

Table 5-4. Gamma activity in particulate air samples, Livermore site perimeter, 1999.(a)

\begin{tabular}{|c|c|c|c|c|c|c|c|}
\hline \multirow{3}{*}{ Month } & \multicolumn{7}{|c|}{ Radiological isotope } \\
\hline & \multirow{2}{*}{$\begin{array}{c}{ }^{7} \mathrm{Be} \\
\left(10^{-3} \mathrm{~Bq} / \mathrm{m}^{3}\right)\end{array}$} & ${ }^{137} \mathrm{Cs}$ & ${ }^{40} K$ & ${ }^{22} \mathrm{Na}$ & ${ }^{226} \mathrm{Ra}$ & ${ }^{228} \mathrm{Ra}$ & ${ }^{228} \mathrm{Th}$ \\
\hline & & \multicolumn{6}{|c|}{$\left(10^{-6} \mathrm{~Bq} / \mathrm{m}^{3}\right)$} \\
\hline Jan & $2.8 \pm 0.056$ & $<0.21^{(b)}$ & $17 \pm 19$ & $<0.21^{(b)}$ & $-0.033 \pm 3.5$ & $-0.46 \pm 2.0$ & $0.038 \pm 1.5$ \\
\hline Feb & $2.8 \pm 0.055$ & $<0.22^{(b)}$ & $26 \pm 22$ & $<0.23^{(b)}$ & $<-2.1^{(b)}$ & $-0.25 \pm 2.5$ & $-0.006 \pm 1.8$ \\
\hline Mar & $3.7 \pm 0.067$ & $<0.20^{(b)}$ & $14 \pm 19$ & $<0.25^{(b)}$ & $-0.62 \pm 2.1$ & $0.20 \pm 1.7$ & $-0.83 \pm 1.7$ \\
\hline Apr & $3.4 \pm 0.096$ & $<0.18^{(b)}$ & $25 \pm 16$ & $<0.23^{(b)}$ & $<-2.1^{(b)}$ & $0.68 \pm 1.8$ & $0.71 \pm 0.83$ \\
\hline May & $3.4 \pm 0.34$ & $0.059 \pm 0.85$ & $5.1 \pm 42$ & $0.38 \pm 1.3$ & $-0.61 \pm 2.1$ & $3.7 \pm 6.3$ & $0.38 \pm 1.1$ \\
\hline Jun & $2.9 \pm 0.29$ & $0.20 \pm 0.75$ & $40 \pm 49$ & $-0.12 \pm 1.3$ & $0.098 \pm 1.6$ & $1.0 \pm 2.5$ & $0.31 \pm 1.1$ \\
\hline Jul & $3.8 \pm 0.38$ & $0.13 \pm 0.63$ & $-22 \pm 43$ & $0.57 \pm 0.96$ & $0.58 \pm 1.4$ & $-0.037 \pm 3.3$ & $-2.8 \pm 2.2$ \\
\hline Aug & $2.7 \pm 0.28$ & $0.57 \pm 0.62$ & $11 \pm 45$ & $-0.39 \pm 1.1$ & $0.26 \pm 1.1$ & $-0.051 \pm 1.2$ & $1.5 \pm 1.2$ \\
\hline Sep & $4.9 \pm 0.49$ & $0.36 \pm 0.69$ & $23 \pm 48$ & $0.39 \pm 0.055$ & $-0.20 \pm 1.1$ & $0.21 \pm 3.6$ & $1.8 \pm 0.73$ \\
\hline Oct & $7.8 \pm 0.77$ & $0.51 \pm 0.70$ & $8.0 \pm 45$ & $0.33 \pm 0.98$ & $0.62 \pm 1.8$ & $3.0 \pm 2.4$ & $0.44 \pm 3.3$ \\
\hline Nov & $2.9 \pm 0.29$ & $0.51 \pm 0.54$ & $-3.2 \pm 45$ & $-0.29 \pm 0.93$ & $0.96 \pm 1.4$ & $0.90 \pm 2.1$ & $-1.7 \pm 0.72$ \\
\hline $\mathrm{Dec}$ & $3.7 \pm 0.38$ & $-0.017 \pm 0.57$ & $-32 \pm 46$ & $0.24 \pm 0.14$ & $0.012 \pm 1.2$ & $0.44 \pm 2.3$ & $0.27 \pm 2.7$ \\
\hline Median & 3.4 & 0.21 & 12 & 0.24 & -0.011 & 0.33 & 0.29 \\
\hline $\operatorname{IQR}^{(c)}$ & 0.85 & $-^{(d)}$ & 20 & $-^{(d)}$ & 0.95 & 0.98 & 0.72 \\
\hline Maximum & 7.8 & 0.57 & 40 & 0.57 & 0.96 & 3.7 & 1.8 \\
\hline $\mathrm{DCG}^{(\mathrm{e})}\left(\mathrm{Bq} / \mathrm{m}^{3}\right)$ & $1.5 \times 10^{3}$ & 15 & 33 & 37 & 0.037 & 0.11 & $1.5 \times 10^{-3}$ \\
\hline Percent of DCG ${ }^{(f)}$ & $2.3 \times 10^{-4}$ & $1.4 \times 10^{-6}$ & $3.7 \times 10^{-5}$ & $6.4 \times 10^{-7}$ & $2.6 \times 10^{-3(g)}$ & $3.0 \times 10^{-4}$ & 0.019 \\
\hline
\end{tabular}

Note: Radioactivities are reported as the measured concentration and either an uncertainty $( \pm 2 \sigma$ counting error) or as being less than or equal to the detection limit. If the concentration is less than the uncertainty or the detection limit, the result is considered to be a nondetection. See the main volume, Chapter 14, Quality Assurance.

a All Livermore site perimeter samples composited. See Figure 5-1, main volume, for sampling locations.

b Error not provided by analytical laboratory.

c $\quad I Q R=$ Interquartile range.

d No measure of dispersion calculated; see Chapter 14, Quality Assurance.

e $\quad$ DCG = Derived Concentration Guide (DOE Order 5400.5). See main volume, Chapter 13, Radiation Dose Assessment.

$f$ Percent of DCG calculated from the median concentration unless otherwise noted.

g The radium-226 percent of DCG was determined by using the maximum value because the median value was negative. 
Table 5-5. Plutonium-239+240 activity in air particulate samples, Livermore site perimeter, 1999.

\begin{tabular}{|c|c|c|c|c|c|c|}
\hline \multirow{3}{*}{ Month } & \multicolumn{6}{|c|}{ Sampling location(a) } \\
\hline & CAFE & cow & MESQ & MET & SALV & VIS \\
\hline & \multicolumn{6}{|c|}{$\left(10^{-9} \mathrm{~Bq} / \mathrm{m}^{3}\right)$} \\
\hline Jan & $4.37 \pm 4.29$ & $5.55 \pm 5.77$ & $4.11 \pm 4.44$ & $5.03 \pm 4.14$ & $4.85 \pm 4.74$ & $8.25 \pm 3.55$ \\
\hline Feb & $3.96 \pm 9.10$ & $1.35 \pm 5.70$ & $1.44 \pm 7.03$ & $1.57 \pm 5.25$ & $3.62 \pm 6.25$ & $6.03 \pm 7.14$ \\
\hline Mar & $3.37 \pm 6.55$ & $3.21 \pm 5.55$ & $3.60 \pm 7.70$ & $1.11 \pm 6.55$ & $-2.12 \pm 2.45$ & $2.48 \pm 4.88$ \\
\hline Apr & $0.077 \pm 3.77$ & $4.29 \pm 4.63$ & $4.29 \pm 4.63$ & $1.38 \pm 2.58$ & $1.47 \pm 2.73$ & $10.7 \pm 6.48$ \\
\hline May & $5.99 \pm 5.74$ & $4.48 \pm 5.62$ & $5.73 \pm 10.5$ & $1.08 \pm 4.59$ & $2.93 \pm 3.39$ & $38.9 \pm 13.2$ \\
\hline Jun & $25.1 \pm 10.8$ & $4.33 \pm 5.03$ & $0.807 \pm 3.41$ & $2.79 \pm 4.33$ & $0 \pm 0^{(b)}$ & $29.3 \pm 11.7$ \\
\hline Jul & $12.5 \pm 6.66$ & $7.88 \pm 5.59$ & $1.47 \pm 3.15$ & $0.888 \pm 1.77$ & $4.14 \pm 4.48$ & $15.4 \pm 8.03$ \\
\hline Aug & $3.85 \pm 5.03$ & $4.81 \pm 5.37$ & $-1.66 \pm 1.92$ & $3.85 \pm 4.44$ & $5.14 \pm 8.73$ & $15.4 \pm 9.73$ \\
\hline Sep & $10.4 \pm 7.40$ & $6.14 \pm 6.40$ & $5.00 \pm 6.25$ & $6.29 \pm 6.99$ & $18.0 \pm 9.32$ & $22.1 \pm 10.4$ \\
\hline Oct & $13.4 \pm 6.96$ & $28.7 \pm 10.4$ & $22.0 \pm 9.44$ & $7.99 \pm 6.36$ & $15.1 \pm 8.36$ & $22.5 \pm 9.21$ \\
\hline Nov & $5.03 \pm 5.00$ & $7.29 \pm 6.22$ & $4.18 \pm 4.18$ & $3.22 \pm 3.7$ & $2.83 \pm 5.59$ & $0.548 \pm 4.40$ \\
\hline Dec & $6.29 \pm 5.59$ & $3.96 \pm 4.96$ & $0 \pm 0^{(b)}$ & $2.25 \pm 3.18$ & $4.33 \pm 6.55$ & $-0.625 \pm 4.37$ \\
\hline Median & 5.51 & 4.64 & 3.85 & 2.52 & 3.88 & 13.0 \\
\hline $\operatorname{IQR}^{(c)}$ & 7.01 & 2.22 & 3.19 & 2.83 & 2.43 & 20.1 \\
\hline Percent of DCG(d) & $7.45 \times 10^{-4}$ & $6.28 \times 10^{-4}$ & $5.21 \times 10^{-4}$ & $3.41 \times 10^{-4}$ & $5.25 \times 10^{-4}$ & $1.76 \times 10^{-3}$ \\
\hline
\end{tabular}

Note: Radioactivities are reported as the measured concentration and either an uncertainty $( \pm 2 \sigma$ counting error) or as being less than or equal to the detection limit. If the concentration is less than the uncertainty or the detection limit, the result is considered to be a nondetection. See the main volume, Chapter 14, Quality Assurance.

a See Figure 5-1, main volume, for sampling locations.

b Actual reported value provided by analytical laboratory.

c IQR $=$ Interquartile range.

d $\quad$ DCG $=$ Derived Concentration Guide of $7.4 \times 10^{-4} \mathrm{~Bq} / \mathrm{m}^{3}$ for Pu-239 activity in air. Percent calculated from the median concentration 


\section{Air Surveillance Monitoring}

Table 5-6. Plutonium-239+240 activity in air particulate samples, Livermore Valley, 1999. (a)

\begin{tabular}{|c|c|c|c|c|c|}
\hline \multirow{3}{*}{ Month } & \multicolumn{4}{|c|}{ Livermore Valley downwind locations } & \multirow{2}{*}{$\begin{array}{c}\text { Special interes } \\
\text { LWRP }\end{array}$} \\
\hline & AMON & PATT & TANK & ZON7 & \\
\hline & \multicolumn{5}{|c|}{$\left(10^{-9} \mathrm{~Bq} / \mathrm{m}^{3}\right)$} \\
\hline Jan & $3.13 \pm 5.85$ & $2.76 \pm 5.37$ & $1.80 \pm 4.29$ & $-1.96 \pm 4.96$ & $0.988 \pm 4.18$ \\
\hline Feb & $-4.11 \pm 8.21$ & $-1.05 \pm 6.07$ & $2.29 \pm 5.44$ & $1.15 \pm 4.85$ & $5.96 \pm 6.44$ \\
\hline Mar & $-2.59 \pm 21.2$ & $5.00 \pm 5.37$ & $-0.570 \pm 4.00$ & $0.810 \pm 6.70$ & $4.37 \pm 5.85$ \\
\hline Apr & $9.21 \pm 7.25$ & $0.614 \pm 2.59$ & $0.138 \pm 1.93$ & $1.41 \pm 3.02$ & $3.43 \pm 3.40$ \\
\hline May & $2.33 \pm 4.33$ & $1.54 \pm 2.87$ & $2.22 \pm 4.11$ & $1.99 \pm 3.37$ & $7.36 \pm 5.85$ \\
\hline Jun & $4.55 \pm 6.11$ & $6.70 \pm 5.99$ & $3.45 \pm 4.00$ & $0.851 \pm 3.61$ & $5.00 \pm 5.99$ \\
\hline Jul & $4.03 \pm 3.92$ & $2.56 \pm 2.96$ & $0.685 \pm 2.89$ & $3.26 \pm 3.74$ & $16.0 \pm 7.73$ \\
\hline Aug & $2.04 \pm 3.44$ & $2.60 \pm 3.59$ & $2.88 \pm 5.33$ & $5.14 \pm 5.55$ & $4.59 \pm 5.48$ \\
\hline Sep & $5.14 \pm 5.70$ & $12.2 \pm 7.73$ & $6.85 \pm 6.11$ & $10.5 \pm 7.33$ & $4.29 \pm 6.14$ \\
\hline Oct & $16.1 \pm 8.47$ & $25.2 \pm 10.58$ & $8.66 \pm 6.59$ & $23.1 \pm 9.25$ & $21.1 \pm 9.21$ \\
\hline Nov & $1.60 \pm 4.77$ & $0.651 \pm 2.42$ & $1.61 \pm 4.81$ & $2.05 \pm 2.90$ & $5.11 \pm 6.11$ \\
\hline Dec & $8.95 \pm 7.96$ & $5.62 \pm 5.03$ & $1.15 \pm 2.30$ & $1.74 \pm 3.23$ & $8.29 \pm 7.03$ \\
\hline Median & 3.58 & 2.68 & 2.01 & 1.87 & 5.06 \\
\hline IQR $(\mathbf{b})$ & 4.16 & 4.57 & 1.99 & 2.65 & 3.24 \\
\hline Percent of DCG(c) & $4.84 \times 10^{-4}$ & $3.62 \times 10^{-4}$ & $2.71 \times 10^{-4}$ & $2.52 \times 10^{-4}$ & $6.83 \times 10^{-4}$ \\
\hline
\end{tabular}


Table 5-6. Plutonium-239+240 activity in air particulate samples, Livermore Valley, 1999(a) (concluded).

\begin{tabular}{|c|c|c|c|c|}
\hline \multirow{3}{*}{ Month } & \multicolumn{4}{|c|}{ Livermore Valley upwind } \\
\hline & CHUR & FCC & FIRE & HOSP \\
\hline & \multicolumn{4}{|c|}{$\left(10^{-9} \mathrm{~Bq} / \mathrm{m}^{3}\right)$} \\
\hline Jan & $-1.58 \pm 4.70$ & $0.022 \pm 2.66$ & $0.040 \pm 3.37$ & $4.63 \pm 5.48$ \\
\hline Feb & $1.24 \pm 3.25$ & $-2.29 \pm 5.18$ & $-0.884 \pm 5.03$ & $-1.78 \pm 3.39$ \\
\hline Mar & $6.22 \pm 6.48$ & $-0.555 \pm 3.89$ & $-0.370 \pm 4.66$ & $1.14 \pm 2.28$ \\
\hline Apr & $1.49 \pm 3.19$ & $-0.437 \pm 3.04$ & $1.69 \pm 2.86$ & $2.06 \pm 4.4$ \\
\hline May & $0.304 \pm 2.99$ & $0.932 \pm 4.00$ & $2.66 \pm 4.11$ & $0.178 \pm 2.48$ \\
\hline Jun & $1.23 \pm 2.46$ & $-3.27 \pm 2.48$ & $2.93 \pm 4.55$ & $-1.43 \pm 2.02$ \\
\hline Jul & $-0.862 \pm 3.38$ & $1.83 \pm 3.09$ & $2.07 \pm 3.50$ & $0.07 \pm 3.44$ \\
\hline Aug & $23.8 \pm 11.0$ & $0.184 \pm 2.56$ & $2.13 \pm 4.92$ & $-0.803 \pm 1.14$ \\
\hline Sep & $6.44 \pm 5.66$ & $2.44 \pm 4.11$ & $6.59 \pm 7.51$ & $0.176 \pm 2.46$ \\
\hline Oct & $21.7 \pm 8.88$ & $11.1 \pm 7.14$ & $11.1 \pm 6.88$ & $6.40 \pm 5.11$ \\
\hline Nov & $4.29 \pm 4.29$ & $-1.79 \pm 3.42$ & $9.44 \pm 7.40$ & $0.855 \pm 3.61$ \\
\hline $\mathrm{Dec}$ & $0.555 \pm 4.48$ & $3.92 \pm 4.92$ & $0.958 \pm 4.11$ & $0.349 \pm 3.43$ \\
\hline Median & 1.37 & 0.103 & 2.10 & 0.264 \\
\hline IQR $(\mathbf{b})$ & 5.78 & 2.85 & 3.12 & 1.52 \\
\hline Percent of DCG(c) & $1.84 \times 10^{-4}$ & $1.39 \times 10^{-5}$ & $2.84 \times 10^{-4}$ & $3.56 \times 10^{-5}$ \\
\hline
\end{tabular}

Note: Radioactivities are reported as the measured concentration and either an uncertainty ( $\pm 2 \sigma$ counting error) or as being less than or equal to the detection limit. If the concentration is less than the uncertainty or the detection limit, the result is considered to be a nondetection. See the main volume, Chapter 14, Quality Assurance.

a See Figure 5-2, main volume for sampling locations.

b $\quad I Q R=$ Interquartile range.

c $\quad$ DCG = Derived Concentration Guide of $7.4 \times 10^{-4} \mathrm{~Bq} / \mathrm{m}^{3}$ for Pu-239 activity in air. Percent calculated from the median concentration. 


\section{Air Surveillance Monitoring}

Table 5-7. Plutonium-239+240 activity in air particulate samples, diffuse sources, 1999.

\begin{tabular}{|c|c|c|}
\hline \multirow{3}{*}{ Month } & \multicolumn{2}{|c|}{ Sampling location ${ }^{(a)}$} \\
\hline & B531 & CRED \\
\hline & \multicolumn{2}{|c|}{$\left(10^{-9} \mathrm{~Bq} / \mathrm{m}^{3}\right)$} \\
\hline Jan & $9.25 \pm 6.81$ & $5.48 \pm 6.66$ \\
\hline Feb & $10.3 \pm 8.21$ & $2.04 \pm 4.37$ \\
\hline Mar & $5.99 \pm 5.74$ & $4.77 \pm 5.11$ \\
\hline Apr & $32.0 \pm 10.9$ & $2.79 \pm 4.33$ \\
\hline May & $94.0 \pm 20.8$ & $5.77 \pm 5.51$ \\
\hline Jun & $57.0 \pm 16.9$ & $8.84 \pm 6.62$ \\
\hline Jul & $69.9 \pm 16.2$ & $7.25 \pm 6.29$ \\
\hline Aug & $81.4 \pm 19.3$ & $8.92 \pm 6.33$ \\
\hline Sep & $21.8 \pm 12.1$ & $33.5 \pm 13.2$ \\
\hline Oct & $53.7 \pm 14.7$ & $25.9 \pm 9.81$ \\
\hline Nov & $6.33 \pm 6.03$ & $3.96 \pm 5.33$ \\
\hline Dec & $12.0 \pm 8.55$ & $6.33 \pm 6.03$ \\
\hline Median & 26.9 & 6.05 \\
\hline $\operatorname{IQR}^{(\mathbf{b})}$ & 50.2 & 4.29 \\
\hline Percent of DCG(c) & $3.64 \times 10^{-3}$ & $8.18 \times 10^{-4}$ \\
\hline
\end{tabular}

Note: Radioactivities are reported as the measured concentration and either an uncertainty $( \pm 2 \sigma$ counting error) or as being less than or equal to the detection limit. If the concentration is less than the uncertainty or the detection limit, the result is considered to be a nondetection. See the main volume, Chapter 14, Quality Assurance.

a See Figure 5-1, main volume, for sampling locations.

b $\quad$ IQR $=$ Interquartile range.

c $\quad$ DCG = Derived Concentration Guide of $7.4 \times 10^{-4} \mathrm{~Bq} / \mathrm{m}^{3}$ for Pu-239 activity in air. Percent calculated from the median concentration. 
Table 5-8. Uranium mass concentration in air particulate samples, Livermore site perimeter 1999.(a)

\begin{tabular}{|c|c|c|c|c|}
\hline Location & Month & $\begin{array}{l}\text { Uranium-235 } \\
\left(10^{-7} \mu \mathrm{g} / \mathrm{m}^{3}\right)\end{array}$ & $\begin{array}{c}\text { Uranium-238(c) } \\
\left(10^{-5} \mu \mathrm{g} / \mathrm{m}^{3}\right)\end{array}$ & $\begin{array}{c}\text { Uranium-235/238 } \\
\left(10^{-3}\right)\end{array}$ \\
\hline \multirow[t]{12}{*}{ CAFE } & Jan & $3.08 \pm 0.108$ & $4.38 \pm 0.151$ & 7.05 \\
\hline & Feb & $1.46 \pm 0.159$ & $2.03 \pm 0.191$ & 7.18 \\
\hline & Mar & $3.65 \pm 0.107$ & $5.11 \pm 0.126$ & 7.14 \\
\hline & Apr & $3.38 \pm 0.230$ & $5.01 \pm 0.064$ & 6.75 \\
\hline & May & $5.22 \pm 0.373$ & $8.04 \pm 0.704$ & 6.49 \\
\hline & Jun & $7.08 \pm 0.362$ & $10.6 \pm 0.695$ & 6.68 \\
\hline & Jul & $6.13 \pm 0.760$ & $9.38 \pm 0.943$ & 6.53 \\
\hline & Aug & $4.93 \pm 0.482$ & $8.34 \pm 0.761$ & 5.91 \\
\hline & Sep & $5.37 \pm 0.776$ & $17.9 \pm 0.883$ & 3.00 \\
\hline & Oct & $11.3 \pm 1.51$ & $16.6 \pm 2.17$ & 6.79 \\
\hline & Nov & $2.72 \pm 1.40$ & $4.06 \pm 1.70$ & 6.71 \\
\hline & Dec & $2.83 \pm 0.839$ & $4.31 \pm 1.02$ & 6.55 \\
\hline Median & & 4.29 & 6.57 & 6.70 \\
\hline $\operatorname{IQR}^{(d)}$ & & 2.54 & 5.32 & 0.335 \\
\hline Maximum & & 11.3 & 17.9 & $N A^{(e)}$ \\
\hline Percent of DCG(f) & & $9.13 \times 10^{-4}$ & $2.19 \times 10^{-2}$ & NA \\
\hline \multirow[t]{12}{*}{ cow } & Jan & $2.51 \pm 0.130$ & $3.48 \pm 0.177$ & 7.20 \\
\hline & Feb & $1.39 \pm 0.157$ & $2.09 \pm 0.190$ & 6.67 \\
\hline & Mar & $1.97 \pm 0.143$ & $2.99 \pm 0.206$ & 6.61 \\
\hline & Apr & $5.30 \pm 0.438$ & $7.48 \pm 0.496$ & 7.09 \\
\hline & May & $5.68 \pm 0.320$ & $8.30 \pm 0.671$ & 6.84 \\
\hline & Jun & $4.61 \pm 0.413$ & $6.79 \pm 0.758$ & 6.79 \\
\hline & Jul & $6.05 \pm 0.504$ & $8.99 \pm 0.875$ & 6.73 \\
\hline & Aug & $6.29 \pm 0.436$ & $10.3 \pm 0.785$ & 6.09 \\
\hline & Sep & $7.87 \pm 0.710$ & $11.2 \pm 1.14$ & 7.03 \\
\hline & Oct & $10.9 \pm 1.51$ & $15.7 \pm 2.07$ & 6.92 \\
\hline & Nov & $4.17 \pm 1.43$ & $5.78 \pm 1.73$ & 7.21 \\
\hline & Dec & $1.46 \pm 1.16$ & $1.92 \pm 1.45$ & 7.63 \\
\hline $\begin{array}{l}\text { Median } \\
\text { IQR }^{(d)}\end{array}$ & & $\begin{array}{l}4.96 \\
3.74\end{array}$ & $\begin{array}{l}7.13 \\
5.96\end{array}$ & $\begin{array}{l}6.88 \\
0.363\end{array}$ \\
\hline Maximum & & 10.9 & 15.7 & NA \\
\hline Percent of DCG(f) & & $1.05 \times 10^{-3}$ & $2.38 \times 10^{-2}$ & NA \\
\hline
\end{tabular}




\section{Air Surveillance Monitoring}

Table 5-8. Uranium mass concentration in air particulate samples, Livermore site perimeter, 1999 (continued). (a)

\begin{tabular}{|c|c|c|c|c|}
\hline Location & Month & $\begin{array}{c}\text { Uranium-235(b) } \\
\left(10^{-7} \mu \mathrm{g} / \mathrm{m}^{3}\right)\end{array}$ & $\begin{array}{c}\text { Uranium-238(c) } \\
\left(10^{-5} \mu \mathrm{g} / \mathrm{m}^{3}\right)\end{array}$ & $\begin{array}{c}\text { Uranium-235/238 } \\
\left(10^{-3}\right)\end{array}$ \\
\hline \multirow[t]{12}{*}{ MESQ } & Jan & $1.39 \pm 0.12$ & $1.97 \pm 0.154$ & 7.08 \\
\hline & Feb & $0.69 \pm 0.13$ & $0.99 \pm 0.164$ & 7.00 \\
\hline & Mar & $3.50 \pm 0.11$ & $4.87 \pm 0.129$ & 7.17 \\
\hline & Apr & $4.97 \pm 0.35$ & $6.81 \pm 0.352$ & 7.29 \\
\hline & May & $4.31 \pm 0.29$ & $6.82 \pm 0.637$ & 6.32 \\
\hline & Jun & $4.60 \pm 0.35$ & $6.85 \pm 0.551$ & 6.72 \\
\hline & Jul & $4.42 \pm 0.34$ & $6.68 \pm 0.538$ & 6.63 \\
\hline & Aug & $3.45 \pm 0.33$ & $6.18 \pm 0.748$ & 5.58 \\
\hline & Sep & $5.13 \pm 0.72$ & $7.27 \pm 0.849$ & 7.06 \\
\hline & Oct & $8.88 \pm 1.43$ & $12.4 \pm 1.84$ & 7.14 \\
\hline & Nov & $1.34 \pm 1.38$ & $2.32 \pm 1.69$ & 5.78 \\
\hline & Dec & $1.70 \pm 1.25$ & $2.45 \pm 1.35$ & 6.94 \\
\hline Median & & 3.90 & 6.43 & 6.97 \\
\hline $\operatorname{IQR}^{(\mathrm{d})}$ & & 3.07 & 4.41 & 0.549 \\
\hline Maximum & & 8.88 & 12.4 & NA \\
\hline Percent of DCG(f) & & $8.30 \times 10^{-4}$ & $2.14 \times 10^{-2}$ & NA \\
\hline \multirow[t]{12}{*}{ MET } & Jan & $1.80 \pm 0.115$ & $2.55 \pm 0.159$ & 7.03 \\
\hline & Feb & $1.86 \pm 0.143$ & $2.76 \pm 0.169$ & 6.73 \\
\hline & Mar & $2.56 \pm 0.098$ & $3.64 \pm 0.126$ & 7.03 \\
\hline & Apr & $2.88 \pm 0.292$ & $3.95 \pm 0.278$ & 7.30 \\
\hline & May & $3.69 \pm 0.285$ & $5.74 \pm 0.715$ & 6.43 \\
\hline & Jun & $3.61 \pm 0.293$ & $5.31 \pm 0.658$ & 6.79 \\
\hline & Jul & $2.00 \pm 0.403$ & $3.00 \pm 0.480$ & 6.67 \\
\hline & Aug & $2.77 \pm 0.429$ & $4.63 \pm 0.923$ & 5.98 \\
\hline & Sep & $2.93 \pm 0.867$ & $3.96 \pm 1.03$ & 7.39 \\
\hline & Oct & $9.32 \pm 1.63$ & $13.5 \pm 1.83$ & 6.90 \\
\hline & Nov & $0.52 \pm 1.51$ & $0.94 \pm 2.02$ & 5.56 \\
\hline & $\mathrm{Dec}$ & $1.75 \pm 0.995$ & $2.51 \pm 1.11$ & 7.00 \\
\hline Median & & 2.66 & 3.80 & 6.85 \\
\hline $\operatorname{IQR}^{(\mathrm{d})}$ & & 1.26 & 2.09 & 0.419 \\
\hline Maximum & & 9.32 & 13.5 & NA \\
\hline Percent of DCG(f) & & $5.67 \times 10^{-4}$ & $1.27 \times 10^{-2}$ & NA \\
\hline
\end{tabular}


Table 5-8. Uranium mass concentration in air particulate samples, Livermore site perimeter, 1999 (concluded). (a)

\begin{tabular}{|c|c|c|c|c|}
\hline Location & Month & $\begin{array}{c}\text { Uranium-235(b) } \\
\left(10^{-7} \mu \mathrm{g} / \mathrm{m}^{3}\right)\end{array}$ & $\begin{array}{c}\text { Uranium-238(c) } \\
\left(10^{-5} \mu \mathrm{g} / \mathrm{m}^{3}\right)\end{array}$ & $\begin{array}{c}\text { Uranium-235/238 } \\
\left(10^{-3}\right)\end{array}$ \\
\hline SALV & $\begin{array}{l}\text { Jan } \\
\text { Feb } \\
\text { Mar } \\
\text { Apr } \\
\text { May } \\
\text { Jun } \\
\text { Jul } \\
\text { Aug } \\
\text { Sep } \\
\text { Oct } \\
\text { Nov } \\
\text { Dec }\end{array}$ & $\begin{aligned} 2.00 & \pm 0.106 \\
1.17 & \pm 0.149 \\
0.889 & \pm 0.110 \\
4.36 & \pm 0.340 \\
3.41 & \pm 0.356 \\
2.52 & \pm 0.343 \\
2.19 & \pm 0.372 \\
2.48 & \pm 0.471 \\
3.42 & \pm 0.905 \\
10.4 & \pm 1.39 \\
1.45 & \pm 1.27 \\
1.90 & \pm 0.865\end{aligned}$ & $\begin{array}{l}2.77 \pm 0.145 \\
1.73 \pm 0.184 \\
1.23 \pm 0.131 \\
6.12 \pm 0.388 \\
5.50 \pm 0.609 \\
3.94 \pm 0.706 \\
3.61 \pm 0.582 \\
4.46 \pm 0.693 \\
4.81 \pm 1.00 \\
14.8 \pm 2.04 \\
2.37 \pm 1.74 \\
2.67 \pm 1.26\end{array}$ & $\begin{array}{l}7.21 \\
6.76 \\
7.20 \\
7.13 \\
6.20 \\
6.41 \\
6.07 \\
5.57 \\
7.11 \\
7.00 \\
6.14 \\
7.12\end{array}$ \\
\hline $\begin{array}{l}\text { Median } \\
\text { IQR }^{(\mathrm{d})} \\
\text { Maximum } \\
\text { Percent of } \mathrm{DCG}^{(\mathrm{f})}\end{array}$ & & $\begin{array}{c}2.34 \\
1.62 \\
10.3 \\
4.97 \times 10^{-4} \\
\end{array}$ & $\begin{array}{c}3.77 \\
2.39 \\
14.8 \\
1.26 \times 10^{-2} \\
\end{array}$ & $\begin{array}{l}6.88 \\
0.931 \\
\text { NA } \\
\text { NA }\end{array}$ \\
\hline VIS & $\begin{array}{l}\text { Jan } \\
\text { Feb } \\
\text { Mar } \\
\text { Apr } \\
\text { May } \\
\text { Jun } \\
\text { Jul } \\
\text { Aug } \\
\text { Sep } \\
\text { Oct } \\
\text { Nov } \\
\text { Dec }\end{array}$ & $\begin{array}{l}2.27 \pm 0.121 \\
1.40 \pm 0.147 \\
2.40 \pm 0.105 \\
3.33 \pm 0.304 \\
9.26 \pm 0.302 \\
5.81 \pm 0.306 \\
3.17 \pm 0.454 \\
4.09 \pm 0.473 \\
4.86 \pm 0.910 \\
11.4 \pm 1.31 \\
2.50 \pm 1.57 \\
1.78 \pm 1.31\end{array}$ & $\begin{array}{l}3.23 \pm 0.166 \\
2.04 \pm 0.180 \\
3.40 \pm 0.131 \\
4.65 \pm 0.275 \\
13.7 \pm 0.878 \\
6.46 \pm 0.594 \\
4.86 \pm 0.623 \\
6.97 \pm 0.756 \\
17.1 \pm 1.39 \\
16.5 \pm 1.81 \\
3.74 \pm 2.11 \\
2.60 \pm 1.38\end{array}$ & $\begin{array}{l}7.04 \\
6.83 \\
7.06 \\
7.16 \\
6.75 \\
8.99 \\
6.53 \\
5.87 \\
2.84 \\
6.92 \\
6.67 \\
6.86\end{array}$ \\
\hline $\begin{array}{l}\text { Median } \\
\text { IQR } \\
\text { Maximum } \\
\text { Percent of } \mathrm{DCG}^{(\mathrm{f})}\end{array}$ & & $\begin{array}{c}3.25 \\
2.72 \\
11.4 \\
6.92 \times 10^{-4}\end{array}$ & $\begin{array}{c}4.76 \\
5.3 \\
17.1 \\
1.59 \times 10^{-2}\end{array}$ & $\begin{array}{l}6.85 \\
0.410 \\
\text { NA } \\
\text { NA }\end{array}$ \\
\hline
\end{tabular}

Note: Radioactivities are reported as the measured concentration and either an uncertainty ( $\pm 2 \sigma$ counting error) or as being less than or equal to the detection limit. If the concentration is less than the uncertainty or the detection limit, the result is considered to be a nondetection. See the main volume, Chapter 14, Quality Assurance.

a See Figure 5-1, main volume, for sampling locations.

b Uranium-235 activities in $\mathrm{Bq} / \mathrm{m}^{3}$ can be determined by dividing the weight in $\mu \mathrm{g} / \mathrm{m}^{3}$ by 12.5 .

c Uranium-238 activities in $\mathrm{Bq} / \mathrm{m}^{3}$ can be determined by dividing the weight in $\mu \mathrm{g} / \mathrm{m}^{3}$ by 80.3 .

d $\quad$ IQR $=$ Interquartile range.

e $N A=$ Not applicable.

f $\quad$ DCG = Derived Concentration Guide for activity in air of $0.3 \mu \mathrm{g} / \mathrm{m}^{3}$ for ${ }^{238} \mathrm{U}$ and $0.047 \mu \mathrm{g} / \mathrm{m}^{3}$ for ${ }^{235} \mathrm{U}$. Percent calculated from the median concentration. 


\section{Air Surveillance Monitoring}

Table 5-9. Monthly median activities for gross alpha summarized from weekly data from low-volume air samplers, 1999. (a)

\begin{tabular}{|c|c|c|}
\hline \multirow{3}{*}{ Month } & \multicolumn{2}{|c|}{ Livermore Valley upwind } \\
\hline & FCC & HOSP \\
\hline & \multicolumn{2}{|c|}{$\left(10^{-6} \mathrm{~Bq} / \mathrm{m}^{3}\right)$} \\
\hline Jan & -2.52 & 44.0 \\
\hline Feb & 16.3 & 66.2 \\
\hline Mar & 29.0 & 37.4 \\
\hline Apr & 17.2 & 35.0 \\
\hline May & 50.5 & 34.5 \\
\hline Jun & 29.3 & 33.3 \\
\hline Jul & 11.8 & -5.88 \\
\hline Aug & 64.8 & 22.8 \\
\hline Sep & 73.1 & 114 \\
\hline Oct & 285 & 144 \\
\hline Nov & 89.2 & 126 \\
\hline Dec & 101 & 50.7 \\
\hline Annual median ${ }^{(\mathbf{b})}$ & 56.4 & 41.8 \\
\hline$I Q R^{(b, c)}$ & 82.9 & 73.3 \\
\hline Annual maximum ${ }^{(\mathbf{b})}$ & 466 & 271 \\
\hline
\end{tabular}

a See Figure 5-2, main volume, for sampling locations.

b Determined by data from the 52-week period.

c $\quad I Q R=$ Interquartile range. 
Table 5-10. Monthly median activities for gross beta summarized from weekly data from low-volume air samplers, 1999.(a)

\begin{tabular}{|c|c|c|}
\hline \multirow{3}{*}{ Month } & \multicolumn{2}{|c|}{ Livermore Valley upwind } \\
\hline & FCC & HOSP \\
\hline & \multicolumn{2}{|c|}{$\left(10^{-6} \mathrm{~Bq} / \mathrm{m}^{3}\right)$} \\
\hline Jan & 847 & 618 \\
\hline Feb & 434 & 540 \\
\hline Mar & 255 & 411 \\
\hline Apr & 382 & 360 \\
\hline May & 372 & 403 \\
\hline Jun & 336 & 347 \\
\hline Jul & 448 & 357 \\
\hline Aug & 273 & 351 \\
\hline Sep & 984 & 790 \\
\hline Oct & 1750 & 1470 \\
\hline Nov & 818 & 951 \\
\hline Dec & 958 & 707 \\
\hline Annual median ${ }^{(\mathbf{b})}$ & 440 & 503 \\
\hline $\mathrm{IQR}^{(\mathbf{b}, \mathbf{c})}$ & 676 & 472 \\
\hline Annual maximum ${ }^{(b)}$ & 2410 & 2200 \\
\hline
\end{tabular}

a See Figure 5-2, main volume, for sampling locations.

b Determined by data from the 52 -week period.

c $\quad I Q R=$ Interquartile range. 


\section{Air Surveillance Monitoring}

Table 5-11. Tritium concentration in air, Livermore Valley, 1999.

\begin{tabular}{|c|c|c|c|c|c|c|}
\hline \multirow{3}{*}{ Month } & \multicolumn{6}{|c|}{ Sampling locations ${ }^{(a)}$} \\
\hline & AMON & FIRE & HOSP & VET & XRDS & ZON7 \\
\hline & \multicolumn{6}{|c|}{$\left(10^{-3} \mathrm{~Bq} / \mathrm{m}^{3}\right)$} \\
\hline \multirow[t]{2}{*}{ Jan } & $21.8 \pm 11.0$ & $20.6 \pm 12.4$ & $21.1 \pm 14.2$ & $40.3 \pm 13.5$ & $10.8 \pm 10.5$ & $34.4 \pm 12.4$ \\
\hline & $22.4 \pm 17.3$ & $17.1 \pm 15.4$ & $-1.35 \pm 15.0$ & $36.6 \pm 17.3$ & $25.9 \pm 15.3$ & $80.3 \pm 18.6$ \\
\hline \multirow[t]{2}{*}{ Feb } & $115 \pm 15.8$ & $119 \pm 13.8$ & -(b) $^{(\mathrm{n}}$ & $179 \pm 17.5$ & $87.7 \pm 13.1$ & $162 \pm 14.2$ \\
\hline & $126 \pm 19.8$ & $147 \pm 18.2$ & $32.7 \pm 16.9$ & $242 \pm 27.6$ & $77.0 \pm 14.1$ & $268 \pm 24.3$ \\
\hline \multirow[t]{2}{*}{ Mar } & $175 \pm 22.2$ & $93.6 \pm 19.4$ & $2.80 \pm 6.25$ & $149 \pm 21.8$ & $93.2 \pm 17.8$ & $251 \pm 22.9$ \\
\hline & $108 \pm 14.9$ & $24.5 \pm 10.5$ & $5.81 \pm 9.55$ & $33.7 \pm 12.2$ & $30.9 \pm 10.1$ & $128 \pm 14.8$ \\
\hline \multirow[t]{3}{*}{ Apr } & $20.0 \pm 16.4$ & $86.2 \pm 20.1$ & $-1.23 \pm 13.9$ & $29.9 \pm 18.9$ & $25.7 \pm 16.0$ & $56.6 \pm 17.8$ \\
\hline & $4.14 \pm 13.4$ & $-1.72 \pm 13.2$ & $3.89 \pm 14.8$ & $5.33 \pm 14.6$ & $10.0 \pm 13.2$ & $14.8 \pm 13.2$ \\
\hline & $11.5 \pm 14.9$ & $11.6 \pm 15.1$ & $10.8 \pm 14.2$ & -(b) $^{(\mathrm{c}}$ & $5.25 \pm 14.1$ & $31.6 \pm 15.0$ \\
\hline \multirow[t]{2}{*}{ May } & $-^{(b)}$ & -(b) $^{(\mathrm{c}}$ & $1.78 \pm 17.0$ & -(b) $^{(\mathrm{c}}$ & $-1.38 \pm 14.9$ & $21.1 \pm 16.4$ \\
\hline & $13.1 \pm 21.5$ & -(b) $^{(\mathrm{c}}$ & -(b) $^{(\mathrm{c}}$ & $-6.29 \pm 22.2$ & -(b) $^{(\mathrm{c}}$ & $39.2 \pm 22.1$ \\
\hline \multirow[t]{2}{*}{ Jun } & $6.11 \pm 17.6$ & $3.42 \pm 18.0$ & -(b) $^{(\mathrm{b}}$ & $-2.48 \pm 17.1$ & $3.02 \pm 17.1$ & $28.4 \pm 18.6$ \\
\hline & $27.6 \pm 29.4$ & $2.35 \pm 27.8$ & $87.0 \pm 31.7$ & $28.2 \pm 30.5$ & $35.3 \pm 27.3$ & $79.2 \pm 30.7$ \\
\hline \multirow[t]{2}{*}{ Jul } & $26.1 \pm 18.5$ & $2.32 \pm 16.7$ & $3.89 \pm 17.3$ & $4.40 \pm 18.9$ & $-0.892 \pm 15.7$ & $36.2 \pm 17.5$ \\
\hline & $12.2 \pm 24.9$ & $5.14 \pm 27.0$ & $38.1 \pm 26.0$ & $-4.40 \pm 24.4$ & $-2.82 \pm 22.0$ & $40.3 \pm 25.2$ \\
\hline \multirow[t]{2}{*}{ Aug } & $5.81 \pm 23.1$ & $-22.5 \pm 24.2$ & $-3.07 \pm 22.8$ & $-11.3 \pm 22.4$ & $11.5 \pm 21.5$ & $59.9 \pm 25.0$ \\
\hline & $1.31 \pm 17.5$ & $-5.96 \pm 17.6$ & $-4.00 \pm 10.9$ & $-12.5 \pm 15.7$ & $0.201 \pm 15.1$ & $13.2 \pm 16.8$ \\
\hline \multirow[t]{2}{*}{ Sep } & $5.70 \pm 20.6$ & $13.2 \pm 22.3$ & $2.60 \pm 20.9$ & $17.8 \pm 21.9$ & $16.7 \pm 19.7$ & $59.9 \pm 22.8$ \\
\hline & $114 \pm 30.9$ & $7.10 \pm 27.5$ & $-4.81 \pm 24.3$ & $-1.08 \pm 24.1$ & $10.2 \pm 23.3$ & $40.7 \pm 25.1$ \\
\hline \multirow[t]{3}{*}{ Oct } & $27.3 \pm 16.9$ & $14.1 \pm 13.4$ & $2.87 \pm 15.1$ & $0.688 \pm 21.2$ & $11.2 \pm 16.8$ & $44.8 \pm 20.8$ \\
\hline & $31.7 \pm 19.6$ & $42.2 \pm 23.2$ & $25.7 \pm 18.1$ & $39.6 \pm 19.1$ & $18.0 \pm 16.6$ & $-^{(\mathrm{b})}$ \\
\hline & $11.0 \pm 17.8$ & $17.1 \pm 20.8$ & $3.27 \pm 16.5$ & $32.9 \pm 17.8$ & $-3.54 \pm 14.2$ & $9.03 \pm 15.8$ \\
\hline \multirow[t]{2}{*}{ Nov } & $3.48 \pm 27.6$ & $5.59 \pm 25.6$ & $-5.66 \pm 20.7$ & $26.8 \pm 24.3$ & $8.70 \pm 22.6$ & $15.5 \pm 23.8$ \\
\hline & $0.807 \pm 26.0$ & $22.8 \pm 27.3$ & $3.40 \pm 21.2$ & $12.7 \pm 22.8$ & $5.74 \pm 21.1$ & $-2.77 \pm 22.5$ \\
\hline \multirow[t]{2}{*}{ Dec } & $43.3 \pm 24.3$ & $12.3 \pm 20.4$ & $8.58 \pm 16.4$ & $3.77 \pm 17.6$ & $-4.66 \pm 15.9$ & $-0.463 \pm 18.6$ \\
\hline & $13.1 \pm 11.0$ & $53.7 \pm 14.8$ & $-0.740 \pm 10.9$ & $8.03 \pm 11.7$ & $0.762 \pm 10.8$ & $3.31 \pm 12.1$ \\
\hline Median(c) & 20.0 & 13.7 & 3.27 & 15.3 & 10.2 & 39.2 \\
\hline $\operatorname{IQR}^{(\mathrm{d})}$ & 25.6 & 24.2 & 10.7 & 34.2 & 24.9 & 44.4 \\
\hline Percent of DCG ${ }^{(e)}$ & $5.4 \times 10^{-4}$ & $3.7 \times 10^{-4}$ & $8.8 \times 10^{-5}$ & $4.1 \times 10^{-4}$ & $2.8 \times 10^{-4}$ & $1.1 \times 10^{-3}$ \\
\hline Dose $(\mathrm{mSv})^{(\mathrm{f})}$ & $4.2 \times 10^{-6}$ & $2.8 \times 10^{-6}$ & $6.8 \times 10^{-7}$ & $3.2 \times 10^{-6}$ & $2.1 \times 10^{-6}$ & $8.1 \times 10^{-6}$ \\
\hline
\end{tabular}

Note: Radioactivities are reported as the measured concentration and either an uncertainty ( $\pm 2 \sigma$ counting error) or as being less than or equal to the detection limit. If the concentration is less than the uncertainty or the detection limit, the result is considered to be a nondetection. See the main volume, Chapter 14, Quality Assurance.

a See Figure 5-2, main volume, for sampling locations.

b No data. See main volume, Chapter 14, Quality Assurance.

C Livermore Valley overall median $=13.2 \times 10^{-3} \mathrm{~Bq} / \mathrm{m}^{3}$.

d $\quad \mathrm{QQR}=$ Interquartile range.

e $\quad \mathrm{DCG}=$ Derived Concentration Guide of $3.7 \times 10^{3} \mathrm{~Bq} / \mathrm{m}^{3}$. Percent calculated from the median concentration.

$f \quad$ This dose is the effective dose equivalent. 
Table 5-12. Tritium concentration in air, Livermore site perimeter, 1999.

\begin{tabular}{|c|c|c|c|c|c|c|c|}
\hline \multirow{3}{*}{ Month } & \multicolumn{7}{|c|}{ Sampling location ${ }^{(a)}$} \\
\hline & CAFE & cow & MESQ & MET & POOL & SALV & VIS \\
\hline & \multicolumn{7}{|c|}{$\left(10^{-3} \mathrm{~Bq} / \mathrm{m}^{3}\right)$} \\
\hline \multirow[t]{2}{*}{ Jan } & $184 \pm 16.6$ & $44.8 \pm 12.0$ & $72.2 \pm 13.3$ & $44.4 \pm 12.4$ & $429 \pm 26.0$ & $61.4 \pm 13.4$ & $50.3 \pm 12.0$ \\
\hline & $131 \pm 17.0$ & $146 \pm 17.9$ & $85.5 \pm 18.5$ & $66.2 \pm 14.7$ & $250 \pm 23.0$ & $179 \pm 21.2$ & $162 \pm 18.0$ \\
\hline \multirow[t]{2}{*}{ Feb } & $807 \pm 28.8$ & $300 \pm 19.1$ & $238 \pm 18.6$ & $150 \pm 16.1$ & $1240 \pm 39.6$ & $298 \pm 20.0$ & $315 \pm 19.6$ \\
\hline & $507 \pm 26.2$ & $618 \pm 30.4$ & $357 \pm 26.2$ & $236 \pm 20.5$ & $1400 \pm 46.3$ & $377 \pm 25.5$ & $555 \pm 27.6$ \\
\hline \multirow[t]{2}{*}{ Mar } & $426 \pm 27.5$ & $688 \pm 34.3$ & $253 \pm 23.3$ & $195 \pm 21.1$ & $944 \pm 42.2$ & $485 \pm 31.1$ & $622 \pm 33.4$ \\
\hline & $107 \pm 12.3$ & $374 \pm 22.4$ & $62.2 \pm 12.2$ & $51.1 \pm 11.3$ & $385 \pm 23.6$ & $151 \pm 16.8$ & $250 \pm 17.5$ \\
\hline \multirow[t]{3}{*}{ Apr } & $102 \pm 18.8$ & $103 \pm 18.5$ & $119 \pm 19.5$ & $23.9 \pm 15.4$ & $172 \pm 22.2$ & $148 \pm 24.9$ & $135 \pm 18.7$ \\
\hline & $45.1 \pm 14.2$ & $24.5 \pm 14.4$ & $23.2 \pm 14.5$ & $16.8 \pm 13.6$ & $133 \pm 17.7$ & 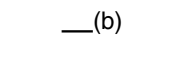 & $40.0 \pm 13.3$ \\
\hline & $132 \pm 18.9$ & $47.7 \pm 15.9$ & $19.1 \pm 14.9$ & $17.9 \pm 15.2$ & $145 \pm 20.8$ & $35.4 \pm 10.1$ & $76.6 \pm 16.9$ \\
\hline \multirow[t]{2}{*}{ May } & $48.5 \pm 13.8$ & $36.2 \pm 15.9$ & 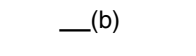 & $-3.77 \pm 14.6$ & $69.2 \pm 13.2$ & $8.18 \pm 22.0$ & $37.7 \pm 15.5$ \\
\hline & $49.2 \pm 23.0$ & $52.5 \pm 23.1$ & $17.5 \pm 21.8$ & $8.44 \pm 21.8$ & $74.7 \pm 25.8$ & $32.7 \pm 22.0$ & $73.3 \pm 21.4$ \\
\hline \multirow[t]{2}{*}{ Jun } & $-7.81 \pm 17.0$ & $34.2 \pm 14.8$ & $12.2 \pm 17.9$ & $76.2 \pm 21.3$ & $32.2 \pm 20.1$ & $15.5 \pm 17.7$ & $68.1 \pm 18.0$ \\
\hline & $35.4 \pm 28.7$ & -(b) $^{(\mathrm{b}}$ & $16.2 \pm 29.0$ & $18.9 \pm 28.9$ & $65.1 \pm 33.4$ & $26.2 \pm 28.5$ & $100 \pm 26.6$ \\
\hline \multirow[t]{2}{*}{ Jul } & $76.2 \pm 20.9$ & $203 \pm 43.7$ & $8.14 \pm 16.2$ & $29.3 \pm 20.0$ & $201 \pm 26.9$ & $50.3 \pm 19.3$ & $132 \pm 21.9$ \\
\hline & $34.9 \pm 18.2$ & $130 \pm 33.3$ & $17.4 \pm 23.6$ & $21.4 \pm 30.0$ & $121 \pm 47.0$ & $50.7 \pm 26.2$ & $149 \pm 28.5$ \\
\hline \multirow[t]{2}{*}{ Aug } & $-11.0 \pm 22.2$ & $31.1 \pm 28.0$ & $-16.8 \pm 20.5$ & $12.6 \pm 28.6$ & $52.5 \pm 44.8$ & $1.57 \pm 22.5$ & $116 \pm 26.6$ \\
\hline & $7.29 \pm 20.5$ & $52.2 \pm 20.5$ & $-4.26 \pm 14.5$ & $-20.3 \pm 18.8$ & $13.2 \pm 27.9$ & $104 \pm 20.4$ & $50.7 \pm 17.6$ \\
\hline \multirow[t]{2}{*}{ Sep } & $94.7 \pm 24.5$ & $122 \pm 37.4$ & $43.3 \pm 21.7$ & $59.2 \pm 26.6$ & $82.9 \pm 15.5$ & $78.4 \pm 24.2$ & $108 \pm 24.5$ \\
\hline & $45.9 \pm 25.1$ & $70.3 \pm 32.1$ & $21.7 \pm 24.1$ & $24.3 \pm 31.3$ & $108 \pm 32.3$ & $34.1 \pm 24.9$ & $94.7 \pm 26.9$ \\
\hline \multirow[t]{3}{*}{ Oct } & $1890 \pm 59.2$ & $89.2 \pm 22.2$ & $83.3 \pm 20.0$ & $68.8 \pm 21.2$ & $385 \pm 31.6$ & $75.9 \pm 20.4$ & $52.5 \pm 19.6$ \\
\hline & $180 \pm 23.9$ & $71.8 \pm 20.0$ & $116 \pm 20.4$ & $117 \pm 24.8$ & $374 \pm 33.6$ & $95.1 \pm 20.3$ & $89.9 \pm 20.1$ \\
\hline & $131 \pm 19.9$ & $44.8 \pm 17.8$ & $150 \pm 20.1$ & $120 \pm 25.5$ & $298 \pm 28.5$ & $57.7 \pm 17.0$ & $58.5 \pm 17.7$ \\
\hline \multirow[t]{2}{*}{ Nov } & $51.4 \pm 21.6$ & $31.2 \pm 23.3$ & $78.8 \pm 22.7$ & $61.4 \pm 25.0$ & $161 \pm 34.8$ & $48.5 \pm 22.1$ & -(b) $^{(\mathrm{b}}$ \\
\hline & $52.9 \pm 21.3$ & $25.6 \pm 22.6$ & $45.9 \pm 20.8$ & $33.1 \pm 23.6$ & $122 \pm 27.7$ & $18.9 \pm 20.8$ & $35.7 \pm 28.0$ \\
\hline \multirow[t]{2}{*}{ Dec } & $53.7 \pm 16.9$ & $34.2 \pm 17.5$ & $46.3 \pm 16.9$ & $30.6 \pm 20.3$ & $96.9 \pm 18.4$ & $36.3 \pm 18.4$ & $29.2 \pm 22.1$ \\
\hline & $47.4 \pm 11.0$ & $11.0 \pm 10.7$ & $2.23 \pm 10.5$ & $38.5 \pm 14.9$ & $98.1 \pm 13.9$ & $30.5 \pm 12.2$ & $40.3 \pm 15.5$ \\
\hline Median(c) & 65.0 & 52.5 & 45.9 & 35.8 & 139 & 50.7 & 89.9 \\
\hline $\operatorname{IQR}^{(\mathrm{d})}$ & 85.5 & 95.8 & 68.1 & 48.6 & 269 & 71.3 & 84.3 \\
\hline Percent of DCG(e) & $1.8 \times 10^{-3}$ & $1.4 \times 10^{-3}$ & $1.2 \times 10^{-3}$ & $9.7 \times 10^{-4}$ & $3.8 \times 10^{-3}$ & $1.4 \times 10^{-3}$ & $2.4 \times 10^{-3}$ \\
\hline Dose (mSv) ${ }^{(f)}$ & $1.3 \times 10^{-5}$ & $1.1 \times 10^{-5}$ & $9.5 \times 10^{-6}$ & $7.4 \times 10^{-6}$ & $2.9 \times 10^{-5}$ & $1.1 \times 10^{-5}$ & $1.9 \times 10^{-5}$ \\
\hline
\end{tabular}

Note: Radioactivities are reported as the measured concentration and either an uncertainty ( $\pm 2 \sigma$ counting error) or as being less than or equal to the detection limit. If the concentration is less than the uncertainty or the detection limit, the result is considered to be a nondetection. See the main volume, Chapter 14, Quality Assurance.

a See Figure 5-1, main volume, for sampling locations.

b No data. See main volume, Chapter 14, Quality Assurance.

c Livermore site overall median $=68.5 \times 10^{-3} \mathrm{~Bq} / \mathrm{m}^{3}$.

d $\quad \mathrm{QQR}=$ Interquartile range.

e $\quad$ DCG = Derived Concentration Guide of $3.7 \times 10^{3} \mathrm{~Bq} / \mathrm{m}^{3}$. Percent calculated from the median concentration.

$f$ This dose is the effective dose equivalent. 


\section{Air Surveillance Monitoring}

Table 5-13. Tritium concentration in air at locations near diffuse sources, 1999.

\begin{tabular}{|c|c|c|c|c|}
\hline \multirow{3}{*}{ Month } & \multicolumn{4}{|c|}{ Sampling locations ${ }^{(a)}$} \\
\hline & B292 & B331 & B514 & B624 \\
\hline & \multicolumn{4}{|c|}{$\left(10^{-3} \mathrm{~Bq} / \mathrm{m}^{3}\right)$} \\
\hline \multirow[t]{2}{*}{ Jan } & $518 \pm 25$ & -(b) $^{(\mathrm{b}}$ & $1,580 \pm 40$ & $4,630 \pm 70$ \\
\hline & $451 \pm 30$ & $8,700 \pm 100$ & $2,350 \pm 57$ & $4,330 \pm 79$ \\
\hline \multirow[t]{2}{*}{ Feb } & $522 \pm 25$ & $9,810 \pm 102$ & $1,880 \pm 44$ & $4,590 \pm 71$ \\
\hline & $522 \pm 31$ & $8,880 \pm 103$ & $2,390 \pm 53$ & $5,140 \pm 88$ \\
\hline \multirow[t]{2}{*}{ Mar } & $540 \pm 33$ & $11,300 \pm 132$ & $2,870 \pm 63$ & $6,220 \pm 104$ \\
\hline & $290 \pm 21$ & $11,800 \pm 108$ & $1,820 \pm 45$ & $3,530 \pm 68$ \\
\hline \multirow[t]{2}{*}{ Apr } & $150 \pm 21$ & -(b) $^{(\mathrm{c}}$ & $1,040 \pm 41$ & $3,430 \pm 77$ \\
\hline & $175 \pm 20$ & $9,470 \pm 108$ & $544 \pm 27$ & $3,960 \pm 75$ \\
\hline \multirow[t]{3}{*}{ May } & $145 \pm 20$ & $13,800 \pm 141$ & $1,100 \pm 42$ & $4,880 \pm 88$ \\
\hline & $57.4 \pm 17.8$ & $2,520 \pm 63$ & $844 \pm 33$ & $2,810 \pm 59$ \\
\hline & $92.1 \pm 24.8$ & $858 \pm 41$ & $1,150 \pm 47$ & $3,490 \pm 76$ \\
\hline \multirow[t]{2}{*}{ Jun } & $47.0 \pm 19.5$ & $124 \pm 22$ & $1,310 \pm 48$ & $2,390 \pm 63$ \\
\hline & $68.8 \pm 30.6$ & $592 \pm 42$ & $2,540 \pm 78$ & $4,440 \pm 102$ \\
\hline \multirow[t]{2}{*}{ Jul } & $156 \pm 22$ & -(b) $^{(\mathrm{c}}$ & $2,080 \pm 59$ & $5,330 \pm 96$ \\
\hline & $153 \pm 28$ & $877 \pm 48$ & $1,760 \pm 62$ & $9,070 \pm 143$ \\
\hline \multirow[t]{2}{*}{ Aug } & $52.9 \pm 25.3$ & $153 \pm 30$ & $1,680 \pm 66$ & $4,260 \pm 103$ \\
\hline & $71.0 \pm 19.6$ & $205 \pm 25$ & $1,620 \pm 57$ & $3,180 \pm 81$ \\
\hline \multirow[t]{2}{*}{ Sep } & $325 \pm 33$ & $2,310 \pm 70$ & $2,830 \pm 77$ & $8,580 \pm 134$ \\
\hline & $155 \pm 31$ & $999 \pm 50$ & $2,950 \pm 83$ & $6,030 \pm 121$ \\
\hline \multirow[t]{3}{*}{ Oct } & $353 \pm 31$ & $4,550 \pm 85$ & $2,360 \pm 70$ & $9,180 \pm 122$ \\
\hline & $422 \pm 32$ & $4,850 \pm 90$ & $2,010 \pm 63$ & $9,100 \pm 125$ \\
\hline & $381 \pm 28$ & -(b) $^{(\mathrm{c}}$ & $1,390 \pm 49$ & $6,880 \pm 106$ \\
\hline \multirow[t]{2}{*}{ Nov } & $247 \pm 31$ & $2,530 \pm 67$ & $1,020 \pm 46$ & $5,000 \pm 100$ \\
\hline & $179 \pm 28$ & $1,550 \pm 53$ & $766 \pm 39$ & $2,800 \pm 64$ \\
\hline \multirow[t]{2}{*}{$\mathrm{Dec}$} & $255 \pm 26$ & $1,410 \pm 45$ & $722 \pm 33$ & $2,290 \pm 53$ \\
\hline & $185 \pm 18$ & $3,380 \pm 57$ & $603 \pm 25$ & $1,270 \pm 34$ \\
\hline Median(c) & 182 & 2,530 & 1,650 & 4,520 \\
\hline $\operatorname{IQR}^{(\mathrm{d})}$ & 228 & 7,930 & 1,230 & 2,410 \\
\hline Percent of DCG(e) & $4.9 \times 10^{-3}$ & .068 & .045 & 0.12 \\
\hline Dose $(m S v)^{(f)}$ & $3.8 \times 10^{-5}$ & $5.2 \times 10^{-4}$ & $3.4 \times 10^{-4}$ & $9.4 \times 10^{-4}$ \\
\hline
\end{tabular}

Note: Radioactivities are reported as the measured concentration and either an uncertainty $( \pm 2 \sigma$ counting error) or as being less than or equal to the detection limit. If the concentration is less than the uncertainty or the detection limit, the result is considered to be a nondetection. See the main volume, Chapter 14, Quality Assurance.

a See Figure 5-1, main volume, for sampling locations.

b No data. See main volume, Chapter 14, Quality Assurance.

c Diffuse source overall median $=1720 \times 10^{-3} \mathrm{~Bq} / \mathrm{m}^{3}$.

d $I Q R=$ Interquartile range.

e $\quad$ DCG $=$ Derived Concentration Guide of $3.7 \times 10^{3} \mathrm{~Bq} / \mathrm{m}^{3}$. Percent calculated from the median concentration.

$f \quad$ This dose is the effective dose equivalent. 
Table 5-14. Monthly beryllium in air particulate composites, Livermore site perimeter, 1999.

\begin{tabular}{|c|c|c|c|c|c|c|}
\hline \multirow{3}{*}{ Month } & \multicolumn{6}{|c|}{ Sampling location ${ }^{(a)}$} \\
\hline & CAFE & cow & MESQ & MET & SALV & VIS \\
\hline & \multicolumn{6}{|c|}{$\left(\mathrm{pg} / \mathrm{m}^{3}\right)$} \\
\hline Jan & 5.95 & 6.39 & 4.15 & 4.43 & 1.78 & 3.59 \\
\hline Feb & 4.50 & 3.75 & 4.00 & 2.24 & 1.96 & 3.52 \\
\hline Mar & 6.52 & 8.37 & 8.86 & 3.51 & 4.55 & 9.69 \\
\hline Apr & 9.10 & 10.6 & 9.07 & 7.39 & 9.69 & 9.51 \\
\hline May & 11.2 & 13.6 & 13.4 & 9.38 & 7.60 & 21.3 \\
\hline Jun & 15.8 & 9.15 & 13.8 & 8.62 & 7.34 & 11.0 \\
\hline Jul & 18.2 & 28.6 & 16.3 & 9.27 & 13.1 & 33.2 \\
\hline Aug & 13.3 & 15.6 & 12.9 & 8.27 & 9.06 & 13.6 \\
\hline Sep & 16.0 & 23.9 & 16.6 & 14.9 & 14.0 & 14.7 \\
\hline Oct & 23.8 & 37.8 & 36.4 & 23.9 & 25.7 & 24.7 \\
\hline Nov & 9.49 & 8.64 & 0.935 & 5.21 & 3.27 & 7.95 \\
\hline Dec & 10.9 & 11.0 & 7.85 & 7.18 & 7.17 & 8.46 \\
\hline Median $^{(b)}$ & 11.1 & 10.8 & 11.0 & 7.83 & 7.47 & 10.3 \\
\hline $\mathrm{IQR}^{(\mathrm{c})}$ & 7.40 & 9.10 & 7.50 & 4.28 & 6.31 & 8.02 \\
\hline Maximum & 23.8 & 37.8 & 36.4 & 23.9 & 25.7 & 33.2 \\
\hline Percent of $A C L^{(d)}$ & 0.111 & 0.108 & 0.110 & 0.0783 & 0.0747 & 0.103 \\
\hline
\end{tabular}

a See Figure 5-1, main volume, for sampling locations.

b Livermore site perimeter overall median is $9.33 \mathrm{pg} / \mathrm{m}^{3}$.

c $\quad$ IQR $=$ Interquartile range.

d The monthly Ambient Concentration Limit (ACL) is $10,000 \mathrm{pg} / \mathrm{m}^{3}$ as set by the Bay Area Air Quality Management District (BAAQMD). Percent calculated from the median concentration. 


\section{Air Surveillance Monitoring}

Table 5-15. Monthly median activities for gross alpha and gross beta summarized from weekly data for Site 300 perimeter and off-site locations, 1999.

\begin{tabular}{|c|c|c|c|c|c|c|c|c|c|}
\hline \multirow{3}{*}{ Month } & \multicolumn{9}{|c|}{ Sampling location(a) } \\
\hline & \multicolumn{7}{|c|}{ Site $\mathbf{3 0 0}$ perimeter } & \multicolumn{2}{|c|}{ Off site Site 300} \\
\hline & $801 E$ & ECP & EOBS & GOLF & NPS & WCP & WOBS & PRIM & TFIR \\
\hline \multirow{2}{*}{$\begin{array}{c}\text { Gross alpha } \\
\text { Jan }\end{array}$} & \multicolumn{9}{|c|}{$\left(10^{-6} \mathrm{~Bq} / \mathrm{m}^{3}\right)$} \\
\hline & 5.67 & 18.1 & 0.797 & 35.3 & 25.3 & 42.1 & 34.4 & 45.8 & 59.2 \\
\hline Feb & -2.83 & 2.03 & 5.42 & 14.1 & 31.3 & 11.0 & 21.6 & 1.53 & 17.5 \\
\hline Mar & 59.9 & 13.8 & 40.9 & 61.4 & 71.2 & 22.9 & 47.9 & 56.6 & 56.2 \\
\hline Apr & 49.6 & 56.6 & 53.3 & 39.6 & 58.8 & 38.9 & 64.8 & 54.4 & 46.3 \\
\hline May & 38.3 & 20.5 & 33.2 & 44.7 & 34.2 & 32.0 & 38.3 & 26.6 & 14.5 \\
\hline Jun & 29.5 & 39.6 & 19.7 & 58.8 & 56.1 & 19.3 & 33.1 & 32.3 & 42.0 \\
\hline Jul & 36.7 & 64.4 & 53.3 & 70.7 & 63.3 & 24.9 & 65.9 & 62.2 & 39.6 \\
\hline Aug & 34.6 & 63.5 & 63.6 & 64.8 & 81.1 & 79.9 & 13.4 & 75.1 & 76.6 \\
\hline Sep & 128 & 87.0 & 99.0 & 98.2 & 90.1 & 77.7 & 124 & 92.9 & 78.8 \\
\hline Oct & 260 & 269 & 187 & 248 & 184 & 203 & 243 & 234 & 196 \\
\hline Nov & 124 & 138 & 106 & 120 & 113 & 133 & 92.5 & 122 & 68.8 \\
\hline Dec & 93.1 & 106 & 87.7 & 92.1 & 77.3 & 79.7 & 97.9 & 54.4 & 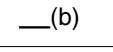 \\
\hline \multirow{3}{*}{$\begin{array}{l}\text { Annual Median } \\
\text { IQR }^{(c, d)} \\
\text { Annual Maximum(c) }\end{array}$} & 43.3 & 54.8 & 48.7 & 63.6 & 59.2 & 54.4 & 52.0 & 50.7 & 48.8 \\
\hline & 79.5 & 72.4 & 65.6 & 56.9 & 53.5 & 61.1 & 65.6 & 76.1 & 60.7 \\
\hline & 352 & 332 & 290 & 294 & 291 & 270 & 365 & 339 & 336 \\
\hline Gross beta & \multicolumn{9}{|c|}{$\left(10^{-6} \mathrm{~Bq} / \mathrm{m}^{3}\right)$} \\
\hline Jan & 580 & 617 & 499 & 825 & 503 & 551 & 500 & 959 & 1306 \\
\hline Feb & 177 & 396 & 419 & 376 & 388 & 422 & 490 & 482 & 567 \\
\hline Mar & 217 & 325 & 275 & 256 & 264 & 231 & 229 & 276 & 280 \\
\hline Apr & 357 & 346 & 369 & 303 & 302 & 335 & 374 & 312 & 308 \\
\hline May & 361 & 370 & 426 & 339 & 364 & 383 & 414 & 426 & 316 \\
\hline Jun & 363 & 260 & 283 & 310 & 377 & 250 & 301 & 331 & 311 \\
\hline Jul & 310 & 301 & 270 & 307 & 293 & 326 & 291 & 308 & 262 \\
\hline Aug & 548 & 500 & 488 & 487 & 389 & 500 & 184 & 511 & 418 \\
\hline Sep & 1034 & 1064 & 840 & 912 & 1070 & 877 & 1050 & 914 & 882 \\
\hline Oct & 1200 & 1370 & 1030 & 1110 & 1150 & 1090 & 1320 & 1120 & 1150 \\
\hline Nov & 781 & 927 & 703 & 921 & 744 & 910 & 882 & 810 & 777 \\
\hline Dec & 605 & 744 & 636 & 685 & 670 & 575 & 670 & 440 & —(b) $^{(\mathrm{n}}$ \\
\hline Annual Median(c) & 424 & 381 & 403 & 361 & 413 & 433 & 364 & 426 & 392 \\
\hline $\operatorname{IQR}^{(\mathrm{c}, \mathrm{d})}$ & 540 & 646 & 447 & 581 & 602 & 606 & 541 & 649 & 513 \\
\hline Annual Maximum(c) & 2080 & 2510 & 2070 & 2300 & 2080 & 1980 & 2300 & 2010 & 1990 \\
\hline \multicolumn{10}{|c|}{ a See Figure 5-3, main volume, for sampling locations. } \\
\hline \multicolumn{10}{|c|}{ b No sample collected due to lack of power at sampling location. } \\
\hline \multicolumn{10}{|c|}{ c Determined by data from the 52 -week period. } \\
\hline
\end{tabular}


Table 5-16. Gamma activity in particulate air samples, Site 300, 1999.(a)

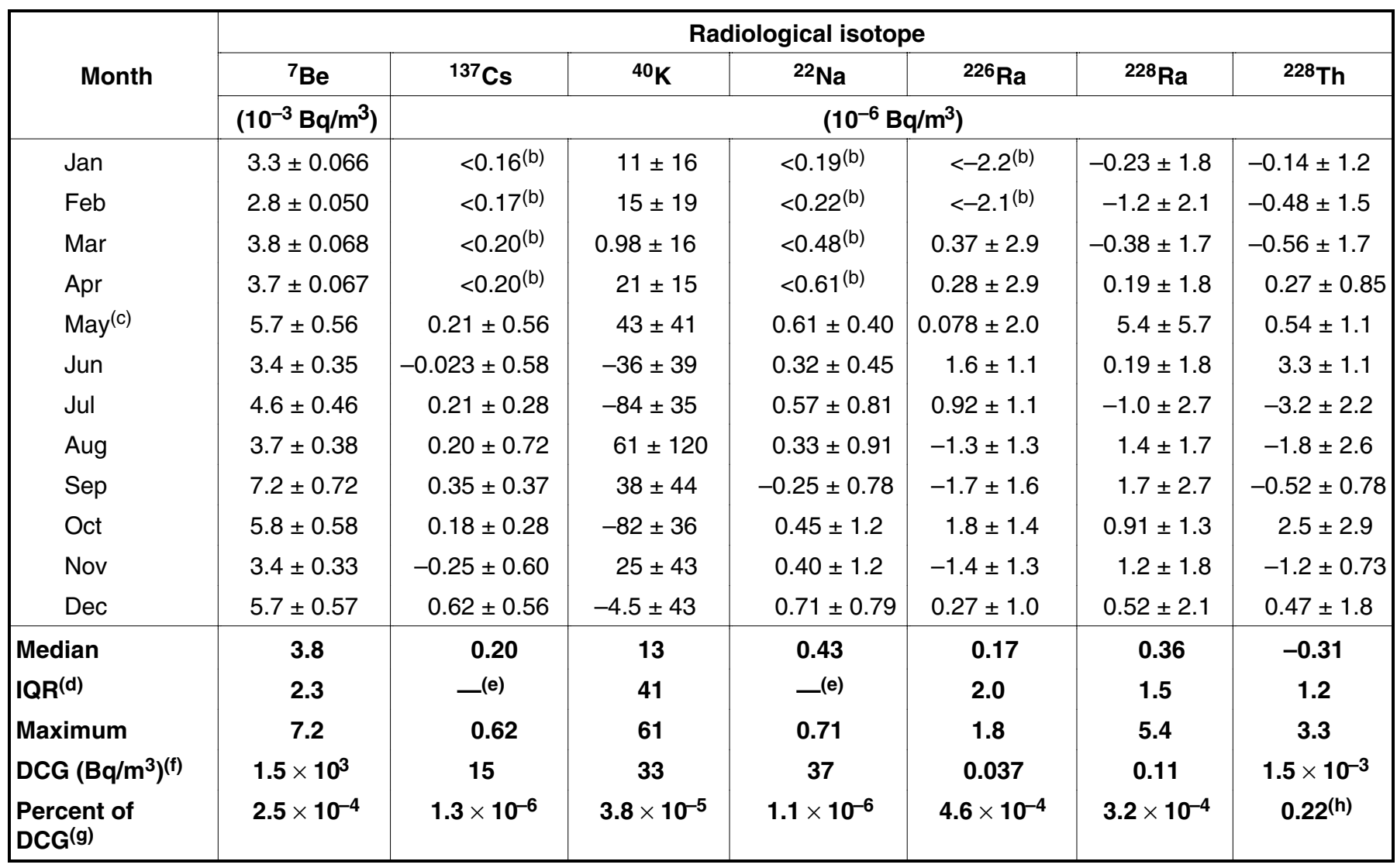

Note: Radioactivities are reported as the measured concentration and either an uncertainty ( \pm 2 s counting error) or as being less than or equal to the detection limit. If the concentration is less than the uncertainty or the detection limit, the result is considered to be a nondetection. See the main volume, Chapter 14, Quality Assurance.

a All Site 300 samples composited. See Figure 5-3, main volume, for sampling locations.

b Error not provided by analytical laboratory.

c The analytical software changed in May 1999.

d $\quad I Q R=$ Interquartile range.

e No measure of dispersion calculated; see Chapter 14, Quality Assurance.

f $\quad$ DCG = Derived Concentration Guide (DOE Order 5400.5). See main volume, Chapter 13, Radiation Dose Assessment.

$g$ Percent of DCG calculated from the median concentration unless otherwise noted.

$\mathrm{h}$ The thorium-228 percent of DCG was determined by using the maximum value because the median value was negative. 


\section{Air Surveillance Monitoring}

Table 5-17. Plutonium-239+240 activity in air particulate samples, Site 300, 1999.

\begin{tabular}{|c|c|c|c|}
\hline \multirow{3}{*}{ Month } & \multicolumn{3}{|c|}{ Sampling location(a) } \\
\hline & Site 300 composite & PRIM & TFIR \\
\hline & \multicolumn{3}{|c|}{$\left(10^{-9} \mathrm{~Bq} / \mathrm{m}^{3}\right)$} \\
\hline Jan & $0.013 \pm 1.13$ & $-1.86 \pm 3.36$ & $-3.42 \pm 4.29$ \\
\hline Feb & $0.918 \pm 1.55$ & $2.39 \pm 6.48$ & $-1.21 \pm 4.33$ \\
\hline Mar & $0.154 \pm 1.24$ & $3.57 \pm 7.59$ & $-0.264 \pm 2.60$ \\
\hline Apr & $2.96 \pm 2.24$ & $0.651 \pm 2.76$ & $0.714 \pm 3.03$ \\
\hline May & $2.50 \pm 2.13$ & $3.54 \pm 5.07$ & $3.50 \pm 4.59$ \\
\hline Jun & $6.99 \pm 3.96$ & $2.21 \pm 3.74$ & $6.77 \pm 6.44$ \\
\hline Jul & $0.755 \pm 1.06$ & $1.60 \pm 2.71$ & $-0.810 \pm 3.17$ \\
\hline Aug & $1.86 \pm 2.07$ & $0.221 \pm 3.08$ & $2.43 \pm 3.44$ \\
\hline Sep & $8.70 \pm 3.92$ & $6.48 \pm 5.70$ & $7.62 \pm 6.70$ \\
\hline Oct & $-0.164 \pm 6.70$ & $11.3 \pm 7.40$ & $12.6 \pm 6.77$ \\
\hline Nov & $1.71 \pm 1.97$ & $-2.05 \pm 1.83$ & $2.52 \pm 3.57$ \\
\hline Dec & $3.62 \pm 3.96$ & $-1.58 \pm 4.00$ & - $^{(\mathrm{b})}$ \\
\hline Median & 1.79 & 1.91 & 2.43 \\
\hline $\operatorname{IQR}^{(c)}$ & 2.52 & 3.78 & 5.67 \\
\hline Percent of DCG(d) & $2.41 \times 10^{-4}$ & $2.58 \times 10^{-4}$ & $3.29 \times 10^{-4}$ \\
\hline
\end{tabular}

Note: Radioactivities are reported as the measured concentration and either an uncertainty ( $\pm 2 \sigma$ counting error) or as being less than or equal to the detection limit. If the concentration is less than the uncertainty or the detection limit, the result is considered to be a nondetection. See the main volume, Chapter 14, Quality Assurance.

a See Figure 5-3, main volume, for sampling locations.

b No data. See Chapter 14, Quality Assurance.

c $\quad I Q R=$ Interquartile range.

d $\quad$ DCG = Derived Concentration Guide of $7.4 \times 10^{-4} \mathrm{~Bq} / \mathrm{m}^{3}$ for Pu-239 activity in air. Percent calculated from the median concentration. 
Table 5-18. Uranium mass in air particulate samples for Site 300 composite and PRIM location, 1999. (a)

\begin{tabular}{|c|c|c|c|c|}
\hline Location $^{(a)}$ & Month & $\begin{array}{c}\text { Uranium-235(b) } \\
\left(10^{-7} \mu \mathrm{g} / \mathrm{m}^{3}\right)\end{array}$ & $\begin{array}{c}\text { Uranium-238(c) } \\
\left(10^{-5} \mu \mathrm{g} / \mathrm{m}^{3}\right)\end{array}$ & $\begin{array}{c}\text { Uranium } 235 / 238 \\
\left(10^{-3}\right)\end{array}$ \\
\hline \multirow[t]{12}{*}{ Site 300} & Jan & $-0.38 \pm 0.23$ & $-0.4 \pm 0.3$ & -(d) $^{(\mathrm{d})}$ \\
\hline & Feb & $0.82 \pm 0.19$ & $1.3 \pm 0.3$ & 6.46 \\
\hline & Mar & $2.94 \pm 0.11$ & $4.7 \pm 0.2$ & 6.29 \\
\hline & Apr & $1.59 \pm 1.10$ & $2.7 \pm 1.5$ & 5.96 \\
\hline & May & $3.99 \pm 0.41$ & $6.0 \pm 0.6$ & 6.59 \\
\hline & Jun & $3.61 \pm 0.49$ & $5.2 \pm 0.8$ & 6.92 \\
\hline & Jul & $2.89 \pm 0.60$ & $4.4 \pm 0.9$ & 6.54 \\
\hline & Aug & $6.04 \pm 0.50$ & $10.7 \pm 1.3$ & 5.62 \\
\hline & Sep & $5.41 \pm 1.28$ & $18.6 \pm 2.2$ & 2.92 \\
\hline & Oct & $9.06 \pm 2.21$ & $13.5 \pm 2.8$ & 2.19 \\
\hline & Nov & $-0.03 \pm 2.24$ & $0.4 \pm 3.0$ & -(d) $^{(\mathrm{n}}$ \\
\hline & $\mathrm{Dec}$ & $0.01 \pm 1.87$ & $-0.01 \pm 2.6$ & -(d) $^{(\mathrm{n}}$ \\
\hline Median & & 2.9 & 4.5 & 6.3 \\
\hline $\operatorname{IQR}^{(\mathrm{e})}$ & & 3.7 & 6.2 & 0.9 \\
\hline Maximum & & 9.1 & 18.6 & $N A^{(f)}$ \\
\hline Percent of DCG(g) & & $6.2 \times 10^{-4}$ & $1.5 \times 10^{-2}$ & NA \\
\hline \multirow[t]{12}{*}{ PRIM } & Jan & $1.17 \pm 0.1$ & $1.7 \pm 0.1$ & 6.98 \\
\hline & Feb & $-0.05 \pm 0.2$ & $0.0 \pm 0.2$ & -(d) $^{(\mathrm{n}}$ \\
\hline & Mar & $14.7 \pm 0.1$ & $6.6 \pm 0.1$ & 22.1 \\
\hline & Apr & $4.11 \pm 0.4$ & $5.7 \pm 0.4$ & 7.25 \\
\hline & May & $4.58 \pm 0.2$ & $6.9 \pm 0.5$ & 6.65 \\
\hline & Jun & $3.90 \pm 0.3$ & $5.7 \pm 0.6$ & 6.81 \\
\hline & Jul & $2.96 \pm 0.3$ & $4.4 \pm 0.5$ & 6.74 \\
\hline & Aug & $5.21 \pm 0.6$ & $8.53 \pm 0.7$ & 6.10 \\
\hline & Sep & $5.46 \pm 1.1$ & $18.2 \pm 1.3$ & 3.00 \\
\hline & Oct & $10.6 \pm 1.8$ & $15.1 \pm 2.4$ & 7.03 \\
\hline & Nov & $1.29 \pm 1.4$ & $1.8 \pm 1.9$ & 7.35 \\
\hline & $\mathrm{Dec}$ & $1.12 \pm 0.9$ & $1.3 \pm 1.3$ & 8.27 \\
\hline Median & & 4.0 & 5.7 & 7.0 \\
\hline IQR & & 4.0 & 5.6 & 0.6 \\
\hline Maximum & & 14.7 & 18.2 & NA \\
\hline Percent of DCG & & $8.5 \times 10^{-4}$ & $1.9 \times 10^{-2}$ & NA \\
\hline
\end{tabular}

Note: Radioactivities are reported as the measured concentration and either an uncertainty ( $\pm 2 \sigma$ counting error) or as being less than or equal to the detection limit. If the concentration is less than the uncertainty or the detection limit, the result is considered to be a nondetection. See the main volume, Chapter 14, Quality Assurance.

a See Figure 5-3, main volume for description of sampling locations.

b Uranium-235 activities in $\mathrm{Bq} / \mathrm{m}^{3}$ can be determined by dividing the weight in $\mu \mathrm{g} / \mathrm{m}^{3}$ by 12.5 .

c Uranium-238 activities in $\mathrm{Bq} / \mathrm{m}^{3}$ can be determined by dividing the weight in $\mu \mathrm{g} / \mathrm{m}^{3}$ by 80.3 .

d Ratio not determined when one of the masses is negative.

e $\quad I Q R=$ Interquartile range.

f $N A=$ Not applicable.

g $\quad$ DCG = Derived Concentration Guide of activity in air of $0.3 \mu \mathrm{g} / \mathrm{m}^{3}$ for ${ }^{238} \mathrm{U}$ and $0.047 \mu \mathrm{g} / \mathrm{m}^{3}$ for ${ }^{235} \mathrm{U}$. Percent calculated from the median concentration. 


\section{Air Surveillance Monitoring}

Table 5-19. Tritium concentration in air, Site 300, 1999.

\begin{tabular}{|c|c|}
\hline Month & $\begin{array}{c}\text { Sampling location }^{(a)} \\
\text { PRIM } \\
\left(10^{-3} \mathrm{~Bq} / \mathrm{m}^{3}\right) \\
\end{array}$ \\
\hline Jan & $\begin{array}{c}4.63 \pm 9.7 \\
-2.19 \pm 11.4\end{array}$ \\
\hline Feb & $\begin{array}{r}6.11 \pm 7.4 \\
-6.22 \pm 9.8\end{array}$ \\
\hline Mar & $\begin{array}{c}4.66 \pm 13.4 \\
0.370 \pm 7.0\end{array}$ \\
\hline Apr & $\begin{array}{r}11.0 \pm 13.8 \\
-4.18 \pm 11.0 \\
17.5 \pm 13.3\end{array}$ \\
\hline May & $\begin{array}{r}-8.25 \pm 12.8 \\
27.4 \pm 76.2\end{array}$ \\
\hline Jun & $\begin{array}{l}7.96 \pm 15.2 \\
20.5 \pm 20.9\end{array}$ \\
\hline Jul & $\begin{array}{l}-1.55 \pm 20.1 \\
-6.73 \pm 18.1\end{array}$ \\
\hline Aug & $\begin{aligned} 8.77 & \pm 21.2 \\
-13.4 & \pm 8.0\end{aligned}$ \\
\hline Sep & $\begin{array}{r}-6.59 \pm 17.6 \\
11.2 \pm 18.4\end{array}$ \\
\hline Oct & $\begin{array}{l}4.11 \pm 15.7 \\
\text { (b) }^{-1}\end{array}$ \\
\hline & $6.92 \pm 22.0$ \\
\hline Nov & $\begin{array}{l}-15.1 \pm 20.4 \\
-17.4 \pm 21.6\end{array}$ \\
\hline Dec & $\begin{array}{l}19.4 \pm 16.8 \\
1.28 \pm 5.9\end{array}$ \\
\hline Median & 4.11 \\
\hline $\operatorname{IQR}^{(\mathbf{c})}$ & 15.0 \\
\hline Percent of DCG(d) & $1.1 \times 10^{-4}$ \\
\hline Dose $(m S v)^{(e)}$ & $8.5 \times 10^{-7}$ \\
\hline
\end{tabular}

Note: $\quad$ Radioactivities are reported as the measured concentration and either an uncertainty $( \pm 2 \sigma$ counting error) or as being less than or equal to the detection limit. If the concentration is less than the uncertainty or the detection limit, the result is considered to be a nondetection. See the main volume, Chapter 14, Quality Assurance.

a See Figure 5-3, main volume, for sampling locations.

b No data; see main volume, Chapter 14, Quality Assurance.

c $\quad I Q R=$ Interquartile range.

d $\quad$ DCG $=$ Derived Concentration Guide of $3.7 \times 10^{3} \mathrm{~Bq} / \mathrm{m}^{3}$. Percent calculated from the median concentration.

e This dose is the effective dose equivalent. 
Table 5-20. Beryllium in air particulate samples, Site 300 network, 1999.

\begin{tabular}{|c|c|c|c|c|}
\hline \multirow{3}{*}{ Month } & \multicolumn{4}{|c|}{ Sampling location(a) } \\
\hline & \multicolumn{4}{|c|}{$\left(\mathrm{pg} / \mathrm{m}^{3}\right)$} \\
\hline & $801 E$ & EOBS & GOLF & TFIR \\
\hline Jan & 0.660 & 1.04 & 2.58 & 3.24 \\
\hline Feb & 2.28 & 2.47 & 2.69 & 4.42 \\
\hline Mar & 12.0 & 4.74 & 4.60 & 7.32 \\
\hline Apr & 13.2 & 5.50 & 7.98 & 11.7 \\
\hline May & 30.9 & 5.55 & 24.8 & 16.1 \\
\hline Jun & 13.1 & 6.54 & 19.0 & 13.4 \\
\hline Jul & 11.5 & 10.1 & 26.4 & 15.3 \\
\hline Aug & 9.21 & 8.15 & 11.4 & 15.4 \\
\hline Sep & 15.4 & 13.2 & 14.4 & 19.5 \\
\hline Oct & 23.4 & 18.2 & 23.1 & 32.3 \\
\hline Nov & 4.09 & 3.04 & 6.17 & 12.0 \\
\hline Dec & 4.54 & 4.15 & 8.97 & 一 $^{(\mathrm{b})}$ \\
\hline $\operatorname{Median}^{(c)}$ & 11.8 & 5.53 & 10.2 & 13.4 \\
\hline$I R^{(d)}$ & 9.32 & 4.77 & 14.3 & 6.24 \\
\hline Maximum & 30.9 & 18.2 & 26.4 & 32.3 \\
\hline Percent of $\mathrm{ACL}^{(\mathrm{e})}$ & 0.118 & 0.055 & 0.102 & 0.134 \\
\hline
\end{tabular}

a See Figure 5-3, main volume, for sampling locations.

b No sample collected due to lack of power at sampling location.

c Median value for all Site 300 locations is $10.1 \mathrm{pg} / \mathrm{m}^{3}$.

d $I Q R=$ Interquartile range.

e The monthly Ambient Concentration Limit (ACL) is $10,000 \mathrm{pg} / \mathrm{m}^{3}$ as set by the Bay Area Air Quality Management District (BAAQMD). Percent calculated from the median concentration. 



\section{Sewerable Water Monitoring}

Jennifer M. Larson Robert J. Vellinger

Robert A. Williams

Ted A. Giesing

\section{Discharges of Treated Ground Water}

Discharges of ground water to Lawrence Livermore National Laboratory's sanitary sewer must comply with the terms and conditions in Permit 1510G(99), issued by the Livermore Water Reclamation Plant (LWRP). Table 6-1 shows discharge dates and monitoring data for discharges of ground water. The self-monitoring program prescribed in this ground water discharge permit requires compliance with the parameters specified in Table 6-2 in the main volume.

\section{Flow Monitoring Methods}

To monitor effluent flow, LLNL installed a flow chart recorder inside the LLNL Sewer Monitoring Station (SMS) and an ultrasonic flow sensor in the adjacent underground sewer vault (main volume Figure 6-1). Every day a flow totalizer reading was recorded on the flow chart recorder when the daily composite sample was acquired from the SMS. Analysts determined the daily total flows by subtracting sequentially recorded flow totalizer readings. Daily flow totals were estimated when flow totalizer readings were not available. Table $6-2 \mathrm{a}$ shows the daily total flows. Table $6-2 \mathrm{~b}$ presents monthly and annual flow summary statistics for 1999.

\section{Sewage Sampling Methods and Analytical Procedures}

LLNL operated a flow-proportional, peristaltic-pump composite sampler in the SMS. This sampler created a 24-hour composite of the Livermore site sewage effluent by taking a sample every $3785 \mathrm{~L}$ of effluent. Every day, technologists transferred 500-mL aliquots of this 24-hour composite to polyethylene bottles and submitted them for analysis. 


\section{Sewerable Water Monitoring}

Two aliquots were submitted to LLNL's Hazards Control Analytical Laboratory (HCAL) for daily analyses of the gross alpha, gross beta, and tritium activity. For the gross alpha and gross beta analyses, HCAL digested a 150-mL aliquot, plated the digestate onto a planchette, and submitted the planchette to the Hazards Control Radiological Measurements Laboratory (HCRML) for a 100-min count in a gas-proportional counter. For the tritium analyses, HCAL distilled a $100-\mathrm{mL}$ aliquot and submitted the distillate to HCRML. HCRML prepared $5 \mathrm{~mL}$ of the distillate with a scintillation cocktail and counted it for $100 \mathrm{~min}$ in a liquid scintillation counter. The analytical results for the gross alpha, gross beta, and tritium analyses are shown in Table 6-3.

A third daily aliquot was submitted to LLNL's Chemistry and Materials Science Environmental Services (CES). From the aliquots submitted for each month, CES created a composite sample and analyzed it, first, for ${ }^{239} \mathrm{Pu}$, and then for ${ }^{137} \mathrm{Cs}$. The ${ }^{239} \mathrm{Pu}$ was analyzed by adding approximately $15 \mathrm{~L}$ of $\mathrm{MnO}_{2}$ to the entire volume of the monthly composite sample to precipitate the plutonium. After the composite volume was digested with concentrated $\mathrm{HNO}_{3}, \mathrm{CES}$ used ion-exchange chromatography to separate the plutonium from the rest of the sample. The plutonium eluted from the ionexchange column was electroplated onto a stainless steel disk, and its activity was measured by alpha spectroscopy.

Before beginning analysis for ${ }^{137} \mathrm{Cs}$ activity in the monthly composite, CES returned any nonplutonium sample material generated from the ion-exchange process to the monthly composite sample in order to prevent ${ }^{137} \mathrm{Cs}$ loss. For the ${ }^{137} \mathrm{Cs}$ analysis, CES added $\mathrm{NH}_{4} \mathrm{MoPO}_{4}$ to the monthly composite sample in order to precipitate the cesium and then counted the composite sample using gamma spectroscopy. The analytical results for the ${ }^{239} \mathrm{Pu}$ and ${ }^{137} \mathrm{Cs}$ analyses are reported in the main volume Table 6-6.

In 1999, LWRP also provided two types of sample - treated effluent and sludge-to LLNL for analysis. LWRP collected two 500-mL aliquots of treated effluent daily and used them to create two different composite samples: (1) a week of daily aliquots, and (2) a month of daily aliquots. LLNL technologists transferred the weekly sample (composited in a 1-gal polyethylene bottle) to HCAL for gross alpha, gross beta, and tritium analyses. Table 6-4 shows the tritium results for the LWRP weekly composite sample.

CES analyzed the monthly sample, which is composited in a 5-gal polyethylene carboy, for ${ }^{137} \mathrm{Cs}$ using gamma spectroscopy and for ${ }^{239} \mathrm{Pu}$ using alpha spectroscopy. The results of the analysis are presented in Chapter 6 of the main volume. 
The other type of sample was sludge from the LWRP digesters. Each month, LWRP employees provided two 500-mL composite samples from each of the digesters. The composites consisted of aliquots taken from the circulating sludge once a week. LLNL collected the composite samples and submitted one 500-mL composite to HCAL and a second 500-mL composite to CES. HCAL analyzed the monthly composite for gross radioactivity and metals. CES composited all of the monthly samples on a quarterly basis and analyzed the quarterly composites for plutonium, cesium, and gammaemitting radionuclides, using alpha spectroscopy for the plutonium and gamma spectroscopy for the cesium and gamma-emitting radionuclides. Table 6-5 in the main volume shows the results for the ${ }^{239} \mathrm{Pu}$ analyses.

Throughout Chapter 6, gross alpha, gross beta, and tritium are displayed in bequerels per unit volume, and the activities shown in Tables 6-3 and 6-4 are the measured concentrations and their associated $\pm 2 \sigma$ counting errors. A $\pm 2 \sigma$ error is not shown when the measured concentration is below the limit of sensitivity (LOS). The LOS is determined individually for each sample analysis according to the following equation:

$$
\operatorname{LOS}=\frac{\mathrm{C}}{\mathrm{Et}}
$$

where

$\mathrm{C}=$ Minimum significant count, above background radiation, for a length of time $(\mathrm{t})$

$\mathrm{E}=$ System counting efficiency

and

$\mathrm{t}=$ Sample counting time $(\mathrm{t})$.

LLNL also operated monitoring station C196 with a flow-proportional, peristaltic pump composite sampler adjacent to the SMS. This sampler functioned as a weekly composite sampler and acquired a 60-mL sample for every 30,280 L of effluent LLNL discharged during a seven-day period. Another sampler operated once a month for 24 hours as a single-day composite sampler and collected a $65-\mathrm{mL}$ sample for every $7570 \mathrm{~L}$ of effluent discharged.

Aliquots were acquired each week from the weekly composite sample and every month from the 24-hour composite sample. From each weekly composite (and each monthly 


\section{Sewerable Water Monitoring}

24-hour composite), analysts transferred one 1-L aliquot to a polyethylene bottle. This aliquot was submitted to an off-site contract laboratory for analyses of aluminum, arsenic, beryllium, cadmium, chromium, copper, iron, lead, mercury, nickel, selenium, silver, and zinc. The results for these analyses are presented in Tables 6-5 and 6-6; the EPA methods used for these analyses are identified by the method numbers 200.7, SM-3114B, 210.2, 200.7, 200.7, 200.7, 200.7, 239.2, 245.2, 249.2, 270.2, 200.7, and 200.7, respectively. In September 1999, sampling for aluminum, beryllium, selenium, and iron was reduced from weekly to twice monthly. LWRP does not require sampling for these parameters, and the change in sampling frequency reduced costs while still allowing continuity with historical records. See the main volume Table 6-2 for constituent analyses required by the LWRP permit.

Two additional aliquots from the weekly composite were submitted each week to HCAL for analyses of gross alpha, beta, and tritium. A subset of these results contributes to the completeness of the daily analytical results for gross alpha, gross beta, and tritium; this subset is reported and footnoted in Table 6-3.

Aliquots were submitted to the contract analytical laboratory for more extensive analyses on the 24-hour composite than on the weekly composite sample. Under the heading of "Composite sample," Table 6-7 lists these results by month, parameters, and the EPA method numbers used for the analyses. (The analytical methods are EPA methods unless otherwise indicated.) It should be noted that only Table 6-6 reports the monthly metals analytical results for those metals mentioned in the previous paragraph.

Concurrent with the monthly acquisition of a 24-hour composite, a portable, peristalticpump sampler collected instantaneous grab samples from the sewage stream in the sewer vault adjacent to SMS. These samples were submitted to a contract analytical laboratory for additional monitoring of water quality parameters and organic compounds. The results of this monitoring are presented in Table 6-7 under the "Grab sample" heading. The table lists the parameters and the EPA method numbers used for the analyses. The last four entries show the oil and grease analysis of samples that were acquired at 4-hour intervals during the day as well as the time of collection of each oil and grease sample. Based on an April 1, 1999, letter from the LWRP, LLNL suspended its monthly oil and grease sampling in May 1999. In a subsequent letter from the LWRP, dated August 18, 1999, oil and grease sampling was reinstated, but the sampling frequency was reduced from monthly to semiannually (every six months). The letter also changed the cyanide sampling frequency from monthly to semiannually. LLNL began semiannual sampling for these parameters in July 1999. 


\section{Quality Assurance Methods}

Standard quality control and quality assurance procedures were followed in the collection of LLNL samples. When each sewage field sample was collected, it was labeled with the sampling location and date of sampling. In the laboratory, each sample was assigned a number that accompanied that sample during analysis. Additionally, split samples accounted for approximately $10 \%$ of the samples submitted for analytical work in 1999. 


\section{Sewerable Water Monitoring}

Table 6-1. Laboratory analytical results for ground water discharges to the sanitary sewer, January 1 through December 31, 1999.

\begin{tabular}{|c|c|c|c|c|c|c|}
\hline \multirow[b]{2}{*}{ Parameter } & \multicolumn{6}{|c|}{ Discharge dates } \\
\hline & 3/1/99 & 3/23/99 & $3 / 26 / 99$ & $5 / 6 / 99$ & $5 / 26 / 99$ & $6 / 14 / 99$ \\
\hline $\mathrm{pH}^{(\mathrm{a})}(\mathrm{pH}$ units) & 6.5 & 7 & 8.2 & 7.5 & 6.5 & 7.5 \\
\hline Arsenic (mg/L) & 0.0029 & 0.01 & $<0.002$ & $<0.002$ & $<0.002$ & 0.0022 \\
\hline Cadmium (mg/L) & $<0.0005$ & $<0.0005$ & $<0.0005$ & $<0.0005$ & $<0.0005$ & $<0.0005$ \\
\hline Nickel (mg/L) & 0.045 & 0.014 & $<0.005$ & $<0.002$ & $<0.005$ & $<0.002$ \\
\hline Total chromium (mg/L) & $<0.001$ & $<0.001$ & 0.43 & $<0.001$ & 0.005 & $<0.001$ \\
\hline Copper (mg/L) & 0.021 & $<0.010$ & $<0.010$ & 0.015 & 0.003 & $<0.001$ \\
\hline Lead (mg/L) & 0.0027 & $<0.002$ & $<0.002$ & $<0.005$ & $<0.005$ & $<0.005$ \\
\hline Mercury (mg/L) & $<0.0002$ & $<0.0002$ & $<0.0002$ & $<0.0002$ & $<0.0002$ & $<0.0002$ \\
\hline Silver (mg/L) & $<0.0005$ & $<0.0005$ & $<0.0025$ & $<0.001$ & $<0.001$ & $<0.001$ \\
\hline Zinc (mg/L) & 0.02 & $<0.02$ & $<0.02$ & 0.047 & $<0.02$ & $<0.02$ \\
\hline Total toxic organics $^{(b)}(\mathrm{mg} / \mathrm{L})$ & $\mathrm{nd}(\mathrm{c})$ & nd & 0.0016 & 0.0013 & nd & nd \\
\hline
\end{tabular}

\begin{tabular}{|c|c|c|c|c|c|c|}
\hline \multirow[b]{2}{*}{ Parameter } & \multicolumn{6}{|c|}{ Discharge dates } \\
\hline & $\begin{array}{c}\text { 6/14/99- } \\
7 / 7 / 99\end{array}$ & $\begin{array}{c}\text { 7/7/99- } \\
\text { 9/8/99 }\end{array}$ & 8/2/99 & $\begin{array}{c}8 / 31 / 99 \\
9 / 29 / 99\end{array}$ & $\begin{array}{c}11 / 13 / 99 \\
11 / 23 / 99\end{array}$ & $12 / 6 / 99$ \\
\hline $\mathrm{pH}^{(\mathrm{a})}(\mathrm{pH}$ units $)$ & 8 & 8 & 7.5 & 7.5 & $6.5-8.0$ & 6.5 \\
\hline Arsenic (mg/L) & $<0.002$ & $<0.002$ & $<0.002$ & $<0.002$ & 0.0074 & 0.0033 \\
\hline Cadmium (mg/L) & $<0.0005$ & $<0.0005$ & 0.002 & $<0.0005$ & $<0.001$ & $<0.0005$ \\
\hline Nickel (mg/L) & $<0.002$ & $<0.002$ & 0.0081 & $<0.005$ & $<0.01$ & $<0.0051$ \\
\hline Total chromium (mg/L) & $<0.0073$ & $<0.001$ & 0.008 & $<0.036$ & $<0.001$ & 0.077 \\
\hline Copper (mg/L) & 0.172 & 0.0087 & $<0.01$ & $<0.01$ & $<0.001$ & 0.0054 \\
\hline Lead (mg/L) & 0.017 & $<0.005$ & 0.073 & $<0.002$ & $<0.005$ & $<0.005$ \\
\hline Mercury (mg/L) & $<0.0002$ & $<0.0002$ & 0.0003 & $<0.0002$ & $<0.0002$ & $<0.0002$ \\
\hline Silver (mg/L) & $<0.001$ & $<0.001$ & 0.0091 & $<0.0005$ & $<0.001$ & $<0.001$ \\
\hline Zinc (mg/L) & $<0.02$ & 0.024 & 0.03 & $<0.02$ & $<0.05$ & 0.023 \\
\hline Total toxic organics $^{(b)}(\mathrm{mg} / \mathrm{L})$ & nd & 0.055 & nd & nd & nd & nd \\
\hline
\end{tabular}

a $\mathrm{pH}$ was verified prior to discharge. The $\mathrm{pH}$ at final discharge could have been slightly different but was always between 5 and 10 .

b Total toxic organics is the sum of concentrations of compounds detected by EPA Method 601 for wastewater.

c $\mathrm{nd}=$ Not detected. Concentrations were not detected by EPA Method 601 for wastewater. 
Table 6-2a. Daily flow totals for LLNL site sanitary sewer effluent (ML), 1999.

\begin{tabular}{|c|c|c|c|c|c|c|c|c|c|c|c|c|}
\hline Day & Jan & Feb & Mar & Apr & May & Jun & Jul & Aug & Sep & Oct & Nov & Dec \\
\hline 1 & 0.472 & 0.535 & 0.550 & 1.659 & 1.437 & 0.670 & 1.394 & 0.508 & 2.844 & 1.140 & 0.632 & 1.089 \\
\hline 2 & 0.442 & 1.100 & 1.016 & 1.768 & 0.932 & 1.741 & 1.353 & 0.528 & 1.716 & 1.079 & 1.120 & 1.177 \\
\hline 3 & 0.441 & 1.058 & 1.024 & 1.871 & 0.991 & 1.941 & 1.072 & 1.249 & 1.314 & 0.568 & 1.075 & 1.275 \\
\hline 4 & 0.528 & 1.428 & 0.991 & 0.986 & 1.679 & 1.577 & 0.568 & 1.200 & 1.135 & 0.587 & 1.130 & 1.095 \\
\hline 5 & 1.131 & 1.063 & 1.444 & 0.855 & 1.317 & 1.640 & 0.578 & 1.151 & 0.551 & 1.260 & 1.215 & 0.511 \\
\hline 6 & 1.225 & 1.077 & 1.641 & 1.173 & 1.185 & 1.127 & 0.679 & 1.270 & 0.510 & 1.172 & 1.184 & 0.493 \\
\hline 7 & 1.294 & 0.370 & 1.204 & 1.073 & 1.094 & 1.172 & 1.245 & 1.220 & 0.519 & 1.193 & 0.649 & 1.168 \\
\hline 8 & 1.205 & 0.560 & 1.327 & 1.089 & 1.169 & 1.373 & 1.792 & 0.818 & 1.103 & 1.226 & 0.705 & 1.534 \\
\hline 9 & 0.977 & 1.258 & 1.283 & 1.098 & 0.569 & 1.386 & 1.772 & 0.667 & 1.230 & 1.056 & 1.147 & 1.619 \\
\hline 10 & 0.519 & 1.292 & 1.544 & 0.936 & 0.532 & 1.353 & 1.211 & 1.169 & 1.270 & 0.587 & 1.213 & 1.167 \\
\hline 11 & 0.404 & 1.130 & 1.763 & 0.377 & 1.207 & 1.261 & 0.614 & 1.246 & 1.125 & 0.610 & 1.000 & 0.937 \\
\hline 12 & 1.162 & 1.009 & 1.656 & 0.393 & 1.216 & 1.130 & 0.775 & 1.224 & 0.614 & 1.137 & 1.173 & 0.467 \\
\hline 13 & 1.234 & 0.874 & 1.023 & 1.184 & 1.244 & 0.597 & 1.207 & 1.231 & 0.666 & 1.135 & 1.093 & 0.425 \\
\hline 14 & 2.335 & 0.377 & 0.382 & 1.224 & 1.294 & 0.743 & 1.099 & 1.012 & 1.175 & 1.100 & 0.526 & 1.196 \\
\hline 15 & 1.072 & 0.358 & 0.413 & 1.142 & 1.009 & 1.350 & 1.691 & 0.483 & 1.195 & 1.179 & 0.610 & 1.394 \\
\hline 16 & 1.167 & 0.454 & 1.184 & 1.012 & 0.578 & 1.173 & 1.288 & 0.557 & 1.233 & 0.992 & 1.169 & 2.088 \\
\hline 17 & 0.418 & 1.155 & 1.189 & 0.967 & 0.573 & 1.255 & 1.033 & 1.102 & 1.553 & 0.625 & 1.189 & 1.375 \\
\hline 18 & 0.392 & 1.054 & 1.104 & 0.372 & 1.286 & 1.229 & 0.634 & 1.116 & 1.138 & 0.634 & 1.109 & 0.953 \\
\hline 19 & 0.698 & 0.918 & 1.205 & 0.415 & 1.346 & 1.090 & 0.651 & 1.127 & 0.620 & 1.133 & 1.230 & 0.439 \\
\hline 20 & 1.291 & 0.912 & 1.317 & 1.091 & 1.292 & 0.581 & 1.191 & 1.216 & 0.647 & 1.202 & 1.068 & 0.491 \\
\hline 21 & 1.170 & 0.549 & 0.681 & 1.235 & 1.384 & 0.637 & 1.227 & 1.883 & 1.285 & 1.225 & 0.554 & 1.382 \\
\hline 22 & 1.086 & 0.444 & 0.503 & 1.416 & 1.136 & 1.228 & 1.231 & 1.470 & 1.229 & 1.159 & 0.502 & 1.213 \\
\hline 23 & 1.034 & 1.058 & 1.326 & 1.269 & 0.484 & 1.186 & 1.270 & 0.604 & 1.261 & 1.084 & 1.152 & 1.177 \\
\hline 24 & 0.544 & 1.156 & 1.266 & 0.984 & 0.506 & 1.502 & 1.246 & 1.229 & 1.335 & 0.560 & 0.812 & 0.580 \\
\hline 25 & 0.502 & 0.778 & 1.246 & 0.378 & 1.202 & 1.217 & 0.769 & 1.143 & 1.237 & 0.606 & 0.935 & 0.473 \\
\hline 26 & 1.088 & 1.073 & 1.329 & 0.386 & 1.248 & 1.159 & 0.622 & 1.150 & 0.670 & 1.244 & 0.420 & 0.473 \\
\hline 27 & 1.202 & 1.006 & 1.728 & 1.172 & 1.309 & 0.806 & 1.216 & 1.312 & 0.503 & 1.190 & 0.448 & 0.506 \\
\hline 28 & 1.136 & 0.522 & 1.263 & 1.163 & 1.349 & 0.799 & 1.206 & 1.156 & 1.248 & 1.199 & 0.542 & 0.917 \\
\hline 29 & 1.310 & & 1.249 & 1.032 & 1.164 & 1.306 & 1.152 & 0.645 & 1.372 & 1.332 & 0.510 & 0.844 \\
\hline 30 & 1.144 & & 1.975 & 1.150 & 0.591 & 1.217 & 1.645 & 0.680 & 1.123 & 1.040 & 1.165 & 0.769 \\
\hline 31 & 0.482 & & 1.909 & & 0.575 & & 1.114 & 2.373 & & 0.626 & & 0.592 \\
\hline
\end{tabular}

Note: Shaded volumes indicate an estimation of the daily flow total; actual volumes are not available. Weekend and holiday daily flow totals are shown in the boxed areas. Note that the majority of the flow volume recorded for a given day was actually discharged on the previous day. 


\section{Sewerable Water Monitoring}

Table 6-2b. Monthly and annual flow summary statistics for LLNL site sanitary sewer effluent (ML), 1999.

\begin{tabular}{|c|c|c|c|c|c|c|c|c|c|c|c|c|c|}
\hline & Jan & Feb & Mar & Apr & May & Jun & Jul & Aug & Sep & Oct & Nov & Dec & 1999 \\
\hline \multicolumn{14}{|c|}{ Weekend days and holidays } \\
\hline Total & 5.842 & 4.169 & 7.572 & 5.335 & 6.331 & 7.132 & 5.890 & 6.960 & 5.300 & 5.403 & 6.098 & 5.450 & 71.482 \\
\hline Minimum & 0.392 & 0.358 & 0.382 & 0.372 & 0.484 & 0.581 & 0.568 & 0.483 & 0.503 & 0.560 & 0.420 & 0.425 & 0.358 \\
\hline Maximum & 0.698 & 0.560 & 1.327 & 1.173 & 0.991 & 1.172 & 0.775 & 1.470 & 0.670 & 0.634 & 0.705 & 0.592 & 1.470 \\
\hline Mean & 0.487 & 0.463 & 0.841 & 0.593 & 0.633 & 0.792 & 0.654 & 0.696 & 0.589 & 0.600 & 0.554 & 0.495 & 0.611 \\
\hline \multicolumn{14}{|c|}{ Weekdays } \\
\hline Total & 23.263 & 20.399 & 30.163 & 25.535 & 26.567 & 28.314 & 28.655 & 26.779 & 28.121 & 25.477 & 21.179 & 24.369 & 308.821 \\
\hline Minimum & 0.977 & 0.778 & 0.991 & 0.936 & 1.009 & 1.090 & 1.033 & 1.012 & 1.103 & 0.992 & 0.812 & 0.769 & 0.769 \\
\hline Maximum & 2.335 & 1.428 & 1.975 & 1.871 & 1.679 & 1.941 & 1.792 & 2.373 & 2.844 & 1.332 & 1.230 & 2.088 & 2.844 \\
\hline Mean & 1.224 & 1.074 & 1.371 & 1.216 & 1.265 & 1.348 & 1.303 & 1.275 & 1.339 & 1.158 & 1.115 & 1.218 & 1.245 \\
\hline \multicolumn{14}{|c|}{ All days } \\
\hline Total & 29.105 & 24.568 & 37.735 & 30.870 & 32.898 & 35.446 & 34.545 & 33.739 & 33.421 & 30.880 & 27.277 & 29.819 & 380.303 \\
\hline Minimum & 0.392 & 0.358 & 0.382 & 0.372 & 0.484 & 0.581 & 0.568 & 0.483 & 0.503 & 0.560 & 0.420 & 0.425 & 0.358 \\
\hline Maximum & 2.335 & 1.428 & 1.975 & 1.871 & 1.679 & 1.941 & 1.792 & 2.373 & 2.844 & 1.332 & 1.230 & 2.088 & 2.844 \\
\hline Mean & 0.939 & 0.877 & 1.217 & 1.029 & 1.061 & 1.182 & 1.114 & 1.088 & 1.114 & 0.996 & 0.909 & 0.962 & 1.042 \\
\hline
\end{tabular}


Table 6-3. Daily monitoring results for gross alpha, gross beta, and tritium in the sanitary sewer effluent, 1999.

\begin{tabular}{|c|c|c|c|c|c|c|c|}
\hline \multirow{2}{*}{\multicolumn{2}{|c|}{ Date }} & \multicolumn{2}{|c|}{ Gross alpha $(\mu B q / m L)$} & \multicolumn{2}{|c|}{ Gross beta $(\mathrm{mBq} / \mathrm{mL})$} & \multicolumn{2}{|c|}{ Tritium (mBq/mL) } \\
\hline & & Activity & $\operatorname{LOS}(a)$ & Activity & LOS & Activity & LOS \\
\hline \multirow[t]{31}{*}{ January } & 1 & 2.94 & 74.4 & $0.138 \pm 0.084$ & 0.124 & 9.88 & 11.1 \\
\hline & 2 & 74.4 & 75.9 & 0.0548 & 0.125 & $14.9 \pm 6.9$ & 11.2 \\
\hline & 3 & $166 \pm 70$ & 70.3 & 0.0326 & 0.124 & 6.03 & 11.4 \\
\hline & 4 & $78.8 \pm 52.0$ & 74.0 & $0.142 \pm 0.084$ & 0.124 & $11.0 \pm 6.3$ & 10.7 \\
\hline & 5 & 31.0 & 100 & $0.792 \pm 0.119$ & 0.133 & -1.70 & 11.4 \\
\hline & 6 & 39.2 & 89.9 & $0.525 \pm 0.105$ & 0.129 & -1.70 & 11.8 \\
\hline & 7 & $148 \pm 68$ & 99.9 & $0.614 \pm 0.111$ & 0.131 & -6.62 & 12.1 \\
\hline & 8 & 67.7 & 113 & $0.725 \pm 0.116$ & 0.135 & -0.585 & 11.5 \\
\hline & 9 & $111 \pm 59$ & 107 & $0.670 \pm 0.114$ & 0.134 & 5.25 & 11.1 \\
\hline & 10 & $133 \pm 70$ & 90.3 & $0.199 \pm 0.087$ & 0.127 & 5.55 & 10.9 \\
\hline & 11 & $182 \pm 80$ & 88.8 & $0.171 \pm 0.085$ & 0.127 & 5.92 & 11.2 \\
\hline & 12 & $110 \pm 53$ & 95.8 & $0.781 \pm 0.117$ & 0.130 & 0.662 & 11.4 \\
\hline & 13 & 32.6 & 91.4 & $0.611 \pm 0.110$ & 0.128 & -1.60 & 11.5 \\
\hline & 14 & $163 \pm 68$ & 84.0 & $0.444 \pm 0.098$ & 0.125 & -8.29 & 12.3 \\
\hline & 15 & $145 \pm 69$ & 114 & $0.966 \pm 0.126$ & 0.136 & 6.51 & 12.0 \\
\hline & 16 & 87.0 & 107 & $0.810 \pm 0.122$ & 0.135 & $14.7 \pm 8.2$ & 11.9 \\
\hline & 17 & $91.4 \pm 55.7$ & 84.0 & $0.370 \pm 0.096$ & 0.126 & $12.3 \pm 7.0$ & 11.5 \\
\hline & 18 & 13.4 & 71.8 & $0.182 \pm 0.086$ & 0.123 & $13.4 \pm 7.0$ & 11.2 \\
\hline & 19 & 62.9 & 93.2 & $0.293 \pm 0.094$ & 0.130 & $15.8 \pm 7.4$ & 11.7 \\
\hline & 20 & 8.51 & 97.3 & $0.692 \pm 0.118$ & 0.131 & 7.25 & 11.4 \\
\hline & 21 & $123 \pm 58$ & 87 & $0.810 \pm 0.113$ & 0.127 & 1.82 & 11.2 \\
\hline & 22 & $107 \pm 57$ & 90.7 & $0.703 \pm 0.112$ & 0.128 & -1.24 & 11.8 \\
\hline & 23 & $135 \pm 62$ & 85.8 & $0.744 \pm 0.112$ & 0.128 & $28.5 \pm 6.5$ & 10.8 \\
\hline & 24 & 46.6 & 79.6 & 0.0755 & 0.127 & 9.81 & 12.0 \\
\hline & 25 & 38.1 & 75.9 & 0.0799 & 0.126 & -1.52 & 12.2 \\
\hline & 26 & 29.4 & 95.8 & $0.644 \pm 0.109$ & 0.132 & $82.5 \pm 9.1$ & 11.9 \\
\hline & 27 & 51.4 & 94.7 & $0.648 \pm 0.110$ & 0.132 & 2.93 & 11.7 \\
\hline & 28 & 35.7 & 98.8 & $0.692 \pm 0.118$ & 0.133 & -6.22 & 12.1 \\
\hline & 29 & $158 \pm 71$ & 92.5 & $0.500 \pm 0.105$ & 0.128 & -9.58 & 12.0 \\
\hline & 30 & $170 \pm 73$ & 92.1 & $0.522 \pm 0.104$ & 0.128 & 8.21 & 11.9 \\
\hline & 31 & 18.7 & 82.9 & 0.103 & 0.125 & $21.5 \pm 8.2$ & 12.5 \\
\hline \multirow[t]{3}{*}{ February } & 1 & $138 \pm 73$ & 87.7 & 0.106 & 0.127 & $20.4 \pm 7.8$ & 11.3 \\
\hline & 2 & 63.3 & 103 & $0.781 \pm 0.117$ & 0.132 & 7.44 & 11.7 \\
\hline & 3 & 80.3 & 86.6 & $0.570 \pm 0.108$ & 0.126 & 2.35 & 11.8 \\
\hline
\end{tabular}




\section{Sewerable Water Monitoring}

Table 6-3. Daily monitoring results for gross alpha, gross beta, and tritium in the sanitary sewer effluent, 1999 (continued).

\begin{tabular}{|c|c|c|c|c|c|c|c|}
\hline \multirow{2}{*}{\multicolumn{2}{|c|}{ Date }} & \multicolumn{2}{|c|}{ Gross alpha $(\mu \mathrm{Bq} / \mathrm{mL})$} & \multicolumn{2}{|c|}{ Gross beta $(\mathrm{mBq} / \mathrm{mL})$} & \multicolumn{2}{|c|}{ Tritium (mBq/mL) } \\
\hline & & Activity & $\operatorname{LOS}^{(a)}$ & Activity & LOS & Activity & LOS \\
\hline \multirow[t]{25}{*}{ February } & 4 & 19.2 & 89.5 & $0.662 \pm 0.113$ & 0.127 & -2.23 & 11.8 \\
\hline & 5 & 45.5 & 94.7 & $0.707 \pm 0.113$ & 0.131 & 1.30 & 11.6 \\
\hline & 6 & $131 \pm 61$ & 93.2 & $0.866 \pm 0.121$ & 0.130 & 5.70 & 11.4 \\
\hline & 7 & 1.07 & 79.2 & $0.210 \pm 0.090$ & 0.126 & $17.3 \pm 7.3$ & 11.3 \\
\hline & 8 & 73.6 & 85.8 & $0.139 \pm 0.085$ & 0.128 & $45.5 \pm 7.7$ & 12.0 \\
\hline & 9 & 91.0 & 91.8 & $0.648 \pm 0.110$ & 0.130 & $12.2 \pm 7.0$ & 11.9 \\
\hline & 10 & 41.8 & 89.5 & $0.599 \pm 0.108$ & 0.129 & -13.9 & 14.1 \\
\hline & 11 & $110 \pm 55$ & 91.4 & $0.847 \pm 0.119$ & 0.130 & $19.2 \pm 8.2$ & 13.5 \\
\hline & 12 & 24.8 & 92.9 & $0.829 \pm 0.116$ & 0.131 & $14.1 \pm 7.7$ & 12.5 \\
\hline & 13 & $108 \pm 52$ & 81.4 & $0.762 \pm 0.114$ & 0.127 & 5.37 & 12.2 \\
\hline & 14 & -27.9 & 72.2 & 0.0907 & 0.124 & $18.4 \pm 8.1$ & 12.8 \\
\hline & 15 & -15.2 & 72.5 & 0.067 & 0.125 & -3.42 & 13.2 \\
\hline & 16 & 34.3 & 75.1 & $0.235 \pm 0.089$ & 0.125 & 0.165 & 12.4 \\
\hline & 17 & -144 & 107 & $5.77 \pm 0.25$ & 0.140 & 5.48 & 11.8 \\
\hline & 18 & $196 \pm 73$ & 94.0 & $1.30 \pm 0.13$ & 0.130 & -0.273 & 12.0 \\
\hline & 19 & $308 \pm 105$ & 117 & $1.09 \pm 0.13$ & 0.137 & 3.77 & 11.3 \\
\hline & 20 & 61.4 & 87.3 & $0.944 \pm 0.123$ & 0.127 & 5.37 & 11.4 \\
\hline & 21 & $151 \pm 73$ & 84.7 & $0.202 \pm 0.087$ & 0.127 & $17.6 \pm 9.4$ & 13.0 \\
\hline & 22 & 36.2 & 81.8 & $0.185 \pm 0.087$ & 0.126 & 8.95 & 13.1 \\
\hline & 23 & 40.0 & 92.9 & $0.744 \pm 0.112$ & 0.129 & $24.0 \pm 7.4$ & 11.9 \\
\hline & 24 & 66.2 & 92.1 & $0.810 \pm 0.113$ & 0.128 & $12.5 \pm 6.1$ & 11.8 \\
\hline & 25 & $111 \pm 50$ & 92.9 & $0.977 \pm 0.127$ & 0.128 & $35.2 \pm 7.7$ & 12.7 \\
\hline & 26 & $146 \pm 61$ & 96.6 & $0.907 \pm 0.118$ & 0.130 & 0.102 & 14.8 \\
\hline & 27 & 52.5 & 87.3 & $0.762 \pm 0.114$ & 0.127 & $14.7 \pm 8.5$ & 11.8 \\
\hline & 28 & $161 \pm 66$ & 70.7 & $0.168 \pm 0.083$ & 0.122 & 5.81 & 11.6 \\
\hline \multirow[t]{9}{*}{ March } & 1 & 27.6 & 71.0 & 0.087 & 0.122 & $16.1 \pm 6.7$ & 11.0 \\
\hline & 2 & $152 \pm 59$ & 85.5 & $0.844 \pm 0.118$ & 0.126 & 10.9 & 11.3 \\
\hline & 3 & $185 \pm 63$ & 83.6 & $1.05 \pm 0.13$ & 0.125 & 4.37 & 11.0 \\
\hline & 4 & 73.6 & 104 & $0.955 \pm 0.124$ & 0.132 & $15.0 \pm 7.0$ & 12.0 \\
\hline & 5 & $102 \pm 54$ & 84.0 & $0.503 \pm 0.101$ & 0.125 & $19.7 \pm 8.3$ & 12.3 \\
\hline & 6 & $182 \pm 82$ & 122 & $0.792 \pm 0.119$ & 0.135 & $71.4 \pm 8.6$ & 12.0 \\
\hline & 7 & $354 \pm 117$ & 114 & $0.455 \pm 0.105$ & 0.135 & $71.0 \pm 8.5$ & 12.1 \\
\hline & 8 & $119 \pm 67$ & 90.7 & $0.217 \pm 0.089$ & 0.127 & $36.9 \pm 8.1$ & 12.5 \\
\hline & 9 & 59.9 & 98.8 & $0.777 \pm 0.117$ & 0.130 & $17.8 \pm 7.1$ & 12.0 \\
\hline
\end{tabular}


Table 6-3. Daily monitoring results for gross alpha, gross beta, and tritium in the sanitary sewer effluent, 1999 (continued).

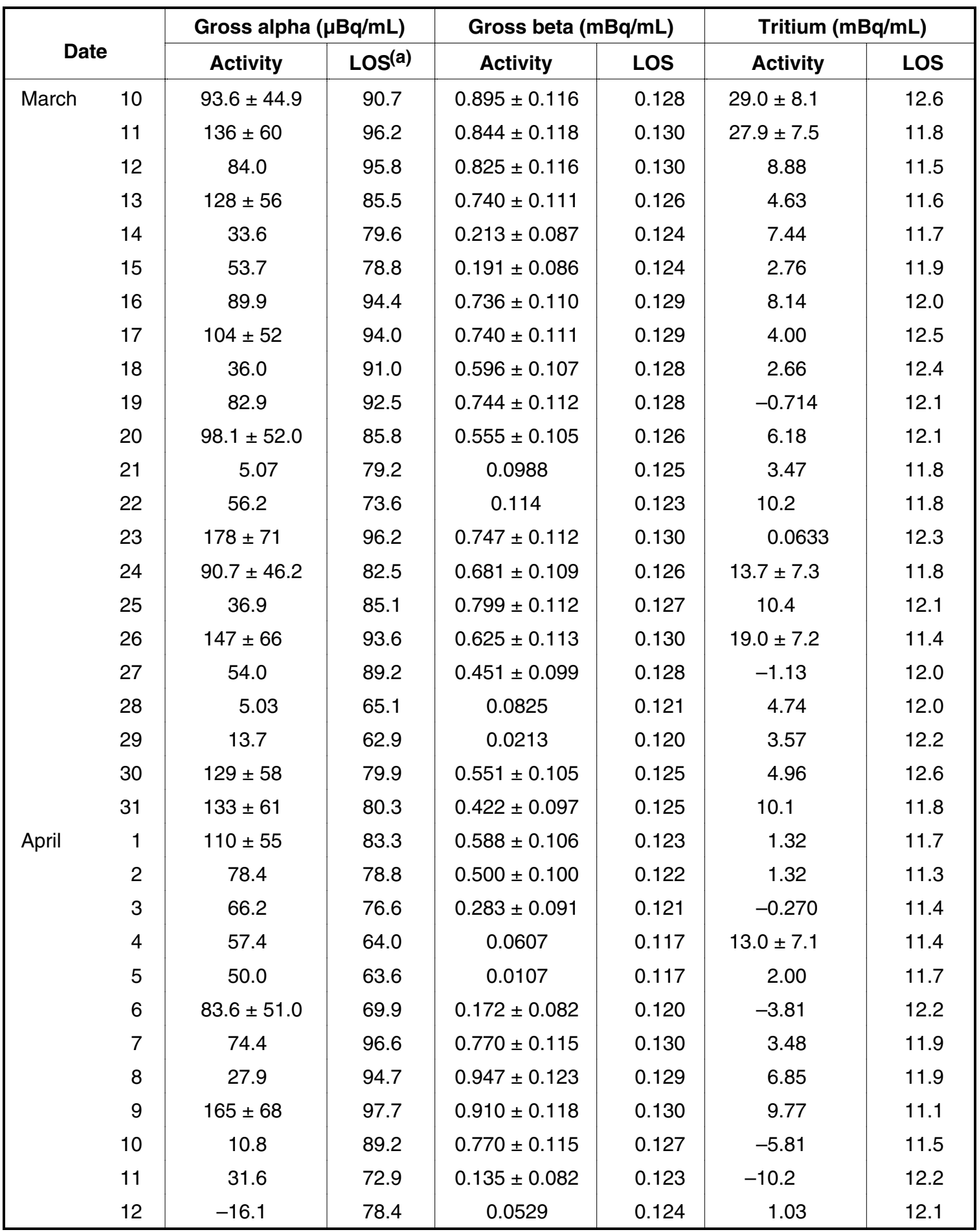




\section{Sewerable Water Monitoring}

Table 6-3. Daily monitoring results for gross alpha, gross beta, and tritium in the sanitary sewer effluent, 1999 (continued).

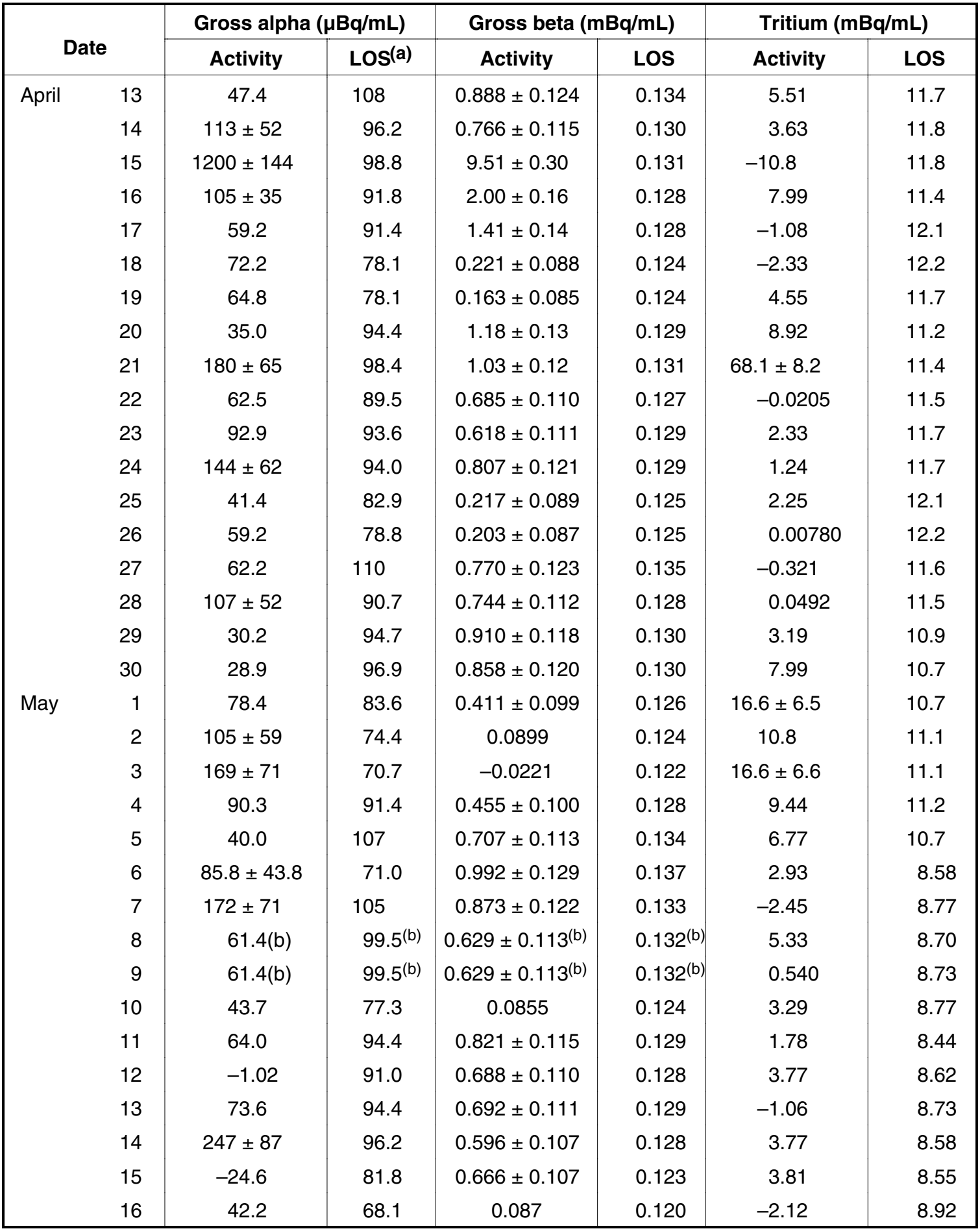


Table 6-3. Daily monitoring results for gross alpha, gross beta, and tritium in the sanitary sewer effluent, 1999 (continued).

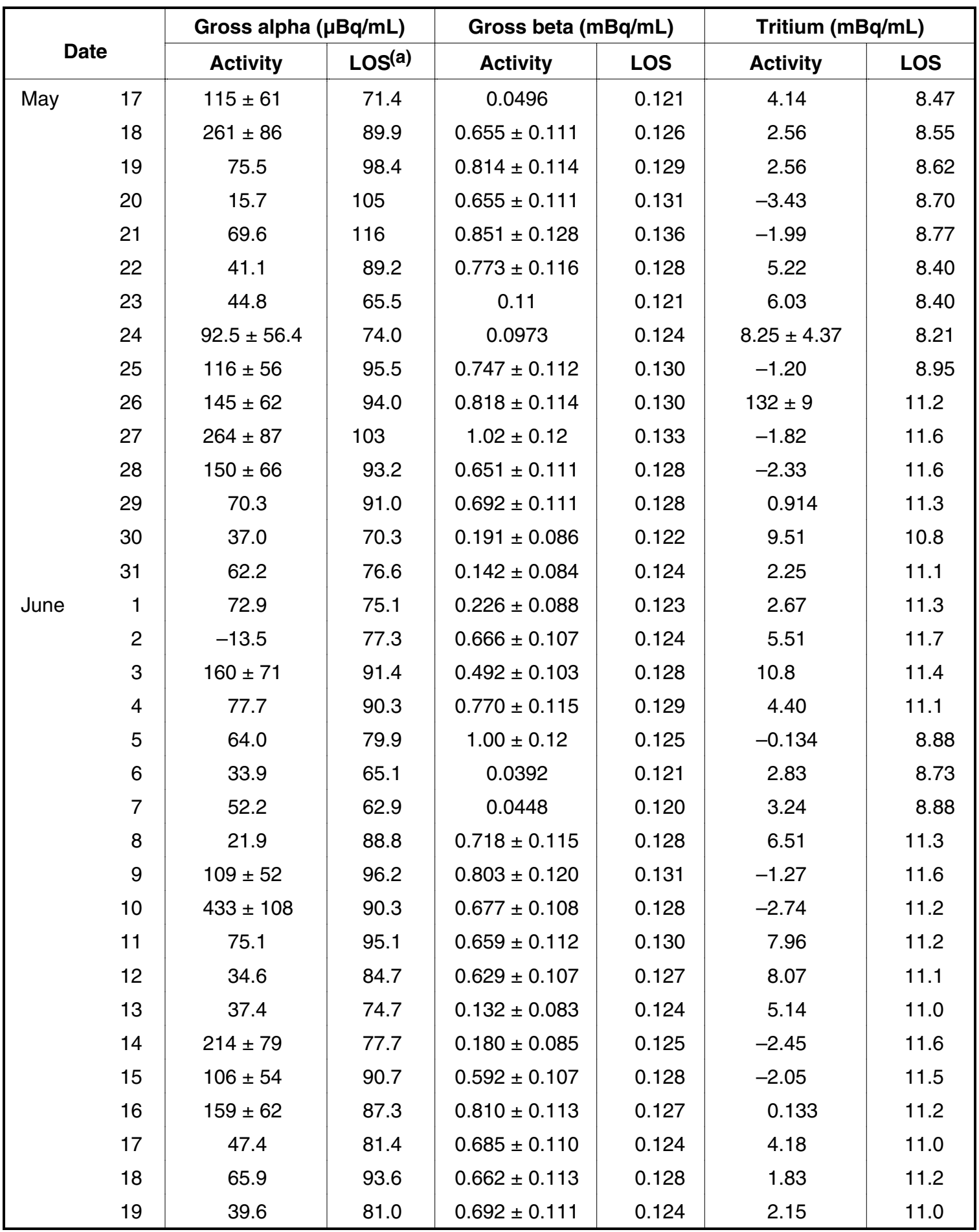




\section{Sewerable Water Monitoring}

Table 6-3. Daily monitoring results for gross alpha, gross beta, and tritium in the sanitary sewer effluent, 1999 (continued).

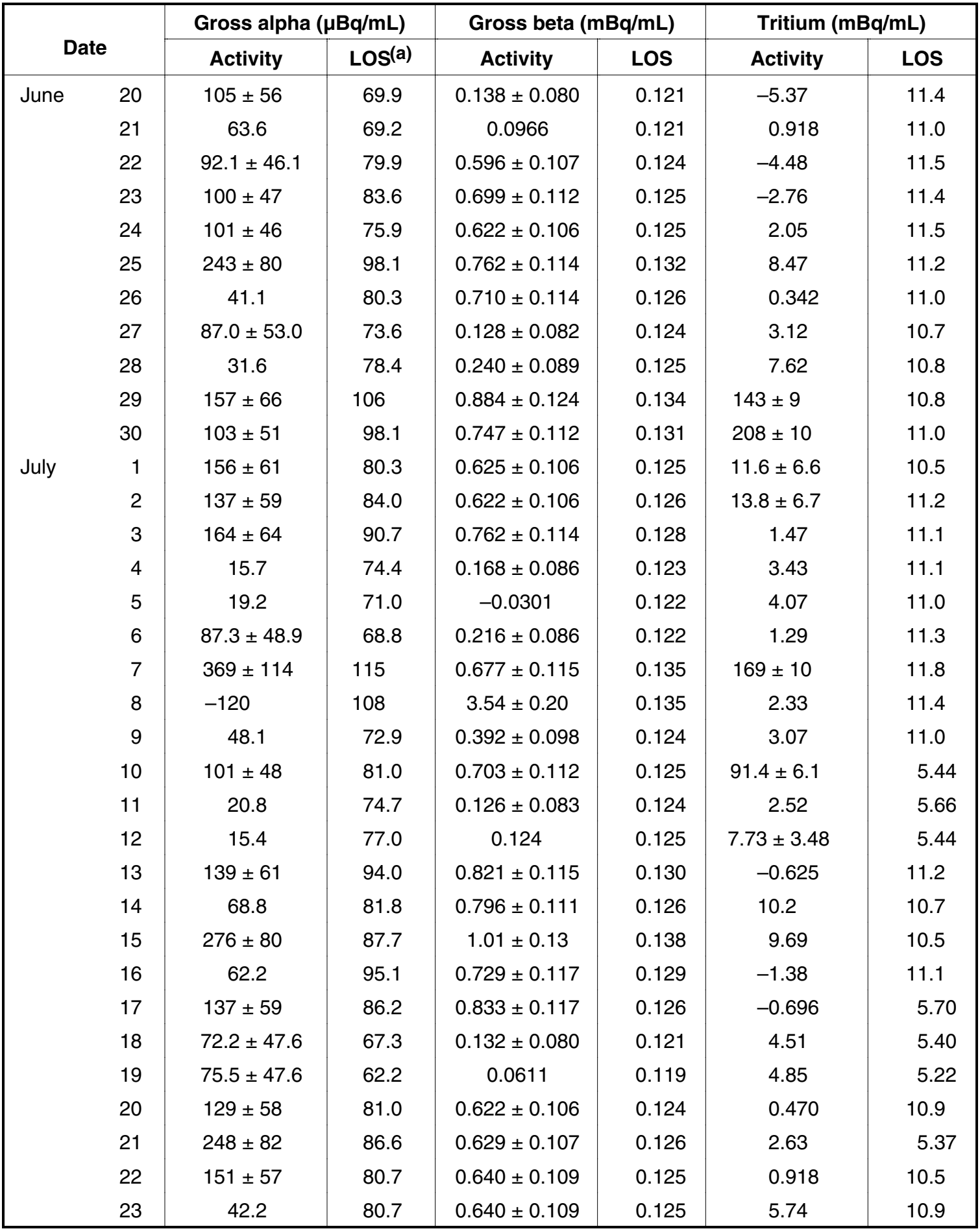


Table 6-3. Daily monitoring results for gross alpha, gross beta, and tritium in the sanitary sewer effluent, 1999 (continued).

\begin{tabular}{|c|c|c|c|c|c|c|c|}
\hline \multirow{2}{*}{\multicolumn{2}{|c|}{ Date }} & \multicolumn{2}{|c|}{ Gross alpha $(\mu \mathrm{Bq} / \mathrm{mL})$} & \multicolumn{2}{|c|}{ Gross beta $(\mathrm{mBq} / \mathrm{mL})$} & \multicolumn{2}{|c|}{ Tritium $(\mathrm{mBq} / \mathrm{mL})$} \\
\hline & & \multirow{2}{*}{$\begin{array}{r}\text { Activity } \\
135 \pm 55\end{array}$} & \multirow{2}{*}{$\begin{array}{c}\operatorname{LOS}^{(a)} \\
84.7\end{array}$} & \multirow{2}{*}{$\begin{array}{c}\text { Activity } \\
0.618 \pm 0.105\end{array}$} & \multirow{2}{*}{$\begin{array}{l}\text { LOS } \\
0.126\end{array}$} & \multirow{2}{*}{$\begin{array}{c}\text { Activity } \\
3.44\end{array}$} & \multirow{2}{*}{$\begin{array}{c}\text { LOS } \\
10.8\end{array}$} \\
\hline July & 24 & & & & & & \\
\hline & 25 & $148 \pm 61$ & 65.1 & $0.122 \pm 0.078$ & 0.121 & 5.37 & 10.9 \\
\hline & 26 & $77.7 \pm 47.4$ & 63.6 & 0.0862 & 0.120 & 0.951 & 10.9 \\
\hline & 27 & -99.2 & 103 & $5.25 \pm 0.24$ & 0.139 & 2.44 & 11.4 \\
\hline & 28 & $260 \pm 73$ & 78.4 & $0.903 \pm 0.117$ & 0.124 & -5.40 & 12.0 \\
\hline & 29 & 64.8 & 102 & $1.21 \pm 0.13$ & 0.130 & -2.59 & 12.6 \\
\hline & 30 & -54.0 & 104 & $1.37 \pm 0.14$ & 0.131 & 3.74 & 11.9 \\
\hline & 31 & $158 \pm 73$ & 137 & $1.01 \pm 0.13$ & 0.139 & $41.1 \pm 7.8$ & 12.1 \\
\hline \multirow[t]{26}{*}{ August } & 1 & $88.8 \pm 53.3$ & 83.3 & $0.302 \pm 0.094$ & 0.126 & -1.85 & 11.6 \\
\hline & 2 & 48.1 & 89.5 & $0.729 \pm 0.109$ & 0.127 & 4.92 & 11.4 \\
\hline & 3 & -54.8 & 95.5 & $0.766 \pm 0.115$ & 0.128 & 3.29 & 12.2 \\
\hline & 4 & $157 \pm 71$ & 121 & $0.899 \pm 0.126$ & 0.136 & $20.6 \pm 7.4$ & 11.8 \\
\hline & 5 & 84.4 & 86.2 & $0.559 \pm 0.106$ & 0.126 & $12.5 \pm 6.7$ & 11.1 \\
\hline & 6 & $126 \pm 63$ & 96.2 & $0.751 \pm 0.105$ & 0.110 & 4.11 & 12.7 \\
\hline & 7 & -46.3 & 106 & $0.759 \pm 0.106$ & 0.113 & $11.7 \pm 7.0$ & 1.15 \\
\hline & 8 & 2.31 & 65.9 & $0.148 \pm 0.073$ & 0.102 & 4.48 & 12.1 \\
\hline & 9 & 32.8 & 66.2 & $0.313 \pm 0.081$ & 0.102 & -2.27 & 12.5 \\
\hline & 10 & -35.3 & 90.3 & $0.525 \pm 0.095$ & 0.108 & 3.67 & 11.8 \\
\hline & 11 & 68.5 & 102 & $0.648 \pm 0.104$ & 0.112 & -0.966 & 11.3 \\
\hline & 12 & 13.7 & 84.0 & $0.932 \pm 0.112$ & 0.107 & 12.0 & 12.2 \\
\hline & 13 & -24.2 & 83.6 & $0.444 \pm 0.089$ & 0.107 & -4.14 & 13.0 \\
\hline & 14 & 16.9 & 83.3 & $0.892 \pm 0.107$ & 0.107 & -11.0 & 12.2 \\
\hline & 15 & 4.85 & 78.1 & $0.437 \pm 0.087$ & 0.105 & -6.92 & 12.0 \\
\hline & 16 & 39.2 & 77.3 & $0.262 \pm 0.079$ & 0.105 & -3.10 & 11.9 \\
\hline & 17 & -95.8 & 86.6 & $1.02 \pm 0.11$ & 0.107 & 4.92 & 13.5 \\
\hline & 18 & 71.8 & 84.4 & $0.951 \pm 0.114$ & 0.107 & -1.75 & 13.6 \\
\hline & 19 & $110 \pm 40$ & 81.8 & $1.61 \pm 0.13$ & 0.106 & 4.51 & 13.2 \\
\hline & 20 & $311 \pm 65$ & 105 & $4.11 \pm 0.21$ & 0.113 & 12.2 & 12.6 \\
\hline & 21 & $127 \pm 43$ & 76.2 & $1.34 \pm 0.13$ & 0.106 & 0.851 & 8.81 \\
\hline & 22 & 16.0 & 59.6 & $0.123 \pm 0.069$ & 0.0999 & 4.29 & 8.47 \\
\hline & 23 & $82.9 \pm 44.8$ & 65.1 & $0.241 \pm 0.077$ & 0.103 & 2.65 & 8.58 \\
\hline & 24 & 48.8 & 87.3 & $0.0692 \pm 0.0104$ & 0.107 & 1.36 & 11.8 \\
\hline & 25 & 16.7 & 85.1 & $0.936 \pm 0.112$ & 0.107 & 0.858 & 11.3 \\
\hline & 26 & 57.7 & 84.7 & $1.10 \pm 0.12$ & 0.107 & -3.12 & 12.8 \\
\hline
\end{tabular}




\section{Sewerable Water Monitoring}

Table 6-3. Daily monitoring results for gross alpha, gross beta, and tritium in the sanitary sewer effluent, 1999 (continued).

\begin{tabular}{|c|c|c|c|c|c|c|}
\hline \multirow[b]{2}{*}{ Date } & \multicolumn{2}{|c|}{ Gross alpha $(\mu \mathrm{Bq} / \mathrm{mL})$} & \multicolumn{2}{|c|}{ Gross beta $(\mathrm{mBq} / \mathrm{mL})$} & \multicolumn{2}{|c|}{ Tritium (mBq/mL) } \\
\hline & Activity & $\operatorname{LOS}^{(a)}$ & Activity & LOS & Activity & LOS \\
\hline August & 4.74 & 90.3 & $0.870 \pm 0.113$ & 0.108 & -7.81 & 13.0 \\
\hline 28 & 77.7 & 85.5 & $1.15 \pm 0.12$ & 0.107 & -11.6 & 12.5 \\
\hline 29 & 41.1 & 66.6 & $0.228 \pm 0.075$ & 0.102 & -11.5 & 12.4 \\
\hline 30 & 64.8 & 68.1 & $0.319 \pm 0.080$ & 0.103 & -15.4 & 12.7 \\
\hline 31 & $104 \pm 46$ & 84.4 & $0.955 \pm 0.115$ & 0.107 & -15.5 & 12.8 \\
\hline September 1 & $134 \pm 54$ & 71.4 & $0.611 \pm 0.098$ & 0.104 & 1.42 & 11.4 \\
\hline 2 & $118 \pm 55$ & 84.4 & $0.707 \pm 0.099$ & 0.107 & -1.81 & 11.2 \\
\hline 3 & 63.6 & 95.5 & $0.947 \pm 0.114$ & 0.110 & -2.22 & 11.5 \\
\hline 4 & 46.3 & 86.6 & $0.829 \pm 0.108$ & 0.108 & 5.66 & 11.1 \\
\hline 5 & 55.1 & 72.5 & $0.260 \pm 0.078$ & 0.105 & -6.62 & 11.7 \\
\hline 6 & $107 \pm 57$ & 81.0 & $0.356 \pm 0.085$ & 0.107 & -2.66 & 11.2 \\
\hline 7 & $103 \pm 55$ & 72.2 & $0.219 \pm 0.077$ & 0.105 & -3.85 & 11.4 \\
\hline 8 & 33.4 & 96.6 & $0.907 \pm 0.109$ & 0.110 & -2.84 & 11.2 \\
\hline 9 & 64.4 & 104 & $0.659 \pm 0.105$ & 0.112 & -2.89 & 11.5 \\
\hline 10 & 103 & 113 & $0.707 \pm 0.106$ & 0.115 & -6.73 & 11.2 \\
\hline 11 & 28.2 & 91.4 & $0.829 \pm 0.108$ & 0.108 & 1.95 & 10.8 \\
\hline 12 & 23.9 & 73.6 & $0.207 \pm 0.077$ & 0.105 & 7.14 & 10.7 \\
\hline 13 & -1.13 & 69.2 & $0.191 \pm 0.074$ & 0.104 & -1.19 & 11.0 \\
\hline 14 & 25.0 & 85.8 & $1.04 \pm 0.11$ & 0.107 & -3.40 & 10.9 \\
\hline 15 & 69.6 & 89.5 & $0.759 \pm 0.106$ & 0.108 & 2.32 & 11.0 \\
\hline 16 & 57.7 & 91.0 & $0.803 \pm 0.104$ & 0.111 & 0.725 & 10.7 \\
\hline 17 & 96.6 & 108 & $0.873 \pm 0.114$ & 0.116 & 6.70 & 10.8 \\
\hline 18 & $268 \pm 86$ & 89.5 & $0.858 \pm 0.112$ & 0.110 & -1.29 & 10.8 \\
\hline 19 & -1.39 & 73.3 & $0.236 \pm 0.080$ & 0.107 & 5.00 & 10.6 \\
\hline 20 & $86.2 \pm 50.0$ & 68.8 & $0.222 \pm 0.078$ & 0.105 & 5.74 & 10.8 \\
\hline 21 & -12.5 & 82.5 & $0.810 \pm 0.105$ & 0.109 & -1.13 & 11.0 \\
\hline 22 & 40.0 & 82.5 & $0.810 \pm 0.105$ & 0.106 & 7.84 & 11.0 \\
\hline 23 & -34.0 & 96.6 & $0.992 \pm 0.119$ & 0.110 & 0.488 & 10.8 \\
\hline 24 & -3.96 & 102 & $0.799 \pm 0.112$ & 0.111 & $15.0 \pm 6.6$ & 11.0 \\
\hline 25 & 61.8 & 84.7 & $0.692 \pm 0.104$ & 0.107 & 6.22 & 10.5 \\
\hline 26 & -13.0 & 68.1 & $0.198 \pm 0.075$ & 0.103 & 5.66 & 10.8 \\
\hline 27 & 62.9 & 67.3 & $0.195 \pm 0.074$ & 0.102 & 7.14 & 10.8 \\
\hline 28 & $157 \pm 66$ & 88.4 & $0.992 \pm 0.119$ & 0.118 & $131 \pm 9$ & 11.0 \\
\hline 29 & 54.4 & 96.9 & $0.892 \pm 0.116$ & 0.111 & 3.96 & 10.7 \\
\hline
\end{tabular}


Table 6-3. Daily monitoring results for gross alpha, gross beta, and tritium in the sanitary sewer effluent, 1999 (continued).

\begin{tabular}{|c|c|c|c|c|c|c|c|}
\hline \multirow{2}{*}{\multicolumn{2}{|c|}{ Date }} & \multicolumn{2}{|c|}{ Gross alpha $(\mu \mathrm{Bq} / \mathrm{mL})$} & \multicolumn{2}{|c|}{ Gross beta $(\mathrm{mBq} / \mathrm{mL})$} & \multicolumn{2}{|c|}{ Tritium $(\mathrm{mBq} / \mathrm{mL})$} \\
\hline & & Activity & $\operatorname{LOS}^{(a)}$ & Activity & LOS & Activity & LOS \\
\hline \multicolumn{2}{|c|}{ September30 } & -42.6 & 96.6 & $0.666 \pm 0.100$ & 0.111 & 3.31 & 11.1 \\
\hline \multirow[t]{31}{*}{ October } & 1 & -26.6 & 91.4 & $0.644 \pm 0.103$ & 0.110 & -2.12 & 11.1 \\
\hline & 2 & 36.3 & 86.6 & $0.710 \pm 0.100$ & 0.109 & 4.29 & 11.0 \\
\hline & 3 & 24.0 & 66.2 & $0.203 \pm 0.075$ & 0.104 & $10.9 \pm 6.3$ & 10.7 \\
\hline & 4 & -18.6 & 63.3 & $0.151 \pm 0.073$ & 0.102 & 8.81 & 10.7 \\
\hline & 5 & 12.4 & 84.4 & $0.777 \pm 0.109$ & 0.108 & 10.5 & 11.1 \\
\hline & 6 & 72.9 & 84.4 & $0.818 \pm 0.106$ & 0.105 & 5.18 & 10.7 \\
\hline & 7 & -1.64 & 80.3 & $0.788 \pm 0.102$ & 0.105 & -1.91 & 11.2 \\
\hline & 8 & 27.6 & 85.8 & $0.807 \pm 0.105$ & 0.106 & 2.22 & 11.0 \\
\hline & 9 & -61.1 & 84.4 & $0.936 \pm 0.112$ & 0.105 & $14.5 \pm 6.8$ & 11.0 \\
\hline & 10 & 15.7 & 68.8 & $0.246 \pm 0.076$ & 0.102 & $14.7 \pm 6.6$ & 10.8 \\
\hline & 11 & 11.4 & 63.6 & $0.152 \pm 0.070$ & 0.0999 & -1.45 & 11.0 \\
\hline & 12 & 43.3 & 92.5 & $0.884 \pm 0.106$ & 0.107 & 1.73 & 10.7 \\
\hline & 13 & 6.96 & 89.9 & $0.844 \pm 0.110$ & 0.107 & 3.56 & 10.8 \\
\hline & 14 & -6.77 & 101 & $0.977 \pm 0.117$ & 0.113 & -1.41 & 10.8 \\
\hline & 15 & 21.8 & 110 & $0.929 \pm 0.121$ & 0.116 & 4.81 & 10.7 \\
\hline & 16 & $161 \pm 61$ & 92.9 & $1.09 \pm 0.12$ & 0.111 & $929 \pm 18$ & 10.6 \\
\hline & 17 & -9.73 & 72.2 & $0.147 \pm 0.074$ & 0.106 & 9.92 & 10.9 \\
\hline & 18 & 37.0 & 65.1 & $0.142 \pm 0.071$ & 0.104 & 0.947 & 10.8 \\
\hline & 19 & 8.29 & 88.1 & $0.570 \pm 0.097$ & 0.110 & -1.13 & 10.6 \\
\hline & 20 & 47.4 & 96.2 & $0.877 \pm 0.114$ & 0.111 & $281 \pm 11$ & 10.8 \\
\hline & 21 & 38.5 & 96.6 & $0.644 \pm 0.103$ & 0.111 & $13.6 \pm 5.5$ & 10.8 \\
\hline & 22 & -76.6 & 102 & $0.685 \pm 0.103$ & 0.113 & $17.4 \pm 5.4$ & 8.66 \\
\hline & 23 & 72.9 & 90.7 & $1.00 \pm 0.11$ & 0.110 & 2.82 & 8.99 \\
\hline & 24 & -14.7 & 75.9 & $0.477 \pm 0.091$ & 0.107 & 2.42 & 9.14 \\
\hline & 25 & 8.47 & 74.4 & $0.359 \pm 0.086$ & 0.107 & -1.66 & 9.47 \\
\hline & 26 & 54.0 & 76.6 & $0.503 \pm 0.091$ & 0.107 & 2.95 & 8.66 \\
\hline & 27 & 52.9 & 84.7 & $0.792 \pm 0.103$ & 0.109 & 4.22 & 10.9 \\
\hline & 28 & 33.0 & 74.7 & $0.481 \pm 0.091$ & 0.107 & 0.570 & 11.2 \\
\hline & 29 & 86.2 & 93.2 & $0.781 \pm 0.109$ & 0.111 & 3.96 & 10.9 \\
\hline & 30 & -20.1 & 84.7 & $0.903 \pm 0.108$ & 0.109 & 1.18 & 11.2 \\
\hline & 31 & 42.9 & 87.3 & $0.339 \pm 0.085$ & 0.110 & -0.725 & 11.0 \\
\hline \multirow[t]{2}{*}{ November } & 1 & -4.92 & 74.4 & $0.239 \pm 0.079$ & 0.107 & 3.15 & 10.9 \\
\hline & 2 & -14.6 & 92.5 & $0.725 \pm 0.102$ & 0.111 & -4.63 & 11.3 \\
\hline
\end{tabular}




\section{Sewerable Water Monitoring}

Table 6-3. Daily monitoring results for gross alpha, gross beta, and tritium in the sanitary sewer effluent, 1999 (continued).

\begin{tabular}{|c|c|c|c|c|c|c|c|}
\hline \multirow{2}{*}{\multicolumn{2}{|c|}{ Date }} & \multicolumn{2}{|c|}{ Gross alpha $(\mu \mathrm{Bq} / \mathrm{mL})$} & \multicolumn{2}{|c|}{ Gross beta $(\mathrm{mBq} / \mathrm{mL})$} & \multicolumn{2}{|c|}{ Tritium (mBq/mL) } \\
\hline & & Activity & $\operatorname{LOS}^{(a)}$ & Activity & LOS & Activity & LOS \\
\hline \multirow[t]{28}{*}{ November } & 3 & 95.1 & 82.9 & $0.947 \pm 0.114$ & 0.112 & 3.60 & 11.1 \\
\hline & 4 & -5.48 & 95.5 & $0.577 \pm 0.098$ & 0.111 & -0.124 & 11.2 \\
\hline & 5 & -23.5 & 101 & $0.648 \pm 0.104$ & 0.113 & 4.14 & 10.7 \\
\hline & 6 & 81.8 & 89.9 & $0.840 \pm 0.109$ & 0.110 & 8.10 & 10.6 \\
\hline & 7 & -1.59 & 76.2 & $0.251 \pm 0.080$ & 0.107 & 2.34 & 10.8 \\
\hline & 8 & -2.59 & 72.5 & $0.155 \pm 0.075$ & 0.106 & 0.803 & 10.8 \\
\hline & 9 & 13.0 & 128 & $0.707 \pm 0.106$ & 0.118 & 6.40 & 10.9 \\
\hline & 10 & 4.70 & 96.2 & $0.762 \pm 0.107$ & 0.111 & 0.733 & 10.5 \\
\hline & 11 & -0.182 & 80.3 & $0.751 \pm 0.105$ & 0.108 & -2.05 & 10.8 \\
\hline & 12 & 61.1 & 78.4 & $0.622 \pm 0.100$ & 0.107 & 0.551 & 10.9 \\
\hline & 13 & 4.48 & 79.6 & $0.659 \pm 0.099$ & 0.108 & -1.28 & 11.0 \\
\hline & 14 & -5.59 & 69.2 & $0.209 \pm 0.077$ & 0.105 & 3.15 & 10.7 \\
\hline & 15 & -12.0 & 64.4 & $0.158 \pm 0.073$ & 0.103 & -6.70 & 10.8 \\
\hline & 16 & -21.7 & 78.4 & $0.629 \pm 0.101$ & 0.107 & 2.27 & 8.73 \\
\hline & 17 & 3.74 & 82.9 & $0.788 \pm 0.102$ & 0.108 & 2.14 & 8.47 \\
\hline & 18 & -31.5 & 86.6 & $0.862 \pm 0.112$ & 0.107 & -0.947 & 8.47 \\
\hline & 19 & 7.59 & 85.8 & $0.599 \pm 0.096$ & 0.107 & -2.30 & 11.0 \\
\hline & 20 & -5.92 & 97.7 & $0.851 \pm 0.111$ & 0.110 & -2.53 & 10.8 \\
\hline & 21 & 7.77 & 92.9 & $0.725 \pm 0.102$ & 0.109 & 0.903 & 10.7 \\
\hline & 22 & -19.4 & 68.1 & $0.192 \pm 0.075$ & 0.103 & 1.05 & 10.7 \\
\hline & 23 & 7.99 & 91.4 & $0.496 \pm 0.094$ & 0.108 & 1.79 & 8.66 \\
\hline & 24 & 57.4 & 94.4 & $0.681 \pm 0.102$ & 0.109 & 3.18 & 8.51 \\
\hline & 25 & 44.8 & 94.0 & $0.814 \pm 0.106$ & 0.108 & 0.903 & 10.8 \\
\hline & 26 & 34.8 & 64.8 & $0.136 \pm 0.069$ & 0.101 & 2.22 & 10.3 \\
\hline & 27 & 4.33 & 66.2 & $0.182 \pm 0.073$ & 0.101 & -2.02 & 10.8 \\
\hline & 28 & 47.0 & 74.0 & $0.178 \pm 0.073$ & 0.104 & -5.66 & 11.1 \\
\hline & 29 & 7.70 & 65.5 & $0.198 \pm 0.073$ & 0.101 & 4.96 & 10.3 \\
\hline & 30 & $145 \pm 62$ & 110 & $0.847 \pm 0.110$ & 0.113 & -0.744 & 10.9 \\
\hline \multirow[t]{6}{*}{ December } & 1 & 28.3 & 74.7 & $0.962 \pm 0.115$ & 0.118 & 2.71 & 1.08 \\
\hline & 2 & -7.14 & 113 & $0.973 \pm 0.117$ & 0.114 & 1.72 & 8.66 \\
\hline & 3 & 21.3 & 85.8 & $0.614 \pm 0.098$ & 0.106 & -4.26 & 8.81 \\
\hline & 4 & 57.0 & 105 & $0.773 \pm 0.108$ & 0.111 & 3.02 & 10.6 \\
\hline & 5 & 26.3 & 77.7 & $0.260 \pm 0.078$ & 0.104 & 2.97 & 8.70 \\
\hline & 6 & 21.8 & 64.8 & $0.126 \pm 0.069$ & 0.101 & -0.910 & 8.70 \\
\hline
\end{tabular}


Table 6-3. Daily monitoring results for gross alpha, gross beta, and tritium in the sanitary sewer effluent, 1999 (concluded).

\begin{tabular}{|c|c|c|c|c|c|c|}
\hline \multirow[b]{2}{*}{ Date } & \multicolumn{2}{|c|}{ Gross alpha $(\mu \mathrm{Bq} / \mathrm{mL})$} & \multicolumn{2}{|c|}{ Gross beta (mBq/mL) } & \multicolumn{2}{|c|}{ Tritium (mBq/mL) } \\
\hline & Activity & $\operatorname{LOS}(a)$ & Activity & LOS & Activity & LOS \\
\hline December 7 & 2.11 & 105 & $0.666 \pm 0.100$ & 0.111 & 1.77 & 10.6 \\
\hline 8 & 17.2 & 93.2 & $0.773 \pm 0.108$ & 0.108 & -2.73 & 8.66 \\
\hline 9 & $106 \pm 51$ & 100 & $0.829 \pm 0.108$ & 0.110 & 1.38 & 8.70 \\
\hline 10 & -16.4 & 105 & $0.629 \pm 0.101$ & 0.111 & 0.422 & 8.84 \\
\hline 11 & 42.6 & 102 & $0.995 \pm 0.119$ & 0.110 & 5.18 & 10.7 \\
\hline 12 & -7.70 & 87.7 & $0.451 \pm 0.090$ & 0.106 & 0.403 & 10.8 \\
\hline 13 & 15.9 & 82.1 & $0.226 \pm 0.077$ & 0.105 & 7.40 & 10.5 \\
\hline 14 & 2.21 & 84.4 & $0.625 \pm 0.100$ & 0.106 & -3.36 & 11.2 \\
\hline 15 & 62.5 & 71.4 & $0.577 \pm 0.104$ & 0.119 & 5.66 & 10.5 \\
\hline 16 & 54.0 & 85.5 & $0.562 \pm 0.096$ & 0.108 & 0.566 & 11.1 \\
\hline 17 & $105 \pm 45$ & 92.5 & $1.44 \pm 0.13$ & 0.110 & 1.11 & 10.8 \\
\hline 18 & -9.32 & 86.6 & $0.710 \pm 0.100$ & 0.108 & -3.24 & 10.9 \\
\hline 19 & -40.0 & 72.9 & $0.149 \pm 0.075$ & 0.105 & -6.92 & 11.1 \\
\hline 20 & 12.1 & 72.5 & $0.228 \pm 0.078$ & 0.105 & -1.08 & 10.7 \\
\hline 21 & $234 \pm 59$ & 97.3 & $2.68 \pm 0.17$ & 0.110 & -2.50 & 11.0 \\
\hline 22 & $159 \pm 60$ & 91.8 & $0.929 \pm 0.111$ & 0.108 & 1.27 & 11.1 \\
\hline 23 & $201 \pm 60$ & 81.4 & $1.35 \pm 0.12$ & 0.106 & 2.25 & 10.7 \\
\hline 24 & $72.9 \pm 39.4$ & 67.0 & $0.422 \pm 0.084$ & 0.102 & 3.74 & 10.4 \\
\hline $25^{(c)}$ & 55.5 & 59.2 & $0.225 \pm 0.074$ & 0.0988 & 4.48 & 10.5 \\
\hline $26^{(c)}$ & 55.5 & 59.2 & $0.225 \pm 0.074$ & 0.0988 & 4.48 & 10.5 \\
\hline 27 & $63.6 \pm 40.1$ & 63.3 & $0.229 \pm 0.076$ & 0.101 & 4.33 & 10.6 \\
\hline 28 & 68.5 & 86.6 & $0.733 \pm 0.103$ & 0.107 & 5.96 & 10.3 \\
\hline 29 & $97.3 \pm 48.7$ & 82.1 & $0.618 \pm 0.099$ & 0.107 & 2.00 & 10.5 \\
\hline 30 & $107 \pm 49$ & 72.9 & $0.522 \pm 0.094$ & 0.105 & -2.09 & 10.6 \\
\hline 31 & 27.9 & 61.8 & $0.317 \pm 0.079$ & 0.101 & 3.24 & 10.5 \\
\hline
\end{tabular}

Note: The activities shown in this table are measured concentrations and their associated $2 \sigma$ counting errors. Activities do not include the $2 \sigma$ counting errors when the measured concentrations are less than the limit of sensitivity (LOS). See the main volume, Chapter 14, Quality Assurance.

a LOS = Limit of sensitivity.

b This value is the monitoring result for the weekly composite sample for the sampling period of May 4-10, 1999. The daily monitoring result is not shown because of a significantly elevated LOS value. (The sample aliquot size was reduced from 150 to $10 \mathrm{~mL}$ to compensate for overchlorination of the daily monitoring samples; the smaller the sample volume, the greater the LOS.) The daily monitoring results for May 8,1999, were 302 and $1640 \mu \mathrm{Bq} / \mathrm{mL}$ for gross alpha activity and LOS, respectively and -0.625 and $2.09 \mathrm{mBq} / \mathrm{mL}$ for gross beta activity and LOS, respectively. The daily monitoring results for May 9,1999, were 1320 and $1780 \mu \mathrm{Bq} / \mathrm{mL}$ for gross alpha activity and LOS, respectively and -0.918 and $2.14 \mathrm{mBq} / \mathrm{mL}$ for gross beta activity and LOS, respectively.

c The daily monitoring results are not available. The results shown for this date are the monitoring results for the weekly composite sample for the sampling period of December 25 and 26, 1999. 


\section{Sewerable Water Monitoring}

Table 6-4. Weekly composite results for tritium $(\mathrm{mBq} / \mathrm{mL})$ for the LWRP effluent,1999.

\begin{tabular}{|c|c|c|c|c|c|}
\hline Composite dates & Activity $^{(a)}$ & $\operatorname{LOS}^{(\mathbf{b})}$ & Composite dates & Activity(a) & $\operatorname{LOS}^{(b)}$ \\
\hline 12/28/1998-1/03/1999 & 8.77 & 10.9 & $7 / 05-7 / 11$ & 3.07 & 10.8 \\
\hline $1 / 04-1 / 10$ & 1.99 & 12.5 & $7 / 12-7 / 18$ & 0.973 & 5.44 \\
\hline $1 / 11-1 / 17$ & 3.36 & 11.5 & $7 / 19-7 / 25$ & -6.14 & 11.8 \\
\hline $1 / 18-1 / 24$ & $16.8 \pm 6.7$ & 11.5 & $7 / 26-8 / 01$ & -7.29 & 11.5 \\
\hline $1 / 25-1 / 31$ & -8.51 & 12.5 & 8/02-8/08 & 4.03 & 11.2 \\
\hline $2 / 01-2 / 07$ & 3.58 & 12.7 & $8 / 09-8 / 15$ & -5.11 & 11.8 \\
\hline $2 / 08-2 / 14$ & 2.46 & 12.0 & 8/16-8/22 & 1.14 & 8.66 \\
\hline $2 / 15-2 / 21$ & 10.1 & 11.8 & 8/23-8/29 & -3.66 & 12.5 \\
\hline $2 / 22-2 / 28$ & 2.87 & 11.7 & 8/30-9/05 & -2.63 & 11.2 \\
\hline $3 / 01-3 / 07$ & -13.3 & 12.6 & $9 / 06-9 / 12$ & -8.81 & 11.0 \\
\hline $3 / 08-3 / 14$ & 0.047 & 12.0 & $9 / 13-9 / 19$ & 0.254 & 10.6 \\
\hline $3 / 15-3 / 21$ & 3.10 & 12.0 & $9 / 20-9 / 26$ & -4.18 & 10.9 \\
\hline $3 / 22-3 / 28$ & 6.85 & 11.8 & $9 / 27-10 / 03$ & 1.31 & 10.9 \\
\hline $3 / 29-4 / 04$ & 1.12 & 11.6 & $10 / 04-10 / 10$ & -5.00 & 11.2 \\
\hline $4 / 05-4 / 11$ & -0.525 & 11.2 & $10 / 11-10 / 17$ & 1.48 & 10.6 \\
\hline $4 / 12-4 / 18$ & -1.44 & 11.5 & $10 / 18-10 / 24$ & 7.07 & 10.8 \\
\hline $4 / 19-4 / 25$ & 5.59 & 11.5 & $10 / 25-10 / 31$ & 2.41 & 10.5 \\
\hline $4 / 26-5 / 02$ & 3.92 & 10.9 & $11 / 01-11 / 07$ & 0.803 & 11.2 \\
\hline $5 / 03-5 / 09$ & 0.955 & 8.77 & $11 / 08-11 / 14$ & 0.426 & 10.6 \\
\hline $5 / 10-5 / 16$ & 1.62 & 8.55 & $11 / 15-11 / 21$ & -1.53 & 8.84 \\
\hline $5 / 17-5 / 23$ & -0.770 & 8.84 & $11 / 22-11 / 28$ & 4.66 & 11.1 \\
\hline $5 / 24-5 / 30$ & -2.49 & 11.5 & $11 / 29-12 / 05$ & 1.44 & 10.7 \\
\hline $5 / 31-6 / 06$ & 2.55 & 8.58 & $12 / 06-12 / 12$ & -2.24 & 11.0 \\
\hline $6 / 07-6 / 13$ & 5.03 & 10.9 & $12 / 13-12 / 19$ & -5.99 & 11.0 \\
\hline $6 / 14-6 / 20$ & -5.33 & 11.6 & $12 / 20-12 / 26$ & 6.07 & 10.8 \\
\hline $6 / 21-6 / 27$ & -2.46 & 11.0 & 12/27/1999-01/02/2000 & 1.71 & 10.7 \\
\hline 6/28-7/04 & 8.33 & 10.7 & & & \\
\hline
\end{tabular}

a The activities shown in this table are measured concentrations and their associated $2 \sigma$ counting errors. Activities do not include the $2 \sigma$ counting errors when the measured concentrations are less than the limit of sensitivity (LOS). See the main volume, Chapter 14, Quality Assurance.

b LOS $=$ Limit of sensitivity. 
Sewerable Water Monitoring

Table 6-5. Weekly composite results for metals in LLNL sanitary sewer effluent, 1999.

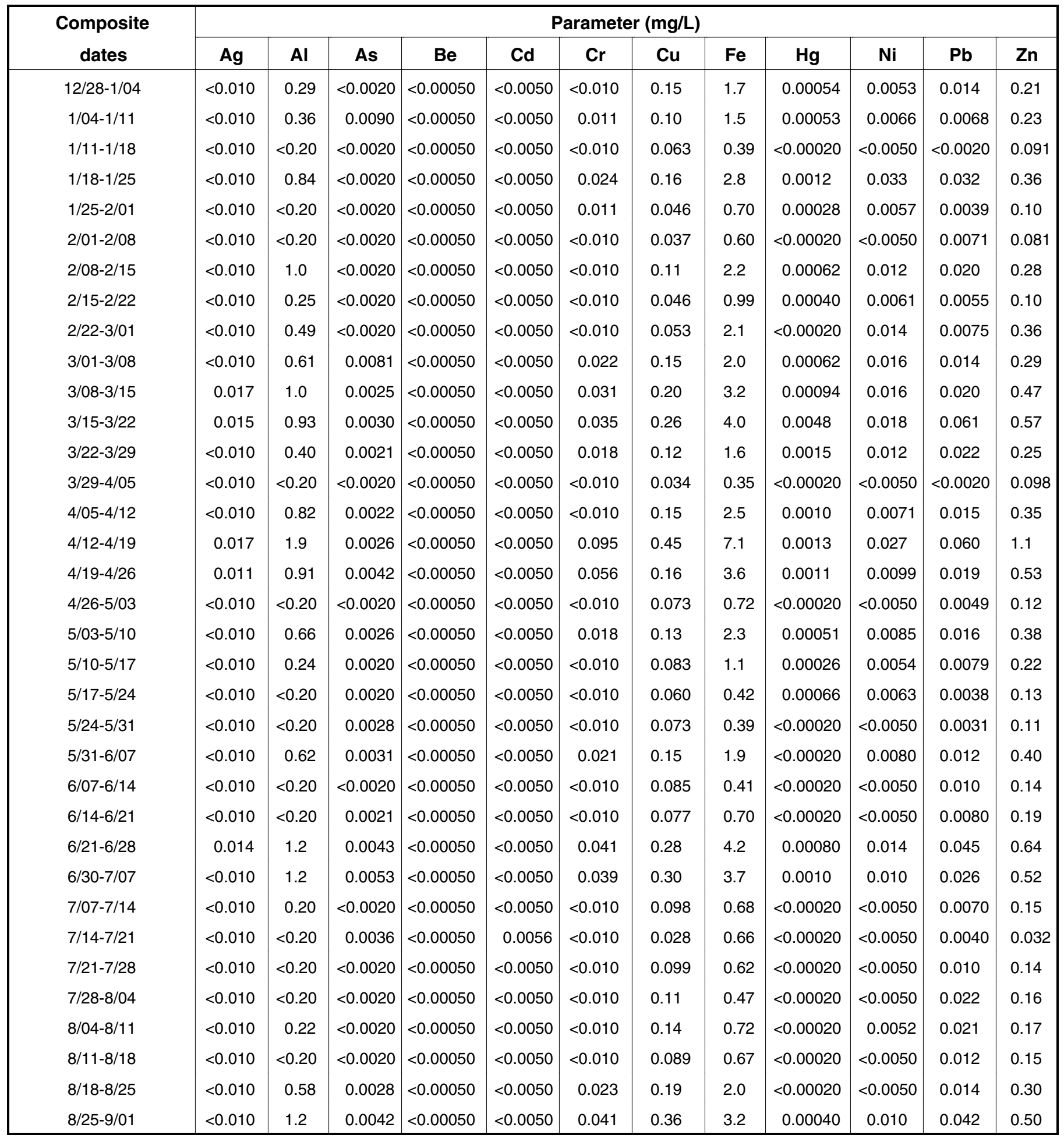




\section{Sewerable Water Monitoring}

Table 6-5. Weekly composite results for metals in LLNL sanitary sewer effluent, 1999 (concluded).

\begin{tabular}{|c|c|c|c|c|c|c|c|c|c|c|c|c|}
\hline \multirow{2}{*}{$\begin{array}{c}\text { Composite } \\
\text { dates }\end{array}$} & \multicolumn{12}{|c|}{ Parameter (mg/L) } \\
\hline & $\mathbf{A g}$ & Al & As & $\mathrm{Be}$ & Cd & $\mathrm{Cr}$ & $\mathrm{Cu}$ & $\mathrm{Fe}$ & $\mathrm{Hg}$ & $\mathrm{Ni}$ & $\mathrm{Pb}$ & $\mathrm{Zn}$ \\
\hline 9/02-9/09 & $<0.010$ & $<0.20$ & $<0.0020$ & $<0.00050$ & $<0.0050$ & $<0.010$ & 0.072 & 0.47 & $<0.00020$ & $<0.0050$ & 0.0055 & 0.11 \\
\hline $9 / 15-9 / 22$ & 0.014 & - (a) & 0.0040 & -(a) & 0.0052 & 0.11 & 0.43 & -(a) $^{(\mathrm{a})}$ & 0.0014 & 0.013 & 0.031 & 0.80 \\
\hline $9 / 22-9 / 29$ & $<0.010$ & — $^{(\mathrm{a})}$ & 0.0058 & -(a) $^{(\mathrm{a}}$ & $<0.0050$ & 0.060 & 0.38 & - $^{(\mathrm{a})}$ & 0.0013 & 0.018 & 0.071 & 0.70 \\
\hline $10 / 06-10 / 13$ & $<0.010$ & 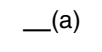 & 0.0041 & -(a) & $<0.0050$ & 0.043 & 0.18 & - (a) $^{(2)}$ & 0.0010 & 0.010 & 0.018 & 0.40 \\
\hline $10 / 13-10 / 20$ & $<0.010$ & — $^{(\mathrm{a})}$ & 0.0042 & -(a) & $<0.0050$ & 0.056 & 0.26 & - $^{(\mathrm{a})}$ & 0.00046 & 0.013 & 0.036 & 0.59 \\
\hline $10 / 20-10 / 27$ & $<0.010$ & — $^{(\mathrm{a})}$ & 0.0052 & -(a) & $<0.0050$ & 0.045 & 0.32 & - (a) $^{(a)}$ & 0.00051 & 0.012 & 0.031 & 0.67 \\
\hline $10 / 27-11 / 03$ & $<0.010$ & -(a) & $<0.0020$ & -(a) & $<0.0050$ & 0.012 & 0.11 & -(a) $^{(\mathrm{a})}$ & 0.00056 & 0.0058 & 0.0084 & 0.26 \\
\hline $11 / 24-12 / 01$ & 0.016 & —(a) $^{(\mathrm{n}}$ & 0.0034 & -(a) & $<0.0050$ & $<0.010$ & 0.14 & —(a) & 0.00025 & $<0.0050$ & 0.017 & 0.91 \\
\hline $12 / 01-12 / 08$ & $<0.010$ & 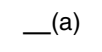 & 0.0054 & -(a) & $<0.0050$ & 0.044 & 0.17 & -(a) & 0.00032 & $<0.0050$ & 0.016 & 0.38 \\
\hline $12 / 08-12 / 15$ & $<0.010$ & —(a) & 0.0055 & -(a) & $<0.0050$ & 0.061 & 0.21 & -(a) & 0.00039 & 0.0087 & 0.062 & 0.47 \\
\hline $12 / 15-12 / 22$ & $<0.010$ & — $^{(\mathrm{a})}$ & $<0.0020$ & -(a) & $<0.0050$ & $<0.010$ & 0.050 & - $^{(\mathrm{a})}$ & $<0.00020$ & $<0.0050$ & 0.0059 & 0.14 \\
\hline $12 / 22-12 / 29$ & $<0.010$ & - (a) & 0.0026 & -(a) & $<0.0050$ & 0.032 & 0.20 & - (a) $^{(\mathrm{n}}$ & 0.00064 & 0.0077 & 0.018 & 0.42 \\
\hline $12 / 29-1 / 05$ & 0.015 & —(a) & $<0.0020$ & -(a) & $<0.0050$ & 0.066 & 0.28 & - $^{(\mathrm{a})}$ & $<0.00020$ & 0.011 & 0.029 & 0.56 \\
\hline \multicolumn{13}{|c|}{ Summary of weekly composite results } \\
\hline Detection frequency & $9 / 53$ & 22/36 & $32 / 53$ & $0 / 36$ & $2 / 53$ & $30 / 53$ & $53 / 53$ & $36 / 36$ & $34 / 53$ & $35 / 53$ & $51 / 53$ & $53 / 53$ \\
\hline Median/50\% of EPL & 0.10 & - (c) $^{(\mathrm{c})}$ & 0.083 & - (c) $^{(\mathrm{c})}$ & 0.071 & 0.058 & 0.30 & -(c) & 0.080 & 0.022 & 0.16 & 0.20 \\
\hline
\end{tabular}

a Weekly sampling for $\mathrm{Al}, \mathrm{Be}$, and Fe was suspended in September, since these are not permitted sampling requirements. Data for these parameters will continue to be collected on a monthly basis.

b $\quad I Q R=$ Interquartile range.

c Because of the large number of nondetects, the interquartile range is omitted. See Chapter 14, Quality Assurance.

d There is no Effluent Pollutant Limit (EPL) for this parameter; therefore, no comparison value can be calculated. 
Table 6-6. Monthly 24-hour composite results for metals in LLNL sanitary sewer effluent, 1999.

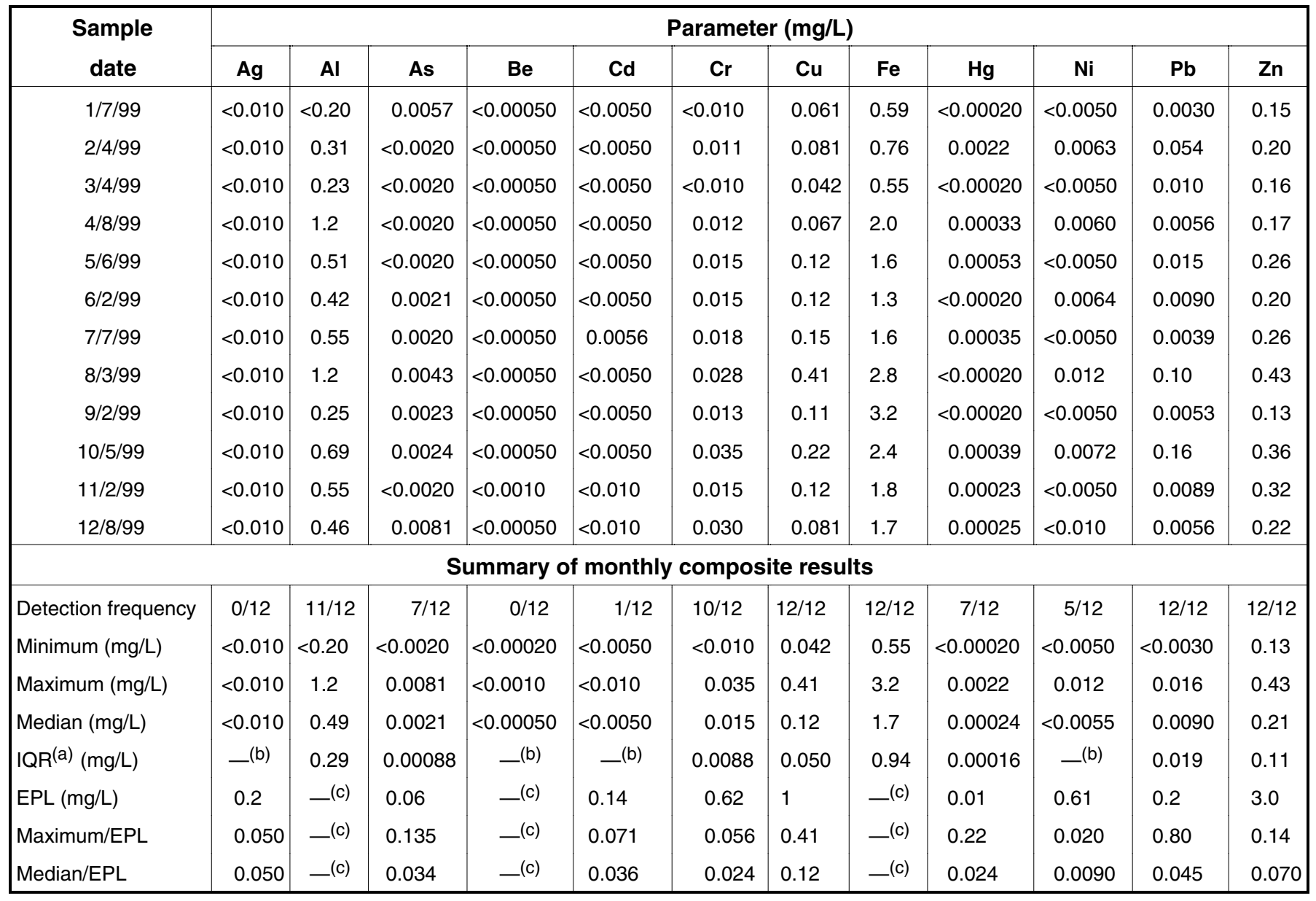

a $I Q R=$ Interquartile range.

b Because of the large number of nondetects, the interquartile range is omitted. See Chapter 14, Quality Assurance.

c There is no Effluent Pollutant Limit (EPL) for this parameter; therefore, no comparison value can be calculated. 


\section{Sewerable Water Monitoring}

Table 6-7. Monthly monitoring results for physical and chemical characteristics of the LLNL sanitary sewer effluent, 1999.

\begin{tabular}{|c|c|c|c|c|c|c|c|}
\hline \multirow{2}{*}{$\begin{array}{c}\text { 24-hour composite sample } \\
\text { parameters }\end{array}$} & \multirow{2}{*}{$\begin{array}{c}\text { EPA } \\
\text { Method }\end{array}$} & \multicolumn{6}{|c|}{ Sample month } \\
\hline & & January & February & March & April & May & June \\
\hline Alkalinity (mg/L) & 310.1 & & & & & & \\
\hline Bicarbonate alkalinity $\left(\right.$ as $\left.\mathrm{CaCO}_{3}\right)$ & & 170 & 180 & 190 & 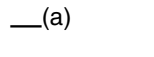 & 180 & 130 \\
\hline Carbonate alkalinity $\left(\right.$ as $\left.\mathrm{CaCO}_{3}\right)$ & & $<1$ & $<1$ & $<1$ & 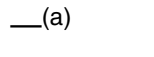 & $<5$ & $<5$ \\
\hline Hydroxide alkalinity (as $\mathrm{CaCO}_{3}$ ) & & $<1$ & $<1$ & $<1$ & - (a) $^{\text {(a) }}$ & $<5$ & $<5$ \\
\hline Total alkalinity $\left(\mathrm{as} \mathrm{CaCO}_{3}\right)$ & & 170 & 180 & 190 & 198 & 180 & 130 \\
\hline Anions (mg/L) & 300.0 & & & & & & \\
\hline Bromide & & $<0.5$ & $<0.5$ & 0.62 & $<0.1$ & $<0.1$ & $<0.1$ \\
\hline Chloride & & 31 & 63 & 43 & 51 & 44 & 22 \\
\hline Fluoride & & 0.095 & $<0.05$ & 0.1 & 0.06 & 0.68 & 0.07 \\
\hline Nitrate (as N) & & $<0.5$ & $<0.5$ & $<0.5$ & $<0.1$ & $<0.1$ & $<0.1$ \\
\hline Nitrate $\left(\right.$ as $\left.\mathrm{NO}_{3}\right)$ & & $<0.5$ & 1.6 & 0.71 & $<0.4$ & $<0.4$ & $<0.4$ \\
\hline Nitrite (as N) & & $<0.5$ & $<0.5$ & $<0.5$ & $<0.02$ & $<0.02$ & $<0.02$ \\
\hline Nitrite $\left(\right.$ as $\left.\mathrm{NO}_{2}\right)$ & & $<0.5$ & $<0.5$ & $<0.5$ & $<0.07$ & $<0.07$ & $<0.07$ \\
\hline Orthophosphate & & 14 & 19 & 17 & 20 & 20 & 12 \\
\hline Sulfate & & 4 & 5.2 & 4.1 & 18 & 15 & 8.4 \\
\hline \multicolumn{8}{|l|}{ Nutrients (mg/L) } \\
\hline Ammonia nitrogen (as $\mathrm{N}$ ) & 350.2 & 35 & 40 & 43 & 51 & 53 & 28 \\
\hline Total Kjeldahl nitrogen & 351.3 & 0.61 & 44 & 2.4 & 53 & 60 & 40 \\
\hline \multicolumn{8}{|l|}{ Oxygen demand (mg/L) } \\
\hline Biochemical oxygen demand & 405.1 & 113 & $<75$ & 160 & 106 & 295 & 137 \\
\hline Chemical oxygen demand & 410.4 & 130 & 130 & 200 & 464 & 542 & 334 \\
\hline \multicolumn{8}{|l|}{ Solids (mg/L) } \\
\hline Solid settling rate $(\mathrm{mL} / \mathrm{L} / \mathrm{h})$ & 160.5 & 60 & 62 & 25 & 25 & 50 & 30 \\
\hline Total dissolved solids (TDS) & 160.1 & 190 & 270 & 240 & 378 & 262 & 120 \\
\hline Total suspended solids (TSS) & 160.2 & 120 & 120 & 350 & 103 & 380 & 398 \\
\hline Volatile solids & 160.4 & 370 & 350 & 210 & 260 & 380 & 218 \\
\hline \multicolumn{8}{|l|}{ Total metals (mg/L) } \\
\hline Calcium & 200.7 & 11 & 12 & 13 & 14 & 18 & 13 \\
\hline Magnesium & 200.7 & 2.7 & 3.2 & 2.9 & 4 & 4.6 & 2.5 \\
\hline Potassium & 200.7 & 14 & 16 & 17 & 23 & 20 & 13 \\
\hline Selenium & 270.2 & $<0.002$ & $<0.002$ & $<0.002$ & $<0.002$ & $<0.002$ & $<0.002$ \\
\hline Sodium & 200.7 & 24 & 40 & 33 & 39 & 38 & 21 \\
\hline
\end{tabular}


Table 6-7. Monthly monitoring results for physical and chemical characteristics of the LLNL sanitary sewer effluent, 1999 (continued).

\begin{tabular}{|c|c|c|c|c|c|c|c|}
\hline \multirow{2}{*}{$\begin{array}{c}\text { 24-hour composite sample } \\
\text { parameters }\end{array}$} & \multirow{2}{*}{$\begin{array}{c}\text { EPA } \\
\text { Method }\end{array}$} & \multicolumn{6}{|c|}{ Sample month } \\
\hline & & July & August & September & October & November & December \\
\hline Alkalinity (mg/L) & 310.1 & & & & & & \\
\hline Bicarbonate alkalinity $\left(\right.$ as $\left.\mathrm{CaCO}_{3}\right)$ & & 221 & 208 & 148 & 185 & 216 & 185 \\
\hline Carbonate alkalinity $\left(\right.$ as $\left.\mathrm{CaCO}_{3}\right)$ & & $<5$ & $<5$ & $<5$ & $<5$ & $<5$ & $<5$ \\
\hline Hydroxide alkalinity $\left(\mathrm{as} \mathrm{CaCO}_{3}\right)$ & & $<5$ & $<5$ & $<5$ & $<5$ & $<5$ & $<5$ \\
\hline Total alkalinity $\left(\mathrm{as} \mathrm{CaCO}_{3}\right)$ & & 221 & 208 & 148 & 185 & 216 & 185 \\
\hline Anions (mg/L) & 300.0 & & & & & & \\
\hline Bromide & & 0.12 & 0.13 & $<0.1$ & $<0.1$ & 0.15 & $<0.1$ \\
\hline Chloride & & 49 & 44 & 36 & 34 & 37 & 47 \\
\hline Fluoride & & 0.08 & 0.14 & 0.12 & 0.06 & $<0.05$ & 0.14 \\
\hline Nitrate (as N) & & $<0.1$ & $<0.1$ & $<0.1$ & $<0.1$ & $<0.1$ & $<0.1$ \\
\hline Nitrate $\left(\right.$ as $\left.\mathrm{NO}_{3}\right)$ & & $<0.4$ & $<0.4$ & $<0.4$ & $<0.4$ & $<0.4$ & $<0.44$ \\
\hline Nitrite (as N) & & $<0.02$ & $<0.02$ & $<0.02$ & $<0.02$ & $<0.02$ & $<0.02$ \\
\hline Nitrite (as $\mathrm{NO}_{2}$ ) & & $<0.07$ & $<0.07$ & $<0.07$ & $<0.07$ & $<0.07$ & $<0.07$ \\
\hline Orthophosphate & & 198 & 18 & 15 & 15 & 20 & 13.8 \\
\hline Sulfate & & 17 & 44 & 7.4 & 9.3 & 10 & 11 \\
\hline \multicolumn{8}{|l|}{ Nutrients (mg/L) } \\
\hline Ammonia nitrogen (as $\mathrm{N}$ ) & 350.2 & 40 & 38 & 28 & 42 & 44 & 31 \\
\hline Total Kjeldahl nitrogen & 351.3 & 47 & 53 & 33 & 56 & 55 & 41 \\
\hline \multicolumn{8}{|l|}{ Oxygen demand (mg/L) } \\
\hline Biochemical oxygen demand & 405.1 & 221 & 214 & 98 & 305 & 96 & 143 \\
\hline Chemical oxygen demand & 410.4 & 363 & 664 & 224 & 644 & 161 & 270 \\
\hline \multicolumn{8}{|l|}{ Solids (mg/L) } \\
\hline Solid settling rate $(\mathrm{mL} / \mathrm{L} / \mathrm{h})$ & 160.5 & 21 & 19 & 34 & 27 & 26 & 23 \\
\hline Total dissolved solids (TDS) & 160.1 & 510 & 317 & 190 & 186 & 202 & 226 \\
\hline Total suspended solids (TSS) & 160.2 & 110 & 222 & 111 & 344 & 84 & 125 \\
\hline Volatile solids & 160.4 & 190 & 280 & 55 & 374 & 120 & 250 \\
\hline \multicolumn{8}{|l|}{ Total metals (mg/L) } \\
\hline Calcium & 200.7 & 14 & 17 & 12 & 14 & 12 & 19 \\
\hline Magnesium & 200.7 & 3.4 & 2.9 & 1.9 & 2.7 & 2.4 & 2.6 \\
\hline Potassium & 200.7 & 99 & 20 & 15 & 20 & 19 & 16 \\
\hline Selenium & 270.2 & $<0.002$ & $<0.002$ & $<0.002$ & $<0.002$ & $<0.002$ & $<0.002$ \\
\hline Sodium & 200.7 & 53 & 64 & 30 & 29 & 27 & 39 \\
\hline
\end{tabular}




\section{Sewerable Water Monitoring}

Table 6-7. Monthly monitoring results for physical and chemical characteristics of the LLNL sanitary sewer effluent, 1999 (continued).

\begin{tabular}{|c|c|c|c|c|c|c|c|}
\hline \multirow{2}{*}{$\begin{array}{l}\text { 24-hour composite sample } \\
\text { parameters }\end{array}$} & \multirow{2}{*}{$\begin{array}{c}\text { EPA } \\
\text { Method }\end{array}$} & \multicolumn{6}{|c|}{ Sample month } \\
\hline & & January & February & March & April & May & June \\
\hline Total organic carbon (TOC) (mg/L) & 415.1 & 56 & 94 & 55 & 64 & 64 & 38 \\
\hline Tributyltin (ng/L) & & 33 & 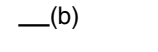 & 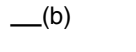 & —(b) & -(b) & -(b) \\
\hline $\begin{array}{l}\text { Semivolatile organic compounds } \\
(\mu \mathrm{g} / \mathrm{L})\end{array}$ & 625 & & & & & & \\
\hline 1,2,4-Trichlorobenzene ${ }^{(\mathrm{c})}$ & & $<5$ & $<50$ & $<5$ & $<10$ & $<50$ & $<10$ \\
\hline 1,2-Dichlorobenzene & & $<5$ & $<50$ & $<5$ & $<10$ & $<50$ & $<10$ \\
\hline 1,2-Diphenylhydrazine ${ }^{(\mathrm{c})}$ & & -(a) & 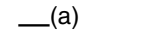 & -(a) & $<10$ & $<50$ & $<10$ \\
\hline 1,3-Dichlorobenzene ${ }^{(c)}$ & & $<5$ & $<50$ & $<5$ & $<10$ & $<50$ & $<10$ \\
\hline 1,4-Dichlorobenzene ${ }^{(c)}$ & & $<5$ & $<50$ & $<5$ & $<10$ & $<50$ & $<10$ \\
\hline 2,4,5-Trichlorophenol & & $<5$ & $<50$ & $<5$ & $<30$ & $<200$ & $<30$ \\
\hline 2,4,6-Trichlorophenol(c) & & $<5$ & $<50$ & $<5$ & $<30$ & $<200$ & $<30$ \\
\hline 2,4-Dichlorophenol(c) & & $<5$ & $<50$ & $<5$ & $<10$ & $<50$ & $<10$ \\
\hline 2,4-Dimethylphenol(c) & & $<5$ & $<50$ & $<5$ & $<10$ & $<50$ & $<10$ \\
\hline 2,4-Dinitrophenol(c) & & $<25$ & $<250$ & $<25$ & $<50$ & $<300$ & $<50$ \\
\hline 2,4-Dinitrotoluene ${ }^{(c)}$ & & $<5$ & $<50$ & $<5$ & $<10$ & $<50$ & $<10$ \\
\hline 2,6-Dinitrotoluene ${ }^{(c)}$ & & $<5$ & $<50$ & $<5$ & $<10$ & $<50$ & $<10$ \\
\hline 2-Chloronaphthalene ${ }^{(c)}$ & & $<5$ & $<50$ & $<5$ & $<10$ & $<50$ & $<10$ \\
\hline 2-Chlorophenol(c) & & $<5$ & $<50$ & $<5$ & $<10$ & $<50$ & $<10$ \\
\hline 2-Methyl-4,6-dinitrophenol & & $<25$ & $<250$ & $<25$ & $<50$ & $<300$ & $<50$ \\
\hline 2-Methylnaphthalene & & $<5$ & $<50$ & $<5$ & $<10$ & $<50$ & $<10$ \\
\hline 2-Naphthylamine & & 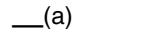 & 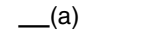 & 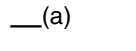 & $<100$ & $<500$ & $<100$ \\
\hline 2-Nitroaniline & & $<25$ & $<250$ & $<25$ & $<10$ & $<50$ & $<10$ \\
\hline 2-Nitrophenol(c) & & $<5$ & $<50$ & $<5$ & $<10$ & $<50$ & $<10$ \\
\hline 3,3-Dichlorobenzidine ${ }^{(c)}$ & & $<10$ & $<100$ & $<10$ & $<30$ & $<200$ & $<30$ \\
\hline 3-Nitroaniline & & $<25$ & $<250$ & $<25$ & $<10$ & $<50$ & $<10$ \\
\hline 4-Bromophenylphenylether & & $<5$ & $<50$ & $<5$ & $<10$ & $<50$ & $<10$ \\
\hline 4-Chloro-3-methylphenol & & $<10$ & $<100$ & $<10$ & $<30$ & $<200$ & $<30$ \\
\hline 4-Chloroaniline & & $<10$ & $<100$ & $<10$ & $<10$ & $<50$ & $<10$ \\
\hline 4-Chlorophenyl phenyl ether ${ }^{(c)}$ & & $<5$ & $<50$ & $<5$ & $<10$ & $<50$ & $<10$ \\
\hline 4-Nitroaniline & & $<25$ & $<250$ & $<25$ & $<30$ & $<200$ & $<30$ \\
\hline 4-Nitrophenol(c) & & $<25$ & $<250$ & $<25$ & $<30$ & $<200$ & $<30$ \\
\hline Acenaphthene ${ }^{(c)}$ & & $<5$ & $<50$ & $<5$ & $<10$ & $<50$ & $<10$ \\
\hline Acenaphthylene $\mathrm{e}^{(\mathrm{c})}$ & & $<5$ & $<50$ & $<5$ & $<10$ & $<50$ & $<10$ \\
\hline
\end{tabular}


Table 6-7. Monthly monitoring results for physical and chemical characteristics of the LLNL sanitary sewer effluent, 1999 (continued).

\begin{tabular}{|c|c|c|c|c|c|c|c|}
\hline \multirow{2}{*}{$\begin{array}{c}\text { 24-hour composite sample } \\
\text { parameters }\end{array}$} & \multirow{2}{*}{$\begin{array}{c}\text { EPA } \\
\text { Method }\end{array}$} & \multicolumn{6}{|c|}{ Sample month } \\
\hline & & July & August & September & October & November & December \\
\hline Total organic carbon (TOC) (mg/L) & 415.1 & 43 & 51 & 34 & 31 & 41 & 42 \\
\hline Tributyltin (ng/L) & & 7 & —(b) & -(b) $^{(\mathrm{n}}$ & -(b) $^{(\mathrm{n}}$ & -(b) $^{(\mathrm{c}}$ & -(b) \\
\hline $\begin{array}{l}\text { Semivolatile organic compounds } \\
(\mu \mathrm{g} / \mathrm{L})\end{array}$ & 625 & & & & & & \\
\hline 1,2,4-Trichlorobenzene ${ }^{(c)}$ & & $<20$ & $<10$ & $<2$ & $<2$ & $<2$ & $<2$ \\
\hline 1,2-Dichlorobenzene & & $<20$ & $<10$ & $<2$ & $<2$ & $<2$ & $<2$ \\
\hline 1,2-Diphenylhydrazine ${ }^{(\mathrm{c})}$ & & $<20$ & $<10$ & $<2$ & $<2$ & $<2$ & $<2$ \\
\hline 1,3-Dichlorobenzene ${ }^{(\mathrm{c})}$ & & $<20$ & $<10$ & $<2$ & $<2$ & $<2$ & $<2$ \\
\hline 1,4-Dichlorobenzene ${ }^{(\mathrm{c})}$ & & $<20$ & $<10$ & $<2$ & $<2$ & $<2$ & $<2$ \\
\hline 2,4,5-Trichlorophenol & & $<30$ & $<30$ & $<5$ & $<5$ & $<5$ & $<5$ \\
\hline 2,4,6-Trichlorophenol $(\mathrm{c})$ & & $<30$ & $<30$ & $<5$ & $<5$ & $<5$ & $<5$ \\
\hline 2,4-Dichlorophenol(c) & & $<20$ & $<10$ & $<2$ & $<2$ & $<2$ & $<2$ \\
\hline 2,4-Dimethylphenol(c) & & $<20$ & $<10$ & $<2$ & $<2$ & $<2$ & $<2$ \\
\hline 2,4-Dinitrophenol(c) & & $<60$ & $<10$ & $<10$ & $<10$ & $<10$ & $<10$ \\
\hline 2,4-Dinitrotoluene $(\mathrm{c})$ & & $<20$ & $<10$ & $<2$ & $<2$ & $<2$ & $<2$ \\
\hline 2,6-Dinitrotoluene ${ }^{(c)}$ & & $<20$ & $<10$ & $<2$ & $<2$ & $<2$ & $<2$ \\
\hline 2-Chloronaphthalene (c) & & $<20$ & $<10$ & $<2$ & $<2$ & $<2$ & $<2$ \\
\hline 2-Chlorophenol(c) & & $<20$ & $<10$ & $<2$ & $<2$ & $<2$ & $<2$ \\
\hline 2-Methyl-4,6-dinitrophenol & & $<60$ & $<50$ & $<10$ & $<10$ & $<10$ & $<10$ \\
\hline 2-Methylnaphthalene & & $<20$ & $<10$ & $<2$ & $<2$ & $<2$ & $<2$ \\
\hline 2-Naphthylamine & & $<200$ & $<100$ & $<20$ & $<20$ & $<20$ & $<20$ \\
\hline 2-Nitroaniline & & $<20$ & $<10$ & $<2$ & $<2$ & $<2$ & $<2$ \\
\hline 2-Nitrophenol(c) & & $<20$ & $<10$ & $<2$ & $<2$ & $<2$ & $<2$ \\
\hline 3,3-Dichlorobenzidine ${ }^{(c)}$ & & $<30$ & $<30$ & $<5$ & $<5$ & $<5$ & $<5$ \\
\hline 3-Nitroaniline & & $<20$ & $<10$ & $<2$ & $<2$ & $<2$ & $<2$ \\
\hline 4-Bromophenylphenylether & & $<20$ & $<10$ & $<2$ & $<2$ & $<2$ & $<2$ \\
\hline 4-Chloro-3-methylphenol & & $<30$ & $<50$ & $<5$ & $<5$ & $<5$ & $<5$ \\
\hline 4-Chloroaniline & & $<20$ & $<10$ & $<2$ & $<2$ & $<2$ & $<2$ \\
\hline 4-Chlorophenyl phenyl ether ${ }^{(\mathrm{c})}$ & & $<20$ & $<10$ & $<2$ & $<2$ & $<2$ & $<2$ \\
\hline 4-Nitroaniline & & $<30$ & $<30$ & $<5$ & $<5$ & $<5$ & $<5$ \\
\hline 4-Nitrophenol(c) & & $<30$ & $<30$ & $<5$ & $<5$ & $<5$ & $<5$ \\
\hline Acenaphthene $\mathrm{e}^{(\mathrm{c})}$ & & $<20$ & $<10$ & $<2$ & $<2$ & $<2$ & $<2$ \\
\hline Acenaphthylene $^{(\mathrm{c})}$ & & $<20$ & $<10$ & $<2$ & $<2$ & $<2$ & $<2$ \\
\hline
\end{tabular}




\section{Sewerable Water Monitoring}

Table 6-7. Monthly monitoring results for physical and chemical characteristics of the LLNL sanitary sewer effluent, 1999 (continued).

\begin{tabular}{|c|c|c|c|c|c|c|c|}
\hline \multirow{2}{*}{$\begin{array}{c}\text { 24-hour composite sample } \\
\text { parameters }\end{array}$} & \multirow{2}{*}{$\begin{array}{c}\text { EPA } \\
\text { Method } \\
\end{array}$} & \multicolumn{6}{|c|}{ Sample month } \\
\hline & & January & February & March & April & May & June \\
\hline $\begin{array}{l}\text { Semivolatile organic compounds } \\
(\mu \mathrm{g} / \mathrm{L}) \text { (continued) }\end{array}$ & 625 & & & & & & \\
\hline $\operatorname{Aldrin}^{(c)}$ & & - (a) $^{(a)}$ & - (a) $^{(\mathrm{n}}$ & $-^{(a)}$ & $<10$ & $<50$ & $<10$ \\
\hline Aniline & & 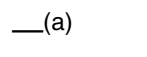 & -(a) $^{(\mathrm{a}}$ & -(a) $^{(\mathrm{a}}$ & $<30$ & $<200$ & $<30$ \\
\hline Anthracene ${ }^{(c)}$ & & $<5$ & $<50$ & $<5$ & $<10$ & $<50$ & $<10$ \\
\hline Benzidine $^{(\mathrm{c})}$ & & - (a) $^{(\mathrm{n}}$ & - (a) $^{(\mathrm{n}}$ & - (a) $^{(\mathrm{n}}$ & $<100$ & $<500$ & $<100$ \\
\hline Benzo(a)anthracene ${ }^{(c)}$ & & $<5$ & $<50$ & $<5$ & $<10$ & $<50$ & $<10$ \\
\hline Benzo(a)pyrene ${ }^{(c)}$ & & $<5$ & $<50$ & $<5$ & $<10$ & $<50$ & $<10$ \\
\hline Benzo(b)fluoranthene ${ }^{(c)}$ & & $<5$ & $<50$ & $<5$ & $<10$ & $<50$ & $<10$ \\
\hline Benzo(g,h,i)perylene ${ }^{(c)}$ & & $<5$ & $<50$ & $<5$ & $<10$ & $<50$ & $<10$ \\
\hline Benzo(k)fluoranthene ${ }^{(c)}$ & & $<5$ & $<50$ & $<5$ & $<10$ & $<50$ & $<10$ \\
\hline Benzoic acid & & $<25$ & $<250$ & $<25$ & $<50$ & $<300$ & $<50$ \\
\hline Benzyl alcohol & & $<10$ & $<100$ & $<10$ & $<5$ & 85 & 22 \\
\hline $\mathrm{BHC}$, alpha isomer ${ }^{(\mathrm{c})}$ & & -(a) & -(a) & -(a) & $<5$ & $<50$ & $<10$ \\
\hline $\mathrm{BHC}$, beta isomer $(\mathrm{c})$ & & - $^{(\mathrm{a})}$ & - $^{(\mathrm{a})}$ & 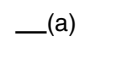 & $<5$ & $<50$ & $<10$ \\
\hline $\mathrm{BHC}$, delta isomer ${ }^{(\mathrm{c})}$ & & 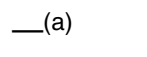 & -(a) & -(a) & $<10$ & $<50$ & $<10$ \\
\hline $\mathrm{BHC}$, gamma isomer (Lindane) ${ }^{(\mathrm{c})}$ & & - (a) $^{(\mathrm{n}}$ & -(a) & (a) $^{(\mathrm{a}}$ & $<10$ & $<50$ & $<10$ \\
\hline Bis(2-chloroethoxy)methane ${ }^{(c)}$ & & $<5$ & $<50$ & $<5$ & $<10$ & $<50$ & $<10$ \\
\hline Bis(2-chloroethyl)ether $(\mathrm{c})$ & & $<5$ & $<50$ & $<5$ & $<10$ & $<50$ & $<10$ \\
\hline Bis(2-chloroisopropyl)ether $(\mathrm{c})$ & & $<5$ & $<50$ & $<5$ & $<10$ & $<50$ & $<10$ \\
\hline Bis(2-ethylhexyl)phthalate ${ }^{(c)}$ & & 14 & $<50$ & 5.3 & $<30$ & $<200$ & $<30$ \\
\hline Butyl benzyl phthalate ${ }^{(c)}$ & & $<5$ & $<50$ & $<5$ & $<5$ & 55 & $<10$ \\
\hline Chrysene ${ }^{(c)}$ & & $<5$ & $<50$ & $<5$ & $<10$ & $<50$ & $<10$ \\
\hline Di-n-butyl phthalate ${ }^{(c)}$ & & $<5$ & $<50$ & $<5$ & 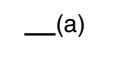 & —(a) & -(a) \\
\hline Di-n-octyl phthalate ${ }^{(c)}$ & & $<5$ & $<50$ & $<5$ & $<10$ & $<50$ & $<10$ \\
\hline Dibenzo(a,h)anthracene ${ }^{(\mathrm{c})}$ & & $<5$ & $<50$ & $<5$ & $<20$ & $<70$ & $<20$ \\
\hline Dibenzofuran & & $<5$ & $<50$ & $<5$ & $<10$ & $<50$ & $<10$ \\
\hline Dibutylphthalate & & - (a) $^{(a)}$ & -(a) & -(a) $^{(\mathrm{n}}$ & $<10$ & $<50$ & $<10$ \\
\hline Dieldrin $^{(\mathrm{c})}$ & & - $^{(\mathrm{a})}$ & - $^{(\mathrm{a})}$ & - $^{(a)}$ & $<20$ & $<70$ & $<20$ \\
\hline Diethyl phthalate ${ }^{(c)}$ & & 13 & $<50$ & $<5$ & $<10$ & $<50$ & $<10$ \\
\hline Dimethyl phthalate ${ }^{(c)}$ & & $<5$ & $<50$ & $<5$ & $<10$ & $<50$ & $<10$ \\
\hline Endosulfan I(c) & & -(a) $^{(\mathrm{a}}$ & -(a) & -(a) & $<50$ & $<300$ & $<50$ \\
\hline Endosulfan II(c) & & - (a) $^{(a)}$ & - (a) $^{(\mathrm{n}}$ & -(a) $^{(\mathrm{n}}$ & $<50$ & $<300$ & $<50$ \\
\hline Endosulfan sulfate ${ }^{(c)}$ & & -(a) $^{(\mathrm{a})}$ & -(a) $^{(\mathrm{a}}$ & -(a) $^{(\mathrm{n}}$ & $<20$ & $<70$ & $<20$ \\
\hline Endrin ${ }^{(c)}$ & & -(a) $^{(\mathrm{n}}$ & -(a) & (a) $^{(\mathrm{a}}$ & $<10$ & $<50$ & $<10$ \\
\hline
\end{tabular}


Table 6-7. Monthly monitoring results for physical and chemical characteristics of the LLNL sanitary sewer effluent, 1999 (continued).

\begin{tabular}{|c|c|c|c|c|c|c|c|}
\hline \multirow{2}{*}{$\begin{array}{c}\text { 24-hour composite sample } \\
\text { parameters }\end{array}$} & \multirow{2}{*}{$\begin{array}{c}\text { EPA } \\
\text { Method }\end{array}$} & \multicolumn{6}{|c|}{ Sample month } \\
\hline & & July & August & September & October & November & December \\
\hline $\begin{array}{l}\text { Semivolatile organic compounds } \\
(\mu \mathrm{g} / \mathrm{L}) \text { (continued) }\end{array}$ & 625 & & & & & & \\
\hline Aldrin(c) $^{(\mathrm{c})}$ & & $<20$ & $<10$ & $<2$ & $<2$ & $<2$ & $<2$ \\
\hline Aniline & & $<30$ & $<30$ & $<5$ & $<5$ & $<5$ & $<5$ \\
\hline Anthracene $e^{(c)}$ & & $<20$ & $<10$ & $<2$ & $<2$ & $<2$ & $<2$ \\
\hline Benzidine ${ }^{(c)}$ & & $<200$ & $<100$ & $<20$ & $<20$ & $<20$ & $<20$ \\
\hline Benzo(a)anthracene ${ }^{(c)}$ & & $<20$ & $<10$ & $<2$ & $<2$ & $<2$ & $<2$ \\
\hline Benzo(a)pyrene ${ }^{(c)}$ & & $<20$ & $<10$ & $<2$ & $<2$ & $<2$ & $<2$ \\
\hline Benzo(b)fluoranthene $e^{(c)}$ & & $<20$ & $<10$ & $<2$ & $<2$ & $<2$ & $<2$ \\
\hline Benzo(g,h,i)perylene ${ }^{(\mathrm{c})}$ & & $<20$ & $<10$ & $<2$ & $<2$ & $<2$ & $<2$ \\
\hline Benzo(k)fluoranthene ${ }^{(\mathrm{c})}$ & & $<20$ & $<10$ & $<2$ & $<2$ & $<2$ & $<2$ \\
\hline Benzoic acid & & $<60$ & $<50$ & 37 & $<10$ & 31 & 11 \\
\hline Benzyl alcohol & & $<20$ & $<10$ & 4.3 & $<2$ & 7.8 & 5.4 \\
\hline $\mathrm{BHC}$, alpha isomer( $\mathrm{c})$ & & $<20$ & $<10$ & $<2$ & $<2$ & $<2$ & $<2$ \\
\hline $\mathrm{BHC}$, beta isomer $\mathrm{c}^{\mathrm{c})}$ & & $<20$ & $<10$ & $<2$ & $<2$ & $<2$ & $<2$ \\
\hline $\mathrm{BHC}$, delta isomer $(\mathrm{c})$ & & $<20$ & $<10$ & $<2$ & $<2$ & $<2$ & $<2$ \\
\hline $\mathrm{BHC}$, gamma isomer (Lindane) ${ }^{(\mathrm{c})}$ & & $<20$ & $<10$ & $<2$ & $<2$ & $<2$ & $<2$ \\
\hline Bis(2-chloroethoxy)methane ${ }^{(\mathrm{c})}$ & & $<20$ & $<10$ & $<2$ & $<2$ & $<2$ & $<2$ \\
\hline Bis(2-chloroethyl)ether $(\mathrm{c})$ & & $<20$ & $<30$ & $<2$ & $<2$ & $<2$ & $<2$ \\
\hline Bis(2-chloroisopropyl)ether ${ }^{(\mathrm{c})}$ & & $<20$ & $<10$ & $<2$ & $<2$ & $<2$ & $<2$ \\
\hline Bis(2-ethylhexyl)phthalate $(\mathrm{c})$ & & 21 & 20 & $<5$ & 7.5 & 8.9 & 5.8 \\
\hline Butyl benzyl phthalate ${ }^{(\mathrm{c})}$ & & $<20$ & $<10$ & $<2$ & $<2$ & $<2$ & $<2$ \\
\hline Chrysene ${ }^{(\mathrm{c})}$ & & $<20$ & $<10$ & $<2$ & $<2$ & $<2$ & $<2$ \\
\hline Di-n-butyl phthalate ${ }^{(c)}$ & & -(a) $^{(\mathrm{a}}$ & -(a) $^{(\mathrm{n}}$ & -(a) $^{(\mathrm{a}}$ & - (a) $^{(a)}$ & -(a) & -(a) $^{(\mathrm{a}}$ \\
\hline Di-n-octyl phthalate ${ }^{(\mathrm{c})}$ & & $<20$ & $<10$ & $<2$ & $<2$ & $<2$ & $<2$ \\
\hline Dibenzo(a,h)anthracene ${ }^{(\mathrm{c})}$ & & $<20$ & $<20$ & $<3$ & $<3$ & $<3$ & $<3$ \\
\hline Dibenzofuran & & $<20$ & $<10$ & $<2$ & $<2$ & $<2$ & $<2$ \\
\hline Dibutylphthalate & & $<20$ & $<10$ & $<2$ & $<2$ & $<2$ & $<2$ \\
\hline $\operatorname{Dieldrin}^{(\mathrm{c})}$ & & $<20$ & $<20$ & $<3$ & $<3$ & $<3$ & $<3$ \\
\hline Diethyl phthalate ${ }^{(c)}$ & & 14 & $<10$ & 7.7 & 8.3 & 4.9 & 5.3 \\
\hline Dimethyl phthalate $(\mathrm{c})$ & & $<20$ & $<10$ & $<2$ & $<2$ & $<2$ & $<2$ \\
\hline Endosulfan I(c) & & $<60$ & $<50$ & $<10$ & $<10$ & $<10$ & $<10$ \\
\hline Endosulfan II (c) & & $<60$ & $<50$ & $<10$ & $<10$ & $<10$ & $<10$ \\
\hline Endosulfan sulfate ${ }^{(\mathrm{c})}$ & & $<20$ & $<20$ & $<3$ & $<3$ & $<3$ & $<3$ \\
\hline Endrin ${ }^{(\mathrm{c})}$ & & $<20$ & $<10$ & $<2$ & $<2$ & $<2$ & $<2$ \\
\hline
\end{tabular}




\section{Sewerable Water Monitoring}

Table 6-7. Monthly monitoring results for physical and chemical characteristics of the LLNL sanitary sewer effluent, 1999 (continued).

\begin{tabular}{|c|c|c|c|c|c|c|c|}
\hline \multirow{2}{*}{$\begin{array}{c}\text { 24-hour composite sample } \\
\text { parameters }\end{array}$} & \multirow{2}{*}{$\begin{array}{c}\text { EPA } \\
\text { Method }\end{array}$} & \multicolumn{6}{|c|}{ Sample month } \\
\hline & & January & February & March & April & May & June \\
\hline $\begin{array}{l}\text { Semivolatile organic compounds } \\
(\mu \mathrm{g} / \mathrm{L}) \text { (continued) }\end{array}$ & 625 & & & & & & \\
\hline Endrin aldehyde ${ }^{(\mathrm{c})}$ & & -(a) $^{(\mathrm{n}}$ & -(a) $^{\text {(a) }}$ & —(a) $^{(\mathrm{n}}$ & $<10$ & $<50$ & $<10$ \\
\hline Fluoranthene $e^{(\mathrm{c})}$ & & $<5$ & $<50$ & $<5$ & $<10$ & $<50$ & $<10$ \\
\hline Fluorene $e^{(\mathrm{c})}$ & & $<5$ & $<50$ & $<5$ & $<10$ & $<50$ & $<10$ \\
\hline Heptachlor(c) & & -(a) $^{(\mathrm{n}}$ & —(a) & —(a) & $<10$ & $<50$ & $<10$ \\
\hline Heptachlor epoxide ${ }^{(\mathrm{c})}$ & & -(a) & —(a) & —(a) & $<10$ & $<50$ & $<10$ \\
\hline Hexachlorobenzene ${ }^{(\mathrm{c})}$ & & $<5$ & $<50$ & $<5$ & $<10$ & $<50$ & $<10$ \\
\hline Hexachlorobutadiene ${ }^{(\mathrm{c})}$ & & $<5$ & $<50$ & $<5$ & $<10$ & $<50$ & $<10$ \\
\hline Hexachlorocyclopentadiene $e^{(c)}$ & & $<5$ & $<50$ & $<5$ & $<10$ & $<50$ & $<10$ \\
\hline Hexachloroethane $e^{(c)}$ & & $<5$ & $<50$ & $<5$ & $<10$ & $<50$ & $<10$ \\
\hline Indeno(1,2,3-c,d)pyrene ${ }^{(c)}$ & & $<5$ & $<50$ & $<5$ & $<10$ & $<50$ & $<10$ \\
\hline Isophorone $e^{(c)}$ & & $<5$ & $<50$ & $<5$ & $<10$ & $<50$ & $<10$ \\
\hline $\mathrm{m}$ - and $\mathrm{p}$ - Cresol & & $<5$ & $<50$ & 19 & -(a) $^{\text {(a) }}$ & —(a) & —(a) \\
\hline N-Nitrosodi-n-propylamine ${ }^{(c)}$ & & $<5$ & $<50$ & $<5$ & $<10$ & $<50$ & $<10$ \\
\hline N-Nitrosodimethylamine $e^{(c)}$ & & -(a) & —(a) $^{\text {(a) }}$ & —(a) $^{(\mathrm{a})}$ & $<10$ & $<50$ & $<10$ \\
\hline N-Nitrosodiphenylamine ${ }^{(\mathrm{c})}$ & & $<5$ & $<50$ & $<5$ & $<10$ & $<50$ & $<10$ \\
\hline Naphthalene & & $<5$ & $<50$ & $<5$ & $<10$ & $<50$ & $<10$ \\
\hline Nitrobenzene ${ }^{(c)}$ & & $<5$ & $<50$ & $<5$ & $<10$ & $<50$ & $<10$ \\
\hline o-Cresol & & $<5$ & $<50$ & $<5$ & $<10$ & $<50$ & $<10$ \\
\hline$p, p-D_{D}^{(c)}$ & & -(a) $^{(\mathrm{n}}$ & —(a) $^{\text {(a) }}$ & —(a) $^{(\mathrm{a})}$ & $<10$ & $<50$ & $<10$ \\
\hline$p, p-D D E^{(c)}$ & & -(a) $^{(\mathrm{n}}$ & —(a) $^{\text {(a) }}$ & —(a) $^{(\mathrm{a})}$ & $<20$ & $<70$ & $<20$ \\
\hline $\mathrm{p}, \mathrm{p}-\mathrm{DDT} \mathrm{T}^{(\mathrm{c})}$ & & -(a) $^{(a)}$ & —(a) $^{\text {(a) }}$ & 一(a) $^{\text {(a) }}$ & $<10$ & $<50$ & $<10$ \\
\hline p-Cresol & & -(a) $^{(a)}$ & —(a) $^{\text {(a) }}$ & —(a) & 23 & $<50$ & $<10$ \\
\hline Pentachlorophenol(c) & & $<25$ & $<250$ & $<25$ & $<50$ & $<300$ & $<50$ \\
\hline Phenanthrene $e^{(c)}$ & & $<5$ & $<50$ & $<5$ & $<10$ & $<50$ & $<10$ \\
\hline Phenol(c) & & $<5$ & $<50$ & $<5$ & $<10$ & $<50$ & $<10$ \\
\hline Pyrene ${ }^{(c)}$ & & $<5$ & $<50$ & $<5$ & $<10$ & $<50$ & $<10$ \\
\hline Total cyanide (mg/L) & 335.2 & $<0.02$ & $<0.02$ & $<0.02$ & $<0.02$ & $<0.02$ & $<0.02$ \\
\hline Total oil and grease $(\mathrm{mg} / \mathrm{L})$ & 413.1 & & & & & & \\
\hline 6:00 a.m. & & 2.3 & 4.8 & 3.1 & 2.7 & -(d) $^{(1}$ & (d) $^{(\mathrm{d})}$ \\
\hline 10:00 a.m. & & 34 & 34 & 21 & 30 & —(d) $^{(\mathrm{n}}$ & —(d) $^{(\mathrm{n}}$ \\
\hline 2:00 p.m. & & 25 & 37 & 14 & 23 & -(d) $^{(1)}$ & (d) $^{(\mathrm{d})}$ \\
\hline 6:00 p.m. & & 18 & 2.2 & 16 & 37 & -(d) $^{\text {(d) }}$ & (d) $^{\text {(d) }}$ \\
\hline
\end{tabular}


Table 6-7. Monthly monitoring results for physical and chemical characteristics of the LLNL sanitary sewer effluent, 1999 (continued).

\begin{tabular}{|c|c|c|c|c|c|c|c|}
\hline \multirow{2}{*}{$\begin{array}{l}\text { 24-hour composite sample } \\
\text { parameters }\end{array}$} & \multirow{2}{*}{$\begin{array}{c}\text { EPA } \\
\text { Method }\end{array}$} & \multicolumn{6}{|c|}{ Sample month } \\
\hline & & July & August & September & October & November & December \\
\hline $\begin{array}{l}\text { Semivolatile organic compounds } \\
(\mu \mathrm{g} / \mathrm{L}) \text { (continued) }\end{array}$ & 625 & & & & & & \\
\hline Endrin aldehyde ${ }^{(c)}$ & & $<20$ & $<10$ & $<2$ & $<2$ & $<2$ & $<2$ \\
\hline Fluoranthene $e^{(\mathrm{c})}$ & & $<20$ & $<10$ & $<2$ & $<2$ & $<2$ & $<2$ \\
\hline Fluorene $e^{(c)}$ & & $<20$ & $<10$ & $<2$ & $<2$ & $<2$ & $<2$ \\
\hline Heptachlor(c) & & $<20$ & $<10$ & $<2$ & $<2$ & $<2$ & $<2$ \\
\hline Heptachlor epoxide ${ }^{(c)}$ & & $<20$ & $<10$ & $<2$ & $<2$ & $<2$ & $<2$ \\
\hline Hexachlorobenzene ${ }^{(\mathrm{c})}$ & & $<20$ & $<10$ & $<2$ & $<2$ & $<2$ & $<2$ \\
\hline Hexachlorobutadiene ${ }^{(\mathrm{c})}$ & & $<20$ & $<10$ & $<2$ & $<2$ & $<2$ & $<2$ \\
\hline Hexachlorocyclopentadiene $\mathrm{e}^{(\mathrm{c})}$ & & $<20$ & $<10$ & $<2$ & $<2$ & $<2$ & $<2$ \\
\hline Hexachloroethane $e^{(c)}$ & & $<20$ & $<10$ & $<2$ & $<2$ & $<2$ & $<2$ \\
\hline Indeno(1,2,3-c,d)pyrene ${ }^{(c)}$ & & $<20$ & $<10$ & $<2$ & $<2$ & $<2$ & $<2$ \\
\hline Isophorone $e^{(c)}$ & & $<20$ & $<10$ & $<2$ & $<2$ & $<2$ & $<2$ \\
\hline $\mathrm{m}$ - and p-Cresol & & -(a) $^{(\mathrm{n}}$ & 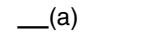 & -(a) $^{(\mathrm{n}}$ & 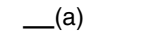 & -(a) $^{(\mathrm{n}}$ & 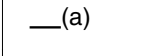 \\
\hline N-Nitrosodi-n-propylamine ${ }^{(c)}$ & & $<20$ & $<10$ & $<2$ & $<2$ & $<2$ & $<2$ \\
\hline N-Nitrosodimethylamine ${ }^{(c)}$ & & $<20$ & $<10$ & $<2$ & $<2$ & $<2$ & $<2$ \\
\hline N-Nitrosodiphenylamine ${ }^{(c)}$ & & $<20$ & $<10$ & $<2$ & $<2$ & $<2$ & $<2$ \\
\hline Naphthalene & & $<20$ & $<10$ & $<2$ & $<2$ & $<2$ & $<2$ \\
\hline Nitrobenzene $e^{(c)}$ & & $<20$ & $<10$ & $<2$ & $<2$ & $<2$ & $<2$ \\
\hline o-Cresol & & $<20$ & $<10$ & $<2$ & $<2$ & $<2$ & $<2$ \\
\hline$p, p-D D D^{(c)}$ & & $<20$ & $<10$ & $<2$ & $<2$ & $<2$ & $<2$ \\
\hline$p, p-D D E^{(c)}$ & & $<20$ & $<20$ & $<3$ & $<3$ & $<3$ & $<3$ \\
\hline$p, p-D D T^{(c)}$ & & $<20$ & $<10$ & $<2$ & $<2$ & $<2$ & $<2$ \\
\hline p-Cresol & & $<20$ & $<10$ & 16 & $<2$ & 29 & $<2$ \\
\hline Pentachlorophenol(c) & & $<60$ & $<50$ & $<10$ & $<10$ & $<10$ & $<10$ \\
\hline Phenanthrene ${ }^{(\mathrm{c})}$ & & $<20$ & $<10$ & $<2$ & $<2$ & $<2$ & $<2$ \\
\hline Phenol(c) & & $<20$ & $<10$ & 2 & $<2$ & 14 & $<2$ \\
\hline Pyrene ${ }^{(c)}$ & & $<20$ & $<10$ & $<2$ & $<2$ & $<2$ & $<2$ \\
\hline Total cyanide (mg/L) & 335.2 & $<0.02$ & $-^{(\mathrm{b})}$ & 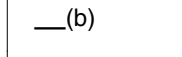 & —(b) & 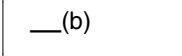 & -(b) $^{(\mathrm{c}}$ \\
\hline Total oil and grease $(\mathrm{mg} / \mathrm{L})$ & 413.1 & & & & & & \\
\hline 6:00 a.m. & & -(d) $^{(\mathrm{n}}$ & 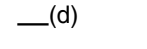 & -(d) $^{(\mathrm{n}}$ & -(d) $^{(\mathrm{n}}$ & -(d) $^{(\mathrm{n}}$ & 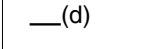 \\
\hline 10:00 a.m. & & -(d) $^{(\mathrm{n}}$ & -(d) $^{(\mathrm{n}}$ & -(d) $^{(\mathrm{n}}$ & -(d) $^{(\mathrm{n}}$ & -(d) $^{(\mathrm{n}}$ & -(d) $^{(\mathrm{n}}$ \\
\hline 2:00 p.m. & & 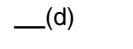 & 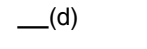 & 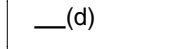 & (d) $^{(\mathrm{d}}$ & (d) $^{(\mathrm{d})}$ & (d) $^{(\mathrm{d})}$ \\
\hline 6:00 p.m. & & -(d) & -(d) $^{(\mathrm{s})}$ & -(d) & 一 (d) $^{\text {(n) }}$ & -(d) & -(d) $^{\text {(d) }}$ \\
\hline
\end{tabular}




\section{Sewerable Water Monitoring}

Table 6-7. Monthly monitoring results for physical and chemical characteristics of the LLNL sanitary sewer effluent, 1999 (continued).

\begin{tabular}{|c|c|c|c|c|c|c|c|}
\hline \multirow{2}{*}{$\begin{array}{c}\text { 24-hour composite sample } \\
\text { parameters }\end{array}$} & \multirow{2}{*}{\begin{tabular}{|c|} 
EPA \\
Method \\
\end{tabular}} & \multicolumn{6}{|c|}{ Sample month } \\
\hline & & January & February & March & April & May & June \\
\hline Total recoverable phenolics (mg/L) & 420.1 & 0.069 & 0.024 & 0.038 & —(e) & —(e) & —(e) \\
\hline Volatile organic compounds ( $\mu \mathrm{g} / \mathrm{L}$ ) & 624 & & & & & & \\
\hline 1,1,1-Trichloroethane ${ }^{(c)}$ & & $<2$ & $<1$ & $<1$ & $<0.5$ & $<0.5$ & $<0.5$ \\
\hline 1,1,2,2-Tetrachloroethane ${ }^{(c)}$ & & $<2$ & $<1$ & $<1$ & $<0.5$ & $<0.5$ & $<0.5$ \\
\hline 1,1,2-Trichloroethane ${ }^{(c)}$ & & $<2$ & $<1$ & $<1$ & $<0.5$ & $<0.5$ & $<0.5$ \\
\hline 1,1-Dichloroethane ${ }^{(c)}$ & & $<2$ & $<1$ & $<1$ & $<0.5$ & $<0.5$ & $<0.5$ \\
\hline 1,1-Dichloroethene ${ }^{(c)}$ & & $<2$ & $<1$ & $<1$ & $<0.5$ & $<0.5$ & $<0.5$ \\
\hline 1,2-Dichlorobenzene(c) & & $<2$ & $<1$ & $<1$ & $<0.5$ & $<0.5$ & $<0.5$ \\
\hline 1,2-Dichloroethane & & $<2$ & $<1$ & $<1$ & $<0.5$ & $<0.5$ & $<0.5$ \\
\hline 1,2-Dichloroethene (total) & & $<2$ & $<1$ & $<1$ & $<1$ & $<1$ & $<1$ \\
\hline 1,2-Dichloropropane ${ }^{(c)}$ & & $<2$ & $<1$ & $<1$ & $<0.5$ & $<0.5$ & $<0.5$ \\
\hline 1,3-Dichlorobenzene & & $<2$ & $<1$ & $<1$ & $<0.5$ & $<0.5$ & $<0.5$ \\
\hline 1,4-Dichlorobenzene & & $<2$ & 1.2 & 1.4 & 1.9 & 3.6 & 3.1 \\
\hline 2-Butanone & & $<80$ & $<40$ & $<40$ & $<20$ & $<20$ & $<20$ \\
\hline 2-Chloroethyl vinylether ${ }^{(c)}$ & & $<80$ & $<40$ & $<40$ & $<5$ & $<5$ & $<5$ \\
\hline 2-Hexanone & & $<20$ & $<10$ & $<10$ & $<20$ & $<20$ & $<20$ \\
\hline 4-Methyl-2-pentanone & & $<20$ & $<10$ & $<10$ & $<20$ & $<20$ & $<20$ \\
\hline Acetone & & 150 & 140 & 54 & 220 & 100 & 57 \\
\hline Benzene $^{(c)}$ & & $<2$ & $<1$ & $<1$ & $<0.5$ & $<0.5$ & $<0.5$ \\
\hline Bromodichloromethane & & $<2$ & $<1$ & $<1$ & $<0.5$ & $<0.5$ & $<0.5$ \\
\hline Bromoform & & $<2$ & $<1$ & $<1$ & $<0.5$ & $<0.5$ & $<0.5$ \\
\hline Bromomethane & & $<4$ & $<2$ & $<2$ & $<0.5$ & $<0.5$ & $<0.5$ \\
\hline Carbon disulfide & & $<2$ & $<1$ & $<1$ & $<5$ & $<5$ & $<5$ \\
\hline Carbon tetrachloride ${ }^{(\mathrm{c})}$ & & $<2$ & $<1$ & $<1$ & $<0.5$ & $<0.5$ & $<0.5$ \\
\hline Chlorobenzene & & $<2$ & $<1$ & $<1$ & $<0.5$ & $<0.5$ & $<0.5$ \\
\hline Chloroethane $^{(\mathrm{c})}$ & & $<4$ & $<2$ & $<2$ & $<1$ & $<1$ & $<1$ \\
\hline Chloroform $^{(\mathrm{c})}$ & & 10 & 12 & 23 & 8.5 & 8.7 & 18 \\
\hline Chloromethane ${ }^{(c)}$ & & $<4$ & $<2$ & $<2$ & $<1$ & $<1$ & $<1$ \\
\hline cis-1,2-Dichloroethene & & -(a) & -(a) $^{(\mathrm{n}}$ & -(a) $^{(\mathrm{n}}$ & $<0.5$ & $<0.5$ & $<0.5$ \\
\hline cis-1,3-Dichloropropene & & $<2$ & $<1$ & $<1$ & $<0.5$ & $<0.5$ & $<0.5$ \\
\hline Dibromochloromethane & & $<2$ & $<1$ & $<1$ & $<0.5$ & $<0.5$ & $<0.5$ \\
\hline Dibromomethane & & $<2$ & $<1$ & $<1$ & -(a) & -(a) $^{(\mathrm{n}}$ & -(a) $^{(\mathrm{n}}$ \\
\hline
\end{tabular}


Table 6-7. Monthly monitoring results for physical and chemical characteristics of the LLNL sanitary sewer effluent, 1999 (continued).

\begin{tabular}{|c|c|c|c|c|c|c|c|}
\hline \multirow{2}{*}{$\begin{array}{c}\text { 24-hour composite sample } \\
\text { parameters }\end{array}$} & \multirow{2}{*}{$\begin{array}{c}\text { EPA } \\
\text { Method }\end{array}$} & \multicolumn{6}{|c|}{ Sample month } \\
\hline & & July & August & September & October & November & December \\
\hline Total recoverable phenolics (mg/L) & 420.1 & -(e) $^{(\mathrm{e})}$ & —(e) & -(e) $^{(\mathrm{e})}$ & -(e) $^{(\mathrm{e})}$ & -(e) $^{(\mathrm{s})}$ & —e) $^{(\mathrm{e})}$ \\
\hline Volatile organic compounds ( $\mu \mathrm{g} / \mathrm{L})$ & 624 & & & & & & \\
\hline 1,1,1-Trichloroethane $\mathrm{e}^{(\mathrm{c})}$ & & $<0.5$ & $<0.5$ & $<0.5$ & $<0.5$ & $<0.5$ & $<0.5$ \\
\hline $1,1,2,2-$ Tetrachloroethane $^{(\mathrm{c})}$ & & $<0.5$ & $<0.5$ & $<0.5$ & $<0.5$ & $<0.5$ & $<0.5$ \\
\hline $1,1,2$-Trichloroethane ${ }^{(\mathrm{c})}$ & & $<0.5$ & $<0.5$ & $<0.5$ & $<0.5$ & $<0.5$ & $<0.5$ \\
\hline 1,1-Dichloroethane ${ }^{(\mathrm{c})}$ & & $<0.5$ & $<0.5$ & $<0.5$ & $<0.5$ & $<0.5$ & $<0.5$ \\
\hline 1,1-Dichloroethene ${ }^{(\mathrm{c})}$ & & $<0.5$ & $<0.5$ & $<0.5$ & $<0.5$ & $<0.5$ & $<0.5$ \\
\hline 1,2-Dichlorobenzene ${ }^{(c)}$ & & $<0.5$ & $<0.5$ & $<0.5$ & $<0.5$ & $<0.5$ & $<0.5$ \\
\hline 1,2-Dichloroethane & & $<0.5$ & $<0.5$ & $<0.5$ & $<0.5$ & $<0.5$ & $<0.5$ \\
\hline 1,2-Dichloroethene (total) & & $<1$ & $<1$ & $<1$ & $<1$ & $<1$ & $<1$ \\
\hline 1,2-Dichloropropane ${ }^{(c)}$ & & $<0.5$ & $<0.5$ & $<0.5$ & $<0.5$ & $<0.5$ & $<0.5$ \\
\hline 1,3-Dichlorobenzene & & $<0.5$ & $<0.5$ & $<0.5$ & $<0.5$ & $<0.5$ & $<0.5$ \\
\hline 1,4-Dichlorobenzene & & 1.2 & 1.9 & $<0.5$ & 0.91 & 1.4 & 1.5 \\
\hline 2-Butanone & & $<20$ & $<20$ & $<20$ & $<20$ & $<20$ & $<20$ \\
\hline 2-Chloroethyl vinylether ${ }^{(\mathrm{c})}$ & & $<5$ & $<5$ & $<5$ & $<5$ & $<5$ & $<5$ \\
\hline 2-Hexanone & & $<20$ & $<20$ & $<20$ & $<20$ & $<20$ & $<20$ \\
\hline 4-Methyl-2-pentanone & & $<20$ & $<20$ & $<20$ & $<20$ & $<20$ & $<20$ \\
\hline Acetone & & 72 & 130 & 360 & 200 & 110 & 100 \\
\hline Benzene ${ }^{(c)}$ & & 1.5 & $<0.5$ & $<0.5$ & $<0.5$ & $<0.5$ & $<0.5$ \\
\hline Bromodichloromethane & & $<0.5$ & $<0.5$ & $<0.5$ & $<0.5$ & $<0.5$ & $<0.5$ \\
\hline Bromoform & & $<0.5$ & $<0.5$ & $<0.5$ & $<0.5$ & $<0.5$ & $<0.5$ \\
\hline Bromomethane & & $<0.5$ & $<0.5$ & $<0.5$ & $<0.5$ & $<0.5$ & $<0.5$ \\
\hline Carbon disulfide & & $<5$ & $<5$ & $<5$ & $<5$ & $<5$ & $<5$ \\
\hline Carbon tetrachloride ${ }^{(c)}$ & & $<0.5$ & $<0.5$ & $<0.5$ & $<0.5$ & $<0.5$ & $<0.5$ \\
\hline Chlorobenzene & & $<0.5$ & $<0.5$ & $<0.5$ & $<0.5$ & $<0.5$ & $<0.5$ \\
\hline Chloroethane $e^{(c)}$ & & $<1$ & $<1$ & $<1$ & $<1$ & $<1$ & $<1$ \\
\hline Chloroform(c) & & 19 & 15 & 11 & 13 & 16 & 14 \\
\hline Chloromethane $(\mathrm{c})$ & & $<1$ & $<1$ & $<1$ & $<1$ & $<1$ & $<1$ \\
\hline cis-1,2-Dichloroethene & & $<0.5$ & $<0.5$ & $<0.5$ & $<0.5$ & $<0.5$ & $<0.5$ \\
\hline cis-1,3-Dichloropropene & & $<0.5$ & $<0.5$ & $<0.5$ & $<0.5$ & $<0.5$ & $<0.5$ \\
\hline Dibromochloromethane & & $<0.5$ & $<0.5$ & $<0.5$ & $<0.5$ & $<0.5$ & $<0.5$ \\
\hline Dibromomethane & & —(a) & -(a) & $<5$ & $<0.5$ & $<0.5$ & $<0.5$ \\
\hline
\end{tabular}




\section{Sewerable Water Monitoring}

Table 6-7. Monthly monitoring results for physical and chemical characteristics of the LLNL sanitary sewer effluent, 1999 (continued).

\begin{tabular}{|c|c|c|c|c|c|c|c|}
\hline \multirow{2}{*}{$\begin{array}{c}\text { 24-hour composite sample } \\
\text { parameters }\end{array}$} & \multirow{2}{*}{$\begin{array}{c}\text { EPA } \\
\text { Method }\end{array}$} & \multicolumn{6}{|c|}{ Sample month } \\
\hline & & January & February & March & April & May & June \\
\hline $\begin{array}{l}\text { Volatile organic compounds ( } \mu \mathrm{g} / \mathrm{L} \text { ) } \\
\text { (continued) }\end{array}$ & 624 & & & & & & \\
\hline Dichlorodifluoromethane & & $<4$ & $<2$ & $<2$ & 0.71 & $<0.5$ & $<0.5$ \\
\hline Ethanol & & -(a) & -(a) $^{\text {(a) }}$ & 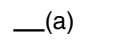 & -(a) & -(a) & -(a) $^{\text {(a) }}$ \\
\hline Ethylbenzene $e^{(\mathrm{c})}$ & & $<2$ & $<1$ & $<1$ & $<0.5$ & $<0.5$ & $<0.5$ \\
\hline Freon 113 & & $<2$ & $<1$ & $<1$ & $<0.5$ & $<0.5$ & $<0.5$ \\
\hline Methylene chloride $\mathrm{c}^{(\mathrm{c})}$ & & $<2$ & $<1$ & $<1$ & 1.1 & $<1$ & $<1$ \\
\hline Naphthalene ${ }^{(\mathrm{c})}$ & & -(a) & -(a) $^{\text {(a) }}$ & -(a) & -(a) & -(a) $^{(\mathrm{a}}$ & -(a) $^{(\mathrm{n}}$ \\
\hline Styrene & & $<2$ & $<1$ & $<1$ & $<0.5$ & $<0.5$ & $<0.5$ \\
\hline Tetrachloroethene $e^{(c)}$ & & $<2$ & $<1$ & $<1$ & $<0.5$ & $<0.5$ & $<0.5$ \\
\hline Toluene ${ }^{(c)}$ & & $<2$ & 1.6 & $<1$ & 1.4 & 1.1 & $<0.5$ \\
\hline Total xylene isomers & & $<4$ & $<2$ & $<2$ & $<1$ & $<1$ & $<1$ \\
\hline trans-1,2-Dichloroethene $(\mathrm{c})$ & & -(a) & -(a) $^{(\mathrm{n}}$ & -(a) $^{(\mathrm{n}}$ & $<0.5$ & $<0.5$ & $<0.5$ \\
\hline trans-1,3-Dichloropropene & & $<2$ & $<1$ & $<1$ & $<0.5$ & $<0.5$ & $<0.5$ \\
\hline Trichloroethene ${ }^{(c)}$ & & $<1$ & $<0.5$ & $<0.5$ & $<0.5$ & $<0.5$ & $<0.5$ \\
\hline Trichlorofluoromethane & & $<2$ & $<1$ & $<1$ & $<0.5$ & $<0.5$ & $<0.5$ \\
\hline Vinyl acetate & & $<20$ & $<10$ & $<10$ & -(a) & -(a) $^{(\mathrm{n}}$ & -(a) $^{(\mathrm{n}}$ \\
\hline Vinyl chloride ${ }^{(\mathrm{c})}$ & & $<4$ & $<2$ & $<2$ & $<0.5$ & $<0.5$ & $<0.5$ \\
\hline
\end{tabular}

a Sample missed because of a change in contract analytical services. Some parameters were not being sampled under the old contract, which ended in March.

b Sampling required by permit on a semiannual basis.

C Indicates priority pollutant parameter used in computing the total organic permit limit of $1 \mathrm{mg} / \mathrm{L}(1000 \mu \mathrm{g} / \mathrm{L})$ issued by the Livermore Water Reclamation Plant. 
Table 6-7. Monthly monitoring results for physical and chemical characteristics of the LLNL sanitary sewer effluent, 1999 (concluded).

\begin{tabular}{|c|c|c|c|c|c|c|c|}
\hline \multirow{2}{*}{$\begin{array}{c}\text { 24-hour composite sample } \\
\text { parameters }\end{array}$} & \multirow{2}{*}{$\begin{array}{c}\text { EPA } \\
\text { Method }\end{array}$} & \multicolumn{6}{|c|}{ Sample month } \\
\hline & & July & August & September & October & November & December \\
\hline $\begin{array}{l}\text { Volatile organic compounds ( } \mu \mathrm{g} / \mathrm{L}) \\
\text { (continued) }\end{array}$ & 624 & & & & & & \\
\hline Dichlorodifluoromethane & & $<0.5$ & $<0.5$ & $<0.5$ & $<0.5$ & $<0.5$ & $<0.5$ \\
\hline Ethanol & & —(a) & —(a) & $<1000$ & $<1000$ & $<1000$ & $<1000$ \\
\hline Ethylbenzene $e^{(c)}$ & & $<0.5$ & $<0.5$ & $<0.5$ & $<0.5$ & $<0.5$ & $<0.5$ \\
\hline Freon 113 & & $<0.5$ & $<0.5$ & $<0.5$ & $<0.5$ & $<0.5$ & $<0.5$ \\
\hline Methylene chloride ${ }^{(\mathrm{c})}$ & & $<1$ & $<1$ & $<1$ & $<1$ & $<1$ & 1.4 \\
\hline Naphthalene ${ }^{(\mathrm{c})}$ & & -(a) $^{(\mathrm{n}}$ & -(a) $^{(\mathrm{n}}$ & $<5$ & $<0.5$ & $<0.5$ & $<0.5$ \\
\hline Styrene & & $<0.5$ & $<0.5$ & $<0.5$ & $<0.5$ & $<0.5$ & $<0.5$ \\
\hline Tetrachloroethene $\mathrm{c}^{(\mathrm{c})}$ & & $<0.5$ & $<0.5$ & $<0.5$ & $<0.5$ & $<0.5$ & $<0.5$ \\
\hline Toluene $^{(\mathrm{c})}$ & & 0.65 & 0.51 & $<0.5$ & 0.76 & 0.58 & $<0.5$ \\
\hline Total xylene isomers & & $<1$ & $<1$ & $<1$ & $<1$ & $<1$ & $<1$ \\
\hline trans-1,2-Dichloroethene ${ }^{(\mathrm{c})}$ & & $<0.5$ & $<0.5$ & $<0.5$ & $<0.5$ & $<0.5$ & $<0.5$ \\
\hline trans-1,3-Dichloropropene & & $<0.5$ & $<0.5$ & $<0.5$ & $<0.5$ & $<0.5$ & $<0.5$ \\
\hline Trichloroethene ${ }^{(\mathrm{c})}$ & & $<0.5$ & $<0.5$ & $<0.5$ & $<0.5$ & $<0.5$ & $<0.5$ \\
\hline Trichlorofluoromethane & & $<0.5$ & $<0.5$ & $<0.5$ & $<0.5$ & $<0.5$ & $<0.5$ \\
\hline Vinyl acetate & & -(a) $^{(\mathrm{n}}$ & -(a) $^{(\mathrm{n}}$ & -(a) $^{(\mathrm{n}}$ & -(a) $^{(\mathrm{n}}$ & -(a) $^{(\mathrm{n}}$ & -(a) $^{(\mathrm{n}}$ \\
\hline Vinyl chloride $(\mathrm{c})$ & & $<0.5$ & $<0.5$ & $<0.5$ & $<0.5$ & $<0.5$ & $<0.5$ \\
\hline
\end{tabular}

d Sampling discontinued as directed by April 1999 letter from LWRPR because of a change in the Environmental Protection Agency sampling method.

e Sampling discontinued because this was not a required permit parameter. 



\section{Surface Water Monitoring}

Sandra Mathews

Erich R. Brandstetter

Karen J. Folks

Ted A. Giesing

Shari Brigdon

\section{Introduction}

Lawrence Livermore National Laboratory monitors surface water at the Livermore site, in surrounding regions of the Livermore Valley, and at Site 300 and vicinity in the nearby Altamont Hills. At the first two locales, LLNL monitors reservoirs and ponds, the LLNL swimming pool, rainfall, tap water, and storm water runoff. Water samples are analyzed for radionuclides and a wide range of nonradioactive constituents. At Site 300 and vicinity, surface water monitoring encompasses rainfall and storm water runoff. Samples of this water are analyzed for radionuclides, high explosives (HE), total organic carbon, total organic halides, total suspended solids, conductivity, and $\mathrm{pH}$.

Chapter 7 of the main volume includes summary data tables and a detailed discussion and analysis of the data. This supplemental chapter presents the complete dataset for 1999, from which the summaries and analyses were prepared. This data supplements material provided in the Surface Water Chapter (Chapter 7) of the main volume.

\section{Storm Water}

LLNL technologists collect storm water samples for nonradiological analysis directly into sample bottles for storm water runoff grab samples. Samples analyzed for tritium are collected in 250-mL, argon-flushed glass containers; samples for gross alpha and gross beta measurements are collected in 1000-mL polyethylene bottles. Numerical comparison criteria for Livermore site storm water are listed in Table 7-1. Results for Livermore site routine tritium, gross alpha, and gross beta are presented in Table 7-2. Results for the tritium source investigation are presented in Table 7-3. Results for plutonium in filtered and unfiltered samples are presented in Table 7-4. Results for metals detected at the Livermore site are presented in Table 7-5. Table 7-6 summarizes results for nonradioactive compounds, physical and chemical properties, and anions in Livermore site storm water. Data for nondetections in Livermore site storm water are summarized in Table 7-7. Table 7-8 shows results for tritium, gross alpha, gross beta, plutonium, and uranium in Site 300 storm water. Results for nonradioactive compounds and physical 


\section{Surface Water}

properties for Site 300 storm water are listed in Table 7-9. Results of dioxin analyses at Site 300 sampling location NLIN are presented in Table 7-10. Analytical results from monitoring of Pit 6 post-closure runoff are in Table 7-11.

\section{Rainfall}

Rainfall is collected in stainless steel buckets mounted about $1 \mathrm{~m}$ above the ground. Samples are decanted into 500-mL argon-flushed amber glass with teflon lined lids and analyzed for tritium. Results are presented in Table 7-12.

\section{Drainage Retention Basin}

DRB discharge sampling locations, which monitor compliance with the Livermore site's Comprehensive Environmental Response, Compensation, and Liability Act (CERCLA), Record of Decision are shown in Figure 7-2, main volume. Figure 7-9 of the main volume shows the sampling locations used to monitor how water quality compares with maintenance goals and action levels. Weekly sampling for dissolved oxygen and temperature occurs at all eight locations identified in Figure 7-9 of the main volume. Weekly turbidity measurements and monthly, quarterly, semiannual, and annual samples are collected at sample location CDBE.

Tables 7-13 and 7-14 show DRB discharge limits and water quality management levels, respectively. Table 7-15 shows the compliance monitoring data for samples collected at sample locations CDBX and WPDC from four DRB releases. Monthly, quarterly, and semiannual maintenance monitoring data for 1998 that were collected at sample location CDBE are shown in Tables 7-16, 17, and 18. Table 7-19 provides the weekly field measurements collected from sample locations CDBA, CDBC, CDBD, CDBE, CDBF, $\mathrm{CDBJ}, \mathrm{CDBK}$, and CDBL. A seasonal inventory of plants and animals observed the Livermore site comprises Table 7-20.

\section{Other Waters}

LLNL technologists sample surface and drinking water near the Livermore site and in the Livermore Valley using a tethered pail to collect water from surface sources; other locations are sampled directly from the outfall. Samples for tritium analysis are collected in 500-mL, argon-flushed glass containers; those for other radiological analyses are collected in acidified 1000-mL polyethylene bottles. Results are presented in Table 7-21. 


\section{Surface Water}

Table 7-1. Numerical comparison criteria for storm water constituents of concern.

\begin{tabular}{|c|c|c|c|}
\hline Constituent & PMCL/SMCL & AWQC/Ag (a) & EPA benchmark \\
\hline \multicolumn{4}{|l|}{ Physical } \\
\hline Specific conductance $(\mu \mathrm{mho} / \mathrm{cm})$ & none/900 $(C A)^{(b)}$ & $700(\mathrm{Ag})$ & none \\
\hline Total suspended solids (TSS) (mg/L) & none/none & none & 100 \\
\hline \multicolumn{4}{|l|}{ Biological (mg/L) } \\
\hline Biochemical oxygen demand (BOD) & none/none & none & 30 \\
\hline \multicolumn{4}{|l|}{ Anions (mg/L) } \\
\hline Bicarbonate alkalinity $\left(\right.$ as $\mathrm{CaCO}_{3}$ ) & none/none & none & none \\
\hline Bromide & none/none & none & none \\
\hline Carbonate alkalinity $\left(\mathrm{as} \mathrm{CaCO}_{3}\right)$ & none/none & none & none \\
\hline Chloride & none/250 (EPA) $)^{(c)}$ & 250 & 860 \\
\hline Fluoride & 2.0/none $(\mathrm{CA})$ & $1.0(\mathrm{Ag})$ & 1.8 \\
\hline & $0.8 /$ none $(\mathrm{SF})^{(\mathrm{d})}$ & & \\
\hline Nitrate (as N) & 10/none (EPA) & none & 0.68 \\
\hline Nitrate $\left(\right.$ as $\left.\mathrm{NO}_{3}\right)$ & 45/none (CA) & none & 3.01 \\
\hline Nitrate plus nitrite (as N) & 10/none (EPA) & none & 0.68 \\
\hline Nitrate plus nitrite $\left(\right.$ as $\left.\mathrm{NO}_{3}\right)$ & 45/none (CA) & none & 3.01 \\
\hline Nitrite (as N) & 1.0/none (EPA) & none & 0.68 \\
\hline Nitrite (as $\mathrm{NO}_{2}$ ) & 3.3/none (EPA) & none & 2.2 \\
\hline Sulfate & none/250 (EPA) & none & none \\
\hline \multicolumn{4}{|l|}{ General minerals (mg/L) } \\
\hline Calcium & none/none & none & none \\
\hline $\mathrm{pH}$ (pH units) & none/<6.5, >8.5 (EPA) & $<6.5,>8.5$ & $<6.0,>9.0$ \\
\hline Orthophosphate & none/none & none & none \\
\hline Potassium & none/none & none & none \\
\hline Sodium & none/none & none & none \\
\hline Surfactants & none/0.5 (CA) & 0.5 (SF) & none \\
\hline Total alkalinity $\left(\mathrm{as} \mathrm{CaCO}_{3}\right)$ & none/none (EPA) & $\geq 20$ & none \\
\hline Total dissolved solids (TDS) & none/500 (EPA) & 500 (SF) & none \\
\hline Total hardness $\left(\mathrm{as} \mathrm{CaCO}_{3}\right)$ & none/none & none & none \\
\hline Total phosphorous & none/none & 0.0001 & 2.0 \\
\hline \multicolumn{4}{|l|}{ Metals (mg/L) } \\
\hline Aluminum & $1.0 / 0.2(\mathrm{CA})$ & 0.75 & 0.75 \\
\hline Antimony & $0.006 /$ none (EPA) & 0.088 & 0.636 \\
\hline Arsenic & 0.05/none (EPA) & 0.36 & 0.168 \\
\hline
\end{tabular}




\section{Surface Water}

Table 7-1. Numerical comparison criteria for storm water constituents of concern (continued).

\begin{tabular}{|c|c|c|c|}
\hline Constituent & PMCL/SMCL & AWQC/Ag(a) & EPA benchmark \\
\hline \multicolumn{4}{|l|}{ Metals (mg/L) (continued) } \\
\hline Barium & 1.0/none $(\mathrm{CA})$ & none & none \\
\hline Beryllium & $0.004 /$ none (EPA) & $0.5(\mathrm{Ag})$ & 0.13 \\
\hline Boron & none/none & $0.7(\mathrm{Ag})$ & none \\
\hline Cadmium & $0.005 /$ none $(E P A)$ & $0.005^{(e)}$ & $0.0159(\mathrm{e})$ \\
\hline Chromium, total & $0.05 /$ none $(C A)$ & none & none \\
\hline Chromium(VI) & none/none & 0.015 & none \\
\hline \multirow[t]{2}{*}{ Copper } & $1.3 / 1.0^{(\mathrm{g})}(\mathrm{EPA})$ & $0.026^{(e)}$ & $0.0636^{(\mathrm{f})}$ \\
\hline & & $0.2(\mathrm{SF}, \mathrm{Ag})$ & \\
\hline Iron & none/0.3 (EPA) & none & 1.0 \\
\hline Lead & $0.015 /$ none (EPA) & $0.11^{(e)}$ & $0.0816^{(\mathrm{f})}$ \\
\hline Magnesium & none/none & none & 0.0636 \\
\hline Manganese & none/0.05 (EPA) & $0.2(\mathrm{Ag})$ & 1.0 \\
\hline Mercury & 0.002/none (EPA) & 0.0021 & 0.0024 \\
\hline Molybdenum & none/none & 0.05 (SF, Ag) & none \\
\hline Nickel & $0.1 /$ none $(C A)$ & $2.1^{(\mathrm{e})}$ & $1.417^{(\mathrm{f})}$ \\
\hline Selenium & $0.05 /$ none $(E P A)$ & 0.02 & 0.2385 \\
\hline Silver & none/0.1 (EPA) & $0.0077^{(e)}$ & $0.0318^{(f)}$ \\
\hline Thallium & $0.002 /$ none (EPA) & $450(\mathrm{Ag})$ & none \\
\hline Vanadium & none/none & 0.1 (SF Ag) & none \\
\hline Zinc & none/5 (EPA) & $0.17^{(e)}$ & $0.117^{(f)}$ \\
\hline \multicolumn{4}{|c|}{ Volatile organic compounds $(\mu \mathrm{g} / \mathrm{L})$} \\
\hline Acetone & none/none & none & none \\
\hline Benzene & 1.0/none $(\mathrm{CA})$ & none & 10 \\
\hline Chloroform & 80/none (EPA) & none & none \\
\hline Chloromethane & none/none & none & none \\
\hline \multicolumn{4}{|c|}{ Semivolatile organic compounds ( $\mu \mathrm{g} / \mathrm{L})$} \\
\hline Benzo[a] pyrene & $0.2 /$ none (EPA) & none & none \\
\hline Bis(2-ethylhexyl)phthalate & $4 /$ none $(\mathrm{CA})$ & none & none \\
\hline Butylbenzylphthalate & none/none & none & none \\
\hline \multicolumn{4}{|l|}{ Herbicides $(\mu \mathrm{g} / \mathrm{L})$} \\
\hline $2,4-D$ & 0.07/none (EPA) & none & none \\
\hline $2,4,5-\mathrm{T}$ & none/none & none & none \\
\hline Bromacil & none/none & none & none \\
\hline Diazinon & none/none & 0.009 & none \\
\hline
\end{tabular}




\section{Surface Water}

Table 7-1. Numerical comparison criteria for storm water constituents of concern (concluded).

\begin{tabular}{|l|c|c|c|}
\hline \multicolumn{1}{|c|}{ Constituent } & PMCL/SMCL & AWQC/Ag & EPA benchmark \\
\hline Herbicides $(\boldsymbol{\mu g} / \mathbf{L})$ (continued) & & & \\
$\quad$ Diuron & none/none & none & none \\
Glyphosate & $700 /$ none (CA) & none & none \\
Simazine & $4 /$ none (EPA) & 10 & none \\
Miscellaneous organics (mg/L) & & & \\
Chemical oxygen demand (mg/L) & none/none & none & 120 \\
Oil and grease & none/none & virtually free & 15 \\
Total organic carbon & none/none & none & 50 \\
Radioactive (Bq/L) & & & \\
Tritium & $740 /$ none (EPA) & 740 & none \\
Gross alpha & $0.56 /$ none (EPA) & 0.56 & none \\
Gross beta & $1.85 /$ none (EPA) & 1.85 & none \\
\hline
\end{tabular}

a $\mathrm{Ag}=$ Criteria for agricultural use.

b CA indicates a state PMCL or SMLL.

c EPA indicates a federal PMCL or SMLL.

d $\mathrm{SF}=$ San Francisco Bay Basin Plan.

e Hardness dependent; based on receiving water hardness of $160 \mathrm{mg} / \mathrm{L}$.

f Hardness dependent benchmark at assumed $100 \mathrm{mg} / \mathrm{L} \mathrm{CaCO}_{3}$.

g 1.3 is U.S. primary maximum contaminant level (PMCL), not to be exceeded in more than $10 \%$ of samples; 1.0 is U.S./CA secondary maximum contaminant level (SMCL). 


\section{Surface Water}

Table 7-2. Routine tritium, gross alpha, and gross beta sampling in storm water runoff at the Livermore site, 1999.

\begin{tabular}{|l|c|c|c|}
\hline \multirow{2}{*}{ Parameter } & \multirow{2}{*}{ Date } & \multicolumn{2}{|c|}{ Arroyo Seco } \\
\cline { 3 - 4 } & & Site influent & Site effluent \\
\cline { 3 - 4 } & & ASS2 & ASW \\
\hline Tritium (Bq/L) & $1 / 26$ & $-0.903 \pm 2.24$ & $25.6 \pm 3.15$ \\
& $2 / 8$ & $14.4 \pm 2.24$ & $38.1 \pm 2.99$ \\
Gross alpha $(\mathrm{Bq} / \mathrm{L})$ & $4 / 8$ & $-0.670 \pm 2.49$ & $-0.703 \pm 2.52$ \\
& $11 / 8$ & - (a) & $0.189 \pm 2.17$ \\
& $1 / 26$ & $0.0146 \pm 0.0237$ & $0.0681 \pm 0.0326$ \\
& $2 / 8$ & $0.0433 \pm 0.0366$ & $0.00 \pm 0.0266$ \\
Gross beta $(\mathrm{Bq} / \mathrm{L})$ & $4 / 8$ & $0.0381 \pm 0.0292$ & $0.00618 \pm 0.0135$ \\
& $11 / 8$ & $-{ }^{*}(\mathrm{a})$ & $0.0235 \pm 0.0191$ \\
& $1 / 26$ & $0.0351 \pm 0.0703$ & $0.125 \pm 0.0666$ \\
& $2 / 8$ & $0.121 \pm 0.0814$ & $0.111 \pm 0.0888$ \\
& $4 / 8$ & $0.133 \pm 0.0392$ & $0.109 \pm 0.0357$ \\
& $11 / 8$ & $-(a)$ & $0.212 \pm 0.0329$ \\
\hline
\end{tabular}

\begin{tabular}{|l|c|c|c|c|c|}
\hline \multirow{3}{*}{ Parameter } & \multirow{3}{*}{ Date } & \multicolumn{3}{|c|}{ Site influent } & Site effluent \\
\cline { 3 - 6 } & & ALPE & ALPO & GRNE & WPDC \\
\cline { 3 - 6 } & $1 / 20$ & $0.350 \pm 1.93$ & $-0.648 \pm 1.77$ & $18.6 \pm 2.53$ & $13.1 \pm 2.34$ \\
\hline Tritium (Bq/L) & $2 / 8$ & $6.77 \pm 1.92$ & $8.14 \pm 1.97$ & $43.7 \pm 3.24$ & $58.5 \pm 3.53$ \\
& $4 / 8$ & $2.66 \pm 2.58$ & $0.278 \pm 2.55$ & $6.44 \pm 2.78$ & $11.1 \pm 2.96$ \\
& $11 / 8$ & $4.70 \pm 2.37$ & $2.13 \pm 2.28$ & $3.51 \pm 2.32$ & $37.7 \pm 3.53$ \\
Gross alpha $(\mathrm{Bq} / \mathrm{L})$ & $1 / 20$ & $0.107 \pm 0.0518$ & $0.0551 \pm 0.0518$ & $0.0710 \pm 0.0444$ & $0.0321 \pm 0.0444$ \\
& $2 / 8$ & $0.160 \pm 0.152$ & $0.255 \pm 0.0962$ & $0.0477 \pm 0.0337$ & $0.044 \pm 0.0407$ \\
& $4 / 8$ & $0.0385 \pm 0.0385$ & $0.152 \pm 0.0755$ & $0.655 \pm 0.0426$ & $0.0629 \pm 0.0459$ \\
& $11 / 8$ & $0.0522 \pm 0.0284$ & $0.231 \pm 0.0981$ & $0.477 \pm 0.159$ & $0.0659 \pm 0.0396$ \\
& $1 / 20$ & $0.337 \pm 0.296$ & $0.223 \pm 0.148$ & $0.183 \pm 0.0703$ & $0.208 \pm 0.0777$ \\
Gross beta $(\mathrm{Bq} / \mathrm{L})$ & $2 / 8$ & $0.299 \pm 0.285$ & $0.279 \pm 0.137$ & $0.120 \pm 0.0666$ & $0.159 \pm 0.0740$ \\
& $4 / 8$ & $0.228 \pm 0.0555$ & $0.374 \pm 0.0722$ & $0.136 \pm 0.0400$ & $0.224 \pm 0.0510$ \\
& $11 / 8$ & $0.162 \pm 0.0287$ & $0.344 \pm 0.0799$ & $0.655 \pm 0.114$ & $0.237 \pm 0.0444$ \\
\hline
\end{tabular}




\section{Surface Water}

Table 7-2. Tritium, gross alpha, and gross beta in storm water runoff at the Livermore site, 1999 (concluded).

\begin{tabular}{|c|c|c|c|c|}
\hline \multirow{3}{*}{ Parameter } & \multirow{3}{*}{ Date } & \multicolumn{3}{|c|}{ Drainage Retention Basin } \\
\hline & & \multicolumn{2}{|c|}{ Site influent } & \multirow{2}{*}{$\begin{array}{c}\text { Site effluent } \\
\text { CDBX }\end{array}$} \\
\hline & & CDB & CDB2 & \\
\hline \multirow[t]{5}{*}{ Tritium $(\mathrm{Bq} / \mathrm{L})$} & $1 / 20$ & $53.3 \pm 3.47$ & —(a) & $21.7 \pm 2.63$ \\
\hline & $1 / 26$ & -(b) $^{(\mathrm{c}}$ & $47.4 \pm 3.77$ & -(b) \\
\hline & $2 / 8$ & $89.5 \pm 4.33$ & $58.5 \pm 3.52$ & $29.5 \pm 2.75$ \\
\hline & $4 / 8$ & $3.07 \pm 2.67$ & $52.9 \pm 4.18$ & $23.3 \pm 3.37$ \\
\hline & $11 / 8$ & $3.67 \pm 2.33$ & $7.03 \pm 2.46$ & $21.5 \pm 3.00$ \\
\hline \multirow[t]{5}{*}{ Gross alpha $(\mathrm{Bq} / \mathrm{L})$} & $1 / 20$ & $0.121 \pm 0.0518$ & 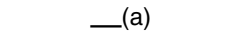 & $0.0633 \pm 0.0518$ \\
\hline & $1 / 26$ & —(b) & $0.0755 \pm 0.0518$ & -(b) \\
\hline & $2 / 8$ & $0.0210 \pm 0.0270$ & $0.149 \pm 0.111$ & $0.170 \pm 0.0851$ \\
\hline & $4 / 8$ & $0.0112 \pm 0.0239$ & $0.0607 \pm 0.0548$ & $0.0944 \pm 0.0640$ \\
\hline & $11 / 8$ & $0.0259 \pm 0.0183$ & $0.114 \pm 0.0477$ & $0.116 \pm 0.0648$ \\
\hline \multirow[t]{5}{*}{ Gross beta $(\mathrm{Bq} / \mathrm{L})$} & $1 / 20$ & $0.295 \pm 0.252$ & —(a) & $0.118 \pm 0.0740$ \\
\hline & $1 / 26$ & —(b) & $0.0755 \pm 0.0777$ & —(b) \\
\hline & $2 / 8$ & $0.118 \pm 0.0666$ & $0.179 \pm 0.137$ & $0.135 \pm 0.0962$ \\
\hline & $4 / 8$ & $0.156 \pm 0.0422$ & $0.248 \pm 0.0559$ & $0.0470 \pm 0.0799$ \\
\hline & $11 / 8$ & $0.192 \pm 0.0295$ & $0.374 \pm 0.0555$ & $0.170 \pm 0.0500$ \\
\hline
\end{tabular}

Note: Radioactivities are reported as the measured concentration and either an uncertainty $( \pm 2 \sigma$ counting error) or as being less than or equal to the detection limit. If the concentration is less than or equal to the uncertainty or the detection limit, the result is considered to be a nondetection. See the main volume, Chapter 14, Quality Assurance.

a Sample not collected because there was no flow.

b Locations sampled 1/20 were not sampled again on 1/26. 


\section{Surface Water}

Table 7-3. Tritium source investigation sampling in storm water runoff $(\mathrm{Bq} / \mathrm{L})$ at the Livermore site, 1999.

\begin{tabular}{|c|c|c|c|c|}
\hline \multirow[b]{2}{*}{ Location } & \multicolumn{4}{|c|}{ Date sampled } \\
\hline & $1 / 2 / 99$ & 2/8/99 & 4/8/99 & $11 / 8 / 99$ \\
\hline $191 E$ & -(a) & $310 \pm 8$ & $31.9 \pm 3.45$ & -(a) \\
\hline $191 S$ & -(a) $^{(\mathrm{a}}$ & $49.2 \pm 4.03$ & $2.28 \pm 2.41$ & -(a) $^{\text {(a) }}$ \\
\hline 196E & $13.2 \pm 2.34$ & $96.9 \pm 4.37$ & $24.2 \pm 3.41$ & -(a) $^{\text {(a) }}$ \\
\hline $196 S$ & $4.70 \pm 2.02$ & $5.62 \pm 1.88$ & $7.55 \pm 2.83$ & - $^{(\mathrm{a})}$ \\
\hline 2582 & - (a) $^{(\mathrm{n}}$ & - (a) $^{(\mathrm{a}}$ & $15.4 \pm 2.91$ & $722 \pm 37.7$ \\
\hline $298 E$ & - (a) $^{(\mathrm{a})}$ & - (a) $^{(a)}$ & $4.96 \pm 2.52$ & $12.4 \pm 2.64$ \\
\hline $298 S$ & - (a) $^{(\mathrm{n}}$ & - $^{(\mathrm{a})}$ & $237 \pm 7.33$ & $414 \pm 9.32$ \\
\hline 3726 & - (a) $^{(a)}$ & - $^{(\mathrm{a})}$ & $511 \pm 10.4$ & $1780 \pm 19.1$ \\
\hline 494E & $23.2 \pm 2.68$ & $43.3 \pm 3.85$ & $5.33 \pm 2.72$ & -(a) $^{(\mathrm{a})}$ \\
\hline $494 S$ & $23.3 \pm 2.68$ & $33.7 \pm 3.57$ & $18.0 \pm 3.18$ & - $^{(\mathrm{a})}$ \\
\hline 591E & - (a) $^{(\mathrm{n}}$ & $18.3 \pm 3.12$ & $2.87 \pm 2.43$ & - (a) $^{(a)}$ \\
\hline $591 S$ & - (a) $^{(\mathrm{n}}$ & $36.5 \pm 3.66$ & $8.03 \pm 2.83$ & —(a) $^{(\mathrm{n}}$ \\
\hline T49E & $21.7 \pm 2.66$ & $71.0 \pm 4.55$ & $5.14 \pm 2.53$ & - (a) $^{(a)}$ \\
\hline T495 & $8.73 \pm 2.19$ & $36.5 \pm 3.67$ & $4.18 \pm 2.49$ & - (a) $^{(a)}$ \\
\hline WPDS & $1.18 \pm 1.91$ & $85.5 \pm 4.14$ & $25.6 \pm 3.44$ & - $^{(\mathrm{a})}$ \\
\hline WPDW & $12.4 \pm 2.29$ & $16.7 \pm 2.33$ & $1.14 \pm 2.35$ & - $^{(\mathrm{a})}$ \\
\hline
\end{tabular}

Note: Radioactivities are reported as the measured concentration and either an uncertainty $( \pm 2 \sigma$ counting error) or as being less than or equal to the detection limit. If the concentration is less than or equal to the uncertainty or the detection limit, the result is considered to be a nondetection.

a Location not sampled on the indicated date. Sample not collected at this location. 
Table 7-4. Plutonium in storm water runoff, Livermore site, 1999.

\begin{tabular}{|c|c|c|c|c|}
\hline \multirow{3}{*}{ Parameter } & \multirow{3}{*}{$\begin{array}{c}\text { Date } \\
\text { Sampled }\end{array}$} & \multicolumn{3}{|c|}{ Arroyo Seco effluent } \\
\hline & & \multicolumn{3}{|c|}{ ASW } \\
\hline & & $\begin{array}{l}\text { Unfiltered water } \\
(\mathrm{Bq} / \mathrm{L})\end{array}$ & $\begin{array}{l}\text { Filtered water } \\
(\mathrm{Bq} / \mathrm{L})\end{array}$ & $\begin{array}{l}\text { Sediments } \\
(\mathrm{Bq} / \mathrm{g})\end{array}$ \\
\hline \multirow[t]{4}{*}{ Plutonium-238 } & $1 / 26$ & $2.47 \times 10^{-6} \pm 3.44 \times 10^{-5}$ & -(a) & -(a) $^{(\mathrm{a}}$ \\
\hline & $2 / 8$ & $1.47 \times 10^{-6} \pm 1.02 \times 10^{-4}$ & -(a) $^{(a)}$ & -(a) $^{\text {(a) }}$ \\
\hline & $4 / 8$ & -(b) $^{(\mathrm{l}}$ & $0.00 \times 10^{0} \pm 0.00 \times 10^{0}$ & $4.18 \times 10^{-4} \pm 4.11 \times 10^{-3}$ \\
\hline & $11 / 8$ & $5.22 \times 10^{-5} \pm 1.56 \times 10^{-4}$ & $4.11 \times 10^{-5} \pm 7.66 \times 10^{-5}$ & $-5.81 \times 10^{-4} \pm 1.11 \times 10^{-3}$ \\
\hline \multirow[t]{4}{*}{ Plutonium-239+240 } & $1 / 26$ & $1.73 \times 10^{-5} \pm 4.55 \times 10^{-5}$ & -(a) $^{(a)}$ & -(a) $^{(a)}$ \\
\hline & $2 / 8$ & $-2.06 \times 10^{-5} \pm 4.14 \times 10^{-5}$ & —(a) & -(a) $^{\text {(a) }}$ \\
\hline & $4 / 8$ & -(b) $^{(\mathrm{c}}$ & $1.13 \times 10^{-5} \pm 4.22 \times 10^{-5}$ & $9.36 \times 10^{-4} \pm 3.96 \times 10^{-3}$ \\
\hline & $11 / 8$ & $4.03 \times 10^{-5} \pm 1.06 \times 10^{-4}$ & $1.03 \times 10^{-5} \pm 3.81 \times 10^{-5}$ & $3.85 \times 10^{-4} \pm 7.73 \times 10^{-4}$ \\
\hline
\end{tabular}

\begin{tabular}{|c|c|c|c|c|}
\hline \multirow{3}{*}{ Parameter } & \multirow{3}{*}{$\begin{array}{c}\text { Date } \\
\text { Sampled }\end{array}$} & \multicolumn{3}{|c|}{ Arroyo Las Positas effluent } \\
\hline & & \multicolumn{3}{|c|}{ WPDC } \\
\hline & & $\begin{array}{l}\text { Unfiltered water } \\
(\mathrm{Bq} / \mathrm{L})\end{array}$ & $\begin{array}{l}\text { Filtered water } \\
\quad(\mathrm{Bq} / \mathrm{L})\end{array}$ & $\begin{array}{l}\text { Sediments } \\
(\mathrm{Bq} / \mathrm{g})\end{array}$ \\
\hline \multirow[t]{4}{*}{ Plutonium-238 } & $1 / 20$ & $5.07 \times 10^{-5} \pm 1.21 \times 10^{-4}$ & -(a) & -(a) \\
\hline & $2 / 8$ & $-4.74 \times 10^{-5} \pm 9.81 \times 10^{-5}$ & -(a) & 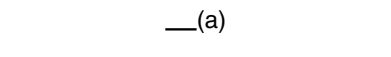 \\
\hline & $4 / 8$ & -(b) & $3.27 \times 10^{-6} \pm 4.55 \times 10^{-5}$ & $-1.15 \times 10^{-3} \pm 1.52 \times 10^{-3}$ \\
\hline & $11 / 8$ & $-2.60 \times 10^{-6} \pm 1.05 \times 10^{-4}$ & $4.59 \times 10^{-5} \pm 7.07 \times 10^{-5}$ & $-5.70 \times 10^{-5} \pm 2.23 \times 10^{-4}$ \\
\hline \multirow[t]{4}{*}{ Plutonium-239+240 } & $1 / 20$ & $-3.04 \times 10^{-5} \pm 3.52 \times 10^{-5}$ & -(a) & -(a) $^{(\mathrm{n}}$ \\
\hline & $2 / 8$ & $8.36 \times 10^{-5} \pm 9.88 \times 10^{-5}$ & -(a) & 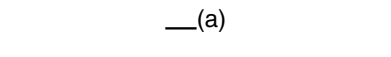 \\
\hline & $4 / 8$ & -(b) $^{(\mathrm{n}}$ & $-5.70 \times 10^{-5} \pm 4.33 \times 10^{-5}$ & $-3.27 \times 10^{-4} \pm 1.28 \times 10^{-3}$ \\
\hline & $11 / 8$ & $9.84 \times 10^{-5} \pm 1.30 \times 10^{-4}$ & $6.66 \times 10^{-5} \pm 6.66 \times 10^{-5}$ & $4.22 \times 10^{-4} \pm 3.64 \times 10^{-4}$ \\
\hline
\end{tabular}

Note: Radioactivities are reported as the measured concentration and either an uncertainty ( $\pm 2 \sigma$ counting error) or as being less than or equal to the detection limit. If the concentration is less than or equal to the uncertainty or the detection limit, the result is considered to be a nondetection. See the main volume, Chapter 14, Quality Assurance.

a Sample was only analyzed on unfiltered water.

b Sample was only analyzed on filtered water and filtrate (sediments). 


\section{Surface Water}

Table 7-5. Metals detected in storm water runoff, Livermore site, 1999.

\begin{tabular}{|c|c|c|c|c|}
\hline \multirow[b]{2}{*}{ Parameter $(\mathrm{mg} / \mathrm{L})$} & \multirow[b]{2}{*}{ Requested analysis } & \multirow[b]{2}{*}{ Storm date } & \multicolumn{2}{|c|}{ Arroyo Seco } \\
\hline & & & $\begin{array}{c}\text { Site influent } \\
\text { ASS2 }\end{array}$ & $\begin{array}{c}\text { Site effluent } \\
\text { ASW }\end{array}$ \\
\hline \multirow[t]{18}{*}{ Aluminum } & GENMIN & $1 / 20$ & - $^{(\mathrm{a})}$ & - $^{(\mathrm{a})}$ \\
\hline & GENMINDISS & & -(a) $^{(\mathrm{n})}$ & - (a) $^{(a)}$ \\
\hline & NPDESDISS & & -(a) & -(a) \\
\hline & NPDESMETAL & & -(a) & -(a) $^{(\mathrm{a})}$ \\
\hline & GENMIN & $1 / 26$ & 0.92 & 3.6 \\
\hline & GENMINDISS & & 0.13 & 0.21 \\
\hline & NPDESDISS & & 0.14 & 0.27 \\
\hline & NPDESMETAL & & 0.88 & 3.3 \\
\hline & GENMIN & $2 / 8$ & 2.9 & 2.5 \\
\hline & GENMINDISS & & 0.17 & 0.15 \\
\hline & NPDESDISS & & 0.15 & 0.13 \\
\hline & NPDESMETAL & & 3 & 2.9 \\
\hline & GENMIN & $4 / 8$ & 2.8 & 1.7 \\
\hline & GENMINDISS & & 0.13 & 0.13 \\
\hline & NPDESDISS & & 0.2 & 0.17 \\
\hline & NPDESMETAL & & 2.8 & 1.6 \\
\hline & GENMIN & $11 / 8$ & - $^{(\mathrm{a})}$ & 2 \\
\hline & NPDESMETAL & & -(a) & 2.2 \\
\hline \multirow[t]{9}{*}{ Arsenic } & NPDESDISS & $1 / 20$ & -(a) & -(a) \\
\hline & NPDESMETAL & & -(a) & -(a) \\
\hline & NPDESDISS & $1 / 26$ & $<0.002$ & $<0.002$ \\
\hline & NPDESMETAL & & $<0.004$ & $<0.004$ \\
\hline & NPDESDISS & $2 / 8$ & $<0.002$ & $<0.002$ \\
\hline & NPDESMETAL & & $<0.002$ & $<0.002$ \\
\hline & NPDESDISS & $4 / 8$ & $<0.002$ & $<0.002$ \\
\hline & NPDESMETAL & & $<0.002$ & $<0.002$ \\
\hline & NPDESMETAL & $11 / 8$ & -(a) & $<0.002$ \\
\hline \multirow[t]{9}{*}{ Barium } & NPDESDISS & $1 / 20$ & - $^{(\mathrm{a})}$ & - $^{(\mathrm{a})}$ \\
\hline & NPDESMETAL & & - (a) $^{(a)}$ & -(a) \\
\hline & NPDESDISS & $1 / 26$ & 0.032 & 0.058 \\
\hline & NPDESMETAL & & $<0.025$ & 0.049 \\
\hline & NPDESDISS & $2 / 8$ & 0.05 & 0.048 \\
\hline & NPDESMETAL & & 0.056 & 0.062 \\
\hline & NPDESDISS & $4 / 8$ & 0.056 & 0.044 \\
\hline & NPDESMETAL & & 0.044 & 0.03 \\
\hline & NPDESMETAL & $11 / 8$ & - (a) $^{(\mathrm{n}}$ & 0.044 \\
\hline
\end{tabular}




\section{Surface Water}

Table 7-5. Metals detected in storm water runoff, Livermore site, 1999 (continued).

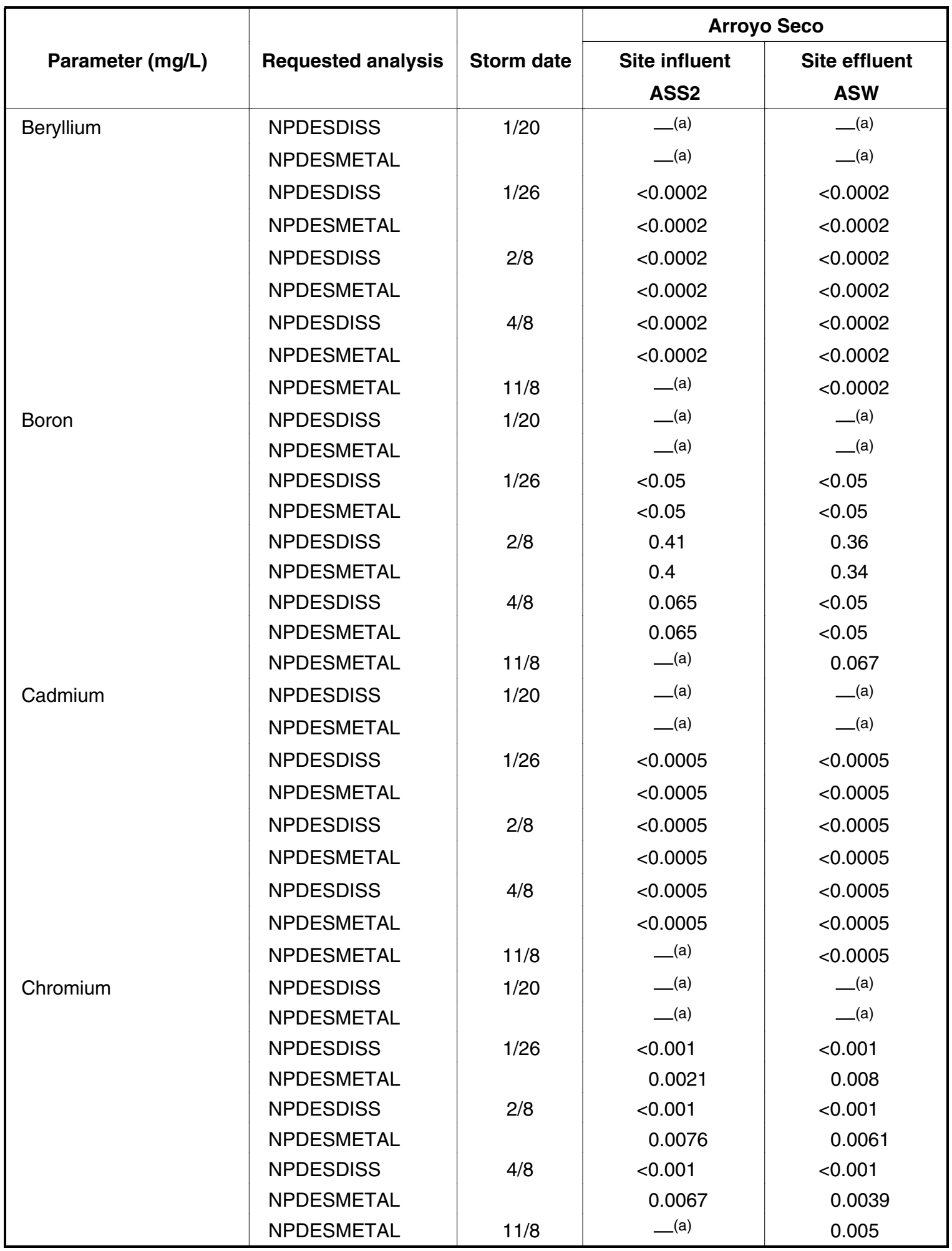




\section{Surface Water}

Table 7-5. Metals detected in storm water runoff, Livermore site, 1999 (continued).

\begin{tabular}{|c|c|c|c|c|}
\hline \multirow[b]{2}{*}{ Parameter (mg/L) } & \multirow[b]{2}{*}{ Requested analysis } & \multirow[b]{2}{*}{ Storm date } & \multicolumn{2}{|c|}{ Arroyo Seco } \\
\hline & & & $\begin{array}{l}\text { Site influent } \\
\text { ASS2 }\end{array}$ & $\begin{array}{c}\text { Site effluent } \\
\text { ASW }\end{array}$ \\
\hline Copper & $\begin{array}{l}\text { GENMIN } \\
\text { GENMINDISS } \\
\text { NPDESDISS } \\
\text { NPDESMETAL } \\
\text { GENMIN } \\
\text { GENMINDISS } \\
\text { NPDESDISS } \\
\text { NPDESMETAL } \\
\text { GENMIN } \\
\text { GENMINDISS } \\
\text { NPDESDISS } \\
\text { NPDESMETAL } \\
\text { GENMIN } \\
\text { GENMINDISS } \\
\text { NPDESDISS } \\
\text { NPDESMETAL } \\
\text { GENMIN } \\
\text { NPDESMETAL }\end{array}$ & 2/8 & $\begin{array}{l}-^{(a)} \\
-^{(a)} \\
-^{(a)} \\
-^{(a)} \\
<0.01 \\
<0.01 \\
0.0037 \\
0.0055 \\
0.014 \\
<0.01 \\
<0.001 \\
0.008 \\
0.017 \\
<0.01 \\
0.0066 \\
0.011 \\
\text { (a) }^{(a)}\end{array}$ & $\begin{array}{l}-^{(a)} \\
-_{(a)}^{(a)} \\
-_{(a)}^{(a)} \\
-^{(a)} \\
0.012 \\
<0.01 \\
0.0051 \\
0.012 \\
0.023 \\
<0.01 \\
<0.001 \\
0.0096 \\
0.017 \\
<0.01 \\
0.0077 \\
0.0097 \\
0.023 \\
0.018\end{array}$ \\
\hline Chromium(VI) & $\begin{array}{l}\text { NPDESDISS } \\
\text { NPDESDISS } \\
\text { NPDESDISS } \\
\text { NPDESDISS } \\
\text { NPDESMETAL }\end{array}$ & $\begin{array}{l}1 / 20 \\
1 / 26 \\
2 / 8 \\
4 / 8 \\
11 / 8\end{array}$ & $\begin{array}{c}\text {-(a) }^{(\mathrm{a}} \\
0.002 \\
<0.002 \\
<0.002 \\
\text { - (a) }^{-}\end{array}$ & $\begin{array}{c}\text {-(a) }^{(\mathrm{a})} \\
0.0043 \\
<0.002 \\
<0.002 \\
<0.002\end{array}$ \\
\hline Iron & $\begin{array}{l}\text { GENMIN } \\
\text { GENMINDISS } \\
\text { NPDESDISS } \\
\text { NPDESMETAL } \\
\text { GENMIN } \\
\text { GENMINDISS } \\
\text { NPDESDISS } \\
\text { NPDESMETAL } \\
\text { GENMIN } \\
\text { GENMINDISS } \\
\text { NPDESDISS } \\
\text { NPDESMETAL }\end{array}$ & $1 / 26$ & $\begin{array}{l}-^{(a)} \\
-^{(a)} \\
-^{(a)} \\
-^{(a)} \\
0.97 \\
0.12 \\
0.12 \\
0.95 \\
3.4 \\
0.16 \\
0.16 \\
3.3 \\
\end{array}$ & $\begin{array}{l}-^{(\mathrm{a})} \\
-^{(\mathrm{a})} \\
-^{(\mathrm{a})} \\
-^{(\mathrm{a})} \\
3.8 \\
0.19 \\
0.23 \\
3.7 \\
3 \\
0.13 \\
0.15 \\
3\end{array}$ \\
\hline
\end{tabular}




\section{Surface Water}

Table 7-5. Metals detected in storm water runoff, Livermore site, 1999 (continued).

\begin{tabular}{|c|c|c|c|c|}
\hline \multirow[b]{2}{*}{ Parameter (mg/L) } & \multirow[b]{2}{*}{ Requested analysis } & \multirow[b]{2}{*}{ Storm date } & \multicolumn{2}{|c|}{ Arroyo Seco } \\
\hline & & & $\begin{array}{c}\text { Site influent } \\
\text { ASS2 }\end{array}$ & $\begin{array}{c}\text { Site effluent } \\
\text { ASW }\end{array}$ \\
\hline \multirow[t]{6}{*}{ Iron (continued) } & GENMIN & $4 / 8$ & 3.1 & 2 \\
\hline & GENMINDISS & & 0.14 & 0.14 \\
\hline & NPDESDISS & & 0.18 & 0.15 \\
\hline & NPDESMETAL & & 3 & 1.8 \\
\hline & GENMIN & $11 / 8$ & -(a) & 1.9 \\
\hline & NPDESMETAL & & -(a) & 2.2 \\
\hline \multirow[t]{9}{*}{ Lead } & NPDESDISS & $1 / 20$ & -(a) & -(a) \\
\hline & NPDESMETAL & & -(a) & -(a) \\
\hline & NPDESDISS & $1 / 26$ & $<0.005$ & $<0.005$ \\
\hline & NPDESMETAL & & $<0.005$ & 0.0061 \\
\hline & NPDESDISS & $2 / 8$ & $<0.005$ & $<0.005$ \\
\hline & NPDESMETAL & & $<0.005$ & $<0.005$ \\
\hline & NPDESDISS & $4 / 8$ & $<0.005$ & $<0.005$ \\
\hline & NPDESMETAL & & $<0.005$ & $<0.005$ \\
\hline & NPDESMETAL & $11 / 8$ & -(a) & $<0.005$ \\
\hline \multirow[t]{9}{*}{ Magnesium } & GENMIN & $1 / 20$ & -(a) & -(a) \\
\hline & GENMINDISS & & -(a) & -(a) \\
\hline & GENMIN & $1 / 26$ & 0.83 & 2.2 \\
\hline & GENMINDISS & & 0.58 & 1.1 \\
\hline & GENMIN & $2 / 8$ & 11 & 9.6 \\
\hline & GENMINDISS & & 11 & 9.6 \\
\hline & GENMIN & $4 / 8$ & 2.3 & 1.7 \\
\hline & GENMINDISS & & 1.4 & 1.1 \\
\hline & GENMIN & $11 / 8$ & - $^{(\mathrm{a})}$ & 2.7 \\
\hline \multirow[t]{11}{*}{ Manganese } & GENMIN & $1 / 20$ & - (a) $^{(2)}$ & -(a) \\
\hline & GENMINDISS & & - $^{(\mathrm{a})}$ & - $^{(\mathrm{a})}$ \\
\hline & NPDESDISS & & -(a) & -(a) \\
\hline & NPDESMETAL & & -(a) & -(a) \\
\hline & GENMIN & $1 / 26$ & 0.021 & 0.074 \\
\hline & GENMINDISS & & $<0.01$ & $<0.01$ \\
\hline & NPDESDISS & & $<0.01$ & $<0.01$ \\
\hline & NPDESMETAL & & 0.02 & 0.073 \\
\hline & GENMIN & $2 / 8$ & 0.067 & 0.065 \\
\hline & GENMINDISS & & $<0.01$ & $<0.01$ \\
\hline & NPDESDISS & & $<0.01$ & $<0.01$ \\
\hline
\end{tabular}




\section{Surface Water}

Table 7-5. Metals detected in storm water runoff, Livermore site, 1999 (continued).

\begin{tabular}{|c|c|c|c|c|}
\hline \multirow[b]{2}{*}{ Parameter (mg/L) } & \multirow[b]{2}{*}{ Requested analysis } & \multirow[b]{2}{*}{ Storm date } & \multicolumn{2}{|c|}{ Arroyo Seco } \\
\hline & & & $\begin{array}{c}\text { Site influent } \\
\text { ASS2 }\end{array}$ & $\begin{array}{c}\text { Site effluent } \\
\text { ASW }\end{array}$ \\
\hline \multirow[t]{7}{*}{ Manganese (continued) } & NPDESMETAL & & 0.064 & 0.06 \\
\hline & GENMIN & $4 / 8$ & 0.059 & 0.039 \\
\hline & GENMINDISS & & $<0.01$ & $<0.01$ \\
\hline & NPDESDISS & & 0.01 & $<0.01$ \\
\hline & NPDESMETAL & & 0.06 & 0.037 \\
\hline & GENMIN & $11 / 8$ & -(a) & 0.064 \\
\hline & NPDESMETAL & & -(a) & 0.071 \\
\hline \multirow[t]{9}{*}{ Mercury } & NPDESDISS & $1 / 20$ & 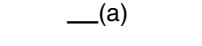 & - (a) $^{(2)}$ \\
\hline & NPDESMETAL & & -(a) & -(a) \\
\hline & NPDESDISS & $1 / 26$ & $<0.0002$ & $<0.0002$ \\
\hline & NPDESMETAL & & $<0.0002$ & $<0.0002$ \\
\hline & NPDESDISS & $2 / 8$ & $<0.0002$ & $<0.0002$ \\
\hline & NPDESMETAL & & $<0.0002$ & $<0.0002$ \\
\hline & NPDESDISS & $4 / 8$ & $<0.0002$ & $<0.0002$ \\
\hline & NPDESMETAL & & $<0.0002$ & $<0.0002$ \\
\hline & NPDESMETAL & $11 / 8$ & -(a) & $<0.0002$ \\
\hline \multirow[t]{9}{*}{ Molybdenum } & NPDESDISS & $1 / 20$ & -(a) & -(a) \\
\hline & NPDESMETAL & & -(a) & -(a) \\
\hline & NPDESDISS & $1 / 26$ & $<0.025$ & $<0.025$ \\
\hline & NPDESMETAL & & $<0.025$ & $<0.025$ \\
\hline & NPDESDISS & 2/8 & $<0.025$ & $<0.025$ \\
\hline & NPDESMETAL & & $<0.025$ & $<0.025$ \\
\hline & NPDESDISS & $4 / 8$ & $<0.025$ & $<0.025$ \\
\hline & NPDESMETAL & & $<0.025$ & $<0.025$ \\
\hline & NPDESMETAL & $11 / 8$ & - $^{(\mathrm{a})}$ & 0.066 \\
\hline \multirow[t]{10}{*}{ Nickel } & GENMIN & $1 / 20$ & -(a) & -(a) \\
\hline & GENMINDISS & & -(a) & -(a) $^{(\mathrm{a})}$ \\
\hline & NPDESDISS & & -(a) & -(a) \\
\hline & NPDESMETAL & & -(a) & -(a) \\
\hline & GENMIN & $1 / 26$ & $<0.05$ & $<0.05$ \\
\hline & GENMINDISS & & $<0.05$ & $<0.05$ \\
\hline & NPDESDISS & & $<0.002$ & $<0.002$ \\
\hline & NPDESMETAL & & 0.0028 & 0.0084 \\
\hline & GENMIN & $2 / 8$ & $<0.05$ & $<0.05$ \\
\hline & GENMINDISS & & $<0.05$ & $<0.05$ \\
\hline
\end{tabular}




\section{Surface Water}

Table 7-5. Metals detected in storm water runoff, Livermore site, 1999 (continued).

\begin{tabular}{|c|c|c|c|c|}
\hline \multirow[b]{2}{*}{ Parameter $(\mathrm{mg} / \mathrm{L})$} & \multirow[b]{2}{*}{ Requested analysis } & \multirow[b]{2}{*}{ Storm date } & \multicolumn{2}{|c|}{ Arroyo Seco } \\
\hline & & & $\begin{array}{c}\text { Site influent } \\
\text { ASS2 }\end{array}$ & $\begin{array}{c}\text { Site effluent } \\
\text { ASW }\end{array}$ \\
\hline Nickel (continued) & $\begin{array}{l}\text { NPDESDISS } \\
\text { NPDESMETAL } \\
\text { GENMIN } \\
\text { GENMINDISS } \\
\text { NPDESDISS } \\
\text { NPDESMETAL } \\
\text { GENMIN } \\
\text { NPDESMETAL }\end{array}$ & $\begin{array}{c}4 / 8 \\
11 / 8\end{array}$ & $\begin{array}{l}<0.002 \\
0.0098 \\
<0.05 \\
<0.05 \\
0.002 \\
0.0083 \\
\ldots \text { (a) } \\
\text { (a) }^{(\mathrm{a})}\end{array}$ & $\begin{array}{c}<0.002 \\
0.0081 \\
<0.05 \\
<0.05 \\
<0.002 \\
0.0049 \\
<0.05 \\
0.0088\end{array}$ \\
\hline Vanadium & $\begin{array}{l}\text { NPDESDISS } \\
\text { NPDESMETAL } \\
\text { NPDESDISS } \\
\text { NPDESMETAL } \\
\text { NPDESDISS } \\
\text { NPDESMETAL } \\
\text { NPDESDISS } \\
\text { NPDESMETAL } \\
\text { NPDESMETAL }\end{array}$ & $\begin{array}{l}1 / 20 \\
1 / 26 \\
2 / 8 \\
4 / 8 \\
11 / 8\end{array}$ & 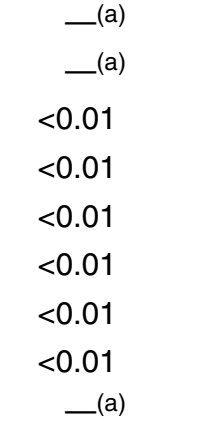 & $\begin{aligned} & { }^{(a)} \\
& \ldots^{(a)} \\
< & 0.01 \\
< & 0.01 \\
< & 0.01 \\
< & 0.01 \\
< & 0.01 \\
< & 0.01 \\
< & 0.01\end{aligned}$ \\
\hline Zinc & $\begin{array}{l}\text { GENMIN } \\
\text { GENMINDISS } \\
\text { NPDESDISS } \\
\text { NPDESMETAL } \\
\text { GENMIN } \\
\text { GENMINDISS } \\
\text { NPDESDISS } \\
\text { NPDESMETAL } \\
\text { GENMIN } \\
\text { GENMINDISS } \\
\text { NPDESDISS } \\
\text { NPDESMETAL } \\
\text { GENMIN } \\
\text { GENMINDISS } \\
\text { NPDESDISS } \\
\text { NPDESMETAL } \\
\text { GENMIN } \\
\text { NPDESMETAL }\end{array}$ & $\begin{array}{l}1 / 26 \\
2 / 8 \\
4 / 8 \\
11 / 8\end{array}$ & $\begin{array}{l}-^{(a)} \\
-_{(a)}^{(a)} \\
-^{(a)} \\
0.081 \\
0.062 \\
0.062 \\
0.078 \\
0.077 \\
0.029 \\
0.03 \\
0.074 \\
0.097 \\
0.056 \\
0.058 \\
0.096 \\
-(a) \\
\ldots(a)\end{array}$ & $\begin{array}{l}-^{(a)} \\
-_{(a)}^{(a)} \\
-^{(a)} \\
0.098 \\
0.048 \\
0.05 \\
0.097 \\
0.085 \\
0.042 \\
0.04 \\
0.08 \\
0.084 \\
0.056 \\
0.062 \\
0.085 \\
0.27 \\
0.27\end{array}$ \\
\hline
\end{tabular}




\section{Surface Water}

Table 7-5. Metals detected in storm water runoff, Livermore site, 1999 (continued).

\begin{tabular}{|c|c|c|c|c|c|c|}
\hline \multirow{3}{*}{ Parameter (mg/L) } & \multirow{3}{*}{ Requested analysis } & \multirow{3}{*}{$\begin{array}{c}\text { Storm } \\
\text { date }\end{array}$} & \multicolumn{4}{|c|}{ Arroyo Las Positas } \\
\hline & & & \multicolumn{3}{|c|}{ Site influent } & \multirow{2}{*}{$\begin{array}{c}\text { Site effluent } \\
\text { WPDC }\end{array}$} \\
\hline & & & ALPE & ALPO & GRNE & \\
\hline \multirow[t]{18}{*}{ Aluminum } & GENMIN & $1 / 20$ & 0.55 & 5.4 & 25 & 1.4 \\
\hline & GENMINDISS & & $<0.05$ & $<0.05$ & 0.18 & $<0.05$ \\
\hline & NPDESDISS & & $<0.05$ & $<0.05$ & 0.15 & $<0.05$ \\
\hline & NPDESMETAL & & 0.55 & 5.6 & 27 & 1.4 \\
\hline & GENMIN & $1 / 26$ & -(b) $^{(\mathrm{n}}$ & $-^{(\mathrm{b})}$ & $-^{(\mathrm{b})}$ & -(b) $^{(\mathrm{c}}$ \\
\hline & GENMINDISS & & -(b) $^{(\mathrm{n}}$ & - (b) $^{(\mathrm{n}}$ & - (b) $^{(\mathrm{n}}$ & -(b) $^{(\mathrm{n}}$ \\
\hline & NPDESDISS & & -(b) $^{(\mathrm{c}}$ & -(b) $^{(\mathrm{c}}$ & -(b) $^{(\mathrm{c}}$ & -(b) $^{(\mathrm{c}}$ \\
\hline & NPDESMETAL & & $-^{(\mathrm{b})}$ & $-^{(\mathrm{b})}$ & -(b) $^{(\mathrm{c}}$ & $-^{(\mathrm{b})}$ \\
\hline & GENMIN & $2 / 8$ & 6.7 & 12 & 4.8 & 4.1 \\
\hline & GENMINDISS & & 0.28 & $<0.05$ & 0.17 & 0.21 \\
\hline & NPDESDISS & & 0.28 & $<0.05$ & 0.14 & 0.28 \\
\hline & NPDESMETAL & & 6.9 & 12 & 6.2 & 3.9 \\
\hline & GENMIN & $4 / 8$ & 3.1 & 9.9 & 3.6 & 4.2 \\
\hline & GENMINDISS & & 0.14 & 0.11 & 0.14 & 0.19 \\
\hline & NPDESDISS & & 0.096 & 0.084 & 0.12 & 0.18 \\
\hline & NPDESMETAL & & 3.2 & 9.3 & 4.8 & 3.7 \\
\hline & GENMIN & $11 / 8$ & 2.6 & 6.4 & 31 & 4.6 \\
\hline & NPDESMETAL & & 2.3 & 5.9 & 28 & 4.5 \\
\hline \multirow[t]{9}{*}{ Arsenic } & NPDESDISS & $1 / 20$ & 0.0067 & 0.011 & 0.0026 & 0.0063 \\
\hline & NPDESMETAL & & 0.0044 & 0.0098 & 0.0058 & 0.0047 \\
\hline & NPDESDISS & $1 / 26$ & $-^{(\mathrm{b})}$ & -(b) $^{(\mathrm{c}}$ & -(b) $^{(\mathrm{n}}$ & -(b) $^{(\mathrm{c}}$ \\
\hline & NPDESMETAL & & -(b) $^{(\mathrm{s}}$ & -(b) $^{(\mathrm{c}}$ & -(b) $^{(\mathrm{s}}$ & $-^{(b)}$ \\
\hline & NPDESDISS & $2 / 8$ & 0.0028 & 0.0063 & $<0.002$ & $<0.002$ \\
\hline & NPDESMETAL & & 0.004 & 0.0094 & $<0.002$ & $<0.002$ \\
\hline & NPDESDISS & $4 / 8$ & $<0.002$ & $<0.002$ & $<0.002$ & $<0.002$ \\
\hline & NPDESMETAL & & $<0.002$ & 0.0029 & $<0.002$ & $<0.002$ \\
\hline & NPDESMETAL & $11 / 8$ & $<0.002$ & 0.0052 & 0.0058 & 0.0026 \\
\hline \multirow[t]{9}{*}{ Barium } & NPDESDISS & $1 / 20$ & 0.077 & 0.11 & 0.093 & 0.11 \\
\hline & NPDESMETAL & & 0.082 & 0.17 & 0.39 & 0.12 \\
\hline & NPDESDISS & $1 / 26$ & -(b) $^{(\mathrm{s})}$ & -(b) $^{(\mathrm{c}}$ & -(b) $^{(\mathrm{c}}$ & $-^{(b)}$ \\
\hline & NPDESMETAL & & -(b) $^{(\mathrm{s})}$ & -(b) $^{(\mathrm{n}}$ & -(b) $^{(\mathrm{n}}$ & -(b) $^{(\mathrm{c}}$ \\
\hline & NPDESDISS & $2 / 8$ & 0.071 & 0.086 & 0.064 & 0.059 \\
\hline & NPDESMETAL & & 0.11 & 0.21 & 0.11 & 0.076 \\
\hline & NPDESDISS & $4 / 8$ & 0.057 & 0.13 & 0.07 & 0.09 \\
\hline & NPDESMETAL & & 0.078 & 0.16 & 0.098 & 0.072 \\
\hline & NPDESMETAL & $11 / 8$ & 0.047 & 0.16 & 0.4 & 0.096 \\
\hline
\end{tabular}


Table 7-5. Metals detected in storm water runoff, Livermore site, 1999 (continued).

\begin{tabular}{|c|c|c|c|c|c|c|}
\hline \multirow{3}{*}{ Parameter $(\mathrm{mg} / \mathrm{L})$} & \multirow{3}{*}{ Requested analysis } & \multirow{3}{*}{$\begin{array}{l}\text { Storm } \\
\text { date }\end{array}$} & \multicolumn{4}{|c|}{ Arroyo Las Positas } \\
\hline & & & \multicolumn{3}{|c|}{ Site influent } & \multirow{2}{*}{$\begin{array}{c}\text { Site effluent } \\
\text { WPDC } \\
\end{array}$} \\
\hline & & & ALPE & ALPO & GRNE & \\
\hline \multirow[t]{9}{*}{ Beryllium } & NPDESDISS & $1 / 20$ & $<0.0002$ & $<0.0002$ & $<0.0002$ & $<0.0002$ \\
\hline & NPDESMETAL & & $<0.0002$ & $<0.0002$ & 0.00074 & $<0.0002$ \\
\hline & NPDESDISS & $1 / 26$ & -(b) $^{(\mathrm{l}}$ & -(b) $^{(\mathrm{l}}$ & -(b) $^{(\mathrm{n}}$ & -(b) $^{(\mathrm{n}}$ \\
\hline & NPDESMETAL & & -(b) $^{(\mathrm{c}}$ & -(b) & -(b) & -(b) $^{(\mathrm{c}}$ \\
\hline & NPDESDISS & $2 / 8$ & $<0.0002$ & $<0.0002$ & $<0.0002$ & $<0.0002$ \\
\hline & NPDESMETAL & & $<0.0002$ & 0.00033 & $<0.0002$ & $<0.0002$ \\
\hline & NPDESDISS & $4 / 8$ & $<0.0002$ & $<0.0002$ & $<0.0002$ & $<0.0002$ \\
\hline & NPDESMETAL & & $<0.0002$ & 0.0002 & $<0.0002$ & $<0.0002$ \\
\hline & NPDESMETAL & $11 / 8$ & $<0.0002$ & $<0.0002$ & 0.0008 & $<0.0002$ \\
\hline \multirow[t]{9}{*}{ Boron } & NPDESDISS & $1 / 20$ & 21 & 5.3 & 0.29 & 2 \\
\hline & NPDESMETAL & & 20 & 5 & 0.28 & 2 \\
\hline & NPDESDISS & $1 / 26$ & -(b) $^{(\mathrm{n}}$ & -(b) $^{(\mathrm{n}}$ & -(b) $^{(\mathrm{n}}$ & -(b) $^{(\mathrm{c}}$ \\
\hline & NPDESMETAL & & -(b) $^{(\mathrm{c}}$ & -(b) $^{(\mathrm{c}}$ & -(b) $^{(\mathrm{n}}$ & -(b) $^{(\mathrm{c}}$ \\
\hline & NPDESDISS & $2 / 8$ & 12 & 4.6 & 0.23 & 0.63 \\
\hline & NPDESMETAL & & 12 & 4.4 & 0.24 & 0.6 \\
\hline & NPDESDISS & $4 / 8$ & 2.8 & 0.18 & 0.2 & 0.21 \\
\hline & NPDESMETAL & & 2.8 & 0.17 & 0.18 & 0.22 \\
\hline & NPDESMETAL & $11 / 8$ & 0.3 & 2.5 & 0.24 & 0.27 \\
\hline \multirow[t]{9}{*}{ Cadmium } & NPDESDISS & $1 / 20$ & $<0.0005$ & $<0.0005$ & $<0.0005$ & $<0.0005$ \\
\hline & NPDESMETAL & & $<0.0005$ & $<0.0005$ & $<0.0005$ & $<0.0005$ \\
\hline & NPDESDISS & $1 / 26$ & -(b) $^{(\mathrm{c}}$ & —(b) $^{(\mathrm{n}}$ & -(b) $^{(\mathrm{s}}$ & $-^{(b)}$ \\
\hline & NPDESMETAL & & —(b) $^{(\mathrm{b}}$ & —(b) $^{(\mathrm{s}}$ & -(b) & -(b) $^{(2)}$ \\
\hline & NPDESDISS & $2 / 8$ & $<0.0005$ & $<0.0005$ & $<0.0005$ & $<0.0005$ \\
\hline & NPDESMETAL & & $<0.0005$ & $<0.0005$ & $<0.0005$ & $<0.0005$ \\
\hline & NPDESDISS & $4 / 8$ & $<0.0005$ & $<0.0005$ & $<0.0005$ & $<0.0005$ \\
\hline & NPDESMETAL & & $<0.0005$ & $<0.0005$ & $<0.0005$ & $<0.0005$ \\
\hline & NPDESMETAL & $11 / 8$ & $<0.0005$ & $<0.0005$ & $<0.0005$ & $<0.0005$ \\
\hline \multirow[t]{9}{*}{ Chromium } & NPDESDISS & $1 / 20$ & $<0.001$ & $<0.001$ & 0.0016 & 0.0022 \\
\hline & NPDESMETAL & & 0.0017 & 0.0095 & 0.041 & 0.0056 \\
\hline & NPDESDISS & $1 / 26$ & -(b) $^{(\mathrm{b}}$ & -(b) $^{(\mathrm{c}}$ & -(b) $^{(\mathrm{c}}$ & -(b) $^{(\mathrm{b}}$ \\
\hline & NPDESMETAL & & $-^{(b)}$ & $-^{(b)}$ & -(b) $^{(b)}$ & -(b) $^{(b}$ \\
\hline & NPDESDISS & $2 / 8$ & $<0.001$ & $<0.001$ & $<0.001$ & 0.0023 \\
\hline & NPDESMETAL & & 0.011 & 0.018 & 0.011 & 0.012 \\
\hline & NPDESDISS & $4 / 8$ & $<0.001$ & $<0.001$ & $<0.001$ & 0.0022 \\
\hline & NPDESMETAL & & 0.007 & 0.017 & 0.0066 & 0.0087 \\
\hline & NPDESMETAL & $11 / 8$ & 0.0057 & 0.011 & 0.055 & 0.013 \\
\hline
\end{tabular}




\section{Surface Water}

Table 7-5. Metals detected in storm water runoff, Livermore site, 1999 (continued).

\begin{tabular}{|c|c|c|c|c|c|c|}
\hline \multirow{3}{*}{ Parameter (mg/L) } & \multirow{3}{*}{ Requested analysis } & \multirow{3}{*}{$\begin{array}{c}\text { Storm } \\
\text { date }\end{array}$} & \multicolumn{4}{|c|}{ Arroyo Las Positas } \\
\hline & & & \multicolumn{3}{|c|}{ Site influent } & \multirow{2}{*}{$\begin{array}{c}\text { Site effluent } \\
\text { WPDC }\end{array}$} \\
\hline & & & ALPE & ALPO & GRNE & \\
\hline \multirow[t]{18}{*}{ Copper } & GENMIN & $1 / 20$ & 0.026 & 0.026 & 0.033 & 0.011 \\
\hline & GENMINDISS & & $<0.01$ & $<0.01$ & $<0.01$ & $<0.01$ \\
\hline & NPDESDISS & & 0.0063 & 0.005 & 0.0033 & 0.0037 \\
\hline & NPDESMETAL & & 0.0056 & 0.011 & 0.019 & 0.005 \\
\hline & GENMIN & $1 / 26$ & $-^{(\mathrm{b})}$ & $-^{(\mathrm{b})}$ & -(b) $^{(\mathrm{n}}$ & -(b) $^{(\mathrm{n}}$ \\
\hline & GENMINDISS & & -(b) $^{(\mathrm{n}}$ & -(b) $^{(\mathrm{n}}$ & -(b) $^{(\mathrm{n}}$ & -(b) $^{(\mathrm{c}}$ \\
\hline & NPDESDISS & & -(b) $^{(\mathrm{c}}$ & -(b) $^{(b)}$ & - (b) $^{(2)}$ & -(b) $^{(\mathrm{c}}$ \\
\hline & NPDESMETAL & & $-^{(\mathrm{b})}$ & $-^{(\mathrm{b})}$ & $-^{(\mathrm{b})}$ & $-_{(\mathrm{b})}$ \\
\hline & GENMIN & $2 / 8$ & 0.015 & 0.025 & $<0.01$ & 0.022 \\
\hline & GENMINDISS & & 0.016 & 0.015 & $<0.01$ & $<0.01$ \\
\hline & NPDESDISS & & $<0.001$ & $<0.001$ & $<0.001$ & $<0.001$ \\
\hline & NPDESMETAL & & 0.016 & 0.026 & 0.0056 & 0.0098 \\
\hline & GENMIN & $4 / 8$ & 0.016 & 0.02 & 0.01 & 0.017 \\
\hline & GENMINDISS & & $<0.01$ & $<0.01$ & $<0.01$ & $<0.01$ \\
\hline & NPDESDISS & & 0.008 & 0.0029 & 0.0023 & 0.0054 \\
\hline & NPDESMETAL & & 0.0092 & 0.012 & 0.0058 & 0.009 \\
\hline & GENMIN & $11 / 8$ & 0.019 & 0.017 & 0.039 & 0.015 \\
\hline & NPDESMETAL & & 0.011 & 0.017 & 0.028 & 0.014 \\
\hline \multirow[t]{5}{*}{ Chromium(VI) } & NPDESDISS & $1 / 20$ & $<0.01$ & $<0.002$ & 0.0027 & 0.0027 \\
\hline & NPDESDISS & $1 / 26$ & 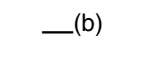 & -(b) $^{(\mathrm{b})}$ & -(b) $^{(\mathrm{c}}$ & -(b) $^{(\mathrm{c}}$ \\
\hline & NPDESDISS & $2 / 8$ & 0.002 & $<0.002$ & 0.002 & 0.004 \\
\hline & NPDESDISS & $4 / 8$ & $<0.002$ & $<0.002$ & $<0.002$ & $<0.002$ \\
\hline & NPDESMETAL & $11 / 8$ & $<0.002$ & $<0.002$ & $<0.002$ & $<0.002$ \\
\hline \multirow[t]{12}{*}{ Iron } & GENMIN & $1 / 20$ & 0.61 & 5.2 & 25 & 1.4 \\
\hline & GENMINDISS & & 0.11 & $<0.05$ & 0.17 & 0.065 \\
\hline & NPDESDISS & & 0.11 & $<0.05$ & 0.15 & 0.066 \\
\hline & NPDESMETAL & & 0.59 & 5.2 & 26 & 1.4 \\
\hline & GENMIN & $1 / 26$ & -(b) $^{(\mathrm{c}}$ & $-^{(\mathrm{b})}$ & $-^{(\mathrm{b})}$ & -(b) $^{(\mathrm{c}}$ \\
\hline & GENMINDISS & & -(b) $^{(\mathrm{n}}$ & -(b) $^{(\mathrm{n}}$ & -(b) $^{(\mathrm{c}}$ & -(b) $^{(\mathrm{c}}$ \\
\hline & NPDESDISS & & -(b) $^{(\mathrm{c}}$ & -(b) $^{(\mathrm{c}}$ & -(b) $^{(\mathrm{c}}$ & -(b) $^{(\mathrm{c}}$ \\
\hline & NPDESMETAL & & $-^{(\mathrm{b})}$ & —(b) $^{(\mathrm{s}}$ & $-^{(b)}$ & -(b) $^{(\mathrm{c}}$ \\
\hline & GENMIN & $2 / 8$ & 5.4 & 11 & 4.7 & 4 \\
\hline & GENMINDISS & & 0.25 & $<0.05$ & 0.16 & 0.18 \\
\hline & NPDESDISS & & 0.27 & $<0.05$ & 0.14 & 0.25 \\
\hline & NPDESMETAL & & 5.6 & 11 & 5.2 & 3.9 \\
\hline
\end{tabular}


Table 7-5. Metals detected in storm water runoff, Livermore site, 1999 (continued).

\begin{tabular}{|c|c|c|c|c|c|c|}
\hline \multirow{3}{*}{ Parameter $(\mathrm{mg} / \mathrm{L})$} & \multirow{3}{*}{ Requested analysis } & \multirow{3}{*}{$\begin{array}{c}\text { Storm } \\
\text { date }\end{array}$} & \multicolumn{4}{|c|}{ Arroyo Las Positas } \\
\hline & & & \multicolumn{3}{|c|}{ Site influent } & \multirow{2}{*}{$\begin{array}{c}\text { Site effluent } \\
\text { WPDC }\end{array}$} \\
\hline & & & ALPE & ALPO & GRNE & \\
\hline \multirow[t]{6}{*}{ Iron (continued) } & GENMIN & $4 / 8$ & 3 & 12 & 3.7 & 4.8 \\
\hline & GENMINDISS & & 0.21 & 0.12 & 0.14 & 0.17 \\
\hline & NPDESDISS & & 0.1 & 0.095 & 0.11 & 0.16 \\
\hline & NPDESMETAL & & 2.8 & 9.9 & 4.6 & 4.1 \\
\hline & GENMIN & $11 / 8$ & 2.4 & 5.9 & 28 & 4.5 \\
\hline & NPDESMETAL & & 2.4 & 5.3 & 25 & 4.6 \\
\hline \multirow[t]{9}{*}{ Lead } & NPDESDISS & $1 / 20$ & $<0.005$ & $<0.005$ & $<0.005$ & $<0.005$ \\
\hline & NPDESMETAL & & $<0.005$ & $<0.005$ & 0.0093 & $<0.005$ \\
\hline & NPDESDISS & $1 / 26$ & —(b) $^{(\mathrm{n})}$ & —(b) $^{(\mathrm{n}}$ & -(b) $^{(\mathrm{b})}$ & -(b) $^{(\mathrm{c}}$ \\
\hline & NPDESMETAL & & —(b) $^{(\mathrm{b})}$ & $-_{(\mathrm{b})}$ & $-^{(\mathrm{b})}$ & 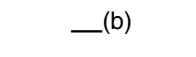 \\
\hline & NPDESDISS & $2 / 8$ & $<0.005$ & $<0.005$ & $<0.005$ & $<0.005$ \\
\hline & NPDESMETAL & & $<0.005$ & 0.0066 & $<0.005$ & $<0.005$ \\
\hline & NPDESDISS & $4 / 8$ & $<0.005$ & $<0.005$ & $<0.005$ & $<0.005$ \\
\hline & NPDESMETAL & & $<0.005$ & $<0.005$ & $<0.005$ & $<0.005$ \\
\hline & NPDESMETAL & $11 / 8$ & $<0.005$ & 0.0066 & 0.0077 & $<0.005$ \\
\hline \multirow[t]{9}{*}{ Magnesium } & GENMIN & $1 / 20$ & 55 & 29 & 12 & 18 \\
\hline & GENMINDISS & & 57 & 28 & 5.6 & 18 \\
\hline & GENMIN & $1 / 26$ & —(b) & —(b) $^{(\mathrm{b})}$ & $-^{(\mathrm{b})}$ & 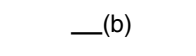 \\
\hline & GENMINDISS & & $—_{(\mathrm{b})}$ & -(b) $^{(\mathrm{c})}$ & $-^{(\mathrm{b})}$ & $-^{(b)}$ \\
\hline & GENMIN & $2 / 8$ & 33 & 30 & 5.3 & 8.2 \\
\hline & GENMINDISS & & 34 & 28 & 4.2 & 7.2 \\
\hline & GENMIN & $4 / 8$ & 11 & 7.3 & 4.6 & 5.6 \\
\hline & GENMINDISS & & 10 & 4 & 3.8 & 4.2 \\
\hline & GENMIN & $11 / 8$ & 2.9 & 21 & 13 & 6.8 \\
\hline \multirow[t]{11}{*}{ Manganese } & GENMIN & $1 / 20$ & 0.054 & 0.15 & 0.39 & 0.028 \\
\hline & GENMINDISS & & 0.027 & $<0.01$ & $<0.01$ & $<0.01$ \\
\hline & NPDESDISS & & 0.027 & $<0.01$ & $<0.01$ & $<0.01$ \\
\hline & NPDESMETAL & & 0.054 & 0.15 & 0.4 & 0.028 \\
\hline & GENMIN & $1 / 26$ & —(b) $^{(\mathrm{n}}$ & -(b) $^{(\mathrm{s})}$ & $-^{(\mathrm{b})}$ & -(b) $^{(\mathrm{c}}$ \\
\hline & GENMINDISS & & -(b) $^{(\mathrm{s}}$ & -(b) $^{(\mathrm{c}}$ & -(b) $^{(\mathrm{c}}$ & -(b) $^{(\mathrm{c}}$ \\
\hline & NPDESDISS & & 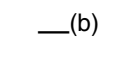 & -(b) $^{(\mathrm{b}}$ & -(b) $^{(\mathrm{b}}$ & -(b) $^{(\mathrm{c}}$ \\
\hline & NPDESMETAL & & $-^{(\mathrm{b})}$ & -(b) $^{(\mathrm{c}}$ & -(b) $^{(\mathrm{c}}$ & -(b) $^{(\mathrm{c})}$ \\
\hline & GENMIN & $2 / 8$ & 0.1 & 0.3 & 0.071 & 0.07 \\
\hline & GENMINDISS & & 0.012 & $<0.01$ & $<0.01$ & $<0.01$ \\
\hline & NPDESDISS & & 0.018 & $<0.01$ & $<0.01$ & $<0.01$ \\
\hline
\end{tabular}




\section{Surface Water}

Table 7-5. Metals detected in storm water runoff, Livermore site, 1999 (continued).

\begin{tabular}{|c|c|c|c|c|c|c|}
\hline \multirow{3}{*}{ Parameter (mg/L) } & \multirow{3}{*}{ Requested analysis } & \multirow{3}{*}{$\begin{array}{c}\text { Storm } \\
\text { date }\end{array}$} & \multicolumn{4}{|c|}{ Arroyo Las Positas } \\
\hline & & & \multicolumn{3}{|c|}{ Site influent } & \multirow{2}{*}{$\begin{array}{c}\text { Site effluent } \\
\text { WPDC }\end{array}$} \\
\hline & & & ALPE & ALPO & GRNE & \\
\hline \multirow{7}{*}{$\begin{array}{l}\text { Manganese } \\
\text { (continued) }\end{array}$} & NPDESMETAL & & 0.1 & 0.29 & 0.076 & 0.065 \\
\hline & GENMIN & $4 / 8$ & 0.063 & 0.21 & 0.062 & 0.1 \\
\hline & GENMINDISS & & 0.043 & $<0.01$ & 0.01 & $<0.01$ \\
\hline & NPDESDISS & & 0.013 & $<0.01$ & $<0.01$ & $<0.01$ \\
\hline & NPDESMETAL & & 0.058 & 0.18 & 0.078 & 0.078 \\
\hline & GENMIN & $11 / 8$ & 0.12 & 0.26 & 0.41 & 0.11 \\
\hline & NPDESMETAL & & 0.12 & 0.25 & 0.36 & 0.11 \\
\hline \multirow[t]{9}{*}{ Mercury } & NPDESDISS & $1 / 20$ & $<0.0002$ & $<0.0002$ & $<0.0002$ & $<0.0002$ \\
\hline & NPDESMETAL & & $<0.0002$ & $<0.0002$ & $<0.0002$ & $<0.0002$ \\
\hline & NPDESDISS & $1 / 26$ & -(b) $^{(\mathrm{b}}$ & —(b) $^{(\mathrm{n}}$ & $-^{(b)}$ & -(b) \\
\hline & NPDESMETAL & & -(b) $^{(\mathrm{c}}$ & -(b) $^{(\mathrm{c}}$ & -(b) $^{(\mathrm{c}}$ & -(b) \\
\hline & NPDESDISS & $2 / 8$ & $<0.0002$ & $<0.0002$ & $<0.0002$ & $<0.0002$ \\
\hline & NPDESMETAL & & $<0.0002$ & $<0.0002$ & $<0.0002$ & $<0.0002$ \\
\hline & NPDESDISS & $4 / 8$ & $<0.0002$ & $<0.0002$ & $<0.0002$ & $<0.0002$ \\
\hline & NPDESMETAL & & $<0.0002$ & $<0.0002$ & $<0.0002$ & $<0.0002$ \\
\hline & NPDESMETAL & $11 / 8$ & $<0.0002$ & $<0.0002$ & $<0.0002$ & $<0.0002$ \\
\hline \multirow[t]{9}{*}{ Molybdenum } & NPDESDISS & $1 / 20$ & $<0.025$ & $<0.025$ & $<0.025$ & $<0.025$ \\
\hline & NPDESMETAL & & $<0.025$ & $<0.025$ & $<0.025$ & $<0.025$ \\
\hline & NPDESDISS & $1 / 26$ & 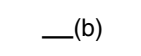 & -(b) $^{(\mathrm{s})}$ & -(b) $^{(\mathrm{n})}$ & -(b) \\
\hline & NPDESMETAL & & —(b) $^{(\mathrm{s}}$ & -(b) $^{(\mathrm{s})}$ & -(b) $^{(\mathrm{c}}$ & -(b) $^{(\text {b }}$ \\
\hline & NPDESDISS & $2 / 8$ & $<0.025$ & $<0.025$ & $<0.025$ & $<0.025$ \\
\hline & NPDESMETAL & & $<0.025$ & $<0.025$ & $<0.025$ & $<0.025$ \\
\hline & NPDESDISS & $4 / 8$ & $<0.025$ & $<0.025$ & $<0.025$ & $<0.025$ \\
\hline & NPDESMETAL & & $<0.025$ & $<0.025$ & $<0.025$ & $<0.025$ \\
\hline & NPDESMETAL & $11 / 8$ & $<0.025$ & $<0.025$ & $<0.025$ & $<0.025$ \\
\hline \multirow[t]{9}{*}{ Nickel } & GENMIN & $1 / 20$ & $<0.05$ & $<0.05$ & $<0.05$ & $<0.05$ \\
\hline & GENMINDISS & & $<0.05$ & $<0.05$ & $<0.05$ & $<0.05$ \\
\hline & NPDESDISS & & 0.0087 & 0.0039 & 0.0029 & 0.0033 \\
\hline & NPDESMETAL & & 0.01 & 0.011 & 0.039 & 0.006 \\
\hline & GENMIN & $1 / 26$ & -(b) $^{(\mathrm{c}}$ & -(b) $^{(\mathrm{c}}$ & -(b) $^{(\mathrm{n}}$ & -(b) $^{(\mathrm{c}}$ \\
\hline & GENMINDISS & & -(b) $^{(\mathrm{n}}$ & $-^{(b)}$ & -(b) $^{(\mathrm{n}}$ & -(b) $^{(\mathrm{c}}$ \\
\hline & NPDESDISS & & $-^{(\mathrm{b})}$ & $-^{(\mathrm{b})}$ & $-_{(\mathrm{b})}$ & $-_{(\text {b) }}$ \\
\hline & NPDESMETAL & & -(b) $^{(\mathrm{s})}$ & $-^{(b)}$ & -(b) $^{(\mathrm{c}}$ & -(b) $^{(\mathrm{c}}$ \\
\hline & GENMIN & $2 / 8$ & $<0.05$ & $<0.05$ & $<0.05$ & $<0.05$ \\
\hline
\end{tabular}


Table 7-5. Metals detected in storm water runoff, Livermore site, 1999 (continued).

\begin{tabular}{|c|c|c|c|c|c|c|}
\hline \multirow{3}{*}{ Parameter $(\mathrm{mg} / \mathrm{L})$} & \multirow{3}{*}{ Requested analysis } & \multirow{3}{*}{$\begin{array}{c}\text { Storm } \\
\text { date }\end{array}$} & \multicolumn{4}{|c|}{ Arroyo Las Positas } \\
\hline & & & \multicolumn{3}{|c|}{ Site influent } & \multirow{2}{*}{$\begin{array}{c}\text { Site effluent } \\
\text { WPDC }\end{array}$} \\
\hline & & & ALPE & ALPO & GRNE & \\
\hline \multirow[t]{9}{*}{ Nickel (continued) } & GENMINDISS & & $<0.05$ & $<0.05$ & $<0.05$ & $<0.05$ \\
\hline & NPDESDISS & & 0.0062 & 0.0032 & 0.0024 & 0.0026 \\
\hline & NPDESMETAL & & 0.016 & 0.017 & 0.0083 & 0.0071 \\
\hline & GENMIN & $4 / 8$ & $<0.05$ & $<0.05$ & $<0.05$ & $<0.05$ \\
\hline & GENMINDISS & & $<0.05$ & $<0.05$ & $<0.05$ & $<0.05$ \\
\hline & NPDESDISS & & 0.0041 & $<0.002$ & $<0.002$ & $<0.002$ \\
\hline & NPDESMETAL & & 0.01 & 0.019 & 0.0078 & 0.0087 \\
\hline & GENMIN & $11 / 8$ & $<0.05$ & $<0.05$ & 0.05 & $<0.05$ \\
\hline & NPDESMETAL & & 0.01 & 0.014 & 0.038 & 0.014 \\
\hline \multirow[t]{9}{*}{ Vanadium } & NPDESDISS & $1 / 20$ & 0.01 & 0.015 & $<0.01$ & $<0.01$ \\
\hline & NPDESMETAL & & 0.01 & 0.023 & 0.058 & $<0.01$ \\
\hline & NPDESDISS & $1 / 26$ & -(b) $^{(\mathrm{c}}$ & -(b) $^{(\mathrm{c}}$ & -(b) $^{(\mathrm{c}}$ & —(b) \\
\hline & NPDESMETAL & & $-^{(\mathrm{b})}$ & -(b) $^{(\mathrm{n}}$ & $-^{(\mathrm{b})}$ & -(b) $^{(\mathrm{c}}$ \\
\hline & NPDESDISS & $2 / 8$ & $<0.01$ & 0.013 & $<0.01$ & $<0.01$ \\
\hline & NPDESMETAL & & 0.016 & 0.035 & 0.013 & 0.012 \\
\hline & NPDESDISS & $4 / 8$ & $<0.01$ & $<0.01$ & $<0.01$ & $<0.01$ \\
\hline & NPDESMETAL & & $<0.01$ & 0.02 & 0.012 & 0.011 \\
\hline & NPDESMETAL & $11 / 8$ & $<0.01$ & 0.018 & 0.058 & 0.014 \\
\hline \multirow[t]{18}{*}{ Zinc } & GENMIN & $1 / 20$ & $<0.01$ & 0.031 & 0.17 & 0.084 \\
\hline & GENMINDISS & & 0.012 & $<0.01$ & 0.014 & 0.055 \\
\hline & NPDESDISS & & $<0.02$ & $<0.02$ & $<0.02$ & 0.058 \\
\hline & NPDESMETAL & & $<0.02$ & 0.034 & 0.17 & 0.087 \\
\hline & GENMIN & $1 / 26$ & —(b) $^{(\mathrm{s})}$ & -(b) $^{(\mathrm{n}}$ & $-^{(b)}$ & -(b) $^{(\mathrm{c}}$ \\
\hline & GENMINDISS & & —(b) $^{(\mathrm{s})}$ & -(b) $^{(\mathrm{s})}$ & -(b) $^{(\mathrm{c}}$ & -(b) $^{(\mathrm{c}}$ \\
\hline & NPDESDISS & & -(b) $^{(\mathrm{s})}$ & -(b) $^{(\mathrm{c}}$ & $-^{(b)}$ & -(b) $^{(\mathrm{s})}$ \\
\hline & NPDESMETAL & & $-_{(\mathrm{b})}$ & $-_{(\mathrm{b})}$ & $-^{(b)}$ & $-^{(\mathrm{b})}$ \\
\hline & GENMIN & $2 / 8$ & 0.021 & 0.047 & 0.083 & 0.086 \\
\hline & GENMINDISS & & 0.016 & $<0.01$ & 0.028 & 0.018 \\
\hline & NPDESDISS & & $<0.02$ & $<0.02$ & 0.037 & 0.022 \\
\hline & NPDESMETAL & & 0.023 & 0.048 & 0.084 & 0.071 \\
\hline & GENMIN & $4 / 8$ & 0.033 & 0.096 & 0.07 & 0.13 \\
\hline & GENMINDISS & & 0.076 & 0.021 & 0.044 & 0.043 \\
\hline & NPDESDISS & & 0.022 & 0.02 & 0.034 & 0.046 \\
\hline & NPDESMETAL & & 0.03 & 0.088 & 0.079 & 0.097 \\
\hline & GENMIN & $11 / 8$ & 0.13 & 0.13 & 0.29 & 0.28 \\
\hline & NPDESMETAL & & 0.16 & 0.17 & 0.24 & 0.29 \\
\hline
\end{tabular}




\section{Surface Water}

Table 7-5. Metals detected in storm water runoff, Livermore site, 1999 (continued).

\begin{tabular}{|c|c|c|c|c|c|}
\hline \multirow{3}{*}{ Parameter (mg/L) } & \multirow{3}{*}{ Requested analysis } & \multirow{3}{*}{$\begin{array}{c}\text { Storm } \\
\text { date }\end{array}$} & \multicolumn{3}{|c|}{ Drainage retention basin } \\
\hline & & & \multicolumn{2}{|c|}{ DRB influent } & \multirow{2}{*}{$\begin{array}{c}\text { DRB effluent } \\
\text { CDBX }\end{array}$} \\
\hline & & & CDB & CDB2 & \\
\hline \multirow[t]{18}{*}{ Aluminum } & GENMIN & $1 / 20$ & 3.1 & -(a) $^{\text {(a) }}$ & 2.2 \\
\hline & GENMINDISS & & 0.11 & -(a) $^{(a)}$ & $<0.05$ \\
\hline & NPDESDISS & & 0.12 & -(a) $^{(a)}$ & $<0.05$ \\
\hline & NPDESMETAL & & 2.8 & -(a) $^{(\mathrm{n}}$ & 1.8 \\
\hline & GENMIN & $1 / 26$ & -(b) $^{(\mathrm{c}}$ & 4.8 & -(b) $^{(\mathrm{b}}$ \\
\hline & GENMINDISS & & -(b) $^{(\mathrm{c}}$ & 0.22 & -(b) $^{(\mathrm{c}}$ \\
\hline & NPDESDISS & & $-^{(b)}$ & 0.26 & $-^{(\mathrm{b})}$ \\
\hline & NPDESMETAL & & $-^{(\mathrm{b})}$ & 4.5 & -(b) $^{(\mathrm{n}}$ \\
\hline & GENMIN & $2 / 8$ & 6 & 3.6 & 17 \\
\hline & GENMINDISS & & 0.55 & 0.11 & $<0.05$ \\
\hline & NPDESDISS & & 0.52 & 0.19 & $<0.05$ \\
\hline & NPDESMETAL & & 6 & 3.4 & 11 \\
\hline & GENMIN & $4 / 8$ & 3.4 & 7.4 & 0.34 \\
\hline & GENMINDISS & & 0.23 & 0.19 & $<0.05$ \\
\hline & NPDESDISS & & 0.24 & 0.31 & $<0.05$ \\
\hline & NPDESMETAL & & 3.3 & 6.7 & 0.27 \\
\hline & GENMIN & $11 / 8$ & 1.5 & 7.5 & 1.5 \\
\hline & NPDESMETAL & & 1.4 & 8.1 & 1.5 \\
\hline \multirow[t]{9}{*}{ Arsenic } & NPDESDISS & $1 / 20$ & 0.0053 & - (a) $^{(2)}$ & 0.0034 \\
\hline & NPDESMETAL & & 0.004 & -(a) $^{(\mathrm{n}}$ & 0.0028 \\
\hline & NPDESDISS & $1 / 26$ & -(b) $^{(\mathrm{c}}$ & $<0.002$ & -(b) $^{(\mathrm{s})}$ \\
\hline & NPDESMETAL & & $-^{(\mathrm{b})}$ & $<0.004$ & -(b) $^{(\mathrm{c}}$ \\
\hline & NPDESDISS & $2 / 8$ & $<0.002$ & 0.0023 & $<0.002$ \\
\hline & NPDESMETAL & & 0.0022 & 0.0039 & 0.0029 \\
\hline & NPDESDISS & $4 / 8$ & $<0.002$ & 0.0027 & $<0.002$ \\
\hline & NPDESMETAL & & 0.0022 & 0.0041 & $<0.002$ \\
\hline & NPDESMETAL & $11 / 8$ & $<0.002$ & 0.011 & 0.0043 \\
\hline \multirow[t]{9}{*}{ Barium } & NPDESDISS & $1 / 20$ & 0.11 & - (a) $^{(2}$ & 0.098 \\
\hline & NPDESMETAL & & 0.14 & -(a) $^{(a)}$ & 0.12 \\
\hline & NPDESDISS & $1 / 26$ & -(b) $^{(\mathrm{c}}$ & 0.057 & $-^{(b)}$ \\
\hline & NPDESMETAL & & $-^{(b)}$ & 0.088 & $-^{(b)}$ \\
\hline & NPDESDISS & $2 / 8$ & 0.14 & 0.089 & 0.11 \\
\hline & NPDESMETAL & & 0.12 & 0.12 & 0.23 \\
\hline & NPDESDISS & $4 / 8$ & 0.1 & 0.086 & 0.095 \\
\hline & NPDESMETAL & & 0.085 & 0.1 & 0.098 \\
\hline & NPDESMETAL & $11 / 8$ & 0.042 & 0.11 & 0.13 \\
\hline
\end{tabular}


Table 7-5. Metals detected in storm water runoff, Livermore site, 1999 (continued).

\begin{tabular}{|c|c|c|c|c|c|}
\hline \multirow{3}{*}{ Parameter (mg/L) } & \multirow{3}{*}{ Requested analysis } & \multirow{3}{*}{$\begin{array}{l}\text { Storm } \\
\text { date }\end{array}$} & \multicolumn{3}{|c|}{ Drainage retention basin } \\
\hline & & & \multicolumn{2}{|c|}{ DRB influent } & \multirow{2}{*}{$\begin{array}{c}\text { DRB effluent } \\
\text { CDBX }\end{array}$} \\
\hline & & & CDB & CDB2 & \\
\hline \multirow[t]{9}{*}{ Beryllium } & NPDESDISS & $1 / 20$ & $<0.0002$ & -(a) & $<0.0002$ \\
\hline & NPDESMETAL & & $<0.0002$ & -(a) & $<0.0002$ \\
\hline & NPDESDISS & $1 / 26$ & —(b) & $<0.0002$ & -(b) $^{(\mathrm{l}}$ \\
\hline & NPDESMETAL & & -(b) & $<0.0002$ & -(b) \\
\hline & NPDESDISS & $2 / 8$ & $<0.0002$ & $<0.0002$ & $<0.0002$ \\
\hline & NPDESMETAL & & $<0.0002$ & $<0.0002$ & 0.00028 \\
\hline & NPDESDISS & $4 / 8$ & $<0.0002$ & $<0.0002$ & $<0.0002$ \\
\hline & NPDESMETAL & & $<0.0002$ & $<0.0002$ & $<0.0002$ \\
\hline & NPDESMETAL & $11 / 8$ & $<0.0002$ & 0.00021 & $<0.0002$ \\
\hline \multirow[t]{9}{*}{ Boron } & NPDESDISS & $1 / 20$ & 10 & -(a) & 1.8 \\
\hline & NPDESMETAL & & 10 & -(a) & 1.8 \\
\hline & NPDESDISS & $1 / 26$ & —(b) & 2.6 & -(b) \\
\hline & NPDESMETAL & & -(b) $^{(\mathrm{c}}$ & 2.8 & -(b) $^{(\mathrm{c}}$ \\
\hline & NPDESDISS & $2 / 8$ & 0.086 & 7.6 & 1.8 \\
\hline & NPDESMETAL & & 0.1 & 10 & 1.7 \\
\hline & NPDESDISS & $4 / 8$ & 0.071 & 0.81 & 1.9 \\
\hline & NPDESMETAL & & 0.079 & 0.8 & 1.9 \\
\hline & NPDESMETAL & $11 / 8$ & 0.082 & 0.21 & 1.6 \\
\hline \multirow[t]{9}{*}{ Cadmium } & NPDESDISS & $1 / 20$ & $<0.0005$ & -(a) & $<0.0005$ \\
\hline & NPDESMETAL & & $<0.0005$ & -(a) & $<0.0005$ \\
\hline & NPDESDISS & $1 / 26$ & (b) $^{(\mathrm{b})}$ & $<0.0005$ & -(b) $^{(\mathrm{n}}$ \\
\hline & NPDESMETAL & & —(b) $^{(\mathrm{b})}$ & $<0.0005$ & - (b) $^{\text {(b) }}$ \\
\hline & NPDESDISS & $2 / 8$ & $<0.0005$ & $<0.0005$ & $<0.0005$ \\
\hline & NPDESMETAL & & $<0.0005$ & $<0.0005$ & $<0.0005$ \\
\hline & NPDESDISS & $4 / 8$ & $<0.0005$ & $<0.0005$ & $<0.0005$ \\
\hline & NPDESMETAL & & $<0.0005$ & $<0.0005$ & $<0.0005$ \\
\hline & NPDESMETAL & $11 / 8$ & $<0.0005$ & 0.00065 & $<0.0005$ \\
\hline \multirow[t]{9}{*}{ Chromium } & NPDESDISS & $1 / 20$ & 0.001 & - (a) & 0.0044 \\
\hline & NPDESMETAL & & 0.006 & -(a) & 0.01 \\
\hline & NPDESDISS & $1 / 26$ & -(b) & 0.0012 & 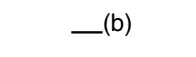 \\
\hline & NPDESMETAL & & -(b) $^{(\mathrm{n}}$ & 0.012 & -(b) $^{(\mathrm{n}}$ \\
\hline & NPDESDISS & $2 / 8$ & $<0.001$ & $<0.001$ & 0.0048 \\
\hline & NPDESMETAL & & 0.016 & 0.0084 & 0.03 \\
\hline & NPDESDISS & $4 / 8$ & 0.0018 & 0.001 & 0.0078 \\
\hline & NPDESMETAL & & 0.0078 & 0.016 & 0.0085 \\
\hline & NPDESMETAL & $11 / 8$ & 0.004 & 0.023 & 0.0068 \\
\hline
\end{tabular}




\section{Surface Water}

Table 7-5. Metals detected in storm water runoff, Livermore site, 1999 (continued).

\begin{tabular}{|c|c|c|c|c|c|}
\hline \multirow{3}{*}{ Parameter (mg/L) } & \multirow{3}{*}{ Requested analysis } & \multirow{3}{*}{$\begin{array}{l}\text { Storm } \\
\text { date }\end{array}$} & \multicolumn{3}{|c|}{ Drainage retention basin } \\
\hline & & & \multicolumn{2}{|c|}{ DRB influent } & \multirow{2}{*}{$\begin{array}{c}\text { DRB effluent } \\
\text { CDBX }\end{array}$} \\
\hline & & & CDB & CDB2 & \\
\hline \multirow[t]{18}{*}{ Copper } & GENMIN & $1 / 20$ & 0.02 & —(a) & 0.014 \\
\hline & GENMINDISS & & $<0.01$ & -(a) $^{(a)}$ & $<0.01$ \\
\hline & NPDESDISS & & 0.0063 & -(a) & 0.0026 \\
\hline & NPDESMETAL & & 0.0078 & -(a) & 0.0042 \\
\hline & GENMIN & $1 / 26$ & $-^{(\mathrm{b})}$ & $<0.01$ & -(b) $^{(\mathrm{c}}$ \\
\hline & GENMINDISS & & $-^{(\mathrm{b})}$ & $<0.01$ & -(b) $^{(\mathrm{c}}$ \\
\hline & NPDESDISS & & -(b) $^{(\mathrm{c}}$ & 0.0051 & -(b) $^{(b}$ \\
\hline & NPDESMETAL & & -(b) $^{(\mathrm{n}}$ & 0.011 & -(b) $^{(\mathrm{c}}$ \\
\hline & GENMIN & $2 / 8$ & 0.012 & 0.019 & 0.038 \\
\hline & GENMINDISS & & $<0.01$ & 0.01 & 0.01 \\
\hline & NPDESDISS & & $<0.001$ & $<0.001$ & $<0.001$ \\
\hline & NPDESMETAL & & 0.0094 & 0.012 & 0.019 \\
\hline & GENMIN & $4 / 8$ & 0.02 & 0.019 & 0.013 \\
\hline & GENMINDISS & & $<0.01$ & $<0.01$ & 0.015 \\
\hline & NPDESDISS & & 0.0052 & 0.0066 & 0.0079 \\
\hline & NPDESMETAL & & 0.0085 & 0.016 & 0.0027 \\
\hline & GENMIN & $11 / 8$ & 0.022 & 0.035 & 0.016 \\
\hline & NPDESMETAL & & 0.0096 & 0.027 & 0.0066 \\
\hline \multirow[t]{5}{*}{ Chromium(VI) } & NPDESDISS & $1 / 20$ & $<0.02$ & - (a) $^{(2}$ & 0.005 \\
\hline & NPDESDISS & $1 / 26$ & -(b) $^{(\mathrm{b}}$ & 0.006 & -(b) $^{(\mathrm{n})}$ \\
\hline & NPDESDISS & $2 / 8$ & $<0.002$ & $<0.002$ & 0.0053 \\
\hline & NPDESDISS & $4 / 8$ & $<0.002$ & $<0.002$ & 0.007 \\
\hline & NPDESMETAL & $11 / 8$ & $<0.002$ & $<0.002$ & $<0.002$ \\
\hline \multirow[t]{13}{*}{ Iron } & GENMIN & $1 / 20$ & 2.8 & -(a) & 2 \\
\hline & GENMINDISS & & 0.17 & -(a) $^{(\mathrm{n}}$ & $<0.05$ \\
\hline & NPDESDISS & & 0.17 & -(a) $^{(a)}$ & $<0.05$ \\
\hline & NPDESMETAL & & 2.7 & - (a) $^{(2}$ & 1.9 \\
\hline & GENMIN & $1 / 26$ & -(b) $^{(\mathrm{c}}$ & 4.8 & -(b) $^{(\mathrm{c}}$ \\
\hline & GENMINDISS & & -(b) $^{(\mathrm{c}}$ & 0.21 & -(b) $^{(\mathrm{n}}$ \\
\hline & NPDESDISS & & -(b) $^{(\mathrm{n}}$ & 0.23 & -(b) $^{(\mathrm{c}}$ \\
\hline & NPDESMETAL & & -(b) $^{(\mathrm{n}}$ & 4.7 & $-^{(b)}$ \\
\hline & GENMIN & $2 / 8$ & 6.6 & 3.6 & 20 \\
\hline & GENMINDISS & & 0.42 & 0.16 & $<0.05$ \\
\hline & NPDESDISS & & 0.42 & 0.21 & $<0.05$ \\
\hline & NPDESMETAL & & 6.3 & 3.4 & 12 \\
\hline & GENMIN & $4 / 8$ & 3.9 & 7.9 & 0.4 \\
\hline
\end{tabular}


Table 7-5. Metals detected in storm water runoff, Livermore site, 1999 (continued).

\begin{tabular}{|c|c|c|c|c|c|}
\hline \multirow{3}{*}{ Parameter (mg/L) } & \multirow{3}{*}{ Requested analysis } & \multirow{3}{*}{$\begin{array}{l}\text { Storm } \\
\text { date }\end{array}$} & \multicolumn{3}{|c|}{ Drainage retention basin } \\
\hline & & & \multicolumn{2}{|c|}{ DRB influent } & \multirow{2}{*}{$\begin{array}{c}\text { DRB effluent } \\
\text { CDBX }\end{array}$} \\
\hline & & & CDB & CDB2 & \\
\hline \multirow[t]{5}{*}{ Iron (continued) } & GENMINDISS & & 0.19 & 0.17 & $<0.05$ \\
\hline & NPDESDISS & & 0.18 & 0.26 & $<0.05$ \\
\hline & NPDESMETAL & & 3.6 & 7.6 & 0.33 \\
\hline & GENMIN & $11 / 8$ & 1.7 & 8.3 & 1.5 \\
\hline & NPDESMETAL & & 1.7 & 8.7 & 1.4 \\
\hline \multirow[t]{9}{*}{ Lead } & NPDESDISS & $1 / 20$ & $<0.005$ & -(a) & $<0.005$ \\
\hline & NPDESMETAL & & $<0.005$ & -(a) $^{\text {(a) }}$ & $<0.005$ \\
\hline & NPDESDISS & $1 / 26$ & —(b) $^{(\mathrm{n}}$ & $<0.005$ & -(b) $^{(\mathrm{c}}$ \\
\hline & NPDESMETAL & & $-^{(\mathrm{b})}$ & 0.0091 & -(b) $^{(\mathrm{c}}$ \\
\hline & NPDESDISS & $2 / 8$ & $<0.005$ & $<0.005$ & $<0.005$ \\
\hline & NPDESMETAL & & $<0.005$ & $<0.005$ & 0.0079 \\
\hline & NPDESDISS & $4 / 8$ & $<0.005$ & $<0.005$ & $<0.005$ \\
\hline & NPDESMETAL & & $<0.005$ & 0.0067 & $<0.005$ \\
\hline & NPDESMETAL & $11 / 8$ & 0.0052 & 0.0086 & $<0.005$ \\
\hline \multirow[t]{9}{*}{ Magnesium } & GENMIN & $1 / 20$ & 29 & -(a) & 23 \\
\hline & GENMINDISS & & 29 & -(a) $^{(\mathrm{n}}$ & 23 \\
\hline & GENMIN & $1 / 26$ & -(b) $^{(\mathrm{n}}$ & 10 & -(b) $^{(\mathrm{c}}$ \\
\hline & GENMINDISS & & —(b) $^{(\mathrm{n}}$ & 9.1 & -(b) $^{(\mathrm{c}}$ \\
\hline & GENMIN & $2 / 8$ & 6.7 & 28 & 30 \\
\hline & GENMINDISS & & 4.7 & 28 & 25 \\
\hline & GENMIN & $4 / 8$ & 5 & 5.6 & 30 \\
\hline & GENMINDISS & & 3.9 & 3.5 & 31 \\
\hline & GENMIN & $11 / 8$ & 2.6 & 5 & 30 \\
\hline \multirow[t]{13}{*}{ Manganese } & GENMIN & $1 / 20$ & 0.073 & -(a) $^{(\mathrm{a}}$ & 0.048 \\
\hline & GENMINDISS & & 0.013 & -(a) $^{\text {(a) }}$ & $<0.01$ \\
\hline & NPDESDISS & & 0.014 & -(a) $^{(a)}$ & $<0.01$ \\
\hline & NPDESMETAL & & 0.072 & -(a) & 0.046 \\
\hline & GENMIN & $1 / 26$ & -(b) $^{(\mathrm{n}}$ & 0.083 & -(b) $^{(\mathrm{c}}$ \\
\hline & GENMINDISS & & -(b) $^{(\mathrm{c}}$ & $<0.01$ & -(b) $^{(\mathrm{c}}$ \\
\hline & NPDESDISS & & -(b) $^{(\mathrm{c}}$ & 0.01 & -(b) $^{(\mathrm{c}}$ \\
\hline & NPDESMETAL & & -(b) $^{(\mathrm{c}}$ & 0.082 & -(b) $^{(\mathrm{c}}$ \\
\hline & GENMIN & $2 / 8$ & 0.14 & 0.089 & 0.68 \\
\hline & GENMINDISS & & $<0.01$ & 0.012 & $<0.01$ \\
\hline & NPDESDISS & & $<0.01$ & $<0.01$ & $<0.01$ \\
\hline & NPDESMETAL & & 0.13 & 0.083 & 0.48 \\
\hline & GENMIN & $4 / 8$ & 0.085 & 0.14 & 0.034 \\
\hline
\end{tabular}




\section{Surface Water}

Table 7-5. Metals detected in storm water runoff, Livermore site, 1999 (continued).

\begin{tabular}{|c|c|c|c|c|c|}
\hline \multirow{3}{*}{ Parameter (mg/L) } & \multirow{3}{*}{ Requested analysis } & \multirow{3}{*}{$\begin{array}{c}\text { Storm } \\
\text { date }\end{array}$} & \multicolumn{3}{|c|}{ Drainage retention basin } \\
\hline & & & \multicolumn{2}{|c|}{ DRB influent } & \multirow{2}{*}{$\begin{array}{c}\text { DRB effluent } \\
\text { CDBX }\end{array}$} \\
\hline & & & CDB & CDB2 & \\
\hline \multirow{5}{*}{$\begin{array}{l}\text { Manganese } \\
\text { (continued) }\end{array}$} & GENMINDISS & & 0.018 & $<0.01$ & $<0.01$ \\
\hline & NPDESDISS & & 0.018 & $<0.01$ & $<0.01$ \\
\hline & NPDESMETAL & & 0.079 & 0.14 & 0.029 \\
\hline & GENMIN & $11 / 8$ & 0.073 & 0.24 & 0.049 \\
\hline & NPDESMETAL & & 0.068 & 0.25 & 0.049 \\
\hline \multirow[t]{9}{*}{ Mercury } & NPDESDISS & $1 / 20$ & $<0.0002$ & 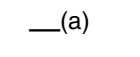 & $<0.0002$ \\
\hline & NPDESMETAL & & $<0.0002$ & -(a) $^{\text {(a) }}$ & $<0.0002$ \\
\hline & NPDESDISS & $1 / 26$ & —(b) $^{(\mathrm{n}}$ & $<0.0002$ & -(b) $^{(\mathrm{c}}$ \\
\hline & NPDESMETAL & & -(b) $^{(\mathrm{n}}$ & $<0.0002$ & $-^{(b)}$ \\
\hline & NPDESDISS & $2 / 8$ & $<0.0002$ & $<0.0002$ & $<0.0002$ \\
\hline & NPDESMETAL & & $<0.0002$ & $<0.0002$ & 0.00043 \\
\hline & NPDESDISS & $4 / 8$ & $<0.0002$ & $<0.0002$ & $<0.0002$ \\
\hline & NPDESMETAL & & $<0.0002$ & $<0.0002$ & $<0.0002$ \\
\hline & NPDESMETAL & $11 / 8$ & $<0.0002$ & $<0.0002$ & $<0.0002$ \\
\hline \multirow[t]{9}{*}{ Molybdenum } & NPDESDISS & $1 / 20$ & $<0.025$ & 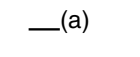 & $<0.025$ \\
\hline & NPDESMETAL & & $<0.025$ & -(a) $^{(\mathrm{a}}$ & $<0.025$ \\
\hline & NPDESDISS & $1 / 26$ & —(b) $^{(\mathrm{s}}$ & $<0.025$ & -(b) $^{(\mathrm{s}}$ \\
\hline & NPDESMETAL & & -(b) $^{(-1}$ & $<0.025$ & -(b) $^{(\mathrm{n}}$ \\
\hline & NPDESDISS & $2 / 8$ & $<0.025$ & $<0.025$ & $<0.025$ \\
\hline & NPDESMETAL & & $<0.025$ & $<0.025$ & $<0.025$ \\
\hline & NPDESDISS & $4 / 8$ & $<0.025$ & $<0.025$ & $<0.025$ \\
\hline & NPDESMETAL & & $<0.025$ & $<0.025$ & $<0.025$ \\
\hline & NPDESMETAL & $11 / 8$ & $<0.025$ & $<0.025$ & $<0.025$ \\
\hline \multirow[t]{13}{*}{ Nickel } & GENMIN & $1 / 20$ & 0.05 & -(a) $^{(a)}$ & $<0.05$ \\
\hline & GENMINDISS & & $<0.05$ & -(a) $^{(a)}$ & $<0.05$ \\
\hline & NPDESDISS & & 0.0068 & -(a) $^{(\mathrm{a}}$ & $<0.002$ \\
\hline & NPDESMETAL & & 0.013 & -(a) $^{(\mathrm{n}}$ & 0.0065 \\
\hline & GENMIN & $1 / 26$ & -(b) $^{(\mathrm{c}}$ & $<0.05$ & -(b) $^{(\mathrm{n}}$ \\
\hline & GENMINDISS & & -(b) $^{(\mathrm{b}}$ & $<0.05$ & -(b) $^{(\mathrm{c}}$ \\
\hline & NPDESDISS & & $-^{(b)}$ & 0.0036 & $-^{(\mathrm{b})}$ \\
\hline & NPDESMETAL & & $-^{(b)}$ & 0.014 & -(b) $^{(\mathrm{c}}$ \\
\hline & GENMIN & $2 / 8$ & $<0.05$ & $<0.05$ & 0.051 \\
\hline & GENMINDISS & & $<0.05$ & $<0.05$ & $<0.05$ \\
\hline & NPDESDISS & & 0.0024 & 0.0057 & $<0.002$ \\
\hline & NPDESMETAL & & 0.016 & 0.012 & 0.028 \\
\hline & GENMIN & $4 / 8$ & $<0.05$ & $<0.05$ & $<0.05$ \\
\hline
\end{tabular}


Table 7-5. Metals detected in storm water runoff, Livermore site, 1999 (continued).

\begin{tabular}{|c|c|c|c|c|c|}
\hline \multirow{3}{*}{ Parameter (mg/L) } & \multirow{3}{*}{ Requested analysis } & \multirow{3}{*}{$\begin{array}{l}\text { Storm } \\
\text { date }\end{array}$} & \multicolumn{3}{|c|}{ Drainage retention basin } \\
\hline & & & \multicolumn{2}{|c|}{ DRB influent } & \multirow{2}{*}{$\begin{array}{c}\text { DRB effluent } \\
\text { CDBX } \\
\end{array}$} \\
\hline & & & CDB & CDB2 & \\
\hline \multirow[t]{5}{*}{ Nickel (continued) } & GENMINDISS & & $<0.05$ & $<0.05$ & $<0.05$ \\
\hline & NPDESDISS & & $<0.002$ & 0.0025 & $<0.002$ \\
\hline & NPDESMETAL & & 0.011 & 0.02 & 0.0023 \\
\hline & GENMIN & $11 / 8$ & $<0.05$ & $<0.05$ & $<0.05$ \\
\hline & NPDESMETAL & & 0.0083 & 0.026 & 0.0065 \\
\hline \multirow[t]{9}{*}{ Vanadium } & NPDESDISS & $1 / 20$ & $<0.01$ & 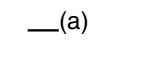 & $<0.01$ \\
\hline & NPDESMETAL & & 0.011 & —(a) $^{(\mathrm{n}}$ & $<0.01$ \\
\hline & NPDESDISS & $1 / 26$ & —(b) $^{(\mathrm{n}}$ & $<0.01$ & -(b) $^{(\mathrm{l}}$ \\
\hline & NPDESMETAL & & $-^{(b)}$ & $<0.01$ & -(b) $^{(\mathrm{c}}$ \\
\hline & NPDESDISS & $2 / 8$ & $<0.01$ & $<0.01$ & $<0.01$ \\
\hline & NPDESMETAL & & 0.014 & 0.012 & 0.028 \\
\hline & NPDESDISS & $4 / 8$ & $<0.01$ & $<0.01$ & $<0.01$ \\
\hline & NPDESMETAL & & $<0.01$ & 0.016 & $<0.01$ \\
\hline & NPDESMETAL & $11 / 8$ & $<0.01$ & 0.021 & 0.012 \\
\hline \multirow[t]{18}{*}{ Zinc } & GENMIN & $1 / 20$ & 0.045 & -(a) & 0.026 \\
\hline & GENMINDISS & & 0.031 & - (a) $^{\text {(a) }}$ & $<0.01$ \\
\hline & NPDESDISS & & 0.03 & - (a) $^{(2}$ & $<0.02$ \\
\hline & NPDESMETAL & & 0.046 & -(a) $^{(\mathrm{n}}$ & 0.026 \\
\hline & GENMIN & $1 / 26$ & -(b) $^{(\mathrm{n}}$ & 0.13 & -(b) $^{(\mathrm{c}}$ \\
\hline & GENMINDISS & & $-^{(b)}$ & 0.048 & $-_{(\mathrm{b})}$ \\
\hline & NPDESDISS & & -(b) $^{(\mathrm{c}}$ & 0.046 & $-^{(b)}$ \\
\hline & NPDESMETAL & & -(b) $^{(\mathrm{c}}$ & 0.12 & -(b) $^{(\mathrm{c}}$ \\
\hline & GENMIN & $2 / 8$ & 0.16 & 0.064 & 0.27 \\
\hline & GENMINDISS & & 0.064 & 0.034 & $<0.01$ \\
\hline & NPDESDISS & & 0.07 & 0.033 & $<0.02$ \\
\hline & NPDESMETAL & & 0.15 & 0.05 & 0.2 \\
\hline & GENMIN & $4 / 8$ & 0.12 & 0.096 & 0.018 \\
\hline & GENMINDISS & & 0.052 & 0.032 & $<0.01$ \\
\hline & NPDESDISS & & 0.054 & 0.033 & $<0.02$ \\
\hline & NPDESMETAL & & 0.11 & 0.099 & $<0.02$ \\
\hline & GENMIN & $11 / 8$ & 0.23 & 0.21 & 0.07 \\
\hline & NPDESMETAL & & 0.24 & 0.22 & 0.11 \\
\hline
\end{tabular}

a Sample not collected because there was no flow.

b Locations sampled on 1/20 were not sampled again on 1/26. 


\section{Surface Water}

Table 7-6. Nonradioactive constituents (other than metals) detected in storm water runoff, Livermore site, 1999.

\begin{tabular}{|c|c|c|c|c|c|c|c|c|c|c|}
\hline \multirow{3}{*}{ Parameter } & \multirow{3}{*}{$\begin{array}{l}\text { Storm } \\
\text { Date }\end{array}$} & \multicolumn{2}{|c|}{ Arroyo Seco } & \multicolumn{4}{|c|}{ Arroyo Las Positas } & \multicolumn{3}{|c|}{ Drainage Retention Basin } \\
\hline & & \multirow{2}{*}{\begin{tabular}{|c}
$\begin{array}{c}\text { Site } \\
\text { influent }\end{array}$ \\
ASS2
\end{tabular}} & \multirow{2}{*}{$\begin{array}{c}\begin{array}{c}\text { Site } \\
\text { effluent }\end{array} \\
\text { ASW }\end{array}$} & \multicolumn{3}{|c|}{$\begin{array}{c}\text { Site } \\
\text { influent }\end{array}$} & \multirow{2}{*}{\begin{tabular}{|c|}
$\begin{array}{c}\text { Site } \\
\text { effluent }\end{array}$ \\
WPDC
\end{tabular}} & \multicolumn{2}{|c|}{$\begin{array}{c}\text { Site } \\
\text { influent }\end{array}$} & \multirow{2}{*}{$\begin{array}{c}\begin{array}{c}\text { Site } \\
\text { effluent }\end{array} \\
\text { CDBX }\end{array}$} \\
\hline & & & & ALPE & ALPO & GRNE & & CDB & CDB2 & \\
\hline \multicolumn{11}{|l|}{ Physical (mg/L) } \\
\hline \multirow[t]{5}{*}{ Chemical oxygen demand } & $1 / 20$ & -(a) & -(a) & 169 & 61 & $<20$ & 30 & 108 & -(a) & 24 \\
\hline & $1 / 26$ & $<4$ & 33 & -(b) $^{(\mathrm{c}}$ & -(b) & -(b) & -(b) & 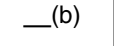 & 46 & -(b) \\
\hline & $2 / 8$ & 27 & 33 & 104 & 64 & $<20$ & 79 & 54 & 116 & 71 \\
\hline & $4 / 8$ & 33 & 50 & 85 & 43 & 28 & 40 & 50 & 84 & 43 \\
\hline & $11 / 8$ & -(a) & 64 & 79 & 56 & 53 & 64 & 61 & 126 & 37 \\
\hline \multirow[t]{5}{*}{ Total suspended solids (TSS) } & $1 / 20$ & -(a) & - (a) $^{(2)}$ & 7.8 & 91 & 206 & 40 & 44 & -(a) & 55 \\
\hline & $1 / 26$ & 21 & 56 & -(b) & - (b) $^{(2)}$ & -(b) & - (b) $^{(2)}$ & -(b) & 66 & -(b) \\
\hline & $2 / 8$ & 47 & 53 & 95 & 240 & 66 & 52 & 87 & 62 & 169 \\
\hline & $4 / 8$ & 34 & 16 & 64 & 219 & 35 & 84 & 48 & 72 & 57 \\
\hline & $11 / 8$ & -(a) $^{(\mathrm{a}}$ & 38 & 29 & 139 & 213 & 94 & 24 & 144 & 28 \\
\hline \multicolumn{11}{|l|}{ Anions (mg/L) } \\
\hline \multirow[t]{5}{*}{ Bromide } & $1 / 20$ & -(a) & -(a) & 0.38 & 0.61 & 0.07 & 0.31 & 0.32 & -(a) & 0.41 \\
\hline & $1 / 26$ & $<0.1$ & $<0.1$ & -(b) & -(b) & -(b) $^{(\mathrm{c}}$ & -(b) $^{(\mathrm{n}}$ & -(b) $^{(\mathrm{n}}$ & 0.28 & -(b) $^{(\mathrm{n}}$ \\
\hline & $2 / 8$ & $<0.1$ & $<0.1$ & 0.62 & 0.9 & $<0.05$ & 0.1 & $<0.05$ & 0.46 & 0.42 \\
\hline & $4 / 8$ & $<0.1$ & 0.16 & 0.19 & 0.22 & 0.11 & $<0.1$ & $<0.1$ & $<0.1$ & 0.5 \\
\hline & $11 / 8$ & -(a) & $<0.1$ & $<0.1$ & 0.96 & 0.14 & $<0.1$ & $<0.1$ & $<0.1$ & 0.51 \\
\hline \multirow[t]{5}{*}{ Chloride } & $1 / 20$ & -(a) & -(a) & 550 & 251 & 7.2 & 114 & 279 & - (a) & 130 \\
\hline & $1 / 26$ & 2.7 & 2.6 & -(b) $^{(\mathrm{n}}$ & - (b) $^{(2)}$ & - (b) $^{(2)}$ & - (b) $^{(2)}$ & - (b) $^{(2)}$ & 85 & - (b) $^{(2)}$ \\
\hline & $2 / 8$ & 26 & 23 & 310 & 223 & 5.2 & 30 & 11 & 304 & 138 \\
\hline & $4 / 8$ & 1.5 & 1.3 & 89 & 12 & 5.2 & 12 & 8.3 & 15 & 76 \\
\hline & $11 / 8$ & - (a) $^{(2}$ & 2.7 & 4.2 & 180 & 13 & 19 & 4.9 & 6.5 & 146 \\
\hline \multirow[t]{5}{*}{ Fluoride } & $1 / 20$ & -(a) & -(a) & 1.2 & 0.92 & 0.38 & 0.38 & 0.57 & -(a) & 0.38 \\
\hline & $1 / 26$ & $<0.05$ & $<0.05$ & -(b) & -(b) & -(b) & -(b) & -(b) & 0.26 & - (b) $^{(2)}$ \\
\hline & $2 / 8$ & 0.12 & 0.1 & 0.97 & 0.88 & 0.17 & 0.17 & 0.09 & 0.92 & 0.38 \\
\hline & $4 / 8$ & $<0.05$ & $<0.05$ & 0.29 & 0.1 & 0.11 & 0.07 & 0.07 & 0.12 & 0.4 \\
\hline & $11 / 8$ & -(a) & 0.06 & 0.08 & 0.66 & 0.27 & 0.11 & 0.06 & 0.07 & 0.35 \\
\hline \multirow[t]{5}{*}{ Nitrate (as N) } & $1 / 20$ & -(a) & -(a) & 0.2 & 2.6 & 6.8 & 2.2 & 0.63 & -(a) & 2.2 \\
\hline & $1 / 26$ & 0.14 & 0.26 & -(b) $^{(\mathrm{s})}$ & -(b) $^{(\mathrm{c}}$ & -(b) $^{(\mathrm{c}}$ & -(b) $^{\left(b^{\prime}\right.}$ & - (b) $^{(2)}$ & 0.38 & - (b) $^{(2)}$ \\
\hline & $2 / 8$ & 0.56 & 0.62 & 0.27 & 2.6 & 3.8 & 1.1 & 0.94 & 0.24 & 2.6 \\
\hline & $4 / 8$ & 0.7 & 0.51 & 0.45 & 5.2 & 3.9 & 0.91 & 0.76 & 0.6 & 2.6 \\
\hline & $11 / 8$ & - (a) $^{(2)}$ & 1.1 & 0.75 & 1.7 & 6.2 & 1 & 0.69 & 1.1 & 2 \\
\hline
\end{tabular}


Table 7-6. Nonradioactive constituents (other than metals) detected in storm water runoff, Livermore site, 1999 (continued).

\begin{tabular}{|c|c|c|c|c|c|c|c|c|c|c|}
\hline \multirow{3}{*}{ Parameter } & \multirow{3}{*}{$\begin{array}{l}\text { Storm } \\
\text { Date }\end{array}$} & \multicolumn{2}{|c|}{ Arroyo Seco } & \multicolumn{4}{|c|}{ Arroyo Las Positas } & \multicolumn{3}{|c|}{ Drainage Retention Basin } \\
\hline & & \multirow{2}{*}{$\begin{array}{c}\begin{array}{c}\text { Site } \\
\text { influent }\end{array} \\
\text { ASS2 }\end{array}$} & \multirow{2}{*}{\begin{tabular}{|c}
$\begin{array}{c}\text { Site } \\
\text { effluent }\end{array}$ \\
ASW
\end{tabular}} & \multicolumn{3}{|c|}{$\begin{array}{c}\text { Site } \\
\text { influent }\end{array}$} & \multirow{2}{*}{\begin{tabular}{c|}
$\begin{array}{c}\text { Site } \\
\text { effluent }\end{array}$ \\
WPDC
\end{tabular}} & \multicolumn{2}{|c|}{$\begin{array}{c}\text { Site } \\
\text { influent }\end{array}$} & \multirow{2}{*}{$\begin{array}{c}\begin{array}{c}\text { Site } \\
\text { effluent }\end{array} \\
\text { CDBX }\end{array}$} \\
\hline & & & & ALPE & ALPO & GRNE & & CDB & CDB2 & \\
\hline \multicolumn{11}{|l|}{ Anions (mg/L) (continued) } \\
\hline \multirow[t]{5}{*}{ Nitrate $\left(\right.$ as $\left.\mathrm{NO}_{3}\right)$} & $1 / 20$ & -(a) & -(a) & 0.89 & 12 & 30 & 9.7 & 2.8 & -(a) & 9.7 \\
\hline & $1 / 26$ & 0.62 & 1.1 & -(b) & —(b) & -(b) & -(b) & -(b) & 1.7 & -(b) \\
\hline & $2 / 8$ & 2.5 & 2.7 & 1.2 & 11 & 17 & 4.9 & 4.1 & 1.1 & 11 \\
\hline & $4 / 8$ & 3.1 & 2.3 & 2 & 23 & 17 & 4.1 & 3.4 & 2.7 & 11 \\
\hline & $11 / 8$ & -(a) & 4.9 & 3.3 & 7.7 & 27 & 4.6 & 3.1 & 4.8 & 9 \\
\hline \multirow[t]{5}{*}{ Nitrite (as N) } & $1 / 20$ & -(a) & -(a) & $<0.02$ & 0.06 & 0.03 & 0.02 & 0.03 & -(a) & $<0.02$ \\
\hline & $1 / 26$ & $<0.02$ & $<0.02$ & 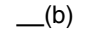 & 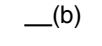 & 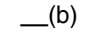 & - (b) $^{(2)}$ & 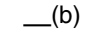 & $<0.02$ & —(b) $^{(\mathrm{b})}$ \\
\hline & $2 / 8$ & $<0.02$ & 0.02 & $<0.02$ & 0.03 & $<0.02$ & $<0.02$ & $<0.02$ & $<0.02$ & $<0.02$ \\
\hline & $4 / 8$ & $<0.02$ & $<0.02$ & 0.03 & 0.02 & 0.02 & 0.03 & $<0.02$ & 0.02 & $<0.02$ \\
\hline & $11 / 8$ & -(a) & 0.05 & 0.07 & 0.08 & 0.06 & 0.02 & $<0.02$ & 0.03 & 0.02 \\
\hline \multirow[t]{5}{*}{ Nitrite (as $\mathrm{NO}_{2}$ ) } & $1 / 20$ & -(a) & -(a) & $<0.07$ & 0.18 & 0.1 & 0.07 & 0.1 & -(a) & $<0.07$ \\
\hline & $1 / 26$ & $<0.07$ & $<0.07$ & -(b) $^{(\mathrm{n})}$ & 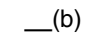 & -(b) $^{(\mathrm{n}}$ & -(b) $^{(\mathrm{s})}$ & -(b) $^{(\mathrm{n}}$ & $<0.07$ & -(b) \\
\hline & $2 / 8$ & $<0.07$ & 0.07 & $<0.07$ & 0.11 & $<0.07$ & $<0.07$ & $<0.07$ & $<0.07$ & $<0.07$ \\
\hline & $4 / 8$ & $<0.07$ & $<0.07$ & 0.09 & 0.07 & 0.07 & 0.11 & $<0.07$ & 0.07 & $<0.07$ \\
\hline & $11 / 8$ & -(a) & 0.15 & 0.24 & 0.28 & 0.2 & 0.07 & $<0.07$ & 0.11 & 0.07 \\
\hline \multirow[t]{5}{*}{ Orthophosphate } & $1 / 20$ & -(a) & -(a) & 4.6 & 1.1 & 0.33 & 0.35 & 2.5 & -(a) & 0.15 \\
\hline & $1 / 26$ & 0.21 & 0.33 & - (b) $^{\text {(b) }}$ & —(b) $^{(\mathrm{s}}$ & - (b) $^{(2)}$ & - (b) $^{(2)}$ & - (b) $^{(2)}$ & 0.64 & -(b) \\
\hline & $2 / 8$ & 0.43 & 0.47 & 3.1 & 0.92 & 0.44 & 0.39 & 0.18 & 2.9 & 0.14 \\
\hline & $4 / 8$ & 0.94 & 0.49 & 0.81 & 0.39 & 0.33 & 0.2 & 0.4 & 0.35 & 0.11 \\
\hline & $11 / 8$ & - (a) $^{(\mathrm{a}}$ & 0.75 & 0.64 & 0.39 & 0.7 & 0.42 & 1 & 1.2 & 0.3 \\
\hline \multirow[t]{5}{*}{ Sulfate } & $1 / 20$ & - (a) & -(a) & 692 & 232 & 10 & 104 & 358 & -(a) & 61 \\
\hline & $1 / 26$ & 1.7 & 1.7 & —(b) $^{(\mathrm{n}}$ & —(b) & -(b) & -(b) & -(b) & 78 & -(b) \\
\hline & $2 / 8$ & 40 & 37 & 349 & 212 & 6.6 & 22 & 3.8 & 323 & 62 \\
\hline & $4 / 8$ & 3 & 1.8 & 69 & 8.3 & 3.2 & 6.2 & 2.6 & 9.6 & 26 \\
\hline & $11 / 8$ & -(a) & 2.9 & 7.8 & 187 & 8.1 & 11 & 2.1 & 6.1 & 51 \\
\hline \multicolumn{11}{|l|}{ General minerals (mg/L) } \\
\hline \multirow[t]{5}{*}{ Bicarbonate alkalinity $\left(\right.$ as $\left.\mathrm{CaCO}_{3}\right)$} & $1 / 20$ & -(a) & -(a) & 342 & 190 & 59 & 129 & 190 & -(a) & 139 \\
\hline & $1 / 26$ & 16 & 19 & -(b) $^{(\mathrm{b})}$ & — (b) $^{(\mathrm{s})}$ & -(b) & - (b) $^{(\mathrm{s})}$ & -(b) $^{(\mathrm{s})}$ & 80 & -(b) \\
\hline & $2 / 8$ & 57 & 56 & 281 & 197 & 55 & 66 & 43 & 257 & 173 \\
\hline & $4 / 8$ & 14 & 14 & 93 & 25 & 34 & 43 & 32 & 37 & 153 \\
\hline & $11 / 8$ & -(a) & 36 & 43 & 146 & 43 & 72 & 25 & 42 & 177 \\
\hline
\end{tabular}




\section{Surface Water}

Table 7-6. Nonradioactive constituents (other than metals) detected in storm water runoff, Livermore site, 1999 (continued).

\begin{tabular}{|c|c|c|c|c|c|c|c|c|c|c|}
\hline \multirow{3}{*}{ Parameter } & \multirow{3}{*}{$\begin{array}{c}\text { Storm } \\
\text { Date }\end{array}$} & \multicolumn{2}{|c|}{ Arroyo Seco } & \multicolumn{4}{|c|}{ Arroyo Las Positas } & \multicolumn{3}{|c|}{ Drainage Retention Basin } \\
\hline & & \multirow{2}{*}{$\begin{array}{c}\begin{array}{c}\text { Site } \\
\text { influent }\end{array} \\
\text { ASS2 }\end{array}$} & \multirow{2}{*}{\begin{tabular}{|c|c}
$\begin{array}{c}\text { Site } \\
\text { effluent }\end{array}$ \\
ASW
\end{tabular}} & \multicolumn{3}{|c|}{$\begin{array}{c}\text { Site } \\
\text { influent }\end{array}$} & \multirow{2}{*}{$\begin{array}{c}\begin{array}{c}\text { Site } \\
\text { effluent }\end{array} \\
\text { WPDC }\end{array}$} & \multicolumn{2}{|c|}{$\begin{array}{c}\text { Site } \\
\text { influent }\end{array}$} & \multirow{2}{*}{$\begin{array}{c}\begin{array}{c}\text { Site } \\
\text { effluent }\end{array} \\
\text { CDBX }\end{array}$} \\
\hline & & & & ALPE & ALPO & GRNE & & CDB & CDB2 & \\
\hline \multicolumn{11}{|l|}{$\begin{array}{l}\text { General minerals (mg/L) } \\
\text { (continued) }\end{array}$} \\
\hline \multirow[t]{5}{*}{ Carbonate alkalinity (as $\mathrm{CaCO}_{3}$ ) } & $1 / 20$ & -(a) & -(a) & $<5$ & $<5$ & $<5$ & $<5$ & $<5$ & -(a) & 25 \\
\hline & $1 / 26$ & $<1$ & $<1$ & -(b) & -(b) $^{(\mathrm{c}}$ & - (b) $^{(2)}$ & - (b) $^{(1)}$ & -(b) & $<5$ & - (b) $^{(2}$ \\
\hline & $2 / 8$ & $<5$ & $<5$ & $<5$ & $<5$ & $<5$ & $<5$ & $<5$ & $<5$ & $<5$ \\
\hline & $4 / 8$ & $<5$ & $<5$ & $<5$ & $<5$ & $<5$ & $<5$ & $<5$ & $<5$ & 38 \\
\hline & $11 / 8$ & -(a) & $<5$ & $<5$ & $<5$ & $<5$ & $<5$ & $<5$ & $<5$ & $<5$ \\
\hline \multicolumn{11}{|l|}{ Herbicides ( $\mu \mathrm{g} / \mathrm{L})$} \\
\hline \multirow[t]{3}{*}{ Atrazine } & $1 / 20$ & -(a) & -(a) & $<0.2$ & $<0.2$ & 0.26 & $<0.2$ & $<0.2$ & -(a) & $<0.2$ \\
\hline & $1 / 26$ & $<0.2$ & $<0.2$ & -(b) $^{(\mathrm{s})}$ & -(b) & -(b) & -(b) $^{(\mathrm{b})}$ & -(b) & $<0.2$ & - (b) $^{(\mathrm{b})}$ \\
\hline & $2 / 8$ & $<0.2$ & $<0.2$ & $<0.2$ & $<0.2$ & $<0.2$ & $<0.2$ & $<0.2$ & $<0.2$ & $<0.2$ \\
\hline \multirow[t]{5}{*}{ Bromacil } & $1 / 20$ & -(a) & -(a) & $<0.5$ & $<0.5$ & $<0.5$ & $<0.5$ & $<0.5$ & -(a) & $<0.5$ \\
\hline & $1 / 26$ & $<0.5$ & $<0.5$ & -(b) $^{(\mathrm{b})}$ & -(b) & -(b) & - (b) $^{(-1)}$ & -(b) & $<0.5$ & -(b) \\
\hline & $2 / 8$ & 2.7 & 2.2 & $<0.5$ & 35 & $<0.5$ & 270 & 20 & 5 & 6 \\
\hline & $4 / 8$ & $<0.5$ & $<0.5$ & 4.2 & 400 & 330 & 3.6 & 2.5 & 5.5 & 4.4 \\
\hline & $11 / 8$ & - (a) $^{(\mathrm{a}}$ & $<0.5$ & 1.9 & $<5$ & 16 & 2.2 & $<5$ & $<5$ & $<5$ \\
\hline \multirow[t]{5}{*}{ Glyphosate } & $1 / 20$ & -(a) & -(a) & $<9$ & 10 & $<9$ & $<9$ & $<9$ & - (a) $^{(-1)}$ & $<9$ \\
\hline & $1 / 26$ & $<9$ & $<9$ & —(b) $^{(\mathrm{s})}$ & - (b) & 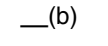 & - (b) $^{\text {(b) }}$ & —(b) & 16 & - (b) $^{(\mathrm{s})}$ \\
\hline & $2 / 8$ & $<9$ & $<9$ & $<9$ & $<9$ & $<9$ & $<9$ & $<9$ & $<9$ & $<9$ \\
\hline & $4 / 8$ & 12 & 14 & 51 & 16 & $<20$ & $<9$ & 24 & 16 & $<9$ \\
\hline & $11 / 8$ & -(a) & 57 & 94 & 95 & 100 & 99 & 11 & 100 & 100 \\
\hline \multirow[t]{5}{*}{ Diuron } & $1 / 20$ & -(a) & - (a) $^{(\mathrm{a}}$ & $<1$ & 190 & 11 & 63 & 6.9 & -(a) & 5.2 \\
\hline & $1 / 26$ & $<1$ & 1.6 & —(b) $^{(\mathrm{b})}$ & -(b) & -(b) & - (b) $^{(-1)}$ & -(b) & 7.5 & -(b) \\
\hline & $2 / 8$ & $<1$ & $<1$ & $<1$ & 34 & 100 & 6.3 & 5.5 & 2.3 & 2.1 \\
\hline & $4 / 8$ & $<1$ & $<1$ & $<2$ & 11 & 12 & $<1$ & $<1$ & 1.6 & $<1$ \\
\hline & $11 / 8$ & -(a) & $<2$ & $<2$ & $<2$ & 2.8 & $<1$ & $<1$ & $<2$ & $<2$ \\
\hline \multirow[t]{5}{*}{ Simazine } & $1 / 20$ & -(a) & -(a) & $<0.2$ & $<0.2$ & 0.97 & $<0.2$ & $<0.2$ & -(a) & $<0.2$ \\
\hline & $1 / 26$ & $<0.2$ & $<0.2$ & - (b) $^{(-1)}$ & -(b) $^{(\mathrm{s})}$ & - (b) $^{(-1)}$ & - (b) $^{(1)}$ & - (b) $^{\left(b^{\prime}\right.}$ & $<0.2$ & - (b) $^{(1)}$ \\
\hline & $2 / 8$ & 0.39 & 0.2 & $<0.2$ & 0.39 & 1.3 & $<0.2$ & $<0.2$ & $<0.2$ & $<0.2$ \\
\hline & $4 / 8$ & 0.21 & $<0.2$ & $<0.2$ & 0.77 & 1.1 & $<0.2$ & $<0.2$ & $<0.2$ & $<0.2$ \\
\hline & $11 / 8$ & -(a) & $<0.2$ & $<0.3$ & $<2$ & 0.34 & $<0.3$ & $<2$ & $<2$ & $<2$ \\
\hline
\end{tabular}


Table 7-6. Nonradioactive constituents (other than metals) detected in storm water runoff, Livermore site, 1999 (concluded).

\begin{tabular}{|c|c|c|c|c|c|c|c|c|c|c|}
\hline \multirow{3}{*}{ Parameter } & \multirow{3}{*}{$\begin{array}{l}\text { Storm } \\
\text { Date }\end{array}$} & \multicolumn{2}{|c|}{ Arroyo Seco } & \multicolumn{4}{|c|}{ Arroyo Las Positas } & \multicolumn{3}{|c|}{ Drainage Retention Basin } \\
\hline & & \multirow{2}{*}{\begin{tabular}{|c|}
$\begin{array}{c}\text { Site } \\
\text { influent }\end{array}$ \\
ASS2 \\
\end{tabular}} & \multirow{2}{*}{$\begin{array}{c}\begin{array}{c}\text { Site } \\
\text { effluent }\end{array} \\
\text { ASW } \\
\end{array}$} & \multicolumn{3}{|c|}{$\begin{array}{c}\text { Site } \\
\text { influent }\end{array}$} & \multirow{2}{*}{\begin{tabular}{|c|}
$\begin{array}{c}\text { Site } \\
\text { effluent }\end{array}$ \\
WPDC \\
\end{tabular}} & \multicolumn{2}{|c|}{$\begin{array}{c}\text { Site } \\
\text { influent }\end{array}$} & \multirow{2}{*}{$\begin{array}{c}\begin{array}{c}\text { Site } \\
\text { effluent }\end{array} \\
\text { CDBX }\end{array}$} \\
\hline & & & & ALPE & ALPO & GRNE & & CDB & CDB2 & \\
\hline \multicolumn{11}{|c|}{$\begin{array}{l}\text { Semivolatile organic compounds } \\
(\mu \mathrm{g} / \mathrm{L})\end{array}$} \\
\hline Anthracene & $11 / 8$ & -(a) & 0.11 & $<0.2$ & $<1$ & $<0.1$ & $<0.1$ & $<1$ & $<1$ & $<1$ \\
\hline Benzo(a)pyrene & $11 / 8$ & -(a) & $<0.1$ & $<0.2$ & $<1$ & 0.23 & 0.24 & $<1$ & $<1$ & $<1$ \\
\hline Benzo $(b)$ fluoranthene & $11 / 8$ & $-(a)$ & $<0.3$ & $<0.5$ & $<3$ & $<0.4$ & 0.44 & $<3$ & $<3$ & $<3$ \\
\hline Butylbenzylphthalate & $11 / 8$ & -(a) & $<1$ & 4.2 & $<10$ & 3.1 & $<1$ & $<10$ & $<10$ & $<10$ \\
\hline Di (2-ethylhexyl) adipate & $11 / 8$ & -(a) & $<1$ & 2.7 & $<10$ & 1.9 & $<1$ & $<10$ & $<10$ & $<10$ \\
\hline \multirow[t]{2}{*}{ Diazinon } & $4 / 8$ & $<0.2$ & $<0.2$ & $<0.2$ & $<0.2$ & $<0.2$ & $<0.2$ & $<0.2$ & $<0.2$ & $<0.2$ \\
\hline & $11 / 8$ & -(a) & 0.26 & $<0.3$ & $<2$ & 4.8 & $<0.3$ & $<2$ & $<2$ & $<2$ \\
\hline Dibutylphthalate & $11 / 8$ & -(a) & $<1$ & $<0.2$ & $<10$ & $<1$ & $<1$ & $<10$ & $<10$ & $<10$ \\
\hline Diethylhexylphthalate & $11 / 8$ & -(a) & $<3$ & 9.6 & $<30$ & 5.6 & 5.4 & $<30$ & $<30$ & $<30$ \\
\hline \multicolumn{11}{|c|}{ Polychlorinated biphenyls ( $\mu \mathrm{g} / \mathrm{L}$ ) } \\
\hline \multirow[t]{3}{*}{ РCB 1260} & $1 / 20$ & -(b) & 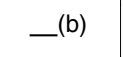 & 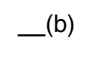 & 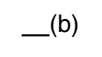 & -(b) & $<0.2$ & -(b) & -(a) & $<0.2$ \\
\hline & $1 / 26$ & -(b) & -(b) $^{(\mathrm{c}}$ & —(b) $^{(\mathrm{n}}$ & -(b) $^{(\mathrm{c}}$ & -(b) & -(b) & -(b) & $<0.3$ & -(b) \\
\hline & $2 / 8$ & -(b) $^{(\mathrm{n}}$ & 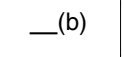 & -(b) $^{(\mathrm{n}}$ & 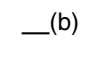 & -(b) & $<0.2$ & 0.24 & $<0.2$ & $<0.2$ \\
\hline \multirow[t]{3}{*}{ Total PCBs } & $1 / 20$ & -(b) $^{(\mathrm{c}}$ & -(b) $^{(\mathrm{c}}$ & -(b) & -(b) $^{(\mathrm{c}}$ & -(b) & $<0.2$ & -(b) & -(b) $^{(\mathrm{n})}$ & $<0.2$ \\
\hline & $1 / 26$ & - (b) $^{(-1}$ & 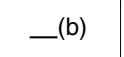 & —(b) & - (b) $^{(-1}$ & -(b) & -(b) & -(b) & $<0.3$ & -(b) \\
\hline & $2 / 8$ & - (b) $^{(\mathrm{n}}$ & -(b) & 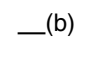 & - (b) $^{(\mathrm{n}}$ & -(b) & $<0.2$ & 0.24 & $<0.2$ & $<0.2$ \\
\hline \multicolumn{11}{|c|}{ Miscellaneous organics (mg/L) } \\
\hline \multirow[t]{5}{*}{ Oil and grease } & $1 / 20$ & -(a) & -(a) & 1.1 & $<1$ & $<1$ & $<1$ & 1.1 & - (a) $^{(\mathrm{n}}$ & 1.1 \\
\hline & $1 / 26$ & $<1$ & 2.2 & 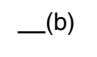 & - (b) $^{(-1}$ & -(b) & -(b) & - (b) $^{(\text {n }}$ & 3.4 & -(b) \\
\hline & $2 / 8$ & 2.7 & $<1$ & 1.2 & 1.1 & $<1$ & $<1$ & 2.1 & 1.6 & $<1$ \\
\hline & $4 / 8$ & 2.1 & $<1$ & 2.4 & 2.2 & 1.1 & $<1$ & $<1$ & 1.6 & $<1$ \\
\hline & $11 / 8$ & -(a) & 1.4 & 110 & 1.9 & 1.9 & $<1$ & $<1$ & 1.2 & 1.9 \\
\hline \multirow[t]{5}{*}{ Total organic carbon (TOC) } & $1 / 20$ & - (a) $^{(a)}$ & -(a) $^{(\mathrm{n}}$ & 60 & 14 & 4.7 & 8.5 & 34 & -(a) & 5.2 \\
\hline & $1 / 26$ & 4.6 & 6.4 & 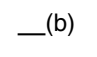 & $-^{(b)}$ & -(b) & -(b) & -(b) & 12 & -(b) \\
\hline & $2 / 8$ & 6 & 6.1 & 28 & 9.1 & 4.1 & 4.5 & 10 & 20 & 4.4 \\
\hline & $4 / 8$ & 6.2 & 6.7 & 20 & 3.5 & 4 & 6.2 & 8.4 & 12 & 3.5 \\
\hline & $11 / 8$ & -(a) & 20 & 24 & 11 & 13 & 17 & 22 & 31 & 8.8 \\
\hline
\end{tabular}

a Sample not collected because there was no flow.

b Not sampled (because, in most cases, locations sampled on 1/20 were not sampled again on 1/26). 


\section{Surface Water}

Table 7-7. Number of nondetects in storm water runoff, Livermore site, 1999.

\begin{tabular}{|c|c|c|c|}
\hline Parameter & $\begin{array}{c}\text { Number of } \\
\text { nondetects } \\
\text { (total analyses) }\end{array}$ & $\begin{array}{c}\text { Number of } \\
\text { samples } \\
\text { taken }\end{array}$ & $\begin{array}{c}\text { Highest } \\
\text { reported } \\
\text { detection limit }\end{array}$ \\
\hline \multicolumn{4}{|l|}{ Anions (mg/L) } \\
\hline Bromide & 15 & 34 & $<0.1$ \\
\hline Fluoride & 12 & 96 & $<0.05$ \\
\hline Nitrate (as N) & 1 & 96 & $<0.1$ \\
\hline Nitrate $\left(\right.$ as $\left.\mathrm{NO}_{3}\right)$ & 1 & 96 & $<0.4$ \\
\hline Nitrite (as N) & 37 & 96 & $<0.02$ \\
\hline Nitrite (as $\mathrm{NO}_{2}$ ) & 17 & 35 & $<0.07$ \\
\hline Sulfate & 2 & 97 & $<1$ \\
\hline \multicolumn{4}{|l|}{ Wet Chemistry } \\
\hline Carbonate alkalinity (as $\mathrm{CaCO}_{3}$ ) & 78 & 88 & $<5$ \\
\hline Chemical oxygen demand & 3 & 34 & $<20$ \\
\hline Hydroxide alkalinity (as $\mathrm{CaCO}_{3}$ ) & 61 & 61 & $<5$ \\
\hline TOX & 11 & 11 & $<20$ \\
\hline \multicolumn{4}{|l|}{ Herbicides ( $\mu \mathrm{g} / \mathrm{L})$} \\
\hline Atrazine & 33 & 34 & $<2$ \\
\hline Bromacil & 18 & 34 & $<5$ \\
\hline Butachlor & 26 & 26 & $<0.3$ \\
\hline Diazinon & 32 & 34 & $<2$ \\
\hline Dimethoate & 34 & 34 & $<20$ \\
\hline Diuron & 18 & 34 & $<2$ \\
\hline Glyphosate & 18 & 34 & $<20$ \\
\hline Metolachlor & 34 & 34 & $<5$ \\
\hline Metribuzin & 34 & 34 & $<5$ \\
\hline Molinate & 34 & 34 & $<5$ \\
\hline Prometryn & 35 & 35 & $<5$ \\
\hline Propachlor & 27 & 27 & $<0.5$ \\
\hline Simazine & 26 & 35 & $<2$ \\
\hline Thiobencarb & 35 & 35 & $<5$ \\
\hline \multicolumn{4}{|l|}{ Volatile organic compounds ( $\mu \mathrm{g} / \mathrm{L})$} \\
\hline 1,1,1-Trichloroethane & 9 & 9 & $<0.5$ \\
\hline 1,1,2,2-Tetrachloroethane & 9 & 9 & $<0.5$ \\
\hline 1,1,2-Trichloroethane & 9 & 9 & $<0.5$ \\
\hline 1,1-Dichloroethane & 9 & 9 & $<0.5$ \\
\hline 1,2-Dichlorobenzene & 11 & 11 & $<3$ \\
\hline 1,2-Dichloroethane & 9 & 9 & $<0.5$ \\
\hline
\end{tabular}




\section{Surface Water}

Table 7-7. Number of nondetects in storm water runoff, Livermore site, 1999 (continued).

\begin{tabular}{|c|c|c|c|}
\hline Parameter & $\begin{array}{c}\text { Number of } \\
\text { nondetects } \\
\text { (total analyses) }\end{array}$ & $\begin{array}{c}\text { Number of } \\
\text { samples } \\
\text { taken }\end{array}$ & $\begin{array}{c}\text { Highest } \\
\text { reported } \\
\text { detection limit }\end{array}$ \\
\hline \multicolumn{4}{|c|}{$\begin{array}{l}\text { Volatile organic compounds ( } \mu \mathrm{g} / \mathrm{L}) \\
\text { (continued) }\end{array}$} \\
\hline 1,2-Dichloroethene (total) & 9 & 9 & $<1$ \\
\hline 1,2-Dichloropropane & 9 & 9 & $<0.5$ \\
\hline 1,3-Dichlorobenzene & 11 & 11 & $<3$ \\
\hline 1,4-Dichlorobenzene & 11 & 11 & $<3$ \\
\hline 2-Butanone & 2 & 2 & $<20$ \\
\hline 2-Chloroethylvinylether & 2 & 2 & $<5$ \\
\hline 2-Hexanone & 2 & 2 & $<20$ \\
\hline 4-Methyl-2-pentanone & 2 & 2 & $<20$ \\
\hline Acenaphthylene & 10 & 10 & $<3$ \\
\hline Acetone & 2 & 2 & $<20$ \\
\hline Alachlor & 8 & 8 & $<2$ \\
\hline Aldrin & 13 & 13 & $<5$ \\
\hline Anthracene & 9 & 10 & $<3$ \\
\hline Atraton & 8 & 8 & $<5$ \\
\hline Benzene & 2 & 2 & $<0.5$ \\
\hline Benzo(a)anthracene & 10 & 10 & $<3$ \\
\hline Benzo(a)pyrene & 6 & 8 & $<1$ \\
\hline Benzo(b)fluoranthene & 7 & 8 & $<3$ \\
\hline Benzo(g,h,i)perylene & 10 & 10 & $<3$ \\
\hline Benzo(k)fluoranthene & 10 & 10 & $<3$ \\
\hline Bromodichloromethane & 9 & 9 & $<0.5$ \\
\hline Bromoform & 9 & 9 & $<0.5$ \\
\hline Bromomethane & 9 & 9 & $<0.5$ \\
\hline Butylbenzylphthalate & 8 & 10 & $<10$ \\
\hline Carbon disulfide & 2 & 2 & $<5$ \\
\hline Carbon tetrachloride & 9 & 9 & $<0.5$ \\
\hline Chlordane & 11 & 11 & $<20$ \\
\hline Chlorobenzene & 9 & 9 & $<0.5$ \\
\hline Chloroethane & 9 & 9 & $<1$ \\
\hline Chloroform & 9 & 9 & $<0.5$ \\
\hline Chloromethane & 9 & 9 & $<1$ \\
\hline Chrysene & 10 & 10 & $<3$ \\
\hline
\end{tabular}




\section{Surface Water}

Table 7-7. Number of nondetects in storm water runoff, Livermore site, 1999 (continued).

\begin{tabular}{|c|c|c|c|}
\hline Parameter & $\begin{array}{c}\text { Number of } \\
\text { nondetects } \\
\text { (total analyses) }\end{array}$ & $\begin{array}{c}\text { Number of } \\
\text { samples } \\
\text { taken }\end{array}$ & $\begin{array}{c}\text { Highest } \\
\text { reported } \\
\text { detection limit }\end{array}$ \\
\hline \multicolumn{4}{|c|}{$\begin{array}{l}\text { Volatile organic compounds ( } \mu \mathrm{g} / \mathrm{L}) \\
\text { (continued) }\end{array}$} \\
\hline cis-1,2-Dichloroethene & 9 & 9 & $<0.5$ \\
\hline cis-1,3-Dichloropropene & 9 & 9 & $<0.5$ \\
\hline Di(2-ethylhexyl) adipate & 6 & 8 & $<10$ \\
\hline Dibenzo(a,h)anthracene & 10 & 10 & $<4$ \\
\hline Dibromochloromethane & 9 & 9 & $<0.5$ \\
\hline Dibromomethane & 1 & 1 & $<0.5$ \\
\hline Dibutylphthalate & 10 & 10 & $<10$ \\
\hline Dichlorodifluoromethane & 9 & 9 & $<0.5$ \\
\hline Diethylhexylphthalate & 5 & 8 & $<30$ \\
\hline Diethylphthalate & 10 & 10 & $<30$ \\
\hline Dimethylphthalate & 10 & 10 & $<10$ \\
\hline Endrin & 13 & 13 & $<3$ \\
\hline Ethanol & 1 & 1 & $<1000$ \\
\hline Ethylbenzene & 2 & 2 & $<0.5$ \\
\hline Fluorene & 10 & 10 & $<3$ \\
\hline Freon 113 & 8 & 9 & $<0.5$ \\
\hline Heptachlor & 13 & 13 & $<3$ \\
\hline Heptachlor epoxide & 13 & 13 & $<3$ \\
\hline Hexachlorobenzene & 10 & 10 & $<5$ \\
\hline Hexachlorocyclopentadiene & 10 & 10 & $<10$ \\
\hline Indeno(1,2,3-c,d)pyrene & 10 & 10 & $<3$ \\
\hline Methoxychlor & 11 & 11 & $<5$ \\
\hline Methylene chloride & 8 & 9 & $<1$ \\
\hline Naphthalene & 3 & 3 & $<3$ \\
\hline Pentachlorophenol & 10 & 10 & $<20$ \\
\hline Phenanthrene & 10 & 10 & $<3$ \\
\hline Prometon & 8 & 8 & $<5$ \\
\hline Pyrene & 10 & 10 & $<3$ \\
\hline Secbumeton & 8 & 8 & $<5$ \\
\hline Styrene & 2 & 2 & $<0.5$ \\
\hline Terbutryn & 8 & 8 & $<5$ \\
\hline Tetrachloroethene & 9 & 9 & $<0.5$ \\
\hline
\end{tabular}




\section{Surface Water}

Table 7-7. Number of nondetects in storm water runoff, Livermore site, 1999 (continued).

\begin{tabular}{|c|c|c|c|}
\hline Parameter & $\begin{array}{c}\text { Number of } \\
\text { nondetects } \\
\text { (total analyses) }\end{array}$ & $\begin{array}{l}\text { Number of } \\
\text { samples } \\
\text { taken }\end{array}$ & $\begin{array}{c}\text { Highest } \\
\text { reported } \\
\text { detection limit }\end{array}$ \\
\hline \multicolumn{4}{|c|}{$\begin{array}{l}\text { Volatile organic compounds ( } \mu \mathrm{g} / \mathrm{L}) \\
\text { (continued) }\end{array}$} \\
\hline Toluene & 2 & 2 & $<0.5$ \\
\hline Total Trihalomethanes & 7 & 7 & $<2$ \\
\hline Total xylene isomers & 2 & 2 & $<1$ \\
\hline trans-1,2-Dichloroethene & 9 & 9 & $<0.5$ \\
\hline trans-1,3-Dichloropropene & 10 & 10 & $<0.5$ \\
\hline Trichloroethene & 9 & 9 & $<0.5$ \\
\hline Trichlorofluoromethane & 10 & 10 & $<0.5$ \\
\hline Vinyl chloride & 9 & 9 & $<0.5$ \\
\hline \multicolumn{4}{|l|}{ Pesticides ( $\mu \mathrm{g} / \mathrm{L}$ ) } \\
\hline Dieldrin & 5 & 5 & $<4$ \\
\hline Endosulfan I & 5 & 5 & $<20$ \\
\hline Endosulfan II & 5 & 5 & $<20$ \\
\hline Endosulfan sulfate & 5 & 5 & $<4$ \\
\hline Endrin aldehyde & 5 & 5 & $<3$ \\
\hline$p, p^{\prime}-D D D$ & 5 & 5 & $<3$ \\
\hline$p, p^{\prime}-D D E$ & 5 & 5 & $<4$ \\
\hline p,p'-DDT & 5 & 5 & $<3$ \\
\hline PCB 1016 & 17 & 17 & $<0.3$ \\
\hline PCB 1221 & 17 & 17 & $<0.3$ \\
\hline PCB 1232 & 17 & 17 & $<0.3$ \\
\hline PCB 1242 & 17 & 17 & $<0.3$ \\
\hline PCB 1248 & 17 & 17 & $<0.3$ \\
\hline PCB 1260 & 16 & 17 & $<0.2$ \\
\hline Total PCBs & 9 & 10 & $<0.3$ \\
\hline Toxaphene & 3 & 3 & $<0.3$ \\
\hline \multicolumn{4}{|c|}{ Semivolatile organic compounds ( $\mu \mathrm{g} / \mathrm{L}$ ) } \\
\hline 1,2,3,7,8-PeCDD & 1 & 1 & $<3.2$ \\
\hline 1,2,4-Trichlorobenzene & 2 & 2 & $<3$ \\
\hline 1,2-Diphenylhydrazine & 2 & 2 & $<3$ \\
\hline 2,3,7,8-TCDD & 1 & 1 & $<1.6$ \\
\hline 2,4,5-Trichlorophenol & 2 & 2 & $<7$ \\
\hline 2,4,6-Trichlorophenol & 2 & 2 & $<7$ \\
\hline
\end{tabular}




\section{Surface Water}

Table 7-7. Number of nondetects in storm water runoff, Livermore site, 1999 (continued).

\begin{tabular}{|c|c|c|c|}
\hline Parameter & $\begin{array}{c}\text { Number of } \\
\text { nondetects } \\
\text { (total analyses) }\end{array}$ & $\begin{array}{c}\text { Number of } \\
\text { samples } \\
\text { taken }\end{array}$ & $\begin{array}{c}\text { Highest } \\
\text { reported } \\
\text { detection limit }\end{array}$ \\
\hline \multicolumn{4}{|c|}{$\begin{array}{l}\text { Semiolatile organic compounds ( } \mu \mathrm{g} / \mathrm{L}) \\
\text { (continued) }\end{array}$} \\
\hline 2,4-Dichlorophenol & 2 & 2 & $<3$ \\
\hline 2,4-Dimethylphenol & 2 & 2 & $<3$ \\
\hline 2,4-Dinitrophenol & 2 & 2 & $<20$ \\
\hline 2,4-Dinitrotoluene & 2 & 2 & $<3$ \\
\hline 2,6-Dinitrotoluene & 2 & 2 & $<3$ \\
\hline 2-Chloronaphthalene & 2 & 2 & $<3$ \\
\hline 2-Chlorophenol & 2 & 2 & $<3$ \\
\hline 2-Methyl-4,6-dinitrophenol & 2 & 2 & $<20$ \\
\hline 2-Methylnaphthalene & 2 & 2 & $<3$ \\
\hline 2-Naphthylamine & 2 & 2 & $<30$ \\
\hline 2-Nitroaniline & 2 & 2 & $<3$ \\
\hline 2-Nitrophenol & 2 & 2 & $<3$ \\
\hline 3,3-Dichlorobenzidine & 2 & 2 & $<7$ \\
\hline 3-Nitroaniline & 2 & 2 & $<3$ \\
\hline 4-Bromophenylphenylether & 2 & 2 & $<3$ \\
\hline 4-Chloro-3-methylphenol & 2 & 2 & $<7$ \\
\hline 4-Chloroaniline & 2 & 2 & $<3$ \\
\hline 4-Chlorophenylphenylether & 2 & 2 & $<3$ \\
\hline 4-Nitroaniline & 2 & 2 & $<7$ \\
\hline 4-Nitrophenol & 2 & 2 & $<7$ \\
\hline Acenaphthene & 2 & 2 & $<3$ \\
\hline Aniline & 2 & 2 & $<7$ \\
\hline Benzidine & 2 & 2 & $<30$ \\
\hline Benzo(a)pyrene & 2 & 2 & $<3$ \\
\hline Benzo(b)fluoranthene & 2 & 2 & $<3$ \\
\hline Benzoic Acid & 2 & 2 & $<20$ \\
\hline Benzyl Alcohol & 2 & 2 & $<3$ \\
\hline Bis(2-chloroethoxy)methane & 2 & 2 & $<3$ \\
\hline Bis(2-chloroethyl)ether & 2 & 2 & $<3$ \\
\hline Bis(2-chloroisopropyl)ether & 2 & 2 & $<3$ \\
\hline Bis(2-ethylhexyl)phthalate & 2 & 2 & $<7$ \\
\hline Di-n-octylphthalate & 2 & 2 & $<3$ \\
\hline Dibenzofuran & 2 & 2 & $<3$ \\
\hline Fluoranthene & 2 & 2 & $<3$ \\
\hline Hexachlorobutadiene & 2 & 2 & $<3$ \\
\hline Hexachloroethane & 2 & 2 & $<3$ \\
\hline
\end{tabular}




\section{Surface Water}

Table 7-7. Number of nondetects in storm water runoff, Livermore site, 1999 (concluded).

\begin{tabular}{|c|c|c|c|}
\hline Parameter & $\begin{array}{c}\text { Number of } \\
\text { nondetects } \\
\text { (total analyses) }\end{array}$ & $\begin{array}{c}\text { Number of } \\
\text { samples } \\
\text { taken }\end{array}$ & $\begin{array}{c}\text { Highest } \\
\text { reported } \\
\text { detection limit }\end{array}$ \\
\hline \multicolumn{4}{|l|}{$\begin{array}{l}\text { Semiolatile organic compounds ( } \mu \mathrm{g} / \mathrm{L}) \\
\text { (continued) }\end{array}$} \\
\hline Isophorone & 2 & 2 & $<3$ \\
\hline N-Nitrosodi-n-propylamine & 2 & 2 & $<3$ \\
\hline N-Nitrosodimethylamine & 2 & 2 & $<3$ \\
\hline N-Nitrosodiphenylamine & 2 & 2 & $<3$ \\
\hline Nitrobenzene & 2 & 2 & $<3$ \\
\hline o-Cresol & 2 & 2 & $<3$ \\
\hline Oil and Grease & 14 & 35 & $<1$ \\
\hline p-Cresol & 2 & 2 & $<3$ \\
\hline Pentachlorinated dibenzo-p-dioxins & 1 & 1 & $<3.2$ \\
\hline Phenol & 2 & 2 & $<3$ \\
\hline Tetrachlorinated dibenzo-p-dioxins & 1 & 1 & $<1.6$ \\
\hline \multicolumn{4}{|l|}{ Metals (mg/L) } \\
\hline Aluminum & 14 & 126 & $<0.05$ \\
\hline Antimony & 63 & 63 & $<0.004$ \\
\hline Arsenic & 33 & 63 & $<0.002$ \\
\hline Barium & 1 & 63 & $<0.025$ \\
\hline Beryllium & 58 & 65 & $<0.0002$ \\
\hline Boron & 6 & 63 & $<0.05$ \\
\hline Cadmium & 62 & 63 & $<0.0005$ \\
\hline Chromium & 16 & 63 & $<0.001$ \\
\hline Cobalt & 63 & 63 & $<0.05$ \\
\hline Copper & 35 & 125 & $<0.01$ \\
\hline Chromium(VI) & 24 & 36 & $<0.02$ \\
\hline Iron & 10 & 125 & $<0.05$ \\
\hline Lead & 10 & 63 & $<0.005$ \\
\hline Manganese & 41 & 125 & $<0.01$ \\
\hline Mercury & 64 & 65 & $<0.0002$ \\
\hline Molybdenum & 62 & 63 & $<0.025$ \\
\hline Nickel & 70 & 125 & $<0.05$ \\
\hline Potassium & 1 & 64 & $<1$ \\
\hline Selenium & 64 & 64 & $<0.002$ \\
\hline Silver & 64 & 64 & $<0.001$ \\
\hline Surfactants & 29 & 62 & $<0.1$ \\
\hline Thallium & 64 & 64 & $<0.001$ \\
\hline Vanadium & 40 & 64 & $<0.01$ \\
\hline Zinc & 18 & 126 & $<0.02$ \\
\hline
\end{tabular}




\section{Surface Water}

Table 7-8. Radioactivity in storm water runoff, Site 300, 1999.

\begin{tabular}{|c|c|c|c|c|}
\hline \multirow{2}{*}{$\begin{array}{l}\text { Parameter } \\
\text { (Bq/L) }\end{array}$} & \multirow{2}{*}{$\begin{array}{c}\text { Sampling } \\
\text { Date }\end{array}$} & \multirow{2}{*}{$\begin{array}{c}\text { Upstream location } \\
\text { CARW }\end{array}$} & \multicolumn{2}{|c|}{ Effluent locations } \\
\hline & & & N883 & NLIN \\
\hline \multirow[t]{2}{*}{ Gross alpha } & $2 / 9$ & $3.89 \times 10^{-1} \pm 2.15 \times 10^{-1}$ & $1.36 \times 10^{-2} \pm 2.22 \times 10^{-2}$ & $2.34 \times 10^{-1} \pm 5.55 \times 10^{-2}$ \\
\hline & $4 / 8$ & $3.67 \pm 1.12$ & $2.46 \times 10^{-2} \pm 2.36 \times 10^{-2}$ & -(a) \\
\hline \multirow[t]{2}{*}{ Gross beta } & $2 / 9$ & $6.25 \times 10^{-1} \pm 3.48 \times 10^{-1}$ & $4.63 \times 10^{-2} \pm 7.77 \times 10^{-2}$ & $4.77 \times 10^{-1} \pm 8.88 \times 10^{-2}$ \\
\hline & $4 / 8$ & $5.11 \pm 8.18 \times 10^{-1}$ & $1.52 \times 10^{-1} \pm 4.14 \times 10^{-2}$ & -(a) \\
\hline \multirow[t]{2}{*}{ Tritium } & $2 / 9$ & $-4.51 \pm 1.56$ & $-5.07 \pm 1.51$ & $-3.15 \pm 1.64$ \\
\hline & $4 / 8$ & $-7.70 \times 10^{-1} \pm 2.47 \times 10$ & $5.48 \times 10^{-1} \pm 2.55$ & -(a) \\
\hline \multirow[t]{2}{*}{ Uranium 234+233 } & $2 / 9$ & $3.26 \times 10^{-1} \pm 4.07 \times 10^{-2}$ & $6.66 \times 10^{-4} \pm 9.62 \times 10^{-4}$ & $6.33 \times 10^{-2} \pm 1.07 \times 10^{-2}$ \\
\hline & $4 / 8$ & $1.75 \times 10^{-1} \pm 3.02 \times 10^{-2}$ & $9.14 \times 10^{-4} \pm 1.20 \times 10^{-3}$ & -(a) \\
\hline \multirow[t]{2}{*}{ Uranium-235+236 } & $2 / 9$ & $1.81 \times 10^{-2} \pm 4.81 \times 10^{-3}$ & $1.18 \times 10^{-3} \pm 9.99 \times 10^{-4}$ & $2.96 \times 10^{-3} \pm 2.26 \times 10^{-3}$ \\
\hline & $4 / 8$ & $1.22 \times 10^{-2} \pm 4.00 \times 10^{-3}$ & $3.16 \times 10^{-4} \pm 8.66 \times 10^{-4}$ & -(a) \\
\hline \multirow[t]{2}{*}{ Uranium-238 } & $2 / 9$ & $3.11 \times 10^{-1} \pm 4.07 \times 10^{-2}$ & $2.22 \times 10^{-3} \pm 1.30 \times 10^{-3}$ & $9.77 \times 10^{-2} \pm 1.48 \times 10^{-2}$ \\
\hline & $4 / 8$ & $1.71 \times 10^{-1} \pm 2.95 \times 10^{-2}$ & $1.51 \times 10^{-3} \pm 1.34 \times 10^{-3}$ & -(a) \\
\hline
\end{tabular}

\begin{tabular}{|l|c|c|c|c|}
\hline \multicolumn{1}{|c}{$\begin{array}{c}\text { Parameter } \\
\mathbf{( B q} / \mathbf{L})\end{array}$} & Sampling & \multicolumn{2}{|c|}{ Effluent locations } & Downstream location \\
\cline { 3 - 5 } & Date & NPT6 & NPT7 & GEOCRK \\
\hline Gross alpha & $2 / 9$ & $1.55 \times 10^{-1} \pm 4.44 \times 10^{-2}$ & $8.10 \times 10^{-2} \pm 4.44 \times 10^{-2}$ & $3.70 \times 10^{-1} \pm 2.04 \times 10^{-1}$ \\
& $4 / 8$ & $1.45 \times 10^{-1} \pm 8.92 \times 10^{-2}$ & $1.30 \times 10^{-1} \pm 6.07 \times 10^{-2}$ & $4.85 \times 10^{-2} \pm 6.03 \times 10^{-2}$ \\
Gross beta & $2 / 9$ & $4.74 \times 10^{-1} \pm 8.14 \times 10^{-2}$ & $1.02 \times 10^{-1} \pm 8.51 \times 10^{-2}$ & $3.52 \times 10^{-1} \pm 2.59 \times 10^{-1}$ \\
& $4 / 8$ & $3.59 \times 10^{-1} \pm 6.70 \times 10^{-2}$ & $2.87 \times 10^{-1} \pm 5.74 \times 10^{-2}$ & $3.62 \times 10^{-1} \pm 1.11 \times 10^{-1}$ \\
Tritium & $2 / 9$ & $-5.55 \pm 1.49$ & $-4.63 \pm 1.56$ & $-3.92 \pm 1.60$ \\
Uranium 234+233 & $4 / 8$ & $1.58 \pm 2.56$ & $-2.86 \times 10^{-1} \pm 2.48$ & $6.73 \times 10^{-1} \pm 2.53$ \\
& $2 / 9$ & $1.81 \times 10^{-2} \pm 4.44 \times 10^{-3}$ & $3.07 \times 10^{-2} \pm 7.03 \times 10^{-3}$ & $8.21 \times 10^{-2} \pm 1.37 \times 10^{-2}$ \\
Uranium-235+236 & $4 / 8$ & $1.64 \times 10^{-2} \pm 4.55 \times 10^{-3}$ & $2.85 \times 10^{-3} \pm 1.44 \times 10^{-3}$ & $7.29 \times 10^{-2} \pm 1.30 \times 10^{-2}$ \\
& $2 / 9$ & $1.11 \times 10^{-3} \pm 1.18 \times 10^{-3}$ & $3.37 \times 10^{-3} \pm 2.44 \times 10^{-3}$ & $4.77 \times 10^{-3} \pm 2.63 \times 10^{-3}$ \\
Uranium-238 & $4 / 8$ & $9.36 \times 10^{-4} \pm 1.02 \times 10^{-3}$ & $4.96 \times 10^{-4} \pm 6.36 \times 10^{-4}$ & $3.38 \times 10^{-3} \pm 1.57 \times 10^{-3}$ \\
& $2 / 9$ & $1.74 \times 10^{-2} \pm 4.44 \times 10^{-3}$ & $1.89 \times 10^{-2} \pm 5.18 \times 10^{-3}$ & $6.99 \times 10^{-2} \pm 1.26 \times 10^{-2}$ \\
& $4 / 8$ & $1.64 \times 10^{-2} \pm 4.51 \times 10^{-3}$ & $2.97 \times 10^{-3} \pm 1.44 \times 10^{-3}$ & $6.03 \times 10^{-2} \pm 1.11 \times 10^{-2}$ \\
\hline
\end{tabular}

Note: Radioactivities are reported as the measured concentration and either an uncertainty ( $\pm 2 \sigma$ counting error) or as being less than the detection limit. If the concentration is less than or equal to the uncertainty or the detection limit, the result is considered to be a nondectection.

a Sample not collected because there was no flow. 


\section{Surface Water}

Table 7-9. Nonradioactive constituents in storm water runoff, Site 300, 1999.

\begin{tabular}{|c|c|c|c|c|c|c|c|}
\hline \multirow{2}{*}{ Parameter } & \multirow{2}{*}{$\begin{array}{c}\text { Sampling } \\
\text { Date }\end{array}$} & \multirow{2}{*}{\begin{tabular}{|c|}
$\begin{array}{l}\text { Upstream } \\
\text { location }\end{array}$ \\
CARW \\
\end{tabular}} & \multicolumn{4}{|c|}{ Effluent location } & \multirow{2}{*}{$\begin{array}{c}\begin{array}{c}\text { Downstream } \\
\text { location }\end{array} \\
\text { GEOCRK } \\
\end{array}$} \\
\hline & & & N883 & NLIN & NPT6 & NPT7 & \\
\hline \multicolumn{8}{|l|}{ General minerals } \\
\hline \multirow[t]{2}{*}{$\mathrm{pH}$ (pH units) } & $2 / 9$ & 7.88 & 6.04 & 7.55 & 7.19 & 8.03 & 8.28 \\
\hline & $4 / 8$ & 7.66 & 6.1 & 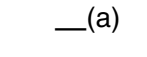 & 7.19 & 8.38 & 8.3 \\
\hline \multirow{2}{*}{$\begin{array}{l}\text { Specific conductance } \\
\quad(\mu \mathrm{mho} / \mathrm{cm})\end{array}$} & $2 / 9$ & 1,120 & 15 & 319 & 69 & 172 & 1,670 \\
\hline & $4 / 8$ & 1,280 & 35 & —(a) & 200 & 93 & 1,610 \\
\hline \multirow{2}{*}{$\begin{array}{l}\text { Total suspended solids } \\
\text { (TSS) (mg/L) }\end{array}$} & $2 / 9$ & 25,000 & 350 & 2,220 & 512 & 64 & 362 \\
\hline & $4 / 8$ & 1,230 & 58 & —(a) & 2,190 & 371 & 118 \\
\hline \multicolumn{8}{|l|}{ Miscellaneous organics } \\
\hline \multirow{2}{*}{$\begin{array}{l}\text { Total organic carbon (TOC) } \\
\quad(\mathrm{mg} / \mathrm{L})\end{array}$} & $2 / 9$ & 11 & 3.4 & 11 & 6.7 & 7.3 & 13 \\
\hline & $4 / 8$ & 7.5 & 13 & —(a) & 13 & 1.9 & 5.7 \\
\hline \multirow{2}{*}{$\begin{array}{l}\text { Total organic halides (TOX) } \\
\quad(\mu \mathrm{g} / \mathrm{L})\end{array}$} & $2 / 9$ & $<20$ & $<20$ & $<20$ & $<20$ & $<20$ & $<20$ \\
\hline & $4 / 8$ & $<20$ & $<20$ & 一 $^{(\mathrm{a})}$ & $<20$ & $<20$ & $<20$ \\
\hline \multicolumn{8}{|l|}{$\begin{array}{l}\text { Polychlorinated biphenyls } \\
(\mu \mathrm{g} / \mathrm{L})\end{array}$} \\
\hline РCB 1016 & $2 / 9$ & -(b) & -(b) & $<0.2$ & —(b) $^{(\mathrm{s})}$ & —(b) $^{(\mathrm{s}}$ & -(b) \\
\hline РCB 1221 & $2 / 9$ & -(b) & -(b) & $<0.2$ & —(b) & (b) $^{(b)}$ & -(b) \\
\hline РCB 1232 & $2 / 9$ & -(b) & -(b) & $<0.2$ & —(b) & (b) $^{(b)}$ & -(b) \\
\hline РCB 1242 & $2 / 9$ & -(b) & -(b) & $<0.2$ & —(b) $^{(\mathrm{s}}$ & —(b) $^{(\mathrm{s})}$ & -(b) \\
\hline РСB 1248 & $2 / 9$ & - (b) $^{\left({ }^{\prime}\right.}$ & -(b) & $<0.2$ & —(b) $^{(\mathrm{s})}$ & —(b) $^{(\mathrm{s})}$ & 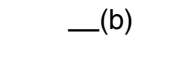 \\
\hline PCB 1254 & $2 / 9$ & -(b) & -(b) & $<0.2$ & —(b) $^{(\mathrm{s})}$ & (b) $^{(b)}$ & -(b) \\
\hline РCB 1260 & $2 / 9$ & -(b) & -(b) & $<0.2$ & —(b) $^{(\mathrm{s})}$ & —(b) $^{(\mathrm{s})}$ & -(b) \\
\hline Total PCBs & $2 / 9$ & -(b) & -(b) & $<0.2$ & —(b) $^{(\mathrm{s})}$ & —(b) & -(b) \\
\hline
\end{tabular}

a Not analyzed because there was no flow at this location.

b PCB analysis not conducted at this location. 


\section{Surface Water}

Table 7-10. Dioxins in storm water runoff, Site 300, 1999.

\begin{tabular}{|c|c|}
\hline Constituents (pg/L) & Sampled 2/9/99 \\
\hline Aldrin & $<0.005$ \\
\hline $\mathrm{BHC}$, alpha isomer & $<0.005$ \\
\hline $\mathrm{BHC}$, beta isomer & $<0.005$ \\
\hline $\mathrm{BHC}$, delta isomer & $<0.005$ \\
\hline $\mathrm{BHC}$, gamma isomer (Lindane) & $<0.005$ \\
\hline Chlordane & $<0.2$ \\
\hline Dieldrin & $<0.005$ \\
\hline Endosulfan I & $<0.005$ \\
\hline Endosulfan II & $<0.005$ \\
\hline Endosulfan sulfate & $<0.005$ \\
\hline Endrin & $<0.005$ \\
\hline Endrin aldehyde & $<0.01$ \\
\hline Heptachlor & $<0.005$ \\
\hline Heptachlor epoxide & $<0.005$ \\
\hline Methoxychlor & $<0.005$ \\
\hline$p, p^{\prime}-D D D$ & $<0.005$ \\
\hline$p, p^{\prime}-D D E$ & $<0.005$ \\
\hline $\mathrm{p}, \mathrm{p}^{\prime}-\mathrm{DDT}$ & $<0.005$ \\
\hline Toxaphene & $<0.2$ \\
\hline 1,2,3,4,6,7,8-HpCDD & 280 \\
\hline $1,2,3,4,6,7,8-\mathrm{HpCDF}$ & 59 \\
\hline $1,2,3,4,6,7,8-O C D D$ & 2800 \\
\hline $1,2,3,4,6,7,8-\mathrm{OCDF}$ & 250 \\
\hline $1,2,3,4,7,8,9-\mathrm{HpCDF}$ & 6.2 \\
\hline $1,2,3,4,7,8-\mathrm{HxCDD}$ & 7 \\
\hline $1,2,3,4,7,8-\mathrm{HxCDF}$ & 8.2 \\
\hline $1,2,3,6,7,8-\mathrm{HxCDD}$ & 17 \\
\hline $1,2,3,6,7,8-\mathrm{HxCDF}$ & 4.4 \\
\hline 1,2,3,7,8,9-HxCDD & 13 \\
\hline $1,2,3,7,8,9-\mathrm{H} \times \mathrm{CDF}$ & 6.8 \\
\hline 1,2,3,7,8-PeCDD & $<3.2$ \\
\hline 1,2,3,7,8-PeCDF & 4.4 \\
\hline $2,3,4,6,7,8-\mathrm{HxCDF}$ & 7.6 \\
\hline 2,3,4,7,8-PeCDF & 8.7 \\
\hline
\end{tabular}




\section{Surface Water}

Table 7-10. Dioxins in storm water runoff, Site 300, 1999 (concluded).

\begin{tabular}{|l|c|}
\hline \multicolumn{1}{|c|}{ Constituents (pg/L) } & Sampled 2/9/99 \\
\hline 2,3,7,8-TCDD & $<1.6$ \\
2,3,7,8-TCDF & 15 \\
Heptachlorinated dibenzo-furans & 200 \\
Heptachlorinated dibenzo-p-dioxins & 450 \\
Hexachlorinated dibenzo-furans & 81 \\
Hexachlorinated dibenzo-p-dioxins & 91 \\
Pentachlorinated dibenzo-furans & 65 \\
Pentachlorinated dibenzo-p-dioxins & $<3.2$ \\
Tetrachlorinated dibenzo-furans & 91 \\
Tetrachlorinated dibenzo-p-dioxins & $<1.6$ \\
\hline
\end{tabular}




\section{Surface Water}

Table 7-11. Pit 6 post-closure storm water monitoring, Site 300, 1999.

\begin{tabular}{|c|c|c|}
\hline Parameters & Sampled 2/9/99 & Sampled 4/8/99 \\
\hline \multicolumn{3}{|l|}{ Inorganics (mg/L) } \\
\hline Beryllium & 0.0011 & $<0.0002$ \\
\hline Mercury & $<0.0002$ & $<0.0002$ \\
\hline Potassium & -(a) $^{(a)}$ & 11 \\
\hline Total dissolved solids (TDS) & 177 & 185 \\
\hline \multicolumn{3}{|l|}{ Volatile organic compounds ( $\mu \mathrm{g} / \mathrm{L}$ ) } \\
\hline 1,1,1-Trichloroethane & $<0.5$ & $<0.5$ \\
\hline 1,1,2,2-Tetrachloroethane & $<0.5$ & $<0.5$ \\
\hline 1,1,2-Trichloroethane & $<0.5$ & $<0.5$ \\
\hline 1,1-Dichloroethane & $<0.5$ & $<0.5$ \\
\hline 1,1-Dichloroethene & $<0.5$ & $<0.5$ \\
\hline 1,2-Dichlorobenzene & $<0.5$ & $<0.5$ \\
\hline 1,2-Dichloroethane & $<0.5$ & $<0.5$ \\
\hline 1,2-Dichloroethene (total) & $<1$ & $<1$ \\
\hline 1,2-Dichloropropane & $<0.5$ & $<0.5$ \\
\hline 1,3-Dichlorobenzene & $<0.5$ & $<0.5$ \\
\hline 1,4-Dichlorobenzene & $<0.5$ & $<0.5$ \\
\hline 2-Butanone & $<20$ & $<20$ \\
\hline 2-Chloroethylvinylether & $<5$ & $<5$ \\
\hline 2-Hexanone & $<20$ & $<20$ \\
\hline 4-Methyl-2-pentanone & $<20$ & $<20$ \\
\hline Acetone & $<20$ & $<20$ \\
\hline Benzene & $<0.5$ & $<0.5$ \\
\hline Bromodichloromethane & $<0.5$ & $<0.5$ \\
\hline Bromoform & $<0.5$ & $<0.5$ \\
\hline Bromomethane & $<0.5$ & $<0.5$ \\
\hline Carbon disulfide & $<5$ & $<5$ \\
\hline Carbon tetrachloride & $<0.5$ & $<0.5$ \\
\hline Chlorobenzene & $<0.5$ & $<0.5$ \\
\hline Chloroethane & $<1$ & $<1$ \\
\hline Chloroform & $<0.5$ & $<0.5$ \\
\hline Chloromethane & $<1$ & $<1$ \\
\hline cis-1,2-Dichloroethene & $<0.5$ & $<0.5$ \\
\hline cis-1,3-Dichloropropene & $<0.5$ & $<0.5$ \\
\hline Dibromochloromethane & $<0.5$ & $<0.5$ \\
\hline Dibromomethane & $<0.5$ & $<0.5$ \\
\hline Dichlorodifluoromethane & $<0.5$ & $<0.5$ \\
\hline
\end{tabular}


Table 7-11. Pit 6 post-closure storm water monitoring, Site 300, 1999 (continued).

\begin{tabular}{|c|c|c|}
\hline Parameters & Sampled 2/9/99 & Sampled 4/8/99 \\
\hline \multicolumn{3}{|c|}{ Volatile organic compounds ( $\mu \mathrm{g} / \mathrm{L})$ (continued) } \\
\hline Ethanol & $<1000$ & $<1000$ \\
\hline Ethylbenzene & $<0.5$ & $<0.5$ \\
\hline Freon 113 & 0.63 & $<0.5$ \\
\hline Methylene chloride & $<1$ & 1.8 \\
\hline Naphthalene & $<0.5$ & $<0.5$ \\
\hline Styrene & $<0.5$ & $<0.5$ \\
\hline Tetrachloroethene & $<0.5$ & $<0.5$ \\
\hline Toluene & $<0.5$ & $<0.5$ \\
\hline Total xylene isomers & $<1$ & $<1$ \\
\hline trans-1,2-Dichloroethene & $<0.5$ & $<0.5$ \\
\hline trans-1,3-Dichloropropene & $<0.5$ & $<0.5$ \\
\hline Trichloroethene & $<0.5$ & $<0.5$ \\
\hline Trichlorofluoromethane & $<0.5$ & $<0.5$ \\
\hline Vinyl chloride & $<0.5$ & $<0.5$ \\
\hline \multicolumn{3}{|c|}{ Semivolatile organic compounds ( $\mu \mathrm{g} / \mathrm{L}$ ) } \\
\hline 1,2,4-Trichlorobenzene & $<3$ & $<3$ \\
\hline 1,2-Dichlorobenzene & $<3$ & $<3$ \\
\hline 1,2-Diphenylhydrazine & $<3$ & $<3$ \\
\hline 1,3-Dichlorobenzene & $<3$ & $<3$ \\
\hline 1,4-Dichlorobenzene & $<3$ & $<3$ \\
\hline 2,4,5-Trichlorophenol & $<6$ & $<7$ \\
\hline 2,4,6-Trichlorophenol & $<6$ & $<7$ \\
\hline 2,4-Dichlorophenol & $<3$ & $<3$ \\
\hline 2,4-Dimethylphenol & $<3$ & $<3$ \\
\hline 2,4-Dinitrophenol & $<20$ & $<20$ \\
\hline 2,4-Dinitrotoluene & $<3$ & $<3$ \\
\hline 2,6-Dinitrotoluene & $<3$ & $<3$ \\
\hline 2-Chloronaphthalene & $<3$ & $<3$ \\
\hline 2-Chlorophenol & $<3$ & $<3$ \\
\hline 2-Methyl-4,6-dinitrophenol & $<20$ & $<20$ \\
\hline 2-Methylnaphthalene & $<3$ & $<3$ \\
\hline 2-Naphthylamine & $<30$ & $<30$ \\
\hline 2-Nitroaniline & $<3$ & $<3$ \\
\hline 2-Nitrophenol & $<3$ & $<3$ \\
\hline 3,3-Dichlorobenzidine & $<6$ & $<7$ \\
\hline 3-Nitroaniline & $<3$ & $<3$ \\
\hline
\end{tabular}




\section{Surface Water}

Table 7-11. Pit 6 post-closure storm water monitoring, Site 300, 1999 (continued).

\begin{tabular}{|c|c|c|}
\hline Parameters & Sampled 2/9/99 & Sampled 4/8/99 \\
\hline \multicolumn{3}{|c|}{$\begin{array}{l}\text { Semivolatile organic compounds ( } \mu \mathrm{g} / \mathrm{L}) \\
\text { (continued) }\end{array}$} \\
\hline 4-Bromophenylphenylether & $<3$ & $<3$ \\
\hline 4-Chloro-3-methylphenol & $<6$ & $<7$ \\
\hline 4-Chloroaniline & $<3$ & $<3$ \\
\hline 4-Chlorophenylphenylether & $<3$ & $<3$ \\
\hline 4-Nitroaniline & $<6$ & $<7$ \\
\hline 4-Nitrophenol & $<6$ & $<7$ \\
\hline Acenaphthene & $<3$ & $<3$ \\
\hline Acenaphthylene & $<3$ & $<3$ \\
\hline Aldrin & $<3$ & $<3$ \\
\hline Aniline & $<6$ & $<7$ \\
\hline Anthracene & $<3$ & $<3$ \\
\hline Benzidine & $<30$ & $<30$ \\
\hline Benzo(a)anthracene & $<3$ & $<3$ \\
\hline Benzo(a)pyrene & $<3$ & $<3$ \\
\hline Benzo(b)fluoranthene & $<3$ & $<3$ \\
\hline Benzo(g,h,i)perylene & $<3$ & $<3$ \\
\hline Benzo(k)fluoranthene & $<3$ & $<3$ \\
\hline Benzoic Acid & $<20$ & $<20$ \\
\hline Benzyl Alcohol & $<3$ & $<3$ \\
\hline $\mathrm{BHC}$, alpha isomer & $<3$ & $<3$ \\
\hline $\mathrm{BHC}$, beta isomer & $<3$ & $<3$ \\
\hline $\mathrm{BHC}$, delta isomer & $<3$ & $<3$ \\
\hline $\mathrm{BHC}$, gamma isomer (Lindane) & $<3$ & $<3$ \\
\hline Bis(2-chloroethoxy)methane & $<3$ & $<3$ \\
\hline Bis(2-chloroethyl)ether & $<3$ & $<3$ \\
\hline Bis(2-chloroisopropyl)ether & $<3$ & $<3$ \\
\hline Bis(2-ethylhexyl)phthalate & $<6$ & $<7$ \\
\hline Butylbenzylphthalate & $<3$ & $<3$ \\
\hline Chrysene & $<3$ & $<3$ \\
\hline Di-n-octylphthalate & $<3$ & $<3$ \\
\hline Dibenzo(a,h)anthracene & $<4$ & $<4$ \\
\hline Dibenzofuran & $<3$ & $<3$ \\
\hline Dibutylphthalate & $<3$ & $<3$ \\
\hline Dieldrin & $<4$ & $<4$ \\
\hline Diethylphthalate & $<3$ & $<3$ \\
\hline Dimethylphthalate & $<3$ & $<3$ \\
\hline
\end{tabular}


Table 7-11. Pit 6 post-closure storm water monitoring, Site 300, 1999 (continued).

\begin{tabular}{|c|c|c|}
\hline Parameters & Sampled 2/9/99 & Sampled 4/8/99 \\
\hline \multicolumn{3}{|c|}{$\begin{array}{l}\text { Semivolatile organic compounds ( } \mu \mathrm{g} / \mathrm{L}) \\
\text { (continued) }\end{array}$} \\
\hline Endosulfan I & $<20$ & $<20$ \\
\hline Endosulfan II & $<20$ & $<20$ \\
\hline Endosulfan sulfate & $<4$ & $<4$ \\
\hline Endrin & $<3$ & $<3$ \\
\hline Endrin aldehyde & $<3$ & $<3$ \\
\hline Fluoranthene & $<3$ & $<3$ \\
\hline Fluorene & $<3$ & $<3$ \\
\hline Heptachlor & $<3$ & $<3$ \\
\hline Heptachlor epoxide & $<3$ & $<3$ \\
\hline Hexachlorobenzene & $<3$ & $<3$ \\
\hline Hexachlorobutadiene & $<3$ & $<3$ \\
\hline Hexachlorocyclopentadiene & $<3$ & $<3$ \\
\hline Hexachloroethane & $<3$ & $<3$ \\
\hline Indeno(1,2,3-c,d)pyrene & $<3$ & $<3$ \\
\hline Isophorone & $<3$ & $<3$ \\
\hline N-Nitrosodi-n-propylamine & $<3$ & $<3$ \\
\hline N-Nitrosodimethylamine & $<3$ & $<3$ \\
\hline N-Nitrosodiphenylamine & $<3$ & $<3$ \\
\hline Naphthalene & $<3$ & $<3$ \\
\hline Nitrobenzene & $<3$ & $<3$ \\
\hline o-Cresol & $<3$ & $<3$ \\
\hline$p, p^{\prime}-D D D$ & $<3$ & $<3$ \\
\hline p.p'-DDE & $<4$ & $<4$ \\
\hline p,p'-DDT & $<3$ & $<3$ \\
\hline p-Cresol & $<3$ & $<3$ \\
\hline Pentachlorophenol & $<20$ & $<20$ \\
\hline Phenanthrene & $<3$ & $<3$ \\
\hline Phenol & $<3$ & $<3$ \\
\hline Pyrene & $<3$ & $<3$ \\
\hline \multicolumn{3}{|l|}{ Pesticides ( $\mu \mathrm{g} / \mathrm{L})$} \\
\hline Aldrin & $<0.006$ & $<0.006$ \\
\hline $\mathrm{BHC}$, alpha isomer & $<0.006$ & $<0.006$ \\
\hline $\mathrm{BHC}$, beta isomer & $<0.006$ & $<0.006$ \\
\hline $\mathrm{BHC}$, delta isomer & $<0.006$ & $<0.006$ \\
\hline $\mathrm{BHC}$, gamma isomer (Lindane) & $<0.006$ & $<0.006$ \\
\hline Chlordane & $<0.3$ & $<0.3$ \\
\hline
\end{tabular}




\section{Surface Water}

Table 7-11. Pit 6 post-closure storm water monitoring, Site 300, 1999 (concluded).

\begin{tabular}{|c|c|c|}
\hline Parameters & Sampled 2/9/99 & Sampled 4/8/99 \\
\hline \multicolumn{3}{|l|}{ Pesticides ( $\mu \mathrm{g} / \mathrm{L})$ (continued) } \\
\hline Dieldrin & $<0.006$ & $<0.006$ \\
\hline Endosulfan I & $<0.006$ & $<0.006$ \\
\hline Endosulfan II & $<0.006$ & $<0.006$ \\
\hline Endosulfan sulfate & $<0.006$ & $<0.006$ \\
\hline Endrin & $<0.006$ & $<0.006$ \\
\hline Endrin aldehyde & $<0.02$ & $<0.02$ \\
\hline Heptachlor & $<0.006$ & $<0.006$ \\
\hline Heptachlor epoxide & $<0.006$ & $<0.006$ \\
\hline Methoxychlor & $<0.006$ & $<0.006$ \\
\hline$p, p^{\prime}-D D D$ & $<0.006$ & $<0.006$ \\
\hline$p, p^{\prime}-D D E$ & $<0.006$ & $<0.006$ \\
\hline$p, p^{\prime}-D D T$ & $<0.006$ & $<0.006$ \\
\hline PCB 1016 & $<0.3$ & $<0.3$ \\
\hline PCB 1221 & $<0.3$ & $<0.3$ \\
\hline PCB 1232 & $<0.3$ & $<0.3$ \\
\hline PCB 1242 & $<0.3$ & $<0.3$ \\
\hline PCB 1248 & $<0.3$ & $<0.3$ \\
\hline PCB 1254 & $<0.3$ & $<0.3$ \\
\hline РCB 1260 & $<0.3$ & $<0.3$ \\
\hline Total PCBs & $<0.3$ & $<0.3$ \\
\hline Toxaphene & $<0.006$ & $<0.3$ \\
\hline
\end{tabular}

a Analyses not requested. See the main volume, Table 14-4. 
Table 7-12. Tritium in rain (Bq/L), Livermore site and Livermore Valley, 1999.

\begin{tabular}{|l|c|c|c|c|}
\hline \multirow{2}{*}{ Location } & \multicolumn{4}{|c|}{ Sampling dates } \\
\cline { 2 - 5 } Livermore site & $\mathbf{1 / 2 1 / 9 9}$ & $\mathbf{2 / 1 0 / 9 9}$ & $\mathbf{3 / 2 3 / 9 9}$ & $\mathbf{4 / 9 / 9 9}$ \\
B343 & $220 \pm 7$ & $466 \pm 10$ & $540 \pm 11$ & $154 \pm 6$ \\
B291 & $1.88 \pm 2.40$ & $18.4 \pm 2.7$ & $25.6 \pm 3.0$ & $2.66 \pm 2.76$ \\
CDB & $88.1 \pm 4.7$ & $303 \pm 10$ & $228 \pm 7$ & $20.0 \pm 3.4$ \\
VIS & $13.3 \pm 2.8$ & $72.2 \pm 4.3$ & $55.9 \pm 4.0$ & $5.11 \pm 2.85$ \\
COW & $8.51 \pm 2.84$ & $65.1 \pm 4.2$ & $48.5 \pm 3.8$ & $3.12 \pm 2.77$ \\
SALV & $8.92 \pm 2.68$ & $18.7 \pm 2.7$ & $19.4 \pm 2.8$ & $0.66 \pm 2.68$ \\
MET & $-5.11 \pm 2.34$ & $4.74 \pm 2.16$ & $8.73 \pm 2.34$ & $0.43 \pm 2.68$ \\
\hline Livermore Valley & & & & \\
ESAN & $-2.19 \pm 2.26$ & $9.55 \pm 2.36$ & $11.3 \pm 2.5$ & $-0.15 \pm 2.67$ \\
ZON7 & $5.07 \pm 2.53$ & $60.7 \pm 7.8$ & $19.4 \pm 2.8$ & $2.49 \pm 2.77$ \\
AQUE & $-(a)$ & $-(a)$ & $16.7 \pm 2.7$ & $0.67 \pm 2.70$ \\
SLST & $-5.66 \pm 2.09$ & $2.56 \pm 2.05$ & $2.91 \pm 2.04$ & $0.01 \pm 2.66$ \\
GTES & $-5.29 \pm 2.16$ & $6.29 \pm 2.25$ & $6.55 \pm 2.24$ & $0.54 \pm 2.69$ \\
VINE & $-7.33 \pm 2.00$ & $0.95 \pm 1.96$ & $6.07 \pm 2.23$ & $-1.59 \pm 2.60$ \\
BVA & $-4.92 \pm 2.12$ & $8.84 \pm 2.34$ & $7.40 \pm 2.28$ & $-0.84 \pm 2.64$ \\
VET & $-6.99 \pm 2.01$ & $10.9 \pm 2.4$ & $8.18 \pm 2.34$ & $-1.91 \pm 2.59$ \\
\hline
\end{tabular}

Note: Radioactivities are reported as the measured concentration and either an uncertainty ( $\pm 2 \sigma$ counting error) or as being less than the detection limit. If the concentration is less than or equal to the uncertainty or the detection limit, the result is considered to be a nondetection.

a Sample not collected at this location. 


\section{Surface Water}

Table 7-13. Drainage Retention Basin discharge limits for CDBX, identified in CERCLA Record of Decision as amended, and sampling frequencies for CDBX and WPDC.

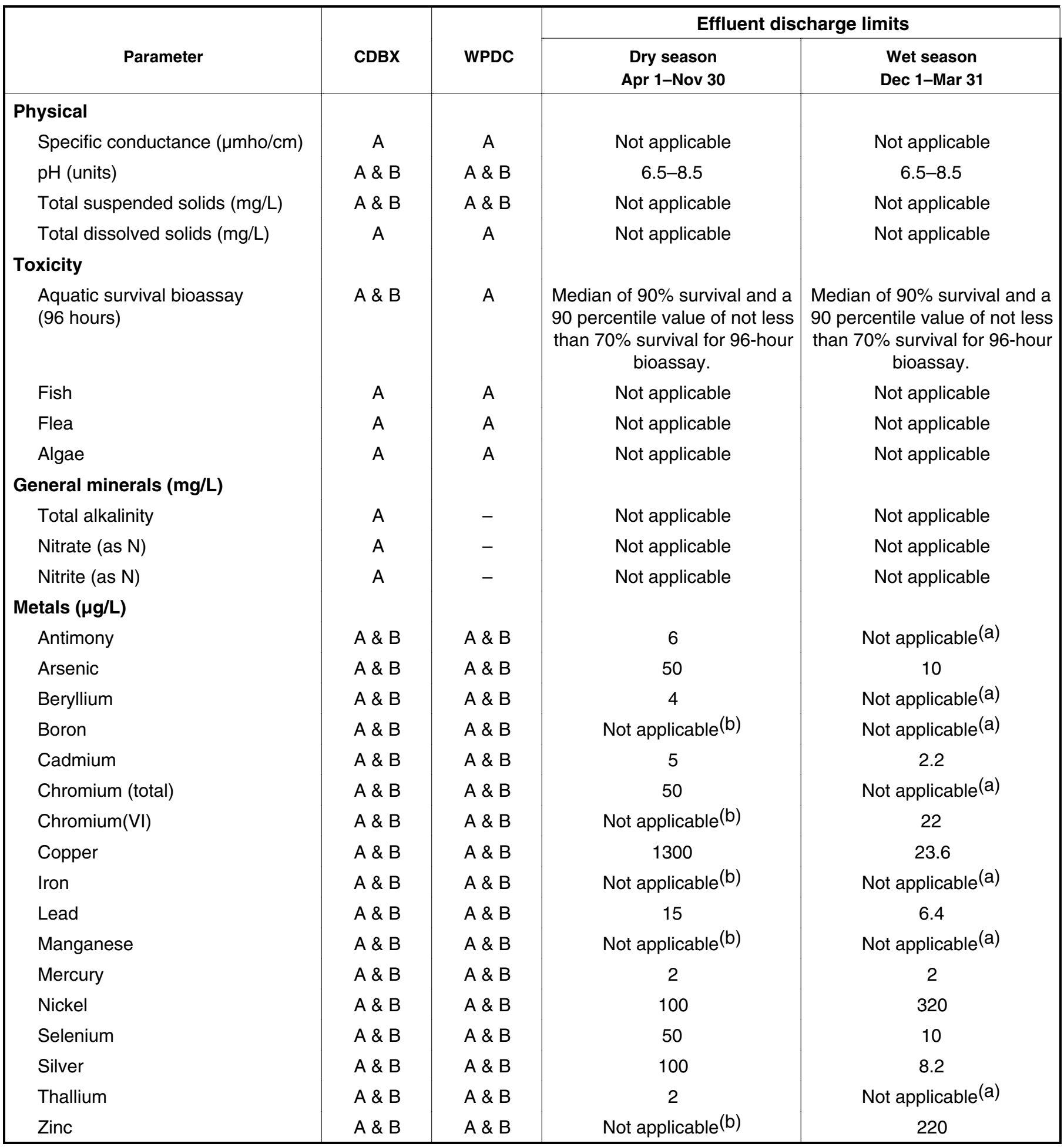


Table 7-13. Drainage Retention Basin discharge limits for CDBX, identified in CERCLA ROD as amended, and sampling frequencies for CDBX and WPDC (concluded).

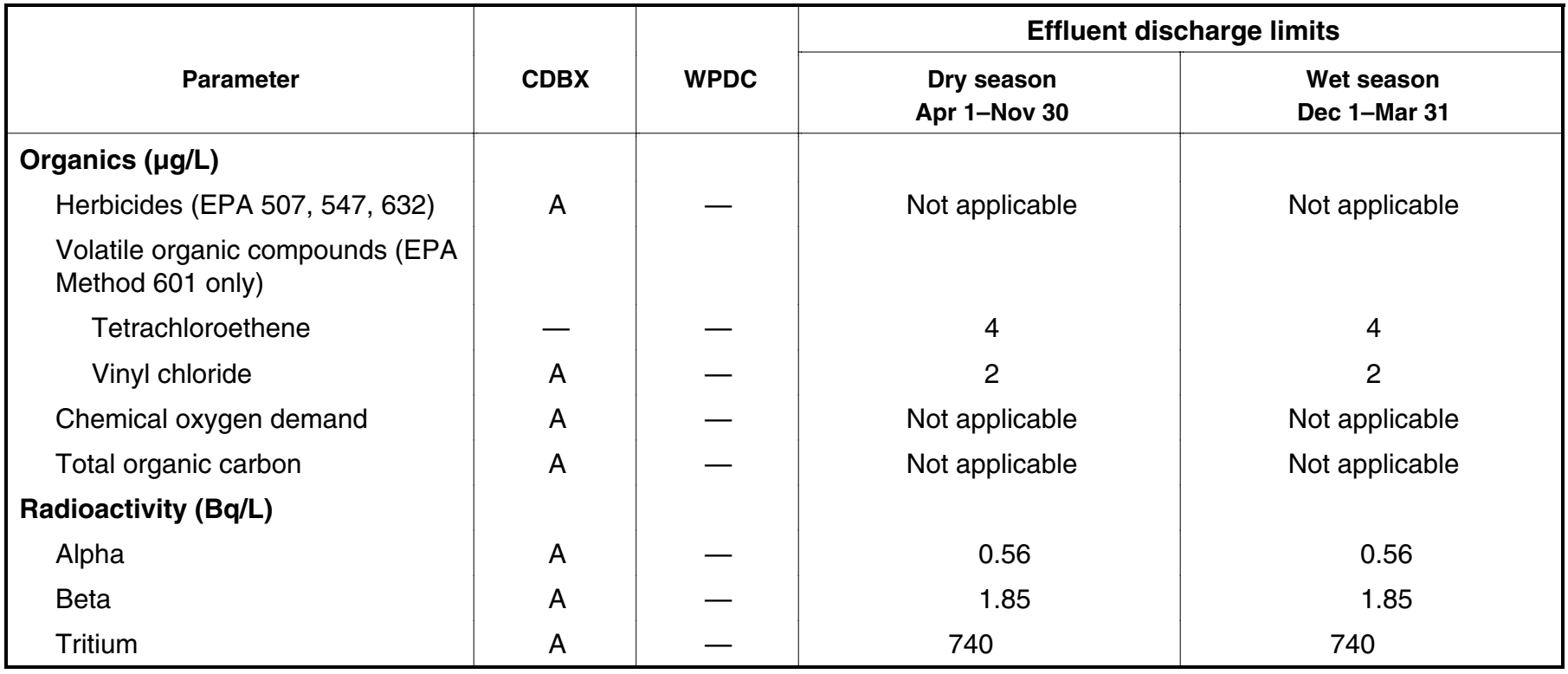

a No limit is established for aquatic life protection; however, aquatic life is protected by bioassay analysis.

b No MCL is established for this metal.

$A=$ Monitoring occurs at the first DRB discharge of the wet season and at one or more additional discharges associated with storm water runoff monitoring. Toxicity testing is required only on the first release.

$\mathrm{B}=$ Monitoring occurs at each dry season release. For purposes of discharge sampling, the dry season is defined to occur from June 1 through September 30. 


\section{Surface Water}

Table 7-14. Routine water quality management action levels and monitoring plan for the Drainage Retention Basin.

\begin{tabular}{|c|c|c|c|c|}
\hline \multirow[b]{2}{*}{ Constituent } & \multirow[b]{2}{*}{ Location } & \multirow{2}{*}{$\begin{array}{l}\text { Sampling } \\
\text { Frequency }\end{array}$} & \multicolumn{2}{|c|}{ Management action levels } \\
\hline & & & $\begin{array}{c}\text { Dry season } \\
\text { Apr 1-Nov } 30\end{array}$ & $\begin{array}{c}\text { Wet season } \\
\text { Dec 1-Mar } 31\end{array}$ \\
\hline \multicolumn{5}{|l|}{ Physical } \\
\hline Dissolved oxygen (mg/L) & $\begin{array}{l}\text { CDBA, CDBC, } \\
\text { CDBD, CDBE, } \\
\text { CDBF, CDFJ, } \\
\text { CDBK, CDBL }\end{array}$ & Weekly & $\begin{array}{c}<80 \% \text { saturation } \\
\text { and }<5 \mathrm{mg} / \mathrm{L}\end{array}$ & $\begin{array}{c}<80 \% \text { saturation } \\
\text { and }<5 \mathrm{mg} / \mathrm{L}\end{array}$ \\
\hline Temperature $\left({ }^{\circ} \mathrm{C}\right)$ & $\begin{array}{l}\text { CDBA, CDBC, } \\
\text { CDBD, CDBE, } \\
\text { CDBF, CDFJ, } \\
\text { CDBK, CDBL }\end{array}$ & Weekly & $<15.6$ and $>26.7$ & $<15.6$ and $>26.7$ \\
\hline Total alkalinity $\left(\right.$ as $\left.\mathrm{CaCO}_{3}\right)(\mathrm{mg} / \mathrm{L})$ & CDBE & Monthly & $<50$ & $<50$ \\
\hline Chlorophyll-a (mg/L) & CDBE & Monthly & $>10$ & $>10$ \\
\hline $\mathrm{pH}$ (pH units) & CDBE & Monthly & $<6.0$ and $>9.0$ & $<6.0$ and $>9.0$ \\
\hline Total dissolved solids (mg/L) & CDBE & Monthly & $>360$ & $>360$ \\
\hline Total suspended solids (mg/L) & CDBE & Monthly & Not applicable & Not applicable \\
\hline Turbidity $(\mathrm{m})$ & CDBE & Weekly & $<0.91$ & $<0.914$ \\
\hline Chemical oxygen demand (mg/L) & CDBE & Quarterly & $>20$ & $>20$ \\
\hline Oil and grease (mg/L) & CDBE & Quarterly & $>15$ & $>15$ \\
\hline Specific Conductance $(\mu \mathrm{mho} / \mathrm{cm})$ & CDBE & Monthly & $>900$ & $>900$ \\
\hline \multicolumn{5}{|l|}{ Nutrients (mg/L) } \\
\hline Nitrate (as N) & CDBE & Monthly & $>0.2$ & $>0.2$ \\
\hline Nitrite (as N) & CDBE & Monthly & $>0.2$ & $>0.2$ \\
\hline Ammonia nitrogen & CDBE & Monthly & $>0.1$ & $>0.1$ \\
\hline Phosphate (as P) & CDBE & Monthly & $>0.02$ & $>0.02$ \\
\hline \multicolumn{5}{|l|}{ Microbiological (MPN(a)/100 mL) } \\
\hline Total coliform & CDBE & Quarterly & $>5000$ & $>5000$ \\
\hline Fecal coliform & CDBE & Quarterly & $>400$ & $>400$ \\
\hline \multicolumn{5}{|l|}{ Metals $(\mu \mathrm{g} / \mathrm{L})$} \\
\hline Antimony & CDBE & Monthly & $>6$ & Not applicable \\
\hline Arsenic & CDBE & Monthly & $>50$ & $>10$ \\
\hline Beryllium & CDBE & Monthly & $>4$ & Not applicable \\
\hline Boron & CDBE & Monthly & Not applicable & Not applicable \\
\hline Cadmium & CDBE & Monthly & $>5$ & $>2.2$ \\
\hline Chromium, total & CDBE & Monthly & $>50$ & Not applicable \\
\hline Chromium(VI) & CDBE & Monthly & Not applicable & $>22$ \\
\hline Copper & CDBE & Monthly & $>1300$ & $>23.6$ \\
\hline Iron & CDBE & Monthly & Not applicable & Not applicable \\
\hline
\end{tabular}


Table 7-14. Routine water quality management action levels and monitoring plan for the Drainage Retention Basin (concluded).

\begin{tabular}{|c|c|c|c|c|}
\hline \multirow[b]{2}{*}{ Constituent } & \multirow[b]{2}{*}{ Location } & \multirow{2}{*}{$\begin{array}{l}\text { Sampling } \\
\text { Frequency }\end{array}$} & \multicolumn{2}{|c|}{ Management action levels } \\
\hline & & & $\begin{array}{l}\text { Dry season } \\
\text { Apr 1-Nov } 30\end{array}$ & $\begin{array}{l}\text { Wet season } \\
\text { Dec 1-Mar } 31\end{array}$ \\
\hline \multicolumn{5}{|l|}{ Metals ( $\mu \mathrm{g} / \mathrm{L})$ (continued) } \\
\hline Lead & CDBE & Monthly & $>15$ & $>6.4$ \\
\hline Manganese & CDBE & Monthly & Not applicable & Not applicable \\
\hline Mercury & CDBE & Monthly & $>2$ & $>2$ \\
\hline Nickel & CDBE & Monthly & $>100$ & $>320$ \\
\hline Selenium & CDBE & Monthly & $>50$ & $>10$ \\
\hline Silver & CDBE & Monthly & $>100$ & $>8.2$ \\
\hline Thallium & CDBE & Monthly & $>2$ & Not applicable \\
\hline Zinc & CDBE & Monthly & Not applicable & $>220$ \\
\hline \multicolumn{5}{|l|}{ Organics ( $\mu \mathrm{g} / \mathrm{L})$} \\
\hline $\begin{array}{l}\text { Total volatile organic compounds (EPA } \\
\text { Method } 601 \text { only) }\end{array}$ & CDBE & Semiannually & $>5$ & $>5$ \\
\hline Tetrachloroethene & CDBE & Semiannually & $>4$ & $>4$ \\
\hline Vinyl chloride & CDBE & Semiannually & $>2$ & $>2$ \\
\hline Herbicides & CDBE & Quarterly & Not applicable & Not applicable \\
\hline \multicolumn{5}{|l|}{ Radiological (Bq/L) } \\
\hline Gross alpha & CDBE & Semiannually & $>0.555$ & $>0.555$ \\
\hline Gross beta & CDBE & Semiannually & $>1.85$ & $>1.85$ \\
\hline Tritium & CDBE & Semiannually & $>740$ & $>740$ \\
\hline \multicolumn{5}{|l|}{ Toxicity (\% survival/96-hour) } \\
\hline Aquatic bioassay, fathead minnow & CDBE & Annually & $\begin{array}{l}90 \% \text { survival } \\
\text { median, } 90 \\
\text { percentile value } \\
\text { of not less than } \\
70 \% \text { survival }\end{array}$ & $\begin{array}{c}90 \% \text { survival } \\
\text { median, } 90 \\
\text { percentile value } \\
\text { of not less than } \\
70 \% \text { survival }\end{array}$ \\
\hline Chronic bioassay, fathead minnow & CDBE & Annually & Not applicable & Not applicable \\
\hline Chronic bioassay, water flea & CDBE & Annually & Not applicable & Not applicable \\
\hline Chronic bioassay, algae & CDBE & Annually & Not applicable & Not applicable \\
\hline
\end{tabular}

a Most probable number. 


\section{Surface Water}

Table 7-15. Compliance monitoring data for releases from the Drainage Retention Basin, 1999.

\begin{tabular}{|c|c|c|c|c|c|c|c|c|}
\hline \multirow[t]{2}{*}{ Parameter } & \multicolumn{8}{|c|}{ CDBX sampling dates } \\
\hline & $1 / 20 / 99$ & $2 / 8 / 99$ & $4 / 8 / 99$ & $6 / 28 / 99$ & 8/19/99 & $10 / 4 / 99$ & 10/8/99 & $11 / 8 / 99$ \\
\hline \multicolumn{9}{|l|}{ Biological } \\
\hline Aquatic bioassay & & & & & & & & \\
\hline $\begin{array}{l}\text { Pimephales promelas survival (percent } \\
\text { survival) }\end{array}$ & $\mathrm{na}^{(\mathrm{a})}$ & na & na & 90 & 100 & na & 100 & na \\
\hline $\begin{array}{l}\text { Pimephales promelas growth (toxic } \\
\text { units) }\end{array}$ & na & na & na & na & na & na & $<1$ & na \\
\hline Pimephales promelas growth IC- $25^{(\mathrm{b})}$ & na & na & na & na & na & na & $>100$ & na \\
\hline Pimephales promelas growth IC-50(c) & na & na & na & na & na & na & $>100$ & na \\
\hline Pimephales promelas growth LOEC ${ }^{(\mathrm{d})}$ & na & na & na & na & na & na & $>100$ & na \\
\hline Pimephales promelas growth NOEC ${ }^{(e)}$ & na & na & na & na & na & na & $>100$ & na \\
\hline $\begin{array}{l}\text { Pimephales promelas survival toxic } \\
\text { units }\end{array}$ & na & na & na & na & na & na & $<1$ & na \\
\hline Pimephales promelas survival LC- $50^{(\mathrm{f})}$ & na & na & na & na & na & na & $>100$ & na \\
\hline Pimephales promelas survival LOEC & na & na & na & na & na & na & $>100$ & na \\
\hline Pimephales promelas survival NOEC & na & na & na & na & na & na & $>100$ & na \\
\hline Ceriodaphnia dubia growth (toxic unit) & na & na & na & na & na & na & $<1$ & na \\
\hline Ceriodaphnia dubia growth IC-25 & na & na & na & na & na & na & $>100$ & na \\
\hline Ceriodaphnia dubia growth IC-50 & na & na & na & na & na & na & $>100$ & na \\
\hline Ceriodaphnia dubia growth LOEC & na & na & na & na & na & na & $>100$ & na \\
\hline Ceriodaphnia dubia growth NOEC & na & na & na & na & na & na & $>100$ & na \\
\hline $\begin{array}{l}\text { Ceriodaphnia dubia survival (toxic } \\
\text { units) }\end{array}$ & na & na & na & na & na & na & $<1$ & na \\
\hline Ceriodaphnia dubia survival LC-50 & na & na & na & na & na & na & $>100$ & na \\
\hline Ceriodaphnia dubia survival LOEC & na & na & na & na & na & na & $>100$ & na \\
\hline Ceriodaphnia dubia survival NOEC & na & na & na & na & na & na & $>100$ & na \\
\hline $\begin{array}{l}\text { Selanastrum capriocomutum growth } \\
\text { (toxic units) }\end{array}$ & na & na & na & na & na & na & $>1$ & na \\
\hline $\begin{array}{l}\text { Selanastrum capriocomutum growth } \\
\text { IC- } 25\end{array}$ & na & na & na & na & na & na & 56.6 & na \\
\hline $\begin{array}{l}\text { Selanastrum capriocomutum growth } \\
\text { IC- } 50\end{array}$ & na & na & na & na & na & na & $>100$ & na \\
\hline $\begin{array}{l}\text { Selanastrum capriocomutum growth } \\
\text { LOEC }\end{array}$ & na & na & na & na & na & na & 100 & na \\
\hline $\begin{array}{l}\text { Selanastrum capriocomutum growth } \\
\text { NOEC }\end{array}$ & na & na & na & na & na & na & $<100$ & na \\
\hline
\end{tabular}


Table 7-15. Compliance monitoring data for releases from the Drainage Retention Basin, 1999 (continued).

\begin{tabular}{|c|c|c|c|c|c|c|c|c|}
\hline \multirow[t]{2}{*}{ Parameter } & \multicolumn{8}{|c|}{ WPDC sampling dates } \\
\hline & $1 / 20 / 99$ & $2 / 8 / 99$ & $4 / 8 / 99$ & $6 / 28 / 99$ & $8 / 19 / 99$ & $10 / 4 / 99$ & $10 / 8 / 99$ & $11 / 8 / 99$ \\
\hline \multicolumn{9}{|l|}{ Biological (continued) } \\
\hline \multicolumn{9}{|l|}{ Aquatic bioassay (continued) } \\
\hline $\begin{array}{l}\text { Pimephales promelas survival (percent } \\
\text { survival) }\end{array}$ & 100 & na & na & 90 & 100 & na & 90 & na \\
\hline $\begin{array}{l}\text { Pimephales promelas growth (toxic } \\
\text { units) }\end{array}$ & $<1$ & na & na & na & na & na & na & na \\
\hline Pimephales promelas growth IC-25(b) & $>100$ & na & na & na & na & na & na & na \\
\hline Pimephales promelas growth IC-50(c) & $>100$ & na & na & na & na & na & na & na \\
\hline Pimephales promelas growth LOEC(d) & $>100$ & na & na & na & na & na & na & na \\
\hline Pimephales promelas growth NOEC $(\mathrm{e})$ & $>100$ & na & na & na & na & na & na & na \\
\hline $\begin{array}{l}\text { Pimephales promelas survival (toxic } \\
\text { units) }\end{array}$ & $<1$ & na & na & na & na & na & na & na \\
\hline Pimephales promelas survival LC-50(f) & $>100$ & na & na & na & na & na & na & na \\
\hline Pimephales promelas survival LOEC & $>100$ & na & na & na & na & na & na & na \\
\hline Pimephales promelas survival NOEC & $>100$ & na & na & na & na & na & na & na \\
\hline Ceriodaphnia dubia growth (toxic unit) & na & na & na & na & na & na & na & na \\
\hline Ceriodaphnia dubia growth IC-25 & na & na & na & na & na & na & na & na \\
\hline Ceriodaphnia dubia growth IC-50 & na & na & na & na & na & na & na & na \\
\hline Ceriodaphnia dubia growth LOEC & na & na & na & na & na & na & na & na \\
\hline Ceriodaphnia dubia growth NOEC & na & na & na & na & na & na & na & na \\
\hline $\begin{array}{l}\text { Ceriodaphnia dubia survival (toxic } \\
\text { units) }\end{array}$ & na & na & na & na & na & na & na & na \\
\hline Ceriodaphnia dubia survival LC-50 & na & na & na & na & na & na & na & na \\
\hline Ceriodaphnia dubia survival LOEC & na & na & na & na & na & na & na & na \\
\hline Ceriodaphnia dubia survival NOEC & na & na & na & na & na & na & na & na \\
\hline $\begin{array}{l}\text { Selanastrum capriocomutum growth } \\
\text { (toxic units) }\end{array}$ & na & na & na & na & na & na & na & na \\
\hline $\begin{array}{l}\text { Selanastrum capriocomutum growth } \\
\text { IC- } 25\end{array}$ & na & na & na & na & na & na & na & na \\
\hline $\begin{array}{l}\text { Selanastrum capriocomutum growth } \\
\text { IC- } 50\end{array}$ & na & na & na & na & na & na & na & na \\
\hline $\begin{array}{l}\text { Selanastrum capriocomutum growth } \\
\text { LOEC }\end{array}$ & na & na & na & na & na & na & na & na \\
\hline $\begin{array}{l}\text { Selanastrum capriocomutum growth } \\
\text { NOEC }\end{array}$ & na & na & na & na & na & na & na & na \\
\hline
\end{tabular}




\section{Surface Water}

Table 7-15. Compliance monitoring data for releases from the Drainage Retention Basin, 1999 (continued).

\begin{tabular}{|c|c|c|c|c|c|c|c|c|}
\hline \multirow[t]{2}{*}{ Parameter } & \multicolumn{8}{|c|}{ CDBX sampling dates } \\
\hline & $1 / 20 / 99$ & 2/8/99 & $4 / 8 / 99$ & $6 / 28 / 99$ & 8/19/99 & $10 / 4 / 99$ & $10 / 8 / 99$ & $11 / 8 / 99$ \\
\hline \multicolumn{9}{|l|}{ Anions (mg/L) } \\
\hline Bicarbonate alkalinity $\left(\right.$ as $\left.\mathrm{CaCO}_{3}\right)$ & 139 & 173 & 153 & na & na & na & na & na \\
\hline Bromide & 0.41 & 0.42 & 0.5 & na & na & na & na & 0.51 \\
\hline Carbonate alkalinity $\left(\right.$ as $\left.\mathrm{CaCO}_{3}\right)$ & 25 & $<5$ & 38 & na & na & na & na & na \\
\hline Chloride & 130 & 138 & 76 & na & na & na & na & 146 \\
\hline Fluoride & 0.38 & 0.38 & 0.4 & na & na & na & na & 0.35 \\
\hline Nitrate (as N) & 2.2 & 2.6 & 2.6 & na & na & na & na & 2 \\
\hline Nitrate $\left(\right.$ as $\left.\mathrm{NO}_{3}\right)$ & 9.7 & 11 & 11 & na & na & na & na & 9 \\
\hline Nitrite (as N) & $<0.02$ & $<0.02$ & $<0.02$ & na & na & na & na & 0.02 \\
\hline Nitrite (as $\mathrm{NO}_{2}$ ) & $<0.07$ & $<0.07$ & $<0.07$ & na & na & na & na & 0.07 \\
\hline Orthophosphate & 0.15 & 0.14 & 0.11 & na & na & na & na & na \\
\hline Sulfate & 61 & 62 & 26 & na & na & na & na & 51 \\
\hline \multicolumn{9}{|l|}{ Dissolved general minerals (mg/L) } \\
\hline Aluminum & $<0.05$ & $<0.05$ & $<0.05$ & na & na & na & na & na \\
\hline Bicarbonate alkalinity (as $\mathrm{CaCO}_{3}$ ) & 141 & 166 & 151 & na & na & na & na & na \\
\hline Calcium & 46 & 51 & 62 & na & na & na & na & na \\
\hline Carbonate alkalinity $\left(\right.$ as $\left.\mathrm{CaCO}_{3}\right)$ & 20 & 10 & 45 & na & na & na & na & na \\
\hline Chloride & 128 & 138 & 157 & na & na & na & na & na \\
\hline Copper & $<0.01$ & 0.01 & 0.015 & na & na & na & na & na \\
\hline Fluoride & 0.36 & 0.38 & 0.4 & na & na & na & na & na \\
\hline Hydroxide alkalinity (as $\mathrm{CaCO}_{3}$ ) & $<5$ & $<5$ & $<5$ & na & na & na & na & na \\
\hline Iron & $<0.05$ & $<0.05$ & $<0.05$ & na & na & na & na & na \\
\hline Magnesium & 23 & 25 & 31 & na & na & na & na & na \\
\hline Manganese & $<0.01$ & $<0.01$ & $<0.01$ & na & na & na & na & na \\
\hline Nickel & $<0.05$ & $<0.05$ & $<0.05$ & na & na & na & na & na \\
\hline Nitrate (as N) & 2.2 & 2.6 & 3.3 & na & na & na & na & na \\
\hline Nitrate $\left(\right.$ as $\left.\mathrm{NO}_{3}\right)$ & 9.5 & 11 & 15 & na & na & na & na & na \\
\hline Nitrite (as N) & $<0.02$ & 0.03 & $<0.02$ & na & na & na & na & na \\
\hline Orthophosphate & 0.16 & 0.14 & 0.11 & na & na & na & na & na \\
\hline $\mathrm{pH}$ (pH units) & 8.74 & 8.39 & 8.7 & na & na & na & na & na \\
\hline Potassium & 2 & 2.1 & 2 & na & na & na & na & na \\
\hline Sodium & 97 & 98 & 109 & na & na & na & na & na \\
\hline Specific conductance & 832 & 900 & 1050 & na & na & na & na & na \\
\hline Sulfate & 60 & 61 & 57 & na & na & na & na & na \\
\hline Surfactants & $<0.1$ & $<0.05$ & 0.06 & na & na & na & na & na \\
\hline
\end{tabular}


Table 7-15. Compliance monitoring data for releases from the Drainage Retention Basin, 1999 (continued).

\begin{tabular}{|c|c|c|c|c|c|c|c|c|}
\hline \multirow[t]{2}{*}{ Parameter } & \multicolumn{8}{|c|}{ WPDC sampling dates } \\
\hline & 1/20/99 & $2 / 8 / 99$ & $4 / 8 / 99$ & $6 / 28 / 99$ & $8 / 19 / 99$ & 10/4/99 & $10 / 8 / 99$ & $11 / 8 / 99$ \\
\hline \multicolumn{9}{|l|}{ Anions (mg/L) } \\
\hline Bicarbonate alkalinity $\left(\right.$ as $\left.\mathrm{CaCO}_{3}\right)$ & 129 & 66 & 43 & na & na & na & na & na \\
\hline Bromide & 0.31 & 0.1 & $<0.1$ & na & na & na & na & $<0.1$ \\
\hline Carbonate alkalinity (as $\left.\mathrm{CaCO}_{3}\right)$ & $<5$ & $<5$ & $<5$ & na & na & na & na & na \\
\hline Chloride & 114 & 30 & 12 & na & na & na & na & 19 \\
\hline Fluoride & 0.38 & 0.17 & 0.07 & na & na & na & na & 0.11 \\
\hline Nitrate (as N) & 2.2 & 1.1 & 0.91 & na & na & na & na & 1 \\
\hline Nitrate (as NO3) & 9.7 & 4.9 & 4.1 & na & na & na & na & 4.6 \\
\hline Nitrite (as N) & 0.02 & $<0.02$ & 0.03 & na & na & na & na & 0.02 \\
\hline Nitrite $\left(\right.$ as $\left.\mathrm{NO}_{2}\right)$ & 0.07 & $<0.07$ & 0.11 & na & na & na & na & 0.07 \\
\hline Orthophosphate & 0.35 & 0.39 & 0.2 & na & na & na & na & na \\
\hline Sulfate & 104 & 22 & 6.2 & na & na & na & na & 11 \\
\hline \multicolumn{9}{|l|}{ Dissolved general minerals (mg/L) } \\
\hline Aluminum & $<0.05$ & 0.21 & 0.19 & na & na & na & na & na \\
\hline Bicarbonate alkalinity (as $\mathrm{CaCO}_{3}$ ) & 134 & 73 & 42 & na & na & na & na & na \\
\hline Calcium & 46 & 20 & 13 & na & na & na & na & na \\
\hline Carbonate alkalinity $\left(\right.$ as $\left.\mathrm{CaCO}_{3}\right)$ & $<5$ & $<5$ & $<5$ & na & na & na & na & na \\
\hline Chloride & 117 & 29 & 6.1 & na & na & na & na & na \\
\hline Copper & $<0.01$ & $<0.01$ & $<0.01$ & na & na & na & na & na \\
\hline Fluoride & 0.38 & 0.17 & 0.08 & na & na & na & na & na \\
\hline Hydroxide alkalinity (as $\mathrm{CaCO}_{3}$ ) & $<5$ & $<5$ & $<5$ & na & na & na & na & na \\
\hline Iron & 0.065 & 0.18 & 0.17 & na & na & na & na & na \\
\hline Magnesium & 18 & 7.2 & 4.2 & na & na & na & na & na \\
\hline Manganese & $<0.01$ & $<0.01$ & $<0.01$ & na & na & na & na & na \\
\hline Nickel & $<0.05$ & $<0.05$ & $<0.05$ & na & na & na & na & na \\
\hline Nitrate (as N) & 2.2 & 1.3 & 1.4 & na & na & na & na & na \\
\hline Nitrate (as $\mathrm{NO}_{3}$ ) & 9.7 & 5.7 & 6.1 & na & na & na & na & na \\
\hline Nitrite (as N) & 0.02 & $<0.02$ & 0.02 & na & na & na & na & na \\
\hline Orthophosphate & 0.48 & 0.33 & 0.2 & na & na & na & na & na \\
\hline $\mathrm{pH}$ (pH units) & 8.07 & 8.17 & 7.34 & na & na & na & na & na \\
\hline Potassium & 4.6 & 1.7 & 1.4 & na & na & na & na & na \\
\hline Sodium & 96 & 32 & 14 & na & na & na & na & na \\
\hline Specific conductance & 835 & 307 & 170 & na & na & na & na & na \\
\hline Sulfate & 89 & 21 & 3.6 & na & na & na & na & na \\
\hline Surfactants & $<0.1$ & $<0.05$ & 0.06 & na & na & na & na & na \\
\hline
\end{tabular}




\section{Surface Water}

Table 7-15. Compliance monitoring data for releases from the Drainage Retention Basin, 1999 (continued).

\begin{tabular}{|c|c|c|c|c|c|c|c|c|}
\hline \multirow[t]{2}{*}{ Parameter } & \multicolumn{8}{|c|}{ CDBX sampling dates } \\
\hline & $1 / 20 / 99$ & $2 / 8 / 99$ & 4/8/99 & $6 / 28 / 99$ & $8 / 19 / 99$ & $10 / 4 / 99$ & $10 / 8 / 99$ & $11 / 8 / 99$ \\
\hline \multicolumn{9}{|l|}{$\begin{array}{l}\text { Dissolved general minerals (mg/L) } \\
\text { (continued) }\end{array}$} \\
\hline Total alkalinity $\left(\right.$ as $\left.\mathrm{CaCO}_{3}\right)$ & 161 & 176 & 196 & na & na & na & na & na \\
\hline Total dissolved solids (TDS) & 473 & 537 & 603 & na & na & na & na & na \\
\hline Total hardness (as $\mathrm{CaCO}_{3}$ ) & 210 & 230 & 282 & na & na & na & na & na \\
\hline Total phosphorus (as P) & 0.16 & 0.64 & 0.12 & na & na & na & na & na \\
\hline Zinc & $<0.01$ & $<0.01$ & $<0.01$ & na & na & na & na & na \\
\hline \multicolumn{9}{|l|}{ Total general minerals (mg/L) } \\
\hline Aluminum & 2.2 & 17 & 0.34 & na & na & 0.78 & na & 1.5 \\
\hline Bicarbonate alkalinity $\left(\right.$ as $\left.\mathrm{CaCO}_{3}\right)$ & 135 & 173 & 152 & na & na & 179 & na & 177 \\
\hline Calcium & 46 & 58 & 61 & na & na & 54 & na & 50 \\
\hline Carbonate alkalinity $\left(\right.$ as $\mathrm{CaCO}_{3}$ ) & 25 & 5 & 38 & na & na & 45 & na & $<5$ \\
\hline Chloride & 129 & 138 & 176 & na & na & 241 & na & 157 \\
\hline Copper & 0.014 & 0.038 & 0.013 & na & na & $<0.01$ & na & 0.016 \\
\hline Fluoride & 0.36 & 0.4 & 0.41 & na & na & 0.52 & na & 0.35 \\
\hline Hydroxide alkalinity $\left(\right.$ as $\left.\mathrm{CaCO}_{3}\right)$ & $<5$ & $<5$ & $<5$ & na & na & $<5$ & na & $<5$ \\
\hline Iron & 2 & 20 & 0.4 & na & na & 0.65 & na & 1.5 \\
\hline Magnesium & 23 & 30 & 30 & na & na & 39 & na & 30 \\
\hline Manganese & 0.048 & 0.68 & 0.034 & na & na & 0.028 & na & 0.049 \\
\hline Nickel & $<0.05$ & 0.051 & $<0.05$ & na & na & $<0.05$ & na & $<0.05$ \\
\hline Nitrate (as N) & 2.2 & 2.6 & 3.3 & na & na & 1.2 & na & 2.2 \\
\hline Nitrate $\left(\right.$ as $\left.\mathrm{NO}_{3}\right)$ & 9.7 & 12 & 15 & na & na & 5.4 & na & 9.8 \\
\hline Nitrite (as N) & 0.02 & 0.02 & 0.02 & na & na & 0.02 & na & 0.02 \\
\hline Orthophosphate & 0.16 & 0.14 & 0.11 & na & na & 0.18 & na & 0.3 \\
\hline $\mathrm{pH}$ (pH units) & 8.77 & 8.17 & 8.75 & 8.82 & 8.35 & 8.45 & na & 8.52 \\
\hline Potassium & 2.5 & 4.4 & 2 & na & na & 2.6 & na & 2.7 \\
\hline Sodium & 93 & 89 & 112 & na & na & 141 & na & 98 \\
\hline Specific conductance & 835 & 901 & 1060 & na & na & 1210 & na & 963 \\
\hline Sulfate & 60 & 61 & 69 & na & na & 82 & na & 57 \\
\hline Surfactants & $<0.1$ & $<0.05$ & 0.06 & na & na & 0.07 & na & 0.06 \\
\hline Total alkalinity $\left(\right.$ as $\mathrm{CaCO}_{3}$ ) & 160 & 173 & 190 & na & na & 224 & na & 177 \\
\hline Total dissolved solids (TDS) & 467 & 513 & 637 & na & na & 720 & na & 546 \\
\hline Total hardness (as $\mathrm{CaCO}_{3}$ ) & 210 & 268 & 276 & na & na & 295 & na & 248 \\
\hline Total phosphorus (as P) & 0.17 & 0.61 & 0.1 & na & na & 0.12 & na & 0.19 \\
\hline Total suspended solids (TSS) & 55 & 169 & 57 & 33 & 18 & 8 & na & 28 \\
\hline Zinc & 0.026 & 0.27 & 0.018 & $<0.02$ & $<0.02$ & $<0.05$ & na & 0.07 \\
\hline
\end{tabular}


Table 7-15. Compliance monitoring data for releases from the Drainage Retention Basin, 1999 (continued).

\begin{tabular}{|c|c|c|c|c|c|c|c|c|}
\hline \multirow[t]{2}{*}{ Parameter } & \multicolumn{8}{|c|}{ WPDC sampling dates } \\
\hline & $1 / 20 / 99$ & 2/8/99 & $4 / 8 / 99$ & $6 / 28 / 99$ & 8/19/99 & $10 / 4 / 99$ & $10 / 8 / 99$ & $11 / 8 / 99$ \\
\hline \multicolumn{9}{|l|}{ Dissolved general minerals (mg/L) } \\
\hline Total alkalinity $\left(\right.$ as $\left.\mathrm{CaCO}_{3}\right)$ & 134 & 73 & 42 & na & na & na & na & na \\
\hline Total dissolved solids (TDS) & 483 & 234 & 131 & na & na & na & na & na \\
\hline Total hardness $\left(\right.$ as $\left.\mathrm{CaCO}_{3}\right)$ & 189 & 80 & 50 & na & na & na & na & na \\
\hline Total phosphorus (as P) & 0.24 & 0.18 & 0.25 & na & na & na & na & na \\
\hline Zinc & 0.055 & 0.018 & 0.043 & na & na & na & na & na \\
\hline \multicolumn{9}{|l|}{ Total general minerals (mg/L) } \\
\hline Aluminum & 1.4 & 4.1 & 4.2 & na & na & 0.33 & na & 4.6 \\
\hline Bicarbonate alkalinity $\left(\right.$ as $\left.\mathrm{CaCO}_{3}\right)$ & 138 & 74 & 34 & na & na & 210 & na & 72 \\
\hline Calcium & 48 & 20 & 17 & na & na & 54 & na & 18 \\
\hline Carbonate alkalinity (as $\left.\mathrm{CaCO}_{3}\right)$ & $<5$ & $<5$ & $<5$ & na & na & 30 & na & $<5$ \\
\hline Chloride & 117 & 29 & 9.4 & na & na & 127 & na & 22 \\
\hline Copper & 0.011 & 0.022 & 0.017 & na & na & $<0.01$ & na & 0.015 \\
\hline Fluoride & 0.37 & 0.17 & 0.08 & na & na & 0.53 & na & 0.12 \\
\hline Hydroxide alkalinity (as $\mathrm{CaCO}_{3}$ ) & $<5$ & $<5$ & $<5$ & na & na & $<5$ & na & $<5$ \\
\hline Iron & 1.4 & 4 & 4.8 & na & na & 0.37 & na & 4.5 \\
\hline Magnesium & 18 & 8.2 & 5.6 & na & na & 25 & na & 6.8 \\
\hline Manganese & 0.028 & 0.07 & 0.1 & na & na & 0.019 & na & 0.11 \\
\hline Nickel & $<0.05$ & $<0.05$ & $<0.05$ & na & na & $<0.05$ & na & $<0.05$ \\
\hline Nitrate (as N) & 2.2 & 1.3 & 1.4 & na & na & 2.3 & na & 1.1 \\
\hline Nitrate $\left(\right.$ as $\left.\mathrm{NO}_{3}\right)$ & 9.7 & 5.7 & 6 & na & na & 10 & na & 4.9 \\
\hline Nitrite (as N) & 0.02 & 0.02 & 0.03 & na & na & 0.02 & na & 0.02 \\
\hline Orthophosphate & 0.48 & 0.32 & 0.23 & na & na & 0.19 & na & 0.42 \\
\hline $\mathrm{pH}$ (pH units) & 8.06 & 7.88 & 7.39 & 8.9 & 8.72 & 8.4 & na & 7.7 \\
\hline Potassium & 4.9 & 3.1 & 2.4 & na & na & 7 & na & 3.7 \\
\hline Sodium & 94 & 32 & 14 & na & na & 122 & na & 18 \\
\hline Specific conductance & 827 & 312 & 175 & na & na & 946 & na & 250 \\
\hline Sulfate & 89 & 21 & 5.2 & na & na & 68 & na & 12 \\
\hline Surfactants & $<0.1$ & $<0.05$ & 0.06 & na & na & 0.05 & na & 0.08 \\
\hline Total alkalinity $\left(\right.$ as $\left.\mathrm{CaCO}_{3}\right)$ & 138 & 74 & 34 & na & na & 240 & na & 72 \\
\hline Total dissolved solids (TDS) & 497 & 220 & 129 & na & na & 583 & na & 182 \\
\hline Total hardness (as $\mathrm{CaCO}_{3}$ ) & 194 & 84 & 66 & na & na & 238 & na & 73 \\
\hline Total phosphorus (as P) & 0.23 & 0.19 & 0.18 & na & na & 0.18 & na & 0.29 \\
\hline Total suspended solids (TSS) & 40 & 52 & 84 & 26 & 85 & 21 & na & 94 \\
\hline Zinc & 0.084 & 0.086 & 0.13 & na & na & $<0.05$ & na & 0.28 \\
\hline
\end{tabular}




\section{Surface Water}

Table 7-15. Compliance monitoring data for releases from the Drainage Retention Basin, 1999 (continued).

\begin{tabular}{|c|c|c|c|c|c|c|c|c|}
\hline \multirow[t]{2}{*}{ Parameter } & \multicolumn{8}{|c|}{ CDBX sampling dates } \\
\hline & $1 / 20 / 99$ & 2/8/99 & $4 / 8 / 99$ & $6 / 28 / 99$ & $8 / 19 / 99$ & $10 / 4 / 99$ & $10 / 8 / 99$ & $11 / 8 / 99$ \\
\hline \multicolumn{9}{|l|}{ Dissolved metals (mg/L) } \\
\hline Aluminum & $<0.05$ & $<0.05$ & $<0.05$ & na & na & na & na & na \\
\hline Antimony & $<0.004$ & $<0.004$ & $<0.004$ & na & na & na & na & na \\
\hline Arsenic & 0.0034 & $<0.002$ & $<0.002$ & na & na & na & na & na \\
\hline Barium & 0.098 & 0.11 & 0.095 & na & na & na & na & na \\
\hline Beryllium & $<0.0002$ & $<0.0002$ & $<0.0002$ & na & na & na & na & na \\
\hline Boron & 1.8 & 1.8 & 1.9 & na & na & na & na & na \\
\hline Cadmium & $<0.0005$ & $<0.0005$ & $<0.0005$ & na & na & na & na & na \\
\hline Chromium & 0.0044 & 0.0048 & 0.0078 & na & na & na & na & na \\
\hline Cobalt & $<0.05$ & $<0.05$ & $<0.05$ & na & na & na & na & na \\
\hline Copper & 0.0026 & $<0.001$ & 0.0079 & na & na & na & na & na \\
\hline Chromium(VI) & 0.005 & 0.0053 & 0.007 & na & na & na & na & na \\
\hline Iron & $<0.05$ & $<0.05$ & $<0.05$ & na & na & na & na & na \\
\hline Lead & $<0.005$ & $<0.005$ & $<0.005$ & na & na & na & na & na \\
\hline Manganese & $<0.01$ & $<0.01$ & $<0.01$ & na & na & na & na & na \\
\hline Mercury & $<0.0002$ & $<0.0002$ & $<0.0002$ & na & na & na & na & na \\
\hline Molybdenum & $<0.025$ & $<0.025$ & $<0.025$ & na & na & na & na & na \\
\hline Nickel & $<0.002$ & $<0.002$ & $<0.002$ & na & na & na & na & na \\
\hline Selenium & $<0.002$ & $<0.002$ & $<0.002$ & na & na & na & na & na \\
\hline Silver & $<0.001$ & $<0.001$ & $<0.001$ & na & na & na & na & na \\
\hline Thallium & $<0.001$ & $<0.001$ & $<0.001$ & na & na & na & na & na \\
\hline Vanadium & $<0.01$ & $<0.01$ & $<0.01$ & na & na & na & na & na \\
\hline Zinc & $<0.02$ & $<0.02$ & $<0.02$ & na & na & na & na & na \\
\hline \multicolumn{9}{|l|}{ Total metals (mg/L) } \\
\hline Aluminum & 1.8 & 11 & 0.27 & 1.6 & 1.6 & 0.74 & na & 1.5 \\
\hline Antimony & $<0.004$ & $<0.004$ & $<0.004$ & $<0.004$ & $<0.004$ & $<0.004$ & na & $<0.004$ \\
\hline Arsenic & 0.0028 & 0.0029 & $<0.002$ & 0.0042 & 0.004 & 0.0023 & na & 0.0043 \\
\hline Barium & 0.12 & 0.23 & 0.098 & 0.14 & 0.15 & 0.18 & na & 0.13 \\
\hline Beryllium & $<0.0002$ & 0.00028 & $<0.0002$ & $<0.0002$ & $<0.0002$ & $<0.0002$ & na & $<0.0002$ \\
\hline Boron & 1.8 & 1.7 & 1.9 & 2.2 & 2.2 & 2.3 & na & 1.6 \\
\hline Cadmium & $<0.0005$ & $<0.0005$ & $<0.0005$ & $<0.0005$ & $<0.0005$ & $<0.0005$ & na & $<0.0005$ \\
\hline Chromium & 0.01 & 0.03 & 0.0085 & 0.0085 & 0.0056 & 0.0024 & na & 0.0068 \\
\hline Cobalt & $<0.05$ & $<0.05$ & $<0.05$ & $<0.05$ & $<0.05$ & $<0.05$ & na & $<0.05$ \\
\hline Copper & 0.0042 & 0.019 & 0.0027 & 0.007 & 0.0042 & 0.0017 & na & 0.0066 \\
\hline Chromium(VI) & na & na & na & 0.014 & 0.0046 & 0.0031 & na & $<0.002$ \\
\hline Iron & 1.9 & 12 & 0.33 & 1.6 & 1.5 & 0.62 & na & 1.4 \\
\hline
\end{tabular}


Table 7-15. Compliance monitoring data for releases from the Drainage Retention Basin, 1999 (continued).

\begin{tabular}{|c|c|c|c|c|c|c|c|c|}
\hline \multirow[t]{2}{*}{ Parameter } & \multicolumn{8}{|c|}{ WPDC sampling dates } \\
\hline & $1 / 20 / 99$ & 2/8/99 & 4/8/99 & $6 / 28 / 99$ & 8/19/99 & $10 / 4 / 99$ & $10 / 8 / 99$ & $11 / 8 / 99$ \\
\hline \multicolumn{9}{|l|}{ Dissolved metals (mg/L) } \\
\hline Aluminum & $<0.05$ & 0.28 & 0.18 & na & na & na & na & na \\
\hline Antimony & $<0.004$ & $<0.004$ & $<0.004$ & na & na & na & na & na \\
\hline Arsenic & 0.0063 & $<0.002$ & $<0.002$ & na & na & na & na & na \\
\hline Barium & 0.11 & 0.059 & 0.09 & na & na & na & na & na \\
\hline Beryllium & $<0.0002$ & $<0.0002$ & $<0.0002$ & na & na & na & na & na \\
\hline Boron & 2 & 0.63 & 0.21 & na & na & na & na & na \\
\hline Cadmium & $<0.0005$ & $<0.0005$ & $<0.0005$ & na & na & na & na & na \\
\hline Chromium & 0.0022 & 0.0023 & 0.0022 & na & na & na & na & na \\
\hline Cobalt & $<0.05$ & $<0.05$ & $<0.05$ & na & na & na & na & na \\
\hline Copper & 0.0037 & $<0.001$ & 0.0054 & na & na & na & na & na \\
\hline Chromium(VI) & 0.0027 & 0.004 & $<0.002$ & na & na & na & na & na \\
\hline Iron & 0.066 & 0.25 & 0.16 & na & na & na & na & na \\
\hline Lead & $<0.005$ & $<0.005$ & $<0.005$ & na & na & na & na & na \\
\hline Manganese & $<0.01$ & $<0.01$ & $<0.01$ & na & na & na & na & na \\
\hline Mercury & $<0.0002$ & $<0.0002$ & $<0.0002$ & na & na & na & na & na \\
\hline Molybdenum & $<0.025$ & $<0.025$ & $<0.025$ & na & na & na & na & na \\
\hline Nickel & 0.0033 & 0.0026 & $<0.002$ & na & na & na & na & na \\
\hline Selenium & $<0.002$ & $<0.002$ & $<0.002$ & na & na & na & na & na \\
\hline Silver & $<0.001$ & $<0.001$ & $<0.001$ & na & na & na & na & na \\
\hline Thallium & $<0.001$ & $<0.001$ & $<0.001$ & na & na & na & na & na \\
\hline Vanadium & $<0.01$ & $<0.01$ & $<0.01$ & na & na & na & na & na \\
\hline Zinc & 0.058 & 0.022 & 0.046 & na & na & na & na & na \\
\hline \multicolumn{9}{|l|}{ Total metals (mg/L) } \\
\hline Aluminum & 1.4 & 3.9 & 3.7 & 0.2 & 0.2 & 0.34 & na & 4.5 \\
\hline Antimony & $<0.004$ & $<0.004$ & $<0.004$ & $<0.004$ & $<0.004$ & $<0.004$ & na & $<0.004$ \\
\hline Arsenic & 0.0047 & $<0.002$ & $<0.002$ & 0.0022 & 0.0028 & $<0.002$ & na & 0.0026 \\
\hline Barium & 0.12 & 0.076 & 0.072 & 0.098 & 0.15 & 0.11 & na & 0.096 \\
\hline Beryllium & $<0.0002$ & $<0.0002$ & $<0.0002$ & $<0.0002$ & $<0.0002$ & $<0.0002$ & na & $<0.0002$ \\
\hline Boron & 2 & 0.6 & 0.22 & 1.6 & 1.7 & 1.6 & na & 0.27 \\
\hline Cadmium & $<0.0005$ & $<0.0005$ & $<0.0005$ & $<0.0005$ & $<0.0005$ & $<0.0005$ & na & $<0.0005$ \\
\hline Chromium & 0.0056 & 0.012 & 0.0087 & 0.013 & 0.018 & 0.0099 & na & 0.013 \\
\hline Cobalt & $<0.05$ & $<0.05$ & $<0.05$ & $<0.05$ & $<0.05$ & $<0.05$ & na & $<0.05$ \\
\hline Copper & 0.005 & 0.0098 & 0.009 & $<0.005$ & 0.0024 & 0.0036 & na & 0.014 \\
\hline Chromium(VI) & na & na & na & 0.014 & 0.014 & 0.013 & na & $<0.002$ \\
\hline Iron & 1.4 & 3.9 & 4.1 & 0.24 & 0.31 & 0.38 & na & 4.6 \\
\hline
\end{tabular}




\section{Surface Water}

Table 7-15. Compliance monitoring data for releases from the Drainage Retention Basin, 1999 (continued).

\begin{tabular}{|c|c|c|c|c|c|c|c|c|}
\hline \multirow[t]{2}{*}{ Parameter } & \multicolumn{8}{|c|}{ CDBX sampling dates } \\
\hline & $1 / 20 / 99$ & 2/8/99 & $4 / 8 / 99$ & 6/28/99 & $8 / 19 / 99$ & $10 / 4 / 99$ & $10 / 8 / 99$ & $11 / 8 / 99$ \\
\hline \multicolumn{9}{|c|}{ Total metals (mg/L) (continued) } \\
\hline Lead & $<0.005$ & 0.0079 & $<0.005$ & $<0.005$ & $<0.005$ & $<0.005$ & na & $<0.005$ \\
\hline Manganese & 0.046 & 0.48 & 0.029 & 0.11 & 0.11 & 0.028 & na & 0.049 \\
\hline Mercury & $<0.0002$ & 0.00043 & $<0.0002$ & $<0.0002$ & $<0.0002$ & $<0.0002$ & na & $<0.0002$ \\
\hline Molybdenum & $<0.025$ & $<0.025$ & $<0.025$ & $<0.025$ & $<0.025$ & $<0.025$ & na & $<0.025$ \\
\hline Nickel & 0.0065 & 0.028 & 0.0023 & 0.0054 & 0.0043 & 0.0024 & na & 0.0065 \\
\hline Selenium & $<0.002$ & $<0.002$ & $<0.002$ & $<0.002$ & $<0.002$ & $<0.002$ & na & $<0.002$ \\
\hline Silver & $<0.001$ & $<0.001$ & $<0.001$ & $<0.001$ & $<0.001$ & $<0.001$ & na & $<0.001$ \\
\hline Thallium & $<0.001$ & $<0.001$ & $<0.001$ & $<0.001$ & $<0.001$ & $<0.001$ & na & $<0.001$ \\
\hline Vanadium & $<0.01$ & 0.028 & $<0.01$ & 0.013 & 0.011 & $<0.01$ & na & 0.012 \\
\hline Zinc & 0.026 & 0.2 & $<0.02$ & $<0.02$ & $<0.02$ & $<0.02$ & na & 0.11 \\
\hline \multicolumn{9}{|c|}{ Volatile organic compounds ( $\mu \mathrm{g} / \mathrm{L})$} \\
\hline 1,1,1-Trichloroethane & $<0.5$ & $<0.5$ & $<0.5$ & $<0.5$ & $<0.5$ & $<0.5$ & na & $<0.5$ \\
\hline 1,1,2,2-Tetrachloroethane & $<0.5$ & $<0.5$ & $<0.5$ & $<0.5$ & $<0.5$ & $<0.5$ & na & $<0.5$ \\
\hline 1,1,2-Trichloroethane & $<0.5$ & $<0.5$ & $<0.5$ & $<0.5$ & $<0.5$ & $<0.5$ & na & $<0.5$ \\
\hline 1,1-Dichloroethane & $<0.5$ & $<0.5$ & $<0.5$ & $<0.5$ & $<0.5$ & $<0.5$ & na & $<0.5$ \\
\hline 1,1-Dichloroethene & $<0.5$ & $<0.5$ & $<0.5$ & $<0.5$ & $<0.5$ & $<0.5$ & na & $<0.5$ \\
\hline 1,2-Dichlorobenzene & $<0.5$ & $<0.5$ & $<0.5$ & $<0.5$ & $<0.5$ & $<0.5$ & na & $<0.5$ \\
\hline 1,2-Dichloroethane & $<0.5$ & $<0.5$ & $<0.5$ & $<0.5$ & $<0.5$ & $<0.5$ & na & $<0.5$ \\
\hline 1,2-Dichloroethene (total) & $<1$ & $<1$ & $<1$ & $<1$ & $<1$ & $<1$ & na & $<1$ \\
\hline 1,2-Dichloropropane & $<0.5$ & $<0.5$ & $<0.5$ & $<0.5$ & $<0.5$ & $<0.5$ & na & $<0.5$ \\
\hline 1,3-Dichlorobenzene & $<0.5$ & $<0.5$ & $<0.5$ & $<0.5$ & $<0.5$ & $<0.5$ & na & $<0.5$ \\
\hline 1,4-Dichlorobenzene & $<0.5$ & $<0.5$ & $<0.5$ & $<0.5$ & $<0.5$ & $<0.5$ & na & $<0.5$ \\
\hline Bromodichloromethane & $<0.5$ & $<0.5$ & $<0.5$ & $<0.5$ & $<0.5$ & $<0.5$ & na & $<0.5$ \\
\hline Bromoform & $<0.5$ & $<0.5$ & $<0.5$ & $<0.5$ & $<0.5$ & $<0.5$ & na & $<0.5$ \\
\hline Bromomethane & $<0.5$ & $<0.5$ & $<0.5$ & $<0.5$ & $<0.5$ & $<0.5$ & na & $<0.5$ \\
\hline Carbon tetrachloride & $<0.5$ & $<0.5$ & $<0.5$ & $<0.5$ & $<0.5$ & $<0.5$ & na & $<0.5$ \\
\hline Chlorobenzene & $<0.5$ & $<0.5$ & $<0.5$ & $<0.5$ & $<0.5$ & $<0.5$ & na & $<0.5$ \\
\hline Chloroethane & $<1$ & $<1$ & $<1$ & $<1$ & $<1$ & $<1$ & na & $<1$ \\
\hline Chloroform & $<0.5$ & $<0.5$ & $<0.5$ & $<0.5$ & $<0.5$ & $<0.5$ & na & $<0.5$ \\
\hline Chloromethane & $<1$ & $<1$ & $<1$ & $<1$ & $<1$ & $<1$ & na & $<1$ \\
\hline cis-1,2-Dichloroethene & $<0.5$ & $<0.5$ & $<0.5$ & $<0.5$ & $<0.5$ & $<0.5$ & na & $<0.5$ \\
\hline cis-1,3-Dichloropropene & $<0.5$ & $<0.5$ & $<0.5$ & $<0.5$ & $<0.5$ & $<0.5$ & na & $<0.5$ \\
\hline Dibromochloromethane & $<0.5$ & $<0.5$ & $<0.5$ & $<0.5$ & $<0.5$ & $<0.5$ & na & $<0.5$ \\
\hline Dichlorodifluoromethane & $<0.5$ & $<0.5$ & $<0.5$ & $<0.5$ & $<0.5$ & $<0.5$ & na & $<0.5$ \\
\hline
\end{tabular}


Table 7-15. Compliance monitoring data for releases from the Drainage Retention Basin, 1999 (continued).

\begin{tabular}{|c|c|c|c|c|c|c|c|c|}
\hline \multirow[t]{2}{*}{ Parameter } & \multicolumn{8}{|c|}{ WPDC sampling dates } \\
\hline & 1/20/99 & 2/8/99 & $4 / 8 / 99$ & 6/28/99 & $8 / 19 / 99$ & 10/4/99 & $10 / 8 / 99$ & $11 / 8 / 99$ \\
\hline \multicolumn{9}{|c|}{ Total metals (mg/L) (continued) } \\
\hline Lead & $<0.005$ & $<0.005$ & $<0.005$ & $<0.005$ & $<0.005$ & $<0.005$ & na & $<0.005$ \\
\hline Manganese & 0.028 & 0.065 & 0.078 & $<0.01$ & 0.034 & 0.022 & na & 0.11 \\
\hline Mercury & $<0.0002$ & $<0.0002$ & $<0.0002$ & $<0.0002$ & $<0.0002$ & $<0.0002$ & na & $<0.0002$ \\
\hline Molybdenum & $<0.025$ & $<0.025$ & $<0.025$ & $<0.025$ & $<0.025$ & $<0.025$ & na & $<0.025$ \\
\hline Nickel & 0.006 & 0.0071 & 0.0087 & $<0.002$ & $<0.002$ & 0.0021 & na & 0.014 \\
\hline Selenium & $<0.002$ & $<0.002$ & $<0.002$ & $<0.002$ & $<0.002$ & $<0.002$ & na & $<0.002$ \\
\hline Silver & $<0.001$ & $<0.001$ & $<0.001$ & $<0.001$ & $<0.001$ & $<0.001$ & na & $<0.001$ \\
\hline Thallium & $<0.001$ & $<0.001$ & $<0.001$ & $<0.001$ & $<0.001$ & $<0.001$ & na & $<0.001$ \\
\hline Vanadium & $<0.01$ & 0.012 & 0.011 & $<0.01$ & 0.013 & $<0.01$ & na & 0.014 \\
\hline Zinc & 0.087 & 0.071 & 0.097 & $<0.02$ & 0.022 & 0.024 & na & 0.29 \\
\hline \multicolumn{9}{|c|}{ Volatile organic compounds $(\mu \mathrm{g} / \mathrm{L})$} \\
\hline 1,1,1-Trichloroethane & na & na & $<0.5$ & $<0.5$ & $<0.5$ & na & na & na \\
\hline 1,1,2,2-Tetrachloroethane & na & na & $<0.5$ & $<0.5$ & $<0.5$ & na & na & na \\
\hline 1,1,2-Trichloroethane & na & na & $<0.5$ & $<0.5$ & $<0.5$ & na & na & na \\
\hline 1,1-Dichloroethane & na & na & $<0.5$ & $<0.5$ & $<0.5$ & na & na & na \\
\hline 1,1-Dichloroethene & na & na & $<0.5$ & $<0.5$ & $<0.5$ & na & na & na \\
\hline 1,2-Dichlorobenzene & na & na & $<0.5$ & $<0.5$ & $<0.5$ & na & na & na \\
\hline 1,2-Dichloroethane & na & na & $<0.5$ & $<0.5$ & $<0.5$ & na & na & na \\
\hline 1,2-Dichloroethene (total) & na & na & $<1$ & $<1$ & $<1$ & na & na & na \\
\hline 1,2-Dichloropropane & na & na & $<0.5$ & $<0.5$ & $<0.5$ & na & na & na \\
\hline 1,3-Dichlorobenzene & na & na & $<0.5$ & $<0.5$ & $<0.5$ & na & na & na \\
\hline 1,4-Dichlorobenzene & na & na & $<0.5$ & $<0.5$ & $<0.5$ & na & na & na \\
\hline Bromodichloromethane & na & na & $<0.5$ & $<0.5$ & $<0.5$ & na & na & na \\
\hline Bromoform & na & na & $<0.5$ & $<0.5$ & $<0.5$ & na & na & na \\
\hline Bromomethane & na & na & $<0.5$ & $<0.5$ & $<0.5$ & na & na & na \\
\hline Carbon tetrachloride & na & na & $<0.5$ & $<0.5$ & $<0.5$ & na & na & na \\
\hline Chlorobenzene & na & na & $<0.5$ & $<0.5$ & $<0.5$ & na & na & na \\
\hline Chloroethane & na & na & $<1$ & $<1$ & $<1$ & na & na & na \\
\hline Chloroform & na & na & $<0.5$ & $<0.5$ & $<0.5$ & na & na & na \\
\hline Chloromethane & na & na & $<1$ & $<1$ & $<1$ & na & na & na \\
\hline cis-1,2-Dichloroethene & na & na & $<0.5$ & $<0.5$ & $<0.5$ & na & na & na \\
\hline cis-1,3-Dichloropropene & na & na & $<0.5$ & $<0.5$ & $<0.5$ & na & na & na \\
\hline Dibromochloromethane & na & na & $<0.5$ & $<0.5$ & $<0.5$ & na & na & na \\
\hline Dichlorodifluoromethane & na & na & $<0.5$ & $<0.5$ & $<0.5$ & na & na & na \\
\hline
\end{tabular}




\section{Surface Water}

Table 7-15. Compliance monitoring data for releases from the Drainage Retention Basin, 1999 (continued).

\begin{tabular}{|c|c|c|c|c|c|c|c|c|}
\hline \multirow[t]{2}{*}{ Parameter } & \multicolumn{8}{|c|}{ CDBX sampling dates } \\
\hline & $1 / 20 / 99$ & 2/8/99 & $4 / 8 / 99$ & $6 / 28 / 99$ & $8 / 19 / 99$ & $10 / 4 / 99$ & $10 / 8 / 99$ & $11 / 8 / 99$ \\
\hline \multicolumn{9}{|c|}{$\begin{array}{l}\text { Volatile organic compounds ( } \mu \mathrm{g} / \mathrm{L} \text { ) } \\
\text { (continued) }\end{array}$} \\
\hline Freon 113 & $<0.5$ & $<0.5$ & $<0.5$ & $<0.5$ & $<0.5$ & $<0.5$ & na & $<0.5$ \\
\hline Methylene chloride & $<1$ & $<1$ & $<1$ & $<1$ & $<1$ & $<1$ & na & $<1$ \\
\hline Tetrachloroethene & $<0.5$ & $<0.5$ & $<0.5$ & $<0.5$ & $<0.5$ & $<0.5$ & na & $<0.5$ \\
\hline Total trihalomethanes & $<2$ & $<2$ & $<2$ & $<2$ & $<2$ & $<2$ & na & $<2$ \\
\hline trans-1,2-Dichloroethene & $<0.5$ & $<0.5$ & $<0.5$ & $<0.5$ & $<0.5$ & $<0.5$ & na & $<0.5$ \\
\hline trans-1,3-Dichloropropene & $<0.5$ & $<0.5$ & $<0.5$ & $<0.5$ & $<0.5$ & $<0.5$ & na & $<0.5$ \\
\hline Trichloroethene & $<0.5$ & $<0.5$ & $<0.5$ & $<0.5$ & $<0.5$ & $<0.5$ & na & $<0.5$ \\
\hline Trichlorofluoromethane & $<0.5$ & $<0.5$ & $<0.5$ & $<0.5$ & $<0.5$ & $<0.5$ & na & $<0.5$ \\
\hline Vinyl chloride & $<0.5$ & $<0.5$ & $<0.5$ & $<0.5$ & $<0.5$ & $<0.5$ & na & $<0.5$ \\
\hline \multicolumn{9}{|l|}{ Polychlorinated biphenyls ( $\mu \mathrm{g} / \mathrm{L}$ ) } \\
\hline РCB 1016 & $<0.2$ & $<0.2$ & $<0.2$ & na & na & na & na & $<0.3$ \\
\hline PCB 1221 & $<0.2$ & $<0.2$ & $<0.2$ & na & na & na & na & $<0.3$ \\
\hline РCB 1232 & $<0.2$ & $<0.2$ & $<0.2$ & na & na & na & na & $<0.3$ \\
\hline PCB 1242 & $<0.2$ & $<0.2$ & $<0.2$ & na & na & na & na & $<0.3$ \\
\hline PCB 1248 & $<0.2$ & $<0.2$ & $<0.2$ & na & na & na & na & $<0.3$ \\
\hline РCB 1254 & $<0.2$ & $<0.2$ & $<0.2$ & na & na & na & na & $<0.3$ \\
\hline РCB 1260 & $<0.2$ & $<0.2$ & $<0.2$ & na & na & na & na & $<0.3$ \\
\hline Total PCBs & $<0.2$ & $<0.2$ & $<0.2$ & na & na & na & na & $<0.3$ \\
\hline \multicolumn{9}{|l|}{ Herbicides $(\mu \mathrm{g} / \mathrm{L})$} \\
\hline Acennaphthylene & na & na & na & na & na & $<0.1$ & na & $<1$ \\
\hline Alachlor & na & na & na & na & na & $<0.2$ & na & $<2$ \\
\hline Aldrin & na & na & na & na & na & $<0.5$ & na & $<5$ \\
\hline Anthracene & na & na & na & na & na & $<0.1$ & na & $<1$ \\
\hline Atraton & na & na & na & na & na & $<0.5$ & na & $<5$ \\
\hline Atrazine & $<0.2$ & $<0.2$ & $<0.2$ & na & na & $<0.2$ & na & $<2$ \\
\hline Benzo(a)anthracene & na & na & na & na & na & $<0.3$ & na & $<3$ \\
\hline Benzo(a)pyrene & na & na & na & na & na & $<0.1$ & na & $<1$ \\
\hline Benzo(b)fluoranthene & na & na & na & na & na & $<0.3$ & na & $<3$ \\
\hline Benzo(g,h,i)perylene & na & na & na & na & na & $<0.3$ & na & $<3$ \\
\hline Benzo(k)fluoranthene & na & na & na & na & na & $<0.3$ & na & $<3$ \\
\hline $\mathrm{BHC}$, delta isomer & na & na & na & na & na & $<0.2$ & na & $<2$ \\
\hline $\mathrm{BHC}$, gamma isomer (Lindane) & na & na & na & na & na & $<0.1$ & na & $<1$ \\
\hline Bromacil & $<0.5$ & 6 & 4.4 & na & na & $<0.5$ & na & $<5$ \\
\hline
\end{tabular}


Table 7-15. Compliance monitoring data for releases from the Drainage Retention Basin, 1999 (continued).

\begin{tabular}{|c|c|c|c|c|c|c|c|c|}
\hline \multirow[t]{2}{*}{ Parameter } & \multicolumn{8}{|c|}{ WPDC sampling dates } \\
\hline & $1 / 20 / 99$ & 2/8/99 & $4 / 8 / 99$ & $6 / 28 / 99$ & 8/19/99 & $10 / 4 / 99$ & $10 / 8 / 99$ & $11 / 8 / 99$ \\
\hline \multicolumn{9}{|c|}{$\begin{array}{l}\text { Volatile organic compounds }(\mu \mathrm{g} / \mathrm{L}) \\
\text { (continued) }\end{array}$} \\
\hline Freon 113 & na & na & $<0.5$ & $<0.5$ & $<0.5$ & na & na & na \\
\hline Methylene chloride & na & na & $<1$ & $<1$ & $<1$ & na & na & na \\
\hline Tetrachloroethene & na & na & $<0.5$ & $<0.5$ & $<0.5$ & na & na & na \\
\hline Total trihalomethanes & na & na & $<2$ & $<2$ & $<2$ & na & na & na \\
\hline trans-1,2-Dichloroethene & na & na & $<0.5$ & $<0.5$ & $<0.5$ & na & na & na \\
\hline trans-1,3-Dichloropropene & na & na & $<0.5$ & $<0.5$ & $<0.5$ & na & na & na \\
\hline Trichloroethene & na & na & $<0.5$ & $<0.5$ & $<0.5$ & na & na & na \\
\hline Trichlorofluoromethane & na & na & $<0.5$ & $<0.5$ & $<0.5$ & na & na & na \\
\hline Vinyl chloride & na & na & $<0.5$ & $<0.5$ & $<0.5$ & na & na & na \\
\hline \multicolumn{9}{|l|}{ Polychlorinated biphenyls ( $\mu \mathrm{g} / \mathrm{L}$ ) } \\
\hline РCB 1016 & $<0.2$ & $<0.2$ & $<0.2$ & na & na & na & na & $<0.2$ \\
\hline РCB 1221 & $<0.2$ & $<0.2$ & $<0.2$ & na & na & na & na & $<0.2$ \\
\hline PCB 1232 & $<0.2$ & $<0.2$ & $<0.2$ & na & na & na & na & $<0.2$ \\
\hline РCB 1242 & $<0.2$ & $<0.2$ & $<0.2$ & na & na & na & na & $<0.2$ \\
\hline РCB 1248 & $<0.2$ & $<0.2$ & $<0.2$ & na & na & na & na & $<0.2$ \\
\hline РCB 1254 & $<0.2$ & $<0.2$ & $<0.2$ & na & na & na & na & $<0.2$ \\
\hline PCB 1260 & $<0.2$ & $<0.2$ & $<0.2$ & na & na & na & na & $<0.2$ \\
\hline Total PCBs & $<0.2$ & $<0.2$ & $<0.2$ & na & na & na & na & $<0.2$ \\
\hline \multicolumn{9}{|l|}{ Herbicides $(\mu \mathrm{g} / \mathrm{L})$} \\
\hline Acennaphthylene & na & na & na & na & na & na & na & $<0.1$ \\
\hline Alachlor & na & na & na & na & na & na & na & $<0.3$ \\
\hline Aldrin & na & na & na & na & na & na & na & $<0.7$ \\
\hline Anthracene & na & na & na & na & na & na & na & $<0.1$ \\
\hline Atraton & na & na & na & na & na & na & na & $<0.7$ \\
\hline Atrazine & $<0.2$ & $<0.2$ & $<0.2$ & na & na & na & na & $<0.3$ \\
\hline Benzo(a)anthracene & na & na & na & na & na & na & na & $<0.4$ \\
\hline Benzo(a)pyrene & na & na & na & na & na & na & na & 0.24 \\
\hline Benzo(b)fluoranthene & na & na & na & na & na & na & na & 0.44 \\
\hline Benzo(g,h,i)perylene & na & na & na & na & na & na & na & $<0.4$ \\
\hline Benzo(k)fluoranthene & na & na & na & na & na & na & na & $<0.4$ \\
\hline $\mathrm{BHC}$, delta isomer & na & na & na & na & na & na & na & $<0.3$ \\
\hline $\mathrm{BHC}$, gamma isomer (Lindane) & na & na & na & na & na & na & na & $<0.1$ \\
\hline Bromacil & $<0.5$ & 270 & 3.6 & na & na & na & na & 2.2 \\
\hline
\end{tabular}




\section{Surface Water}

Table 7-15. Compliance monitoring data for releases from the Drainage Retention Basin, 1999 (continued).

\begin{tabular}{|c|c|c|c|c|c|c|c|c|}
\hline \multirow[t]{2}{*}{ Parameter } & \multicolumn{8}{|c|}{ CDBX sampling dates } \\
\hline & $1 / 20 / 99$ & $2 / 8 / 99$ & $4 / 8 / 99$ & $6 / 28 / 99$ & $8 / 19 / 99$ & $10 / 4 / 99$ & $10 / 8 / 99$ & $11 / 8 / 99$ \\
\hline \multicolumn{9}{|c|}{$\begin{array}{l}\text { Volatile organic compounds ( } \mu \mathrm{g} / \mathrm{L} \text { ) } \\
\text { (continued) }\end{array}$} \\
\hline Butachlor & $<0.3$ & $<0.3$ & $<0.3$ & na & na & $<0.3$ & na & na \\
\hline Butylbenzylphthalate & na & na & na & na & na & $<1$ & na & $<10$ \\
\hline Chlordane & na & na & na & na & na & $<2$ & na & $<20$ \\
\hline Chrysene & na & na & na & na & na & $<0.3$ & na & $<3$ \\
\hline Di (2-ethylhexyl) adipate & na & na & na & na & na & $<1$ & na & $<10$ \\
\hline Diazinon & $<0.2$ & $<0.2$ & $<0.2$ & na & na & $<0.2$ & na & $<2$ \\
\hline Dibenzo( $a, h)$ anthracene & na & na & na & na & na & $<0.3$ & na & $<3$ \\
\hline Dibutylphthalate & na & na & na & na & na & $<1$ & na & $<10$ \\
\hline Diethylhexylphthalate & na & na & na & na & na & $<3$ & na & $<30$ \\
\hline Diethylphthalate & na & na & na & na & na & $<3$ & na & $<30$ \\
\hline Dimethoate & $<2$ & $<2$ & $<2$ & na & na & $<2$ & na & $<20$ \\
\hline Dimethylphthalate & na & na & na & na & na & $<1$ & na & $<10$ \\
\hline Diuron & 5.2 & 2.1 & $<1$ & na & na & $<1$ & na & $<2$ \\
\hline Endrin & na & na & na & na & na & $<0.2$ & na & $<2$ \\
\hline Fluorene & na & na & na & na & na & $<0.1$ & na & $<1$ \\
\hline Glyphosate & $<9$ & $<9$ & $<9$ & na & na & $<9$ & na & 100 \\
\hline Heptachlor & na & na & na & na & na & $<0.1$ & na & $<1$ \\
\hline Heptachlor epoxide & na & na & na & na & na & $<0.1$ & na & $<1$ \\
\hline Hexachlorobenzene & na & na & na & na & na & $<0.5$ & na & $<5$ \\
\hline Hexachlorocyclopentadiene & na & na & na & na & na & $<1$ & na & $<10$ \\
\hline Indeno(1,2,3-c,d)pyrene & na & na & na & na & na & $<0.3$ & na & $<3$ \\
\hline Methoxychlor & na & na & na & na & na & $<0.5$ & na & $<5$ \\
\hline Metolachlor & $<0.5$ & $<0.5$ & $<0.5$ & na & na & $<0.5$ & na & $<5$ \\
\hline Metribuzin & $<0.5$ & $<0.5$ & $<0.5$ & na & na & $<0.5$ & na & $<5$ \\
\hline Molinate & $<0.5$ & $<0.5$ & $<0.5$ & na & na & $<0.5$ & na & $<5$ \\
\hline Pentachlorophenol & na & na & na & na & na & $<1$ & na & $<10$ \\
\hline Phenanthrene & na & na & na & na & na & $<0.1$ & na & $<1$ \\
\hline Prometon & na & na & na & na & na & $<0.5$ & na & $<5$ \\
\hline Prometryn & $<0.5$ & $<0.5$ & $<0.5$ & na & na & $<0.5$ & na & $<5$ \\
\hline Propachlor & $<0.5$ & $<0.5$ & $<0.5$ & na & na & $<0.5$ & na & na \\
\hline Pyrene & na & na & na & na & na & $<0.1$ & na & $<1$ \\
\hline Secbumeton & na & na & na & na & na & $<0.5$ & na & $<5$ \\
\hline Simazine & $<0.2$ & $<0.2$ & $<0.2$ & na & na & $<0.2$ & na & $<2$ \\
\hline
\end{tabular}


Table 7-15. Compliance monitoring data for releases from the Drainage Retention Basin, 1999 (continued).

\begin{tabular}{|c|c|c|c|c|c|c|c|c|}
\hline \multirow[t]{2}{*}{ Parameter } & \multicolumn{8}{|c|}{ WPDC sampling dates } \\
\hline & $1 / 20 / 99$ & $2 / 8 / 99$ & $4 / 8 / 99$ & $6 / 28 / 99$ & 8/19/99 & $10 / 4 / 99$ & $10 / 8 / 99$ & $11 / 8 / 99$ \\
\hline \multicolumn{9}{|c|}{$\begin{array}{l}\text { Volatile organic compounds }(\mu \mathrm{g} / \mathrm{L}) \\
\text { (continued) }\end{array}$} \\
\hline Butachlor & $<0.3$ & $<0.3$ & $<0.3$ & na & na & na & na & na \\
\hline Butylbenzylphthalate & na & na & na & na & na & na & na & $<1$ \\
\hline Chlordane & na & na & na & na & na & na & na & $<3$ \\
\hline Chrysene & na & na & na & na & na & na & na & $<0.4$ \\
\hline Di (2-ethylhexyl) adipate & na & na & na & na & na & na & na & $<1$ \\
\hline Diazinon & na & na & $<0.2$ & na & na & na & na & $<0.3$ \\
\hline Dibenzo $(a, h)$ anthracene & na & na & na & na & na & na & na & $<0.4$ \\
\hline Dibutylphthalate & na & na & na & na & na & na & na & $<1$ \\
\hline Diethylhexylphthalate & na & na & na & na & na & na & na & 5.4 \\
\hline Diethylphthalate & na & na & na & na & na & na & na & $<4$ \\
\hline Dimethoate & na & na & $<2$ & na & na & na & na & $<3$ \\
\hline Dimethylphthalate & na & na & na & na & na & na & na & $<1$ \\
\hline Diuron & 63 & 6.3 & $<1$ & na & na & na & na & $<1$ \\
\hline Endrin & na & na & na & na & na & na & na & $<0.3$ \\
\hline Fluorene & na & na & na & na & na & na & na & $<0.1$ \\
\hline Glyphosate & $<9$ & $<9$ & $<9$ & na & na & na & na & 99 \\
\hline Heptachlor & na & na & na & na & na & na & na & $<0.1$ \\
\hline Heptachlor epoxide & na & na & na & na & na & na & na & $<0.1$ \\
\hline Hexachlorobenzene & na & na & na & na & na & na & na & $<0.7$ \\
\hline Hexachlorocyclopentadiene & na & na & na & na & na & na & na & $<1$ \\
\hline Indeno(1,2,3-c,d)pyrene & na & na & na & na & na & na & na & $<0.4$ \\
\hline Methoxychlor & na & na & na & na & na & na & na & $<0.7$ \\
\hline Metolachlor & $<0.5$ & $<0.5$ & $<0.5$ & na & na & na & na & $<0.7$ \\
\hline Metribuzin & $<0.5$ & $<0.5$ & $<0.5$ & na & na & na & na & $<0.7$ \\
\hline Molinate & $<0.5$ & $<0.5$ & $<0.5$ & na & na & na & na & $<0.7$ \\
\hline Pentachlorophenol & na & na & na & na & na & na & na & $<1$ \\
\hline Phenanthrene & na & na & na & na & na & na & na & $<0.1$ \\
\hline Prometon & na & na & na & na & na & na & na & $<0.7$ \\
\hline Prometryn & $<0.5$ & $<0.5$ & $<0.5$ & na & na & na & na & $<0.7$ \\
\hline Propachlor & $<0.5$ & $<0.5$ & $<0.5$ & na & na & na & na & na \\
\hline Pyrene & na & na & na & na & na & na & na & $<0.1$ \\
\hline Secbumeton & na & na & na & na & na & na & na & $<0.7$ \\
\hline Simazine & $<0.2$ & $<0.2$ & $<0.2$ & na & na & na & na & $<0.3$ \\
\hline
\end{tabular}




\section{Surface Water}

Table 7-15. Compliance monitoring data for releases from the Drainage Retention Basin, 1999 (continued).

\begin{tabular}{|c|c|c|c|c|c|c|c|c|}
\hline \multirow[t]{2}{*}{ Parameter } & \multicolumn{8}{|c|}{ CDBX sampling dates } \\
\hline & $1 / 20 / 99$ & $2 / 8 / 99$ & $4 / 8 / 99$ & 6/28/99 & $8 / 19 / 99$ & $10 / 4 / 99$ & $10 / 8 / 99$ & $11 / 8 / 99$ \\
\hline \multicolumn{9}{|c|}{$\begin{array}{l}\text { Volatile organic compounds ( } \mu \mathrm{g} / \mathrm{L}) \\
\text { (continued) }\end{array}$} \\
\hline Terbutryn & na & na & na & na & na & $<0.5$ & na & $<5$ \\
\hline Thiobencarb & $<0.5$ & $<0.5$ & $<0.5$ & na & na & $<0.5$ & na & $<5$ \\
\hline \multicolumn{9}{|l|}{ Miscellaneous organics (mg/L) } \\
\hline Chemical oxygen demand & 24 & 71 & 43 & na & na & $<20$ & na & 37 \\
\hline Oil and grease & 1.1 & $<1$ & $<1$ & na & na & na & na & 1.9 \\
\hline Total organic carbon (TOC) & 5.2 & 4.4 & 3.5 & na & na & 3.7 & na & 8.8 \\
\hline
\end{tabular}

\begin{tabular}{|l|c|c|c|c|c|c|c|c|}
\hline \multirow{2}{*}{ Parameter } & \multicolumn{9}{c|}{ Sampling dates } \\
\cline { 2 - 8 } & $\mathbf{1 / 2 0 / 9 9}$ & $\mathbf{2 / 8 / 9 9}$ & $\mathbf{4 / 8 / 9 9}$ & $\mathbf{6 / 2 8 / 9 9}$ & $\mathbf{8 / 1 9 / 9 9}$ & $\mathbf{1 0 / 4 / 9 9}$ & $\mathbf{1 0 / 8 / 9 9}$ & $\mathbf{1 1 / 8 / 9 9}$ \\
\hline Radioactivity (Bq/L) & & & & & & & & \\
Gross alpha & $0.063 \pm 0.052$ & $0.17 \pm 0.085$ & $0.094 \pm 0.064$ & na & na & $0.096 \pm 0.044$ & na & $0.116 \pm 0.065$ \\
Gross beta & $0.118 \pm 0.074$ & $0.135 \pm 0.096$ & $0.047 \pm 0.08$ & na & na & $0.085 \pm 0.044$ & na & $0.17 \pm 0.05$ \\
Tritium & $21.7 \pm 2.6$ & $29.6 \pm 2.7$ & $23.3 \pm 3.4$ & na & na & 31.4 & na & $21.6 \pm 3.0$ \\
\hline
\end{tabular}

Note: Radioactivities are reported as the measured concentration and either an uncertainty $( \pm 2 \sigma$ counting error) or as being less than or equal to the detection limit. If the concentration is less than or equal to the uncertainty or the detection limit, the result is considered to be a nondetection. See the main volume, Chapter 14, Quality Assurance.

a $\mathrm{na}=$ Not analyzed because the analysis was not required.

b $\quad \mathrm{IC}_{25}=25 \%$ inhibition concentration; concentration at which $25 \%$ of the organisms show inhibition responses. 
Table 7-15. Compliance monitoring data for releases from the Drainage Retention Basin, 1999 (concluded).

\begin{tabular}{|c|c|c|c|c|c|c|c|c|}
\hline \multirow{2}{*}{ Parameter } & \multicolumn{8}{|c|}{ WPDC sampling dates } \\
\hline & $1 / 20 / 99$ & $2 / 8 / 99$ & $4 / 8 / 99$ & $6 / 28 / 99$ & $8 / 19 / 99$ & $10 / 4 / 99$ & 10/8/99 & $11 / 8 / 99$ \\
\hline \multicolumn{9}{|c|}{$\begin{array}{l}\text { Volatile organic compounds }(\mu \mathrm{g} / \mathrm{L}) \\
\text { (continued) }\end{array}$} \\
\hline Terbutryn & na & na & na & na & na & na & na & $<0.7$ \\
\hline Thiobencarb & $<0.5$ & $<0.5$ & $<0.5$ & na & na & na & na & $<0.7$ \\
\hline \multicolumn{9}{|l|}{ Miscellaneous organics (mg/L) } \\
\hline Chemical oxygen demand & 30 & 79 & 40 & na & na & na & na & 64 \\
\hline Oil and grease & $<1$ & $<1$ & $<1$ & na & na & na & na & $<1$ \\
\hline Total organic carbon (TOC) & 8.5 & 4.5 & 6.2 & na & na & na & na & 17 \\
\hline
\end{tabular}

\begin{tabular}{|l|c|c|c|c|c|c|c|c|}
\hline \multirow{2}{*}{ Parameter } & \multicolumn{9}{c|}{ Sampling dates } \\
\cline { 2 - 8 } & $\mathbf{1 / 2 0 / 9 9}$ & $\mathbf{2 / 8 / 9 9}$ & $\mathbf{4 / 8 / 9 9}$ & $\mathbf{6 / 2 8 / 9 9}$ & $\mathbf{8 / 1 9 / 9 9}$ & $\mathbf{1 0 / 4 / 9 9}$ & $\mathbf{1 0 / 8 / 9 9}$ & $\mathbf{1 1 / 8 / 9 9}$ \\
\hline Radioactivity (Bq/L) & & & & & & & & \\
Gross alpha & $0.032 \pm 0.044$ & $0.044 \pm 0.041$ & $0.063 \pm 0.046$ & na & na & na & na & $0.066 \pm 0.04$ \\
Gross beta & $0.208 \pm 0.078$ & $0.16 \pm 0.074$ & $0.224 \pm 0.051$ & na & na & na & na & $0.237 \pm 0.044$ \\
Tritium & $13.1 \pm 2.3$ & $70.7 \pm 5.6$ & $11.2 \pm 3.0$ & na & na & na & na & $37.8 \pm 3.5$ \\
\hline
\end{tabular}

c $\quad \mathrm{IC}_{50}=50 \%$ inhibition concentration: concentration at which $50 \%$ of the organisms show inhibition responses.

d LOEC = Lowest observed effect concentration.

e $\mathrm{NOEC}=$ No observed effect concentration.

$f \quad L_{50}=50 \%$ lethal concentration; concentration at which $50 \%$ of the organisms die. 


\section{Surface Water}

Table 7-16. Monthly analyses of water samples collected from the Drainage Retention Basin location CDBE, 1999.

\begin{tabular}{|c|c|c|c|c|c|c|}
\hline \multirow[b]{2}{*}{ Parameter } & \multicolumn{6}{|c|}{ Sampling dates } \\
\hline & 1/13/99 & $2 / 4 / 99$ & $3 / 11 / 99$ & 4/26/99 & $5 / 4 / 99$ & $6 / 14 / 99$ \\
\hline \multicolumn{7}{|l|}{ Nutrients (mg/L) } \\
\hline Ammonia nitrogen (as $\mathrm{N}$ ) & 0.03 & 0.02 & 0.06 & 0.07 & 0.22 & 0.07 \\
\hline Nitrate (as N) & 1.7 & 1.2 & 0.54 & $<0.1$ & 0.33 & 0.77 \\
\hline Nitrate $\left(\right.$ as $\left.\mathrm{NO}_{3}\right)$ & 7.7 & 5.4 & 2.4 & $<0.4$ & 1.4 & 3.4 \\
\hline Nitrite (as N) & 0.03 & 0.02 & 0.02 & $<0.02$ & $<0.02$ & $<0.02$ \\
\hline Nitrite (as $\mathrm{NO}_{2}$ ) & 0.09 & 0.07 & 0.07 & $<0.07$ & $<0.07$ & $<0.07$ \\
\hline Total Kjeldahl nitrogen & 1.0 & 0.94 & 1.2 & 1.0 & 1.2 & 1.3 \\
\hline \multicolumn{7}{|l|}{ General minerals (mg/L) } \\
\hline Aluminum & 0.15 & 0.61 & 0.6 & 0.3 & 1.4 & 1.5 \\
\hline Bicarbonate alkalinity $\left(\right.$ as $\left.\mathrm{CaCO}_{3}\right)$ & 147 & 137 & 116 & 152 & 125 & 176 \\
\hline Calcium & 53 & 42 & 32 & 37 & 40 & 53 \\
\hline Carbonate alkalinity $\left(\mathrm{as} \mathrm{CaCO}_{3}\right)$ & 62 & 22 & 30 & $<5$ & 33 & 28 \\
\hline Chloride & 134 & 111 & 89 & 108 & 116 & 160 \\
\hline Copper & $<0.01$ & $<0.01$ & $<0.01$ & $<0.01$ & 0.026 & 0.033 \\
\hline Fluoride & 0.44 & 0.32 & 0.32 & 0.40 & 0.36 & 0.49 \\
\hline Hydroxide alkalinity $\left(\mathrm{as} \mathrm{CaCO}_{3}\right)$ & $<5$ & $<5$ & $<5$ & $<5$ & $<5$ & $<5$ \\
\hline Iron & 0.16 & 0.6 & 0.6 & 0.29 & 1.4 & 1.6 \\
\hline Magnesium & 26 & 21 & 16 & 22 & 24 & 31 \\
\hline Manganese & 0.016 & 0.025 & 0.059 & 0.039 & 0.23 & 0.12 \\
\hline Nickel & $<0.05$ & $<0.05$ & $<0.05$ & $<0.05$ & $<0.05$ & $<0.05$ \\
\hline Nitrate (as N) & 1.7 & 1.2 & 0.57 & $<0.1$ & 0.33 & 0.77 \\
\hline Nitrate $\left(\right.$ as $\left.\mathrm{NO}_{3}\right)$ & 7.6 & 5.4 & 2.5 & $<0.4$ & 1.4 & 3.4 \\
\hline Nitrite (as N) & 0.03 & 0.02 & 0.02 & $<0.02$ & $<0.02$ & $<0.02$ \\
\hline Orthophosphate & $<0.05$ & 0.12 & 0.25 & $<0.05$ & 0.32 & $<0.05$ \\
\hline $\mathrm{pH}$ (pH units) & 9.1 & 8.7 & 9.2 & 9.1 & 9.1 & 9.1 \\
\hline Potassium & 2.0 & 1.9 & 2.2 & 2.1 & 2.2 & 2.4 \\
\hline Sodium & 94 & 76 & 70 & 80 & 86 & 115 \\
\hline Specific conductance $(\mu \mathrm{mho} / \mathrm{cm})$ & 910 & 736 & 648 & 710 & 773 & 1000 \\
\hline Sulfate & 48 & 51 & 48 & 45 & 45 & 56 \\
\hline Surfactants & $<0.05$ & $<0.1$ & 0.09 & $<0.05$ & $<0.05$ & $<0.05$ \\
\hline Total alkalinity $\left(\mathrm{as} \mathrm{CaCO}_{3}\right)$ & 209 & 159 & 146 & 152 & 158 & 204 \\
\hline Total dissolved solids (TDS) & 510 & 457 & 372 & 414 & 435 & 600 \\
\hline Total hardness (as $\mathrm{CaCO}_{3}$ ) & 239 & 191 & 146 & 183 & 199 & 260 \\
\hline Total phosphorus (as P) & 0.08 & 0.12 & 0.17 & 0.1 & 0.21 & 0.21 \\
\hline Total solids & na & na & 398 & 458 & 390 & 623 \\
\hline
\end{tabular}


Table 7-16. Monthly analyses of water samples collected from the Drainage Retention Basin location CDBE, 1999 (continued).

\begin{tabular}{|c|c|c|c|c|c|c|}
\hline \multirow[b]{2}{*}{ Parameter } & \multicolumn{6}{|c|}{ Sampling dates } \\
\hline & 1/13/99 & 2/4/99 & 3/11/99 & $4 / 26 / 99$ & $5 / 4 / 99$ & $6 / 14 / 99$ \\
\hline \multicolumn{7}{|l|}{ General minerals (mg/L) (continued) } \\
\hline Total suspended solids (TSS) & 14 & 16 & 7.6 & 15 & 14 & 59 \\
\hline Volatile solids & na & 15 & 4.8 & 114 & 90 & 217 \\
\hline Volatile suspended solids & na & 87 & 76 & 10 & 4.5 & 20 \\
\hline Zinc & $<0.01$ & 0.016 & 0.012 & $<0.01$ & 0.028 & 0.019 \\
\hline \multicolumn{7}{|l|}{ Total metals (mg/L) } \\
\hline Aluminum & 0.13 & 0.56 & 0.6 & 0.29 & 0.74 & 1.3 \\
\hline Antimony & $<0.004$ & $<0.004$ & $<0.004$ & $<0.004$ & $<0.004$ & $<0.004$ \\
\hline Arsenic & 0.0026 & $<0.002$ & 0.0021 & 0.0031 & 0.0037 & 0.0044 \\
\hline Barium & 0.13 & 0.12 & 0.093 & 0.093 & 0.1 & 0.15 \\
\hline Beryllium & $<0.0002$ & $<0.0002$ & $<0.0002$ & $<0.0002$ & $<0.0002$ & $<0.0002$ \\
\hline Boron & 1.4 & 1.5 & 1.5 & 1.5 & 1.5 & 1.8 \\
\hline Cadmium & $<0.0005$ & $<0.0005$ & $<0.0005$ & $<0.0005$ & $<0.0005$ & $<0.0005$ \\
\hline Chromium & 0.0058 & 0.005 & 0.0049 & 0.0036 & 0.0055 & 0.0062 \\
\hline Chromium(VI) & 0.0062 & 0.0071 & 0.0056 & 0.0048 & $<0.002$ & 0.0073 \\
\hline Cobalt & $<0.05$ & $<0.05$ & $<0.05$ & $<0.05$ & $<0.05$ & $<0.05$ \\
\hline Copper & 0.0018 & 0.0068 & 0.004 & 0.0047 & 0.0063 & 0.0068 \\
\hline Iron & 0.16 & 0.55 & 0.59 & 0.28 & 0.79 & 1.6 \\
\hline Lead & $<0.005$ & 0.0093 & $<0.005$ & $<0.005$ & $<0.005$ & $<0.005$ \\
\hline Manganese & 0.016 & 0.023 & 0.059 & 0.038 & 0.16 & 0.11 \\
\hline Mercury & $<0.0002$ & $<0.0002$ & $<0.0002$ & $<0.0002$ & $<0.0002$ & $<0.0002$ \\
\hline Molybdenum & $<0.025$ & $<0.025$ & $<0.025$ & $<0.025$ & $<0.025$ & $<0.025$ \\
\hline Nickel & 0.0033 & 0.0031 & 0.0038 & 0.0028 & 0.0045 & 0.007 \\
\hline Selenium & $<0.002$ & $<0.002$ & $<0.002$ & $<0.002$ & $<0.002$ & $<0.002$ \\
\hline Silver & $<0.001$ & $<0.001$ & $<0.001$ & $<0.001$ & $<0.001$ & $<0.001$ \\
\hline Thallium & $<0.001$ & $<0.001$ & $<0.001$ & $<0.001$ & $<0.001$ & $<0.001$ \\
\hline Vanadium & $<0.01$ & $<0.01$ & $<0.01$ & $<0.01$ & $<0.01$ & 0.013 \\
\hline Zinc & $<0.02$ & $<0.02$ & $<0.02$ & $<0.02$ & 0.029 & $<0.02$ \\
\hline \multicolumn{7}{|l|}{ Miscellaneous organics $(\mu \mathrm{g} / \mathrm{L})$} \\
\hline Chlorophyll-a & 54.5 & 28 & 36.7 & 16 & 5.0 & 73.7 \\
\hline
\end{tabular}




\section{Surface Water}

Table 7-16. Monthly analyses of water samples collected from the Drainage Retention Basin location CDBE, 1999 (continued).

\begin{tabular}{|c|c|c|c|c|c|c|}
\hline \multirow[b]{2}{*}{ Parameter } & \multicolumn{6}{|c|}{ Sampling dates } \\
\hline & 7/29/99 & $8 / 19 / 99$ & 9/9/99 & $10 / 4 / 99$ & $11 / 4 / 99$ & $12 / 2 / 99$ \\
\hline \multicolumn{7}{|l|}{ Nutrients (mg/L) } \\
\hline Ammonia nitrogen (as $\mathrm{N}$ ) & $<0.02$ & 0.04 & 0.14 & 0.09 & 0.04 & 0.10 \\
\hline Nitrate (as N) & 1.20 & 0.59 & 0.46 & 1.1 & 2.0 & 1.9 \\
\hline Nitrate $\left(\right.$ as $\left.\mathrm{NO}_{3}\right)$ & 5.2 & 2.6 & 2.1 & 4.9 & 8.9 & 8.6 \\
\hline Nitrite (as N) & 0.03 & $<0.02$ & 0.04 & 0.02 & 0.03 & 0.03 \\
\hline Nitrite (as $\mathrm{NO}_{2}$ ) & 0.09 & $<0.07$ & 0.15 & $<0.50$ & $<0.50$ & $<0.50$ \\
\hline Total Kjeldahl nitrogen & 0.96 & 0.96 & 0.95 & 0.57 & 0.68 & 0.71 \\
\hline \multicolumn{7}{|l|}{ General minerals (mg/L) } \\
\hline Aluminum & 1.7 & 2.0 & 2.0 & 0.56 & 0.95 & 0.64 \\
\hline Bicarbonate alkalinity (as $\mathrm{CaCO}_{3}$ ) & 211 & 191 & 206 & 226 & 204 & 182 \\
\hline Calcium & 58 & 45 & 51 & 52 & 59 & 47 \\
\hline Carbonate alkalinity $\left(\mathrm{as} \mathrm{CaCO}_{3}\right)$ & 18 & 28 & 17 & $<5$ & 22 & 35 \\
\hline Chloride & 202 & 212 & 223 & 215 & 208 & 186 \\
\hline Copper & 0.019 & 0.023 & $<0.01$ & $<0.01$ & $<0.01$ & $<0.01$ \\
\hline Fluoride & 0.49 & 0.51 & 0.54 & 0.56 & 0.52 & 0.43 \\
\hline Hydroxide alkalinity $\left(\mathrm{as} \mathrm{CaCO}_{3}\right)$ & $<5$ & $<5$ & $<5$ & $<5$ & $<5$ & $<5$ \\
\hline Iron & 1.7 & 1.8 & 1.9 & 0.49 & 0.91 & 0.62 \\
\hline Magnesium & 36 & 37 & 41 & 38 & 39 & 29 \\
\hline Manganese & 0.081 & 0.11 & 0.095 & 0.018 & 0.034 & 0.025 \\
\hline Nickel & $<0.05$ & $<0.05$ & $<0.05$ & $<0.05$ & $<0.05$ & $<0.05$ \\
\hline Nitrate (as N) & 1.2 & 0.59 & 0.49 & 1.2 & 2.0 & 1.9 \\
\hline Nitrate $\left(\right.$ as $\left.\mathrm{NO}_{3}\right)$ & 5.1 & 2.6 & 2.2 & 5.2 & 8.9 & 8.6 \\
\hline Nitrite (as N) & 0.02 & 0.02 & 0.04 & 0.02 & 0.03 & 0.03 \\
\hline Orthophosphate & 0.32 & 0.06 & 0.18 & 0.22 & 0.16 & 0.13 \\
\hline $\mathrm{pH}$ (pH units) & 8.6 & 8.4 & 8.3 & 8.3 & 8.6 & 8.7 \\
\hline Potassium & 2.7 & 2.9 & 3.0 & 2.5 & 2.5 & 1.9 \\
\hline Sodium & 128 & 132 & 142 & 132 & 132 & 100 \\
\hline Specific conductance $(\mu \mathrm{mho} / \mathrm{cm})$ & 1180 & 1160 & 1200 & 1210 & 1210 & 1100 \\
\hline Sulfate & 69 & 71 & 77 & 74 & 74 & 65 \\
\hline Surfactants & $<0.1$ & 0.05 & $<0.05$ & $<0.05$ & $<0.05$ & $<0.05$ \\
\hline Total alkalinity $\left(\right.$ as $\left.\mathrm{CaCO}_{3}\right)$ & 229 & 219 & 223 & 226 & 226 & 217 \\
\hline Total dissolved solids (TDS) & 745 & 680 & 695 & 700 & 735 & 653 \\
\hline Total hardness $\left(\mathrm{as} \mathrm{CaCO}_{3}\right)$ & 293 & 265 & 296 & 286 & 308 & 237 \\
\hline Total phosphorus (as P) & 0.21 & 0.12 & 0.14 & 0.14 & 0.13 & 0.1 \\
\hline Total solids & 760 & 720 & 735 & 760 & 765 & 670 \\
\hline
\end{tabular}


Table 7-16. Monthly analyses of water samples collected from the Drainage Retention Basin location CDBE, 1999 (continued).

\begin{tabular}{|c|c|c|c|c|c|c|}
\hline \multirow[b]{2}{*}{ Parameter } & \multicolumn{6}{|c|}{ Sampling dates } \\
\hline & $7 / 29 / 99$ & $8 / 19 / 99$ & 9/9/99 & 10/4/99 & $11 / 4 / 99$ & 12/2/99 \\
\hline \multicolumn{7}{|l|}{ General minerals (mg/L) (continued) } \\
\hline Total suspended solids (TSS) & 30 & 35 & 21 & 17 & 13 & 9.2 \\
\hline Volatile solids & 190 & 195 & 180 & 190 & 165 & 133 \\
\hline Volatile suspended solids & 6.5 & 7.3 & 4 & 6 & 3.4 & 2 \\
\hline Zinc & 0.045 & 0.03 & 0.021 & $<0.05$ & 0.082 & 0.03 \\
\hline \multicolumn{7}{|l|}{ Total metals (mg/L) } \\
\hline Aluminum & 1.8 & na & 2.2 & 1.0 & 1.0 & 0.49 \\
\hline Antimony & $<0.004$ & $<0.004$ & $<0.004$ & $<0.004$ & $<0.004$ & $<0.004$ \\
\hline Arsenic & 0.006 & 0.0029 & 0.0035 & 0.0022 & 0.0023 & 0.003 \\
\hline Barium & 0.18 & 0.15 & 0.19 & 0.16 & 0.18 & 0.14 \\
\hline Beryllium & $<0.0002$ & $<0.0002$ & $<0.0002$ & $<0.0002$ & $<0.0002$ & $<0.0002$ \\
\hline Boron & 2.1 & 2.1 & 2.3 & 2.0 & 2.0 & 1.5 \\
\hline Cadmium & $<0.0005$ & $<0.0005$ & $<0.0005$ & $<0.0005$ & $<0.0005$ & $<0.0005$ \\
\hline Chromium & 0.0084 & 0.0077 & 0.0048 & 0.0028 & 0.0051 & 0.0057 \\
\hline Chromium(VI) & 0.002 & 0.006 & 0.002 & 0.0031 & 0.0046 & 0.006 \\
\hline Cobalt & $<0.05$ & $<0.05$ & $<0.05$ & $<0.05$ & $<0.05$ & $<0.05$ \\
\hline Copper & 0.014 & 0.01 & 0.006 & 0.0024 & 0.0039 & 0.003 \\
\hline Iron & 1.8 & 1.8 & 2.0 & 0.88 & 0.88 & 0.47 \\
\hline Lead & $<0.005$ & $<0.005$ & $<0.005$ & $<0.005$ & $<0.005$ & $<0.005$ \\
\hline Manganese & 0.083 & na & 0.095 & 0.038 & 0.033 & 0.022 \\
\hline Mercury & $<0.0002$ & $<0.0002$ & $<0.0002$ & $<0.0002$ & 0.0005 & $<0.0002$ \\
\hline Molybdenum & $<0.025$ & $<0.025$ & $<0.025$ & $<0.025$ & $<0.025$ & $<0.025$ \\
\hline Nickel & 0.0065 & 0.0057 & 0.007 & 0.0032 & 0.003 & 0.0029 \\
\hline Selenium & $<0.002$ & $<0.002$ & $<0.002$ & $<0.002$ & $<0.002$ & $<0.002$ \\
\hline Silver & $<0.001$ & $<0.001$ & $<0.001$ & $<0.001$ & $<0.001$ & $<0.001$ \\
\hline Thallium & $<0.001$ & $<0.001$ & $<0.001$ & $<0.001$ & $<0.001$ & $<0.001$ \\
\hline Vanadium & 0.014 & 0.012 & 0.01 & $<0.01$ & 0.01 & $<0.01$ \\
\hline Zinc & 0.13 & 0.024 & $<0.02$ & $<0.02$ & 0.082 & 0.046 \\
\hline \multicolumn{7}{|l|}{ Miscellaneous organics ( $\mu \mathrm{g} / \mathrm{L})$} \\
\hline Chlorophyll-a & 84.6 & 35.4 & 10.4 & 3.36 & 13 & 2.35 \\
\hline
\end{tabular}




\section{Surface Water}

Table 7-16. Monthly analyses of water samples collected from the Drainage Retention Basin location CDBE, 1999 (continued).

\begin{tabular}{|c|c|c|c|c|c|}
\hline Parameter & $\begin{array}{c}\text { Number of } \\
\text { samples }\end{array}$ & Minimum & Maximum & Median & $\begin{array}{c}\text { Interquartile } \\
\text { range }\end{array}$ \\
\hline \multicolumn{6}{|l|}{ Nutrients (mg/L) } \\
\hline Ammonia nitrogen (as $\mathrm{N}$ ) & 12 & $<0.02$ & 0.22 & 0.07 & 0.06 \\
\hline Nitrate (as N) & 12 & $<0.1$ & 2.0 & 0.94 & 0.81 \\
\hline Nitrate $\left(\right.$ as $\left.\mathrm{NO}_{3}\right)$ & 12 & $<0.4$ & 8.9 & 4.2 & 3.7 \\
\hline Nitrite (as N) & 12 & $<0.02$ & 0.04 & 0.02 & 0.01 \\
\hline Nitrite (as $\mathrm{NO}_{2}$ ) & 12 & $<0.07$ & $<0.5$ & 0.08 & 0.17 \\
\hline Total Kjeldahl nitrogen & 12 & 0.57 & 1.30 & 0.96 & 0.17 \\
\hline \multicolumn{6}{|l|}{ General minerals (mg/L) } \\
\hline Aluminum & 12 & 0.15 & 2.0 & 0.8 & 0.96 \\
\hline Bicarbonate alkalinity $\left(\right.$ as $\left.\mathrm{CaCO}_{3}\right)$ & 12 & 116 & 226 & 179 & 60 \\
\hline Calcium & 12 & 32 & 59 & 49 & 12 \\
\hline Carbonate alkalinity $\left(\mathrm{as} \mathrm{CaCO}_{3}\right)$ & 12 & $<5$ & 62 & 25 & 13 \\
\hline Chloride & 12 & 89 & 223 & 173 & 94 \\
\hline Copper & 12 & $<0.01$ & 0.033 & 0.01 & 0.01 \\
\hline Fluoride & 12 & 0.32 & 0.56 & 0.47 & 0.12 \\
\hline Hydroxide alkalinity $\left(\mathrm{as} \mathrm{CaCO}_{3}\right)$ & 12 & $<5$ & $<5$ & $<5$ & 0 \\
\hline Iron & 12 & 0.16 & 1.90 & 0.77 & 1.05 \\
\hline Magnesium & 12 & 16 & 41 & 30 & 14 \\
\hline Manganese & 12 & 0.016 & 0.23 & 0.049 & 0.074 \\
\hline Nickel & 12 & $<0.05$ & $<0.05$ & $<0.05$ & 0.00 \\
\hline Nitrate (as N) & 12 & $<0.1$ & 2.0 & 0.99 & 0.78 \\
\hline Nitrate $\left(\right.$ as $\left.\mathrm{NO}_{3}\right)$ & 12 & $<0.4$ & 8.9 & 4.3 & 3.5 \\
\hline Nitrite (as N) & 12 & $<0.02$ & 0.04 & 0.02 & 0.01 \\
\hline Orthophosphate & 12 & $<0.05$ & 0.32 & 0.15 & 0.17 \\
\hline $\mathrm{pH}$ (pH units) & 12 & 8.2 & 9.2 & 8.7 & 0.5 \\
\hline Potassium & 12 & 1.9 & 3.0 & 2.3 & 0.5 \\
\hline Sodium & 12 & 70 & 142 & 108 & 48 \\
\hline Specific conductance $(\mu \mathrm{mho} / \mathrm{cm})$ & 12 & 648 & 1210 & 1050 & 421 \\
\hline Sulfate & 12 & 45 & 77 & 61 & 24 \\
\hline Surfactants & 12 & $<0.05$ & 0.10 & 0.05 & 0.01 \\
\hline Total alkalinity $\left(\mathrm{as} \mathrm{CaCO}_{3}\right)$ & 12 & 146 & 229 & 213 & 65 \\
\hline Total dissolved solids (TDS) & 12 & 372 & 745 & 627 & 245 \\
\hline Total hardness (as $\mathrm{CaCO}_{3}$ ) & 12 & 146 & 308 & 250 & 91 \\
\hline Total phosphorus (as P) & 12 & 0.08 & 0.21 & 0.14 & 0.07 \\
\hline Total solids & 10 & 390 & 765 & 695 & 255 \\
\hline
\end{tabular}


Table 7-16. Monthly analyses of water samples collected from the Drainage Retention Basin location CDBE, 1999 (concluded).

\begin{tabular}{|c|c|c|c|c|c|}
\hline Parameter & $\begin{array}{l}\text { Number of } \\
\text { samples }\end{array}$ & Minimum & Maximum & Median & $\begin{array}{c}\text { Interquartile } \\
\text { range }\end{array}$ \\
\hline \multicolumn{6}{|l|}{ General minerals (mg/L) (continued) } \\
\hline Total suspended solids (TSS) & 12 & 7.6 & 59.0 & 15.5 & 9.5 \\
\hline Volatile solids & 11 & 5 & 217 & 165 & 88 \\
\hline Volatile suspended solids & 11 & 2.0 & 87 & 6.5 & 10.8 \\
\hline Zinc & 12 & $<0.01$ & 0.082 & 0.025 & 0.019 \\
\hline \multicolumn{6}{|l|}{ Total metals (mg/L) } \\
\hline Aluminum & 12 & 0.13 & 2.20 & 0.87 & 0.86 \\
\hline Antimony & 12 & $<0.004$ & $<0.004$ & $<0.004$ & -0.004 \\
\hline Arsenic & 12 & 0.002 & 0.006 & 0.003 & 0.001 \\
\hline Barium & 12 & 0.093 & 0.19 & 0.15 & 0.05 \\
\hline Beryllium & 12 & $<0.0002$ & $<0.0002$ & $<0.0002$ & 0.000 \\
\hline Boron & 12 & 1.4 & 2.3 & 1.7 & 0.5 \\
\hline Cadmium & 12 & $<0.0005$ & $<0.0005$ & $<0.0005$ & 0 \\
\hline Chromium & 12 & 0.0028 & 0.0084 & 0.0053 & 0.001 \\
\hline Chromium(VI) & 12 & $<0.002$ & 0.007 & 0.005 & 0.003 \\
\hline Cobalt & 12 & $<0.05$ & $<0.05$ & $<0.05$ & 0 \\
\hline Copper & 12 & 0.0018 & 0.0140 & 0.0054 & 0.0031 \\
\hline Iron & 12 & 0.160 & 2.000 & 0.835 & 1.12 \\
\hline Lead & 12 & $<0.005$ & 0.009 & $<0.005$ & 0 \\
\hline Manganese & 12 & 0.016 & 0.16 & 0.049 & 0.056 \\
\hline Mercury & 12 & $<0.0002$ & 0.0005 & $<0.0002$ & 0 \\
\hline Molybdenum & 12 & $<0.025$ & $<0.025$ & $<0.025$ & 0 \\
\hline Nickel & 12 & 0.0028 & 0.0070 & 0.0036 & 0.0028 \\
\hline Selenium & 12 & $<0.002$ & $<0.002$ & $<0.002$ & 0 \\
\hline Silver & 12 & $<0.001$ & $<0.001$ & $<0.001$ & 0 \\
\hline Thallium & 12 & $<0.001$ & $<0.001$ & $<0.001$ & 0 \\
\hline Vanadium & 12 & $<0.01$ & 0.014 & $<0.01$ & 0.001 \\
\hline Zinc & 12 & $<0.02$ & 0.130 & $<0.02$ & 0.013 \\
\hline \multicolumn{6}{|l|}{ Miscellaneous organics ( $\mu \mathrm{g} / \mathrm{L})$} \\
\hline Chlorophyll-a & 12 & 2.35 & 84.6 & 22 & 32.1 \\
\hline
\end{tabular}

a na $=$ Sample not analyzed for this constituent. 


\section{Surface Water}

Table 7-17. Quarterly analyses of water samples collected from the Drainage Retention Basin location CDBE, 1999.

\begin{tabular}{|c|c|c|c|c|}
\hline \multirow[b]{2}{*}{ Parameter } & \multicolumn{4}{|c|}{ Sampling dates } \\
\hline & 1/13/99 & $4 / 26 / 99$ & $8 / 19 / 99$ & $10 / 4 / 99$ \\
\hline \multicolumn{5}{|l|}{ Biological } \\
\hline Fecal coliform MPN/100ml & $<2$ & $<2$ & $<2$ & 49 \\
\hline Total coliform MPN/100ml & 8 & 50 & 240 & 240 \\
\hline \multicolumn{5}{|l|}{ Herbicides $(\mu \mathrm{g} / \mathrm{L})^{(\mathrm{a})}$} \\
\hline Acenaphthylene & -(a) $^{\text {(a) }}$ & $<0.1$ & $<0.1$ & $<0.1$ \\
\hline Alachlor & -(a) & $<0.2$ & $<0.2$ & $<0.2$ \\
\hline Aldrin & -(a) $^{(a)}$ & $<0.5$ & $<0.5$ & $<0.5$ \\
\hline Anthracene & -(a) $^{\text {(a) }}$ & $<0.1$ & $<0.1$ & $<0.1$ \\
\hline Atraton & - (a) $^{(1)}$ & $<0.5$ & $<0.5$ & $<0.5$ \\
\hline Atrazine & $<0.2$ & $<0.2$ & $<0.2$ & $<0.2$ \\
\hline Benzo(a)anthracene & - $^{(\mathrm{a})}$ & $<0.3$ & $<0.3$ & $<0.3$ \\
\hline Benzo(a)pyrene & 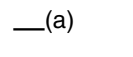 & 0.14 & $<0.1$ & 0.12 \\
\hline Benzo(b)fluoranthene & -(a) $^{(\mathrm{n}}$ & $<0.3$ & $<0.3$ & $<0.3$ \\
\hline Benzo $(g, h, i)$ perylene & -(a) $^{\text {(a) }}$ & $<0.3$ & $<0.3$ & $<0.3$ \\
\hline Benzo(k)fluoranthene & 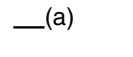 & $<0.3$ & $<0.3$ & $<0.3$ \\
\hline $\mathrm{BHC}$, delta isomer & -(a) $^{(a)}$ & $<0.2$ & $<0.2$ & $<0.2$ \\
\hline $\mathrm{BHC}$, gamma isomer (Lindane) & -(a) $^{(2}$ & $<0.1$ & $<0.1$ & $<0.1$ \\
\hline Bromacil & $<0.5$ & 1.8 & $<0.5$ & $<0.5$ \\
\hline Butachlor & $<0.3$ & $<0.3$ & $<0.3$ & $<0.3$ \\
\hline Butylbenzylphthalate & -(a) & $<1$ & $<1$ & $<1$ \\
\hline Chlordane & -(a) $^{(\mathrm{n}}$ & $<2$ & $<2$ & $<2$ \\
\hline Chrysene & 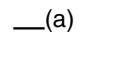 & $<0.3$ & $<0.3$ & $<0.3$ \\
\hline Di(2-ethylhexyl)adipate & 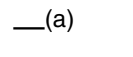 & $<1$ & $<1$ & $<1$ \\
\hline Diazinon & $<0.2$ & $<0.2$ & $<0.2$ & $<0.2$ \\
\hline Dibenzo( $(a, h)$ anthracene & -(a) & $<0.3$ & $<0.3$ & $<0.3$ \\
\hline Dibutylphthalate & -(a) $^{(\mathrm{n}}$ & $<1$ & $<1$ & $<1$ \\
\hline Diethylhexylphthalate & -(a) $^{(\mathrm{n}}$ & $<3$ & $<3$ & $<3$ \\
\hline Diethylphthalate & -(a) $^{\text {(a) }}$ & $<3$ & $<3$ & $<3$ \\
\hline Dimethoate & $<2$ & $<2$ & $<2$ & $<2$ \\
\hline Dimethylphthalate & -(a) $^{(\mathrm{n}}$ & $<1$ & $<1$ & $<1$ \\
\hline Diuron & $<1$ & 1.8 & 0.3 & $<1$ \\
\hline Endrin & -(a) $^{\text {(a) }}$ & $<0.2$ & $<0.2$ & $<0.2$ \\
\hline Fluorene & - (a) $^{\text {(a) }}$ & $<0.1$ & $<0.1$ & $<0.1$ \\
\hline Glyphosate & $<9$ & $<9$ & $<9$ & $<9$ \\
\hline Heptachlor & -(a) $^{\text {(a) }}$ & $<0.1$ & $<0.1$ & $<0.1$ \\
\hline
\end{tabular}


Table 7-17. Quarterly analyses of water samples collected from the Drainage Retention Basin location CDBE, 1999 (concluded).

\begin{tabular}{|c|c|c|c|c|}
\hline \multirow[b]{2}{*}{ Parameter } & \multicolumn{4}{|c|}{ Sampling dates } \\
\hline & 1/13/99 & $4 / 26 / 99$ & 8/19/99 & $10 / 4 / 99$ \\
\hline \multicolumn{5}{|l|}{ Herbicides ( $\mu \mathrm{g} / \mathrm{L})$ (continued) } \\
\hline Heptachlor epoxide & -(a) & $<0.1$ & $<0.1$ & $<0.1$ \\
\hline Hexachlorobenzene & - (a) $^{(2)}$ & $<0.5$ & $<0.5$ & $<0.5$ \\
\hline Hexachlorocyclopentadiene & -(a) & $<1$ & $<1$ & $<1$ \\
\hline Indeno(1,2,3-c, $d)$ pyrene & 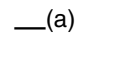 & $<0.3$ & $<0.3$ & $<0.3$ \\
\hline Methoxychlor & $<0.5$ & $<0.5$ & $<0.5$ & $<0.5$ \\
\hline Metolachlor & $<0.5$ & $<0.5$ & $<0.5$ & $<0.5$ \\
\hline Metribuzin & -(a) $^{(\mathrm{a}}$ & $<0.5$ & $<0.5$ & $<0.5$ \\
\hline Molinate & $<0.5$ & $<0.5$ & $<0.5$ & $<0.5$ \\
\hline Pentachlorophenol & -(a) & $<1$ & $<1$ & $<1$ \\
\hline Phenanthrene & 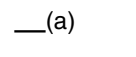 & $<0.1$ & $<0.1$ & $<0.1$ \\
\hline Prometon & -(a) & $<0.5$ & $<0.5$ & $<0.5$ \\
\hline Prometryn & $<0.5$ & $<0.5$ & $<0.5$ & $<0.5$ \\
\hline Propachlor & $<0.5$ & $<0.5$ & $<0.5$ & $<0.5$ \\
\hline Pyrene & -(a) $^{(\mathrm{n}}$ & $<0.1$ & $<0.1$ & $<0.1$ \\
\hline Secbumeton & - (a) $^{(2)}$ & $<0.5$ & $<0.5$ & $<0.5$ \\
\hline Simazine & $<0.2$ & $<0.2$ & $<0.2$ & $<0.2$ \\
\hline Terbutryn & 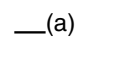 & $<0.5$ & $<0.5$ & $<0.5$ \\
\hline Thiobencarb & $<0.5$ & $<0.5$ & $<0.5$ & $<0.5$ \\
\hline Toxaphene & -(a) & $<5$ & -(a) & -(a) $^{(\mathrm{a}}$ \\
\hline \multicolumn{5}{|l|}{ Miscellaneous organics (mg/L) } \\
\hline Chemical oxygen demand & $<20$ & 46 & 27 & $<20$ \\
\hline Oil and grease & 1.4 & $<1$ & —(b) $^{(\mathrm{c}}$ & —(b) $^{(\mathrm{c}}$ \\
\hline
\end{tabular}

a Herbicide analyses are mainly directed at bromacil, diazinon, diuron, and glyphosate. Other analyses may be reported by the analytical laboratory.

b No sample collected. 


\section{Surface Water}

Table 7-18. Semiannual/annual analyses of water samples collected from the Drainage Retention Basin location CDBE, 1999.

\begin{tabular}{|c|c|c|}
\hline \multirow[b]{2}{*}{ Parameter } & \multicolumn{2}{|c|}{ Sampling dates } \\
\hline & 4/26/99 & $10 / 4 / 99$ \\
\hline \multicolumn{3}{|l|}{ Biological } \\
\hline \multicolumn{3}{|l|}{ Aqueous bioassay } \\
\hline Pimephales promelas acute survival (percent) & $\mathrm{na}^{(\mathrm{a})}$ & 95 \\
\hline Pimephales promelas growth (toxic units) & na & $<1$ \\
\hline Pimephales promelas growth, IC-25(b) & na & $>100$ \\
\hline Pimephales promelas growth, IC-50(c) & na & $>100$ \\
\hline Pimephales promelas growth, LOEC(d) & na & $>100$ \\
\hline Pimephales promelas growth, NOEC(e) & na & $>100$ \\
\hline Pimephales promelas chronic survival (toxic units) & na & $<1$ \\
\hline Pimephales promelas chronic survival, LC-50(f) & na & $>100$ \\
\hline Pimephales promelas chronic survival, LOEC & na & $>100$ \\
\hline Pimephales promelas chronic survival, NOEC & na & $>100$ \\
\hline Ceriodaphnia dubia growth (toxic units) & na & $>1$ \\
\hline Ceriodaphnia dubia growth, IC-25 & na & $>100$ \\
\hline Ceriodaphnia dubia growth, IC-50 & na & $>100$ \\
\hline Ceriodaphnia dubia growth, LOEC & na & 100 \\
\hline Ceriodaphnia dubia growth, NOEC & na & $<100$ \\
\hline Ceriodaphnia dubia chronic survival (toxic units) & na & $<1$ \\
\hline Ceriodaphnia dubia chronic survival, LC-50 & na & $>100$ \\
\hline Ceriodaphnia dubia chronic survival, LOEC & na & $>100$ \\
\hline Ceriodaphnia dubia chronic survival, NOEC & na & $>100$ \\
\hline Selanastrum capriocornutum growth (toxic units) & na & $>1$ \\
\hline Selanastrum capriocornutum growth, IC-25 & na & $>100$ \\
\hline Selanastrum capriocornutum growth, IC-50 & na & $>100$ \\
\hline Selanastrum capriocornutum growth, LOEC & na & 100 \\
\hline Selanastrum capriocornutum growth, NOEC & na & $<100$ \\
\hline \multicolumn{3}{|l|}{ Volatile organic compounds ( $\mu \mathrm{g} / \mathrm{L})$} \\
\hline 1,1,1-Trichloroethane & $<0.5$ & $<0.5$ \\
\hline $1,1,2,2$-Tetrachloroethane & $<0.5$ & $<0.5$ \\
\hline $1,1,2$-Trichloroethane & $<0.5$ & $<0.5$ \\
\hline 1,1-Dichloroethane & $<0.5$ & $<0.5$ \\
\hline 1,1-Dichloroethene & $<0.5$ & $<0.5$ \\
\hline 1,2-Dichlorobenzene & $<0.5$ & $<0.5$ \\
\hline 1,2-Dichloroethane & $<0.5$ & $<0.5$ \\
\hline 1,2-Dichloroethene (total) & $<1$ & $<1$ \\
\hline 1,2-Dichloropropane & $<0.5$ & $<0.5$ \\
\hline
\end{tabular}


Table 7-18. Semiannual/annual analyses of water samples collected from the Drainage Retention Basin location CDBE, 1999 (concluded).

\begin{tabular}{|c|c|c|}
\hline \multirow[b]{2}{*}{ Parameter } & \multicolumn{2}{|c|}{ Sampling dates } \\
\hline & $4 / 26 / 99$ & 10/4/99 \\
\hline \multicolumn{3}{|c|}{ Volatile organic compounds $(\mu \mathrm{g} / \mathrm{L})$ (continued) } \\
\hline 1,3-Dichlorobenzene & $<0.5$ & $<0.5$ \\
\hline 1,4-Dichlorobenzene & $<0.5$ & $<0.5$ \\
\hline Bromodichloromethane & $<0.5$ & $<0.5$ \\
\hline Bromoform & $<0.5$ & $<0.5$ \\
\hline Bromomethane & $<0.5$ & $<0.5$ \\
\hline Carbon tetrachloride & $<0.5$ & $<0.5$ \\
\hline Chlorobenzene & $<0.5$ & $<0.5$ \\
\hline Chloroethane & $<1$ & $<1$ \\
\hline Chloroform & $<0.5$ & $<0.5$ \\
\hline Chloromethane & $<1$ & $<1$ \\
\hline cis-1,2-Dichloroethene & $<0.5$ & $<0.5$ \\
\hline cis-1,3-Dichloropropene & $<0.5$ & $<0.5$ \\
\hline Dibromochloromethane & $<0.5$ & $<0.5$ \\
\hline Dichlorodifluoromethane & $<0.5$ & $<0.5$ \\
\hline Freon 113 & $<0.5$ & $<0.5$ \\
\hline Methylene chloride & $<1$ & $<1$ \\
\hline Tetrachloroethene & $<0.5$ & $<0.5$ \\
\hline Total Trihalomethanes & $<2$ & $<2$ \\
\hline trans-1,2-Dichloroethene & $<0.5$ & $<0.5$ \\
\hline trans-1,3-Dichloropropene & $<0.5$ & $<0.5$ \\
\hline Trichloroethene & $<0.5$ & $<0.5$ \\
\hline Trichlorofluoromethane & $<0.5$ & $<0.5$ \\
\hline Vinyl chloride & $<0.5$ & $<0.5$ \\
\hline \multicolumn{3}{|l|}{ Miscellaneous organics } \\
\hline Total organic carbon (TOC) (mg/L) & 6.4 & 3.8 \\
\hline \multicolumn{3}{|l|}{ Radioactive (Bq/L) } \\
\hline Gross alpha & $0.072 \pm 0.037$ & $0.138 \pm 0.056$ \\
\hline Gross beta & $0.08 \pm 0.036$ & $0.1 \pm 0.044$ \\
\hline Tritium & $43.7 \pm 3.8$ & $31.4 \pm 3.4$ \\
\hline
\end{tabular}

Note: Radioactivities are reported as the measured concentration and either an uncertainty ( $\pm 2 \sigma$ counting error) or as being less than or equal to the detection limit. If the concentration is less than or equal to the uncertainty or the detection limit, the result is considered to be a nondetection. See the main volume, Chapter 14, Quality Assurance.

a $n a=$ Not analyzed.

b IC $25=25 \%$ Inhibition concentration; concentration at which $25 \%$ of the organisms show inhibition responses.

c IC $50=50 \%$ Inhibition concentration; concentration at which $50 \%$ of the organisms show inhibition responses.

d LOEC = Lowest observed effect concentration.

e $\mathrm{NOEC}=$ No observed effect concentration.

f LC $50=50 \%$ Lethal concentration; concentration at which $50 \%$ of the organisms die. 
Table 7-19. Field data collected from the Drainage Retention Basin at eight locations, 1999.

\begin{tabular}{|c|c|c|c|c|c|c|c|c|c|}
\hline \multirow[b]{2}{*}{ Date } & \multicolumn{2}{|c|}{ CDBA } & \multicolumn{2}{|c|}{ CDBC } & \multicolumn{2}{|c|}{ CDBD } & \multicolumn{3}{|c|}{ CDBE } \\
\hline & $\begin{array}{c}\text { Dissolved } \\
\text { oxygen } \\
(\mathrm{mg} / \mathrm{L})\end{array}$ & $\begin{array}{c}\text { Temper- } \\
\text { ature } \\
\left({ }^{\circ} \mathrm{C}\right)\end{array}$ & $\begin{array}{c}\text { Dissolved } \\
\text { oxygen } \\
(\mathrm{mg} / \mathrm{L})\end{array}$ & $\begin{array}{c}\text { Temper- } \\
\text { ature } \\
\left({ }^{\circ} \mathrm{C}\right)\end{array}$ & $\begin{array}{c}\text { Dissolved } \\
\text { oxygen } \\
(\mathrm{mg} / \mathrm{L})\end{array}$ & $\begin{array}{c}\text { Temper- } \\
\text { ature } \\
\left({ }^{\circ} \mathrm{C}\right)\end{array}$ & $\begin{array}{c}\text { Dissolved } \\
\text { oxygen } \\
(\mathrm{mg} / \mathrm{L})\end{array}$ & $\begin{array}{c}\text { Temper- } \\
\text { ature } \\
\left({ }^{\circ} \mathrm{C}\right)\end{array}$ & $\begin{array}{c}\text { Turbidity } \\
\text { (m) }\end{array}$ \\
\hline 1/7/99 & 13.7 & 7.3 & 17.8 & 8.2 & 17.7 & 7.8 & 12.9 & 7.8 & 0.76 \\
\hline 1/13/99 & 16.7 & 11.3 & 17.5 & 10.1 & 17.1 & 8.8 & 13.3 & 8.6 & 0.61 \\
\hline 1/22/99 & 10.1 & 14.2 & 12.2 & 11.9 & 13.8 & 10.7 & 10 & 10.1 & 0.38 \\
\hline 1/29/99 & 8.47 & 13.3 & 11.3 & 10.1 & 13.6 & 8.4 & 8.38 & 8.4 & 0.53 \\
\hline 2/4/99 & 13.3 & 13.4 & 16.2 & 11 & 13.6 & 10.8 & 10.2 & 10.9 & 0.46 \\
\hline 2/12/99 & 11.2 & 10.2 & 10.3 & 9.3 & 9.54 & 9.6 & 9.56 & 9.5 & 0.33 \\
\hline 2/19/99 & 9.35 & 13.1 & 11.1 & 12.7 & 11.4 & 11.5 & 7.63 & 10.6 & 0.48 \\
\hline 2/26/99 & 7.29 & 14.9 & 8.56 & 10.5 & 8.67 & 10.6 & 8.23 & 10.6 & 0.46 \\
\hline 3/5/99 & 12.9 & 13.4 & 15.9 & 10.5 & 13.1 & 11.6 & 10.3 & 10.7 & 0.43 \\
\hline 3/11/99 & 12.7 & 15.8 & 15.0 & 14.7 & 14.8 & 13.6 & 11.5 & 13.8 & 0.41 \\
\hline 3/19/99 & 12.4 & 12.6 & 12.0 & 12.6 & 11.4 & 12.3 & 9.04 & 12.1 & 0.48 \\
\hline 3/26/99 & 12.6 & 18.9 & 10.0 & 15.1 & 8.38 & 13.8 & 7.28 & 13.6 & 1.19 \\
\hline 4/2/99 & 10.7 & 13.9 & 13.9 & 14 & 13.9 & 13.5 & 10.5 & 13.4 & 0.71 \\
\hline 4/9/99 & 10.0 & 14 & 12.7 & 10.4 & 11.4 & 10.9 & 8.11 & 10.9 & 0.75 \\
\hline 4/16/99 & 10.8 & 21.6 & 11.5 & 22 & 13.3 & 18.3 & 4.96 & 14.3 & 0.91 \\
\hline 4/21/99 & 16 & 17.8 & 14.8 & 16.9 & 14.6 & 15.5 & 12.6 & 13.4 & 0.69 \\
\hline 4/26/99 & 11.2 & 21.8 & 11.6 & 18.1 & 11.3 & 17.5 & 8.92 & 16.4 & 0.56 \\
\hline 5/4/99 & 9.66 & 19.4 & 8.42 & 15.6 & 7.26 & 15.1 & 6.52 & 14.8 & 0.48 \\
\hline $5 / 13 / 99$ & 11.3 & 22.2 & 6.82 & 18.9 & 6.48 & 18.5 & 6.18 & 18.4 & 0.76 \\
\hline $5 / 21 / 99$ & 12.3 & 22.8 & 8.05 & 20.1 & 8.1 & 20.2 & 7.72 & 7.62 & 0.64 \\
\hline $5 / 27 / 99$ & 9.6 & 24.6 & 6.9 & 22.4 & 6.5 & 21.8 & 5.9 & 21.6 & 0.56 \\
\hline 6/4/99 & 10.3 & 21.3 & 8.34 & 20.4 & 8.35 & 18.9 & 7.02 & 19 & 0.56 \\
\hline $6 / 11 / 99$ & 6.95 & 21 & 5.88 & 22.2 & 6.55 & 20.6 & 6.05 & 20.4 & 0.46 \\
\hline 6/15/99 & 12.7 & 22.9 & 9.66 & 22.1 & 8.95 & 21.8 & 7.5 & 21.7 & 0.38 \\
\hline 6/24/99 & 3.63 & 23.6 & 5.37 & 24.4 & 5.52 & 24.3 & 5.02 & 24.1 & 0.41 \\
\hline 7/2/99 & 12.7 & 24.5 & 12.2 & 24.4 & 9.78 & 25.1 & 4.85 & 25.1 & 0.20 \\
\hline 7/8/99 & 8.56 & 22.1 & 7.07 & 24.3 & 6.55 & 23.3 & 6.24 & 23.1 & 0.23 \\
\hline 7/15/99 & 10.2 & 23.3 & 9.81 & 24.3 & 7.88 & 25.6 & 7 & 25.5 & 0.28 \\
\hline 7/22/99 & 9.01 & 20.5 & 7.98 & 22.2 & 8.01 & 22.3 & 5.82 & 22.1 & 0.27 \\
\hline 7/29/99 & 12.3 & 28.9 & 14.5 & 24.9 & 11.1 & 22.6 & 10.4 & 22.6 & 0.33 \\
\hline 8/6/99 & 7.67 & 21.2 & 11.5 & 22 & 9.27 & 20.8 & 8.95 & 20.8 & 0.38 \\
\hline 8/12/99 & 10.1 & 23.3 & 10.1 & 22.7 & 10.2 & 20.7 & 6.25 & 20.7 & 0.41 \\
\hline 8/19/99 & 8.96 & 24.6 & 9.72 & 23.1 & 7.27 & 21.6 & 4.78 & 21.5 & 0.36 \\
\hline 8/27/99 & 6.27 & 24 & 5.21 & 23.9 & 4.23 & 23.7 & 3.25 & 23.6 & 0.38 \\
\hline 9/3/99 & 9.75 & 22.4 & 9.06 & 22.2 & 7.06 & 21.8 & 3.78 & 21.7 & 0.51 \\
\hline
\end{tabular}


Table 7-19. Field data collected from the Drainage Retention Basin at eight locations, 1999 (continued).

\begin{tabular}{|c|c|c|c|c|c|c|c|c|}
\hline \multirow[b]{2}{*}{ Date } & \multicolumn{2}{|c|}{ CDBF } & \multicolumn{2}{|c|}{ CDBJ } & \multicolumn{2}{|c|}{ CDBK } & \multicolumn{2}{|c|}{ CDBL } \\
\hline & $\begin{array}{c}\text { Dissolved } \\
\text { oxygen } \\
\text { (mg/L) }\end{array}$ & $\begin{array}{c}\text { Temper- } \\
\text { ature } \\
\left({ }^{\circ} \mathrm{C}\right)\end{array}$ & $\begin{array}{c}\text { Dissolved } \\
\text { oxygen } \\
\text { (mg/L) }\end{array}$ & $\begin{array}{l}\text { Temper- } \\
\text { ature } \\
\left({ }^{\circ} \mathrm{C}\right)\end{array}$ & $\begin{array}{c}\text { Dissolved } \\
\text { oxygen } \\
\text { (mg/L) }\end{array}$ & $\begin{array}{c}\text { Temper- } \\
\text { ature } \\
\left({ }^{\circ} \mathrm{C}\right)\end{array}$ & $\begin{array}{c}\text { Dissolved } \\
\text { oxygen } \\
\text { (mg/L) }\end{array}$ & $\begin{array}{c}\text { Temper- } \\
\text { ature } \\
\left({ }^{\circ} \mathbf{C}\right)\end{array}$ \\
\hline $1 / 7 / 99$ & 12.4 & 7.7 & 18.0 & 8 & 12.9 & 8 & 12.9 & 8.5 \\
\hline $1 / 13 / 99$ & 12.3 & 9.1 & 17.5 & 8.8 & 12.1 & 8.5 & 10.1 & 8.8 \\
\hline 1/22/99 & 12.2 & 10.3 & 13.4 & 11.5 & 9.37 & 9.7 & 10.9 & 9.6 \\
\hline 1/29/99 & 7.52 & 8.4 & 18.6 & 8.5 & 6.85 & 8.5 & 9.25 & 9 \\
\hline 2/4/99 & 7.49 & 12.4 & 11.8 & 9.6 & 8.71 & 8.5 & 9.42 & 10.7 \\
\hline 2/12/99 & 9.25 & 9.4 & 9.36 & 9.6 & 10.3 & 9.3 & 9.05 & 9.5 \\
\hline 2/19/99 & 4.98 & 10.3 & 9.56 & 11.5 & 6.97 & 10.7 & 3.57 & 11 \\
\hline 2/26/99 & 8.64 & 10.5 & 7.99 & 10.8 & 7.12 & 10.6 & 5.81 & 10.6 \\
\hline 3/5/99 & 3.9 & 10.6 & 13.4 & 11.5 & 10.2 & 11 & 3.95 & 10.8 \\
\hline $3 / 11 / 99$ & 7.5 & 12.2 & 14.8 & 11.6 & 9 & 11.5 & 6.28 & 12.8 \\
\hline 3/19/99 & 5.52 & 11.9 & 11.5 & 12.1 & 7.93 & 11.8 & 6.72 & 11.6 \\
\hline 3/26/99 & 5.35 & 14 & 9.71 & 15.2 & 8.34 & 14.9 & 4.45 & 13.6 \\
\hline $4 / 2 / 99$ & 9.15 & 13.2 & 13.5 & 12.9 & 7.94 & 12.8 & 6.39 & 12.2 \\
\hline 4/9/99 & 8.57 & 10.8 & 11.6 & 11 & 8.23 & 11.1 & 8.35 & 11.1 \\
\hline 4/16/99 & 3.43 & 14.9 & 10.9 & 16.8 & 3.41 & 15.5 & 3.67 & 16.3 \\
\hline 4/21/99 & 3.25 & 13.4 & 15.4 & 16.3 & 13.9 & 15.9 & 3.27 & 15.6 \\
\hline 4/26/99 & 3.54 & 17.3 & 11.0 & 17.4 & 8.98 & 15.6 & 3.36 & 15.6 \\
\hline $5 / 4 / 99$ & 6.26 & 15.1 & 7.44 & 15.5 & 6.95 & 15.2 & 6.38 & 15.3 \\
\hline $5 / 13 / 99$ & 6.04 & 18.7 & 7.26 & 19.3 & 6.9 & 18.8 & 6.57 & 18.8 \\
\hline $5 / 21 / 99$ & 8.01 & 19.4 & 8.88 & 20.1 & 7.86 & 19.6 & 7.31 & 20.3 \\
\hline $5 / 27 / 99$ & 5.85 & 21.7 & 6.48 & 22.3 & 5.8 & 22 & 5.61 & 22.3 \\
\hline 6/4/99 & 6.81 & 19.1 & 8.15 & 19.8 & 6.9 & 19.9 & 6.55 & 21.3 \\
\hline $6 / 11 / 99$ & 5.99 & 20.4 & 6.84 & 21.4 & 5.92 & 21.4 & 5.23 & 21.4 \\
\hline $6 / 15 / 99$ & 6.65 & 20.8 & 9.49 & 22.6 & 8.97 & 22.4 & 8.85 & 22.4 \\
\hline $6 / 24 / 99$ & 4.96 & 24.1 & 6.35 & 24.4 & 6.16 & 24.2 & 6.05 & 24.1 \\
\hline 7/2/99 & 4.53 & 25.2 & 7.03 & 25.4 & 5.42 & 24.9 & 4.5 & 24.8 \\
\hline 7/8/99 & 6 & 22.8 & 6.91 & 23.1 & 6.06 & 23.1 & 5.83 & 22.8 \\
\hline $7 / 15 / 99$ & 7.11 & 25.2 & 8.41 & 25.5 & 7.7 & 25.1 & 7.5 & 24.8 \\
\hline $7 / 22 / 99$ & 5.69 & 22.3 & 7.69 & 22.2 & 6.29 & 22.1 & 5.8 & 20.8 \\
\hline 7/29/99 & 10.2 & 23.3 & 12.3 & 22.9 & 11.0 & 22.5 & 10.4 & 22.6 \\
\hline 8/6/99 & 8.15 & 20.9 & 10.9 & 21.1 & 9.53 & 21 & 7.99 & 21.1 \\
\hline $8 / 12 / 99$ & 5.35 & 20.8 & 10.1 & 21.1 & 7.07 & 21 & 5.9 & 21.1 \\
\hline 8/19/99 & 3.94 & 21.6 & 11.4 & 21.8 & 5.16 & 21.3 & 4.45 & 21.6 \\
\hline 8/27/99 & 3.12 & 23.7 & 4.41 & 23.6 & 3.38 & 23.5 & 3.35 & 23.5 \\
\hline 9/3/99 & 3.32 & 21.8 & 7.94 & 21.8 & 4.06 & 21.8 & 3.67 & 21.9 \\
\hline
\end{tabular}




\section{Surface Water}

Table 7-19. Field data collected from the Drainage Retention Basin at eight locations, 1999 (continued).

\begin{tabular}{|c|c|c|c|c|c|c|c|c|c|}
\hline \multirow[b]{2}{*}{ Date } & \multicolumn{2}{|c|}{ CDBA } & \multicolumn{2}{|c|}{ CDBC } & \multicolumn{2}{|c|}{ CDBD } & \multicolumn{3}{|c|}{ CDBE } \\
\hline & $\begin{array}{c}\text { Dissolved } \\
\text { oxygen } \\
(p p m)\end{array}$ & $\begin{array}{c}\text { Temper- } \\
\text { ature } \\
\left({ }^{\circ} \mathrm{C}\right)\end{array}$ & $\begin{array}{c}\text { Dissolved } \\
\text { Oxygen } \\
\text { (ppm) }\end{array}$ & $\begin{array}{c}\text { Temper- } \\
\text { ature } \\
\left({ }^{\circ} \mathrm{C}\right)\end{array}$ & $\begin{array}{c}\text { Dissolved } \\
\text { Oxygen } \\
\text { (ppm) }\end{array}$ & $\begin{array}{c}\text { Temper- } \\
\text { ature } \\
\left({ }^{\circ} \mathrm{C}\right)\end{array}$ & $\begin{array}{c}\text { Dissolved } \\
\text { Oxygen } \\
(p p m)\end{array}$ & $\begin{array}{c}\text { Temper- } \\
\text { ature } \\
\left({ }^{\circ} \mathrm{C}\right)\end{array}$ & $\begin{array}{c}\text { Turbidity } \\
\text { (m) }\end{array}$ \\
\hline 9/9/99 & 11.1 & 21.4 & 7.38 & 22.8 & 8.7 & 22.1 & 4.55 & 22.2 & 0.41 \\
\hline 9/17/99 & 7.8 & 17.7 & 5.47 & 19.4 & 4.86 & 21 & 4.4 & 20.9 & 0.46 \\
\hline 9/30/99 & 9.01 & 22.8 & 8.14 & 22.1 & 7.2 & 20.9 & 5.28 & 20.8 & 0.64 \\
\hline $10 / 4 / 99$ & 8.35 & 25 & 7.53 & 20.4 & 5.42 & 20.1 & 3.92 & 20 & 0.64 \\
\hline $10 / 15 / 99$ & 7.78 & 22.4 & 6.8 & 18.8 & 6.38 & 19.3 & 4.3 & 19.3 & 0.61 \\
\hline 10/22/99 & 11.6 & 17.2 & 7.55 & 17.1 & 9.12 & 16.7 & 5.66 & 16.5 & 0.53 \\
\hline $10 / 30 / 99$ & 10.6 & 15.3 & 7.59 & 14.6 & 9.62 & 16 & 5.77 & 15.9 & 0.48 \\
\hline $11 / 4 / 99$ & 15.4 & 18.3 & 10.4 & 18.1 & 8.54 & 16.1 & 6.94 & 15.9 & 0.61 \\
\hline $11 / 11 / 99$ & 10.7 & 16.2 & 8.47 & 16.1 & 7.78 & 15.2 & 4.27 & 15 & 0.48 \\
\hline $11 / 19 / 99$ & 11.5 & 11.6 & 9.92 & 12.7 & 8.79 & 12.8 & 6.86 & 12.8 & 0.56 \\
\hline $11 / 24 / 99$ & 12.7 & 10.5 & 12.2 & 11.2 & 12.3 & 10.9 & 8.75 & 10.7 & 0.38 \\
\hline 12/2/99 & 9.69 & 11.2 & 8.7 & 11.7 & 9.22 & 11.1 & 5.96 & 11.2 & 0.48 \\
\hline 12/3/99 & 9.52 & 9.6 & 8.03 & 9.3 & 7.84 & 10.3 & 5.3 & 10.3 & 0.51 \\
\hline $12 / 10 / 99$ & 8.15 & 7.7 & 9.45 & 9.3 & 9.6 & 7.97 & 7.75 & 9.6 & 0.48 \\
\hline $12 / 17 / 99$ & 9.44 & 10 & 8.22 & 10.9 & 8.17 & 8.9 & 7.66 & 8.9 & 0.53 \\
\hline $12 / 21 / 99$ & 9.3 & 10.8 & 9 & 10 & 10.3 & 9.8 & 8.14 & 9.7 & 0.43 \\
\hline $12 / 28 / 99$ & 10.9 & 8.4 & 10.3 & 9.3 & 10.07 & 8.1 & 8.87 & 8.7 & 0.28 \\
\hline \multicolumn{10}{|c|}{ Data Summary } \\
\hline Number of samples & 52 & 52 & 52 & 52 & 52 & 52 & 52 & 52 & 52 \\
\hline Minimum & 3.63 & 7.3 & 5.2 & 8.2 & 4.2 & 7.8 & 3.3 & 7.6 & 0.20 \\
\hline Maximum & 16.7 & 28.9 & 17.8 & 24.9 & 17.7 & 25.6 & 13.3 & 25.5 & 1.19 \\
\hline Median & 10.2 & 17.8 & 9.7 & 17.0 & 9.0 & 16.1 & 7.0 & 14.9 & 0.48 \\
\hline 75th percentile & 12.3 & 22.4 & 11.7 & 22.1 & 11.4 & 20.9 & 8.88 & 20.8 & 0.57 \\
\hline 25th percentile & 9.0 & 13.3 & 8.0 & 11.0 & 7.7 & 10.9 & 5.57 & 10.7 & 0.40 \\
\hline Interquartile range & 3.3 & 9.2 & 3.7 & 11.0 & 3.7 & 10.0 & 3.3 & 10.2 & 0.17 \\
\hline
\end{tabular}


Table 7-19. Field data collected from the Drainage Retention Basin at eight locations, 1999 (concluded).

\begin{tabular}{|c|c|c|c|c|c|c|c|c|}
\hline \multirow[b]{2}{*}{ Date } & \multicolumn{2}{|c|}{ CDBF } & \multicolumn{2}{|c|}{ CDBJ } & \multicolumn{2}{|c|}{ CDBK } & \multicolumn{2}{|c|}{ CDBL } \\
\hline & $\begin{array}{c}\text { Dissolved } \\
\text { oxygen } \\
(p p m)\end{array}$ & $\begin{array}{c}\text { Temper- } \\
\text { ature } \\
\left({ }^{\circ} \mathrm{C}\right)\end{array}$ & $\begin{array}{c}\text { Dissolved } \\
\text { oxygen } \\
\text { (ppm) }\end{array}$ & $\begin{array}{c}\text { Temper- } \\
\text { ature } \\
\left({ }^{\circ} \mathrm{C}\right)\end{array}$ & $\begin{array}{c}\text { Dissolved } \\
\text { oxygen } \\
\text { (ppm) }\end{array}$ & $\begin{array}{c}\text { Temper- } \\
\text { ature } \\
\left({ }^{\circ} \mathrm{C}\right)\end{array}$ & $\begin{array}{c}\text { Dissolved } \\
\text { oxygen } \\
\text { (ppm) }\end{array}$ & $\begin{array}{c}\text { Temper- } \\
\text { ature } \\
\left({ }^{\circ} \mathbf{C}\right)\end{array}$ \\
\hline 9/9/99 & 4.18 & 22.3 & 8.57 & 22.5 & 4.97 & 22.5 & 4.43 & 22.6 \\
\hline 9/17/99 & 4.19 & 21 & 4.71 & 21.1 & 4.33 & 21.1 & 4.01 & 21.1 \\
\hline 9/30/99 & 4.21 & 20.9 & 7.48 & 20.7 & 5.09 & 20.7 & 4.33 & 20.6 \\
\hline $10 / 4 / 99$ & 3.25 & 20.1 & 8.4 & 20.3 & 4.86 & 20.3 & 4.4 & 20.5 \\
\hline $10 / 15 / 99$ & 3.79 & 19.4 & 6.65 & 19.3 & 4.9 & 19.2 & 4.14 & 19.7 \\
\hline 10/22/99 & 5.12 & 16.5 & 8.01 & 16.4 & 6.26 & 16.4 & 5.66 & 16.3 \\
\hline 10/30/99 & 5.56 & 15 & 7.32 & 16.1 & 5.2 & 16 & 4.74 & 15.8 \\
\hline $11 / 4 / 99$ & 5.7 & 16.1 & 9.84 & 16.1 & 7.23 & 15.8 & 4.76 & 15.9 \\
\hline $11 / 11 / 99$ & 3.22 & 15.1 & 8.14 & 14.6 & 4.26 & 14.6 & 3.78 & 14.6 \\
\hline $11 / 19 / 99$ & 3.95 & 12.9 & 7.36 & 12.7 & 4.53 & 12.6 & 3.4 & 12.6 \\
\hline $11 / 24 / 99$ & 6.59 & 10.6 & 9.75 & 11 & 9.43 & 11 & 8.47 & 11.1 \\
\hline 12/2/99 & 4.67 & 11.5 & 8.39 & 11 & 5.5 & 11.1 & 4.88 & 11.3 \\
\hline 12/3/99 & 4.26 & 10 & 7.56 & 10.4 & 4.93 & 10.3 & 4.2 & 10.2 \\
\hline 12/10/99 & 7.67 & 9.6 & 8.82 & 9.8 & 9.2 & 9.7 & 8.4 & 9.6 \\
\hline $12 / 17 / 99$ & 7.57 & 9.2 & 8.52 & 8.9 & 7.94 & 9 & 7.4 & 9.3 \\
\hline 12/21/99 & 8.13 & 9.9 & 8.07 & 9.8 & 7.69 & 9.9 & 7.32 & 10 \\
\hline 12/28/99 & 8.75 & 8 & 4.18 & 9.2 & 4.06 & 9.3 & 4.44 & 8.2 \\
\hline \multicolumn{9}{|c|}{ Data Summary } \\
\hline Number of samples & 52 & 52 & 52 & 52 & 52 & 52 & 52 & 52 \\
\hline Minimum & 3.1 & 7.7 & 4.18 & 8 & 3.38 & 8 & 3.27 & 8.2 \\
\hline Maximum & 12.4 & 25.2 & 18.6 & 25.5 & 13.9 & 25.1 & 12.9 & 24.8 \\
\hline Median & 5.8 & 15.1 & 8.5 & 16.2 & 7.0 & 15.7 & 5.8 & 15.7 \\
\hline 75th percentile & 7.6 & 20.9 & 11.1 & 21.2 & 8.78 & 21.2 & 7.45 & 21.2 \\
\hline 25th percentile & 4.21 & 10.6 & 7.47 & 11 & 5.18 & 10.9 & 4.38 & 11.0 \\
\hline Interquartile range & 3.4 & 10.3 & 3.6 & 10.2 & 3.6 & 10.2 & 3.0 & 10.2 \\
\hline
\end{tabular}




\section{Surface Water}

Table 7-20. Seasonal inventory of plants and animals, Livermore site, 1999.

\begin{tabular}{|c|c|c|c|c|c|c|c|}
\hline \multirow{3}{*}{ Common Name } & \multirow{3}{*}{ Scientific Name } & \multicolumn{6}{|c|}{ Location } \\
\hline & & \multicolumn{2}{|c|}{ DRB } & \multicolumn{2}{|c|}{ Arroyo Las Positas } & \multicolumn{2}{|c|}{$\begin{array}{c}\text { Tributaries } \\
\text { (CDB and CDB2) }\end{array}$} \\
\hline & & Spring(a) $^{(a)}$ & Fall(b) & Spring & Fall & Spring & Fall \\
\hline \multicolumn{8}{|l|}{ Birds } \\
\hline American crow & Corvus bachyrhynchos & $P^{(c)}$ & $P$ & $P$ & $P$ & $P$ & $P$ \\
\hline American kestrel & Falco sparverius & $P$ & $P$ & $\mathrm{NO}(\mathrm{d})$ & $P$ & NO & NO \\
\hline American robin & Turdus migratorius & $P$ & $P$ & $P$ & $P$ & NO & NO \\
\hline Anna's hummingbird & Calypte anna & $P$ & $P$ & $P$ & $P$ & $P$ & $P$ \\
\hline Black phoebe & Sayornis saya & NO & NO & $P$ & $P$ & NO & NO \\
\hline Black-chinned hummingbird & Archilochus alexandri & $P$ & NO & NO & NO & NO & NO \\
\hline Black-necked stilt & Himantopus mexicanus & $\mathrm{P}$ & NO & NO & NO & NO & NO \\
\hline Brewer's blackbird & Euphagus cyanocephalus & $P$ & $P$ & $P$ & $P$ & $P$ & $\mathrm{P}$ \\
\hline Bufflehead & Bucephala & $\mathrm{P}$ & $P$ & NO & NO & NO & NO \\
\hline Bushtit & Psaltriparus minimus & NO & NO & NO & NO & $P$ & $P$ \\
\hline Canada goose & Branta canadensis & $P$ & $P$ & NO & NO & NO & NO \\
\hline Cedar waxwing & Bombycilla garrulus & $P$ & NO & NO & NO & $P$ & NO \\
\hline Chestnut-backed chickadee & Parus rufescens & $\mathrm{P}$ & NO & $P$ & NO & NO & NO \\
\hline Cliff swallow & Hirundo pyrrhonota & $P$ & NO & NO & NO & NO & NO \\
\hline Common raven & Corvus corax & NO & NO & $P$ & $P$ & NO & NO \\
\hline Double-crested cormorant & Phalacrocorax auritus & $\mathrm{P}$ & $P$ & NO & NO & NO & NO \\
\hline European starling & Sturnus vulgaris & $\mathrm{P}$ & $\mathrm{P}$ & $P$ & $P$ & $P$ & $\mathrm{P}$ \\
\hline Great egret & Casmerodius albus & $P$ & $\mathrm{P}$ & $\mathrm{P}$ & $\mathrm{P}$ & NO & NO \\
\hline Greater scaup & Aythya marila & $\mathrm{P}$ & NO & NO & NO & NO & NO \\
\hline Greater-yellow legs & Tringa melanoleuca & $P$ & $\mathrm{P}$ & NO & NO & NO & NO \\
\hline House finch & Carpodacus mexicanus & $P$ & $\mathrm{P}$ & $\mathrm{P}$ & $\mathrm{P}$ & $P$ & $\mathrm{P}$ \\
\hline Killdeer & Haradrius vociferus & $\mathrm{P}$ & $\mathrm{P}$ & NO & NO & NO & NO \\
\hline Loggerhead shrike & Lanius Iudovicianus & NO & NO & $P$ & NO & NO & NO \\
\hline Mallard & Anas platyrhynchos & $\mathrm{P}$ & $\mathrm{P}$ & NO & NO & $P$ & $\mathrm{P}$ \\
\hline Mourning dove & Zenaida macroura & $\mathrm{P}$ & $\mathrm{P}$ & $P$ & $P$ & $\mathrm{P}$ & $P$ \\
\hline Pied-billed grebe & Podilymbus podiceps & $\mathrm{P}$ & $P$ & NO & NO & NO & NO \\
\hline Red-shafted flicker & Colaptes auratus & $P$ & $P$ & $P$ & $P$ & NO & NO \\
\hline Red-shouldered hawk & Buteo lineatus & NO & NO & $P$ & $P$ & NO & NO \\
\hline
\end{tabular}


Table 7-20. Seasonal inventory of plants and animals, Livermore site, 1999 (continued).

\begin{tabular}{|c|c|c|c|c|c|c|c|}
\hline \multirow{3}{*}{ Common Name } & \multirow{3}{*}{ Scientific Name } & \multicolumn{6}{|c|}{ Location } \\
\hline & & \multicolumn{2}{|c|}{ DRB } & \multicolumn{2}{|c|}{ Arroyo Las Positas } & \multicolumn{2}{|c|}{$\begin{array}{c}\text { Tributaries } \\
\text { (CDB and CDB2) }\end{array}$} \\
\hline & & Spring(a) & Fall(b) & Spring & Fall & Spring & Fall \\
\hline Red-tailed hawk & Buteo jamaicensis & NO & NO & $\mathrm{P}$ & $P$ & $\mathrm{P}$ & $\mathrm{P}$ \\
\hline Red-winged blackbird & Agelaius phoeniceus & $P$ & $P$ & $P$ & $P$ & $P$ & $P$ \\
\hline Rock dove & Columba livia & $\mathrm{P}$ & $\mathrm{P}$ & $P$ & $P$ & $P$ & $P$ \\
\hline Ruby-crowned kinglet & Regulus caledula & $\mathrm{P}$ & $\mathrm{P}$ & NO & NO & NO & NO \\
\hline Scrub jay & Aphelocoma coerulescens & $P$ & $P$ & $\mathrm{P}$ & $P$ & $P$ & $P$ \\
\hline Snowy egret & Egretta thula & NO & NO & $P$ & $P$ & NO & NO \\
\hline Song sparrow & Zmelospiza melodia & $P$ & $P$ & $P$ & $P$ & $P$ & $P$ \\
\hline Turkey vulture & Cathartes aura & NO & NO & $\mathrm{P}$ & $\mathrm{P}$ & NO & NO \\
\hline Western gull & Larus occidentalis & $P$ & $P$ & NO & NO & NO & NO \\
\hline Western meadowlark & Sturnella neglecta & NO & NO & $P$ & $P$ & NO & NO \\
\hline White-breasted nuthatch & Sitta carolinensis & NO & NO & NO & NO & $P$ & NO \\
\hline White-crowned sparrow & Zonotrichia leucophrys & $P$ & $P$ & $P$ & $P$ & $P$ & $P$ \\
\hline White-tailed kite & Elanus leucurus & NO & NO & $P$ & $\mathrm{P}$ & NO & NO \\
\hline Yellow-rumped warbler & Dendroica coronata & $P$ & $P$ & $P$ & $\mathrm{P}$ & $P$ & $P$ \\
\hline \multicolumn{8}{|l|}{ Amphibians and reptiles } \\
\hline Bullfrog & Rana catesbeiana & $P$ & $P$ & NO & NO & $P$ & $P$ \\
\hline Pacific tree frog & Hyla regilla & $P$ & $P$ & $P$ & $P$ & $P$ & $P$ \\
\hline Red-legged frog & Rana aurora draytonii & NO & NO & $P$ & $P$ & $P$ & $P$ \\
\hline Western fence lizard & Sceloporus occidentailis & $P$ & $P$ & NO & NO & $P$ & $P$ \\
\hline Western pond turtle & Clemmys marmorata & NO & $P$ & NO & NO & NO & NO \\
\hline Western toad & Bufo borieus & $P$ & $P$ & $P$ & $P$ & $P$ & $P$ \\
\hline \multicolumn{8}{|l|}{ Fish } \\
\hline Catfish & Ictalurus sp. & $P$ & $\mathrm{P}$ & NO & NO & NO & NO \\
\hline Mosquito fish & Gambusia affinis & $\mathrm{P}$ & $\mathrm{P}$ & $P$ & $\mathrm{P}$ & $\mathrm{P}$ & $\mathrm{P}$ \\
\hline Prickly sculpin & Cottus asper & NO & NO & $P$ & $P$ & $P$ & $P$ \\
\hline
\end{tabular}




\section{Surface Water}

Table 7-20. Seasonal inventory of plants and animals, Livermore site, 1999 (concluded).

\begin{tabular}{|c|c|c|c|}
\hline Common Name & Scientific Name & Spring $^{(a)}$ & Fall(b) \\
\hline \multicolumn{4}{|l|}{ Mammals } \\
\hline California ground squirrel & Spermophilus beecheyi & $\mathrm{P}$ & $P$ \\
\hline California meadow vole & Microtus californicus & $\mathrm{P}$ & $\mathrm{P}$ \\
\hline Feral house cat & Felis domesticus & $\mathrm{P}$ & $\mathrm{P}$ \\
\hline Gray fox & Urocyon cinereoargenteus & $\mathrm{P}$ & $\mathrm{P}$ \\
\hline House mouse & Mus musculus & $\mathrm{P}$ & $\mathrm{P}$ \\
\hline Red fox & Vulps vulpes & $\mathrm{P}$ & $\mathrm{P}$ \\
\hline \multicolumn{4}{|l|}{ Vegetation } \\
\hline American water-plantain & Alisma plantago-aquatica & $\mathrm{P}$ & $\mathrm{P}$ \\
\hline Bullrush & Scripus spp. & $\mathrm{P}$ & $P$ \\
\hline Cattail & Typha latifolia & $\mathrm{P}$ & $\mathrm{P}$ \\
\hline Cocklebur & Xanthium spinosum & $P$ & $\mathrm{P}$ \\
\hline Coontail & Ceratophyllum demersum & $\mathrm{P}$ & $\mathrm{P}$ \\
\hline Curly dock & Rumex crispus & $\mathrm{P}$ & $\mathrm{P}$ \\
\hline Harding grass & Phalaris aquatica & $\mathrm{P}$ & $\mathrm{P}$ \\
\hline Leafy pondweed & Potamogeton foliosus & $P$ & $\mathrm{P}$ \\
\hline Nutsedge & Carex spp. & $\mathrm{P}$ & $\mathrm{P}$ \\
\hline Spearscale & Atriplex triangularis & $\mathrm{P}$ & $P$ \\
\hline Water velvet & Azolla mexicana & $\mathrm{P}$ & $\mathrm{P}$ \\
\hline Watercress & Rorippa nasturium-aquaticum & $\mathrm{P}$ & $\mathrm{P}$ \\
\hline Waterpepper & Polygonum hydropiperoides & $\mathrm{P}$ & $\mathrm{P}$ \\
\hline Willow & Salix spp. & $\mathrm{P}$ & $P$ \\
\hline
\end{tabular}

a Spring survey dates are as follows: amphibians 2/22/99, 3/5/99, 3/10/99, and 3/16/99; birds 2/21/99, 2/25/99, 3/10/99, and 3/16/99; fish 3/1/99; mammals 3/13/99; and vegetation 3/99.

b Fall survey dates are as follows: amphibians 3/27/99, 10/21/99, 11/5/99, and 11/18/99; birds 10/21/99, 10/28/99, 1/11/99, and 11/18/99; fish 11/11/99; mammals 10/29/99; and vegetation 9/99.

c $P=$ present.

d $\quad \mathrm{NO}=$ Not observed. 
Table 7-21. Radioactivity in surface and drinking water (Bq/L) in the Livermore Valley, 1999.

\begin{tabular}{|c|c|c|c|c|}
\hline Locations & Date & Tritium & Gross alpha & Gross beta \\
\hline \multicolumn{5}{|l|}{ Drinking waters } \\
\hline \multirow[t]{2}{*}{ BELL } & $1 / 14$ & $-5.74 \pm 2.14$ & $0.0132 \pm 0.0481$ & $1.03 \pm 0.178$ \\
\hline & $7 / 16$ & $-1.48 \pm 1.38$ & $0.0191 \pm 0.0206$ & $0.0718 \pm 0.0168$ \\
\hline \multirow[t]{2}{*}{ GAS } & $1 / 14$ & $-5.25 \pm 2.15$ & $0.168 \pm 0.0703$ & $0.389 \pm 0.155$ \\
\hline & $7 / 15$ & $-1.64 \pm 1.41$ & $0.0159 \pm 0.0236$ & $0.0611 \pm 0.0312$ \\
\hline \multirow[t]{3}{*}{ PALM } & $1 / 14$ & $-5.96 \pm 2.15$ & $0.0762 \pm 0.0629$ & $0.192 \pm 0.141$ \\
\hline & $7 / 16$ & $-1.21 \pm 1.4$ & -(a) & - (a) $^{(2}$ \\
\hline & $7 / 30$ & - (a) & $0.0114 \pm 0.0264$ & $0.0929 \pm 0.0418$ \\
\hline \multirow[t]{2}{*}{$\mathrm{ORCH}$} & $1 / 14$ & $-4.96 \pm 2.18$ & $0.0463 \pm 0.0629$ & $0.132 \pm 0.126$ \\
\hline & $7 / 15$ & $0.356 \pm 1.48$ & $0.0366 \pm 0.0411$ & $0.381 \pm 0.091$ \\
\hline \multirow[t]{3}{*}{ TAP } & $1 / 14$ & $-5 \pm 2.16$ & $0.0403 \pm 0.0444$ & $<2.9$ \\
\hline & $7 / 15$ & - $^{(\mathrm{a})}$ & $0.00622 \pm 0.0105$ & $0.0353 \pm 0.0118$ \\
\hline & $7 / 16$ & $-0.377 \pm 1.44$ & -(a) & -(a) \\
\hline \multicolumn{5}{|l|}{ Surface waters } \\
\hline \multirow[t]{2}{*}{ CAL } & $1 / 14$ & $-4.59 \pm 2.26$ & $0.057 \pm 0.0481$ & $0.071 \pm 0.133$ \\
\hline & $7 / 15$ & $-0.574 \pm 1.42$ & $-0.00181 \pm 0.0114$ & $0.074 \pm 0.0175$ \\
\hline \multirow[t]{2}{*}{ DEL } & $1 / 14$ & $-5.22 \pm 2.18$ & $0.109 \pm 0.0518$ & $0.396 \pm 0.137$ \\
\hline & $7 / 15$ & $-0.71 \pm 1.46$ & $0.0293 \pm 0.0273$ & $0.14 \pm 0.0335$ \\
\hline \multirow[t]{2}{*}{ DUCK } & $1 / 14$ & $-4.63 \pm 2.19$ & $<5.3$ & $0.651 \pm 0.252$ \\
\hline & $7 / 15$ & $0.87 \pm 1.5$ & $0.232 \pm 0.188$ & $0.326 \pm 0.188$ \\
\hline \multirow[t]{2}{*}{ ALAG } & $1 / 14$ & $-4.14 \pm 2.22$ & $0.0847 \pm 0.0666$ & $0.136 \pm 0.141$ \\
\hline & $7 / 15$ & $-1.04 \pm 1.41$ & $0.0237 \pm 0.0256$ & $0.091 \pm 0.0275$ \\
\hline \multirow[t]{2}{*}{ SHAD } & $1 / 14$ & $-4.55 \pm 2.19$ & $0.196 \pm 0.0925$ & $0.193 \pm 0.13$ \\
\hline & $7 / 15$ & $1.18 \pm 1.56$ & $0.0459 \pm 0.0429$ & $0.181 \pm 0.0481$ \\
\hline \multirow[t]{2}{*}{ ZON7 } & $1 / 14$ & $-5.74 \pm 2.14$ & $0.374 \pm 0.0999$ & $0.555 \pm 0.163$ \\
\hline & $7 / 16$ & $-0.518 \pm 1.43$ & $0.0208 \pm 0.0194$ & $0.0592 \pm 0.016$ \\
\hline \multicolumn{5}{|l|}{ On-site pool } \\
\hline \multirow[t]{4}{*}{ POOL } & $1 / 14$ & $0.0807 \pm 2.4$ & $0.102 \pm 0.118$ & $0.201 \pm 0.137$ \\
\hline & $4 / 2$ & $10.1 \pm 1.91$ & —(b) $^{(\mathrm{n}}$ & -(b) \\
\hline & $7 / 16$ & $4.66 \pm 1.72$ & $0.0574 \pm 0.0821$ & $0.225 \pm 0.105$ \\
\hline & $10 / 12$ & $5.29 \pm 2.35$ & -(b) & -(b) \\
\hline
\end{tabular}

Note: Radioactivities are reported as the measured concentration and either an uncertainty ( $\pm 2 \sigma$ counting error) or as being less than or equal to the detection limit. If the concentration is less than or equal to the uncertainty or the detection limit, the result is considered to be a nondetection. See the main volume, Chapter 14, Quality Assurance.

a Third-quarter-samples for all analytes were not collected on the same day (procedures do not require same-day sample collection).

b Sampled semiannually_Pool samples are collected quarterly for tritium, semiannually for gross alpha and gross beta radiation. 

There are no supplemental data in this chapter. Please see the main volume for details about Ground Water Investigation and Remediation. 



\section{Ground Water Monitoring}

Eric Christofferson

Richard A. Brown

Sandra Mathews

Rebecca Ward

\section{Methods}

Representative samples of ground water from monitoring wells were obtained by following the written protocols contained in the LLNL Environmental Restoration Project Standard Operating Procedures (Dibley and Depue 1999), which are updated annually. The protocols cover sampling techniques and specific information for the analytes that are routinely searched for in ground water. Different sampling techniques were applied to different wells depending on whether they were fitted with submersible pumps, had to be bailed, or contained Barcad devices, where we used nitrogen gas under pressure to extract water samples.

Typically, sampling technologists purged wells of standing water and waited for the wells to recover before they collected water samples. They wore disposable vinyl gloves to prevent accidental contamination during sampling and cleaned $\mathrm{pH}$ and depth-to-water probes with deionized water after each use. For quality assurance purposes, they obtained field blank samples and equipment blank samples to test the cleanliness of the sampling methods. They used clean sample containers and, where required, they used ultrapure chemicals (mostly acids) to preserve the samples.

Off-site laboratories performed most of the water analyses during 1999, under contract with LLNL. LLNL personnel primarily measured tritium activity in an on-site laboratory dedicated to that purpose. (Note that the ground water radioactivity data for 1999 include some small negative values [in Bq/L]. These can occur when the independently determined correction for background radioactivity is subtracted from measurements of ground waters that contain little or no radioactive material.)

At Site 300, wastewater samples were collected in accordance with written protocols outlined in Operations and Regulatory Affairs Division, Water Guidance and Monitoring Group procedure EMP-W-S (Rev. 4), Water Sampling. The procedure details several sample collection methodologies appropriate for wastewater sampling, and the field technologist selected the exact methodology for sampling the process discharge. 


\section{Ground Water Monitoring}

As with ground water sampling, standard sample handling and hygiene procedures were employed to prevent cross-contamination (e.g., wearing disposable gloves, decontaminating sampling equipment, and maintaining samples at $4 \pm 2^{\circ}$ Celsius). Replicates, field blanks, and trip blanks were collected for quality assurance/quality control purposes. Most analyses were performed off site by contract analytical laboratories except when the on-site laboratory offered better capabilities and/or detection limits.

Technologists sampled wastewater from the chemistry area and sampled retention tanks associated with Buildings 825, 826, and 827 using Hazardous Waste Management Procedure 411. Wastewater was held in retention tanks until analytical results were reviewed for compliance with Waste Discharge Requirements No. 96-248.

\section{Livermore Site}

Table 9-1 lists the ground water constituents monitored at the Livermore site and at Site 300, the EPA-approved methods used to measure them, and the detection limits (reporting limits) employed.

Tables 9-2 to 9-11 report routine surveillance monitoring for wells along the Livermore site perimeter; wells W-008, W-017, and W-221 are upgradient and the remaining seven wells are downgradient from the site. Tables 9-12 and 9-13 contain analytical data obtained from monitoring wells downgradient from the Taxi Strip Area, and Tables 9-14 through 9-18 contain analytical data obtained from monitoring wells downgradient from the East Traffic Circle Area. Table 9-19 contains data from W-593, downgradient from the Decontamination and Waste Treatment Facility. Tables 9-20 through 9-22 contain data from wells downgradient from the Hazardous Waste Management facilities near Buildings 514 and 612. Tables 9-23 through 9-25 list results of metals analyses from wells downgradient from where metal wastes are managed. Table 9-26 contains data from monitoring well W-305 just upgradient from Superblock, containing the Plutonium and Tritium facilities. Data from SIP-331-001, just downgradient from the Plutonium Facility is contained in Table 9-27; data from well W-148, downgradient from both the Plutonium and Tritium Facilities, is contained in Table 9-28.

Tritium activities in ground water at 18 monitoring wells in the Livermore Valley are listed in Table 9-29. 


\section{Ground Water Monitoring}

\section{Site 300}

Tables 9-30 through 9-43 contain chemical data for Site 300 surveillance monitoring wells (Elk ravine drainage area, including closed landfill pits 2, 8, and 9, and Corral Hollow creek drainage area, including closed high-explosives burn pit, standby water supply, active water supply, and off-site wells).

Additional chemical data for Site 300 ground water that was obtained during 1999 from compliance monitoring of closed landfill pits 1, 6, and 7, active surface water impoundments, and sewage ponds can be found in published compliance monitoring reports (Brown et al. 2000; Christofferson and MacQueen 2000; and Christofferson and Taffet 2000). 
Table 9-1a. Analytical methods and reporting limits for inorganic constituents of concern in ground water. (a)

\begin{tabular}{|c|c|c|}
\hline $\begin{array}{l}\text { Constituents of } \\
\text { concern }\end{array}$ & $\begin{array}{l}\text { Analytical } \\
\text { method }\end{array}$ & $\begin{array}{l}\text { Reporting } \\
\text { limit }\end{array}$ \\
\hline \multicolumn{3}{|l|}{ Metals and minerals (mg/L) } \\
\hline All alkalinities & EPA 310.1 & 1 \\
\hline Aluminum & EPA 200.7 & 0.02 \\
\hline Ammonia nitrogen (as $\mathrm{N}$ ) & EPA 350.3 & 0.03 \\
\hline Antimony & EPA 204.2 & 0.005 \\
\hline Arsenic & EPA 206.2 & 0.002 \\
\hline Barium & EPA 200.7 & 0.025 \\
\hline Beryllium & EPA 210.2 & 0.0005 \\
\hline Cadmium & EPA 213.2 & 0.0005 \\
\hline Calcium & EPA 200.7 & 0.5 \\
\hline Chloride & EPA 325.3 or 300.0 & 1 \\
\hline Chromium & EPA 218.2 & 0.001 \\
\hline Cobalt & EPA 200.7 & 0.025 \\
\hline Copper & EPA 200.7 & 0.01 \\
\hline Fluoride & EPA 340.2 & 0.1 \\
\hline Hardness, total $\left(\mathrm{as} \mathrm{CaCO}_{3}\right)$ & SM 2320B & 1 \\
\hline Iron & EPA 200.7 & 0.1 \\
\hline Lead & EPA 239.2 & 0.002 \\
\hline Magnesium & EPA 200.7 & 0.5 \\
\hline Manganese & EPA 200.7 & 0.03 \\
\hline Mercury & EPA 245.2 & 0.0002 \\
\hline Molybdenum & EPA 200.7 & 0.025 \\
\hline Nickel & EPA 249.2 & 0.005 \\
\hline Nitrate $\left(\right.$ as $\left.\mathrm{NO}_{3}\right)$ & EPA 353.2 & 0.1 \\
\hline Perchlorate & EPA 300.0-IC & 0.004 \\
\hline Potassium & EPA 200.7 & 1 \\
\hline Selenium & EPA 270.2 & 0.002 \\
\hline Silver & EPA 272.2 & 0.001 \\
\hline Sodium & EPA 200.7 & 1 \\
\hline Sulfate & EPA 300.0 & 1 \\
\hline Surfactants & EPA 425.1 & 0.5 \\
\hline Thallium & EPA 279.2 & 0.001 \\
\hline Total dissolved solids & EPA 160.1 & 1 \\
\hline Total Kjeldahl nitrogen & EPA 351.4 & 0.2 \\
\hline Total suspended solids & EPA 160.2 & 1 \\
\hline
\end{tabular}


Table 9-1a. Analytical methods and reporting limits for inorganic constituents of concern in ground water (concluded). (a)

\begin{tabular}{|c|c|c|}
\hline $\begin{array}{c}\text { Constituents of } \\
\text { concern }\end{array}$ & $\begin{array}{l}\text { Analytical } \\
\text { method }\end{array}$ & $\begin{array}{l}\text { Reporting } \\
\text { limit }\end{array}$ \\
\hline \multicolumn{3}{|c|}{ Metals and minerals (mg/L) (continued) } \\
\hline Vanadium & EPA 200.7 & 0.025 \\
\hline Zinc & EPA 200.7 & 0.02 \\
\hline \multicolumn{3}{|l|}{ Phenolics (mg/L) } \\
\hline Phenolics & EPA 420.1 & 0.005 \\
\hline \multicolumn{3}{|l|}{ General indicator parameters } \\
\hline $\mathrm{pH}$ (pH units) & EPA 150.1 & none \\
\hline Specific conductance $(\mu \mathrm{mho} / \mathrm{cm})$ & EPA 120.1 & 1 \\
\hline Total organic carbon (mg/L) & EPA 415.1 & 0.5 \\
\hline Total organic halides (mg/L) & EPA 9020 & 0.01 \\
\hline \multicolumn{3}{|l|}{ Explosive compounds ( $\mu \mathrm{g} / \mathrm{L})$} \\
\hline $\mathrm{HMX}^{(\mathrm{b})}$ & HPLC & 5 \\
\hline $\mathrm{RDX}^{(\mathrm{c})}$ & HPLC & 5 \\
\hline $\mathrm{TNT}^{(\mathrm{d})}$ & HPLC & 5 \\
\hline \multicolumn{3}{|l|}{ Radioactivity (Bq/L) } \\
\hline Gross alpha & EPA 900 & 0.1 \\
\hline Gross beta & EPA 900 & 0.1 \\
\hline \multicolumn{3}{|l|}{ Radioisotopes (Bq/L) } \\
\hline Americium-241 & U-NAS-NS-3050 & 0.0037 \\
\hline Plutonium-238 & U-NAS-NS-3050 & 0.0037 \\
\hline Plutonium-239+240 & U-NAS-NS-3050 & 0.0037 \\
\hline Radon-222 & EPA 913 & 0.4 \\
\hline Radium-226 & EPA 903 & 0.0037 \\
\hline Radium-228 & EPA 904 & 0.037 \\
\hline Strontium-90 & SM 7500 & $0.1-0.15$ \\
\hline Thorium-228 & U-NAS-NS-3050 & 0.009 \\
\hline Thorium-230 & U-NAS-NS-3050 & 0.006 \\
\hline Thorium-232 & U-NAS-NS-3050 & 0.006 \\
\hline Tritium & LLNL-RAS-011 & 2 \\
\hline Uranium-234 & U-NAS-NS-3050 & 0.0037 \\
\hline Uranium-235 & U-NAS-NS-3050 & 0.0037 \\
\hline Uranium-238 & U-NAS-NS-3050 & 0.0037 \\
\hline
\end{tabular}

a The significant figures displayed in this table vary by constituents of concern. These variations reflect regulatory agency permit stipulations, or the applicable analytical laboratory contract under which the work was performed, or both.

b HMX is octahydro-1,3,5,7-tetranitro-1,3,5,7-tetrazocine.

c RDX is hexahydro-1,3,5-trinitro-1,3,5-triazine.

d TNT is 2,4,6-trinitrotoluene. 
Table 9-1b. Analytical methods and reporting limits for organic constituents of concern in ground water. (a)

\begin{tabular}{|l|c|}
\hline \multicolumn{1}{|c|}{$\begin{array}{c}\text { Constituents } \\
\text { of concern }\end{array}$} & $\begin{array}{c}\text { Reporting limit } \\
(\boldsymbol{\mu g} / \mathbf{L})\end{array}$ \\
\hline EPA Method 502.2 & \\
1,1,1,2-Tetrachloroethane & 0.2 \\
1,1,1-Trichloroethane & 0.2 \\
1,1,2,2-Tetrachloroethane & 0.2 \\
1,1,2-Trichloroethane & 0.2 \\
1,1-Dichloroethane & 0.2 \\
1,1-Dichloroethene & 0.2 \\
1,1-Dichloropropene & 0.2 \\
1,2,3-Trichlorobenzene & 0.2 \\
1,2,3-Trichloropropane & 0.2 \\
1,2,4-Trichlorobenzene & 0.2 \\
1,2,4-Trimethylbenzene & 0.2 \\
1,2-Dichlorobenzene & 0.2 \\
1,2-Dichloroethane & 0.2 \\
1,2-Dichloropropane & 0.2 \\
1,3,5-Trimethylbenzene & 0.2 \\
1,3-Dichlorobenzene & 0.2 \\
1,3-Dichloropropane & 0.2 \\
1,4-Dichlorobenzene & 0.2 \\
2,2-Dichloropropane & 0.2 \\
2-Chlorotoluene & 0.2 \\
4-Chlorotoluene & 0.2 \\
Benzene & 0.2 \\
Bromobenzene & 0.2 \\
Bromochloromethane & 0.2 \\
Bromodichloromethane & 0.2 \\
Bromoform & 0.2 \\
Bromomethane & 0.2 \\
Carbon tetrachloride & 0.2 \\
Chlorobenzene & 0.2 \\
Chloroethane & 0.2 \\
\hline
\end{tabular}

\begin{tabular}{|l|c|}
\hline \multicolumn{1}{|c|}{$\begin{array}{c}\text { Constituents } \\
\text { of concern }\end{array}$} & $\begin{array}{c}\text { Reporting limit } \\
(\boldsymbol{\mu g} / \mathbf{L})\end{array}$ \\
\hline Chloroform & 0.2 \\
Chloromethane & 0.2 \\
cis-1,2-Dichloroethene & 0.2 \\
cis-1,3-Dichloropropene & 0.5 \\
Dibromochloromethane & 0.2 \\
Dibromomethane & 0.2 \\
Dichlorodifluoromethane & 0.2 \\
Ethylbenzene & 0.2 \\
Freon 113 & 0.2 \\
Hexachlorobutadiene & 0.2 \\
Isopropylbenzene & 0.2 \\
$m$ - and $p$-Xylene isomers & 0.2 \\
Methylene chloride & 0.2 \\
$n$-Butylbenzene & 0.2 \\
$n$-Propylbenzene & 0.2 \\
Naphthalene & 0.2 \\
o-Xylene & 0.2 \\
Isopropyl toluene & 0.2 \\
sec-Butylbenzene & 0.2 \\
Styrene & 0.2 \\
tert-Butylbenzene & 0.2 \\
Tetrachloroethene & 0.2 \\
Toluene & 0.2 \\
trans-1,2-Dichloroethene & \\
trans-1,3-Dichloropropene & 0.2 \\
Trichloroethene & 0.2 \\
Trichlorofluoromethane & 0.2 \\
Bromacil & 0 Method 525.2 \\
\hline
\end{tabular}


Table 9-1b. Analytical methods and reporting limits for organic constituents of concern in ground water (continued). (a)

\begin{tabular}{|c|c|c|c|}
\hline $\begin{array}{l}\text { Constituents } \\
\text { of concern }\end{array}$ & $\begin{array}{c}\text { Reporting limit } \\
(\mu \mathrm{g} / \mathrm{L})\end{array}$ & $\begin{array}{c}\text { Constituents } \\
\text { of concern }\end{array}$ & $\begin{array}{c}\text { Reporting limit } \\
(\mu \mathrm{g} / \mathrm{L})\end{array}$ \\
\hline EPA Method 525.2 & & 2-Chlorotoluene & 1 \\
\hline Butachlor & 0.3 & 4-Chlorotoluene & 1 \\
\hline Diazinon & 0.2 & Benzene & 1 \\
\hline Dimethoate & 2 & Bromobenzene & 1 \\
\hline Metolachlor & 0.5 & Bromodichloromethane & 1 \\
\hline Metribuzin & 0.5 & Bromoform & 1 \\
\hline Molinate & 0.5 & Bromomethane & 2 \\
\hline Prometryn & 0.5 & Carbon tetrachloride & 1 \\
\hline Propachlor & 0.5 & Chlorobenzene & 1 \\
\hline Simazine & 0.2 & Chloroethane & 2 \\
\hline Thiobencarb & 0.5 & Chloroform & 1 \\
\hline EPA Method 524.2 & & Chloromethane & 2 \\
\hline 1,1,1,2-Tetrachloroethane & 1 & cis-1,2-Dichloroethene & 1 \\
\hline 1,1,1-Trichloroethane & 1 & cis-1,3-Dichloropropene & 1 \\
\hline 1,1,2,2-Tetrachloroethane & 1 & Dibromochloromethane & 1 \\
\hline 1,1,2-Trichloroethane & 1 & Dibromomethane & 1 \\
\hline 1,1-Dichloroethane & 1 & Dichlorodifluoromethane & 2 \\
\hline 1,1-Dichloroethene & 1 & Ethylbenzene & 1 \\
\hline 1,1-Dichloropropene & 1 & Ethylene dibromide & 1 \\
\hline 1,2,3-Trichlorobenzene & 1 & Freon 113 & 1 \\
\hline 1,2,3-Trichloropropane & 1 & Hexachlorobutadiene & 1 \\
\hline 1,2,4-Trichlorobenzene & 1 & Isopropylbenzene & 1 \\
\hline 1,2,4-Trimethylbenzene & 1 & $m$ - and $p$-Xylene isomers & 1 \\
\hline 1,2-Dibromo-3-chloropropane & 2 & Methylene chloride & 1 \\
\hline 1,2-Dichlorobenzene & 1 & $n$-Butylbenzene & 1 \\
\hline 1,2-Dichloroethane & 1 & $n$-Propylbenzene & 1 \\
\hline 1,2-Dichloropropane & 1 & Naphthalene & 1 \\
\hline 1,3,5-Trimethylbenzene & 1 & o-Xylene & 1 \\
\hline 1,3-Dichlorobenzene & 1 & Isopropyl toluene & 1 \\
\hline 1,3-Dichloropropane & 1 & sec-Butylbenzene & 1 \\
\hline 1,4-Dichlorobenzene & 1 & Styrene & 1 \\
\hline
\end{tabular}


Table 9-1b. Analytical methods and reporting limits for organic constituents of concern in ground water (continued). (a)

\begin{tabular}{|c|c|c|c|}
\hline $\begin{array}{c}\text { Constituents } \\
\text { of concern }\end{array}$ & $\begin{array}{c}\text { Reporting limit } \\
(\mu \mathrm{g} / \mathrm{L})\end{array}$ & $\begin{array}{c}\text { Constituents } \\
\text { of concern }\end{array}$ & $\begin{array}{c}\text { Reporting limit } \\
(\mu \mathrm{g} / \mathrm{L})\end{array}$ \\
\hline EPA Method 524.2 (cont'd) & & cis-1,3-Dichloropropene & 0.5 \\
\hline tert-Butylbenzene & 1 & Dibromochloromethane & 0.5 \\
\hline Tetrachloroethene & 1 & Dichlorodifluoromethane & 0.5 \\
\hline Toluene & 1 & Freon 113 & 0.5 \\
\hline trans-1,2-Dichloroethene & 1 & Methylene chloride & 0.5 \\
\hline trans-1,3-Dichloropropene & 1 & Tetrachloroethene & 0.5 \\
\hline Trichloroethene & 0.5 & trans-1,3-Dichloropropene & 0.5 \\
\hline Trichlorofluoromethane & 1 & Trichloroethene & 0.5 \\
\hline Vinyl chloride & 2 & Trichlorofluoromethane & 0.5 \\
\hline EPA Method 547 & & Vinyl chloride & 0.5 \\
\hline Glyphosate & 9 & 1,2-Dichlorobenzene & 0.5 \\
\hline EPA Method 601 & & EPA Method 602 & \\
\hline 1,1,1-Trichloroethane & 0.5 & 1,3-Dichlorobenzene & 0.3 \\
\hline $1,1,2,2-$ Tetrachloroethane & 0.5 & 1,4-Dichlorobenzene & 0.3 \\
\hline 1,1,2-Trichloroethane & 0.5 & Benzene & 0.4 \\
\hline 1,1-Dichloroethane & 0.5 & Chlorobenzene & 0.3 \\
\hline 1,1-Dichloroethene & 0.5 & Ethylbenzene & 0.3 \\
\hline 1,2-Dichlorobenzene & 0.5 & $m$ - and $p$-Xylene isomers & 0.4 \\
\hline 1,2-Dichloroethane & 0.5 & o-Xylene & 0.4 \\
\hline 1,2-Dichloroethene (total) & 0.5 & Toluene & 0.3 \\
\hline 1,2-Dichloropropane & 0.5 & Total xylene isomers & 0.4 \\
\hline 1,3-Dichlorobenzene & 0.5 & EPA Method 608 & \\
\hline 1,4-Dichlorobenzene & 0.5 & Aldrin & 0.05 \\
\hline 2-Chloroethylvinylether & 0.5 & $\mathrm{BHC}$, alpha isomer & 0.05 \\
\hline Bromodichloromethane & 0.5 & $\mathrm{BHC}$, beta isomer & 0.05 \\
\hline Bromoform & 0.5 & $\mathrm{BHC}$, delta isomer & 0.05 \\
\hline Bromomethane & 0.5 & $\mathrm{BHC}$, gamma isomer (Lindane) & 0.05 \\
\hline Carbon tetrachloride & 0.5 & Chlordane & 0.5 \\
\hline Chlorobenzene & 0.5 & Dieldrin & 0.1 \\
\hline Chloroethane & 0.5 & Endosulfan I & 0.05 \\
\hline Chloroform & 0.5 & Endosulfan II & 0.1 \\
\hline Chloromethane & 0.5 & Endosulfan sulfate & 0.1 \\
\hline
\end{tabular}


Table 9-1b. Analytical methods and reporting limits for organic constituents of concern in ground water (continued). (a)

\begin{tabular}{|c|c|c|c|}
\hline $\begin{array}{l}\text { Constituents } \\
\text { of concern }\end{array}$ & $\begin{array}{c}\text { Reporting limit } \\
(\mu \mathrm{g} / \mathrm{L})\end{array}$ & $\begin{array}{l}\text { Constituents } \\
\text { of concern }\end{array}$ & $\begin{array}{l}\text { Reporting limit } \\
(\mu \mathrm{g} / \mathrm{L})\end{array}$ \\
\hline EPA Method 608 (cont'd) & & 1,2-Dichloropropane & 0.5 \\
\hline Endrin & 0.1 & 1,3-Dichlorobenzene & 0.5 \\
\hline Endrin aldehyde & 0.1 & 1,4-Dichlorobenzene & 0.5 \\
\hline Heptachlor & 0.05 & 2-Butanone & 20 \\
\hline Heptachlor epoxide & 0.05 & 2-Chloroethylvinylether & 5 \\
\hline Methoxychlor & 0.5 & 2-Hexanone & 20 \\
\hline 4,4'-DDD & 0.1 & 4-Methyl-2-pentanone & 20 \\
\hline 4,4'-DDE & 0.1 & Acetone & 10 \\
\hline 4,4'-DDT & 0.1 & Benzene & 0.5 \\
\hline PCBs & 0.5 & Bromodichloromethane & 0.5 \\
\hline Toxaphene & 1 & Bromoform & 0.5 \\
\hline EPA Method 615 & & Bromomethane & 0.5 \\
\hline $2,4,5-\mathrm{T}$ & 0.5 & Carbon disulfide & 5 \\
\hline 2,4,5-TP (Silvex) & 0.2 & Carbon tetrachloride & 0.5 \\
\hline 2,4-D & 1 & Chlorobenzene & 0.5 \\
\hline 2,4-Dichlorophenoxy acetic acid & 2 & Chloroethane & 1 \\
\hline Dalapon & 2 & Chloroform & 0.5 \\
\hline Dicamba & 1 & Chloromethane & 1 \\
\hline Dichloroprop & 2 & cis-1,3-Dichloropropene & 0.5 \\
\hline Dinoseb & 1 & Dibromochloromethane & 1 \\
\hline MCPA & 250 & Dibromomethane & 1 \\
\hline MCPP & 250 & Dichlorodifluoromethane & 0.5 \\
\hline EPA Method 624 & & Ethylbenzene & 0.5 \\
\hline 1,1,1-Trichloroethane & 0.5 & Freon 113 & 0.5 \\
\hline $1,1,2,2$-Tetrachloroethane & 0.5 & Methylene chloride & 1.0 \\
\hline 1,1,2-Trichloroethane & 0.5 & Styrene & 0.5 \\
\hline 1,1-Dichloroethane & 0.5 & Tetrachloroethene & 0.5 \\
\hline 1,1-Dichloroethene & 0.5 & Toluene & 0.5 \\
\hline 1,2-Dichlorobenzene & 0.5 & Total xylene isomers & 1 \\
\hline 1,2-Dichloroethane & 0.5 & trans-1,3-Dichloropropene & 0.5 \\
\hline 1,2-Dichloroethene (total) & 0.5 & Trichloroethene & 0.5 \\
\hline
\end{tabular}


Table 9-1b. Analytical methods and reporting limits for organic constituents of concern in ground water (continued). (a)

\begin{tabular}{|c|c|c|c|}
\hline $\begin{array}{l}\text { Constituents } \\
\text { of concern }\end{array}$ & $\begin{array}{c}\text { Reporting limit } \\
(\mu \mathrm{g} / \mathrm{L})\end{array}$ & $\begin{array}{l}\text { Constituents } \\
\text { of concern }\end{array}$ & $\begin{array}{c}\text { Reporting limit } \\
(\mu \mathrm{g} / L)\end{array}$ \\
\hline EPA Method 624 (cont'd) & & Acenaphthene & 5 \\
\hline Trichlorofluoromethane & 0.5 & Acenaphthylene & 5 \\
\hline Vinyl chloride & 0.5 & Anthracene & 5 \\
\hline EPA Method 625(b) & & Benzo[a]anthracene & 5 \\
\hline 1,2,4-Trichlorobenzene & 5 & Benzo[a]pyrene & 5 \\
\hline 1,2-Dichlorobenzene & 5 & Benzo[b]fluoranthene & 5 \\
\hline 1,3-Dichlorobenzene & 5 & Benzo[ $g, h, i]$ perylene & 5 \\
\hline 1,4-Dichlorobenzene & 5 & Benzo[k]fluoranthene & 5 \\
\hline 2,4,5-Trichlorophenol & 5 & Benzoic acid & 25 \\
\hline 2,4,6-Trichlorophenol & 5 & Benzyl alcohol & 10 \\
\hline 2,4-Dichlorophenol & 5 & Bis(2-chloroethoxy)methane & 5 \\
\hline 2,4-Dimethylphenol & 5 & Bis(2-chloroisopropyl)ether & 5 \\
\hline 2,4-Dinitrophenol & 25 & Bis(2-ethylhexyl)phthalate & 5 \\
\hline 2,4-Dinitrotoluene & 5 & Butylbenzylphthalate & 5 \\
\hline 2,6-Dinitrotoluene & 5 & Chrysene & 5 \\
\hline 2-Chloronaphthalene & 5 & Di- $n$-butylphthalate & 5 \\
\hline 2-Chlorophenol & 5 & Di-n-octylphthalate & 5 \\
\hline 2-Methylphenol & 5 & Dibenzo[a,h]anthracene & 5 \\
\hline 2-Methyl-4,6-dinitrophenol & 25 & Dibenzofuran & 5 \\
\hline 2-Methylnaphthalene & 5 & Diethylphthalate & 5 \\
\hline 2-Nitroaniline & 25 & Dimethylphthalate & 5 \\
\hline 2-Nitrophenol & 5 & Fluoranthene & 5 \\
\hline 3,3'-Dichlorobenzidine & 10 & Fluorene & 5 \\
\hline 3-Nitroaniline & 25 & Hexachlorobenzene & 5 \\
\hline 4-Bromophenylphenylether & 5 & Hexachlorobutadiene & 5 \\
\hline 4-Chloro-3-methylphenol & 10 & Hexachlorocyclopentadiene & 5 \\
\hline 4-Chloroaniline & 10 & Hexachloroethane & 5 \\
\hline 4-Chlorophenylphenylether & 5 & Indeno[ $1,2,3-c, d]$ pyrene & 5 \\
\hline 4-Nitroaniline & 25 & Isophorone & 5 \\
\hline 4-Nitrophenol & 25 & $m$ - and $p$-Cresol & 5 \\
\hline
\end{tabular}


Table 9-1b. Analytical methods and reporting limits for organic constituents of concern in ground water (concluded). (a)

\begin{tabular}{|l|c|}
\hline \multicolumn{1}{|c|}{$\begin{array}{c}\text { Constituents } \\
\text { of concern }\end{array}$} & $\begin{array}{c}\text { Reporting limit } \\
(\mu \mathrm{g} / \mathrm{L})\end{array}$ \\
\hline EPA Method 625 (cont'd) & 5 \\
N-Nitroso-di- $n$-propylamine & 5 \\
N-Nitrosodiphenylamine & 5 \\
Naphthalene & 5 \\
Nitrobenzene & 25 \\
Pentachlorophenol & 5 \\
Phenanthrene & 5 \\
\hline
\end{tabular}

\begin{tabular}{|l|c|}
\hline \multicolumn{1}{|c|}{$\begin{array}{c}\text { Constituents } \\
\text { of concern }\end{array}$} & $\begin{array}{c}\text { Reporting limit } \\
(\mu \mathrm{g} / \mathrm{L})\end{array}$ \\
\hline Phenol & 5 \\
Pyrene & 5 \\
EPA Method 632 & \\
Diuron & 0.1 \\
EPA Method 8080 & \\
$\quad$ Polychlorinated biphenyls & 0.2 \\
\hline
\end{tabular}

a The significant figures displayed in this table vary by constituents of concern. These variations reflect regulatory agency permit stipulations, or the applicable analytical laboratory contract under which the work was performed, or both.

b Analytical reporting limits for analytes in EPA Method 625 varied by laboratory used.

Table 9-1c. Radioisotopes and reporting limits for gamma spectroscopic analysis of constituents of concern in ground water. (a)

\begin{tabular}{|l|l|}
\hline $\begin{array}{c}\text { Constituents } \\
\text { of concern }\end{array}$ & $\begin{array}{c}\text { Reporting limit } \\
\text { (Bq/L) }\end{array}$ \\
\hline Actinium-228 & 0.52 \\
Americum-241 & 0.28 \\
Beryllium-7 & 0.9 \\
Bismuth-214 & 0.56 \\
Cesium-137 & 0.56 \\
Cobalt-57 & 1.0 \\
Cobalt-60 & 0.9 \\
Europium-152 & 0.96 \\
Potassium-40 & 1.66 \\
Lead-212 & 0.9 \\
Lead-214 & 0.9 \\
Thorium-234 & 0.23 \\
Thallium-208 & 0.15 \\
Uranium-235 & 0.89 \\
Zirconium-95 & 0.9 \\
\hline
\end{tabular}

a The significant figures displayed in this table vary by constituents of concern. These variations reflect the applicable analytical laboratory contract under which the work was performed. 
Table 9-2. Livermore site surveillance well W-008.

\begin{tabular}{|c|c|c|c|c|}
\hline \multirow{2}{*}{$\begin{array}{l}\text { Constituents } \\
\text { of concern }\end{array}$} & \multicolumn{4}{|c|}{ Sampling dates } \\
\hline & 2/3/99 & 6/9/99 & 7/21/99 & $10 / 18 / 99$ \\
\hline \multicolumn{5}{|l|}{ Inorganic ( $\mu \mathrm{g} / \mathrm{L})$} \\
\hline $\mathrm{pH}$ (pH units) & 7.7 & na ${ }^{(a)}$ & na & 7.2 \\
\hline Field $\mathrm{pH}$ (pH units) & 7.3 & 7.4 & 7.32 & 7.4 \\
\hline Specific conductance $(\mu \mathrm{mho} / \mathrm{cm})$ & 2500 & na & na & 2400 \\
\hline Field specific conductance $(\mu \mathrm{mho} / \mathrm{cm})$ & 1000 & 540 & 2600 & 2600 \\
\hline Total dissolved solids (TDS) & 1500 & na & na & 1600 \\
\hline Water temperature $\left({ }^{\circ} \mathrm{C}\right)$ & 20 & 20 & 20 & 20 \\
\hline Aluminum & $<50$ & $<100$ & $<100$ & $<100$ \\
\hline Antimony & $<4$ & $<5$ & $<5$ & $<5$ \\
\hline Arsenic & $<2$ & $<2$ & 2 & 2 \\
\hline Barium & $<25$ & $<25$ & $<25$ & $<25$ \\
\hline Beryllium & $<0.2$ & $<0.5$ & $<0.5$ & $<0.5$ \\
\hline Cadmium & $<0.5$ & $<0.5$ & $<0.5$ & $<0.5$ \\
\hline Chromium & 6.8 & 5 & 5 & 3 \\
\hline Cobalt & $<50$ & $<50$ & $<50$ & $<50$ \\
\hline Copper & $<1$ & $<2$ & $<2$ & $<1$ \\
\hline Chromium(VI) & 9.5 & 5 & 8 & 10 \\
\hline Iron & $<50$ & $<100$ & $<100$ & $<100$ \\
\hline Lead & $<5$ & $<5$ & $<5$ & $<5$ \\
\hline Manganese & $<10$ & $<30$ & $<30$ & $<30$ \\
\hline Mercury & $<0.2$ & $<0.2$ & $<0.2$ & $<0.2$ \\
\hline Molybdenum & $<25$ & $<25$ & $<25$ & $<25$ \\
\hline Nickel & $<2$ & $<5$ & $<5$ & $<5$ \\
\hline Selenium & $<2$ & $<2$ & $<2$ & $<2$ \\
\hline Silver & $<1$ & $<1$ & $<1$ & $<1$ \\
\hline Thallium & $<1$ & $<2$ & $<2$ & $<2$ \\
\hline Vanadium & 16 & $<20$ & $<20$ & $<20$ \\
\hline Zinc & $<10$ & $<20$ & $<20$ & $<20$ \\
\hline \multicolumn{5}{|l|}{ General minerals (mg/L) } \\
\hline Bicarbonate alkalinity $\left(\text { as } \mathrm{CaCO}_{3}\right)^{(\mathrm{b})}$ & 230 & na & na & 220 \\
\hline Boron & 9.4 & 9.1 & 9 & 8.3 \\
\hline Calcium & 100 & na & na & 98 \\
\hline Chloride & 510 & na & na & 560 \\
\hline Fluoride & 1.3 & na & na & 1.28 \\
\hline Magnesium & 54 & na & na & 49 \\
\hline Nitrate & 19 & na & na & $<0.5$ \\
\hline Orthophosphate & 0.06 & na & na & $<0.02$ \\
\hline Potassium & 2 & na & na & 2 \\
\hline
\end{tabular}


Table 9-2. Livermore site surveillance well W-008 (concluded).

\begin{tabular}{|c|c|c|c|c|}
\hline \multirow{2}{*}{$\begin{array}{c}\text { Constituents } \\
\text { of concern }\end{array}$} & \multicolumn{4}{|c|}{ Sampling dates } \\
\hline & 2/3/99 & $6 / 9 / 99$ & 7/21/99 & 10/18/99 \\
\hline \multicolumn{5}{|c|}{ General minerals (mg/L) (continued) } \\
\hline Sodium & 370 & na & na & 320 \\
\hline Sulfate & 340 & na & na & 330 \\
\hline Surfactants & $<0.05$ & na & na & $<0.5$ \\
\hline Total hardness (as $\mathrm{CaCO}_{3}$ ) & 470 & na & na & 450 \\
\hline Total phosphorus & 0.05 & na & na & na \\
\hline \multicolumn{5}{|l|}{ Organic $(\mu \mathrm{g} / \mathrm{L})$} \\
\hline EPA Method 625 & na & na & na & $\mathrm{nd}(\mathrm{c})$ \\
\hline \multicolumn{5}{|l|}{ Radioactive (Bq/L) } \\
\hline Gross alpha & $0.38 \pm 0.10$ & $0.13 \pm 0.13$ & $0.27 \pm 0.15$ & $0.17 \pm 0.052$ \\
\hline Gross beta & $0.20 \pm 0.10$ & $0.085 \pm 0.088$ & $0.20 \pm 0.12$ & $0.082 \pm 0.053$ \\
\hline Americium-241 & $0.0005 \pm 0.001$ & $0.0004 \pm 0.001$ & $0.001 \pm 0.0004$ & na \\
\hline Plutonium-238 & $-0.00002 \pm 0.00002$ & na & $<0.19$ & $0.0004 \pm 0.001$ \\
\hline Plutonium-239+240 & $0.00002 \pm 0.00006$ & na & $0.0002 \pm 0.0002$ & $0.0001 \pm 0.001$ \\
\hline Radium-226 & $0.020 \pm 0.013$ & $0.001 \pm 0.001$ & $0.003 \pm 0.001$ & na \\
\hline Radium-228 & $0.011 \pm 0.011$ & $0.036 \pm 0.013$ & $0.009 \pm 0.010$ & na \\
\hline Thorium-228 & $0.003 \pm 0.003$ & $0.0002 \pm 0.001$ & $0.001 \pm 0.002$ & na \\
\hline Thorium-230 & $0.003 \pm 0.002$ & $-0.0001 \pm 0.0001$ & $0.001 \pm 0.002$ & na \\
\hline Thorium-232 & $0.00001 \pm 0.001$ & $<0.006$ & $-0.0001 \pm 0.0001$ & na \\
\hline Tritium & $-2.8 \pm 2.0$ & $0.18 \pm 2.7$ & $-1.3 \pm 1.4$ & $0.74 \pm 2.3$ \\
\hline Uranium (total) & $0.21 \pm 0.02$ & $0.20 \pm 0.02$ & $0.20 \pm 0.02$ & $0.19 \pm 0.02$ \\
\hline
\end{tabular}

Note: Radioactivities are reported as the measured concentration and either an uncertainty $( \pm 2 \sigma$ counting error) or as being less than or equal to the detection limit. If the concentration is less than or equal to the uncertainty or the detection limit, the result is considered to be a nondetection. See the main volume, Chapter 14, Quality Assurance.

a $\mathrm{na}=$ Not analyzed (analysis not required).

b Bicarbonate alkalinity = total alkalinity.

c $n d=$ None detected above reporting limits. See Table 9-1 for analytical methods and their constituents. 
Table 9-3. Livermore site surveillance well W-221.

\begin{tabular}{|c|c|c|c|c|}
\hline \multirow{2}{*}{$\begin{array}{l}\text { Constituents } \\
\text { of concern }\end{array}$} & \multicolumn{4}{|c|}{ Sampling dates } \\
\hline & 2/3/99 & $6 / 14 / 99$ & $7 / 22 / 99$ & $10 / 18 / 99$ \\
\hline \multicolumn{5}{|l|}{ Inorganic ( $\mu \mathrm{g} / \mathrm{L})$} \\
\hline $\mathrm{pH}$ (pH units) & 7.5 & na(a) & na & 7.1 \\
\hline Field $\mathrm{pH}$ (pH units) & 7.0 & 7.3 & 7.2 & 7.3 \\
\hline Specific conductance $(\mu \mathrm{mho} / \mathrm{cm})$ & 1600 & na & na & 1600 \\
\hline Field specific conductance $(\mu \mathrm{mho} / \mathrm{cm})$ & 1700 & 1700 & 1700 & 1600 \\
\hline Total dissolved solids (TDS) (mg/L) & 1000 & na & na & 1000 \\
\hline Water temperature $\left({ }^{\circ} \mathrm{C}\right)$ & 20 & 21 & 20 & 21 \\
\hline Aluminum & $<50$ & $<100$ & 300 & $<100$ \\
\hline Antimony & $<4$ & $<5$ & $<5$ & $<5$ \\
\hline Arsenic & $<2$ & $<2$ & $<2$ & $<2$ \\
\hline Barium & 110 & 120 & 110 & 110 \\
\hline Beryllium & $<0.2$ & $<0.5$ & $<0.5$ & $<0.5$ \\
\hline Cadmium & $<0.5$ & $<0.5$ & $<0.5$ & $<0.5$ \\
\hline Chromium & 5.2 & 3 & 3 & 2 \\
\hline Cobalt & $<50$ & $<50$ & $<50$ & $<50$ \\
\hline Chromium(VI) & 4.6 & 4 & 5 & 4 \\
\hline Lead & $<5$ & $<5$ & $<5$ & $<5$ \\
\hline Mercury & $<0.2$ & $<0.2$ & $<0.2$ & $<0.2$ \\
\hline Nickel & 24 & 21 & 14 & 18 \\
\hline Selenium & $<2$ & $<2$ & $<2$ & $<2$ \\
\hline Silver & $<1$ & $<1$ & $<1$ & $<1$ \\
\hline Thallium & $<1$ & $<2$ & $<2$ & $<2$ \\
\hline Vanadium & $<10$ & $<20$ & $<20$ & $<20$ \\
\hline Zinc & $<10$ & $<20$ & 30 & $<20$ \\
\hline \multicolumn{5}{|l|}{ General minerals (mg/L) } \\
\hline Bicarbonate alkalinity (as $\left.\mathrm{CaCO}_{3}\right)^{(\mathrm{b})}$ & 330 & na & na & 310 \\
\hline Boron & 2.5 & 2.5 & 2.4 & 2.3 \\
\hline Calcium & 120 & na & na & 130 \\
\hline Chloride & 290 & na & na & 280 \\
\hline Fluoride & 0.65 & na & na & 0.7 \\
\hline Magnesium & 45 & na & na & 43 \\
\hline Nitrate & 31 & na & na & $<0.5$ \\
\hline Orthophosphate & $<0.05$ & na & na & $<0.02$ \\
\hline Potassium & 1.9 & na & na & 1 \\
\hline Sodium & 150 & na & na & 140 \\
\hline Sulfate & 88 & na & na & 77 \\
\hline Surfactants & 0.12 & na & na & $<0.5$ \\
\hline Total hardness (as $\mathrm{CaCO}_{3}$ ) & 490 & na & na & 500 \\
\hline Total phosphorus & $<0.05$ & na & na & na \\
\hline
\end{tabular}


Table 9-3. Livermore site surveillance well W-221 (continued).

\begin{tabular}{|c|c|c|c|c|}
\hline \multirow{2}{*}{$\begin{array}{l}\text { Constituents } \\
\text { of concern }\end{array}$} & \multicolumn{4}{|c|}{ Sampling dates } \\
\hline & 2/3/99 & $6 / 14 / 99$ & $7 / 22 / 99$ & 10/18/99 \\
\hline \multicolumn{5}{|l|}{ Organic ( $\mu g / L)$} \\
\hline EPA Method 625 & na & na & na & $\mathrm{nd}^{(\mathrm{c})}$ \\
\hline \multicolumn{5}{|l|}{ Radioactive (Bq/L) } \\
\hline Gross alpha & $0.31 \pm 0.06$ & $0.23 \pm 0.11$ & $0.21 \pm 0.11$ & $0.33 \pm 0.07$ \\
\hline Gross beta & $0.15 \pm 0.05$ & $0.23 \pm 0.09$ & $0.21 \pm 0.10$ & $0.36 \pm 0.06$ \\
\hline Americium-241 & $0.001 \pm 0.001$ & $0.0005 \pm 0.001$ & $0.0003 \pm 0.0003$ & na \\
\hline Plutonium-238 & $0.0001 \pm 0.00009$ & na & $-0.00004 \pm 0.0001$ & $0.002 \pm 0.002$ \\
\hline Plutonium-239+240 & $-0.000004 \pm 0.00004$ & na & $-0.0001 \pm 0.0001$ & $-0.0004 \pm 0.001$ \\
\hline Radium-226 & $0.033 \pm 0.016$ & $0.001 \pm 0.001$ & $0.001 \pm 0.001$ & na \\
\hline Radium-228 & $0.012 \pm 0.020$ & $0.010 \pm 0.008$ & $0.017 \pm 0.011$ & na \\
\hline Thorium-228 & $0.003 \pm 0.002$ & $0.001 \pm 0.001$ & $-0.0003 \pm 0.0011$ & na \\
\hline Thorium-230 & $0.001 \pm 0.001$ & $<0.006 \pm 0.001$ & $-0.00004 \pm 0.0001$ & na \\
\hline Thorium-232 & $-0.0002 \pm 0.0003$ & $<0.006 \pm 0.001$ & $<0.006 \pm 0.001$ & na \\
\hline Tritium & $-1.3 \pm 2.0$ & $4.4 \pm 2.7$ & $1.4 \pm 1.6$ & $1.7 \pm 2.3$ \\
\hline Uranium (total) & $0.30 \pm 0.03$ & $0.28 \pm 0.03$ & $0.26 \pm 0.03$ & $0.27 \pm 0.02$ \\
\hline
\end{tabular}

Note: Radioactivities are reported as the measured concentration and either an uncertainty ( $\pm 2 \sigma$ counting error) or as being less than or equal to the detection limit. If the concentration is less than or equal to the uncertainty or the detection limit, the result is considered to be a nondetection. See the main volume, Chapter 14, Quality Assurance.

a $\mathrm{na}=$ Not analyzed (analysis not required).

b Bicarbonate alkalinity = total alkalinity.

C $\mathrm{nd}=$ None detected by above method reporting limits. See Table 9-1 for analytical methods and their constituents. 


\section{Ground Water Monitoring}

Table 9-4. Livermore site surveillance well W-017.

\begin{tabular}{|c|c|}
\hline $\begin{array}{l}\text { Constituents } \\
\text { of concern }\end{array}$ & $\begin{array}{c}\text { Sampling date } \\
7 / 26 / 99\end{array}$ \\
\hline \multicolumn{2}{|l|}{ Inorganic ( $\mu \mathrm{g} / \mathrm{L})$} \\
\hline Aluminum & $<100$ \\
\hline Antimony & $<5$ \\
\hline Arsenic & 3 \\
\hline Barium & 230 \\
\hline Beryllium & $<0.5$ \\
\hline Boron & 600 \\
\hline Cadmium & $<0.5$ \\
\hline Chromium & 9 \\
\hline Cobalt & $<50$ \\
\hline Copper & $<2$ \\
\hline Chromium(VI) & 11 \\
\hline Iron & $<100$ \\
\hline Lead & $<5$ \\
\hline Manganese & $<30$ \\
\hline Mercury & $<0.2$ \\
\hline Molybdenum & $<25$ \\
\hline Nickel & $<5$ \\
\hline Selenium & $<2$ \\
\hline Silver & $<1$ \\
\hline Thallium & $<2$ \\
\hline Vanadium & $<20$ \\
\hline Zinc & $<20$ \\
\hline \multicolumn{2}{|l|}{ Radioactive (Bq/L) } \\
\hline Gross alpha & $0.24 \pm 0.08$ \\
\hline Gross beta & $0.12 \pm 0.05$ \\
\hline Americium 241 & $0.0001 \pm 0.0002$ \\
\hline Plutonium 238 & $-0.00002 \pm 0.0002$ \\
\hline Plutonium $239+240$ & $0.00008 \pm 0.0002$ \\
\hline Radium 226 & $0.029 \pm 0.007$ \\
\hline Radium 228 & $0.011 \pm 0.010$ \\
\hline Thorium 228 & $-0.000001 \pm 0.001$ \\
\hline Thorium 230 & $0.0002 \pm 0.0005$ \\
\hline Thorium 232 & $<0.006$ \\
\hline Tritium & $-1.8 \pm 1.4$ \\
\hline Uranium (total) & $0.19 \pm 0.01$ \\
\hline
\end{tabular}

Note: Radioactivities are reported as the measured concentration and either an uncertainty $( \pm 2 \sigma$ counting error) or as being less than or equal to the detection limit. If the concentration is less than or equal to the uncertainty or the detection limit, the result is considered to be a nondetection. See the main volume, Chapter 14, Quality Assurance. 
Table 9-5. Livermore off-site surveillance well 14B1.

\begin{tabular}{|c|c|c|c|c|}
\hline \multirow{2}{*}{$\begin{array}{l}\text { Constituents } \\
\text { of concern }\end{array}$} & \multicolumn{4}{|c|}{ Sampling dates } \\
\hline & 2/3/99 & $6 / 14 / 99$ & $7 / 20 / 99$ & $10 / 19 / 99$ \\
\hline \multicolumn{5}{|l|}{ Inorganic ( $\mu \mathrm{g} / \mathrm{L})$} \\
\hline $\mathrm{pH}$ (pH units) & 7.7 & na ${ }^{(a)}$ & na & na \\
\hline Specific conductance $(\mu \mathrm{mho} / \mathrm{cm})$ & 820 & na & na & na \\
\hline Total dissolved solids (TDS) (mg/L) & 520 & na & na & na \\
\hline Aluminum & $<50$ & na & na & na \\
\hline Antimony & $<4$ & na & na & na \\
\hline Arsenic & $<2$ & na & na & na \\
\hline Barium & 110 & na & na & na \\
\hline Beryllium & $<0.2$ & na & na & na \\
\hline Cadmium & $<0.5$ & na & na & na \\
\hline Chromium & 12 & na & na & na \\
\hline Cobalt & $<50$ & na & na & na \\
\hline Copper & $<1$ & na & na & na \\
\hline Chromium(VI) & 8.9 & na & na & na \\
\hline Iron & 68 & na & na & 20 \\
\hline Lead & $<5$ & na & na & na \\
\hline Manganese & $<10$ & na & na & na \\
\hline Mercury & $<0.2$ & na & na & na \\
\hline Molybdenum & $<25$ & na & na & na \\
\hline Nickel & $<2$ & na & na & na \\
\hline Selenium & $<2$ & na & na & na \\
\hline Silver & $<1$ & na & na & na \\
\hline Thallium & $<1$ & na & na & na \\
\hline Vanadium & $<10$ & na & na & na \\
\hline Zinc & 36 & na & na & na \\
\hline \multicolumn{5}{|l|}{ General minerals (mg/L) } \\
\hline Bicarbonate alkalinity $\left(\text { as } \mathrm{CaCO}_{3}\right)^{(\mathrm{b})}$ & 240 & na & na & na \\
\hline Boron & 0.78 & na & na & na \\
\hline Calcium & 58 & na & na & na \\
\hline Chloride & 82 & na & na & na \\
\hline Fluoride & 0.25 & na & na & na \\
\hline Magnesium & 33 & na & na & na \\
\hline Nitrate & 30 & na & na & na \\
\hline Orthophosphate & 0.24 & na & na & na \\
\hline Potassium & 2 & na & na & na \\
\hline Sodium & 69 & na & na & na \\
\hline Sulfate & 48 & na & na & na \\
\hline Surfactants & 0.11 & na & na & na \\
\hline
\end{tabular}


Table 9-5. Livermore off-site surveillance well 14B1 (concluded).

\begin{tabular}{|c|c|c|c|c|}
\hline \multirow{2}{*}{$\begin{array}{l}\text { Constituents } \\
\text { of concern }\end{array}$} & \multicolumn{4}{|c|}{ Sampling dates } \\
\hline & 2/3/99 & $6 / 14 / 99$ & $7 / 20 / 99$ & 10/19/99 \\
\hline \multicolumn{5}{|c|}{ General minerals (mg/L) (continued) } \\
\hline Total hardness (as $\mathrm{CaCO}_{3}$ ) & 280 & na & na & na \\
\hline Total phosphorus & 0.06 & na & na & na \\
\hline \multicolumn{5}{|l|}{ Organic ( $\mu g / L)$} \\
\hline EPA Method 507 & $\mathrm{nd}(\mathrm{c})$ & nd & nd & na \\
\hline EPA Method 547 & nd & nd & na & na \\
\hline EPA Method 632 & nd & nd & na & na \\
\hline EPA Method 625 & na & nd & na & na \\
\hline \multicolumn{5}{|l|}{ Radioactive (Bq/L) } \\
\hline Gross alpha & $0.12 \pm 0.03$ & $0.075 \pm 0.038$ & $0.091 \pm 0.052$ & $0.12 \pm 0.04$ \\
\hline Gross beta & $0.15 \pm 0.03$ & $0.087 \pm 0.041$ & $0.091 \pm 0.058$ & $0.12 \pm 0.04$ \\
\hline Plutonium-238 & $0.00002 \pm 0.00004$ & na & na & na \\
\hline Plutonium-239+240 & $0.00002 \pm 0.00004$ & na & na & na \\
\hline Radium-226 & $0.013 \pm 0.011$ & $0.001 \pm 0.001$ & na & na \\
\hline Radium-228 & $0.012 \pm 0.019$ & $0.021 \pm 0.010$ & na & na \\
\hline Thorium-228 & $0.004 \pm 0.003$ & na & na & na \\
\hline Thorium-230 & $0.0005 \pm 0.001$ & na & na & na \\
\hline Thorium-232 & $0.00001 \pm 0.001$ & na & na & na \\
\hline Tritium & $-1.3 \pm 2.0$ & $3.3 \pm 2.7$ & $2.8 \pm 1.6$ & na \\
\hline Uranium (total) & $0.087 \pm 0.013$ & $0.068 \pm 0.010$ & na & na \\
\hline
\end{tabular}

Note: Radioactivities are reported as the measured concentration and either an uncertainty ( $\pm 2 \sigma$ counting error) or as being less than or equal to the detection limit. If the concentration is less than or equal to the uncertainty or the detection limit, the result is considered to be a nondetection. See the main volume, Chapter 14, Quality Assurance.

a $n a=$ Not analyzed (analysis not required).

b Bicarbonate alkalinity $=$ total alkalinity.

c $n d=$ None detected above reporting limits. See Table 9-1 for analytical methods and their constituents. 
Table 9-6. Livermore off-site surveillance well W-121.

\begin{tabular}{|c|c|c|c|c|}
\hline \multirow{2}{*}{$\begin{array}{c}\text { Constituents } \\
\text { of concern }\end{array}$} & \multicolumn{4}{|c|}{ Sampling dates } \\
\hline & 1/27/99 & 6/10/99 & $7 / 20 / 99$ & $10 / 20 / 99$ \\
\hline \multicolumn{5}{|l|}{ Inorganic $(\mu \mathrm{g} / \mathrm{L})$} \\
\hline $\mathrm{pH}$ (pH units) & 8.0 & $\mathrm{na}^{(\mathrm{a})}$ & na & na \\
\hline Field $\mathrm{pH}$ (pH units) & 7.8 & 8.1 & 7.9 & 8.1 \\
\hline Specific conductance ( $\mu \mathrm{mho} / \mathrm{cm})$ & 720 & na & na & na \\
\hline Field specific conductance $(\mu \mathrm{mho} / \mathrm{cm})$ & 740 & 730 & 730 & 730 \\
\hline Total dissolved solids (TDS) (mg/L) & 440 & na & na & na \\
\hline Water temperature $\left({ }^{\circ} \mathrm{C}\right)$ & 19 & 21 & 20.1 & 19.7 \\
\hline Aluminum & $<50$ & na & na & na \\
\hline Antimony & $<4$ & na & na & na \\
\hline Arsenic & $<2$ & na & na & na \\
\hline Barium & 66 & na & na & na \\
\hline Beryllium & $<0.2$ & na & na & na \\
\hline Cadmium & $<0.5$ & na & na & na \\
\hline Chromium & 12 & na & na & na \\
\hline Cobalt & $<50$ & na & na & na \\
\hline Copper & $<1$ & na & na & na \\
\hline Chromium(VI) & 12 & 13 & na & 9 \\
\hline Iron & $<50$ & na & na & na \\
\hline Lead & $<5$ & na & na & na \\
\hline Manganese & $<10$ & na & na & na \\
\hline Mercury & $<0.2$ & na & na & na \\
\hline Molybdenum & $<25$ & na & na & na \\
\hline Nickel & $<2$ & na & na & na \\
\hline Selenium & $<2$ & na & na & na \\
\hline Silver & $<1$ & na & na & na \\
\hline Thallium & $<1$ & na & na & na \\
\hline Vanadium & $<10$ & na & na & na \\
\hline Zinc & $<10$ & na & na & na \\
\hline \multicolumn{5}{|l|}{ General minerals (mg/L) } \\
\hline Bicarbonate alkalinity $\left(\text { as } \mathrm{CaCO}_{3}\right)^{(b)}$ & 190 & na & na & na \\
\hline Boron & 0.9 & na & na & na \\
\hline Calcium & 37 & na & na & na \\
\hline Chloride & 76 & na & na & na \\
\hline Fluoride & 0.34 & na & na & na \\
\hline Magnesium & 30 & na & na & na \\
\hline Nitrate & $27^{(\mathrm{c})}$ & na & na & na \\
\hline
\end{tabular}


Table 9-6. Livermore off-site surveillance well W-121 (concluded).

\begin{tabular}{|c|c|c|c|c|}
\hline \multirow{2}{*}{$\begin{array}{c}\text { Constituents } \\
\text { of concern }\end{array}$} & \multicolumn{4}{|c|}{ Sampling dates } \\
\hline & $1 / 27 / 99$ & 6/10/99 & $7 / 20 / 99$ & $10 / 20 / 99$ \\
\hline \multicolumn{5}{|c|}{ General minerals (mg/L) (continued) } \\
\hline Orthophosphate & 0.24 & na & na & na \\
\hline Potassium & 1.6 & na & na & na \\
\hline Sodium & 69 & na & na & na \\
\hline Sulfate & 12 & na & na & na \\
\hline Surfactants & $<0.05$ & na & na & na \\
\hline Total hardness (as $\mathrm{CaCO}_{3}$ ) & 220 & na & na & na \\
\hline Total phosphorus & 0.07 & na & na & na \\
\hline \multicolumn{5}{|l|}{ Organic ( $\mu \mathrm{g} / \mathrm{L})$} \\
\hline EPA Method 507 & $\mathrm{nd}^{(\mathrm{d})}$ & nd & nd & na \\
\hline EPA Method 547 & nd & nd & na & na \\
\hline EPA Method 625 & na & nd & na & na \\
\hline EPA Method 632 & nd & nd & na & na \\
\hline \multicolumn{5}{|l|}{ Radioactive (Bq/L) } \\
\hline Gross alpha & $0.20 \pm 0.05$ & $0.011 \pm 0.034$ & $0.015 \pm 0.025$ & $0.030 \pm 0.029$ \\
\hline Gross beta & $0.068 \pm 0.032$ & $0.083 \pm 0.040$ & $0.10 \pm 0.047$ & $0.061 \pm 0.034$ \\
\hline Radium 226 & $0.018 \pm 0.008$ & $0.001 \pm 0.001$ & na & na \\
\hline Radium 228 & $0.007 \pm 0.022$ & $0.015 \pm 0.011$ & na & na \\
\hline Tritium & na & $-1.1 \pm 2.5$ & $-0.53 \pm 1.5$ & na \\
\hline Thorium 228 & $0.003 \pm 0.002$ & na & na & na \\
\hline Thorium 230 & $0.0002 \pm 0.001$ & na & na & na \\
\hline Thorium 232 & $0.0002 \pm 0.0004$ & na & na & na \\
\hline Uranium (total) & $0.032 \pm 0.008$ & $0.002 \pm 0.005$ & na & na \\
\hline
\end{tabular}

Note: Radioactivities are reported as the measured concentration and either an uncertainty ( $\pm 2 \sigma$ counting error) or as being less than or equal to the detection limit. If the concentration is less than or equal to the uncertainty or the detection limit, the result is considered to be a nondetection. See the main volume, Chapter 14, Quality Assurance.

a $\quad$ na $=$ Not analyzed (analysis not required).

b Bicarbonate alkalinity $=$ total alkalinity.

c Samples were analyzed in five days, outside the holding time of two days.

d $n d=$ None detected above reporting limits. See Table 9-1 for analytical methods and their constituents. 
Table 9-7. Livermore off-site surveillance well W-151.

\begin{tabular}{|c|c|c|c|c|}
\hline \multirow[b]{2}{*}{ Constituents of concern } & \multicolumn{4}{|c|}{ Sampling dates } \\
\hline & $1 / 27 / 99$ & 6/10/99 & 7/20/99 & $10 / 20 / 99$ \\
\hline \multicolumn{5}{|l|}{ Inorganic $(\mu \mathrm{g} / \mathrm{L})$} \\
\hline $\mathrm{pH}$ (pH units) & 7.9 & $\mathrm{na}^{(\mathrm{a})}$ & na & na \\
\hline Field $\mathrm{pH}$ (pH units) & 7.4 & 7.7 & 7.5 & 7.8 \\
\hline Specific conductance ( $\mu \mathrm{mho} / \mathrm{cm})$ & 850 & na & na & na \\
\hline Field specific conductance $(\mu \mathrm{mho} / \mathrm{cm})$ & 860 & 870 & 870 & 870 \\
\hline Total dissolved solids (TDS) (mg/L) & 530 & na & na & na \\
\hline Water temperature $\left({ }^{\circ} \mathrm{C}\right)$ & 18 & 20 & 19 & 19 \\
\hline Aluminum & $<50$ & na & na & na \\
\hline Antimony & $<4$ & na & na & na \\
\hline Arsenic & $<2$ & na & na & na \\
\hline Barium & 87 & na & na & na \\
\hline Beryllium & $<0.2$ & na & na & na \\
\hline Cadmium & $<0.5$ & na & na & na \\
\hline Chromium & 16 & na & na & na \\
\hline Cobalt & $<50$ & na & na & na \\
\hline Copper & 1 & na & na & na \\
\hline Chromium(VI) & 18 & 22 & na & 19 \\
\hline Iron & $<50$ & na & na & na \\
\hline Lead & $<5$ & na & na & na \\
\hline Manganese & $<10$ & na & na & na \\
\hline Mercury & $<0.2$ & na & na & na \\
\hline Molybdenum & $<25$ & na & na & na \\
\hline Nickel & $<2$ & na & na & na \\
\hline Selenium & $<2$ & na & na & na \\
\hline Silver & $<1$ & na & na & na \\
\hline Thallium & $<1$ & na & na & na \\
\hline Vanadium & $<10$ & na & na & na \\
\hline Zinc & $<10$ & na & na & na \\
\hline \multicolumn{5}{|l|}{ General minerals (mg/L) } \\
\hline Bicarbonate alkalinity $\left(\text { as } \mathrm{CaCO}_{3}\right)^{(\mathrm{b})}$ & 240 & na & na & na \\
\hline Boron & 0.77 & na & na & na \\
\hline Calcium & 52 & na & na & na \\
\hline Chloride & 87 & na & na & na \\
\hline Fluoride & 0.3 & na & na & na \\
\hline Magnesium & 38 & na & na & na \\
\hline Nitrate & $31^{(\mathrm{c})}$ & na & na & na \\
\hline Orthophosphate & 0.25 & na & na & na \\
\hline
\end{tabular}




\section{Ground Water Monitoring}

Table 9-7. Livermore off-site surveillance well W-151 (concluded).

\begin{tabular}{|c|c|c|c|c|}
\hline \multirow{2}{*}{$\begin{array}{c}\text { Constituents } \\
\text { of concern }\end{array}$} & \multicolumn{4}{|c|}{ Sampling dates } \\
\hline & $1 / 27 / 99$ & $6 / 10 / 99$ & 7/20/99 & 10/20/99 \\
\hline \multicolumn{5}{|c|}{ General minerals (mg/L) (continued) } \\
\hline Potassium & 1.8 & na & na & na \\
\hline Sodium & 70 & na & na & na \\
\hline Sulfate & 44 & na & na & na \\
\hline Surfactants & $<0.05$ & na & na & na \\
\hline Total hardness $\left(\mathrm{as} \mathrm{CaCO}_{3}\right)$ & 290 & na & na & na \\
\hline Total phosphorus & 0.07 & na & na & na \\
\hline \multicolumn{5}{|l|}{ Organic $(\mu \mathrm{g} / \mathrm{L})$} \\
\hline EPA Method 507 & $n d^{(d)}$ & nd & nd & na \\
\hline EPA Method 547 & nd & nd & na & na \\
\hline EPA Method 625 & na & nd & na & na \\
\hline EPA Method 632 & nd & nd & na & na \\
\hline \multicolumn{5}{|l|}{ Radioactive (Bq/L) } \\
\hline Gross alpha & $0.066 \pm 0.035$ & $0.062 \pm 0.049$ & $0.11 \pm 0.06$ & $0.047 \pm 0.035$ \\
\hline Gross beta & $0.19 \pm 0.04$ & $0.15 \pm 0.05$ & $0.084 \pm 0.056$ & $0.073 \pm 0.036$ \\
\hline Plutonium-238 & $-0.0001 \pm 0.0001$ & na & na & na \\
\hline Plutonium-239+240 & $-0.00004 \pm 0.0001$ & na & na & na \\
\hline Radium-226 & $0.023 \pm 0.007$ & $0.001 \pm 0.001$ & na & na \\
\hline Radium-228 & $-0.011 \pm 0.017$ & $0.027 \pm 0.011$ & na & na \\
\hline Thorium-228 & $0.004 \pm 0.003$ & na & na & na \\
\hline Thorium-230 & $0.001 \pm 0.002$ & na & na & na \\
\hline Thorium-232 & $-0.0002 \pm 0.001$ & na & na & na \\
\hline Tritium & $-4.4 \pm 2.1$ & $0.88 \pm 2.6$ & $-0.32 \pm 1.5$ & na \\
\hline Uranium (total) & $0.060 \pm 0.012$ & $0.048 \pm 0.007$ & na & na \\
\hline
\end{tabular}

Note: Radioactivities are reported as the measured concentration and either an uncertainty $( \pm 2 \sigma$ counting error) or as being less than or equal to the detection limit. If the concentration is less than or equal to the uncertainty or the detection limit, the result is considered to be a nondetection. See the main volume, Chapter 14, Quality Assurance.

a $n a=$ Not analyzed (analysis not required).

b Bicarbonate alkalinity $=$ total alkalinity.

c Samples were analyzed in five days, outside the holding time of two days.

d $n d=$ None detected above reporting limits. See Table 9-1 for analytical methods and their constituents. 
Table 9-8. Livermore off-site surveillance well W-571.

\begin{tabular}{|c|c|c|c|c|}
\hline \multirow[b]{2}{*}{ Constituents of concern } & \multicolumn{4}{|c|}{ Sampling dates } \\
\hline & $1 / 27 / 99$ & $6 / 10 / 99$ & $7 / 26 / 99$ & $10 / 20 / 99$ \\
\hline \multicolumn{5}{|l|}{ Inorganic ( $\mu \mathrm{g} / \mathrm{L})$} \\
\hline $\mathrm{pH}$ (units) & 7.6 & $\mathrm{na}^{(\mathrm{a})}$ & na & na \\
\hline Field pH (units) & 7.3 & 7.5 & na & 7.6 \\
\hline Specific conductance ( $\mu \mathrm{mho} / \mathrm{cm})$ & 830 & na & na & na \\
\hline Field specific conductance $(\mu \mathrm{mho} / \mathrm{cm})$ & 860 & 840 & na & 850 \\
\hline Total dissolved solids (TDS, mg/L) & 510 & na & na & na \\
\hline Field temperature $\left({ }^{\circ} \mathrm{C}\right)$ & 18 & 19 & na & 19 \\
\hline Aluminum & $<50$ & na & na & na \\
\hline Antimony & $<4$ & na & na & na \\
\hline Arsenic & $<2$ & na & na & na \\
\hline Barium & 96 & na & na & na \\
\hline Beryllium & $<0.2$ & na & na & na \\
\hline Cadmium & $<0.5$ & na & na & na \\
\hline Chromium & 20 & na & na & na \\
\hline Cobalt & $<50$ & na & na & na \\
\hline Copper & 1 & na & na & na \\
\hline Chromium(VI) & 20 & 24 & na & 21 \\
\hline Iron & $<50$ & na & na & na \\
\hline Lead & $<5$ & na & na & na \\
\hline Manganese & $<10$ & na & na & na \\
\hline Mercury & $<0.2$ & na & na & na \\
\hline Molybdenum & $<25$ & na & na & na \\
\hline Nickel & 2.6 & na & na & na \\
\hline Selenium & 2 & na & na & na \\
\hline Silver & $<1$ & na & na & na \\
\hline Thallium & $<1$ & na & na & na \\
\hline Vanadium & $<10$ & na & na & na \\
\hline Zinc & $<10$ & na & na & na \\
\hline \multicolumn{5}{|l|}{ General minerals (mg/L) } \\
\hline Bicarbonate alkalinity $\left(\text { as } \mathrm{CaCO}_{3}\right)^{(b)}$ & 250 & na & na & na \\
\hline Boron & 0.67 & na & na & na \\
\hline Calcium & 64 & na & na & na \\
\hline Chloride & 87 & na & na & na \\
\hline Fluoride & 0.34 & na & na & na \\
\hline Magnesium & 25 & na & na & na \\
\hline
\end{tabular}


Table 9-8. Livermore off-site surveillance well W-571 (concluded).

\begin{tabular}{|c|c|c|c|c|}
\hline \multirow{2}{*}{$\begin{array}{c}\text { Constituents } \\
\text { of concern }\end{array}$} & \multicolumn{4}{|c|}{ Sampling dates } \\
\hline & $1 / 27 / 99$ & 6/10/99 & $7 / 26 / 99$ & $10 / 20 / 99$ \\
\hline \multicolumn{5}{|c|}{ General minerals (mg/L) (continued) } \\
\hline Nitrate & $31^{(\mathrm{c})}$ & na & na & na \\
\hline Orthophosphate & 0.21 & na & na & na \\
\hline Potassium & 2 & na & na & na \\
\hline Sodium & 73 & na & na & na \\
\hline Sulfate & 35 & na & na & na \\
\hline Surfactants & $<0.05$ & na & na & na \\
\hline Total hardness (as $\mathrm{CaCO}_{3}$ ) & 260 & na & na & na \\
\hline Total phosphorus & 0.06 & na & na & na \\
\hline \multicolumn{5}{|l|}{ Radioactive (Bq/L) } \\
\hline Gross alpha & $0.11 \pm 0.04$ & $0.061 \pm 0.059$ & $0.12 \pm 0.05$ & $0.058 \pm 0.031$ \\
\hline Gross beta & $0.22 \pm 0.04$ & $0.12 \pm 0.05$ & $0.14 \pm 0.05$ & $0.15 \pm 0.04$ \\
\hline Americium-241 & $0.002 \pm 0.002$ & na & na & na \\
\hline Plutonium-238 & $0.000002 \pm 0.0001$ & na & na & na \\
\hline Plutonium-239+240 & $0.00003 \pm 0.0001$ & na & na & na \\
\hline Radium-226 & $0.007 \pm 0.006$ & $0.002 \pm 0.001$ & na & na \\
\hline Radium-228 & $0.016 \pm 0.022$ & $0.032 \pm 0.012$ & na & na \\
\hline Thorium-228 & $-0.003 \pm 0.003$ & na & na & na \\
\hline Thorium-230 & $-0.0003 \pm 0.001$ & na & na & na \\
\hline Thorium-232 & $0.0005 \pm 0.001$ & na & na & na \\
\hline Tritium & $-1.8 \pm 2.2$ & $4.5 \pm 2.8$ & $2.2 \pm 1.6$ & na \\
\hline Uranium (total) & $0.10 \pm 0.02$ & $0.098 \pm 0.013$ & na & na \\
\hline
\end{tabular}

Note: Radioactivities are reported as the measured concentration and either an uncertainty ( $\pm 2 \sigma$ counting error) or as being less than or equal to the detection limit. If the concentration is less than or equal to the uncertainty or the detection limit, the result is considered to be a nondetection. See the main volume, Chapter 14, Quality Assurance.

a $n a=$ Not analyzed (analysis not required).

b Bicarbonate alkalinity = total alkalinity.

c Samples were analyzed in five days, outside the holding time of two days. 
Table 9-9. Livermore site surveillance well W-1012.

\begin{tabular}{|c|c|c|c|c|}
\hline \multirow{2}{*}{$\begin{array}{l}\text { Constituents } \\
\text { of concern }\end{array}$} & \multicolumn{4}{|c|}{ Sampling dates } \\
\hline & 2/11/99 & 6/8/99 & $7 / 20 / 99$ & $10 / 19 / 99$ \\
\hline \multicolumn{5}{|l|}{ Inorganic $(\mu \mathrm{g} / \mathrm{L})$} \\
\hline $\mathrm{pH}$ (pH units) & 7.8 & $\mathrm{na}^{(\mathrm{a})}$ & na & na \\
\hline Field $\mathrm{pH}$ (pH units) & na & 7.5 & 7.3 & 7.3 \\
\hline Specific conductance ( $\mu \mathrm{mho} / \mathrm{cm})$ & 1100 & na & na & na \\
\hline Field specific conductance $(\mu \mathrm{mho} / \mathrm{cm})$ & na & 970 & 1100 & 1100 \\
\hline Total dissolved solids (TDS, mg/L) & 650 & na & na & na \\
\hline Water temperature $\left({ }^{\circ} \mathrm{C}\right)$ & na & 19.2 & 20 & 19 \\
\hline Aluminum & $<50$ & na & na & na \\
\hline Antimony & $<4$ & na & na & na \\
\hline Arsenic & $<2$ & na & na & na \\
\hline Barium & 140 & na & na & na \\
\hline Beryllium & $<0.2$ & na & na & na \\
\hline Cadmium & $<0.5$ & na & na & na \\
\hline Chromium & 20 & na & na & na \\
\hline Cobalt & 22 & na & na & na \\
\hline Copper & $<1$ & na & na & na \\
\hline Chromium(VI) & 18 & 15 & 21 & 24 \\
\hline Iron & $<50$ & na & na & na \\
\hline Lead & $<5$ & na & na & na \\
\hline Manganese & $<10$ & na & na & na \\
\hline Mercury & $<0.2$ & na & na & na \\
\hline Molybdenum & $<25$ & na & na & na \\
\hline Nickel & 2.6 & na & na & na \\
\hline Selenium & 3.5 & na & na & na \\
\hline Silver & $<2$ & na & na & na \\
\hline Thallium & $<1$ & na & na & na \\
\hline Vanadium & $<10$ & na & na & na \\
\hline Zinc & $<10$ & na & na & na \\
\hline \multicolumn{5}{|l|}{ General minerals (mg/L) } \\
\hline Bicarbonate alkalinity $\left(\text { as } \mathrm{CaCO}_{3}\right)^{(b)}$ & 270 & na & na & na \\
\hline Boron & 0.69 & na & na & na \\
\hline Calcium & 89 & na & na & na \\
\hline Chloride & 130 & na & na & na \\
\hline Fluoride & 0.20 & na & na & na \\
\hline Magnesium & 34 & na & na & na \\
\hline Nitrate & 79 & na & na & na \\
\hline Orthophosphate & 0.15 & na & na & na \\
\hline
\end{tabular}




\section{Ground Water Monitoring}

Table 9-9. Livermore site surveillance well W-1012 (concluded).

\begin{tabular}{|c|c|c|c|c|}
\hline \multirow{2}{*}{$\begin{array}{l}\text { Constituents } \\
\text { of concern }\end{array}$} & \multicolumn{4}{|c|}{ Sampling dates } \\
\hline & 2/11/99 & 6/8/99 & 7/20/99 & $10 / 19 / 99$ \\
\hline \multicolumn{5}{|c|}{ General minerals (mg/L) (continued) } \\
\hline Potassium & 2.8 & na & na & na \\
\hline Sodium & 79 & na & na & na \\
\hline Sulfate & 28 & na & na & na \\
\hline Surfactants & $<0.05$ & na & na & na \\
\hline Total hardness (as $\mathrm{CaCO}_{3}$ ) & 360 & na & na & na \\
\hline Total phosphorus & 0.05 & na & na & na \\
\hline \multicolumn{5}{|l|}{ Organic $(\mu \mathrm{g} / \mathrm{L})$} \\
\hline EPA Method 507 & $\mathrm{nd}(\mathrm{c})$ & nd & nd & na \\
\hline EPA Method 547 & nd & nd & na & na \\
\hline EPA Method 632 & nd & nd & na & na \\
\hline EPA Method 625 & na & nd & na & na \\
\hline \multicolumn{5}{|l|}{ Radioactive (Bq/L) } \\
\hline Gross alpha & $0.23 \pm 0.05$ & $0.087 \pm 0.058$ & $0.11 \pm 0.07$ & $0.165 \pm 0.045$ \\
\hline Gross beta & $0.14 \pm 0.03$ & $0.14 \pm 0.06$ & $0.14 \pm 0.06$ & $0.175 \pm 0.053$ \\
\hline Plutonium-238 & $-0.00002 \pm 0.0001$ & na & na & na \\
\hline Plutonium-239+240 & $0.00002 \pm 0.0001$ & na & na & na \\
\hline Radium-226 & $0.011 \pm 0.010$ & $0.001 \pm 0.001$ & na & na \\
\hline Radium-228 & $0.039 \pm 0.021$ & $0.028 \pm 0.012$ & na & na \\
\hline Thorium-228 & $0.006 \pm 0.002$ & na & na & na \\
\hline Thorium-230 & $0.002 \pm 0.001$ & na & na & na \\
\hline Thorium-232 & $-0.0001 \pm 0.0003$ & na & na & na \\
\hline Tritium & $0.27 \pm 2.5$ & $-1.1 \pm 2.7$ & $-0.32 \pm 1.5$ & na \\
\hline Uranium (total) & $0.14 \pm 0.02$ & $0.12 \pm 0.02$ & na & na \\
\hline
\end{tabular}

Note: Radioactivities are reported as the measured concentration and either an uncertainty ( $\pm 2 \sigma$ counting error) or as being less than or equal to the detection limit. If the concentration is less than or equal to the uncertainty or the detection limit, the result is considered to be a nondetection. See the main volume, Chapter 14, Quality Assurance.

a $n a=$ Not analyzed (analysis not required).

b Bicarbonate alkalinity $=$ total alkalinity.

c $n d=$ None detected above reporting limits. See Table 9-1 for analytical methods and their constituents. 
Table 9-10. Livermore site surveillance well W-556.

\begin{tabular}{|c|c|c|c|c|}
\hline \multirow{2}{*}{$\begin{array}{l}\text { Constituents } \\
\text { of concern }\end{array}$} & \multicolumn{4}{|c|}{ Sampling dates } \\
\hline & $1 / 13 / 99$ & 6/8/99 & 7/21/99 & $10 / 19 / 99$ \\
\hline \multicolumn{5}{|l|}{ Inorganic ( $\mu \mathrm{g} / \mathrm{L})$} \\
\hline $\mathrm{pH}$ (pH units) & 7.7 & $\mathrm{na}^{(\mathrm{a})}$ & na & na \\
\hline Field $\mathrm{pH}$ (pH units) & 7.4 & 7.6 & 7.5 & 7.5 \\
\hline Specific conductance ( $\mu \mathrm{mho} / \mathrm{cm})$ & 720 & 850 & 970 & 980 \\
\hline Field specific conductance $(\mu \mathrm{mho} / \mathrm{cm})$ & 960 & na & na & na \\
\hline Total dissolved solids (TDS, mg/L) & 560 & na & na & na \\
\hline Field temperature $\left({ }^{\circ} \mathrm{C}\right)$ & 18 & 19 & 19 & 18 \\
\hline Aluminum & $<50$ & na & na & na \\
\hline Antimony & $<4$ & na & na & na \\
\hline Arsenic & $<2$ & na & na & na \\
\hline Barium & 81 & na & na & na \\
\hline Beryllium & $<0.2$ & na & na & na \\
\hline Cadmium & $<0.5$ & na & na & na \\
\hline Chromium & 29 & na & na & na \\
\hline Cobalt & $<50$ & na & na & na \\
\hline Copper & $<1$ & na & na & na \\
\hline Chromium(VI) & 32 & na & na & 27 \\
\hline Iron & $<50$ & na & na & na \\
\hline Lead & $<5$ & na & na & na \\
\hline Manganese & $<10$ & na & na & na \\
\hline Mercury & $<0.2$ & na & na & na \\
\hline Molybdenum & $<25$ & na & na & na \\
\hline Nickel & $<2$ & na & na & na \\
\hline Selenium & 5.4 & na & na & na \\
\hline Silver & $<1$ & na & na & na \\
\hline Thallium & $<1$ & na & na & na \\
\hline Vanadium & $<10$ & na & na & na \\
\hline Zinc & $<10$ & na & na & na \\
\hline \multicolumn{5}{|l|}{ General minerals (mg/L) } \\
\hline Bicarbonate alkalinity $\left(\text { as } \mathrm{CaCO}_{3}\right)^{(b)}$ & 230 & na & na & na \\
\hline Boron & 1100 & na & na & na \\
\hline Calcium & 58 & na & na & na \\
\hline Chloride & 130 & na & na & na \\
\hline Fluoride & 0.3 & na & na & na \\
\hline Magnesium & 22 & na & na & na \\
\hline Nitrate & 29 & na & na & na \\
\hline Orthophosphate & 0.17 & na & na & na \\
\hline
\end{tabular}




\section{Ground Water Monitoring}

Table 9-10. Livermore site surveillance well W-556 (concluded).

\begin{tabular}{|c|c|c|c|c|}
\hline \multirow{2}{*}{$\begin{array}{l}\text { Constituents } \\
\text { of concern }\end{array}$} & \multicolumn{4}{|c|}{ Sampling dates } \\
\hline & 1/13/99 & $6 / 8 / 99$ & 7/21/99 & 10/19/99 \\
\hline \multicolumn{5}{|c|}{ General minerals (mg/L) (continued) } \\
\hline Potassium & 1.6 & na & na & na \\
\hline Sodium & 100 & na & na & na \\
\hline Sulfate & 38 & na & na & na \\
\hline Surfactants & $<0.05$ & na & na & na \\
\hline Total hardness $\left(\right.$ as $\mathrm{CaCO}_{3}$ ) & 240 & na & na & na \\
\hline Total phosphorus & 0.05 & na & na & na \\
\hline \multicolumn{5}{|l|}{ Organic constituents $(\mu \mathrm{g} / \mathrm{L})$} \\
\hline EPA Method 507 & na & $n d^{(c)}$ & nd & na \\
\hline EPA Method 525 & no(d) & na & na & na \\
\hline Di(2-ethylhexyl)adipate & 3.9 & na & na & na \\
\hline EPA Method 547 & nd & nd & na & na \\
\hline EPA Method 625 & na & nd & na & na \\
\hline EPA Method 632 & nd & nd & na & na \\
\hline \multicolumn{5}{|l|}{ Radioactive (Bq/L) } \\
\hline Gross alpha & $0.098 \pm 0.037$ & $0.15 \pm 0.07$ & $0.029 \pm 0.043$ & $0.16 \pm 0.05$ \\
\hline Gross beta & $0.19 \pm 0.04$ & $0.11 \pm 0.05$ & $0.15 \pm 0.06$ & $0.067 \pm 0.048$ \\
\hline Plutonium-238 & $-0.0001 \pm 0.0001$ & na & na & na \\
\hline Plutonium-239+240 & $-0.0001 \pm 0.0001$ & na & na & na \\
\hline Radium-226 & $0.018 \pm 0.007$ & $0.004 \pm 0.001$ & na & na \\
\hline Radium-228 & $0.027 \pm 0.016$ & $0.039 \pm 0.015$ & na & na \\
\hline Thorium-228 & $-0.001 \pm 0.002$ & na & na & na \\
\hline Thorium-230 & $0.002 \pm 0.001$ & na & na & na \\
\hline Thorium-232 & $-0.0002 \pm 0.001$ & na & na & na \\
\hline Tritium & $-3.4 \pm 2.7$ & $2.0 \pm 2.8$ & $0.095 \pm 1.5$ & na \\
\hline Uranium (total) & $0.089 \pm 0.009$ & $0.092 \pm 0.013$ & na & na \\
\hline
\end{tabular}

Note: Radioactivities are reported as the measured concentration and either an uncertainty ( $\pm 2 \sigma$ counting error) or as being less than or equal to the detection limit. If the concentration is less than or equal to the uncertainty or the detection limit, the result is considered to be a nondetection. See the main volume, Chapter 14, Quality Assurance.

a $\mathrm{na}=$ Not analyzed (analysis not required).

b Bicarbonate alkalinity = total alkalinity.

c $\mathrm{nd}=$ None detected above reporting limits (exceptions are listed). See Table 9-1 for analytical methods and their constituents.

d $n o=$ No analytes other than di(2-ethylhexvl)adipate were detected by this method. 
Table 9-11. Livermore site surveillance well W-373.

\begin{tabular}{|c|c|c|c|c|}
\hline \multirow{2}{*}{$\begin{array}{l}\text { Constituents } \\
\text { of concern }\end{array}$} & \multicolumn{4}{|c|}{ Sampling dates } \\
\hline & $1 / 13 / 99$ & 6/8/99 & 7/21/99 & $10 / 19 / 99$ \\
\hline \multicolumn{5}{|l|}{ Inorganic $(\mu \mathrm{g} / \mathrm{L})$} \\
\hline $\mathrm{pH}$ (units) & 7.8 & $n a^{(a)}$ & na & na \\
\hline Field pH (units) & 7.4 & 7.7 & 7.5 & 7.5 \\
\hline Specific conductance ( $\mu \mathrm{mho} / \mathrm{cm})$ & 930 & na & na & na \\
\hline Field specific conductance $(\mu \mathrm{mho} / \mathrm{cm})$ & 800 & 810 & 930 & 930 \\
\hline Total dissolved solids (TDS, mg/L) & 560 & na & na & na \\
\hline Field temperature $\left({ }^{\circ} \mathrm{C}\right)$ & 18 & 18 & 19 & 18 \\
\hline Aluminum & $<50$ & na & na & na \\
\hline Antimony & $<4$ & na & na & na \\
\hline Arsenic & $<2$ & na & na & na \\
\hline Barium & 48 & na & na & na \\
\hline Beryllium & $<0.2$ & na & na & na \\
\hline Cadmium & $<0.5$ & na & na & na \\
\hline Chromium & 65 & na & na & na \\
\hline Cobalt & $<50$ & na & na & na \\
\hline Copper & $<1$ & na & na & na \\
\hline Chromium(VI) & 76 & 54 & na & 65 \\
\hline Iron & $<50$ & na & na & na \\
\hline Lead & $<5$ & na & na & na \\
\hline Manganese & $<10$ & na & na & na \\
\hline Mercury & $<0.2$ & na & na & na \\
\hline Molybdenum & $<25$ & na & na & na \\
\hline Nickel & $<2$ & na & na & na \\
\hline Selenium & $<2$ & na & na & na \\
\hline Silver & $<1$ & na & na & na \\
\hline Thallium & $<1$ & na & na & na \\
\hline Vanadium & $<10$ & na & na & na \\
\hline Zinc & $<10$ & na & na & na \\
\hline \multicolumn{5}{|l|}{ General minerals (mg/L) } \\
\hline Bicarbonate alkalinity $\left(\text { as } \mathrm{CaCO}_{3}\right)^{(b)}$ & 210 & na & na & na \\
\hline Boron & 1800 & na & na & na \\
\hline Calcium & 50 & na & na & na \\
\hline Chloride & 120 & na & na & na \\
\hline Fluoride & 0.49 & na & na & na \\
\hline Magnesium & 19 & na & na & na \\
\hline Nitrate & 12 & na & na & na \\
\hline Orthophosphate & 0.12 & na & na & na \\
\hline
\end{tabular}


Table 9-11. Livermore site surveillance well W-373 (concluded).

\begin{tabular}{|c|c|c|c|c|}
\hline \multirow{2}{*}{$\begin{array}{l}\text { Constituents } \\
\text { of concern }\end{array}$} & \multicolumn{4}{|c|}{ Sampling dates } \\
\hline & 1/13/99 & 6/8/99 & $7 / 21 / 99$ & $10 / 19 / 99$ \\
\hline \multicolumn{5}{|c|}{ General minerals (mg/L) (continued) } \\
\hline Potassium & 1.4 & na & na & na \\
\hline Sodium & 110 & na & na & na \\
\hline Sulfate & 65 & na & na & na \\
\hline Surfactants & $<0.05$ & na & na & na \\
\hline Total hardness (as $\mathrm{CaCO}_{3}$ ) & 200 & na & na & na \\
\hline Total phosphorus & $<0.05$ & na & na & na \\
\hline \multicolumn{5}{|l|}{ Organic $(\mu \mathrm{g} / \mathrm{L})$} \\
\hline EPA Method 507 & na & nd $(c)$ & nd & na \\
\hline EPA Method 525 & no(d) & na & na & na \\
\hline Di(2-ethylhexyl)adipate & 5.2 & na & na & na \\
\hline EPA Method 547 & nd & nd & na & na \\
\hline EPA Method 625 & na & nd & na & na \\
\hline EPA Method 632 & nd & nd & na & na \\
\hline \multicolumn{5}{|l|}{ Radioactive (Bq/L) } \\
\hline Gross alpha & $0.093 \pm 0.036$ & $0.065 \pm 0.063$ & $0.066 \pm 0.045$ & $0.26 \pm 0.07$ \\
\hline Gross beta & $0.076 \pm 0.032$ & $0.048 \pm 0.046$ & $0.13 \pm 0.05$ & $0.076 \pm 0.060$ \\
\hline Plutonium 238 & $0.00002 \pm 0.00006$ & na & na & na \\
\hline Plutonium 239+240 & $0.000003 \pm 0.00005$ & na & na & na \\
\hline Radium 226 & $0.032 \pm 0.010$ & $0.001 \pm 0.001$ & na & na \\
\hline Radium 228 & $0.013 \pm 0.017$ & $0.014 \pm 0.013$ & na & na \\
\hline Thorium 228 & $-0.001 \pm 0.002$ & na & na & na \\
\hline Thorium 230 & $0.002 \pm 0.001$ & na & na & na \\
\hline Thorium 232 & $-0.0003 \pm 0.001$ & na & na & na \\
\hline Tritium & $7.6 \pm 3.1$ & $10 \pm 3.1$ & $9.7 \pm 1.9$ & na \\
\hline Uranium (total) & $0.092 \pm 0.009$ & $0.081 \pm 0.011$ & na & na \\
\hline
\end{tabular}

Note: Radioactivities are reported as the measured concentration and either an uncertainty ( $\pm 2 \sigma$ counting error) or as being less than or equal to the detection limit. If the concentration is less than or equal to the uncertainty or the detection limit, the result is considered to be a nondetection. See the main volume, Chapter 14, Quality Assurance.

a $n a=$ Not analyzed (analysis not required).

b Bicarbonate alkalinity $=$ total alkalinity.

c $\quad \mathrm{nd}=$ None detected above reporting limits (exceptions are listed). See Table 9-1 for analytical methods and their constituents.

d $n o=$ No analytes other than di(2-ethylhexvl)adipate were detected by this method. 
Table 9-12. Livermore site surveillance well W-204.

\begin{tabular}{|c|c|c|c|c|}
\hline \multirow{2}{*}{$\begin{array}{c}\text { Constituents } \\
\text { of concern }\end{array}$} & \multicolumn{4}{|c|}{ Sampling dates } \\
\hline & $2 / 4 / 99$ & 6/15/99 & $7 / 26 / 99$ & $11 / 3 / 99$ \\
\hline \multicolumn{5}{|l|}{ Inorganic $(\mu \mathrm{g} / \mathrm{L})$} \\
\hline $\mathrm{pH}$ (pH units) & 8.0 & $\mathrm{na}^{(\mathrm{a})}$ & na & na \\
\hline Field $\mathrm{pH}$ (pH units) & 7.7 & 8.1 & na & 7.6 \\
\hline Specific conductance $(\mu \mathrm{mho} / \mathrm{cm})$ & 400 & na & na & na \\
\hline Field specific conductance $(\mu \mathrm{mho} / \mathrm{cm})$ & 420 & 410 & na & 430 \\
\hline Total dissolved solids (TDS) (mg/L) & 250 & na & na & na \\
\hline Water temperature $\left({ }^{\circ} \mathrm{C}\right)$ & 20 & 21 & na & 20 \\
\hline Aluminum & $<50$ & 100 & na & 100 \\
\hline Antimony & $<4$ & 5 & na & 5 \\
\hline Arsenic & $<2$ & 2 & na & 3 \\
\hline Barium & 170 & 190 & na & 180 \\
\hline Beryllium & $<0.2$ & 0.5 & na & 0.5 \\
\hline Cadmium & $<0.5$ & 0.5 & na & 0.5 \\
\hline Chromium & 17 & 12 & na & 12 \\
\hline Cobalt & $<50$ & $<50$ & na & $<50$ \\
\hline Copper & $<1$ & 2 & na & 2 \\
\hline Chromium(VI) & 18 & 21 & na & 14 \\
\hline Iron & $<50$ & $<100$ & na & $<100$ \\
\hline Lead & $<5$ & $<5$ & na & $<5$ \\
\hline Manganese & $<10$ & 30 & na & 30 \\
\hline Mercury & $<0.2$ & $<0.2$ & na & $<0.2$ \\
\hline Molybdenum & $<25$ & $<25$ & na & $<25$ \\
\hline Nickel & $<2$ & $<5$ & na & $<5$ \\
\hline Selenium & $<2$ & $<2$ & na & $<2$ \\
\hline Silver & $<1$ & $<1$ & na & $<1$ \\
\hline Thallium & $<1$ & $<2$ & na & $<2$ \\
\hline Vanadium & 12 & $<20$ & na & $<20$ \\
\hline Zinc & $<10$ & $<20$ & na & $<20$ \\
\hline \multicolumn{5}{|l|}{ General minerals (mg/L) } \\
\hline Bicarbonate alkalinity $\left(\text { as } \mathrm{CaCO}_{3}\right)^{(\mathrm{b})}$ & 130 & na & na & na \\
\hline Boron & 0.14 & 0.18 & na & 0.1 \\
\hline Calcium & 33 & na & na & na \\
\hline Chloride & 39 & na & na & na \\
\hline Fluoride & 0.22 & na & na & na \\
\hline
\end{tabular}


Table 9-12. Livermore site surveillance well W-204 (concluded).

\begin{tabular}{|c|c|c|c|c|}
\hline \multirow{2}{*}{$\begin{array}{c}\text { Constituents } \\
\text { of concern }\end{array}$} & \multicolumn{4}{|c|}{ Sampling dates } \\
\hline & 2/4/99 & $6 / 15 / 99$ & $7 / 26 / 99$ & 11/3/99 \\
\hline \multicolumn{5}{|c|}{ General minerals (mg/L) (continued) } \\
\hline Magnesium & 8.4 & na & na & na \\
\hline Nitrate & $6.1^{(c)}$ & na & na & na \\
\hline Orthophosphate & 0.1 & na & na & na \\
\hline Potassium & 1.6 & na & na & na \\
\hline Sodium & 37 & na & na & na \\
\hline Sulfate & 9.3 & na & na & na \\
\hline Surfactants & $<0.05$ & na & na & na \\
\hline Total hardness (as $\mathrm{CaCO}_{3}$ ) & 120 & na & na & na \\
\hline Total phosphorus & 0.06 & na & na & na \\
\hline \multicolumn{5}{|l|}{ Organic ( $\mu \mathrm{g} / \mathrm{L})$} \\
\hline EPA Method 8082 (for PCBs) & na & na & $n d^{(d)}$ & na \\
\hline \multicolumn{5}{|l|}{ Radioactive (Bq/L) } \\
\hline Gross alpha & $0.083 \pm 0.022$ & $0.023 \pm 0.027$ & na & $0.040 \pm 0.020$ \\
\hline Gross beta & $0.086 \pm 0.025$ & $0.056 \pm 0.029$ & na & $0.076 \pm 0.025$ \\
\hline Americium 241 & $0.0001 \pm 0.0004$ & $0.0001 \pm 0.000$ & na & $0.001 \pm 0.001$ \\
\hline Plutonium 238 & $-0.00001 \pm 0.0001$ & na & na & na \\
\hline Plutonium 239+240 & $0.0001 \pm 0.0001$ & na & na & na \\
\hline Radium 226 & $0.014 \pm 0.012$ & $0.003 \pm 0.001$ & na & $0.083 \pm 0.032$ \\
\hline Radium 228 & $0.016 \pm 0.014$ & $0.005 \pm 0.008$ & na & $-0.065 \pm 0.026$ \\
\hline Thorium 228 & $0.001 \pm 0.002$ & $0.001 \pm 0.001$ & na & $-0.005 \pm 0.006$ \\
\hline Thorium 230 & $0.001 \pm 0.001$ & $-0.0001 \pm 0.0001$ & na & $0.0004 \pm 0.004$ \\
\hline Thorium 232 & $0.0002 \pm 0.001$ & $<0.006$ & na & $0.002 \pm 0.003$ \\
\hline Tritium & $-2.4 \pm 2.0$ & $1.0 \pm 2.6$ & na & $-0.7 \pm 2.2$ \\
\hline Uranium 238 & $0.044 \pm 0.008$ & $0.044 \pm 0.007$ & na & $0.037 \pm 0.006$ \\
\hline
\end{tabular}

Note: Radioactivities are reported as the measured concentration and either an uncertainty ( $\pm 2 \sigma$ counting error) or as being less than or equal to the detection limit. If the concentration is less than or equal to the uncertainty or the detection limit, the result is considered to be a nondetection. See the main volume, Chapter 14, Quality Assurance.

a $\quad$ na $=$ Not analyzed (analysis not required).

b Bicarbonate alkalinity = total alkalinity.

c Samples were analyzed in 13 days, outside the holding time of two days.

d $n d=$ None detected above reporting limits. See Table 9-1 for analytical methods and their constituents. 
Table 9-13. Livermore site surveillance well W-363.

\begin{tabular}{|c|c|c|c|c|}
\hline \multirow{2}{*}{$\begin{array}{c}\text { Constituents } \\
\text { of concern }\end{array}$} & \multicolumn{4}{|c|}{ Sampling dates } \\
\hline & $2 / 4 / 99$ & $6 / 15 / 99$ & $7 / 26 / 99$ & $11 / 3$ and $11 / 4 / 99$ \\
\hline \multicolumn{5}{|l|}{ Inorganic $(\mu \mathrm{g} / \mathrm{L})$} \\
\hline $\mathrm{pH}$ (units) & 7.4 & $\mathrm{na}^{(\mathrm{a})}$ & na & na \\
\hline Field pH (units) & 7.1 & 7.4 & na & 7.6 \\
\hline Specific conductance $(\mu \mathrm{mho} / \mathrm{cm})$ & 470 & na & na & na \\
\hline Field specific conductance $(\mu \mathrm{mho} / \mathrm{cm})$ & 490 & 470 & na & 490 \\
\hline Total dissolved solids (TDS, mg/L) & 270 & na & na & na \\
\hline Field temperature $\left({ }^{\circ} \mathrm{C}\right)$ & 20 & 21 & na & 20 \\
\hline Aluminum & $<50$ & $<100$ & na & $<100$ \\
\hline Antimony & $<4$ & $<5$ & na & $<5$ \\
\hline Arsenic & $<2$ & $<2$ & na & $<2$ \\
\hline Barium & 220 & 220 & na & 220 \\
\hline Beryllium & $<0.2$ & $<0.5$ & na & $<0.5$ \\
\hline Cadmium & $<0.5$ & $<0.5$ & na & $<0.5$ \\
\hline Chromium & 12 & 10 & na & 14 \\
\hline Cobalt & $<50$ & $<50$ & na & $<50$ \\
\hline Copper & $<1$ & $<2$ & na & 2 \\
\hline Chromium(VI) & 9.1 & 17 & na & $<2$ \\
\hline Iron & $<50$ & $<100$ & na & $<100$ \\
\hline Lead & $<5$ & $<5$ & na & $<5$ \\
\hline Manganese & $<10$ & $<30$ & na & $<30$ \\
\hline Mercury & $<0.2$ & $<0.2$ & na & $<0.2$ \\
\hline Molybdenum & $<25$ & $<25$ & na & $<25$ \\
\hline Nickel & $<2$ & $<5$ & na & 12 \\
\hline Selenium & $<2$ & $<2$ & na & $<2$ \\
\hline Silver & $<1$ & $<1$ & na & $<1$ \\
\hline Thallium & $<1$ & $<2$ & na & $<2$ \\
\hline Vanadium & 10 & $<20$ & na & $<20$ \\
\hline Zinc & $<10$ & $<20$ & na & $<20$ \\
\hline \multicolumn{5}{|l|}{ General minerals (mg/L) } \\
\hline Bicarbonate alkalinity $\left(\text { as } \mathrm{CaCO}_{3}\right)^{(\mathrm{b})}$ & 150 & na & na & na \\
\hline Boron & 0.18 & 0.19 & na & 0.1 \\
\hline Calcium & 41 & na & na & na \\
\hline Chloride & 40 & na & na & na \\
\hline Fluoride & 0.24 & na & na & na \\
\hline
\end{tabular}


Table 9-13. Livermore site surveillance well W-363 (concluded).

\begin{tabular}{|c|c|c|c|c|}
\hline \multirow{2}{*}{$\begin{array}{l}\text { Constituents } \\
\text { of concern }\end{array}$} & \multicolumn{4}{|c|}{ Sampling dates } \\
\hline & $2 / 4 / 99$ & $6 / 15 / 99$ & 7/26/99 & $11 / 3$ and $11 / 4 / 99$ \\
\hline \multicolumn{5}{|c|}{ General minerals (mg/L) (continued) } \\
\hline Magnesium & 14 & na & na & na \\
\hline Nitrate & $14^{(\mathrm{c})}$ & na & na & na \\
\hline Orthophosphate & 0.12 & na & na & na \\
\hline Potassium & 1.5 & na & na & na \\
\hline Sodium & 35 & na & na & na \\
\hline Sulfate & 9.1 & na & na & na \\
\hline Surfactants & $<0.05$ & na & na & na \\
\hline Total hardness $\left(\mathrm{as} \mathrm{CaCO}_{3}\right)$ & 160 & na & na & na \\
\hline Total phosphorus & 0.05 & na & na & na \\
\hline \multicolumn{5}{|l|}{ Organic $(\mu g / L)$} \\
\hline EPA Method 8082 (for PCBs) & na & na & $\mathrm{nd}^{(\mathrm{d})}$ & na \\
\hline \multicolumn{5}{|l|}{ Radioactive (Bq/L) } \\
\hline Gross alpha & $0.076 \pm 0.031$ & $0.035 \pm 0.034$ & na & $0.042 \pm 0.020$ \\
\hline Gross beta & $0.076 \pm 0.032$ & $0.082 \pm 0.032$ & na & $0.061 \pm 0.023$ \\
\hline Americium 241 & $0.001 \pm 0.001$ & $0.0004 \pm 0.001$ & na & $0.0002 \pm 0.0004$ \\
\hline Plutonium 238 & $0.0000 \pm 0.0002$ & na & na & $<0.037$ \\
\hline Plutonium $239+240$ & $-0.00004 \pm 0.0001$ & na & na & $<0.037$ \\
\hline Radium 226 & $0.013 \pm 0.012$ & $0.003 \pm 0.001$ & na & $0.058 \pm 0.030$ \\
\hline Radium 228 & $0.014 \pm 0.019$ & $0.026 \pm 0.011$ & na & $-0.057 \pm 0.025$ \\
\hline Thorium 228 & $0.002 \pm 0.002$ & $-0.0003 \pm 0.001$ & na & $0.002 \pm 0.008$ \\
\hline Thorium 230 & $0.002 \pm 0.001$ & $<0.006$ & na & $0.005 \pm 0.006$ \\
\hline Thorium 232 & $-0.0005 \pm 0.0005$ & $-0.00004 \pm 0.0001$ & na & $0.003 \pm 0.005$ \\
\hline Tritium & $140 \pm 6$ & $100 \pm 5.1$ & na & $140 \pm 6$ \\
\hline Uranium (total) & $0.018 \pm 0.005$ & $0.035 \pm 0.006$ & na & $0.044 \pm 0.007$ \\
\hline
\end{tabular}

Note: Radioactivities are reported as the measured concentration and either an uncertainty $( \pm 2 \sigma$ counting error) or as being less than or equal to the detection limit. If the concentration is less than or equal to the uncertainty or the detection limit, the result is considered to be a nondetection. See the main volume, Chapter 14, Quality Assurance.

a $\quad$ na $=$ Not analyzed (analysis not required).

b Bicarbonate alkalinity = total alkalinity.

c Samples were analyzed in 13 days, outside the holding time of two days.

d $n d=$ None detected above reporting limits. See Table 9-1 for analytical methods and their constituents. 
Ground Water Monitoring

Table 9-14. Livermore site surveillance well W-1308.

\begin{tabular}{|c|c|c|c|}
\hline \multirow{2}{*}{$\begin{array}{l}\text { Constituents } \\
\text { of concern }\end{array}$} & \multicolumn{3}{|c|}{ Sampling dates } \\
\hline & $1 / 2 / 99$ and $1 / 19 / 99$ & $4 / 15 / 99$ and $6 / 7 / 99$ & $7 / 15 / 99$ and $7 / 19 / 99$ \\
\hline \multicolumn{4}{|l|}{ Inorganic $(\mu \mathrm{g} / \mathrm{L})$} \\
\hline $\mathrm{pH}$ (pH units) & 7.5 & $\mathrm{na}{ }^{(\mathrm{a})}$ & na \\
\hline Specific conductance $(\mu \mathrm{mho} / \mathrm{cm})$ & 910 & na & na \\
\hline Total dissolved solids (TDS) (mg/L) & 520 & na & na \\
\hline Aluminum & $<50$ & $<100$ & na \\
\hline Antimony & $<4$ & $<5$ & na \\
\hline Arsenic & $<2$ & $<2$ & na \\
\hline Barium & 340 & 410 & na \\
\hline Beryllium & $<0.2$ & $<0.5$ & na \\
\hline Cadmium & $<0.5$ & $<0.5$ & na \\
\hline Chromium & 17 & 13 & na \\
\hline Cobalt & $<50$ & $<50$ & na \\
\hline Copper & $<1$ & $<2$ & na \\
\hline Chromium(VI) & 21 & 19 & na \\
\hline Iron & $<50$ & $<100$ & na \\
\hline Lead & $<5$ & $<5$ & na \\
\hline Manganese & $<10$ & $<30$ & na \\
\hline Mercury & $<0.2$ & $<0.2$ & na \\
\hline Molybdenum & $<25$ & $<25$ & na \\
\hline Nickel & $<2$ & $<5$ & na \\
\hline Selenium & $<2$ & $<2$ & na \\
\hline Silver & $<1$ & $<1$ & na \\
\hline Thallium & $<2$ & $<2$ & na \\
\hline Vanadium & $<10$ & $<20$ & na \\
\hline Zinc & $<10$ & $<20$ & na \\
\hline \multicolumn{4}{|l|}{ General minerals (mg/L) } \\
\hline Bicarbonate alkalinity $\left(\mathrm{as} \mathrm{CaCO}_{3}\right)^{(b)}$ & 330 & na & na \\
\hline Boron & 0.54 & 0.59 & na \\
\hline Calcium & 70 & na & na \\
\hline Chloride & 72 & na & na \\
\hline Fluoride & 0.48 & na & na \\
\hline Magnesium & 27 & na & na \\
\hline Nitrate & 29 & na & na \\
\hline Orthophosphate & 0.08 & na & na \\
\hline
\end{tabular}




\section{Ground Water Monitoring}

Table 9-14. Livermore site surveillance well W-1308 (concluded).

\begin{tabular}{|c|c|c|c|}
\hline \multirow{2}{*}{$\begin{array}{l}\text { Constituents } \\
\text { of concern }\end{array}$} & \multicolumn{3}{|c|}{ Sampling date } \\
\hline & $1 / 2 / 99$ and $1 / 19 / 99$ & $4 / 15 / 99$ and $6 / 7 / 99$ & $7 / 15 / 99$ and $7 / 19 / 99$ \\
\hline \multicolumn{4}{|c|}{ General minerals (mg/L) (continued) } \\
\hline Potassium & 1.9 & na & na \\
\hline Sodium & 85 & na & na \\
\hline Sulfate & 21 & na & na \\
\hline Surfactants & $<0.05$ & na & na \\
\hline Total hardness $\left(\mathrm{as} \mathrm{CaCO}_{3}\right)$ & 290 & na & na \\
\hline Total phosphorus & $<0.05$ & na & na \\
\hline \multicolumn{4}{|l|}{ Organic $(\mu \mathrm{g} / \mathrm{L})$} \\
\hline EPA Method 8082 (for PCBs) & na & na & $n d^{(c)}$ \\
\hline \multicolumn{4}{|l|}{ Radioactive (Bq/L) } \\
\hline Gross alpha & $0.15 \pm 0.03$ & $0.22 \pm 0.09$ & na \\
\hline Gross beta & $0.10 \pm 0.03$ & $0.17 \pm 0.06$ & na \\
\hline Americium-241 & $-0.0004 \pm 0.0005$ & $0.0004 \pm 0.001$ & na \\
\hline Plutonium-238 & $0.00003 \pm 0.0001$ & na & na \\
\hline Plutonium-239+240 & $0.0001 \pm 0.0001$ & na & na \\
\hline Radium-226 & $0.018 \pm 0.012$ & $0.001 \pm 0.001$ & na \\
\hline Radium-228 & $-0.019 \pm 0.020$ & $0.023 \pm 0.011$ & na \\
\hline Thorium-228 & $0.003 \pm 0.003$ & $-0.001 \pm 0.001$ & na \\
\hline Thorium-230 & $0.003 \pm 0.003$ & $<0.006$ & na \\
\hline Thorium-232 & $0.002 \pm 0.002$ & $<0.006$ & na \\
\hline Tritium & $19 \pm 3.5$ & $23 \pm 3.4$ & na \\
\hline Uranium (total) & $0.22 \pm 0.02$ & $0.16 \pm 0.02$ & na \\
\hline
\end{tabular}

Note: Radioactivities are reported as the measured concentration and either an uncertainty ( $\pm 2 \sigma$ counting error) or as being less than or equal to the detection limit. If the concentration is less than or equal to the uncertainty or the detection limit, the result is considered to be a nondetection. See the main volume, Chapter 14, Quality Assurance.

a $\mathrm{na}=$ Not analyzed (analysis not required).

b Bicarbonate alkalinity = total alkalinity.

c $n d=$ None detected above reporting limits. See Table 9-1 for analytical methods and their constituents. 


\section{Ground Water Monitoring}

Table 9-15. Livermore site surveillance well W-1303.

\begin{tabular}{|c|c|c|c|}
\hline \multirow{2}{*}{$\begin{array}{l}\text { Constituents } \\
\text { of concern }\end{array}$} & \multicolumn{3}{|c|}{ Sampling dates } \\
\hline & $1 / 21 / 99$ and $1 / 26 / 99$ & 4/14/99 and 6/7/99 & $7 / 19 / 99$ \\
\hline \multicolumn{4}{|l|}{ Inorganic ( $\mu \mathrm{g} / \mathrm{L})$} \\
\hline $\mathrm{pH}$ (pH units) & 7.5 & $\mathrm{na}^{(\mathrm{a})}$ & na \\
\hline Specific conductance $(\mu \mathrm{mho} / \mathrm{cm})$ & 1300 & na & na \\
\hline Total dissolved solids (TDS) (mg/L) & 760 & na & na \\
\hline Aluminum & $<50$ & $<100$ & na \\
\hline Antimony & $<4$ & $<5$ & na \\
\hline Arsenic & $<2$ & $<2$ & na \\
\hline Barium & 740 & 750 & na \\
\hline Beryllium & $<0.2$ & $<0.5$ & na \\
\hline Cadmium & $<0.5$ & $<0.5$ & na \\
\hline Chromium & 6.1 & 4 & na \\
\hline Cobalt & $<50$ & $<50$ & na \\
\hline Copper & $<1$ & $<2$ & na \\
\hline Chromium(VI) & $<2$ & 10 & na \\
\hline Iron & 76 & $<100$ & na \\
\hline Lead & $<5$ & $<5$ & na \\
\hline Manganese & $<10$ & $<30$ & na \\
\hline Mercury & $<0.2$ & $<0.2$ & na \\
\hline Molybdenum & $<25$ & $<25$ & na \\
\hline Nickel & $<50$ & $<5$ & na \\
\hline Selenium & $<2$ & $<2$ & na \\
\hline Silver & $<1$ & $<1$ & na \\
\hline Thallium & $<1$ & $<2$ & na \\
\hline Vanadium & $<10$ & $<20$ & na \\
\hline Zinc & $<10$ & $<20$ & na \\
\hline \multicolumn{4}{|l|}{ General minerals (mg/L) } \\
\hline Bicarbonate alkalinity $\left(\mathrm{as} \mathrm{CaCO}_{3}\right)^{(\mathrm{b})}$ & 500 & na & na \\
\hline Boron & 0.48 & na & na \\
\hline Calcium & 120 & na & na \\
\hline Chloride & 110 & na & na \\
\hline Fluoride & 0.45 & na & na \\
\hline Magnesium & 43 & na & na \\
\hline Nitrate & 31 & na & na \\
\hline
\end{tabular}




\section{Ground Water Monitoring}

Table 9-15. Livermore site surveillance well W-1303 (concluded).

\begin{tabular}{|c|c|c|c|}
\hline \multirow{2}{*}{$\begin{array}{l}\text { Constituents } \\
\text { of concern }\end{array}$} & \multicolumn{3}{|c|}{ Sampling dates } \\
\hline & $1 / 21 / 99$ and $1 / 26 / 99$ & 4/14/99 and 6/7/99 & 7/19/99 \\
\hline \multicolumn{4}{|c|}{ General minerals (mg/L) (continued) } \\
\hline Orthophosphate & 0.05 & na & na \\
\hline Potassium & 2 & na & na \\
\hline Sodium & 80 & na & na \\
\hline Sulfate & 21 & na & na \\
\hline Surfactants & $<0.05$ & na & na \\
\hline Total hardness $\left(\right.$ as $\left.\mathrm{CaCO}_{3}\right)$ & 480 & na & na \\
\hline Total phosphorus & $<0.05$ & na & na \\
\hline \multicolumn{4}{|l|}{ Organic $(\mu g / L)$} \\
\hline EPA Method 8082 (for PCBs) & na & na & $n d^{(c)}$ \\
\hline \multicolumn{4}{|l|}{ Radioactive (Bq/L) } \\
\hline Gross alpha & $0.39 \pm 0.07$ & $0.35 \pm 0.13$ & na \\
\hline Gross beta & $0.14 \pm 0.05$ & $0.20 \pm 0.06$ & na \\
\hline Americium-241 & $0.002 \pm 0.003$ & $<0.0004$ & na \\
\hline Plutonium-238 & $0.0001 \pm 0.0001$ & na & na \\
\hline Plutonium-239+240 & $0.0002 \pm 0.0001$ & na & na \\
\hline Radium-226 & $0.006 \pm 0.006$ & $0.008 \pm 0.002$ & na \\
\hline Radium-228 & $0.025 \pm 0.016$ & $0.036 \pm 0.013$ & na \\
\hline Thorium-228 & $0.0002 \pm 0.003$ & $<0.009$ & na \\
\hline Thorium-230 & $0.001 \pm 0.001$ & $<0.006$ & na \\
\hline Thorium-232 & $-0.0003 \pm 0.0004$ & $<0.006$ & na \\
\hline Tritium & $43 \pm 3.6$ & $47 \pm 4.1$ & na \\
\hline Uranium (total) & $0.30 \pm 0.04$ & $0.32 \pm 0.04$ & na \\
\hline
\end{tabular}

Note: Radioactivities are reported as the measured concentration and either an uncertainty $( \pm 2 \sigma$ counting error) or as being less than or equal to the detection limit. If the concentration is less than or equal to the uncertainty or the detection limit, the result is considered to be a nondetection. See the main volume, Chapter 14, Quality Assurance.

a $\mathrm{na}=$ Not analyzed (analysis not required).

b Bicarbonate alkalinity $=$ total alkalinity.

c $n d=$ None detected above reporting limits. See Table 9-1 for analytical methods and their constituents. 


\section{Ground Water Monitoring}

Table 9-16. Livermore site surveillance well W-119.

\begin{tabular}{|c|c|c|c|}
\hline \multirow{2}{*}{$\begin{array}{c}\text { Constituents } \\
\text { of concern }\end{array}$} & \multicolumn{3}{|c|}{ Sampling dates } \\
\hline & 2/4/99 & $6 / 14 / 99$ & $7 / 22 / 99$ \\
\hline \multicolumn{4}{|l|}{ Inorganic ( $\mu \mathrm{g} / \mathrm{L})$} \\
\hline $\mathrm{pH}$ (pH units) & 7.4 & $\mathrm{na}^{(\mathrm{a})}$ & na \\
\hline Field $\mathrm{pH}$ (pH units) & na & 7.4 & 7.3 \\
\hline Specific conductance $(\mu \mathrm{mho} / \mathrm{cm})$ & 900 & na & na \\
\hline Field specific conductance $(\mu \mathrm{mho} / \mathrm{cm})$ & na & 830 & 830 \\
\hline Total dissolved solids (TDS) (mg/L) & 530 & na & na \\
\hline Water temperature $\left({ }^{\circ} \mathrm{C}\right)$ & na & 19 & 19 \\
\hline Aluminum & $<50$ & $<100$ & 200 \\
\hline Antimony & $<4$ & $<5$ & $<5$ \\
\hline Arsenic & $<2$ & $<2$ & $<2$ \\
\hline Barium & 390 & 370 & 350 \\
\hline Beryllium & $<0.2$ & $<0.5$ & $<0.5$ \\
\hline Cadmium & $<0.5$ & $<0.5$ & 1.1 \\
\hline Chromium & 5.4 & 2 & 4 \\
\hline Cobalt & $<50$ & $<50$ & $<50$ \\
\hline Copper & $<1$ & $<2$ & $<2$ \\
\hline Chromium(VI) & 5 & 3 & 4 \\
\hline Iron & $<50$ & $<100$ & $<100$ \\
\hline Lead & $<5$ & $<5$ & $<5$ \\
\hline Manganese & $<10$ & $<30$ & $<30$ \\
\hline Mercury & $<0.2$ & $<0.2$ & $<0.2$ \\
\hline Molybdenum & $<25$ & $<25$ & $<25$ \\
\hline Nickel & $<2$ & $<5$ & $<5$ \\
\hline Selenium & $<2$ & $<2$ & $<2$ \\
\hline Silver & $<1$ & $<1$ & $<1$ \\
\hline Thallium & $<1$ & $<2$ & $<2$ \\
\hline Vanadium & $<10$ & $<20$ & $<20$ \\
\hline Zinc & $<10$ & $<20$ & 30 \\
\hline \multicolumn{4}{|l|}{ General minerals $(\mathrm{mg} / \mathrm{L})$} \\
\hline Bicarbonate alkalinity $\left(\mathrm{as} \mathrm{CaCO}_{3}\right)^{(\mathrm{b})}$ & 290 & na & na \\
\hline Boron & 0.46 & 0.51 & 0.48 \\
\hline Calcium & 81 & na & na \\
\hline Chloride & 95 & na & na \\
\hline Fluoride & 0.32 & na & na \\
\hline
\end{tabular}


Table 9-16. Livermore site surveillance well W-119 (concluded).

\begin{tabular}{|c|c|c|c|}
\hline \multirow{2}{*}{$\begin{array}{l}\text { Constituents } \\
\text { of concern }\end{array}$} & \multicolumn{3}{|c|}{ Sampling dates } \\
\hline & 2/4/99 & 6/14/99 & $7 / 22 / 99$ \\
\hline \multicolumn{4}{|c|}{ General minerals (mg/L) (continued) } \\
\hline Magnesium & 29 & na & na \\
\hline Nitrate & $35^{(c)}$ & na & na \\
\hline Orthophosphate & 0.09 & na & na \\
\hline Potassium & 1.5 & na & na \\
\hline Sodium & 69 & na & na \\
\hline Sulfate & 25 & na & na \\
\hline Surfactants & $<0.05$ & na & na \\
\hline Total hardness (as $\mathrm{CaCO}_{3}$ ) & 320 & na & na \\
\hline Total phosphorus & $<0.05$ & na & na \\
\hline \multicolumn{4}{|l|}{ Radioactive (Bq/L) } \\
\hline Gross alpha & $0.23 \pm 0.05$ & $0.11 \pm 0.05$ & $0.076 \pm 0.055$ \\
\hline Gross beta & $0.098 \pm 0.031$ & $0.11 \pm 0.05$ & $0.081 \pm 0.051$ \\
\hline Americium-241 & $0.001 \pm 0.001$ & $0.001 \pm 0.001$ & $0.0001 \pm 0.0003$ \\
\hline Plutonium-238 & $<0.0002$ & na & $-0.00004 \pm 0.0001$ \\
\hline Plutonium-239+240 & $-0.00001 \pm 0.0001$ & na & $-0.0000 \pm 0.0001$ \\
\hline Radium-226 & $0.027 \pm 0.014$ & $0.006 \pm 0.002$ & $0.004 \pm 0.001$ \\
\hline Radium-228 & $0.017 \pm 0.016$ & $0.017 \pm 0.010$ & $0.009 \pm 0.009$ \\
\hline Thorium-228 & $0.002 \pm 0.002$ & $0.001 \pm 0.002$ & $0.001 \pm 0.002$ \\
\hline Thorium-230 & $0.001 \pm 0.001$ & $0.001 \pm 0.001$ & $-0.00003 \pm 0.0001$ \\
\hline Thorium-232 & $0.0005 \pm 0.001$ & $0.0003 \pm 0.001$ & $<0.006$ \\
\hline Tritium & $19 \pm 2.8$ & $25 \pm 3.4$ & $24 \pm 2.5$ \\
\hline Uranium (total) & $0.15 \pm 0.02$ & $0.12 \pm 0.02$ & $0.13 \pm 6.00$ \\
\hline
\end{tabular}

Note: Radioactivities are reported as the measured concentration and either an uncertainty ( $\pm 2 \sigma$ counting error) or as being less than or equal to the detection limit. If the concentration is less than or equal to the uncertainty or the detection limit, the result is considered to be a nondetection. See the main volume, Chapter 14, Quality Assurance.
a $n a=$ Not analyzed (analysis not required).
b Bicarbonate alkalinity = total alkalinity.
c Samples were analyzed in 13 days, outside the holding time of two days. 
Table 9-17. Livermore site surveillance well W-1306.

\begin{tabular}{|c|c|c|c|}
\hline \multirow{2}{*}{$\begin{array}{l}\text { Constituents } \\
\text { of concern }\end{array}$} & \multicolumn{3}{|c|}{ Sampling dates } \\
\hline & $1 / 21 / 99$ and $1 / 26 / 99$ & $4 / 14 / 99$ and $6 / 7 / 99$ & $7 / 14 / 99$ and $7 / 19 / 99$ \\
\hline \multicolumn{4}{|l|}{ Inorganic $(\mu g / L)$} \\
\hline $\mathrm{pH}$ (pH units) & 7.8 & $\mathrm{na}^{(\mathrm{a})}$ & na \\
\hline Specific conductance ( $\mu \mathrm{mho} / \mathrm{cm})$ & 1300 & na & na \\
\hline Total dissolved solids (TDS) (mg/L) & 770 & na & na \\
\hline Aluminum & $<50$ & $<100$ & na \\
\hline Antimony & $<4$ & $<5$ & na \\
\hline Arsenic & $<2$ & 2 & na \\
\hline Barium & 480 & 490 & na \\
\hline Beryllium & $<0.2$ & $<0.5$ & na \\
\hline Cadmium & $<0.5$ & $<0.5$ & na \\
\hline Chromium & 6.4 & 4 & na \\
\hline Cobalt & $<50$ & $<50$ & na \\
\hline Copper & 1.1 & $<2$ & na \\
\hline Chromium(VI) & 3.2 & 8 & na \\
\hline Iron & $<50$ & $<100$ & na \\
\hline Lead & $<5$ & $<5$ & na \\
\hline Manganese & $<10$ & $<30$ & na \\
\hline Mercury & $<0.2$ & $<0.2$ & na \\
\hline Molybdenum & $<25$ & $<25$ & na \\
\hline Nickel & 4 & $<5$ & na \\
\hline Selenium & $<2$ & $<2$ & na \\
\hline Silver & $<1$ & $<1$ & na \\
\hline Thallium & $<1$ & $<2$ & na \\
\hline Vanadium & $<10$ & $<20$ & na \\
\hline Zinc & $<10$ & $<20$ & na \\
\hline \multicolumn{4}{|l|}{ General minerals (mg/L) } \\
\hline Bicarbonate alkalinity (as $\left.\mathrm{CaCO}_{3}\right)^{(\mathrm{b})}$ & 370 & na & na \\
\hline Boron & 0.45 & 0.4 & na \\
\hline Calcium & 110 & na & na \\
\hline Chloride & 160 & na & na \\
\hline Fluoride & 0.48 & na & na \\
\hline Magnesium & 41 & na & na \\
\hline Nitrate & 41 & na & na \\
\hline Orthophosphate & 0.09 & na & na \\
\hline
\end{tabular}




\section{Ground Water Monitoring}

Table 9-17. Livermore site surveillance well W-1306 (concluded).

\begin{tabular}{|c|c|c|c|}
\hline \multirow{2}{*}{$\begin{array}{l}\text { Constituents } \\
\text { of concern }\end{array}$} & \multicolumn{3}{|c|}{ Sampling dates } \\
\hline & $1 / 21 / 99$ and $1 / 26 / 99$ & $4 / 14 / 99$ and $6 / 7 / 99$ & $7 / 14 / 99$ and $7 / 19 / 99$ \\
\hline \multicolumn{4}{|c|}{ General minerals (mg/L) (continued) } \\
\hline Potassium & 2 & na & na \\
\hline Sodium & 91 & na & na \\
\hline Sulfate & 43 & na & na \\
\hline Surfactants & $<0.05$ & na & na \\
\hline Total hardness (as $\mathrm{CaCO}_{3}$ ) & 450 & na & na \\
\hline Total phosphorus & $<0.05$ & na & na \\
\hline \multicolumn{4}{|l|}{ Organic ( $\mu g / L)$} \\
\hline EPA Method 8082 (for PCBs) & na & na & $\mathrm{nd}^{(\mathrm{c})}$ \\
\hline \multicolumn{4}{|l|}{ Radioactivity (Bq/L) } \\
\hline Gross alpha & $0.31 \pm 0.06$ & $0.32 \pm 0.12$ & na \\
\hline Gross beta & $0.13 \pm 0.05$ & $0.20 \pm 0.07$ & na \\
\hline Americium-241 & $<0.004$ & $<0.004$ & na \\
\hline Plutonium-238 & $-0.0001 \pm 0.0001$ & na & na \\
\hline Plutonium-239+240 & $0.00002 \pm 0.0001$ & na & na \\
\hline Radium-226 & $0.013 \pm 0.007$ & $0.006 \pm 0.002$ & na \\
\hline Radium-228 & $0.020 \pm 0.018$ & $0.045 \pm 0.014$ & na \\
\hline Thorium-228 & $0.003 \pm 0.002$ & $-0.0003 \pm 0.001$ & na \\
\hline Thorium-230 & $0.0002 \pm 0.001$ & $-0.0001 \pm 0.0001$ & na \\
\hline Thorium-232 & $<0.006$ & $<0.006$ & na \\
\hline Tritium & $7.4 \pm 2.6$ & $13 \pm 3.2$ & na \\
\hline Uranium (total) & $0.34 \pm 0.03$ & $0.33 \pm 0.04$ & na \\
\hline
\end{tabular}

Note: Radioactivities are reported as the measured concentration and either an uncertainty $( \pm 2 \sigma$ counting error) or as being less than or equal to the detection limit. If the concentration is less than or equal to the uncertainty or the detection limit, the result is considered to be a nondetection. See the main volume, Chapter 14, Quality Assurance.

a $n a=$ Not analyzed (analysis not required).

b Bicarbonate alkalinity = total alkalinity.

c $\quad$ nd $=$ None detected above reporting limits. See Table 9-1 for analytical methods and their constituents. 
Table 9-18. Livermore site surveillance well W-906.

\begin{tabular}{|c|c|c|c|c|}
\hline \multirow{2}{*}{$\begin{array}{l}\text { Constituents } \\
\text { of concern }\end{array}$} & \multicolumn{4}{|c|}{ Sampling dates } \\
\hline & $\begin{array}{c}1 / 13 / 99 \text { and } \\
1 / 19 / 99\end{array}$ & $\begin{array}{c}4 / 14 / 99 \text { and } \\
6 / 9 / 99\end{array}$ & $\begin{array}{c}7 / 14 / 99 \text { and } \\
7 / 19 / 99\end{array}$ & $\begin{array}{c}10 / 12 / 99 \text { and } \\
10 / 18 / 99\end{array}$ \\
\hline \multicolumn{5}{|l|}{ Inorganic $(\mu \mathrm{g} / \mathrm{L})$} \\
\hline $\mathrm{pH}$ (units) & 7.6 & $\mathrm{na}^{(\mathrm{a})}$ & na & na \\
\hline Specific conductance $(\mu \mathrm{mho} / \mathrm{cm})$ & 1800 & na & na & na \\
\hline Total dissolved solids (TDS, mg/L) & 1100 & na & na & na \\
\hline Aluminum & $<50$ & $<100$ & na & na \\
\hline Antimony & $<4$ & $<5$ & na & na \\
\hline Arsenic & $<2$ & $<2$ & na & na \\
\hline Barium & 350 & 380 & na & na \\
\hline Beryllium & $<0.2$ & $<0.5$ & na & na \\
\hline Cadmium & 0.59 & $<0.5$ & na & na \\
\hline Chromium & 12 & 5 & na & na \\
\hline Cobalt & $<50$ & $<50$ & na & na \\
\hline Copper & 160 & $<2$ & 10 & na \\
\hline Chromium(VI) & 8.3 & 2 & na & na \\
\hline Iron & 130 & $<100$ & na & na \\
\hline Lead & 73 & $<5$ & $<5$ & na \\
\hline Manganese & $<10$ & $<30$ & na & na \\
\hline Mercury & $<0.2$ & $<0.2$ & na & na \\
\hline Molybdenum & $<25$ & $<25$ & na & na \\
\hline Nickel & 6.9 & $<5$ & na & na \\
\hline Selenium & $<2$ & $<2$ & na & na \\
\hline Silver & $<1$ & $<1$ & na & na \\
\hline Thallium & $<2$ & $<2$ & na & na \\
\hline Vanadium & $<10$ & $<20$ & na & na \\
\hline Zinc & 3400 & $<20$ & 110 & na \\
\hline \multicolumn{5}{|l|}{ General minerals (mg/L) } \\
\hline Bicarbonate alkalinity $\left(\text { as } \mathrm{CaCO}_{3}\right)^{(b)}$ & 330 & na & na & na \\
\hline Boron & 0.9 & 0.88 & na & na \\
\hline Calcium & 140 & na & na & na \\
\hline Chloride & 330 & na & na & na \\
\hline Fluoride & 0.49 & na & na & na \\
\hline Magnesium & 60 & na & na & na \\
\hline Nitrate & 35 & na & na & na \\
\hline Orthophosphate & 0.09 & na & na & na \\
\hline Potassium & 1.9 & na & na & na \\
\hline Sodium & 120 & na & na & na \\
\hline
\end{tabular}


Table 9-18. Livermore site surveillance well W-906 (concluded).

\begin{tabular}{|c|c|c|c|c|}
\hline \multirow{2}{*}{$\begin{array}{l}\text { Constituents } \\
\text { of concern }\end{array}$} & \multicolumn{4}{|c|}{ Sampling dates } \\
\hline & $\begin{array}{c}1 / 13 / 99 \text { and } \\
1 / 19 / 99\end{array}$ & $\begin{array}{c}4 / 14 / 99 \text { and } \\
6 / 9 / 99\end{array}$ & $\begin{array}{c}7 / 14 / 99 \text { and } \\
7 / 19 / 99\end{array}$ & $\begin{array}{c}10 / 12 / 99 \text { and } \\
10 / 18 / 99\end{array}$ \\
\hline \multicolumn{5}{|c|}{ General minerals (mg/L) (continued) } \\
\hline Sulfate & 53 & na & na & na \\
\hline Surfactants & $<0.05$ & na & na & na \\
\hline Total hardness (as $\mathrm{CaCO}_{3}$ ) & 600 & na & na & na \\
\hline Total phosphorus & $<0.05$ & na & na & na \\
\hline \multicolumn{5}{|l|}{ Organic constituents $(\mu \mathrm{g} / \mathrm{L})$} \\
\hline EPA Method 8082 (for PCBs) & na & na & na & $\mathrm{nd}^{(\mathrm{c})}$ \\
\hline \multicolumn{5}{|l|}{ Radioactive (Bq/L) } \\
\hline Gross alpha & $0.30 \pm 0.06$ & $0.36 \pm 0.13$ & na & na \\
\hline Gross beta & $0.095 \pm 0.047$ & $0.13 \pm 0.07$ & na & na \\
\hline Americium-241 & $0.013 \pm 0.010$ & $<0.004$ & na & $0.0004 \pm 0.001$ \\
\hline Plutonium-238 & $0.0001 \pm 0.0002$ & na & na & na \\
\hline Plutonium-239+240 & $-0.00002 \pm 0.0001$ & na & na & na \\
\hline Radium-226 & $0.016 \pm 0.012$ & $0.005 \pm 0.002$ & na & na \\
\hline Radium-228 & $-0.003 \pm 0.018$ & $0.023 \pm 0.011$ & na & na \\
\hline Thorium-228 & $-0.001 \pm 0.002$ & $0.0003 \pm 0.000$ & na & na \\
\hline Thorium-230 & $0.001 \pm 0.001$ & $0.0002 \pm 0.0004$ & na & na \\
\hline Thorium-232 & $0.001 \pm 0.001$ & $0.0002 \pm 0.0004$ & na & na \\
\hline Tritium & $-2.5 \pm 2.8$ & $3.4 \pm 2.8$ & na & na \\
\hline Uranium (total) & $0.31 \pm 0.03$ & $0.31 \pm 0.04$ & na & na \\
\hline
\end{tabular}

Note: Radioactivities are reported as the measured concentration and either an uncertainty ( $\pm 2 \sigma$ counting error) or as being less than or equal to the detection limit. If the concentration is less than or equal to the uncertainty or the detection limit, the result is considered to be a nondetection. See the main volume, Chapter 14, Quality Assurance.

a $n a=$ Not analyzed (analysis not required).

b Bicarbonate alkalinity $=$ total alkalinity.

c $\mathrm{nd}=$ None detected above reporting limits. See Table 9-1 for analytical methods and their constituents. 
Table 9-19. Livermore site surveillance well W-593.

\begin{tabular}{|c|c|c|}
\hline \multirow{2}{*}{$\begin{array}{l}\text { Constituents } \\
\text { of concern }\end{array}$} & \multicolumn{2}{|c|}{ Sampling dates } \\
\hline & $1 / 12 / 99$ & $6 / 9 / 99$ \\
\hline \multicolumn{3}{|l|}{ Inorganic $(\mu \mathrm{g} / \mathrm{L})$} \\
\hline $\mathrm{pH}$ (units) & 7.9 & $n a^{(a)}$ \\
\hline Field $\mathrm{pH}$ (units) & na & 7.4 \\
\hline Specific conductance $(\mu \mathrm{mho} / \mathrm{cm})$ & 2300 & na \\
\hline Field specific conductance $(\mu \mathrm{mho} / \mathrm{cm})$ & na & 490 \\
\hline Total dissolved solids (TDS, mg/L) & na & 20 \\
\hline Field temperature $\left({ }^{\circ} \mathrm{C}\right)$ & 1400 & na \\
\hline Aluminum & $<50$ & $<100$ \\
\hline Antimony & $<4$ & $<5$ \\
\hline Arsenic & 3 & 2 \\
\hline Barium & $<25$ & $<25$ \\
\hline Beryllium & $<0.2$ & $<0.5$ \\
\hline Cadmium & $<0.5$ & $<0.5$ \\
\hline Chromium & 6.2 & 6 \\
\hline Cobalt & $<50$ & $<50$ \\
\hline Copper & 1.6 & $<2$ \\
\hline Chromium(VI) & 11 & 5 \\
\hline Iron & $<50$ & $<100$ \\
\hline Lead & $<5$ & $<5$ \\
\hline Manganese & $<10$ & $<30$ \\
\hline Mercury & $<0.2$ & $<0.2$ \\
\hline Molybdenum & $<25$ & $<25$ \\
\hline Nickel & 14 & 9 \\
\hline Selenium & $<2$ & $<2$ \\
\hline Silver & $<1$ & $<1$ \\
\hline Thallium & $<1$ & $<2$ \\
\hline Vanadium & 16 & $<20$ \\
\hline Zinc & 28 & $<20$ \\
\hline \multicolumn{3}{|l|}{ General minerals (mg/L) } \\
\hline Bicarbonate alkalinity $\left(\text { as } \mathrm{CaCO}_{3}\right)^{(\mathrm{b})}$ & 260 & na \\
\hline Boron & 8.5 & 8.1 \\
\hline Calcium & 88 & na \\
\hline Chloride & 460 & na \\
\hline Fluoride & 1.2 & na \\
\hline
\end{tabular}


Table 9-19. Livermore site surveillance well W-593 (concluded).

\begin{tabular}{|c|c|c|}
\hline \multirow{2}{*}{$\begin{array}{l}\text { Constituents } \\
\text { of concern }\end{array}$} & \multicolumn{2}{|c|}{ Sampling dates } \\
\hline & 1/12/99 & 6/9/99 \\
\hline \multicolumn{3}{|c|}{ General minerals (mg/L) (continued) } \\
\hline Magnesium & 49 & na \\
\hline Nitrate & 21 & na \\
\hline Orthophosphate & 0.05 & na \\
\hline Potassium & 1.7 & na \\
\hline Sodium & 300 & na \\
\hline Sulfate & 210 & na \\
\hline Surfactants & $<0.05$ & na \\
\hline Total hardness $\left(\mathrm{as} \mathrm{CaCO}_{3}\right)$ & 420 & na \\
\hline Total phosphorus & $<0.05$ & na \\
\hline \multicolumn{3}{|l|}{ Radioactive (Bq/L) } \\
\hline Gross alpha & $0.41 \pm 0.12$ & $0.13 \pm 0.12$ \\
\hline Gross beta & $0.16 \pm 0.10$ & $0.20 \pm 0.10$ \\
\hline Americium-241 & $0.0003 \pm 0.001$ & $-0.0002 \pm 0.0003$ \\
\hline Plutonium-238 & $0.000005 \pm 0.0001$ & na \\
\hline Plutonium-239+240 & $0.0001 \pm 0.0001$ & na \\
\hline Radium-226 & $0.016 \pm 0.008$ & $0.001 \pm 0.001$ \\
\hline Radium-228 & $-0.005 \pm 0.018$ & $0.019 \pm 0.010$ \\
\hline Thorium-228 & $-0.0004 \pm 0.001$ & $-0.0002 \pm 0.001$ \\
\hline Thorium-230 & $0.003 \pm 0.001$ & $<0.006 \pm 0.001$ \\
\hline Thorium-232 & $<0.006$ & $0.0002 \pm 0.0004$ \\
\hline Tritium & $-2.7 \pm 2.7$ & $-0.46 \pm 2.7$ \\
\hline Uranium(total) & $0.21 \pm 0.02$ & $0.23 \pm 0.03$ \\
\hline
\end{tabular}

Note: Radioactivities are reported as the measured concentration and either an uncertainty $( \pm 2 \sigma$ counting error) or as being less than or equal to the detection limit. If the concentration is less than or equal to the uncertainty or the detection limit, the result is considered to be a nondetection. See the main volume, Chapter 14, Quality Assurance.

a $\quad$ na $=$ Not analyzed (analysis not required).

b Bicarbonate alkalinity $=$ total alkalinity. 
Table 9-20. Livermore site surveillance well W-270.

\begin{tabular}{|c|c|}
\hline \multirow{2}{*}{$\begin{array}{l}\text { Constituents } \\
\text { of concern }\end{array}$} & Sampling date \\
\hline & 7/27/99 \\
\hline \multicolumn{2}{|l|}{ Inorganic $(\mu \mathrm{g} / \mathrm{L})$} \\
\hline Field temperature $\left({ }^{\circ} \mathrm{C}\right)$ & 21 \\
\hline Field specific conductance $(\mu \mathrm{mho} / \mathrm{cm})$ & 780 \\
\hline Field $\mathrm{pH}$ ( $\mathrm{pH}$ units) & 7.5 \\
\hline Aluminum & $<100$ \\
\hline Antimony & $<5$ \\
\hline Arsenic & 2 \\
\hline Barium & 97 \\
\hline Beryllium & $<0.5$ \\
\hline Boron & 200 \\
\hline Cadmium & $<0.5$ \\
\hline Chromium & 15 \\
\hline Cobalt & $<50$ \\
\hline Copper & $<2$ \\
\hline Chromium(VI) & 4 \\
\hline Iron & $<100$ \\
\hline Lead & $<5$ \\
\hline Manganese & $<30$ \\
\hline Mercury & $<0.2$ \\
\hline Molybdenum & $<25$ \\
\hline Nickel & $<5$ \\
\hline Selenium & 6 \\
\hline Silver & $<1$ \\
\hline Thallium & $<2$ \\
\hline Vanadium & $<20$ \\
\hline Zinc & $<20$ \\
\hline \multicolumn{2}{|l|}{ Radioactive (Bq/L) } \\
\hline Gross alpha & $0.14 \pm 0.06$ \\
\hline Gross beta & $0.12 \pm 0.04$ \\
\hline Americium-241 & $0.0006 \pm 0.0004$ \\
\hline Plutonium-238 & $-0.00002 \pm 0.0002$ \\
\hline Plutonium-239+240 & $0.0001 \pm 0.0002$ \\
\hline
\end{tabular}




\section{Ground Water Monitoring}

Table 9-20. Livermore site surveillance well W-270 (concluded).

\begin{tabular}{|l|c|}
\multicolumn{1}{|c|}{$\begin{array}{c}\text { Constituents } \\
\text { of concern }\end{array}$} & Sampling date \\
\cline { 2 - 2 } Radioactive (Bq/L) (continued) & $\mathbf{7 / 2 7 / 9 9}$ \\
Radium-226 & $0.004 \pm 0.002$ \\
Radium-228 & $0.014 \pm 0.010$ \\
Thorium-228 & $0.001 \pm 0.001$ \\
Thorium-230 & $0.0003 \pm 0.0005$ \\
Thorium-232 & $<0.006$ \\
Tritium & $0.03 \pm 1.5$ \\
Uranium (total) & $0.13 \pm 0.02$ \\
\hline
\end{tabular}

Note: Radioactivities are reported as the measured concentration and either an uncertainty ( $\pm 2 \sigma$ counting error) or as being less than or equal to the detection limit. If the concentration is less than or equal to the uncertainty or the detection limit, the result is considered to be a nondetection. See the main volume, Chapter 14, Quality Assurance. 
Table 9-21. Livermore site surveillance well W-359.

\begin{tabular}{|c|c|c|}
\hline \multirow{2}{*}{$\begin{array}{l}\text { Constituents } \\
\text { of concern }\end{array}$} & \multicolumn{2}{|c|}{ Sampling dates } \\
\hline & 2/1/99 & $10 / 26 / 99$ \\
\hline \multicolumn{3}{|l|}{ Inorganic ( $\mu \mathrm{g} / \mathrm{L})$} \\
\hline $\mathrm{pH}$ (units) & $\mathrm{na}^{(\mathrm{a})}$ & 7.2 \\
\hline Field pH (units) & na & 7.6 \\
\hline Field specific conductance $(\mu \mathrm{mho} / \mathrm{cm})$ & na & 590 \\
\hline Specific conductance $(\mu \mathrm{mho} / \mathrm{cm})$ & na & 560 \\
\hline Total dissolved solids (TDS, mg/L) & na & 760 \\
\hline Field temperature $\left({ }^{\circ} \mathrm{C}\right)$ & na & 20 \\
\hline Aluminum & $<200$ & 100 \\
\hline Antimony & $<5$ & $<5$ \\
\hline Arsenic & $<2$ & $<2$ \\
\hline Barium & 160 & 140 \\
\hline Beryllium & $<0.5$ & $<0.5$ \\
\hline Cadmium & $<0.5$ & $<0.5$ \\
\hline Chromium & 9.7 & 5 \\
\hline Cobalt & $<50$ & $<50$ \\
\hline Copper & 16 & $<2$ \\
\hline Chromium(VI) & 8.7 & 7 \\
\hline Iron & $<100$ & $<100$ \\
\hline Lead & $<2$ & $<5$ \\
\hline Manganese & $<30$ & $<30$ \\
\hline Mercury & $<0.2$ & $<0.2$ \\
\hline Molybdenum & $<50$ & $<25$ \\
\hline Nickel & 7.9 & $<5$ \\
\hline Selenium & $<2$ & $<2$ \\
\hline Silver & $<0.5$ & $<1$ \\
\hline Thallium & $<1$ & $<2$ \\
\hline Vanadium & $<50$ & $<20$ \\
\hline Zinc & $<20$ & $<20$ \\
\hline \multicolumn{3}{|l|}{ General minerals (mg/L) } \\
\hline Bicarbonate alkalinity $\left(\text { as } \mathrm{CaCO}_{3}\right)^{(\mathrm{b})}$ & na & 150 \\
\hline Boron & 0.38 & 0.3 \\
\hline Calcium & na & 41 \\
\hline Chloride & na & 69 \\
\hline Fluoride & na & 0.32 \\
\hline
\end{tabular}




\section{Ground Water Monitoring}

Table 9-21. Livermore site surveillance well W-359 (concluded).

\begin{tabular}{|c|c|c|}
\hline \multirow{2}{*}{$\begin{array}{l}\text { Constituents } \\
\text { of concern }\end{array}$} & \multicolumn{2}{|c|}{ Sampling dates } \\
\hline & 2/1/99 & 10/26/99 \\
\hline \multicolumn{3}{|c|}{ General minerals (mg/L) (continued) } \\
\hline Magnesium & na & 17 \\
\hline Nitrate & na & $50^{(\mathrm{c})}$ \\
\hline Orthophosphate & na & 0.04 \\
\hline Potassium & na & 2 \\
\hline Sodium & na & 49 \\
\hline Sulfate & na & 15 \\
\hline Surfactants & na & $<0.5$ \\
\hline Total hardness $\left(\right.$ as $\left.\mathrm{CaCO}_{3}\right)$ & na & 170 \\
\hline Total phosphorus & na & $<0.05$ \\
\hline \multicolumn{3}{|l|}{ Organic $(\mu \mathrm{g} / \mathrm{L})$} \\
\hline EPA Method 625 & na & $n d^{(d)}$ \\
\hline \multicolumn{3}{|l|}{ Radioactive (Bq/L) } \\
\hline Gross alpha & na & $0.053 \pm 0.022$ \\
\hline Gross beta & na & $0.075 \pm 0.026$ \\
\hline \multirow[t]{2}{*}{ Plutonium 238} & & $0.012 \pm 0.004$ \\
\hline & na & $0.000002^{(e)} \pm 0.00008$ \\
\hline \multirow[t]{2}{*}{ Plutonium 239+240 } & na & $0.00003 \pm 0.00073$ \\
\hline & na & $0.00003^{(\mathrm{e})} \pm 0.00003$ \\
\hline Tritium & $4.9^{(f)} \pm 2.7$ & $6.8 \pm 2.6$ \\
\hline Uranium 238 & na & $0.037 \pm 0.005$ \\
\hline
\end{tabular}

Note: Radioactivities are reported as the measured concentration and either an uncertainty $( \pm 2 \sigma$ counting error) or as being less than or equal to the detection limit. If the concentration is less than or equal to the uncertainty or the detection limit, the result is considered to be a nondetection. See the main volume, Chapter 14, Quality Assurance.

a $n a=$ Not analyzed (analysis not required).

b Bicarbonate alkalinity = total alkalinity.

c Concentration is supect; it is much greater than nitrate concentrations previously measured in ground water samples collected from this well.

d $n d=$ None detected above reporting limits. See Table 9-1 for analytical methods and their constituents.

e Retest sample collected on 12/1/99 and analyzed by LLNL on-site laboratory. (Original sample collected on 10/26/99 was analyzed by off-site laboratory.)

f Concentration is approximate. 
Table 9-22. Livermore site surveillance well GSW-011.

\begin{tabular}{|c|c|}
\hline \multirow{2}{*}{$\begin{array}{l}\text { Constituents } \\
\text { of concern }\end{array}$} & Sampling date \\
\hline & $7 / 27 / 99$ \\
\hline \multicolumn{2}{|l|}{ Inorganic ( $\mu \mathrm{g} / \mathrm{L})$} \\
\hline Field temperature $\left({ }^{\circ} \mathrm{C}\right)$ & 21 \\
\hline Field specific conductance $(\mu \mathrm{mho} / \mathrm{cm})$ & 960 \\
\hline Field $\mathrm{pH}$ (pH units) & 7.3 \\
\hline Aluminum & $<100$ \\
\hline Antimony & $<5$ \\
\hline Arsenic & $<2$ \\
\hline Barium & 370 \\
\hline Beryllium & $<0.5$ \\
\hline Boron & 1100 \\
\hline Cadmium & $<0.5$ \\
\hline Chromium & $<1$ \\
\hline Cobalt & $<50$ \\
\hline Copper & $<2$ \\
\hline Chromium(VI) & $<2$ \\
\hline Iron & $<100$ \\
\hline Lead & $<5$ \\
\hline Manganese & 690 \\
\hline Mercury & $<0.2$ \\
\hline Molybdenum & $<25$ \\
\hline Nickel & 24 \\
\hline Selenium & $<2$ \\
\hline Silver & $<1$ \\
\hline Thallium & $<2$ \\
\hline Vanadium & $<20$ \\
\hline Zinc & $<20$ \\
\hline \multicolumn{2}{|l|}{ Radioactive (Bq/L) } \\
\hline Gross alpha & $0.25 \pm 0.09$ \\
\hline Gross beta & $0.13 \pm 0.05$ \\
\hline Americium-241 & $0.0002 \pm 0.0002$ \\
\hline Plutonium-238 & $-0.0001 \pm 0.0002$ \\
\hline Plutonium-239+240 & $0.0001 \pm 0.0002$ \\
\hline
\end{tabular}




\section{Ground Water Monitoring}

Table 9-22. Livermore site surveillance well GSW-011 (concluded).

\begin{tabular}{|l|c|}
\hline \multicolumn{1}{|c|}{$\begin{array}{c}\text { Constituents } \\
\text { of concern }\end{array}$} & Sampling date \\
\cline { 2 - 2 } Radioactive (Bq/L) (continued) & $\mathbf{7 / 2 7 / 9 9}$ \\
Radium-226 & $0.004 \pm 0.001$ \\
Radium-228 & $0.008 \pm 0.009$ \\
Thorium-228 & $0.001 \pm 0.001$ \\
Thorium-230 & $0.001 \pm 0.001$ \\
Thorium-232 & $-0.00004 \pm 0.0001$ \\
Tritium & $3.8 \pm 1.7$ \\
Uranium (total) & $0.24 \pm 0.03$ \\
\hline
\end{tabular}

Note: Radioactivities are reported as the measured concentration and either an uncertainty ( $\pm 2 \sigma$ counting error) or as being less than or equal to the detection limit. If the concentration is less than or equal to the uncertainty or the detection limit, the result is considered to be a nondetection. See the main volume, Chapter 14, Quality Assurance. 
Table 9-23. Livermore site surveillance well W-307.

\begin{tabular}{|c|c|c|}
\hline \multirow{2}{*}{$\begin{array}{l}\text { Constituents } \\
\text { of concern }\end{array}$} & \multicolumn{2}{|c|}{ Sampling dates } \\
\hline & 2/11/99 & $10 / 27 / 99$ \\
\hline \multicolumn{3}{|l|}{ Inorganic ( $\mu \mathrm{g} / \mathrm{L})$} \\
\hline Aluminum & $<50$ & $n a^{(a)}$ \\
\hline Antimony & $<4$ & na \\
\hline Arsenic & $<2$ & na \\
\hline Barium & 300 & na \\
\hline Beryllium & $<0.2$ & na \\
\hline Boron & 690 & na \\
\hline Cadmium & $<0.5$ & na \\
\hline Chromium & 13 & na \\
\hline Chromium(VI) & 12 & 13 \\
\hline Cobalt & $<50$ & na \\
\hline Copper & $<1$ & na \\
\hline Iron & $<50$ & na \\
\hline Lead & $<5$ & na \\
\hline Manganese & $<10$ & na \\
\hline Mercury & $<0.2$ & na \\
\hline Molybdenum & $<25$ & na \\
\hline Nickel & $<2$ & na \\
\hline Selenium & $<2$ & na \\
\hline Silver & $<2$ & na \\
\hline Thallium & $<1$ & na \\
\hline Vanadium & $<10$ & na \\
\hline Zinc & $<20$ & na \\
\hline
\end{tabular}

Note: Radioactivities are reported as the measured concentration and either an uncertainty ( $\pm 2 \sigma$ counting error) or as being less than or equal to the detection limit. If the concentration is less than or equal to the uncertainty or the detection limit, the result is considered to be a nondetection. See the main volume, Chapter 14, Quality Assurance.

a na $=$ Not analyzed (analysis not required). 
Table 9-24. Livermore site surveillance well W-226.

\begin{tabular}{|l|c|c|}
\hline \multirow{2}{*}{$\begin{array}{c}\text { Constituents } \\
\text { of concern }\end{array}$} & \multicolumn{2}{|c|}{ Sampling dates } \\
\cline { 2 - 3 } Inorganic ( $\boldsymbol{\mu g} / \mathrm{L}$ ) & $\mathbf{2 / 1 1 / 9 9}$ & $\mathbf{7 / 2 9 / 9 9}$ \\
Aluminum & & \\
Antimony & $<50$ & na(a) \\
Arsenic & $<4$ & na \\
Barium & $<2$ & na \\
Beryllium & 180 & na \\
Boron & $<0.2$ & na \\
Cadmium & 470 & na \\
Chromium & $<0.5$ & na \\
Chromium(VI) & 71 & 19 \\
Cobalt & 27 & 24 \\
Copper & $<50$ & na \\
Iron & 3.2 & na \\
Lead & $<50$ & na \\
Manganese & $<5$ & na \\
Mercury & $<10$ & na \\
Molybdenum & $<0.2$ & na \\
Nickel & $<25$ & na \\
Selenium & $<2$ & na \\
Silver & $<2$ & na \\
Thallium & $<2$ & na \\
Vanadium & $<2$ & na \\
Zinc & $<2$ & na \\
\hline
\end{tabular}

Note: Radioactivities are reported as the measured concentration and either an uncertainty ( $\pm 2 \sigma$ counting error) or as being less than or equal to the detection limit. If the concentration is less than or equal to the uncertainty or the detection limit, the result is considered to be a nondetection. See the main volume, Chapter 14, Quality Assurance.

a $\quad$ na $=$ Not analyzed (analysis not required). 
Table 9-25. Livermore site surveillance well W-306.

\begin{tabular}{|c|c|c|c|}
\hline \multirow{2}{*}{$\begin{array}{l}\text { Constituents } \\
\text { of concern }\end{array}$} & \multicolumn{3}{|c|}{ Sampling dates } \\
\hline & 2/11/99 & 7/29/99 & $7 / 29 / 99$ \\
\hline \multicolumn{4}{|l|}{ Inorganic ( $\mu \mathrm{g} / \mathrm{L})$} \\
\hline Aluminum & $<50$ & $n a^{(a)}$ & na \\
\hline Antimony & $<4$ & na & na \\
\hline Arsenic & $<2$ & na & na \\
\hline Barium & 92 & na & na \\
\hline Beryllium & $<0.2$ & na & na \\
\hline Boron & 1200 & na & na \\
\hline Cadmium & $<0.5$ & na & na \\
\hline Chromium & 37 & na & na \\
\hline Chromium(VI) & 25 & 33 & 13 \\
\hline Cobalt & $<50$ & 50 & na \\
\hline Copper & $<1$ & na & na \\
\hline Iron & $<50$ & na & na \\
\hline Lead & $<5$ & na & na \\
\hline Manganese & $<10$ & na & na \\
\hline Mercury & $<0.2$ & na & na \\
\hline Molybdenum & $<25$ & na & na \\
\hline Nickel & $<2$ & na & na \\
\hline Selenium & $<2$ & na & na \\
\hline Silver & $<2$ & na & na \\
\hline Thallium & $<1$ & na & na \\
\hline Vanadium & $<10$ & na & na \\
\hline Zinc & $<20$ & na & na \\
\hline
\end{tabular}

Note: Radioactivities are reported as the measured concentration and either an uncertainty ( $\pm 2 \sigma$ counting error) or as being less than or equal to the detection limit. If the concentration is less than or equal to the uncertainty or the detection limit, the result is considered to be a nondetection. See the main volume, Chapter 14, Quality Assurance.

a $n a=$ Not analyzed (analysis not required). 


\section{Ground Water Monitoring}

Table 9-26. Livermore site surveillance well W-305.

\begin{tabular}{|c|c|}
\hline \multirow{2}{*}{$\begin{array}{l}\text { Constituents } \\
\text { of concern }\end{array}$} & Sampling date \\
\hline & $11 / 3 / 99$ \\
\hline \multicolumn{2}{|l|}{ Inorganic $(\mu \mathrm{g} / \mathrm{L})$} \\
\hline $\mathrm{pH}$ (units) & 7.2 \\
\hline Field $\mathrm{pH}$ ( $\mathrm{pH}$ units) & 7.6 \\
\hline Specific conductance $(\mu \mathrm{mho} / \mathrm{cm})$ & 660 \\
\hline Field specific conductance $(\mu \mathrm{mho} / \mathrm{cm})$ & 690 \\
\hline Total dissolved solids (TDS, mg/L) & 400 \\
\hline Field temperature $\left({ }^{\circ} \mathrm{C}\right)$ & 20 \\
\hline Aluminum & $<100$ \\
\hline Antimony & $<5$ \\
\hline Arsenic & $<2$ \\
\hline Barium & 310 \\
\hline Beryllium & $<0.5$ \\
\hline Cadmium & $<0.5$ \\
\hline Chromium & 11 \\
\hline Cobalt & $<50$ \\
\hline Copper & $<2$ \\
\hline Chromium & 10 \\
\hline Iron & $<100$ \\
\hline Lead & $<5$ \\
\hline Manganese & $<30$ \\
\hline Mercury & $<0.2$ \\
\hline Molybdenum & $<25$ \\
\hline Nickel & $<5$ \\
\hline Selenium & $<2$ \\
\hline Silver & $<1$ \\
\hline Thallium & $<2$ \\
\hline Vanadium & $<20$ \\
\hline Zinc & $<20$ \\
\hline \multicolumn{2}{|l|}{ General minerals (mg/L) } \\
\hline Bicarbonate alkalinity $\left(\mathrm{as} \mathrm{CaCO}_{3}\right)^{(a)}$ & 280 \\
\hline Boron & 0.3 \\
\hline Calcium & 64 \\
\hline Chloride & 37 \\
\hline Fluoride & 0.26 \\
\hline
\end{tabular}




\section{Ground Water Monitoring}

Table 9-26. Livermore site surveillance well W-305 (concluded).

\begin{tabular}{|l|c|}
\multicolumn{1}{|c|}{$\begin{array}{c}\text { Constituents } \\
\text { of concern }\end{array}$} & Sampling date \\
\cline { 2 - 2 } General minerals (mg/L) (continued) & $\mathbf{1 1 / 3 / 9 9}$ \\
Magnesium & 20 \\
Nitrate & $<0.5$ \\
Orthophosphate & 0.02 \\
Potassium & 2 \\
Sodium & 50 \\
Sulfate & 11 \\
Surfactants & $<0.5$ \\
Total hardness (as CaCO $\left.{ }_{3}\right)$ & 240 \\
Total phosphorus & 0.05 \\
Organic constituents ( $\mathrm{Hg} / \mathrm{L})$ & \\
EPA Method 625 & $\mathrm{nd}(\mathrm{b})$ \\
Radioactive (Bq/L) & \\
Gross alpha & $0.17 \pm 0.03$ \\
Gross beta & $0.064 \pm 0.025$ \\
Plutonium-238 & $0.001 \pm 0.001$ \\
Plutonium-239+240 & $<.037$ \\
Tritium & 2.5 \\
Uranium (total) & 0.01 \\
\hline
\end{tabular}

Note: Radioactivities are reported as the measured concentration and either an uncertainty ( $\pm 2 \sigma$ counting error) or as being less than or equal to the detection limit. If the concentration is less than or equal to the uncertainty or the detection limit, the result is considered to be a nondetection. See the main volume, Chapter 14, Quality Assurance.

a Bicarbonate alkalinity = total alkalinity.

b $n d=$ None detected above reporting limits. See Table 9-1 for analytical methods and their constituents. 


\section{Ground Water Monitoring}

Table 9-27. Livermore site surveillance well SIP-331-001.

\begin{tabular}{|c|c|c|}
\hline \multirow{2}{*}{$\begin{array}{c}\text { Constituents } \\
\text { of concern }\end{array}$} & \multicolumn{2}{|c|}{ Sampling dates } \\
\hline & 6/23/99 & 10/21/99 \\
\hline \multicolumn{3}{|l|}{ Inorganic $(\mu \mathrm{g} / \mathrm{L})$} \\
\hline $\mathrm{pH}$ (pH units) & $n a^{(a)}$ & 7 \\
\hline Field $\mathrm{pH}$ (pH units) & na & 7.3 \\
\hline Specific conductance $(\mu \mathrm{mho} / \mathrm{cm})$ & na & 780 \\
\hline Field specific conductance $(\mu \mathrm{mho} / \mathrm{cm})$ & na & 880 \\
\hline Total dissolved solids (TDS) (mg/L) & na & 570 \\
\hline Water temperature $\left({ }^{\circ} \mathrm{C}\right)$ & na & 24 \\
\hline Aluminum & 970 & 1600 \\
\hline Antimony & 15 & $<5$ \\
\hline Arsenic & 7.4 & 9 \\
\hline Barium & 140 & 440 \\
\hline Beryllium & $<0.2$ & $<0.5$ \\
\hline Cadmium & $<0.5$ & $<0.5$ \\
\hline Chromium & 4.2 & 1 \\
\hline Cobalt & $<50$ & $<50$ \\
\hline Copper & 8 & $<2$ \\
\hline Chromium(VI) & $<2$ & $<2$ \\
\hline Iron & 2600 & 1600 \\
\hline Lead & $<5$ & $<5$ \\
\hline Manganese & 880 & 1500 \\
\hline Mercury & $<0.2$ & $<0.2$ \\
\hline Molybdenum & $<25$ & $<25$ \\
\hline Nickel & 3 & 6 \\
\hline Selenium & $<2$ & $<2$ \\
\hline Silver & $<1$ & $<1$ \\
\hline Thallium & $<1$ & $<2$ \\
\hline Vanadium & $<10$ & $<20$ \\
\hline Zinc & 53 & $<20$ \\
\hline \multicolumn{3}{|l|}{ General minerals (mg/L) } \\
\hline Bicarbonate alkalinity $\left(\text { as } \mathrm{CaCO}_{3}\right)^{(\mathrm{b})}$ & na & 460 \\
\hline Boron & 0.31 & 0.5 \\
\hline Calcium & na & 68 \\
\hline Chloride & na & 65 \\
\hline Fluoride & na & 0.6 \\
\hline
\end{tabular}




\section{Ground Water Monitoring}

Table 9-27. Livermore site surveillance well SIP-331-001 (concluded).

\begin{tabular}{|c|c|c|}
\hline \multirow{2}{*}{$\begin{array}{c}\text { Constituents } \\
\text { of concern }\end{array}$} & \multicolumn{2}{|c|}{ Sampling dates } \\
\hline & 6/23/99 & $10 / 21 / 99$ \\
\hline \multicolumn{3}{|c|}{ General minerals (mg/L) (continued) } \\
\hline Magnesium & na & 27 \\
\hline Nitrate & na & $<0.5$ \\
\hline Orthophosphate & na & 0.03 \\
\hline Potassium & na & 3 \\
\hline Sodium & na & 69 \\
\hline Sulfate & na & 8 \\
\hline Surfactants & na & $<0.5$ \\
\hline Total hardness (as $\mathrm{CaCO}_{3}$ ) & na & 280 \\
\hline Total phosphorus & na & 13 \\
\hline \multicolumn{3}{|l|}{ Organic $(\mu \mathrm{g} / \mathrm{L})$} \\
\hline EPA Method 625 & na & $\mathrm{nd}(\mathrm{c})$ \\
\hline Bis(2-ethylhexyl)phthalate & na & 29 \\
\hline Phenol & na & 10 \\
\hline \multicolumn{3}{|l|}{ Radioactive (Bq/L) } \\
\hline Gross alpha & na & $0.092 \pm 0.038$ \\
\hline Gross beta & na & $0.19 \pm 0.04$ \\
\hline Plutonium-238 & na & $0.001 \pm 0.003$ \\
\hline Plutonium-239+240 & na & $0.004 \pm 0.002$ \\
\hline Tritium & $11 \pm 2.5$ & $18 \pm 3.0$ \\
\hline Uranium (total) & na & $0.061 \pm 0.010$ \\
\hline
\end{tabular}

Note: Radioactivities are reported as the measured concentration and either an uncertainty $( \pm 2 \sigma$ counting error) or as being less than or equal to the detection limit. If the concentration is less than or equal to the uncertainty or the detection limit, the result is considered to be a nondetection. See the main volume, Chapter 14, Quality Assurance.

a $n a=$ Not analyzed (analysis not required).

b Bicarbonate alkalinity = total alkalinity.

c $n d=$ None detected above reporting limits. See Table 9-1 for analytical methods and their constituents. 


\section{Ground Water Monitoring}

Table 9-28. Livermore site surveillance well W-148.

\begin{tabular}{|c|c|c|}
\hline \multirow{2}{*}{$\begin{array}{l}\text { Constituents } \\
\text { of concern }\end{array}$} & \multicolumn{2}{|c|}{ Sampling dates } \\
\hline & $5 / 27 / 99$ & $10 / 21 / 99$ \\
\hline \multicolumn{3}{|l|}{ Inorganic $(\mu \mathrm{g} / \mathrm{L})$} \\
\hline $\mathrm{pH}$ (pH units) & $n a^{(a)}$ & 7.4 \\
\hline Field pH (units) & na & 7.8 \\
\hline Specific conductance $(\mu \mathrm{mho} / \mathrm{cm})$ & na & 460 \\
\hline Field specific conductance $(\mu \mathrm{mho} / \mathrm{cm})$ & na & 480 \\
\hline Total dissolved solids (TDS, mg/L) & na & 310 \\
\hline Field temperature $\left({ }^{\circ} \mathrm{C}\right)$ & na & 19 \\
\hline Aluminum & 1400 & $<100$ \\
\hline Antimony & $<4$ & $<5$ \\
\hline Arsenic & 3.5 & $<2$ \\
\hline Barium & 300 & 230 \\
\hline Beryllium & $<0.2$ & $<0.5$ \\
\hline Cadmium & $<0.5$ & $<0.5$ \\
\hline Chromium & 4.1 & $<1$ \\
\hline Cobalt & $<50$ & $<50$ \\
\hline Copper & 3.4 & $<2$ \\
\hline Chromium(VI) & $<2$ & $<2$ \\
\hline Iron & 1300 & $<100$ \\
\hline Lead & $<5$ & $<5$ \\
\hline Manganese & 180 & 240 \\
\hline Mercury & $<0.2$ & $<0.2$ \\
\hline Molybdenum & $<25$ & $<25$ \\
\hline Nickel & 6.5 & $<5$ \\
\hline Selenium & $<2$ & $<2$ \\
\hline Silver & $<1$ & $<1$ \\
\hline Thallium & $<1$ & $<2$ \\
\hline Vanadium & 18 & $<20$ \\
\hline Zinc & 35 & $<20$ \\
\hline \multicolumn{3}{|l|}{ General minerals (mg/L) } \\
\hline Bicarbonate alkalinity $\left(\text { as } \mathrm{CaCO}_{3}\right)^{(\mathrm{b})}$ & na & 180 \\
\hline Boron & 0.19 & $<0.1$ \\
\hline Calcium & na & 54 \\
\hline Chloride & na & 20 \\
\hline
\end{tabular}




\section{Ground Water Monitoring}

Table 9-28. Livermore site surveillance well W-148 (concluded).

\begin{tabular}{|c|c|c|}
\hline \multirow{2}{*}{$\begin{array}{c}\text { Constituents } \\
\text { of concern }\end{array}$} & \multicolumn{2}{|c|}{ Sampling dates } \\
\hline & $5 / 27 / 99$ & $10 / 21 / 99$ \\
\hline \multicolumn{3}{|c|}{ General minerals (mg/L) (continued) } \\
\hline Fluoride & na & 0.1 \\
\hline Magnesium & na & 16 \\
\hline Nitrate & na & 24 \\
\hline Orthophosphate & na & 0.06 \\
\hline Potassium & na & 2 \\
\hline Sodium & na & 21 \\
\hline Sulfate & na & 9 \\
\hline Surfactants & na & $<0.5$ \\
\hline Total hardness $\left(\right.$ as $\left.\mathrm{CaCO}_{3}\right)$ & na & 200 \\
\hline Total phosphorus & na & $<0.05$ \\
\hline \multicolumn{3}{|l|}{ Organic $(\mu \mathrm{g} / \mathrm{L})$} \\
\hline EPD Method 625 & na & $n d^{(c)}$ \\
\hline \multicolumn{3}{|l|}{ Radioactive (Bq/L) } \\
\hline Gross alpha & na & $0.15 \pm 0.05$ \\
\hline Gross beta & na & $0.11 \pm 0.04$ \\
\hline Plutonium-238 & na & $0.006^{(d)} \pm 0.002$ \\
\hline Plutonium-239+240 & na & $0.0002^{(\mathrm{e})} \pm 0.0004$ \\
\hline Tritium & $59 \pm 6.7$ & $46 \pm 3.8$ \\
\hline Uranium (total) & na & $0.10 \pm 0.01$ \\
\hline
\end{tabular}

Note: Radioactivities are reported as the measured concentration and either an uncertainty ( $\pm 2 \sigma$ counting error) or as being less than or equal to the detection limit. If the concentration is less than or equal to the uncertainty or the detection limit, the result is considered to be a nondetection. See the main volume, Chapter 14, Quality Assurance.

a $n a=$ Not analyzed (analysis not required).

b Bicarbonate alkalinity = total alkalinity.

c $\mathrm{nd}=$ None detected above reporting limits. See Table 9-1 for analytical methods and their constituents.

d Reanalysis result from off-site laboratory was $0.012 \pm 0.012$ for Pu-238.

e Reanalysis result from off-site laboratory was $-0.0011 \pm 0.0012 \mathrm{Pu}-239+240$. 
Table 9-29. Tritium activity in Livermore Valley wells, 1999.

\begin{tabular}{|c|c|c|}
\hline Location & Sampling date & Tritium activity (Bq/L) \\
\hline $11 \mathrm{~B} 1$ & $8 / 31$ & $8.5 \pm 2.4$ \\
$12 \mathrm{~A} 2$ & $8 / 31$ & $1.9 \pm 2.1$ \\
$12 \mathrm{D} 2$ & $8 / 31$ & $8.1 \pm 2.4$ \\
$12 \mathrm{G} 1$ & $8 / 31$ & $2.4 \pm 2.1$ \\
$16 \mathrm{~L} 5$ & $9 / 17$ & $0.8 \pm 2.2$ \\
$16 \mathrm{~L} 7$ & $9 / 17$ & $1.4 \pm 2.2$ \\
$17 \mathrm{D} 2$ & No sample & na(a) \\
18A1 & $6 / 24$ & $0.4 \pm 2.2$ \\
1H3 & $8 / 31$ & $0.2 \pm 2.1$ \\
1P2 & $8 / 31$ & $2.9 \pm 2.2$ \\
1R2 & $8 / 31$ & $1.3 \pm 2.1$ \\
2R1 & $8 / 31$ & $2.4 \pm 2.2$ \\
004 & $9 / 17$ & $0.2 \pm 2.1$ \\
9M2 & $6 / 24$ & $-0.01 \pm 2.2$ \\
9M3 & $6 / 24$ & $0.43 \pm 2.2$ \\
16B1 & No sample & na \\
7C2 & $8 / 31$ & $0.9 \pm 2.1$ \\
7P3 & $6 / 24$ & $0.5 \pm 2.1$ \\
8P1 & $6 / 24$ & $0.3 \pm 2.2$ \\
$9 \mathrm{Q} 1$ & $6 / 24$ & $1.6 \pm 2.2$ \\
\hline
\end{tabular}

a $n a=$ Not analyzed (analysis not required). 
Table 9-30. Site 300, Elk Ravine surveillance wells.

\begin{tabular}{|c|c|c|c|c|c|c|}
\hline \multirow{4}{*}{$\begin{array}{l}\text { Constituents } \\
\text { of concern }\end{array}$} & \multicolumn{6}{|c|}{ Well } \\
\hline & K7-07(a) & \multicolumn{2}{|c|}{ NC7-61 } & NC7-69(b) & \multicolumn{2}{|c|}{ K2-04D } \\
\hline & \multicolumn{6}{|c|}{ Sampling dates } \\
\hline & $5 / 26 / 99$ & $6 / 1 / 99$ & $11 / 9 / 99$ & $11 / 10 / 99$ & $5 / 26 / 99$ & $11 / 8 / 99$ \\
\hline \multicolumn{7}{|l|}{ Inorganic ( $\mu \mathrm{g} / \mathrm{L})$} \\
\hline Antimony & $<5$ & $<5$ & $<5$ & $<5$ & $<5$ & $<5$ \\
\hline Arsenic & 18 & 18 & 19 & $<2$ & 12 & 12 \\
\hline Barium & 81 & 97 & 94 & 28 & 39 & 39 \\
\hline Beryllium & $<0.5$ & $<0.5$ & $<0.5$ & $<0.5$ & $<0.5$ & $<0.5$ \\
\hline Cadmium & $<0.5$ & $<0.5$ & $<0.5$ & $<0.5$ & $<0.5$ & $<0.5$ \\
\hline Chromium & $<1$ & $<1$ & $<1$ & $<1$ & 2 & $<1$ \\
\hline Cobalt & $<25$ & $<25$ & $<25$ & $<25$ & $<25$ & $<25$ \\
\hline Copper & $<10$ & $<10$ & $<10$ & $<10$ & $<10$ & $<10$ \\
\hline Lead & $<2$ & $<2$ & $<2$ & $<2$ & 2 & $<2$ \\
\hline Mercury & $<0.2$ & $<0.2$ & $<0.2$ & $<0.2$ & $<0.2$ & $<0.2$ \\
\hline Molybdenum & $<25$ & $<25$ & $<25$ & $<25$ & $<25$ & $<25$ \\
\hline Nickel & $<5$ & $<5$ & $<5$ & $<5$ & $<5$ & $<5$ \\
\hline Potassium (mg/L) & 1.1 & 4.4 & 3.3 & 4.4 & 2.8 & 3 \\
\hline Selenium & $<2$ & $<2$ & $<2$ & $<2$ & 2 & $<2$ \\
\hline Silver & $<0.5$ & $<0.5$ & $<0.5$ & $<0.5$ & $<0.5$ & $<0.5$ \\
\hline Thallium & $<2$ & $<2$ & $<2$ & $<2$ & $<2$ & $<2$ \\
\hline Vanadium & 33 & 96 & 93 & $<25$ & 54 & 54 \\
\hline Zinc & $<20$ & $<20$ & $<20$ & $<20$ & $<20$ & $<20$ \\
\hline \multicolumn{7}{|l|}{ Organic $(\mu \mathrm{g} / \mathrm{L})$} \\
\hline $\begin{array}{l}\text { EPA Method } 601 \\
\text { (volatile) }\end{array}$ & $\mathrm{nd}^{(\mathrm{c})}$ & nd & nd & nd & nd & nd \\
\hline \multicolumn{7}{|l|}{ Explosives ( $\mu \mathrm{g} / \mathrm{L})$} \\
\hline HMX & $<1$ & 5 & 4 & $<1$ & $<1$ & $<1$ \\
\hline RDX & $<1$ & 7 & 6 & $<1$ & $<1$ & $<1$ \\
\hline \multicolumn{7}{|l|}{ Radioactive (Bq/L) } \\
\hline Gross alpha & $0.16 \pm 0.04$ & $0.07 \pm 0.03$ & $0.11 \pm 0.04$ & $0.13 \pm 0.04$ & $0.04 \pm 0.02$ & $0.06 \pm 0.03$ \\
\hline Gross beta & $0.17 \pm 0.04$ & $0.14 \pm 0.04$ & $0.16 \pm 0.04$ & $0.41 \pm 0.07$ & $0.13 \pm 0.03$ & $0.14 \pm 0.03$ \\
\hline Tritium & $260 \pm 7.7$ & $3900 \pm 28$ & $3600 \pm 27$ & $0.26 \pm 2.1$ & $140 \pm 5.7$ & $150 \pm 5.9$ \\
\hline Uranium (total) & $0.38 \pm 0.03$ & $0.14 \pm 0.01$ & $0.08 \pm 0.01$ & $0.002 \pm 0.001$ & $0.10 \pm 0.01$ & $0.07 \pm 0.01$ \\
\hline
\end{tabular}


Table 9-30. Site 300, Elk Ravine surveillance wells (continued).

\begin{tabular}{|c|c|c|c|c|c|c|}
\hline \multirow{4}{*}{$\begin{array}{l}\text { Constituents } \\
\text { of concern }\end{array}$} & \multicolumn{6}{|c|}{ Well } \\
\hline & \multicolumn{2}{|c|}{ K2-04S } & \multicolumn{2}{|c|}{$\mathrm{K} 2-01 \mathrm{C}$} & \multicolumn{2}{|c|}{ NC2-12D } \\
\hline & \multicolumn{6}{|c|}{ Sampling dates } \\
\hline & $6 / 2 / 99$ & $11 / 9 / 99$ & $5 / 26 / 99$ & $11 / 8 / 99$ & $5 / 24 / 99$ & $11 / 10 / 99$ \\
\hline \multicolumn{7}{|l|}{ Inorganic $(\mu \mathrm{g} / \mathrm{L})$} \\
\hline Antimony & $<5$ & $<5$ & $<5$ & $<5$ & $<5$ & $<5$ \\
\hline Arsenic & 15 & 18 & 8 & 7 & 12 & 10 \\
\hline Barium & 53 & 70 & 380 & 39 & $<25$ & $<25$ \\
\hline Beryllium & $<0.5$ & $<0.5$ & $<0.5$ & $<0.5$ & $<0.5$ & $<0.5$ \\
\hline Cadmium & $<0.5$ & $<0.5$ & $<0.5$ & $<0.5$ & $<0.5$ & $<0.5$ \\
\hline Chromium & $<1$ & $<1$ & 1 & $<1$ & 1 & $<1$ \\
\hline Cobalt & $<25$ & $<25$ & $<25$ & $<25$ & $<25$ & $<25$ \\
\hline Copper & $<10$ & $<10$ & $<10$ & 40 & $<10$ & $<10$ \\
\hline Lead & $<2$ & $<2$ & $<2$ & $<2$ & $<2$ & $<2$ \\
\hline Mercury & $<0.2$ & $<0.2$ & $<0.2$ & $<0.2$ & $<0.2$ & $<0.2$ \\
\hline Molybdenum & $<25$ & $<25$ & $<25$ & $<25$ & $<25$ & $<25$ \\
\hline Nickel & $<5$ & $<5$ & 5 & $<5$ & $<5$ & $<5$ \\
\hline Potassium (mg/L) & 2.7 & 2.7 & 3.5 & 3.4 & 3.4 & 3.7 \\
\hline Selenium & 3 & 2 & $<2$ & $<2$ & 2 & 3 \\
\hline Silver & $<0.5$ & $<0.5$ & $<0.5$ & $<0.5$ & $<0.5$ & $<0.5$ \\
\hline Thallium & $<2$ & $<2$ & $<2$ & $<2$ & $<2$ & $<2$ \\
\hline Vanadium & 67 & 71 & 50 & 48 & 48 & 48 \\
\hline Zinc & $<20$ & 20 & $<20$ & 200 & $<20$ & $<20$ \\
\hline \multicolumn{7}{|l|}{ Organic $(\mu \mathrm{g} / \mathrm{L})$} \\
\hline $\begin{array}{l}\text { EPA Method } 601 \\
\text { (volatile) }\end{array}$ & nd & nd & nd & nd & nd & nd \\
\hline \multicolumn{7}{|l|}{ Explosives ( $\mu \mathrm{g} / \mathrm{L})$} \\
\hline HMX & $<1$ & $<1$ & $<1$ & $<1$ & $<1$ & $<1$ \\
\hline $\mathrm{RDX}$ & $<1$ & $<1$ & $<1$ & $<1$ & $<1$ & $<1$ \\
\hline \multicolumn{7}{|l|}{ Radioactive (Bq/L) } \\
\hline Gross alpha & $<0.07 \pm 0.03$ & $0.06 \pm 0.03$ & $0.10 \pm 0.04$ & $0.11 \pm 0.04$ & $0.08 \pm 0.03$ & $0.03 \pm 0.02$ \\
\hline Gross beta & $<0.11 \pm 0.04$ & $0.11 \pm 0.04$ & $0.18 \pm 0.04$ & $0.18 \pm 0.04$ & $0.15 \pm 0.04$ & $0.07 \pm 0.03$ \\
\hline Tritium & $490 \pm 10$ & $700 \pm 12$ & $680 \pm 12$ & $710 \pm 12$ & $310 \pm 7.2$ & $350 \pm 8.6$ \\
\hline Uranium (total) & $0.11 \pm 0.01$ & $0.10 \pm 0.01$ & $0.21 \pm 0.02$ & $0.16 \pm 0.01$ & $0.13 \pm 0.01$ & $0.09 \pm .01$ \\
\hline
\end{tabular}




\section{Ground Water Monitoring}

Table 9-30. Site 300, Elk Ravine surveillance wells (concluded).

\begin{tabular}{|c|c|c|c|c|c|c|}
\hline \multirow{4}{*}{$\begin{array}{l}\text { Constituents } \\
\text { of concern }\end{array}$} & \multicolumn{6}{|c|}{ Well } \\
\hline & \multicolumn{2}{|c|}{ NC2-11D } & \multicolumn{2}{|c|}{ 812CRK (SPRING6) } & \multicolumn{2}{|c|}{ NC2-07 } \\
\hline & \multicolumn{6}{|c|}{ Sampling dates } \\
\hline & $5 / 25 / 99$ & $11 / 10 / 99$ & $5 / 24 / 99$ & $11 / 9 / 99$ & $6 / 3 / 99$ & $11 / 15 / 99$ \\
\hline \multicolumn{7}{|l|}{ Inorganic $(\mu \mathrm{g} / \mathrm{L})$} \\
\hline Antimony & $<5$ & $<5$ & $<5$ & $<5$ & $<5$ & $<5$ \\
\hline Arsenic & 12 & 11 & 24 & 30 & 34 & 34 \\
\hline Barium & $<25$ & $<25$ & 78 & 56 & 34 & 35 \\
\hline Beryllium & $<0.5$ & $<0.5$ & $<0.5$ & $<0.5$ & $<0.5$ & $<0.5$ \\
\hline Cadmium & $<0.5$ & $<0.5$ & $<0.5$ & $<0.5$ & $<0.5$ & $<0.5$ \\
\hline Chromium & $<1$ & $<1$ & 1 & $<1$ & $<1$ & $<1$ \\
\hline Cobalt & $<25$ & $<25$ & $<25$ & $<25$ & $<25$ & $<25$ \\
\hline Copper & $<10$ & $<10$ & $<10$ & $<10$ & $<10$ & $<10$ \\
\hline Lead & $<2$ & $<2$ & $<2$ & $<2$ & $<2$ & $<2$ \\
\hline Mercury & $<0.2$ & $<0.2$ & $<0.2$ & $<0.2$ & $<0.2$ & $<0.2$ \\
\hline Molybdenum & $<25$ & $<25$ & $<25$ & $<25$ & $<25$ & $<25$ \\
\hline Nickel & $<5$ & $<5$ & $<5$ & $<5$ & $<5$ & $<5$ \\
\hline Potassium (mg/L) & 4.5 & 4.1 & 6.7 & 4.8 & 4 & 4.6 \\
\hline Selenium & $<2$ & 2 & 2 & 4 & 3 & 2 \\
\hline Silver & $<0.5$ & $<0.5$ & $<0.5$ & $<0.5$ & $<0.5$ & $<0.5$ \\
\hline Thallium & $<2$ & $<2$ & $<2$ & $<2$ & $<2$ & $<2$ \\
\hline Vanadium & 52 & 51 & 40 & 67 & 48 & 49 \\
\hline Zinc & $<20$ & $<20$ & $<20$ & $<20$ & $<20$ & $<20$ \\
\hline \multicolumn{7}{|l|}{ Organic ( $\mu \mathrm{g} / \mathrm{L})$} \\
\hline $\begin{array}{l}\text { EPA Method } 601 \\
\text { (volatile) }\end{array}$ & nd & nd & nd & nd & nd & nd \\
\hline \multicolumn{7}{|l|}{ Explosives ( $\mu \mathrm{g} / \mathrm{L})$} \\
\hline HMX & $<1$ & $<1$ & $<1$ & $<1$ & $<1$ & $<1$ \\
\hline $\mathrm{RDX}$ & $<1$ & $<1$ & $<1$ & $<1$ & $<1$ & $<1$ \\
\hline \multicolumn{7}{|l|}{ Radioactive (Bq/L) } \\
\hline Gross alpha & $0.07 \pm 0.03$ & $0.07 \pm 0.03$ & $0.10 \pm 0.04$ & $0.10 \pm 0.04$ & $0.14 \pm 0.05$ & $0.15 \pm 0.05$ \\
\hline Gross beta & $0.20 \pm 0.04$ & $0.14 \pm 0.03$ & $0.25 \pm 0.05$ & $0.25 \pm 0.06$ & $0.23 \pm 0.05$ & $0.24 \pm 0.06$ \\
\hline Tritium & $120 \pm 5.5$ & $140 \pm 5.6$ & $0.65 \pm 1.40$ & $0.93 \pm 2.1$ & $-1.2 \pm 2.1$ & $0.27 \pm 2.1$ \\
\hline Uranium (total) & $0.20 \pm 0.02$ & $0.15 \pm 0.01$ & $0.21 \pm 0.02$ & $0.15 \pm 0.01$ & $0.28 \pm 0.02$ & $0.22 \pm 0.02$ \\
\hline
\end{tabular}

Note: Radioactivities are reported as the measured concentration and either an uncertainty ( $\pm 2 \sigma$ counting error) or as being less than or equal to the detection limit. If the concentration is less than or equal to the uncertainty or the detection limit, the result is considered to be a nondetection. See the main volume, Chapter 14, Quality Assurance.

a Well was dry during fourth quarter.

b Well was dry during fourth quarter.

c $\mathrm{nd}=$ None detected above reporting limits. See Table 9-1 for analytical methods and their constituents.. 
Table 9-31. Site 300, Pit 2 surveillance wells. (a)

\begin{tabular}{|c|c|c|c|c|}
\hline \multirow{4}{*}{$\begin{array}{l}\text { Constituents } \\
\text { of concern }\end{array}$} & \multicolumn{4}{|c|}{ Barcad } \\
\hline & \multicolumn{2}{|c|}{ K1-02A } & \multicolumn{2}{|c|}{ K2-01A } \\
\hline & \multicolumn{4}{|c|}{ Sampling dates } \\
\hline & $5 / 21 / 99$ & $11 / 2 / 99$ & $5 / 21 / 99$ & 11/2/99 \\
\hline \multicolumn{5}{|l|}{ Inorganic $(\mu \mathrm{g} / \mathrm{L})$} \\
\hline Antimony & $<5$ & $<5$ & $<5$ & $<5$ \\
\hline Arsenic & 14 & 14 & $<2$ & $<2$ \\
\hline Barium & 40 & 43 & 25 & 27 \\
\hline Beryllium & $<0.5$ & $<0.5$ & $<0.5$ & $<0.5$ \\
\hline Cadmium & $<0.5$ & $<0.5$ & $<0.5$ & $<0.5$ \\
\hline Chromium & $<1$ & $<1$ & $<1$ & $<1$ \\
\hline Cobalt & $<25$ & $<25$ & $<25$ & $<25$ \\
\hline Copper & $<10$ & $<10$ & $<10$ & $<10$ \\
\hline Lead & $<2$ & $<2$ & $<2$ & $<2$ \\
\hline Mercury & $<0.2$ & $<0.2$ & $<0.2$ & $<0.2$ \\
\hline Molybdenum & $<25$ & $<25$ & $<25$ & $<25$ \\
\hline Nickel & $<5$ & $<5$ & $<5$ & $<5$ \\
\hline Potassium (mg/L) & 3 & 3.3 & 3.7 & 3.8 \\
\hline Selenium & $<2$ & $<2$ & $<2$ & $<2$ \\
\hline Silver & $<0.5$ & $<0.5$ & $<0.5$ & $<0.5$ \\
\hline Thallium & $<2$ & $<2$ & $<2$ & $<2$ \\
\hline Vanadium & $<25$ & $<25$ & $<25$ & $<25$ \\
\hline Zinc & $<20$ & $<20$ & $<20$ & $<20$ \\
\hline \multicolumn{5}{|l|}{ Explosive ( $\mu \mathrm{g} / \mathrm{L})$} \\
\hline HMX & $<1$ & $<1$ & $<1$ & $<1$ \\
\hline $\mathrm{RDX}$ & $<1$ & $<1$ & $<1$ & $<1$ \\
\hline \multicolumn{5}{|l|}{ Radioactive (Bq/L) } \\
\hline Gross alpha & $0.064 \pm 0.027$ & $0.022 \pm 0.016$ & $0.007 \pm 0.022$ & $0.003 \pm 0.016$ \\
\hline Gross beta & $0.13 \pm 0.04$ & $0.077 \pm 0.028$ & $0.14 \pm 0.04$ & $0.11 \pm 0.03$ \\
\hline Tritium & $0.66 \pm 1.5$ & $-0.53 \pm 2.2$ & $-0.39 \pm 1.3$ & $-2.4 \pm 2.1$ \\
\hline Uranium (total) & $0.092 \pm 0.009$ & $0.033 \pm 0.004$ & $0.004 \pm 0.001$ & $0.007 \pm 0.001$ \\
\hline
\end{tabular}




\section{Ground Water Monitoring}

Table 9-31. Site 300, Pit 2 surveillance wells (concluded).

\begin{tabular}{|c|c|c|c|c|}
\hline \multirow{4}{*}{$\begin{array}{l}\text { Constituents } \\
\text { of concern }\end{array}$} & \multicolumn{4}{|c|}{ Barcad } \\
\hline & \multicolumn{2}{|c|}{ K2-02A } & \multicolumn{2}{|c|}{ K2-02B } \\
\hline & \multicolumn{4}{|c|}{ Sampling dates } \\
\hline & $6 / 22 / 99$ & $12 / 16 / 99$ & $6 / 2 / 99$ & $12 / 16 / 99$ \\
\hline \multicolumn{5}{|l|}{ Inorganic ( $\mu \mathrm{g} / \mathrm{L})$} \\
\hline Antimony & $<5$ & $<5$ & $<5$ & $<5$ \\
\hline Arsenic & 42 & 33 & $<2$ & $<2$ \\
\hline Barium & 27 & 27 & 28 & 27 \\
\hline Beryllium & $<0.5$ & $<0.5$ & $<0.5$ & $<0.5$ \\
\hline Cadmium & $<0.5$ & $<0.5$ & $<0.5$ & $<0.5$ \\
\hline Chromium & $<1$ & $<1$ & $<1$ & $<1$ \\
\hline Cobalt & $<25$ & $<25$ & $<25$ & $<25$ \\
\hline Copper & $<10$ & $<10$ & $<10$ & $<10$ \\
\hline Lead & $<2$ & $<2$ & $<2$ & $<2$ \\
\hline Mercury & $<0.2$ & 0.3 & $<0.2$ & $<0.2$ \\
\hline Molybdenum & $<25$ & $<25$ & $<25$ & $<25$ \\
\hline Nickel & $<5$ & $<5$ & $<5$ & $<5$ \\
\hline Potassium (mg/L) & 4 & 3.8 & 3.5 & 3.7 \\
\hline Selenium & $<2$ & $<2$ & $<2$ & $<2$ \\
\hline Silver & $<0.5$ & $<0.5$ & $<0.5$ & $<0.5$ \\
\hline Thallium & $<2$ & $<2$ & $<2$ & $<2$ \\
\hline Vanadium & $<25$ & $<25$ & $<25$ & $<25$ \\
\hline Zinc & $<20$ & $<20$ & $<20$ & $<20$ \\
\hline \multicolumn{5}{|l|}{ Explosive $(\mu \mathrm{g} / \mathrm{L})$} \\
\hline HMX & $<1$ & $<1$ & $<1$ & $<1$ \\
\hline RDX & $<1$ & $<1$ & $<1$ & $<1$ \\
\hline \multicolumn{5}{|l|}{ Radioactive (Bq/L) } \\
\hline Gross alpha & $0.13 \pm 0.03$ & $0.037 \pm 0.019$ & $0.022 \pm 0.019$ & $-0.002 \pm 0.013$ \\
\hline Gross beta & $0.15 \pm 0.03$ & $0.088 \pm 0.031$ & $0.13 \pm 0.03$ & $0.083 \pm 0.028$ \\
\hline Tritium & $-0.61 \pm 1.4$ & $-1.9 \pm 2.2$ & $-0.57 \pm 1.4$ & $-1.2 \pm 2.2$ \\
\hline Uranium (total) & $0.12 \pm 0.007$ & $0.081 \pm 0.009$ & $0.001 \pm 0.001$ & $0.001 \pm 0.001$ \\
\hline
\end{tabular}

Note: Radioactivities are reported as the measured concentration and either an uncertainty $( \pm 2 \sigma$ counting error) or as being less than or equal to the detection limit. If the concentration is less than or equal to the uncertainty or the detection limit, the result is considered to be a nondetection. See the main volume, Chapter 14, Quality Assurance.

a Other barcads were inoperative during 1999. 


\section{Ground Water Monitoring}

Table 9-32. Site 300, Pit 8 surveillance wells. (a)

\begin{tabular}{|c|c|c|c|c|}
\hline \multirow{4}{*}{$\begin{array}{c}\text { Constituents } \\
\text { of concern }\end{array}$} & \multicolumn{4}{|c|}{ Wells } \\
\hline & \multicolumn{2}{|c|}{ K8-01 } & \multicolumn{2}{|c|}{ K8-02B } \\
\hline & \multicolumn{4}{|c|}{ Sampling dates } \\
\hline & $6 / 4 / 99$ & $11 / 4 / 99$ & $6 / 4 / 99$ & $11 / 4 / 99$ \\
\hline \multicolumn{5}{|l|}{ Inorganic ( $\mu \mathrm{g} / \mathrm{L})$} \\
\hline Antimony & $<5$ & $<5$ & $<5$ & $<5$ \\
\hline Arsenic & 19 & 18 & 25 & 25 \\
\hline Barium & $<25$ & $<25$ & $<25$ & $<25$ \\
\hline Beryllium & $<0.5$ & $<0.5$ & $<0.5$ & $<0.5$ \\
\hline Cadmium & $<0.5$ & $<0.5$ & $<0.5$ & $<0.5$ \\
\hline Chromium & 13 & 17 & $<1$ & 1 \\
\hline Cobalt & $<25$ & $<25$ & $<25$ & $<25$ \\
\hline Copper & $<10$ & $<10$ & $<10$ & $<10$ \\
\hline Lead & $<2$ & $<2$ & $<2$ & $<2$ \\
\hline Mercury & $<0.2$ & $<0.2$ & $<0.2$ & $<0.2$ \\
\hline Molybdenum & $<25$ & $<25$ & $<25$ & $<25$ \\
\hline Nickel & $<5$ & $<5$ & $<5$ & $<5$ \\
\hline Selenium & 4 & 4 & 7 & 6 \\
\hline Silver & 1 & $<0.5$ & $<0.5$ & $<0.5$ \\
\hline Thallium & $<2$ & $<2$ & $<2$ & $<2$ \\
\hline Vanadium & 75 & 74 & 70 & 71 \\
\hline Zinc & $<20$ & $<20$ & $<20$ & $<20$ \\
\hline Potassium (mg/L) & 4.6 & 3.9 & 4.2 & 4.5 \\
\hline \multicolumn{5}{|l|}{ Organics ( $\mu \mathrm{g} / \mathrm{L})$} \\
\hline EPA Method 601 & $\mathrm{nd}(\mathrm{b})$ & nd & nd & nd \\
\hline 1,2-dichloroethane & 2.3 & 1.6 & $<0.5$ & $<0.5$ \\
\hline Trichloreoethene & 3.9 & 3.7 & 1.4 & 1.6 \\
\hline EPA Method 608 & $\mathrm{na}^{(\mathrm{c})}$ & nd & na & nd \\
\hline \multicolumn{5}{|l|}{ Explosive ( $\mu \mathrm{g} / \mathrm{L})$} \\
\hline $\mathrm{HMX}$ & $<1$ & na & $<1$ & $<1$ \\
\hline RDX & $<1$ & na & $<1$ & $<1$ \\
\hline \multicolumn{5}{|l|}{ Radioactivity (Bq/L) } \\
\hline Gross alpha & $0.19 \pm 0.06$ & $0.21 \pm 0.06$ & $0.25 \pm 0.06$ & $0.26 \pm 0.07$ \\
\hline Gross beta & $0.28 \pm 0.05$ & $0.22 \pm 0.04$ & $0.27 \pm 0.05$ & $0.29 \pm 0.06$ \\
\hline Tritium & $1.39 \pm 1.6$ & $3.3 \pm 2.1$ & $-1.48 \pm 1.5$ & $-0.28 \pm 2.1$ \\
\hline Uranium (total) & $0.32 \pm 0.03$ & $0.24 \pm 0.02$ & $0.41 \pm 0.03$ & $0.29 \pm 0.02$ \\
\hline
\end{tabular}

a Well K8-03B was inaccessible during 1999 because of construction activities there.

b $n d=$ None detected above reporting limits. See Table 9-1 for analytical methods and their constituents.

c $n a=$ Not analyzed (analysis not required). 
Table 9-33. Site 300, Pit 9 surveillance wells.

\begin{tabular}{|c|c|c|c|c|}
\hline \multirow{4}{*}{$\begin{array}{l}\text { Constituents } \\
\text { of concern }\end{array}$} & \multicolumn{4}{|c|}{ Well } \\
\hline & K9-01 & K9-02 & K9-03 & K9-04 \\
\hline & \multicolumn{4}{|c|}{ Sampling dates } \\
\hline & 9/28/99 & 9/27/99 & 9/27/99 & 9/28/99 \\
\hline \multicolumn{5}{|l|}{ Inorganic $(\mu \mathrm{g} / \mathrm{L})$} \\
\hline Antimony & $<5$ & $<5$ & $<5$ & $<5$ \\
\hline Arsenic & 4 & 35 & 13 & $<2$ \\
\hline Barium & $<25$ & $<25$ & $<25$ & $<25$ \\
\hline Beryllium & $<0.5$ & $<0.5$ & $<0.5$ & $<0.5$ \\
\hline Cadmium & $<0.5$ & $<0.5$ & $<0.5$ & $<0.5$ \\
\hline Chromium & $<1$ & $<1$ & $<1$ & 1 \\
\hline Cobalt & $<25$ & $<25$ & $<25$ & $<25$ \\
\hline Copper & $<10$ & $<10$ & $<10$ & $<10$ \\
\hline Lead & $<2$ & $<2$ & $<2$ & $<2$ \\
\hline Mercury & $<0.2$ & $<0.2$ & $<0.2$ & $<0.2$ \\
\hline Molybdenum & 26 & 53 & 29 & 32 \\
\hline Nickel & $<5$ & $<5$ & $<5$ & $<5$ \\
\hline Potassium (mg/L) & 7.6 & 8 & 8.4 & 10 \\
\hline Selenium & $<2$ & $<2$ & $<2$ & $<2$ \\
\hline Silver & $<0.5$ & $<0.5$ & $<0.5$ & $<0.5$ \\
\hline Thallium & $<2$ & $<2$ & $<2$ & $<2$ \\
\hline Vanadium & $<25$ & $<25$ & $<25$ & $<25$ \\
\hline Zinc & $<20$ & $<20$ & $<20$ & 20 \\
\hline \multicolumn{5}{|l|}{ Organic } \\
\hline EPA Method 601 (volatile) & $n d^{(a)}$ & nd & nd & nd \\
\hline EPA Method 625 (volatile) & nd & nd & nd & nd \\
\hline \multicolumn{5}{|l|}{ Explosive ( $\mu \mathrm{g} / \mathrm{L})$} \\
\hline HMX & $<1$ & $<1$ & $<1$ & $<1$ \\
\hline RDX & $<1$ & $<1$ & $<1$ & $<1$ \\
\hline \multicolumn{5}{|l|}{ Radioactive (Bq/L) } \\
\hline Gross alpha & $-0.040 \pm 0.070$ & $0.002 \pm 0.052$ & $-0.050 \pm 0.048$ & $0.029 \pm 0.044$ \\
\hline Gross beta & $0.29 \pm 0.06$ & $0.27 \pm 0.07$ & $0.28 \pm 0.06$ & $0.24 \pm 0.06$ \\
\hline Tritium & $-1.6 \pm 2.6$ & $-0.64 \pm 2.6$ & $-1.8 \pm 2.6$ & $-2.0 \pm 2.6$ \\
\hline Uranium (total) & $0.003 \pm 0.001$ & $0.013 \pm 0.002$ & $0.013 \pm 0.003$ & $0.017 \pm 0.003$ \\
\hline
\end{tabular}

Note: Radioactivities are reported as the measured concentration and either an uncertainty $( \pm 2 \sigma$ counting error) or as being less than or equal to the detection limit. If the concentration is less than or equal to the uncertainty or the detection limit, the result is considered to be a nondetection. See the main volume, Chapter 14, Quality Assurance.

a $n d=$ None detected above reporting limits. See Table 9-1 for analytical methods and their constituents. 
Table 9-34. 1999 analytical results for Site 300 Building 829 area deep monitoring wells. (a)

\begin{tabular}{|c|c|c|c|c|}
\hline \multirow{2}{*}{$\begin{array}{l}\text { Constituents } \\
\text { of concern }\end{array}$} & \multicolumn{4}{|c|}{ Sampling dates for W-827-05 } \\
\hline & $3 / 11 / 99$ & 6/14/99 & 9/2/99 & $11 / 22-23 / 99$ \\
\hline \multicolumn{5}{|l|}{ Inorganic ( $\mu \mathrm{g} / \mathrm{L})$} \\
\hline $\mathrm{pH}$ (pH units) & $n a^{(b)}$ & na & na & na \\
\hline Field pH & 7.5 & 7.2 & 7.0 & 8.2 \\
\hline Specific conductance $(\mu \mathrm{mho} / \mathrm{cm})$ & na & na & na & na \\
\hline Field specific conductance $(\mu \mathrm{mho} / \mathrm{cm})$ & 1800 & 1900 & 1800 & 1100 \\
\hline Total dissolved solids (TDS) & na & na & na & na \\
\hline Field temperature $\left({ }^{\circ} \mathrm{C}\right)$ & 22 & 23 & 23 & 21 \\
\hline Aluminum & na & na & na & na \\
\hline Antimony & $<5$ & $<5$ & $<5$ & $<5$ \\
\hline Arsenic & $<2$ & $<2$ & $<4$ & $<2$ \\
\hline Barium & $<25$ & $<25$ & $<25$ & $<25$ \\
\hline Beryllium & $<0.5$ & $<0.5$ & $<0.5$ & $<0.5$ \\
\hline Cadmium & $<0.5$ & $<0.5$ & $<0.5$ & $<0.5$ \\
\hline Chromium & 1.4 & 1.5 & 1.4 & $<1$ \\
\hline Cobalt & $<25$ & $<25$ & $<25$ & $<25$ \\
\hline Copper & $<10$ & $<10$ & $<10$ & $<10$ \\
\hline Iron & $<50$ & 58 & $<50$ & $<50$ \\
\hline Lead & $<2$ & $<2$ & $<2$ & $<2$ \\
\hline Manganese & 250 & 230 & 240 & 200 \\
\hline Mercury & $<0.2$ & $<0.2$ & $<0.2$ & $<0.2$ \\
\hline Molybdenum & $<25$ & $<25$ & $<25$ & $<25$ \\
\hline Nickel & $<5$ & $<5$ & $<5$ & $<5$ \\
\hline Selenium & $<2$ & $<2$ & $<4$ & $<2$ \\
\hline Silver & $<0.5$ & $<1$ & $<0.5$ & $<0.5$ \\
\hline Thallium & $<1$ & $<1$ & $<1$ & $<1$ \\
\hline Vanadium & $<25$ & $<25$ & $<25$ & $<25$ \\
\hline Zinc & $<50$ & na & $<20$ & $<20$ \\
\hline \multicolumn{5}{|l|}{ General Minerals (mg/L) } \\
\hline Bicarbonate alkalinity $\left(\mathrm{as} \mathrm{CaCO}_{3}\right)^{(\mathrm{c})}$ & na & na & na & na \\
\hline Bromide & 0.57 & 0.78 & 0.82 & 0.7 \\
\hline Calcium & na & na & na & na \\
\hline
\end{tabular}


Table 9-34. 1999 analytical results for Site 300 Building 829 area deep monitoring wells (continued).

\begin{tabular}{|c|c|c|c|c|}
\hline \multirow{2}{*}{$\begin{array}{l}\text { Constituents } \\
\text { of concern }\end{array}$} & \multicolumn{4}{|c|}{ Sampling dates for W-827-05 } \\
\hline & $3 / 11 / 99$ & $6 / 14 / 99$ & 9/2/99 & 11/22-23/99 \\
\hline \multicolumn{5}{|l|}{ General minerals (mg/L) (continued) } \\
\hline Chloride & 180 & 170 & 170 & 170 \\
\hline Fluoride & 0.22 & 0.2 & 0.21 & 0.2 \\
\hline Magnesium & na & na & na & na \\
\hline Nitrate & $<0.4$ & $<0.4$ & $<0.4$ & $<0.4$ \\
\hline Orthophosphate & 0.1 & $<0.05$ & 0.09 & 0.09 \\
\hline Potassium & na & na & na & 23 \\
\hline Sodium & 260 & 250 & 260 & 250 \\
\hline Sulfate & 630 & 610 & 620 & 590 \\
\hline Surfactants & na & na & na & na \\
\hline Perchlorate & na & $<4$ & na & $<4$ \\
\hline Total hardness $\left(\mathrm{as} \mathrm{CaCO}_{3}\right)$ & na & na & na & na \\
\hline Total phosphorus & na & na & na & na \\
\hline \multicolumn{5}{|l|}{ Organic $(\mu \mathrm{g} / \mathrm{L})$} \\
\hline EPA Method 624 & $\mathrm{nd}^{(\mathrm{d})}$ & nd & nd & nd \\
\hline EPA Method 625 & nd & nd & nd & nd \\
\hline EPA Method 608 & nd & nd & nd & nd \\
\hline Total organic halides (TOX, $\mu \mathrm{g} / \mathrm{L}$ ) & $<20$ & $<20$ & $<20$ & 55 \\
\hline Total organic carbon (TOC, mg/L) & 1.5 & 1.7 & $<1$ & $<1$ \\
\hline Fecal coliform (MPN(e)/100 mL) & $<1.1$ & na & $<2$ & $<2$ \\
\hline Total coliform (MPN/100 mL) & $<1.1$ & $<2$ & $<2$ & $<2$ \\
\hline \multicolumn{5}{|l|}{ Explosive compounds ( $\mu \mathrm{g} / \mathrm{L})$} \\
\hline HMX & $<1$ & $<5$ & $<5$ & $<5$ \\
\hline RDX & $<0.8$ & $<5$ & $<5$ & $<5$ \\
\hline TNT & na & $<5$ & $<5$ & $<5$ \\
\hline 1,3,5-trinitrobenzene & $<0.3$ & na & na & na \\
\hline \multicolumn{5}{|l|}{ Radioactive (Bq/L) } \\
\hline Gross alpha & $0.043 \pm 0.033$ & $-0.001 \pm 0.025$ & $-0.011 \pm 0.017$ & $-0.025 \pm 0.048$ \\
\hline Gross beta & $0.310 \pm 0.042$ & $0.28 \pm 0.06$ & $0.22 \pm 0.05$ & $0.81 \pm 0.13$ \\
\hline Radium 226 & $0.009 \pm 0.004$ & $0.002 \pm 0.003$ & $0.002 \pm 0.003$ & $0.003 \pm 0.004$ \\
\hline Radium 228 & $0.009 \pm 0.010$ & na & na & na \\
\hline Tritium & $0.37 \pm 1.9$ & $1.2 \pm 2.6$ & $-0.36 \pm 2.04$ & $-0.3 \pm 2.1$ \\
\hline Uranium (total) & $0.004 \pm 0.001$ & $0.003 \pm 0.001$ & $0.005 \pm 0.001$ & $0.003 \pm 0.001$ \\
\hline
\end{tabular}


Table 9-34. 1999 analytical results for Site 300 Building 829 area deep monitoring wells (continued).

\begin{tabular}{|c|c|c|c|c|}
\hline \multirow{2}{*}{$\begin{array}{l}\text { Constituents } \\
\text { of concern }\end{array}$} & \multicolumn{4}{|c|}{ Sampling dates for $\mathrm{W}-829-15$} \\
\hline & $3 / 17-23 / 99$ & 6/10/99 & 9/1/99 & $11 / 29 / 99$ \\
\hline \multicolumn{5}{|l|}{ Inorganic ( $\mu \mathrm{g} / \mathrm{L})$} \\
\hline $\mathrm{pH}$ (pH units) & na & 9.9 & 10 & na \\
\hline Field pH & 9.8 & 9.9 & 9.7 & 9.9 \\
\hline Specific conductance $(\mu \mathrm{mho} / \mathrm{cm})$ & na & 1300 & 1300 & na \\
\hline Field specific conductance $(\mu \mathrm{mho} / \mathrm{cm})$ & 1500 & 1100 & 1200 & 1300 \\
\hline Total dissolved solids (TDS) & na & 850 & 850 & na \\
\hline Field temperature $\left({ }^{\circ} \mathrm{C}\right)$ & 23 & 20 & 22 & 21 \\
\hline Aluminum & na & 57 & $<50$ & na \\
\hline Antimony & $<10$ & $<5$ & $<5$ & $<5$ \\
\hline Arsenic & 13 & 13 & 11 & 12 \\
\hline Barium & $<25$ & $<25$ & $<25$ & $<25$ \\
\hline Beryllium & $<0.5$ & $<0.5$ & $<0.5$ & $<0.5$ \\
\hline Cadmium & $<0.5$ & $<0.5$ & $<0.5$ & $<0.5$ \\
\hline Chromium & $<1$ & 1.4 & $<1$ & $<1$ \\
\hline Cobalt & $<25$ & $<25$ & $<25$ & $<25$ \\
\hline Copper & $<10$ & $<10$ & $<10$ & $<10$ \\
\hline Iron & $<50$ & $<50$ & $<50$ & $<50$ \\
\hline Lead & $<2$ & $<2$ & $<2$ & $<5$ \\
\hline Manganese & $<10$ & $<10$ & $<10$ & $<10$ \\
\hline Mercury & $<0.2$ & $<0.2$ & $<0.2$ & $<0.2$ \\
\hline Molybdenum & $<25$ & $<25$ & $<25$ & $<25$ \\
\hline Nickel & $<5$ & $<5$ & $<5$ & $<5$ \\
\hline Selenium & $<2$ & $<2$ & $<4$ & $<2$ \\
\hline Silver & $<0.5$ & $<0.5$ & $<0.5$ & $<0.5$ \\
\hline Thallium & $<1$ & $<1$ & $<1$ & $<1$ \\
\hline Vanadium & $<25$ & $<25$ & $<25$ & $<25$ \\
\hline Zinc & na & $<10$ & $<10$ & $<20$ \\
\hline \multicolumn{5}{|l|}{ General minerals (mg/L) } \\
\hline Bicarbonate alkalinity $\left(\text { as } \mathrm{CaCO}_{3}\right)^{(\mathrm{c})}$ & na & 300 & 260 & na \\
\hline Bromide & 0.33 & 0.4 & 0.34 & 0.35 \\
\hline Calcium & na & 3.1 & 4.3 & na \\
\hline
\end{tabular}


Table 9-34. 1999 analytical results for Site 300 Building 829 area deep monitoring wells (continued).

\begin{tabular}{|c|c|c|c|c|}
\hline \multirow{2}{*}{$\begin{array}{l}\text { Constituents } \\
\text { of concern }\end{array}$} & \multicolumn{4}{|c|}{ Sampling dates for W-829-15 } \\
\hline & $3 / 17-23 / 99$ & 6/10/99 & 9/1/99 & $11 / 29 / 99$ \\
\hline \multicolumn{5}{|l|}{ General minerals (mg/L) (continued) } \\
\hline Chloride & 90 & 87 & 90 & 86 \\
\hline Fluoride & 0.32 & 0.28 & 0.28 & 0.22 \\
\hline Magnesium & na & $<0.5$ & $<0.5$ & na \\
\hline Nitrate & $<0.4$ & $<0.4$ & $<0.4$ & 0.9 \\
\hline Orthophosphate & 0.19 & 0.17 & 0.15 & 0.14 \\
\hline Potassium & na & 73 & 72 & 72 \\
\hline Sodium & 280 & 240 & 230 & 220 \\
\hline Sulfate & 180 & 180 & 190 & 190 \\
\hline Surfactants & na & $<0.05$ & $<0.05$ & na \\
\hline Perchlorate & na & $<4$ & $<4$ & $<4$ \\
\hline Total hardness $\left(\mathrm{as} \mathrm{CaCO}_{3}\right)$ & na & 8.1 & 11 & na \\
\hline Total phosphorus & na & 0.06 & 0.16 & na \\
\hline \multicolumn{5}{|l|}{ Organic ( $\mu \mathrm{g} / \mathrm{L})$} \\
\hline EPA Method 624 & nd & nd & nd & nd \\
\hline EPA Method 625 & nd & nd & nd & nd \\
\hline EPA Method 608 & nd & nd & nd & nd \\
\hline Total organic halides (TOX, $\mu \mathrm{g} / \mathrm{L}$ ) & $<20$ & $<20$ & $<20$ & $<20$ \\
\hline Total organic carbon (TOC, mg/L) & 1.7 & 1.6 & 1.4 & 1.2 \\
\hline Fecal coliform (MPN/100 mL) & $<1.1$ & na & $<2$ & na \\
\hline Total coliform (MPN/100 mL) & $<1.1$ & $<2$ & $<2$ & $<2$ \\
\hline \multicolumn{5}{|l|}{ Explosive compounds ( $\mu \mathrm{g} / \mathrm{L}$ ) } \\
\hline HMX & $<1$ & $<5$ & $<5$ & $<5$ \\
\hline RDX & $<0.8$ & na & na & $<5$ \\
\hline TNT & $<0.1$ & $<5$ & $<5$ & na \\
\hline \multicolumn{5}{|l|}{ Radioactive (Bq/L) } \\
\hline Gross alpha & $0.040 \pm 0.043$ & $-0.011 \pm 0.019$ & $-0.028 \pm 0.041$ & $0.020 \pm 0.052$ \\
\hline Gross beta & $2.0 \pm 0.2$ & $0.54 \pm 0.09$ & $1.0 \pm 0.2$ & $2.4 \pm 0.37$ \\
\hline Radium 226 & $0.001 \pm 0.003$ & $0.003 \pm 0.002$ & $-0.001 \pm 0.004$ & $-0.005 \pm 0.004$ \\
\hline Radium 228 & $-0.006 \pm 0.014$ & na & na & na \\
\hline Tritium & $-1.5 \pm 1.3$ & $-0.24 \pm 2.5$ & $-0.39 \pm 2.0$ & $-0.67 \pm 2.0$ \\
\hline Uranium (total) & $0.005 \pm 0.001$ & $0.003 \pm 0.002$ & $0.004 \pm 0.002$ & $<0.007 \pm 0.001$ \\
\hline
\end{tabular}


Table 9-34. 1999 analytical results for Site 300 Building 829 area deep monitoring wells (continued).

\begin{tabular}{|c|c|c|c|c|}
\hline \multirow{2}{*}{$\begin{array}{l}\text { Constituents } \\
\text { of concern }\end{array}$} & \multicolumn{4}{|c|}{ Sampling dates for W-829-22 } \\
\hline & $3 / 24 / 99$ & 6/17/99 & 9/2/99 & $11 / 22 / 99$ \\
\hline \multicolumn{5}{|l|}{ Inorganic $(\mu \mathrm{g} / \mathrm{L})$} \\
\hline $\mathrm{pH}$ (pH units) & na & 8.5 & 8.4 & na \\
\hline Field $\mathrm{pH}$ (pH units) & 8.5 & 8.8 & 7.9 & 8.5 \\
\hline Specific conductance $(\mu \mathrm{mho} / \mathrm{cm})$ & na & 970 & 940 & na \\
\hline Field specific conductance $(\mu \mathrm{mho} / \mathrm{cm})$ & 1000 & 1200 & 900 & 1000 \\
\hline Total dissolved solids (TDS) & na & 620 & 650 & na \\
\hline Field temperature $\left({ }^{\circ} \mathrm{C}\right)$ & 22 & 24 & 23 & 21 \\
\hline Aluminum & na & $<50$ & $<50$ & na \\
\hline Antimony & $<5$ & $<5$ & $<5$ & $<5$ \\
\hline Arsenic & 2.9 & $<2$ & $<4$ & $<2$ \\
\hline Barium & $<25$ & $<25$ & $<25$ & $<25$ \\
\hline Beryllium & $<0.5$ & $<0.5$ & $<0.5$ & $<0.5$ \\
\hline Cadmium & $<0.5$ & $<0.5$ & $<0.5$ & $<0.5$ \\
\hline Chromium & 1.2 & $<1$ & $<1$ & $<1$ \\
\hline Cobalt & $<25$ & $<25$ & $<25$ & $<25$ \\
\hline Copper & $<10$ & $<10$ & $<10$ & $<10$ \\
\hline Iron & $<50$ & $<50$ & $<50$ & $<50$ \\
\hline Lead & $<2$ & $<2$ & $<2$ & $<2$ \\
\hline Manganese & $<10$ & $<10$ & $<10$ & $<10$ \\
\hline Mercury & $<0.2$ & $<0.2$ & $<0.2$ & $<0.2$ \\
\hline Molybdenum & $<25$ & $<25$ & $<25$ & $<25$ \\
\hline Nickel & $<5$ & $<5$ & $<5$ & $<5$ \\
\hline Selenium & $<2$ & $<2$ & $<2$ & $<2$ \\
\hline Silver & $<0.5$ & $<1$ & $<0.5$ & $<0.5$ \\
\hline Thallium & $<1$ & $<1$ & $<1$ & $<1$ \\
\hline Vanadium & $<25$ & $<25$ & $<25$ & $<25$ \\
\hline Zinc & na & $<10$ & $<10$ & $<20$ \\
\hline \multicolumn{5}{|l|}{ General minerals (mg/L) } \\
\hline Bicarbonate alkalinity (as $\mathrm{CaCO}_{3}$ ) & na & 220 & 220 & na \\
\hline Carbonate alkalinity $\left(\mathrm{as} \mathrm{CaCO}_{3}\right)$ & na & 10 & $<5$ & na \\
\hline Total alkalinity $\left(\right.$ as $\left.\mathrm{CaCO}_{3}\right)$ & na & 230 & 220 & na \\
\hline Bromide & 0.32 & 0.38 & 0.38 & 0.46 \\
\hline Calcium & na & 6.2 & 6.5 & na \\
\hline Chloride & 95 & 89 & 90 & 87 \\
\hline
\end{tabular}


Table 9-34. 1999 analytical results for Site 300 Building 829 area deep monitoring wells (concluded).

\begin{tabular}{|c|c|c|c|c|}
\hline \multirow{2}{*}{$\begin{array}{l}\text { Constituents } \\
\text { of concern }\end{array}$} & \multicolumn{4}{|c|}{ Sampling dates for W-829-22 } \\
\hline & $3 / 24 / 99$ & 6/17/99 & 9/2/99 & $11 / 22 / 99$ \\
\hline \multicolumn{5}{|l|}{ General minerals ( $\mu \mathrm{g} / \mathrm{L})$ (continued) } \\
\hline Fluoride & 0.38 & 0.38 & 0.4 & 0.36 \\
\hline Magnesium & na & 0.9 & 0.93 & na \\
\hline Nitrate & $<0.4$ & $<0.4$ & $<0.4$ & $<0.4$ \\
\hline Orthophosphate & 0.21 & 0.16 & 0.16 & 0.14 \\
\hline Potassium & na & 9 & 8.6 & 8.3 \\
\hline Sodium & 180 & 210 & 210 & 190 \\
\hline Sulfate & 130 & 120 & 120 & 120 \\
\hline Surfactants & na & $<0.05$ & $<0.05$ & na \\
\hline Perchlorate & na & $<4$ & $<4$ & $<4$ \\
\hline Total hardness $\left(\mathrm{as} \mathrm{CaCO}_{3}\right)$ & na & 19 & 20 & na \\
\hline Total phosphorus & na & 0.07 & 0.06 & na \\
\hline \multicolumn{5}{|l|}{ Organic $(\mu g / L)$} \\
\hline EPA Method 624 & nd & nd & nd & nd \\
\hline EPA Method 625 & nd & nd & nd & nd \\
\hline EPA Method 608 & nd & nd & nd & nd \\
\hline Total organic halides (TOX, $\mu \mathrm{g} / \mathrm{L})$ & $<20$ & 45 & $<20$ & $<20$ \\
\hline Total organic carbon (TOC, mg/L) & 1.1 & 1.4 & 1.5 & 1.2 \\
\hline Fecal coliform (MPN/100 mL) & $<1.1$ & $<2$ & $<2$ & $<2$ \\
\hline Total coliform (MPN/100 mL) & 23 & 44 & $<2$ & 8 \\
\hline \multicolumn{5}{|l|}{ Explosive compounds ( $\mu \mathrm{g} / \mathrm{L})$} \\
\hline $\mathrm{HMX}$ & $<1$ & $<5$ & $<5$ & $<5$ \\
\hline RDX & $<0.8$ & $<5$ & $<5$ & $<5$ \\
\hline TNT & $<1.2$ & $<5$ & $<5$ & $<5$ \\
\hline 1,3,5-Trinitrobenzene & na & na & na & na \\
\hline \multicolumn{5}{|l|}{ Radioactive (Bq/L) } \\
\hline Gross alpha & $0.027 \pm 0.027$ & $0.010 \pm 0.031$ & $-0.008 \pm 0.035$ & $-0.006 \pm 0.028$ \\
\hline Gross beta & $0.39 \pm 0.05$ & $0.29 \pm 0.06$ & $0.26 \pm 0.05$ & $0.30 \pm 0.06$ \\
\hline Radium 226 & $0.006 \pm 0.005$ & $0.002 \pm 0.004$ & $0.003 \pm 0.004$ & $0.005 \pm 0.004$ \\
\hline Radium 228 & $0.001 \pm 0.016$ & na & na & na \\
\hline Tritium & $-1.6 \pm 1.3$ & $-0.59 \pm 2.7$ & $0.04 \pm 2.1$ & $0.07 \pm 2.1$ \\
\hline Uranium (total) & $0.005 \pm 0.002$ & $<0.007 \pm 0.001$ & $<0.006 \pm 0.002$ & $0.004 \pm 0.002$ \\
\hline
\end{tabular}

a W-827-04 dry all year. No samples obtained.

b $n a=$ Not analyzed (analysis not required).

c Bicarbonate alkalinity = total alkalinity.

d $n d=$ None detected above reporting limits (exceptions are listed). See Table 9-1 for analytical methods and their constituents.

e $\mathrm{MPN}=$ Most probable number 


\section{Ground Water Monitoring}

Table 9-35. 1999 analytical results for Site 300 Building 829 area shallow monitoring wells.

\begin{tabular}{|c|c|c|c|c|c|c|c|}
\hline \multirow{4}{*}{$\begin{array}{l}\text { Constituents } \\
\text { of concern }\end{array}$} & \multicolumn{7}{|c|}{ Well } \\
\hline & \multicolumn{4}{|c|}{ W-829-06 } & \multicolumn{3}{|c|}{ W-829-08(a) } \\
\hline & \multicolumn{7}{|c|}{ Sampling dates } \\
\hline & 3/9/99 & 6/11/99 & $8 / 26 / 99$ & 12/1/99 & 3/15/99 & 8/26/99 & $12 / 2 / 99$ \\
\hline \multicolumn{8}{|l|}{ Inorganic $(\mu \mathrm{g} / \mathrm{L})$} \\
\hline Field pH (units) & 7.9 & 7.6 & 7.4 & 7.4 & 7.6 & 7.5 & 7.8 \\
\hline $\begin{array}{l}\text { Field specific conductance } \\
(\mu \mathrm{mho} / \mathrm{cm})\end{array}$ & 2800 & 2800 & 2700 & 1000 & 2900 & 2800 & 1100 \\
\hline Field temperature $\left({ }^{\circ} \mathrm{C}\right)$ & 15 & 21 & 23 & 19 & 19 & 21 & 17 \\
\hline Antimony & $n a^{(b)}$ & na & na & $<5$ & na & na & $<5$ \\
\hline Arsenic & na & na & na & 3 & na & na & $<2$ \\
\hline Barium & na & na & na & 74 & na & na & 29 \\
\hline Beryllium & na & na & na & $<0.5$ & na & na & $<0.5$ \\
\hline Cadmium & na & na & na & $<0.5$ & na & na & $<1$ \\
\hline Chromium & na & na & na & 2 & na & na & 2 \\
\hline Cobalt & na & na & na & $<50$ & na & na & $<50$ \\
\hline Copper & na & na & na & $<10$ & na & na & 34 \\
\hline Iron & na & na & na & 270 & na & na & $<50$ \\
\hline Lead & na & na & na & $<5$ & na & na & $<5$ \\
\hline Manganese & na & na & na & 74 & na & na & $<10$ \\
\hline Mercury & na & na & na & $<0.2$ & na & na & $<0.2$ \\
\hline Molybdenum & na & na & na & $<25$ & na & na & $<25$ \\
\hline Nickel & na & na & na & $<5$ & na & na & 10 \\
\hline Perchlorate $(\mu \mathrm{g} / \mathrm{L})^{(\mathrm{c})}$ & 18 & 21 & na & 24 & 12 & na & 14 \\
\hline Potassium & na & na & na & 25 & na & na & 27 \\
\hline Selenium & na & na & na & 410 & na & na & 520 \\
\hline Silver & na & na & na & $<1$ & na & na & $<1$ \\
\hline Thallium & na & na & na & $<1$ & na & na & $<1$ \\
\hline Vanadium & na & na & na & $<25$ & na & na & $<25$ \\
\hline Zinc & na & na & na & 32 & na & na & 260 \\
\hline \multicolumn{8}{|l|}{ General minerals (mg/L) } \\
\hline Bromide & na & na & na & 1.7 & na & na & 2 \\
\hline Chloride & na & na & na & 410 & na & na & 500 \\
\hline Fluoride & na & na & na & 0.52 & na & na & 0.52 \\
\hline Nitrate & na & na & na & 230 & na & na & 230 \\
\hline Orthophosphate & na & na & na & 0.24 & na & na & 0.16 \\
\hline Sodium & na & na & na & 520 & na & na & 540 \\
\hline Sulfate & na & na & na & 320 & na & na & 300 \\
\hline
\end{tabular}


Table 9-35. 1999 analytical results for Site 300 Building 829 area shallow monitoring wells (concluded).

\begin{tabular}{|c|c|c|c|c|c|c|c|}
\hline \multirow{4}{*}{$\begin{array}{l}\text { Constituents } \\
\text { of concern }\end{array}$} & \multicolumn{7}{|c|}{ Well } \\
\hline & \multicolumn{4}{|c|}{ W-829-06 } & \multicolumn{3}{|c|}{ W-829-08(a) } \\
\hline & \multicolumn{7}{|c|}{ Sampling dates } \\
\hline & $3 / 9 / 99$ & $6 / 11 / 99$ & $8 / 26 / 99$ & $12 / 1 / 99$ & $3 / 15 / 99$ & $8 / 26 / 99$ & 12/2/99 \\
\hline \multicolumn{8}{|l|}{ General minerals (mg/L) } \\
\hline \multicolumn{8}{|l|}{ Organic $(\mu \mathrm{g} / \mathrm{L})$} \\
\hline EPA Method 624 & $\mathrm{nd}^{(\mathrm{d})}$ & nd & nd & nd & nd & nd & nd \\
\hline 1,2-Dichloroethene (total) & $<3$ & 2.6 & $<3$ & 2.9 & $<1$ & $<1$ & $<1$ \\
\hline cis-1,2-Dichloroethene & 2.5 & 2.6 & 2.4 & 2.9 & $<0.5$ & $<0.5$ & $<0.5$ \\
\hline Trichloroethene & 260 & 310 & 280 & 280 & 28 & 31 & 29 \\
\hline EPA Method 625 & na & na & na & nd & na & na & nd \\
\hline EPA Method 608 & na & na & na & nd & na & na & nd \\
\hline $\begin{array}{l}\text { Total organic halides (TOX, } \\
\mu \mathrm{g} / \mathrm{L} \text { ) }\end{array}$ & na & na & na & 192 & na & na & $<20$ \\
\hline 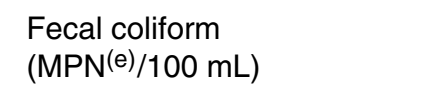 & na & na & na & na & na & na & $<2$ \\
\hline Total coliform (MPN/100 mL) & na & na & na & $<2$ & na & na & $<2$ \\
\hline $\begin{array}{l}\text { Total organic carbon (TOC, } \\
\mathrm{mg} / \mathrm{L} \text { ) }\end{array}$ & na & na & na & 1.8 & na & na & 1.9 \\
\hline \multicolumn{8}{|l|}{ Explosive compounds ( $\mu \mathrm{g} / \mathrm{L})$} \\
\hline HMX & $<1$ & $<5$ & $<5$ & $<5$ & $<1$ & $<5$ & $<5$ \\
\hline RDX & $<0.8$ & $<5$ & $<5$ & $<5$ & $<0.8$ & $<5$ & $<5$ \\
\hline TNT & na & na & na & $<5$ & na & na & $<5$ \\
\hline \multicolumn{8}{|l|}{ Radioactivity (Bq/L) } \\
\hline Gross alpha & na & na & na & $1.7 \pm 0.41$ & na & na & $1.2 \pm 0.33$ \\
\hline Gross beta & na & na & na & $1.0 \pm 0.25$ & na & na & $1.3 \pm 0.26$ \\
\hline Radium-226 & na & na & na & $0.011 \pm 0.004$ & na & na & $0.002 \pm 0.003$ \\
\hline Tritium & na & na & na & $-0.63 \pm 2.0$ & na & na & $0.70 \pm 2.1$ \\
\hline Uranium (total) & na & na & na & $0.43 \pm 0.03$ & na & na & $1.1 \pm 0.08$ \\
\hline
\end{tabular}

a Second quarter sampling not done because pump down.

b $n a=$ Not analyzed (analysis not required).

c Perchlorate sampled on $3 / 5 / 99$ for first quarter.

d $\quad n d=$ None detected above reporting limits (exceptions are listed). See Table 9-1 for analytical methods and their constituents.

e $\quad$ MPN $=$ Most probable number 


\section{Ground Water Monitoring}

Table 9-36. Site 300 potable standby supply well 18.

\begin{tabular}{|c|c|c|c|c|}
\hline \multirow{2}{*}{$\begin{array}{l}\text { Constituents } \\
\text { of concern }\end{array}$} & \multicolumn{4}{|c|}{ Sampling dates } \\
\hline & $1 / 13 / 99$ & 4/14/99(a) & 9/15/99 & $11 / 10 / 99$ \\
\hline \multicolumn{5}{|l|}{ Organic $(\mu \mathrm{g} / \mathrm{L})$} \\
\hline EPA Method 502.2 (volatile) & $n d^{(b)}$ & nd & -(c) $^{(\mathrm{c}}$ & $-(\mathrm{c})$ \\
\hline EPA Method 601 (volatile) & nd & nd & nd & nd \\
\hline Trichloroethene (TCE) & $<0.5$ & 0.63 & 0.54 & $<0.5$ \\
\hline EPA Method 624 (volatile) & nd & na ${ }^{(d)}$ & na & na \\
\hline \multicolumn{5}{|l|}{ Radioactive (Bq/L) } \\
\hline Gross alpha & $-0.024 \pm 0.034$ & $-0.014 \pm 0.026$ & -(c) & $-0.0004 \pm 0.041$ \\
\hline Gross beta & $0.064 \pm 0.056$ & $0.23 \pm 0.03$ & -(c) & $0.23 \pm 0.06$ \\
\hline Tritium & $-3.9 \pm 2.6$ & $-0.53 \pm 1.7$ & -(c) & $0.008 \pm 2.1$ \\
\hline
\end{tabular}

Note: Radioactivities are reported as the measured concentration and either an uncertainty ( $\pm 2 \sigma$ counting error) or as being less than or equal to the detection limit. If the concentration is less than or equal to the uncertainty or the detection limit, the result is considered to be a nondetection. See the main volume, Chapter 14, Quality Assurance.

a There was one analysis for perchlorate, resulting in a nondetection of $<4 \mu \mathrm{g} / \mathrm{L}$.

b $\quad$ nd $=$ None detected above reporting limits (exceptions are listed). See Table 9-1 for analytical methods and their constituents.

c Construction prohibited sampling.

d $n a=$ Not analyzed (analysis not required). 
Table 9-37. Site 300 potable supply well 20.

\begin{tabular}{|c|c|c|c|c|}
\hline \multirow{2}{*}{$\begin{array}{l}\text { Constituents } \\
\text { of concern }\end{array}$} & \multicolumn{4}{|c|}{ Sampling dates } \\
\hline & 1/28/99 & $4 / 30 / 99$ & 7/29/99 & 12/28/99 \\
\hline \multicolumn{5}{|l|}{ Inorganic ( $\mu \mathrm{g} / \mathrm{L})$} \\
\hline Antimony & $<5$ & $<5$ & $<5$ & $<5$ \\
\hline Arsenic & $<2$ & $<2$ & $<2$ & $<2$ \\
\hline Barium & $<25$ & $<25$ & $<25$ & $<25$ \\
\hline Beryllium & $<0.5$ & $<0.5$ & $<0.5$ & $<0.5$ \\
\hline Cadmium & $<0.5$ & $<0.5$ & $<0.5$ & $<0.5$ \\
\hline Chromium & $<1$ & $<1$ & $<1$ & $<1$ \\
\hline Cobalt & $<25$ & $<25$ & $<25$ & $<25$ \\
\hline Copper & $<10$ & $<10$ & $<10$ & $<10$ \\
\hline Lead & 2.1 & $<4$ & $<2$ & $<2$ \\
\hline Mercury & $<0.2$ & $<0.2$ & $<0.2$ & $<0.2$ \\
\hline Molybdenum & $<25$ & $<25$ & $<25$ & $<25$ \\
\hline Nickel & $<5$ & $<5$ & $<5$ & $<5$ \\
\hline Nitrate (mg/L) & $\mathrm{na}^{(\mathrm{a})}$ & $<0.4$ & $<0.4$ & $<0.4$ \\
\hline Perchlorate & $<4$ & na & na & na \\
\hline Potassium (mg/L) & 8 & 8.4 & 7.6 & 8.1 \\
\hline Selenium & $<2$ & $<2$ & $<4$ & $<2$ \\
\hline Silver & $<0.5$ & $<0.5$ & $<2$ & $<1$ \\
\hline Thallium & $<1$ & $<1$ & $<1$ & $<1$ \\
\hline Vanadium & $<25$ & $<25$ & $<25$ & $<25$ \\
\hline Zinc & $<20$ & na & na & $<20$ \\
\hline \multicolumn{5}{|l|}{ Organic ( $\mu \mathrm{g} / \mathrm{L})$} \\
\hline EPA Method 502.2 (volatile) & $\mathrm{nd}^{(\mathrm{b})}$ & nd & nd & nd \\
\hline \multicolumn{5}{|l|}{ Explosive ( $\mu \mathrm{g} / \mathrm{L})$} \\
\hline HMX & $<5$ & $<5$ & $<5$ & $<5$ \\
\hline RDX & $<5$ & $<5$ & $<5$ & $<5$ \\
\hline \multicolumn{5}{|l|}{ Radioactive (Bq/L) } \\
\hline Gross alpha & $-0.031 \pm 0.043$ & $-0.003 \pm 0.031$ & $-0.012 \pm 0.030$ & $0.004 \pm 0.034$ \\
\hline Gross beta & $0.28 \pm 0.06$ & $0.23 \pm 0.04$ & $0.22 \pm 0.05$ & $0.24 \pm 0.06$ \\
\hline Tritium & $-6.0 \pm 2.0$ & $-0.18 \pm 1.8$ & $-0.72 \pm 1.5$ & $0.7 \pm 2.1$ \\
\hline
\end{tabular}

Note: Radioactivities are reported as the measured concentration and either an uncertainty ( $\pm 2 \sigma$ counting error) or as being less than or equal to the detection limit. If the concentration is less than or equal to the uncertainty or the detection limit, the result is considered to be a nondetection. See the main volume, Chapter 14, Quality Assurance.

a $n a=$ Not analyzed (analysis not required).

b $n d=$ None detected above reporting limits (exceptions are listed). See Table 9-1 for analytical methods and their constituents. 


\section{Ground Water Monitoring}

Table 9-38. Site 300 off-site well CARNRW1.

\begin{tabular}{|c|c|c|c|c|}
\hline \multirow{2}{*}{$\begin{array}{l}\text { Constituents } \\
\text { of concern }\end{array}$} & \multicolumn{4}{|c|}{ Sampling dates } \\
\hline & $1 / 21 / 99$ & 4/29/99 & $7 / 29 / 99$ & $10 / 14 / 99$ \\
\hline Organic ( $\mu g / L)$ & & & & \\
\hline $\begin{array}{l}\text { EPA Method } 601 \\
\text { (volatile) }\end{array}$ & $n d^{(a)}$ & nd & nd & nd \\
\hline
\end{tabular}

a $n d=$ None detected above reporting limits. See Table 9-1 for analytical methods and their constituents. 
Table 9-39. Site 300 off-site well CDF1.

\begin{tabular}{|c|c|c|c|c|}
\hline \multirow{2}{*}{$\begin{array}{l}\text { Constituents } \\
\text { of concern }\end{array}$} & \multicolumn{4}{|c|}{ Sampling date } \\
\hline & $1 / 20 / 99$ & 4/28/99 & 7/30/99 & $10 / 14 / 99$ \\
\hline \multicolumn{5}{|l|}{ Inorganic $(\mu \mathrm{g} / \mathrm{L})$} \\
\hline Antimony & $<5$ & ${ }_{-}^{(a)}$ & $<5$ & $<5$ \\
\hline Arsenic & 5 & $-^{\text {(a) }}$ & 4.7 & 6.5 \\
\hline Barium & 28 & ${ }_{-}^{(a)}$ & 38 & 30 \\
\hline Beryllium & $<0.5$ & ${ }_{-}^{(a)}$ & $<0.5$ & $<0.5$ \\
\hline Cadmium & $<0.5$ & ${ }_{-}^{(a)}$ & $<0.5$ & $<0.5$ \\
\hline Chromium & $<1$ & ${ }_{-}^{(a)}$ & $<1$ & $<1$ \\
\hline Cobalt & $<25$ & - (a) $^{\text {(a) }}$ & $<25$ & $<25$ \\
\hline Copper & $<10$ & - (a) $^{\text {(a) }}$ & $<10$ & $<10$ \\
\hline Lead & 6 & - (a) & 2.4 & $<4$ \\
\hline Mercury & $<0.2$ & - (a) $^{\text {(a) }}$ & $<0.2$ & $<0.2$ \\
\hline Molybdenum & $<25$ & -(a) & $<25$ & $<25$ \\
\hline Nickel & $<5$ & - (a) $^{\text {(a) }}$ & $<5$ & $<5$ \\
\hline Nitrate (mg/L) & 0.6 & 2.0 & 3.0 & 0.5 \\
\hline Potassium (mg/L) & 8 & 8 & 7.6 & 8.9 \\
\hline Selenium & $<2$ & $-^{(a)}$ & $<2$ & $<2$ \\
\hline Silver & $<0.5$ & ${ }_{-}^{(a)}$ & $<0.5$ & $<2$ \\
\hline Thallium & $<1$ & - (a) $^{\text {(a) }}$ & $<1$ & $<1$ \\
\hline Vanadium & $<25$ & ${ }_{-}^{(a)}$ & $<25$ & $<25$ \\
\hline Zinc & 38 & - (a) $^{\text {(a) }}$ & $n a^{(b)}$ & $<20$ \\
\hline \multicolumn{5}{|l|}{ Organic $(\mu g / L)$} \\
\hline EPA Method 524.2 (volatile) & $n d^{(c)}$ & nd & nd & nd \\
\hline EPA Method 608 (pesticides and PCBs) & $-(d)$ & ${ }_{-}^{(d)}$ & nd & nd \\
\hline EPA Method 615 (herbicides) & $-^{(d)}$ & $-^{(d)}$ & nd & nd \\
\hline EPA Method 625 (semivolatile) & - (d) $^{(\mathrm{n}}$ & ${ }_{-}^{(d)}$ & nd & nd \\
\hline \multicolumn{5}{|l|}{ Explosive $(\mu \mathrm{g} / \mathrm{L})$} \\
\hline HMX & $<5$ & $<5$ & $<5$ & $<5$ \\
\hline RDX & $<5$ & $<5$ & $<5$ & $<5$ \\
\hline \multicolumn{5}{|l|}{ Radioactive (Bq/L) } \\
\hline Gross alpha & $0.041 \pm 0.041$ & $0.029 \pm 0.037$ & $0.092 \pm 0.052$ & $0.036 \pm 0.044$ \\
\hline Gross beta & $0.34 \pm 0.06$ & $0.26 \pm 0.06$ & $0.25 \pm 0.05$ & $0.29 \pm 0.06$ \\
\hline Tritium & $-7.1 \pm 2.2$ & $0.24 \pm 1.8$ & $-1.2 \pm 1.4$ & $-1.1 \pm 2.6$ \\
\hline Uranium (total) & $\ldots$ & (d) & $0.064 \pm 0.007$ & $0.016 \pm 0.003$ \\
\hline
\end{tabular}

Note: Radioactivities are reported as the measured concentration and either an uncertainty ( $\pm 2 \sigma$ counting error) or as being less than or equal to the detection limit. If the concentration is less than or equal to the uncertainty or the detection limit, the result is considered to be a nondetection. See the main volume, Chapter 14, Quality Assurance.

a Analysis not requested (sampling error).

b $n a=$ Not analyzed (analysis not required).

c $n d=$ None detected above reporting limits. See Table 9-1 for analytical methods and their constituents.

d Analysis not planned. 
Table 9-40. Off-site surveillance well CON1.

\begin{tabular}{|c|c|c|c|c|}
\hline \multirow{2}{*}{$\begin{array}{c}\text { Constituents } \\
\text { of concern }\end{array}$} & \multicolumn{4}{|c|}{ Sampling dates } \\
\hline & $1 / 20 / 99$ & $4 / 29 / 99$ & 7/30/99 & $10 / 15 / 99$ \\
\hline \multicolumn{5}{|l|}{ Inorganic $(\mu \mathrm{g} / \mathrm{L})$} \\
\hline Antimony & $<5$ & $<5$ & $<5$ & $<5$ \\
\hline Arsenic & $<2$ & 2.6 & $<2$ & 2.6 \\
\hline Barium & $<25$ & $<25$ & $<25$ & $<25$ \\
\hline Beryllium & $<0.5$ & $<0.5$ & $<0.5$ & $<0.5$ \\
\hline Cadmium & $<0.5$ & $<0.5$ & $<0.5$ & $<0.5$ \\
\hline Chromium & 4.3 & $<1$ & $<1$ & $<1$ \\
\hline Cobalt & $<25$ & $<25$ & $<25$ & $<25$ \\
\hline Copper & $<10$ & $<10$ & $<10$ & $<10$ \\
\hline Lead & $<2$ & $<4$ & $<2$ & $<5$ \\
\hline Mercury & $<0.2$ & $<0.2$ & $<0.2$ & $<0.2$ \\
\hline Molybdenum & $<25$ & $<25$ & $<25$ & $<25$ \\
\hline Nickel & 18 & $<5$ & $<5$ & $<5$ \\
\hline Selenium & $<2$ & $<2$ & $<2$ & $<2$ \\
\hline Silver & $<0.5$ & $<0.5$ & $<0.5$ & $<0.5$ \\
\hline Thallium & $<1$ & $<1$ & $<1$ & $<1$ \\
\hline Vanadium & $<25$ & $<25$ & $<25$ & $<25$ \\
\hline Zinc & $<20$ & -(a) & —(a) & $<20$ \\
\hline Potassium (mg/L) & 8.1 & 9.2 & 8.9 & 8.9 \\
\hline Nitrate (mg/L) & $<0.5$ & $<0.9$ & $<0.9$ & $<0.9$ \\
\hline \multicolumn{5}{|l|}{ Organic ( $\mu \mathrm{g} / \mathrm{L})$} \\
\hline EPA Method 524.2 (volatile) & $n d^{(b)}$ & nd & nd & nd \\
\hline EPA Method 608 (pesticides and PCBs) & -(c) & -(c) & nd & -(c) \\
\hline EPA Method 615 (herbicides) & -(c) & -(c) & nd & -(c) \\
\hline EPA Method 625 (semivolatile) & -(c) & -(c) & nd & -(c) \\
\hline \multicolumn{5}{|l|}{ Energetic $(\mu \mathrm{g} / \mathrm{L})$} \\
\hline $\mathrm{HMX}$ & $<5$ & $<5$ & $<5$ & $<5$ \\
\hline RDX & $<5$ & $<5$ & $<5$ & $<5$ \\
\hline \multicolumn{5}{|l|}{ Radioactive (Bq/L) } \\
\hline Gross alpha & $0.056 \pm 0.061$ & $0.001 \pm 0.015$ & $-0.018 \pm 0.030$ & $-0.001 \pm 0.028$ \\
\hline Gross beta & $0.32 \pm 0.07$ & $0.004 \pm 0.027$ & $0.15 \pm 0.04$ & $0.13 \pm 0.05$ \\
\hline Tritium & $-8.1 \pm 2.1$ & $-0.54 \pm 1.8$ & $-2.3 \pm 1.4$ & $-0.4 \pm 2.6$ \\
\hline Uranium (total) & -(c) & -(c) & $0.002 \pm 0.001$ & -(c) \\
\hline
\end{tabular}

Note: Radioactivities are reported as the measured concentration and either an uncertainty ( $\pm 2 \sigma$ counting error) or as being less than or equal to the detection limit. If the concentration is less than or equal to the uncertainty or the detection limit, the result is considered to be a nondetection. See the main volume, Chapter 14, Quality Assurance.

a Analysis not requested (sampling error).

b $n d=$ None detected above reporting limits. See Table 9-1 for analytical methods and their constituents.

c Analysis not planned. 
Table 9-41. Site 300, off-site surveillance well GALLO1.

\begin{tabular}{|c|c|c|c|c|}
\hline \multirow{2}{*}{$\begin{array}{c}\text { Constituents } \\
\text { of concern }\end{array}$} & \multicolumn{4}{|c|}{ Sampling date } \\
\hline & $1 / 21 / 99$ & 4/30/99 & $7 / 29 / 99$ & $10 / 14 / 99$ \\
\hline \multicolumn{5}{|l|}{ Inorganic ( $\mu \mathrm{g} / \mathrm{L})$} \\
\hline Antimony & $<5$ & $<5$ & $<5$ & $<5$ \\
\hline Arsenic & 2.7 & 5.4 & 3.5 & 5.4 \\
\hline Barium & $<25$ & $<25$ & $<25$ & $<25$ \\
\hline Beryllium & $<0.5$ & $<0.5$ & $<0.5$ & $<0.5$ \\
\hline Cadmium & $<0.5$ & $<0.5$ & $<0.5$ & $<0.5$ \\
\hline Chromium & $<1$ & $<1$ & $<1$ & $<1$ \\
\hline Cobalt & $<25$ & $<25$ & $<25$ & $<25$ \\
\hline Copper & $<10$ & $<10$ & $<10$ & $<10$ \\
\hline Lead & 2.7 & $<4$ & $<2$ & $<4$ \\
\hline Mercury & $<0.2$ & $<0.2$ & $<0.2$ & $<0.2$ \\
\hline Molybdenum & 42 & 44 & 37 & 45 \\
\hline Nickel & $<5$ & $<5$ & $<5$ & $<5$ \\
\hline Selenium & $<2$ & $<2$ & $<4$ & $<2$ \\
\hline Silver & $<0.5$ & $<0.5$ & $<2$ & $<2$ \\
\hline Thallium & $<1$ & $<1$ & $<1$ & $<1$ \\
\hline Vanadium & $<25$ & $<25$ & $<25$ & $<25$ \\
\hline Zinc & $<20$ & -(a) $^{(\mathrm{n}}$ & -(a) $^{\text {(a) }}$ & $<20$ \\
\hline Potassium (mg/L) & 3.6 & 3.9 & 3.8 & 3.9 \\
\hline Nitrate (mg/L) & 2.9 & $<0.4$ & $<0.4$ & $<0.4$ \\
\hline \multicolumn{5}{|l|}{ Organic $(\mu \mathrm{g} / \mathrm{L})$} \\
\hline EPA Method 524.2 (volatile) & $\mathrm{nd} \mathrm{d}^{(\mathrm{b})}$ & nd & nd & nd \\
\hline Trichloroethene & 0.57 & 0.74 & 0.58 & 0.52 \\
\hline EPA Method 608 (pesticides and PCBs) & 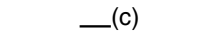 & 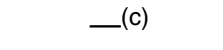 & nd & 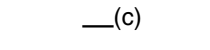 \\
\hline EPA Method 615 (herbicides) & 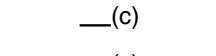 & 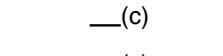 & nd & 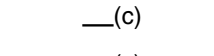 \\
\hline EPA Method 625 (semivolatile) & 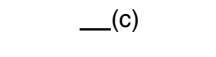 & 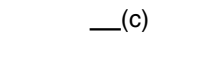 & nd & -(c) $^{(\mathrm{c}}$ \\
\hline \multicolumn{5}{|l|}{ Explosive $(\mu \mathrm{g} / \mathrm{L})$} \\
\hline HMX & $<5$ & $<5$ & $<5$ & $<5$ \\
\hline RDX & $<5$ & $<5$ & $<5$ & $<5$ \\
\hline \multicolumn{5}{|l|}{ Radioactive (Bq/L) } \\
\hline Gross alpha & $0.034 \pm 0.040$ & $0.006 \pm 0.026$ & $-0.043 \pm 0.031$ & $0.005 \pm 0.036$ \\
\hline Gross beta & $0.17 \pm 0.05$ & $0.094 \pm 0.034$ & $0.074 \pm 0.030$ & $0.078 \pm 0.048$ \\
\hline Tritium & $-7.0 \pm 2.1$ & $-0.74 \pm 1.8$ & $-2.8 \pm 1.3$ & $-0.58 \pm 2.6$ \\
\hline Uranium (total) & -(c) $^{(\mathrm{c}}$ & -(c) & $0.006 \pm 0.001$ & -(c) $^{(\mathrm{s})}$ \\
\hline
\end{tabular}

Note: Radioactivities are reported as the measured concentration and either an uncertainty $( \pm 2 \sigma$ counting error) or as being less than or equal to the detection limit. If the concentration is less than or equal to the uncertainty or the detection limit, the result is considered to be a nondetection. See the main volume, Chapter 14, Quality Assurance.

a Analysis not requested (sampling error).

b $n d=$ None detected above reporting limits (exceptions are listed). See Table 9-1 for analytical methods and their constituents.

c Analysis not planned. 
Table 9-42. Site 300 off-site surveillance well CARNRW2.

\begin{tabular}{|c|c|c|c|c|}
\hline \multirow{2}{*}{$\begin{array}{l}\text { Constituents } \\
\text { of concern }\end{array}$} & \multicolumn{4}{|c|}{ Sampling date } \\
\hline & $1 / 21 / 99$ & 4/29/99 & 7/29/99 & $10 / 14 / 99$ \\
\hline \multicolumn{5}{|l|}{ Inorganic $(\mu \mathrm{g} / \mathrm{L})$} \\
\hline Antimony & $<5$ & $<5$ & $<5$ & $<5$ \\
\hline Arsenic & 2.6 & 3.7 & 3 & 4.5 \\
\hline Barium & $<25$ & $<25$ & $<25$ & $<25$ \\
\hline Beryllium & $<0.5$ & $<0.5$ & $<0.5$ & $<0.5$ \\
\hline Cadmium & $<0.5$ & $<0.5$ & $<0.5$ & $<0.5$ \\
\hline Chromium & $<1$ & $<1$ & $<1$ & $<1$ \\
\hline Cobalt & $<25$ & $<25$ & $<25$ & $<25$ \\
\hline Copper & $<10$ & $<10$ & $<10$ & $<10$ \\
\hline Lead & 2.8 & $<4$ & $<2$ & $<4$ \\
\hline Mercury & $<0.2$ & $<0.2$ & $<0.2$ & $<0.2$ \\
\hline Molybdenum & $<25$ & $<25$ & $<25$ & 31 \\
\hline Nickel & $<5$ & $<5$ & $<5$ & $<5$ \\
\hline Nitrate $(\mathrm{mg} / \mathrm{L})$ & 1.9 & -(a) & $<0.4$ & $<0.4$ \\
\hline Perchlorate & $n a^{(b)}$ & $<4$ & na & na \\
\hline Potassium (mg/L) & 8.5 & 9 & 8.1 & 8.9 \\
\hline Selenium & $<2$ & $<2$ & $<4$ & $<2$ \\
\hline Silver & $<0.5$ & $<0.5$ & $<2$ & $<2$ \\
\hline Thallium & $<1$ & $<1$ & $<1$ & $<1$ \\
\hline Vanadium & $<25$ & $<25$ & $<25$ & $<25$ \\
\hline Zinc & 26 & -(c) & -(c) $^{(\mathrm{c})}$ & $<20$ \\
\hline \multicolumn{5}{|l|}{ Organic ( $\mu \mathrm{g} / \mathrm{L})$} \\
\hline EPA Method 524.2 (volatile) & $n d^{(d)}$ & nd & nd & nd \\
\hline EPA Method 608 (pesticides and PCBs) & -(e) & —(e) $^{(\mathrm{n}}$ & nd & —(e) \\
\hline EPA Method 615 (herbicides) & -(e) $^{(\mathrm{n}}$ & -(e) $^{(\mathrm{e}}$ & nd & -(e) $^{(\mathrm{e}}$ \\
\hline EPA Method 625 (semivolatile) & 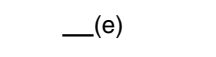 & -(e) & nd & -(e) \\
\hline \multicolumn{5}{|l|}{ Explosive ( $\mu \mathrm{g} / \mathrm{L})$} \\
\hline $\mathrm{HMX}$ & $<5$ & $<5$ & $<5$ & $<5$ \\
\hline RDX & $<5$ & $<5$ & $<5$ & $<5$ \\
\hline \multicolumn{5}{|l|}{ Radioactive (Bq/L) } \\
\hline Gross alpha & $0.019 \pm 0.033$ & $-0.020 \pm 0.037$ & $-0.022 \pm 0.036$ & $-0.025 \pm 0.033$ \\
\hline Gross beta & $0.37 \pm 0.05$ & $0.29 \pm 0.03$ & $0.27 \pm 0.06$ & $0.26 \pm 0.06$ \\
\hline Tritium & $-6.6 \pm 2.2$ & $-0.44 \pm 1.8$ & $-0.87 \pm 1.4$ & —(a) \\
\hline Uranium (total) & —(e) & —(e) & $<0.008 \pm 0.001$ & -(e) \\
\hline
\end{tabular}

Note: Radioactivities are reported as the measured concentration and either an uncertainty ( $\pm 2 \sigma$ counting error) or as being less than or equal to the detection limit. If the concentration is less than or equal to the uncertainty or the detection limit, the result is considered to be a nondetection. See the main volume, Chapter 14, Quality Assurance.

a Analysis not requested (sampling error).

b $\quad$ na $=$ Not analyzed (analysis not required).

c Analysis not reported (analytical error)

d $n d=$ None detected above reporting limits. See Table 9-1 for analytical methods and their constituents.

e Analysis not planned 


\section{Ground Water Monitoring}

Table 9-43. Site 300 off-site surveillance well CON2.

\begin{tabular}{|c|c|c|c|c|}
\hline \multirow{2}{*}{$\begin{array}{c}\text { Constituents } \\
\text { of concern }\end{array}$} & $1 / 20 / 99$ & $4 / 29 / 99$ & $7 / 29 / 99$ & $10 / 15 / 99$ \\
\hline $\begin{array}{c}\text { Organic }(\mu \mathrm{g} / \mathrm{L}) \\
\text { EPA Method 601 (volatile) }\end{array}$ & $\mathrm{nd}(\mathrm{a})$ & $\mathrm{nd}$ & $\mathrm{nd}$ & nd \\
\hline
\end{tabular}

a $n d=$ None detected above reporting limits. See Table 9-1 for analytical methods and their constituents. 


\section{Ground Water Monitoring}

Table 9-44. Annually monitored off-site surveillance wells.

\begin{tabular}{|c|c|c|c|c|c|c|}
\hline \multirow{4}{*}{$\begin{array}{l}\text { Constituents } \\
\text { of concern }\end{array}$} & \multicolumn{6}{|c|}{ Well } \\
\hline & MUL1 & MUL2 & STONEHAM1 & VIE1 & VIE2 & W-35A-04 \\
\hline & \multicolumn{6}{|c|}{ Sampling dates } \\
\hline & 9/8/99 & 9/8/99 & 9/8/99 & 9/10/99 & 9/8/99 & $8 / 6 / 99$ \\
\hline \multicolumn{7}{|l|}{ Inorganic ( $\mu \mathrm{g} / \mathrm{L})$} \\
\hline Antimony & $<5$ & $<5$ & $<5$ & $<5$ & $<5$ & $<5$ \\
\hline Arsenic & 4 & $<2$ & $<2$ & 12 & $<2$ & 2.4 \\
\hline Barium & 30 & $<25$ & 61 & 54 & 38 & 57 \\
\hline Beryllium & $<0.5$ & $<0.5$ & $<0.5$ & $<0.5$ & $<0.5$ & $<0.5$ \\
\hline Cadmium & $<0.5$ & $<0.5$ & $<0.5$ & $<0.5$ & $<0.5$ & $<0.5$ \\
\hline Chromium & $<1$ & $<1$ & $<1$ & $<1$ & $<1$ & 1.7 \\
\hline Cobalt & $<25$ & $<25$ & $<25$ & $<25$ & $<25$ & $<25$ \\
\hline Copper & $<10$ & $<10$ & $<10$ & $<10$ & 20 & $<10$ \\
\hline Lead & $<2$ & $<2$ & $<2$ & $<2$ & 3 & $<2$ \\
\hline Mercury & $<0.2$ & $<0.2$ & $<0.2$ & $<0.2$ & $<0.2$ & $<0.2$ \\
\hline Molybdenum & $<25$ & $<25$ & $<25$ & $<25$ & $<25$ & $<25$ \\
\hline Nickel & $<5$ & 8 & $<5$ & $<5$ & $<5$ & $<5$ \\
\hline Potassium (mg/L) & 5.1 & 7.7 & 6.9 & 11 & 2 & 5.6 \\
\hline Selenium & $<2$ & 3 & $<2$ & 5 & $<2$ & 2 \\
\hline Silver & $<0.5$ & $<0.5$ & $<0.5$ & $<0.5$ & $<0.5$ & $<0.5$ \\
\hline Thallium & $<2$ & $<2$ & $<2$ & $<2$ & $<2$ & $<1$ \\
\hline Vanadium & $<25$ & $<25$ & $<25$ & 32 & $<25$ & $<25$ \\
\hline Zinc & 40 & 20 & 90 & 40 & 40 & 0 \\
\hline \multicolumn{7}{|l|}{ Organic $(\mu \mathrm{g} / \mathrm{L})$} \\
\hline EPA Method 502.2 & $n d^{(a)}$ & nd & nd & nd & nd & nd \\
\hline EPA Method 608 & nd & nd & nd & nd & nd & nd \\
\hline EPA Method 615 & nd & nd & nd & $\mathrm{na}^{(\mathrm{b})}$ & nd & nd \\
\hline EPA Method 625 & nd & nd & nd & nd & nd & nd \\
\hline \multicolumn{7}{|l|}{ Explosive ( $\mu \mathrm{g} / \mathrm{L})$} \\
\hline HMX & $<1$ & $<1$ & $<1$ & $<1$ & $<1$ & na \\
\hline RDX & $<1$ & $<1$ & $<1$ & $<1$ & $<1$ & na \\
\hline \multicolumn{7}{|l|}{ Radioactive (Bq/L) } \\
\hline Gross alpha & $0.080 \pm 0.034$ & $-0.006 \pm 0.023$ & $0.051 \pm 0.048$ & $0.051 \pm 0.037$ & $0.096 \pm 0.052$ & $0.032 \pm 0.037$ \\
\hline Gross beta & $0.21 \pm 0.04$ & $0.11 \pm 0.03$ & $0.10 \pm 0.03$ & $0.41 \pm 0.07$ & $0.095 \pm 0.034$ & $0.056 \pm 0.048$ \\
\hline Tritium & $-0.78 \pm 2.0$ & $1.0 \pm 2.1$ & $-0.01 \pm 2.0$ & $-0.58 \pm 2.0$ & $-0.84 \pm 2.0$ & $1.1 \pm 2.1$ \\
\hline Uranium (total) & $0.13 \pm 0.01$ & $0.035 \pm 0.004$ & $0.22 \pm 0.02$ & $0.11 \pm 0.01$ & $0.093 \pm 0.008$ & $0.14 \pm 0.01$ \\
\hline
\end{tabular}

Note: Radioactivities are reported as the measured concentration and either an uncertainty $( \pm 2 \sigma$ counting error) or as being less than or equal to the detection limit. If the concentration is less than or equal to the uncertainty or the detection limit, the result is considered to be a nondetection. See the main volume, Chapter 14, Quality Assurance.

a $\mathrm{nd}=$ None detected above reporting limits. See Table 9-1 for analytical methods and their constituents..

b $n a=$ Not analyzed (sampling error). 


\section{Soil and Sediment Monitoring}

Gretchen M. Gallegos

Erich R. Brandstetter

\section{Surface Soil Methods}

Prior to 1988, surface soil samples were collected at sites selected at random from Livermore Valley locations previously sampled for a 1971-1972 study. That earlier study was conducted to determine background concentrations of radionuclides in area soils. In 1988, Livermore Valley surface soil sampling locations were chosen to coincide with air sampling locations, to cover areas with contaminants from past incidents, or to sample other areas of special concern (see Figure 10-1, in the main volume). In 1991, five additional soil sampling locations associated with air sampling locations were established. The 1999 Livermore Valley surface soil samples were collected from generally the same locations as those in 1991 to 1998. The 1999 Site 300 soil samples were collected from the same 14 locations as those sampled between 1990 and 1998. In 1998, the soil sampling program added the PRIM location at Site 300 to complement air monitoring at that location. The PRIM site is downwind of Site 300 and off site but sufficiently close to the Site 300 boundary to potentially be affected by Site 300 operations. Analysis for plutonium in Site 300 soils was discontinued in 1997 because plutonium has not been used at the site, and sample results have continuously been at background levels since sampling began in 1972. The use of established sampling locations is preferred, when possible, from year to year because it allows us to determine more meaningful trends in data.

Sampling locations at areas with known or suspected contaminants were monitored to delimit the extent of the contaminants and to track the contaminants from year to year. For example, six surface soil sampling locations are located near the Livermore Water Reclamation Plant (LWRP) to monitor soils that contain slightly elevated plutonium levels originating from resuspension of sludge that contained plutonium contamination from accidental releases to the sewer, from 1967 and earlier years.

Surface soil sampling is conducted according to written, standardized procedures contained in the Environmental Monitoring Plan (Tate et al. 1999). Samples are collected from undisturbed areas near the permanent sampling location marker. These areas generally are level, free of rocks, and unsheltered by trees or buildings. The sampling technicians choose two 1-m squares from which to collect the sample and record how far away and in what direction from the permanent marker the sample is collected. Each 


\section{Soil and Sediment Monitoring}

sample is a composite consisting of 10 subsamples that are collected with an $8.25-\mathrm{cm}-$ diameter stainless steel core sampler at the corners and the center of each square. All subsamples are collected from the top $5 \mathrm{~cm}$ of soil because surface deposition from the air is the primary pathway for potential contamination.

Quality assurance (QA) duplicate samples are submitted with each batch of soil samples. At locations chosen for duplicate sampling, two identical samples are obtained by collecting adjacent cores from the corners and center of the sampling squares. Separate composites of 10 cores each are made, and the duplicate samples are identified with unique sample identifier codes.

Surface soil samples are dried, ground, sieved, and homogenized. Samples are analyzed by LLNL's Chemistry and Materials Science Environmental Services (CES) laboratory. The plutonium content of a 100-g sample aliquot is determined by alpha spectroscopy (Hall and Edwards 1994c). Other sample aliquots (300 g) are analyzed for more than 150 radionuclides by gamma spectroscopy, using a high-purity germanium (HPGe) detector (Hall and Edwards 1994a, b, and c). Only those nuclides measured above detection limits or of particular interest are reported. The 10-g subsamples of samples from Site 300 are sent to a contract analytical laboratory and are analyzed by graphitefurnace atomic absorption spectroscopy for beryllium. Chain-of-custody procedures are followed throughout the sampling, delivery, and analytical processes.

\section{Surface Sediment Methods}

Surface samples of a sediment are collected from arroyos and storm water drainages at and around the Livermore site after the cessation of spring runoff. For 1999, samples were analyzed for radionuclides.

Sediment was sampled from seven Livermore site drainages. Location ALPO was covered in water throughout the sampling period and was not sampled (see Figure 10-3, in the main volume). The sediment sampling locations coincide with storm water runoff sampling locations so that the sampling results from these two media can be compared.

All surface sediment locations are marked by a permanent location marker, which serves as a reference point for each sampling location. Ten subsamples, 5-cm deep, are collected at 1-m intervals along a transect of the arroyo or drainage channel. At one of the subsample locations, a 15-cm-deep sample is acquired for tritium analysis. The sample collection technicians record how far away and in what direction from the 
permanent marker the samples are actually collected. As with soil samples, QA samples are submitted with each batch of sediment samples.

Samples are analyzed by LLNL's CES laboratory. For samples collected for tritium analyses, CES uses freeze-drying techniques to recover water from the samples and determines the tritium content of the water by liquid-scintillation counting. The plutonium content of a sample aliquot is determined by alpha spectroscopy. Other sample aliquots are analyzed for radionuclides using gamma spectroscopy as described above for surface soil samples. The radioanalytical methods employed by the CES laboratory enable detection of concentrations at levels far more sensitive than regulatory limits. Chain-of-custody procedures are followed throughout the sampling, delivery, and analytical processes.

\section{Vadose Zone Soil Methods}

Vadose zone soil samples are collected at the same locations as the surface sediments. One of the 10 surface subsample locations is selected for collection of the deeper vadose zone samples. A hand auger is used to collect a 30- to 45-cm-deep sample, which is submitted for analysis for total and soluble metals by EPA Methods 200.7, 245.2, 7471A and 6010B. Using an electric drive, a sample is collected at $45-65 \mathrm{~cm}$ deep for analysis for organic compounds by EPA Method 8240. Chain-of-custody procedures are followed throughout the sampling, delivery, and analytical processes.

\section{Data}

Table 10-1 presents the analytical data for radionuclides for surface soil and sediment samples collected in 1999 in the Livermore Valley and Livermore site. Table 10-2 presents the data, which include radionuclides and beryllium, for samples collected at Site 300. The data generally reflect historic data values for these analytes at these locations. A detailed discussion of these results is provided in the main volume of this report. Tables 10-3 through 10-5 list background levels for total and soluble metals in soils and sediments and de minimis concentrations for soluble metals and organics. Table 10-6 presents analytical values for semi-volatile organic compounds measured by EPA Method 8240 in Livermore site sediments. Tables 10-7 and 10-8 give results for total and dissolved metals, respectively. 


\section{Soil and Sediment Monitoring}

Table 10-1. Radionuclides in soils and sediments in the Livermore Valley, 1999.

\begin{tabular}{|c|c|c|c|c|}
\hline $\begin{array}{l}\text { Location } \\
\text { identifier }\end{array}$ & $\begin{array}{l}\text { Plutonium-238 } \\
(\mu \mathrm{Bq} / \text { dry g) }\end{array}$ & $\begin{array}{c}\text { Plutonium-239+240 } \\
(\mu \mathrm{Bq} / \text { dry } \mathrm{g})\end{array}$ & $\begin{array}{c}\text { Americium-241 } \\
\text { (mBq/dry g) }\end{array}$ & $\begin{array}{l}\text { Cesium-137 } \\
\text { (mBq/dry g) }\end{array}$ \\
\hline \multicolumn{5}{|c|}{ Livermore Valley soils } \\
\hline L-AMON-SO & $2.7 \pm 1.4$ & $72.5 \pm 7.7$ & -(d) $^{(\mathrm{d})}$ & $2.9 \pm 0.3$ \\
\hline L-CHUR-SO & $4.4 \pm 1.8$ & $73.6 \pm 7.8$ & -(d) $^{\text {(d) }}$ & $6.1 \pm 0.5$ \\
\hline L-COW-SO & $2.1 \pm 1.4$ & $23.1 \pm 4.4$ & -(d) $^{(\mathrm{C}}$ & $<0.13$ \\
\hline L-FCC-SO & $2.6 \pm 1.6$ & $79.6 \pm 9.0$ & -(d) $^{(\mathrm{C}}$ & $2.3 \pm 0.3$ \\
\hline L-HOSP-SO & $1.4 \pm 1.1$ & $42.6 \pm 6.0$ & -(d) $^{(\mathrm{C}}$ & $1.5 \pm 0.4$ \\
\hline L-MESQ-SO & $1.2 \pm 1.1$ & $28.3 \pm 4.9$ & -(d) $^{(\mathrm{C}}$ & $0.69 \pm 0.20$ \\
\hline L-MET-SO & $1.2 \pm 1.1$ & $52.5 \pm 6.7$ & -(d) $^{(\mathrm{n}}$ & $1.6 \pm 0.3$ \\
\hline L-NEP-SO & $3.2 \pm 1.7$ & $64.4 \pm 7.6$ & $-^{(d)}$ & $2.0 \pm 0.4$ \\
\hline L-PATT-SO & $0.64 \pm 0.84$ & $18.9 \pm 4.0$ & -(d) $^{(\mathrm{n}}$ & $0.64 \pm 0.20$ \\
\hline L-SALV-SO & $4.4 \pm 1.9$ & $93.2 \pm 9.0$ & -(d) $^{(\mathrm{c}}$ & $1.0 \pm 0.2$ \\
\hline L-TANK-SO & $6.6 \pm 2.5$ & $125 \pm 11$ & -(d) $^{(\mathrm{C}}$ & $4.2 \pm 0.4$ \\
\hline L-VIS-SO & $22.8 \pm 4.4$ & $507 \pm 28$ & $-^{(d)}$ & $1.0 \pm 0.2$ \\
\hline L-ZON7-SO & $6.1 \pm 2.3$ & $156 \pm 13$ & -(d) $^{(\mathrm{n}}$ & $3.3 \pm 0.4$ \\
\hline Median & 2.7 & 72.5 & -(f) $^{(\mathrm{r}}$ ( & 1.6 \\
\hline Interquartile range & 3.0 & 51 & 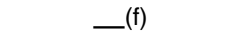 & 1.9 \\
\hline Maximum & 22.8 & 507 & -(f) $^{(\mathrm{n}}$ & 6.1 \\
\hline \multicolumn{5}{|c|}{ LWRP soils } \\
\hline L-WRP1-SO & $354 \pm 22$ & $6960 \pm 290$ & $2.5 \pm 2.1$ & $4.0 \pm 0.3$ \\
\hline L-WRP2-SO & $196 \pm 14$ & $3550 \pm 150$ & $2.9 \pm 2.1$ & $2.4 \pm 0.3$ \\
\hline L-WRP3-SO & $27.6 \pm 4.7$ & $544 \pm 29$ & $<1.0$ & $0.50 \pm 0.32$ \\
\hline L-WRP4-SO & $124 \pm 10$ & $2170 \pm 90$ & $<0.7$ & $1.0 \pm 0.2$ \\
\hline L-WRP5-SO & $91.4 \pm 91$ & $2120 \pm 90$ & $<0.7$ & $1.2 \pm 0.3$ \\
\hline L-WRP6-SO & $33.8 \pm 5.3$ & $588 \pm 32$ & $<1.2$ & $0.46 \pm 0.17$ \\
\hline Median & 108 & 2150 & $<1.1$ & 1.1 \\
\hline Interquartile range & 130 & 2230 & —(g) & 1.4 \\
\hline Maximum & 354 & 6960 & 2.9 & 4.0 \\
\hline \multicolumn{5}{|c|}{ Livermore site sediments } \\
\hline L-ALPE-SD & $6.2 \pm 2.2$ & $173 \pm 13$ & -(d) $^{(\mathrm{d})}$ & $0.72 \pm 0.26$ \\
\hline L-ASS2-SD & $-0.12 \pm 0.48$ & $7.0 \pm 2.3$ & -(d) $^{(\mathrm{n}}$ & $0.24 \pm 0.19$ \\
\hline L-ASW-SD & $0.75 \pm 0.83$ & $7.2 \pm 2.4$ & -(d) $^{(\mathrm{n}}$ & $<0.10$ \\
\hline L-CDB-SD & $42.2 \pm 5.8$ & $607 \pm 32$ & -(d) $^{(\mathrm{n}}$ & $0.53 \pm 0.28$ \\
\hline L-ESB-SD & $211 \pm 15$ & $2180 \pm 90$ & -(d) $^{(\mathrm{n}}$ & $0.46 \pm 0.19$ \\
\hline L-GRNE-SD & $5.0 \pm 2.1$ & $84 \pm 9$ & -(d) $^{(\mathrm{c}}$ & $1.3 \pm 0.2$ \\
\hline L-WPDC-SD & $0.44 \pm 0.82$ & $7.4 \pm 2.4$ & -(d) $^{(\mathrm{n}}$ & $<0.12$ \\
\hline Median & 5.0 & 84 & -(f) $^{(\mathrm{f})}$ & 0.46 \\
\hline Interquartile range & 23.6 & 382 & 一(f) $^{(\mathrm{f}}$ & 0.44 \\
\hline Maximum & 211 & 2180 & 一(f) $^{(\mathrm{f}}$ & 1.3 \\
\hline
\end{tabular}

Note: Radioactivities are reported as the measured concentration and either an uncertainty ( $\pm 2 \sigma$ counting error) or as being less than or equal to the detection limit. If the concentration is less than or equal to the uncertainty or the detection limit, the result is considered to be a nondetection. See the main volume, Chapter 14, Quality Assurance.

a Thorium-232 activities in Bq/dry g can be determined by dividing the weight in $\mu \mathrm{g} / \mathrm{dry} \mathrm{g}$ by 247.3 , and $\mathrm{pCi} / \mathrm{dry} \mathrm{g}$ can be determined by dividing by 9.15 .

b Uranium-235 activities in Bq/dry g were determined by dividing the weight in $\mu \mathrm{g} / \mathrm{dry} \mathrm{g}$ by 12.5 , and in $\mathrm{pCi} / \mathrm{dry} \mathrm{g}$ by dividing by 0.463 . 


\begin{tabular}{|c|c|c|c|c|c|}
\hline $\begin{array}{c}\text { Potassium-40 } \\
\text { (Bq/dry g) }\end{array}$ & $\begin{array}{c}\text { Tritium } \\
(\mathrm{Bq} / \mathrm{L})\end{array}$ & $\begin{array}{c}\text { Thorium-232(a) } \\
(\mu \mathrm{g} / \mathrm{dry} \mathrm{g})\end{array}$ & $\begin{array}{c}\text { Uranium-235(b) } \\
(\mu \mathrm{g} / \mathrm{dry} \mathrm{g})\end{array}$ & $\begin{array}{c}\text { Uranium-238(c) } \\
(\mu \mathrm{g} / \mathrm{dry} \mathrm{g})\end{array}$ & $\begin{array}{l}\text { Uranium- } \\
235 / 238\end{array}$ \\
\hline \multicolumn{6}{|c|}{ Livermore Valley soils } \\
\hline $0.581 \pm 0.013$ & -(e) $^{(\mathrm{e})}$ & $9.2 \pm 0.2$ & $0.022 \pm 0.009$ & $1.8 \pm 1.3$ & $0.012 \pm 0.010$ \\
\hline $0.485 \pm 0.014$ & -(e) & $8.0 \pm 0.2$ & $0.024 \pm 0.012$ & $2.5 \pm 1.7$ & $0.009 \pm 0.008$ \\
\hline $0.466 \pm 0.013$ & —(e) & $7.5 \pm 0.2$ & $0.015 \pm 0.008$ & $1.3 \pm 0.9$ & $0.012 \pm 0.010$ \\
\hline $0.385 \pm 0.012$ & -(e) $^{(\mathrm{e})}$ & $6.1 \pm 0.2$ & $0.017 \pm 0.009$ & $1.2 \pm 1.1$ & $0.013 \pm 0.014$ \\
\hline $0.418 \pm 0.013$ & -(e) $^{(\mathrm{e})}$ & $5.4 \pm 0.2$ & $0.015 \pm 0.012$ & $1.2 \pm 1.0$ & $0.013 \pm 0.015$ \\
\hline $0.474 \pm 0.014$ & -(e) $^{(\mathrm{e})}$ & $7.9 \pm 0.2$ & $0.023 \pm 0.011$ & $2.2 \pm 1.7$ & $0.011 \pm 0.010$ \\
\hline $0.544 \pm 0.017$ & —(e) & $7.7 \pm 0.2$ & $0.019 \pm 0.011$ & $1.7 \pm 1.2$ & $0.011 \pm 0.010$ \\
\hline $0.477 \pm 0.014$ & —(e) & $7.2 \pm 0.2$ & $0.020 \pm 0.011$ & $2.0 \pm 1.2$ & $0.010 \pm 0.008$ \\
\hline $0.518 \pm 0.011$ & —(e) $^{(\mathrm{e}}$ & $7.4 \pm 0.2$ & $0.017 \pm 0.011$ & $1.5 \pm 0.8$ & $0.011 \pm 0.009$ \\
\hline $0.422 \pm 0.012$ & -(e) $^{(\mathrm{e})}$ & $8.6 \pm 0.2$ & $0.024 \pm 0.010$ & $2.5 \pm 0.9$ & $0.010 \pm 0.005$ \\
\hline $0.295 \pm 0.011$ & -(e) $^{(\mathrm{e})}$ & $6.7 \pm 0.2$ & $0.020 \pm 0.013$ & $1.5 \pm 1.2$ & $0.013 \pm 0.014$ \\
\hline $0.403 \pm 0.010$ & - $^{(\mathrm{e})}$ & $6.8 \pm 0.2$ & $0.016 \pm 0.008$ & $1.6 \pm 0.8$ & $0.010 \pm 0.007$ \\
\hline $0.418 \pm 0.011$ & -(e) $^{(\mathrm{e})}$ & $7.7 \pm 0.2$ & $0.020 \pm 0.008$ & $1.6 \pm 1.2$ & $0.012 \pm 0.010$ \\
\hline 0.466 & - (f) $^{(\mathrm{f})}$ & 7.5 & 0.020 & 1.6 & - (f) $^{(\mathrm{s})}$ \\
\hline 0.067 & 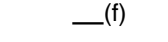 & 1.2 & 0.005 & 0.5 & -(f) \\
\hline 0.581 & - (f) $^{(1)}$ & 9.2 & 0.024 & 2.5 & -(f) $^{(\mathrm{f}}$ \\
\hline \multicolumn{6}{|c|}{ LWRP soils } \\
\hline $0.407 \pm 0.011$ & -(e) $^{(\mathrm{e})}$ & $7.8 \pm 0.2$ & $0.020 \pm 0.011$ & $1.9 \pm 1.0$ & $0.011 \pm 0.008$ \\
\hline $0.323 \pm 0.012$ & -(e) $^{(\mathrm{e})}$ & $7.1 \pm 0.2$ & $0.019 \pm 0.009$ & $2.2 \pm 1.0$ & $0.009 \pm 0.006$ \\
\hline $0.345 \pm 0.013$ & -(e) $^{(\mathrm{e})}$ & $7.7 \pm 0.2$ & $0.018 \pm 0.010$ & $2.1 \pm 1.3$ & $0.009 \pm 0.007$ \\
\hline $0.337 \pm 0.013$ & -(e) $^{(\mathrm{e})}$ & $7.3 \pm 0.2$ & $0.018 \pm 0.009$ & $1.6 \pm 1.0$ & $0.011 \pm 0.009$ \\
\hline $0.374 \pm 0.013$ & -(e) $^{(\mathrm{e})}$ & $7.0 \pm 0.2$ & $0.020 \pm 0.005$ & $2.0 \pm 1.4$ & $0.010 \pm 0.007$ \\
\hline $0.381 \pm 0.012$ & -(e) & $6.1 \pm 0.2$ & $0.017 \pm 0.008$ & $2.0 \pm 1.5$ & $0.008 \pm 0.007$ \\
\hline 0.359 & -(f) $^{(\mathrm{f}}$ & 7.2 & 0.018 & 2.0 & -(f) $^{(\mathrm{f})}$ \\
\hline 0.040 & -(f) $^{(1)}$ & 0.6 & 0.002 & 0.2 & -(f) $^{(\mathrm{n}}$ \\
\hline 0.407 & 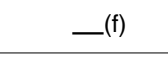 & 7.8 & 0.020 & 2.2 & 一(f) $^{(\mathrm{n}}$ \\
\hline \multicolumn{6}{|c|}{ Livermore site sediments } \\
\hline $0.361 \pm 0.012$ & $20.9 \pm 3.0$ & $5.9 \pm 0.2$ & $0.017 \pm 0.009$ & $1.9 \pm 1.1$ & $0.009 \pm 0.007$ \\
\hline $0.529 \pm 0.014$ & $<0.5 \pm 2.2$ & $3.9 \pm 0.1$ & $0.011 \pm 0.008$ & $<0.6$ & $0.019 \pm 0.014$ \\
\hline $0.466 \pm 0.014$ & $<1.4 \pm 2.3$ & $3.9 \pm 0.2$ & $0.014 \pm 0.013$ & $1.2 \pm 0.9$ & $0.012 \pm 0.014$ \\
\hline $0.440 \pm 0.016$ & $75.1 \pm 4.4$ & $7.5 \pm 0.3$ & $0.024 \pm 0.007$ & $2.0 \pm 0.8$ & $0.012 \pm 0.006$ \\
\hline $0.407 \pm 0.009$ & $68.1 \pm 4.3$ & $6.2 \pm 0.1$ & $0.015 \pm 0.009$ & $2.7 \pm 1.3$ & $0.006 \pm 0.004$ \\
\hline $0.466 \pm 0.010$ & $<2.7 \pm 2.3$ & $5.8 \pm 0.2$ & $0.016 \pm 0.010$ & $1.2 \pm 0.8$ & $0.013 \pm 0.012$ \\
\hline $0.485 \pm 0.014$ & $4.5 \pm 2.5$ & $8.6 \pm 0.2$ & $0.024 \pm 0.014$ & $2.0 \pm 1.4$ & $0.012 \pm 0.011$ \\
\hline 0.466 & 4.5 & 5.9 & 0.016 & 1.9 & -(f) $^{(\mathrm{n}}$ \\
\hline 0.052 & -(g) & 2.0 & 0.006 & 0.8 & -(f) $^{(1)}$ \\
\hline 0.529 & 75.1 & 8.6 & 0.024 & 2.7 & -(f) $^{(1)}$ \\
\hline
\end{tabular}

c Uranium-238 activities in Bq/dry g were determined by dividing the weight in $\mu \mathrm{g} / \mathrm{dry} \mathrm{g}$ by 80.3 , and in pCi/dry $\mathrm{g}$ by dividing by 2.97 .

d Americium-241was detected only in LWRP samples.

e Only sediment samples are analyzed for tritium.

f Not calculated.

$\mathrm{g}$ Interquartile range not calculated because of high incidence of reported values below detection limits. 


\section{Soil and Sediment Monitoring}

Table 10-2. Radionuclides and beryllium in soils at Site 300, 1999.

\begin{tabular}{|c|c|c|c|c|c|c|c|}
\hline $\begin{array}{l}\text { Location } \\
\text { identifier }\end{array}$ & $\begin{array}{l}\text { Cesium-137 } \\
\text { (mBq/dry g) }\end{array}$ & $\begin{array}{l}\text { Potassium-40 } \\
\text { (Bq/dry g) }\end{array}$ & $\begin{array}{c}\text { Thorium-232 }^{(a)} \\
(\mu \mathrm{g} / \mathrm{dry} g)\end{array}$ & $\begin{array}{l}\text { Uranium-235 } \\
(\mu \mathrm{g}) \\
(\mathrm{dry} g)\end{array}$ & $\begin{array}{l}\text { Uranium-238 } \\
(\mu \mathrm{c}) \\
\left.{ }^{\prime} / \mathrm{dry} g\right)\end{array}$ & $\begin{array}{l}\text { Beryllium } \\
\text { (mg/kg) }\end{array}$ & $\begin{array}{l}\text { Uranium } \\
235 / 238\end{array}$ \\
\hline 3-801E-SO & $1.4 \pm 0.3$ & $0.396 \pm 0.012$ & $9.6 \pm 0.2$ & $0.021 \pm 0.010$ & $1.5 \pm 1.2$ & 0.62 & $0.014 \pm 0.012$ \\
\hline $3-801 \mathrm{~N}-\mathrm{SO}$ & $2.4 \pm 0.4$ & $0.440 \pm 0.013$ & $12.4 \pm 0.3$ & $0.037 \pm 0.014$ & $6.9 \pm 3.0$ & 1.0 & $0.005 \pm 0.003$ \\
\hline 3-801W-SO & $3.6 \pm 0.4$ & $0.474 \pm 0.015$ & $10.1 \pm 0.3$ & $0.028 \pm 0.013$ & $4.8 \pm 1.7$ & 0.64 & $0.006 \pm 0.003$ \\
\hline $3-812 N-S O$ & $1.1 \pm 0.3$ & $0.392 \pm 0.015$ & $12.6 \pm 0.4$ & $0.148 \pm 0.008$ & $71.3 \pm 2.8$ & 2.5 & $0.002 \pm 0.0001$ \\
\hline 3-834W-SO & $3.2 \pm 0.4$ & $0.444 \pm 0.017$ & $11.6 \pm 0.3$ & $0.016 \pm 0.013$ & $1.1 \pm 0.9$ & 0.9 & $0.014 \pm 0.016$ \\
\hline $3-851 \mathrm{~N}-\mathrm{SO}$ & $1.8 \pm 0.3$ & $0.433 \pm 0.016$ & $14.2 \pm 0.4$ & $0.033 \pm 0.019$ & $2.9 \pm 1.6$ & 0.8 & $0.011 \pm 0.009$ \\
\hline $3-856 \mathrm{~N}-\mathrm{SO}$ & $3.2 \pm 0.3$ & $0.377 \pm 0.013$ & $10.6 \pm 0.3$ & $0.023 \pm 0.012$ & $2.0 \pm 1.2$ & 0.8 & $0.012 \pm 0.009$ \\
\hline 3-858S-SO & $5.4 \pm 0.3$ & $0.440 \pm 0.018$ & $9.5 \pm 0.3$ & $0.029 \pm 0.014$ & $2.8 \pm 1.8$ & 0.56 & $0.010 \pm 0.008$ \\
\hline 3-DSW-SO & $5.1 \pm 0.5$ & $0.414 \pm 0.013$ & $9.3 \pm 0.3$ & $0.033 \pm 0.011$ & $3.5 \pm 1.5$ & 0.6 & $0.009 \pm 0.005$ \\
\hline 3-EOBS-SO & $1.4 \pm 0.3$ & $0.500 \pm 0.013$ & $10.2 \pm 0.2$ & $0.025 \pm 0.011$ & $1.3 \pm 1.2$ & 0.8 & $0.019 \pm 0.019$ \\
\hline 3-EVAP-SO & $2.3 \pm 0.4$ & $0.377 \pm 0.009$ & $12.3 \pm 0.2$ & $0.035 \pm 0.013$ & $4.0 \pm 1.1$ & 0.8 & $0.009 \pm 0.004$ \\
\hline 3-GOLF-SO & $5.6 \pm 0.4$ & $0.492 \pm 0.013$ & $8.9 \pm 0.2$ & $0.019 \pm 0.008$ & $1.4 \pm 1.0$ & 0.55 & $0.014 \pm 0.012$ \\
\hline 3-NPS-SO & $4.5 \pm 0.5$ & $0.574 \pm 0.017$ & $8.5 \pm 0.2$ & $0.020 \pm 0.011$ & $2.3 \pm 1.5$ & 0.54 & $0.009 \pm 0.008$ \\
\hline 3-PRIM-SO & $1.0 \pm 0.3$ & $0.525 \pm 0.014$ & $10.8 \pm 0.2$ & $0.025 \pm 0.011$ & $2.2 \pm 1.3$ & 0.56 & $0.011 \pm 0.008$ \\
\hline 3-WOBS -SO & $4.0 \pm 0.3$ & $0.396 \pm 0.013$ & $8.0 \pm 0.2$ & $0.017 \pm 0.010$ & $1.9 \pm 1.5$ & 0.63 & $0.009 \pm 0.009$ \\
\hline Median & 3.2 & 0.440 & 10.2 & 0.025 & 2.3 & 0.6 & -(d) \\
\hline $\begin{array}{l}\text { Interquartile } \\
\text { range }\end{array}$ & 2.6 & 0.087 & 2.5 & 0.012 & 2.1 & 0.2 & -(d) $^{(\mathrm{d})}$ \\
\hline Maximum & 5.6 & 0.574 & 14.2 & 0.148 & 71.3 & 2.5 & - (d) $^{(-1)}$ \\
\hline
\end{tabular}

Note: Radioactivities are reported as the measured concentration and either an uncertainty ( $\pm 2 \sigma$ counting error) or as being less than or equal to the detection limit. If the concentration is less than or equal to the uncertainty or the detection limit, the result is considered to be a nondetection. See the main volume, Chapter 14, Quality Assurance.

a Thorium-232 activities in Bq/dry g can be determined by dividing the weight in $\mu \mathrm{g} / \mathrm{dry} \mathrm{g}$ by 247.3 , and $\mathrm{pCi} / \mathrm{dry} \mathrm{g}$ can be determined by dividing by 9.15 .

b Uranium-235 activities in Bq/dry g can be determined by dividing the weight in $\mu \mathrm{g} / \mathrm{dry} \mathrm{g}$ by 12.5 , and $\mathrm{pCi} / \mathrm{dry} \mathrm{g}$ can be determined by dividing by 0.463 .

c Uranium-238 activities in Bq/dry g can be determined by dividing the weight in $\mu \mathrm{g} / \mathrm{dry} \mathrm{g}$ by 80.3 , and $\mathrm{pCi} / \mathrm{dry} \mathrm{g}$ can be determined by dividing by 2.97 .

d Not calculated. 
Table 10-3. Background screening concentration values for metals in soils at the Livermore site.

\begin{tabular}{|c|c|c|c|}
\hline Metal & $\begin{array}{l}\text { Background } \\
\text { screening value } \\
\text { Total (mg/kg) }\end{array}$ & Metal & $\begin{array}{c}\text { Background } \\
\text { screening value } \\
\text { Soluble (mg/L) }\end{array}$ \\
\hline Antimony & 1.12 & Antimony & Any detection \\
\hline Arsenic & 8.51 & Arsenic & 0.237 \\
\hline Barium & 308 & Barium & 16.7 \\
\hline Beryllium & 0.62 & Beryllium & Any detection \\
\hline Cadmium & 1.59 & Boron & To be determined \\
\hline Chromium & 72.4 & Cadmium & Any detection \\
\hline Chromium(VI) & Any detection & Chromium & 0.727 \\
\hline Cobalt & 14.6 & Cobalt & 0.985 \\
\hline Copper & 62.5 & Copper & 2.6 \\
\hline Lead & 43.7 & Iron & To be determined \\
\hline Mercury & 0.14 & Lead & 0.987 \\
\hline Molybdenum & Any detection & Manganese & To be determined \\
\hline Nickel & 82.8 & Mercury & 0.0063 \\
\hline Selenium & Any detection & Molybdenum & Any detection \\
\hline Silver & Any detection & Nickel & 1.68 \\
\hline Thallium & Any detection & Selenium & Any detection \\
\hline Vanadium & 65.2 & Silver & Any detection \\
\hline \multirow[t]{3}{*}{ Zinc } & 75.3 & Thallium & Any detection \\
\hline & & Vanadium & 1.22 \\
\hline & & Zinc & 4.52 \\
\hline
\end{tabular}

Note: Background values were developed for all soils and sediments at the Livermore site but are used here as a basis for comparison for analytical results for vadose zone soils. 


\section{Soil and Sediment Monitoring}

Table 10-4. De minimis concentration levels for soluble metals found in Livermore site soils.

\begin{tabular}{|l|l|l|c|c|}
\hline Constituents & $\begin{array}{c}\text { Water quality } \\
\text { objective } \\
\text { (mg/L) }\end{array}$ & \multicolumn{1}{|c|}{ Reference } & $\begin{array}{c}\text { Attenuation } \\
\text { factor }\end{array}$ & $\begin{array}{c}\text { De minimis } \\
\text { level } \\
\text { (mg/L) }\end{array}$ \\
\hline $\begin{array}{l}\text { Metals } \\
\text { Antimony }\end{array}$ & 0.006 & Cal Primary MCL (a) & 100 & 0.06 \\
Arsenic & 0.050 & Cal Primary MCL & 100 & 0.5 \\
Barium & 1.0 & Cal Primary MCL & 100 & 10 \\
Beryllium & 0.004 & Cal Primary MCL & 100 & 0.04 \\
Cadmium & 0.005 & Cal Primary MCL & 100 & 0.05 \\
Chromium & 0.05 & Cal Primary MCL & 100 & 0.5 \\
Cobalt & 5 & RWQCB Basin Plan & 100 & 50 \\
Copper & 1 & RWQCB Basin Plan & 1000 & 100 (b) \\
Lead & 0.015 & EPA & 1000 & 1.5 \\
Mercury & 0.002 & Cal Primary MCL & 100 & 0.02 \\
Molybdenum & 0.05 & RWQCB Basin Plan & 100 & 0.5 \\
Nickel & 0.1 & Cal Primary MCL & 100 & 1 \\
Selenium & 0.05 & Cal Primary MCL & 100 & 0.5 \\
Silver & 0.1 & Cal Secondary MCL & 100 & 1 \\
Thallium & 0.002 & Cal Primary MCL & 100 & 0.02 \\
Vanadium & 1 & RWQCB Basin Plan & 100 & 10 \\
Zinc & 5 & Cal Secondary MCL & 1000 & $500^{(\mathbf{b})}$ \\
\hline
\end{tabular}

Note: De minimis values were developed for all soils and sediments at the Livermore site but are used here as a basis for comparison for analytical results for vadose zone soils.

a $\mathrm{MCL}=$ maximum contaminant level in drinking water.

b Although the de minimis leavels are $100 \mathrm{mg} / \mathrm{L}$ for copper and $500 \mathrm{mg} / \mathrm{L}$ for zinc, the hazardous waste limit is $25 \mathrm{mg} / \mathrm{L}$ for copper and $250 \mathrm{mg} / \mathrm{L}$ for zinc. Consequently, soils with soluble concentrations at or above the hazardous waste limit are disposed of as a hazardous waste and not reused on site. 
Table 10-5. De minimis concentration levels for organic and radioactive constituents of concern found in Livermore site soils and sediments.

\begin{tabular}{|c|c|c|c|c|}
\hline Constituents & $\begin{array}{l}\text { Water quality } \\
\text { objective }\end{array}$ & Reference & $\begin{array}{l}\text { Attenuation } \\
\text { factor }\end{array}$ & $\begin{array}{c}\text { De minimis } \\
\text { level }\end{array}$ \\
\hline \multicolumn{5}{|l|}{ Organics $(\mu g / L)$} \\
\hline 1,2-Dichlorobenzene & 600 & EPA Primary $M \mathrm{CL}^{(\mathrm{a})}$ & 100 & 3000 \\
\hline 1,3-Dichlorobenzene & 130 & CA DHS Action Level & 100 & 650 \\
\hline 1-4-Dichlorobenzene & 5 & Cal Primary MCL & 100 & 25 \\
\hline 1,1-Dichloroethane & 5 & Cal Primary MCL & 100 & 25 \\
\hline 1-2-Dichloroethane & 0.5 & Cal Primary MCL & 100 & 2.5 \\
\hline 1,1-Dichloroethene & 6 & Cal Primary MCL & 100 & 30 \\
\hline 1,2-Dichloroethene & 6 & Cal Primary MCL & 100 & 30 \\
\hline cis-1,2-Dichloroethene & 6 & Cal Primary MCL & 100 & 30 \\
\hline trans-1,2-Dichloroethene & 10 & Cal Primary MCL & 100 & 50 \\
\hline 1,1,1-Trichloroethane & 200 & Cal Primary MCL & 100 & 1000 \\
\hline 1,1,2-Trichloroethane & 5 & Cal Primary MCL & 100 & 25 \\
\hline Benzene & 1 & Cal Primary MCL & 100 & 5 \\
\hline Carbon tetrachloride & 0.5 & Cal Primary MCL & 100 & 2.5 \\
\hline Chloroform & 100 & EPA Primary MCL & 100 & 500 \\
\hline Diesel oil/kerosene & 100 & SNARL $(b)$ & 100 & 500 \\
\hline Ethyl benzene & 700 & Cal Primary MCL & 100 & 3500 \\
\hline Freon 11 (trichlorofluoromethane) & 150 & Cal Primary MCL & 100 & 750 \\
\hline Freon 12 (dichlorodifluoromethane) & 1000 & CA DHS Action Level & 100 & 5000 \\
\hline Freon 113 (1,1,2-trichloro-1,2,2-trifluoroethane) & 1200 & Cal Primary MCL & 100 & 6000 \\
\hline Gasoline & 5 & Other $(\mathrm{c})$ & 100 & 25 \\
\hline Methylene chloride & 5 & Cal Primary MCL & 100 & 25 \\
\hline Methyl tertiary-butyl ether (MTBE) & 35 & CA DHS Action Level & 100 & 175 \\
\hline Oil and grease & 25,000 & Other & 100 & 125,000 \\
\hline Tetrachloroethene (PCE) & 5 & Cal Primary MCL & 100 & 25 \\
\hline Toluene & 150 & Cal Primary MCL & 100 & 750 \\
\hline Trichloroethene (TCE) & 5 & Cal Primary MCL & 100 & 25 \\
\hline Xylene(s) & 1750 & Cal Primary MCL & 100 & 8750 \\
\hline PCB (total) & 0.5 & Cal Primary MCL & 100 & 2.5 \\
\hline Vinyl chloride & 0.5 & Cal Primary MCL & 100 & 2.5 \\
\hline
\end{tabular}




\section{Soil and Sediment Monitoring}

Table 10-5. De minimis concentration levels for organic and radioactive constituents of concern found in Livermore site soils and sediments (concluded).

\begin{tabular}{|l|c|l|r|r|}
\hline \multicolumn{1}{|c|}{ Constituents } & $\begin{array}{c}\text { Water quality } \\
\text { objective }\end{array}$ & Reference & $\begin{array}{c}\text { Attenuation } \\
\text { factor }\end{array}$ & $\begin{array}{c}\text { De minimis } \\
\text { level }\end{array}$ \\
\hline Radioactivity (Bq/L) & & & & \\
Gross alpha & 0.56 & Cal Primary MCL & 100 & 5.6 \\
Gross beta & 1.9 & Cal Primary MCL & 100 & 19 \\
Tritium & 740 & Cal Primary MCL & 100 & 7400 \\
\hline
\end{tabular}

Note: De minimis values were developed for all soils and sediments at the Livermore site but are used here as a basis for comparison for analytical results for vadose zone soils.

a $\mathrm{MCL}=$ Maximum contaminant level.

b SNARL = Suggested No Adverse Response Level.

c Other $=$ Taste and odor threshold for gasoline, and the California Ocean Plan Water Quality Objectives for oil and grease.

Table 10-6. Semi-volatile organic compounds measured by EPA Method 8240 in Livermore site vadose zone soil, 1999.

\begin{tabular}{|l|c|c|c|c|c|c|c|}
\hline \multicolumn{1}{|c|}{ Organic compounds $(\boldsymbol{\mu g} / \mathbf{L})$} & ASS2 & ASW & ALPE & GRNE & WPDC & CDB & ESB \\
\hline 1,1-Dichloroethene & $<10$ & $<10$ & $<10$ & $<10$ & $<10$ & $<10$ & $<10$ \\
1,2-Dichloroethane & $<2.5$ & $<2.5$ & $<2.5$ & $<2.5$ & $<2.5$ & $<2.5$ & $<2.5$ \\
1-4-Dichlorobenzene & $<10$ & $<10$ & $<10$ & $<10$ & $<10$ & $<10$ & $<10$ \\
2-Butanone & $<10$ & $<10$ & $<10$ & $<10$ & $<10$ & $<10$ & $<10$ \\
Benzene & $<5$ & $<5$ & $<5$ & $<5$ & $<5$ & $<5$ & $<5$ \\
Carbon tetrachloride & $<2.5$ & $<2.5$ & $<2.5$ & $<2.5$ & $<2.5$ & $<2.5$ & $<2.5$ \\
Chlorobenzene & $<10$ & $<10$ & $<10$ & $<10$ & $<10$ & $<10$ & $<10$ \\
Chloroform & $<10$ & $<10$ & $<10$ & $<10$ & $<10$ & $<10$ & $<10$ \\
Trichloroethene & $<10$ & $<10$ & $<10$ & $<10$ & $<10$ & $<10$ & $<10$ \\
Vinyl chloride & $<10$ & $<10$ & $<10$ & $<10$ & $<10$ & $<10$ & $<10$ \\
\hline
\end{tabular}


Table 10-7. Total metals in Livemore site vadose zone soil, 1999.

\begin{tabular}{|c|c|c|c|c|c|c|c|}
\hline \multirow{3}{*}{$\begin{array}{l}\text { Total } \\
\text { metals } \\
(\mathrm{mg} / \mathrm{kg})\end{array}$} & \multicolumn{2}{|c|}{ Arroyo Seco } & \multicolumn{3}{|c|}{ Arroyo Las Positas } & \multirow{2}{*}{\multicolumn{2}{|c|}{$\begin{array}{c}\text { Drainage Retention Basin } \\
\text { Influent }\end{array}$}} \\
\hline & Influent & Effluent & & & Effluent & & \\
\hline & ASS2 & ASW & ALPE & GRNE & WPDC & CDB & ESB \\
\hline Antimony & $<1$ & $<1$ & $<1$ & $<1$ & $<1$ & $<1$ & $<1$ \\
\hline Arsenic & 3.3 & 3.5 & 3.8 & 2.7 & 5.8 & 4.8 & 3.5 \\
\hline Barium & 53 & 100 & 520 & 140 & 220 & 150 & 130 \\
\hline Beryllium & $<0.5$ & $<0.5$ & $<0.5$ & $<0.5$ & $<0.5$ & $<0.5$ & $<0.5$ \\
\hline Cadmium & $<0.1$ & $<0.1$ & 0.1 & 0.1 & 0.4 & $<0.1$ & 0.2 \\
\hline Chromium & 22 & 22 & 21 & 11 & 35 & 27 & 24 \\
\hline Cobalt & 6 & 7 & 9 & 6 & 11 & 8 & 10 \\
\hline Copper & 11 & 14 & 13 & 7 & 25 & 17 & 13 \\
\hline Lead & $<10$ & $<10$ & $<10$ & 10 & $<10$ & $<10$ & $<10$ \\
\hline Mercury & $<0.05$ & $<0.05$ & $<0.05$ & $<0.05$ & $<0.05$ & $<0.05$ & $<0.05$ \\
\hline Molybdenum & $<5$ & $<5$ & $<5$ & $<5$ & $<5$ & $<5$ & $<5$ \\
\hline Nickel & 30 & 30 & 30 & 10 & 40 & 30 & 40 \\
\hline Potassium & 800 & 800 & 900 & 600 & 1800 & 1800 & 800 \\
\hline Selenium & $<2.5$ & $<2.5$ & $<2.5$ & $<2.5$ & $<2.5$ & $<2.5$ & $<2.5$ \\
\hline Silver & $<2.5$ & $<2.5$ & $<2.5$ & $<2.5$ & $<2.5$ & $<2.5$ & $<2.5$ \\
\hline Thallium & $<1$ & $<1$ & $<1$ & $<1$ & $<1$ & $<1$ & $<1$ \\
\hline Vanadium & 18 & 19 & 32 & 18 & 37 & 34 & 25 \\
\hline Zinc & 47 & 41 & 25 & 20 & 47 & 37 & 45 \\
\hline
\end{tabular}




\section{Soil and Sediment Monitoring}

Table 10-8. Soluble metals in Livermore site vadose zone soil, 1999.

\begin{tabular}{|c|c|c|c|c|c|c|c|}
\hline \multirow{3}{*}{$\begin{array}{c}\text { Soluable } \\
\text { metals } \\
\text { (mg/L) }\end{array}$} & \multicolumn{2}{|c|}{ Arroyo Seco } & \multicolumn{3}{|c|}{ Arroyo Las Positas } & \multirow{2}{*}{\multicolumn{2}{|c|}{$\begin{array}{c}\text { Drainage Retention Basin } \\
\text { Influent }\end{array}$}} \\
\hline & Influent & Effluent & \multicolumn{2}{|c|}{ Influent } & \multirow{2}{*}{$\begin{array}{c}\text { Effluent } \\
\text { WPDC }\end{array}$} & & \\
\hline & ASS2 & ASW & ALPE & GRNE & & CDB & ESB \\
\hline Antimony & $<0.06$ & $<0.06$ & $<0.06$ & $<0.06$ & $<0.06$ & $<0.06$ & $<0.06$ \\
\hline Arsenic & 0.07 & 0.08 & $<0.05$ & 0.1 & $<0.05$ & $<0.05$ & 0.06 \\
\hline Barium & 3.3 & 4.2 & 6.3 & 5.6 & 7.6 & 5.7 & 6.2 \\
\hline Beryllium & $<0.04$ & $<0.04$ & $<0.04$ & $<0.04$ & $<0.04$ & $<0.04$ & $<0.04$ \\
\hline Cadmium & $<0.05$ & $<0.05$ & $<0.05$ & $<0.05$ & $<0.05$ & $<0.05$ & $<0.05$ \\
\hline Chromium & $<0.5$ & $<0.5$ & $<0.5$ & $<0.5$ & $<0.5$ & $<0.5$ & $<0.5$ \\
\hline Cobalt & $<0.5$ & $<0.5$ & $<0.5$ & $<0.5$ & $<0.5$ & $<0.5$ & $<0.5$ \\
\hline Copper & $<0.5$ & 0.6 & $<0.5$ & $<0.5$ & $<0.5$ & $<0.5$ & $<0.5$ \\
\hline Lead & $<0.5$ & $<0.5$ & $<0.5$ & 0.5 & $<0.5$ & $<0.5$ & $<0.5$ \\
\hline Mercury & $<0.005$ & $<0.005$ & $<0.005$ & $<0.005$ & $<0.005$ & $<0.005$ & $<0.005$ \\
\hline Molybdenum & $<0.5$ & $<0.5$ & $<0.5$ & $<0.5$ & $<0.5$ & $<0.5$ & $<0.5$ \\
\hline Nickel & $<0.5$ & $<0.5$ & 0.6 & $<0.5$ & 0.5 & $<0.5$ & 0.6 \\
\hline Potassium & 17 & 16 & 7 & 13 & 9 & 13 & 13 \\
\hline Selenium & $<0.2$ & $<0.2$ & $<0.2$ & $<0.2$ & $<0.2$ & $<0.2$ & $<0.2$ \\
\hline Silver & $<0.5$ & $<0.5$ & $<0.5$ & $<0.5$ & $<0.5$ & $<0.5$ & $<0.5$ \\
\hline Thallium & $<0.03$ & $<0.02$ & $<0.02$ & $<0.02$ & $<0.02$ & $<0.02$ & $<0.02$ \\
\hline Vanadium & $<0.5$ & $<0.5$ & $<0.5$ & $<0.5$ & $<0.5$ & $<0.5$ & $<0.5$ \\
\hline Zinc & 1 & 0.7 & $<0.5$ & $<0.5$ & $<0.5$ & $<0.5$ & 0.9 \\
\hline
\end{tabular}




\section{0}

\section{Vegetation and Foodstuff Monitoring}

S. Ring Peterson

\section{Introduction}

This chapter discusses the sampling methods Lawrence Livermore National Laboratory uses to monitor vegetation and wine, including a description of how sampling locations are selected, what types and amounts of samples are collected, and the process for handling the samples. Of the radionuclides released to the environment from LLNL activities, tritium is the only one that can be measured in vegetation and foodstuff and, therefore, is the only radionuclide monitored.

\section{Vegetation Sampling Methods}

The vegetation sampling locations that Lawrence Livermore National Laboratory chose were areas with ample living vegetation during most of the year. Sampling locations were distant from buildings or other obstructions that could have caused unusual patterns of airflow or precipitation. Irrigated or shaded areas were also avoided. Practical considerations, such as access during inclement weather and personnel safety during vehicle operation or sample collection, also affected selection of sampling locations.

Sampling locations for 1999 were the same as in 1998 and are listed in Table 11-1. The routine vegetation sampling locations are designated with permanent location markers. Consistent use of the same general sampling locations allows LLNL to determine more meaningful trends in data and to monitor areas of concern more closely. For example, every year at Site 300, LLNL examines vegetation from areas where tritium is known to be present in the subsurface soil.

Vegetation sampling is conducted according to written, standardized procedures contained in the Environmental Monitoring Plan (Tate et al. 1999). In 1999, vegetation samples consisted of annual grasses and weeds. LLNL collected approximately 340 to $450 \mathrm{~g}$ of vegetation with relatively high water content for each analysis. Standard chainof-custody procedures were followed. 


\section{Vegetation and Foodstuff Monitoring}

Samples were delivered on the day of collection to LLNL's Chemistry and Materials Science Environmental Services laboratory and were kept frozen prior to processing. Water from the vegetation was collected using freeze-drying techniques (lyophilization), and the tritium content of the water was determined by liquid-scintillation counting.

Approximately $10 \%$ of the sites was sampled in duplicate to comply with quality assurance protocols. Duplicate samples were preserved, stored, processed, and analyzed with methods identical to those employed for all other samples.

\section{Wine Sampling Methods}

California is divided into nine wine-growing regions (including Livermore), and Europe is divided into 13 (Tate et al. 1999). LLNL purchased 12 wines from the Livermore Valley, one wine from each of six California wine growing regions (excluding Livermore), and one wine from each of four European wine growing regions and submitted them for tritium analyses (see Table 11-2). The selection of wines from a particular wine-growing region was random. An equal mix of red and white wines was selected from the Livermore Valley, other California wine-growing areas, and Europe. Any estate wine from a designated area was considered representative of that area. Samples were purchased in 750-mL or 1-L bottles. Approximately $10 \%$ of the total complement of wines was sampled in duplicate to comply with quality assurance protocols. Because of the importance of the wine-sampling network, LLNL either samples or archives as many of the available Livermore Valley wines as possible.

Wine sampling is conducted according to written, standardized procedures contained in the Environmental Monitoring Plan (Tate et al. 1999). The wine samples were submitted unopened to the laboratory for analysis to prevent potential airborne tritium contamination. Chain-of-custody procedures were followed when delivering the samples and throughout the analytical process. LLNL analyzed wines for tritium using ${ }^{3} \mathrm{He}$ mass spectrometry in the Isotope Sciences Division Noble Gas Mass Spectrometry Laboratory (Surano et al. 1991). Using this highly sensitive method, the minimum detectable tritium concentration is about $0.056 \mathrm{~Bq} / \mathrm{L}(1.5 \mathrm{pCi} / \mathrm{L})$. Conventional scintillation detection systems typically have detection limits between 3.7 and 19 Bq/L (100-500 pCi/L) depending on sample size and counting times. With great care, a scintillation detection system's sensitivity can reach about $1 \mathrm{~Bq} / \mathrm{L}(27 \mathrm{pCi} / \mathrm{L})$; however, this detection level is still not sensitive enough to detect small differences in wine samples. 
Table 11-1. Concentrations of tritium in plant water (Bq/L), 1999.

\begin{tabular}{|c|c|c|c|c|c|c|c|c|}
\hline & \multirow{2}{*}{$\begin{array}{c}\text { First } \\
\text { quarter }\end{array}$} & \multirow{2}{*}{$\begin{array}{l}\text { Second } \\
\text { quarter }\end{array}$} & \multirow{2}{*}{$\begin{array}{l}\text { Third } \\
\text { quarter }\end{array}$} & \multirow{2}{*}{$\begin{array}{l}\text { Fourth } \\
\text { quarter }\end{array}$} & \multirow[b]{2}{*}{ Median } & \multirow{2}{*}{$\begin{array}{l}\text { Inter- } \\
\text { quartile } \\
\text { range }\end{array}$} & \multicolumn{2}{|c|}{ Dose $(\mu S v / y)^{(a)}$} \\
\hline & & & & & & & Median & Maximum \\
\hline \multicolumn{9}{|c|}{ Sampling locations near Livermore site } \\
\hline AQUE & $7.0 \pm 2.0$ & $5.9 \pm 1.8$ & $9.7 \pm 2.9$ & $6.1 \pm 2.6$ & 6.6 & 1.6 & 0.032 & 0.047 \\
\hline VIS & $100 \pm 4.4$ & $19 \pm 2.3$ & $16 \pm 3.1$ & $6.7 \pm 2.7$ & 18 & 26 & 0.085 & 0.48 \\
\hline NPER & $18 \pm 2.3$ & $7.5 \pm 1.9$ & $9.1 \pm 2.9$ & $6.6 \pm 2.6$ & 8.3 & 4.1 & 0.040 & 0.087 \\
\hline MET & $3.4 \pm 1.7$ & $1.8 \pm 1.6$ & $4.7 \pm 2.8$ & $19 \pm 3.1$ & 4.1 & 5.3 & 0.020 & 0.092 \\
\hline MESQ & $6.9 \pm 1.9$ & $1.6 \pm 1.6$ & $0.37 \pm 2.6$ & $8.2 \pm 2.7$ & 4.3 & 5.9 & 0.021 & 0.040 \\
\hline GARD & $5.9 \pm 1.8$ & $2.5 \pm 2.6$ & $0.60 \pm 2.6$ & $2.2 \pm 2.5$ & 2.4 & 1.6 & 0.011 & 0.029 \\
\hline PIN1 & $110 \pm 4.6$ & $26 \pm 2.6$ & $180 \pm 6.5$ & $280 \pm 7.8$ & 150 & 120 & -(b) & -(b) \\
\hline PIN2 & $89 \pm 4.1$ & $15 \pm 2.2$ & $14 \pm 3.1$ & $11 \pm 2.8$ & 15 & 20 & -(b) & -(b) \\
\hline \multicolumn{9}{|c|}{ Sampling locations at an intermediate distance from Livermore site } \\
\hline PATT & $-0.75 \pm 1.5$ & $1.4 \pm 1.6$ & $4.9 \pm 2.8$ & $0.93 \pm 2.4$ & 1.2 & 1.8 & 0.0056 & 0.024 \\
\hline ZON7 & $21 \pm 2.5$ & $6.7 \pm 1.8$ & $5.4 \pm 2.8$ & $2.5 \pm 2.5$ & 6.1 & 5.6 & 0.029 & 0.010 \\
\hline 1580 & $100 \pm 4.4$ & $0.83 \pm 1.5$ & $1.3 \pm 2.6$ & $0.076 \pm 2.4$ & 1.1 & 25 & 0.0052 & 0.49 \\
\hline TESW & $0.52 \pm 1.6$ & $1.4 \pm 1.6$ & $0.70 \pm 2.6$ & $3.0 \pm 2.7$ & 1.1 & 1.1 & 0.0051 & 0.015 \\
\hline \multicolumn{9}{|c|}{ Sampling locations far from Livermore site } \\
\hline FCC & $0.68 \pm 1.6$ & $-1.8 \pm 1.4$ & $-0.79 \pm 2.6$ & $-2.0 \pm 2.3$ & -1.3 & 1.4 & -(c) & 0.0033 \\
\hline CAL & $-2.8 \pm 1.4$ & $0.34 \pm 1.5$ & $0.065 \pm 2.6$ & $-0.54 \pm 2.4$ & -0.24 & 1.2 & $-(c)$ & 0.0016 \\
\hline PARK & $-1.8 \pm 1.4$ & $-0.070 \pm 1.5$ & $-0.64 \pm 2.6$ & $-3.2 \pm 2.2$ & -1.2 & 1.7 & -(c) & -(c) \\
\hline \multicolumn{9}{|c|}{ Sampling locations at Site $\mathbf{3 0 0}$} \\
\hline CARN & $1.7 \pm 1.5$ & $-1.0 \pm 1.4$ & $1.1 \pm 2.6$ & $-1.3 \pm 2.7$ & 0.050 & 2.3 & 0.00024 & 0.0082 \\
\hline GOLF & $4.0 \pm 1.6$ & $-1.1 \pm 1.4$ & $-0.68 \pm 2.6$ & $-0.88 \pm 2.7$ & -0.78 & 1.4 & $-(c)$ & 0.019 \\
\hline GEO & $1.7 \pm 1.5$ & $-0.0055 \pm 1.4$ & $0.51 \pm 2.6$ & $-0.025 \pm 2.7$ & 0.25 & 0.82 & 0.0012 & 0.0082 \\
\hline DSW & $3.1 \pm 1.6$ & $1.1 \pm 1.6$ & $2.1 \pm 2.7$ & $1.1 \pm 2.8$ & 1.6 & 1.3 & 0.0078 & 0.015 \\
\hline $801 E$ & $-0.45 \pm 1.4$ & $-1.5 \pm 1.4$ & $0.38 \pm 2.6$ & $-0.52 \pm 2.7$ & -0.49 & 0.52 & -(c) & 0.0018 \\
\hline EVAP & $2.9 \pm 1.6$ & $-0.90 \pm 1.4$ & $330 \pm 8.6$ & $480 \pm 10$ & 170 & 370 & 0.810 & 2.3 \\
\hline PRIM & $2.0 \pm 1.5$ & $0.17 \pm 1.5$ & $0.51 \pm 2.6$ & $-1.4 \pm 2.7$ & 1.6 & 1.3 & 0.0078 & 0.0097 \\
\hline
\end{tabular}

Note: Radioactivities are reported as the measured concentration and either an uncertainty $( \pm 2 \sigma$ counting error) or as being less than or equal to the detection limit. If the concentration is less than or equal to the uncertainty or the detection limit, the result is considered to be a nondetection. See the main volume, Chapter 14, Quality Assurance.

a Ingestion dose is based on conservative assumptions that an adult's diet is exclusively vegetables with this tritium concentration, and that meat and milk are derived from livestock fed on grasses with the same concentration of tritium. See Appendix A, Methods of Dose Calculations.

b Doses were not calculated because pine trees are not ingested by human beings; an ingestion dose to the maximally exposed individual was calculated with CAP88 using evapotranspiration from PIN1 as a diffuse source of tritium. The median dose was $8.0 \times 10^{-6} \mu \mathrm{Sv} / \mathrm{y}$, and the maximum dose was $1.5 \times 10^{-5} \mu \mathrm{Sv} / \mathrm{y}$.

c Doses resulting from negative median concentrations are not calculated. 


\section{Vegetation and Foodstuff Monitoring}

Table 11-2. Tritium in retail wine (Bq/L), 1999. (a)

\begin{tabular}{|c|c|c|c|}
\hline \multirow[b]{2}{*}{ Sample } & \multicolumn{3}{|c|}{ Area of production } \\
\hline & Livermore Valley & California & Europe \\
\hline 1 & $0.94 \pm 0.21$ & $0.30 \pm 0.19$ & $0.72 \pm 0.20$ \\
\hline 2 & $1.0 \pm 0.21$ & $0.39 \pm 0.19$ & $1.4 \pm 0.23$ \\
\hline 3 & $1.3 \pm 0.23$ & $0.39 \pm 0.19$ & $1.5 \pm 0.24$ \\
\hline 4 & $1.5 \pm 0.24$ & $0.47 \pm 0.19$ & $1.6 \pm 0.25$ \\
\hline 5 & $1.5 \pm 0.24$ & $0.56 \pm 0.19$ & \\
\hline 6 & $1.7 \pm 0.25$ & $0.57 \pm 0.19$ & \\
\hline 7 & $1.7 \pm 0.25$ & & \\
\hline 8 & $1.9 \pm 0.26$ & & \\
\hline 9 & $2.1 \pm 0.28$ & & \\
\hline 10 & $2.2 \pm 0.29$ & & \\
\hline 11 & $4.9 \pm 0.52$ & & \\
\hline 12 & $8.3 \pm 0.85$ & & \\
\hline Median & 1.7 & 0.43 & 1.5 \\
\hline Interquartile range & 0.68 & 0.15 & 0.30 \\
\hline Mean & 2.4 & 0.45 & 1.3 \\
\hline Standard deviation & 2.1 & 0.11 & 0.40 \\
\hline nSv/y ${ }^{(b)}$ from median concentration & 1.5 & 0.38 & 1.3 \\
\hline $\mathrm{nSv} / \mathrm{y}^{(\mathrm{b})}$ from mean concentration & 2.2 & 0.40 & 1.2 \\
\hline nSv/y ${ }^{(b)}$ from maximum concentration & 7.4 & 0.51 & 1.4 \\
\hline
\end{tabular}

Note: Radioactivities are reported as the measured concentration and either an uncertainty ( $\pm 2 \sigma$ counting error) or as being less than or equal to the detection limit. If the concentration is less than or equal to the uncertainty or the detection limit, the result is considered to be a nondetection. See the main volume, Chapter 14, Quality Assurance.

a A variety of vintages was purchased and analyzed during 1999. The tritium concentrations reported are those at the time the bottle was opened.

b This dose is calculated based on consumption of $52 \mathrm{~L}$ wine per year (see Appendix $A$ ). 


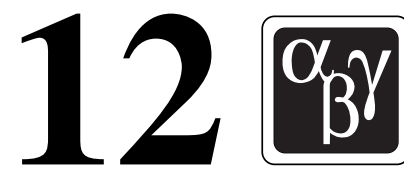

\section{Environmental Radiation Monitoring}

Nicholas A. Bertoldo

\section{Methods of Gamma Radiation Monitoring}

The environmental gamma-radiation dose from terrestrial and cosmic sources is monitored at 14 locations on the laboratory site perimeter, twenty-three locations in the Livermore Valley, eight locations on the Site 300 perimeter, five sites in the vicinity of Site 300, and at two locations in the City of Tracy. Thermoluminescent dosimeters (TLDs) are deployed to the field on a quarterly basis following laboratory preparation. Each TLD is labeled with a Lawrence Livermore National Laboratory dosimeter identification number and placed into a mylar foil sample pouch for protection.

Each sample pouch is then numbered by its field location and mounted on preexisting structures (such as fences) at approximately $1 \mathrm{~m}$ above ground to comply with Environmental Regulatory Guide for Radiological Effluent Monioring and Environmental Surveillance (U.S. Department of Energy 1991). Duplicate trip blanks, transit control TLDs, and calibration control TLDs are also prepared. Upon their removal from the site locations at the end of each quarter, the exposed TLDs are taken to the LLNL Hazards Control dosimetry laboratory for processing. A chain-of-custody form accompanies the field deployment and the collection of the TLDs. Details of the TLD calculations and reporting of external gamma-radiation dose are described in an Operations and Regulatory Affairs Division procedure.

LLNL uses the Panasonic Model UD-814AS1 TLD, which contains three thalliumactivated calcium sulfate crystals $\left(\mathrm{CaSO}_{4}\right)$ and one lithium borate crystal $\left(\mathrm{Li}_{2} \mathrm{~B}_{4} \mathrm{O}_{7}\right)$. The gamma-ray energy imparted to the TLD's crystal elements excite the electrons in the valence band to a higher energy state, creating a vacancy in the valence band known as a "hole." These electron holes are trapped in impurity sites within the crystal. When the TLDs are heated in the analytical laboratory, the thermal energy of the process raises the electron trap to the conduction band or the hole trap to the valence band, causing thermoluminescence. This light intensity is proportional to the original gamma-ray energy imparted to the TLD crystal elements (that is, the TLD absorbed dose) and is measured by the photomultiplier tube output signal. After the TLD is measured, it is reheated and remeasured. A near-zero reading indicates that all the stored energy has been released. This process, called annealing, also verifies that the TLD is again ready 


\section{Environmental Radiation Monitoring}

for field deployment. When a TLD is found open on the ground, damaged, or lost, the associated annual dose reported is calculated from the average of the available mean quarterly dose values for that given location.

Gamma-radiation exposure is measured in roentgens $(\mathrm{R})$, which are defined as the electronic charge required to ionize a given volume of air $\left(2.54 \times 10^{-4}\right.$ coulombs $/ \mathrm{kg}$ air $)$. The equivalent absorbed dose is $8.7 \times 10^{-3} \mathrm{~Gy}(0.87 \mathrm{rad})$ in air. The tissue equivalent absorbed dose is $9.6 \times 10^{-3} \mathrm{~Gy}$ ( $0.96 \mathrm{rad}$ ). The measured exposure is converted to dose equivalent by calibrating the dosimeters against sources that deliver a known absorbed dose and then applying the gamma-radiation quality factor of 1 . The resultant doseequivalent is reported for environmental dose in submultiple factors of $1 \times 10^{-3}$ sieverts or millisieverts (mSv) and compared to Department of Energy (DOE) Order 5400.5 radiation protection standards. Site boundary doses are compared to environmental background measurements to assess the contribution or impact, if any, from LLNL operations.

To ensure accuracy in TLD measurements, some TLDs are irradiated each quarter to specific exposures for calibration purposes, and others are irradiated to specific exposures to serve as quality-control accuracy checks. Duplicate TLDs are located in the field at several locations each quarter to assess TLD measurement precision. When the field deployment time is either less than or exceeds 90 days, the data is normalized to a standard, 90-day quarter or 360 days per year for the purpose of comparison. LLNL participates in the National Intercomparison Laboratory Study for external gamma radiation measurements, and LLNL processing complies with the DOE Environmental Measurement Laboratory standards.

\section{Tables}

Data tables for the 1999 gamma-radiation monitoring network are presented below. Table 12-1 presents the Livermore site perimeter data, Table 12-2 presents the Livermore Valley data, Table 12-3 presents the Site 300 perimeter data, and Table 12-4 presents Tracy and other Site 300 off-site data. Summary data are discussed in detail in Chapter 12 of the main volume of this report. 


\section{Environmental Radiation Monitoring}

Table 12-1. Calculated dose from TLD environmental radiation measurements, Livermore site perimeter, 1999.

\begin{tabular}{|c|c|c|c|c|c|}
\hline \multirow{2}{*}{ Location $^{(\mathbf{a})}$} & \multicolumn{4}{|c|}{ Quarterly dose $(\mathbf{m S v})^{(\mathbf{b})}$} & $\begin{array}{c}\text { Annual Dose } \\
\text { (c) }\end{array}$ \\
\cline { 2 - 5 } & Jan-Mar & Apr-Jun & Jul-Sep & Oct-Dec & $0.612 \pm 0.015$ \\
L-001-TD & $0.159 \pm 0.010$ & $0.148 \pm 0.001$ & $0.149 \pm 0.011$ & $0.156 \pm 0.004$ & $0.608 \pm 0.010$ \\
L-004-TD & $0.154 \pm 0.006$ & $0.149 \pm 0.005$ & $0.153 \pm 0.001$ & $0.152 \pm 0.006$ & $0.631 \pm 0.012$ \\
L-005-TD & $0.159 \pm 0.005$ & $0.159 \pm 0.006$ & $0.156 \pm 0.007$ & $0.157 \pm 0.005$ & $0.630 \pm 0.018$ \\
L-006-TD & $0.156 \pm 0.010$ & $0.160 \pm 0.011$ & $0.158 \pm 0.007$ & $0.156 \pm 0.008$ & $0.475 \pm 0.019$ \\
L-011-TD & $0.117 \pm 0.004$ & $0.120 \pm 0.011$ & $0.119 \pm 0.014$ & $0.119 \pm 0.005$ & $0.553 \pm 0.025$ \\
L-014-TD & $0.136 \pm 0.018$ & $0.140 \pm 0.004$ & $-(\mathrm{d})$ & $0.139 \pm 0.005$ & $0.556 \pm 0.019$ \\
L-016-TD & $0.135 \pm 0.009$ & $0.141 \pm 0.006$ & $0.14 \pm 0.012$ & $0.140 \pm 0.010$ & $0.586 \pm 0.011$ \\
L-042-TD & $0.137 \pm 0.008$ & $0.152 \pm 0.004$ & $0.142 \pm 0.004$ & $0.155 \pm 0.005$ & $0.617 \pm 0.022$ \\
L-043-TD & $0.150 \pm 0.019$ & $0.149 \pm 0.005$ & $0.154 \pm 0.002$ & $0.164 \pm 0.010$ & $0.520 \pm 0.015$ \\
L-047-TD & $0.134 \pm 0.009$ & $0.129 \pm 0.009$ & $0.126 \pm 0.005$ & $0.131 \pm 0.007$ & $0.566 \pm 0.015$ \\
L-052-TD & $0.137 \pm 0.003$ & $0.141 \pm 0.008$ & $0.134 \pm 0.008$ & $0.154 \pm 0.009$ & $0.567 \pm 0.016$ \\
L-056-TD & $0.139 \pm 0.005$ & $0.140 \pm 0.008$ & $0.147 \pm 0.012$ & $0.141 \pm 0.004$ & $0.583 \pm 0.013$ \\
L-068-TD & $0.145 \pm 0.008$ & $0.144 \pm 0.007$ & $0.15 \pm 0.008$ & $0.144 \pm 0.000$ & $0.548 \pm 0.019$ \\
L-069-TD & $0.136 \pm 0.004$ & $0.138 \pm 0.009$ & $0.139 \pm 0.011$ & $0.135 \pm 0.012$ & $\mathbf{0 . 5 7 7} \pm \mathbf{0 . 0 2 5}$ \\
\hline Mean(e) & $\mathbf{0 . 1 4 2} \pm \mathbf{0 . 0 0 6}$ & $\mathbf{0 . 1 4 4} \pm \mathbf{0 . 0 0 6}$ & $\mathbf{0 . 1 4 4} \pm \mathbf{0 . 0 0 7}$ & $\mathbf{0 . 1 4 6} \pm \mathbf{0 . 0 0 6}$ & \\
\hline
\end{tabular}

Note: Measurement represents the TLD absorbed dose in $\mathrm{mR}$ converted to $\mathrm{mSv}$.

a See Figure 12-1 in the main volume for locations.

b Measurement uncertainty is reported as $\pm 2 \sigma$ of the data.

c Uncertainty is reported as twice the propagated error of the quarterly means.

d Data are not available due to a missing or damaged TLD.

e Uncertainty associated with the quarterly means is reported as two standard errors of the location data. 


\section{Environmental Radiation Monitoring}

Table 12-2. Calculated dose from TLD environmental radiation measurements, Livermore Valley, 1999.

\begin{tabular}{|c|c|c|c|c|c|}
\hline \multirow[b]{2}{*}{ Location $^{(a)}$} & \multicolumn{4}{|c|}{ Quarterly dose (mSv) ${ }^{(b)}$} & \multirow{2}{*}{$\begin{array}{c}\text { Annual dose }{ }^{(\mathrm{c})} \\
(\mathrm{mSv})\end{array}$} \\
\hline & Jan-Mar & Apr-Jun & Jul-Sep & Oct-Dec & \\
\hline V-018-TD & $0.119 \pm 0.013$ & $0.114 \pm 0.011$ & $0.117 \pm 0.003$ & $0.121 \pm 0.009$ & $0.471 \pm 0.019$ \\
\hline V-019-TD & $0.132 \pm 0.008$ & $0.133 \pm 0.010$ & $0.130 \pm 0.006$ & $0.131 \pm 0.005$ & $0.526 \pm 0.015$ \\
\hline V-022-TD & $0.151 \pm 0.011$ & $0.161 \pm 0.011$ & $0.159 \pm 0.002$ & $0.151 \pm 0.002$ & $0.622 \pm 0.016$ \\
\hline V-024-TD & $0.147 \pm 0.007$ & $0.153 \pm 0.006$ & $0.145 \pm 0.011$ & $0.148 \pm 0.008$ & $0.593 \pm 0.016$ \\
\hline V-027-TD & $0.137 \pm 0.004$ & $0.138 \pm 0.009$ & $0.136 \pm 0.011$ & $0.131 \pm 0.010$ & $0.542 \pm 0.018$ \\
\hline V-028-TD & $0.160 \pm 0.014$ & $0.168 \pm 0.011$ & $0.159 \pm 0.004$ & $0.171 \pm 0.006$ & $0.658 \pm 0.019$ \\
\hline V-030-TD & $0.134 \pm 0.010$ & $0.142 \pm 0.003$ & $0.146 \pm 0.007$ & $0.149 \pm 0.009$ & $0.571 \pm 0.015$ \\
\hline V-032-TD & $0.139 \pm 0.005$ & $0.143 \pm 0.007$ & $0.142 \pm 0.003$ & $0.145 \pm 0.003$ & $0.569 \pm 0.010$ \\
\hline V-033-TD & $0.145 \pm 0.009$ & $0.149 \pm 0.006$ & $0.152 \pm 0.006$ & $0.152 \pm 0.016$ & $0.598 \pm 0.020$ \\
\hline V-035-TD & $0.149 \pm 0.016$ & $0.150 \pm 0.007$ & $0.159 \pm 0.006$ & $0.150 \pm 0.011$ & $0.608 \pm 0.021$ \\
\hline V-037-TD & $0.145 \pm 0.011$ & $0.144 \pm 0.009$ & (d) $^{(\mathrm{d}}$ & (d) $^{(\mathrm{d}}$ & $0.578 \pm 0.028$ \\
\hline V-045-TD & $0.131 \pm 0.006$ & $0.142 \pm 0.004$ & $0.145 \pm 0.008$ & $0.138 \pm 0.004$ & $0.556 \pm 0.011$ \\
\hline V-057-TD & $0.148 \pm 0.002$ & $0.153 \pm 0.007$ & $0.162 \pm 0.013$ & $0.159 \pm 0.010$ & $0.622 \pm 0.018$ \\
\hline V-060-TD & $0.143 \pm 0.005$ & $0.152 \pm 0.008$ & $0.147 \pm 0.007$ & $0.148 \pm 0.009$ & $0.590 \pm 0.015$ \\
\hline V-061-TD & $0.137 \pm 0.009$ & $-^{(d)}$ & $0.127 \pm 0.004$ & $0.127 \pm 0.007$ & $0.521 \pm 0.016$ \\
\hline V-066-TD & $0.150 \pm 0.008$ & $0.149 \pm 0.008$ & $0.152 \pm 0.006$ & $0.150 \pm 0.007$ & $0.601 \pm 0.015$ \\
\hline V-070-TD & $0.142 \pm 0.007$ & $0.143 \pm 0.002$ & $-^{(d)}$ & $0.143 \pm 0.006$ & $0.571 \pm 0.013$ \\
\hline V-072-TD & $0.170 \pm 0.014$ & $0.166 \pm 0.004$ & $0.170 \pm 0.013$ & $0.172 \pm 0.007$ & $0.678 \pm 0.021$ \\
\hline V-073-TD & -(d) $^{(\mathrm{d}}$ & $0.151 \pm 0.004$ & $0.156 \pm 0.008$ & $0.144 \pm 0.008$ & $0.601 \pm 0.016$ \\
\hline V-074-TD & $0.135 \pm 0.009$ & $0.151 \pm 0.004$ & $0.156 \pm 0.008$ & $0.144 \pm 0.008$ & $0.586 \pm 0.015$ \\
\hline V-075-TD & $0.117 \pm 0.005$ & $0.130 \pm 0.012$ & $0.131 \pm 0.009$ & $0.136 \pm 0.009$ & $0.514 \pm 0.018$ \\
\hline V-076-TD & $0.124 \pm 0.005$ & $0.113 \pm 0.008$ & $0.112 \pm 0.004$ & $0.117 \pm 0.004$ & $0.466 \pm 0.011$ \\
\hline V-077-TD & $0.133 \pm 0.009$ & $0.119 \pm 0.015$ & $0.120 \pm 0.003$ & $0.123 \pm 0.005$ & $0.495 \pm 0.018$ \\
\hline Mean(e) & $0.140 \pm 0.005$ & $0.143 \pm 0.006$ & $0.143 \pm 0.007$ & $0.143 \pm 0.006$ & $0.571 \pm 0.022$ \\
\hline
\end{tabular}

Note: Measurement represents the TLD absorbed dose in $\mathrm{mR}$ converted to $\mathrm{mSv}$.

a See Figure 12-2 in the main volume for locations.

b Measurement uncertainty is reported as $\pm 2 \sigma$ of the data.

c Uncertainty is reported as twice the propagated error of the quarterly mean.

d Data are not available due to a missing or damaged TLD.

e Uncertainty associated with the quarterly mean is reported as two standard errors of the location data. 


\section{Environmental Radiation Monitoring}

Table 12-3. Calculated dose from TLD environmental radiation measurements, Site 300 perimeter, 1999.

\begin{tabular}{|c|c|c|c|c|c|}
\hline \multirow{2}{*}{ Location $^{(a)}$} & \multicolumn{4}{|c|}{ Quarterly dose (mSv) ${ }^{(b)}$} & \multirow{2}{*}{$\begin{array}{c}\text { Annual dose } \text { (c) }^{(\mathrm{mSv})} \\
\text { (m) }\end{array}$} \\
\hline & Jan-Mar & Apr-Jun & Jul-Sep & Oct-Dec & \\
\hline 3-078-TD & $0.148 \pm 0.001$ & $0.148 \pm 0.007$ & $0.151 \pm 0.004$ & $0.154 \pm 0.011$ & $0.601 \pm 0.014$ \\
\hline 3-081-TD & $0.197 \pm 0.036$ & $0.189 \pm 0.014$ & $0.234 \pm 0.019$ & $0.190 \pm 0.009$ & $0.810 \pm 0.044$ \\
\hline 3-082-TD & $0.161 \pm 0.012$ & $0.174 \pm 0.008$ & $0.169 \pm 0.010$ & $0.173 \pm 0.005$ & $0.677 \pm 0.018$ \\
\hline 3-085-TD & $0.163 \pm 0.012$ & $0.168 \pm 0.008$ & $0.175 \pm 0.004$ & $0.173 \pm 0.009$ & $0.679 \pm 0.017$ \\
\hline 3-086-TD & $0.169 \pm 0.012$ & $0.179 \pm 0.012$ & $0.174 \pm 0.011$ & $0.170 \pm 0.012$ & $0.692 \pm 0.024$ \\
\hline 3-088-TD & $0.174 \pm 0.012$ & $0.165 \pm 0.012$ & $0.169 \pm 0.002$ & $0.170 \pm 0.009$ & $0.678 \pm 0.019$ \\
\hline 3-089-TD & $0.186 \pm 0.012$ & $0.187 \pm 0.017$ & $0.180 \pm 0.012$ & $0.184 \pm 0.007$ & $0.737 \pm 0.025$ \\
\hline 3-091-TD & $0.168 \pm 0.007$ & $0.176 \pm 0.004$ & $0.188 \pm 0.001$ & $0.181 \pm 0.003$ & $0.713 \pm 0.009$ \\
\hline 3-121-TD & $0.182 \pm 0.008$ & $0.189 \pm 0.009$ & $0.192 \pm 0.023$ & $0.208 \pm 0.013$ & $0.771 \pm 0.029$ \\
\hline $\operatorname{Mean}(d)$ & $0.172 \pm 0.009$ & $0.175 \pm 0.008$ & $0.181 \pm 0.015$ & $0.178 \pm 0.010$ & $0.706 \pm 0.040$ \\
\hline
\end{tabular}

Note: Measurement represents the TLD absorbed dose in $\mathrm{mR}$ converted to $\mathrm{mSv}$.

a See Figure 12-3 in the main volume for locations.

b Measurement uncertainty is reported as $\pm 2 \sigma$ of the data.

c Uncertainty is reported as twice the propagated error of the quarterly means.

d Uncertainty associated with the quarterly means is reported as two standard errors of the location data. 


\section{Environmental Radiation Monitoring}

Table 12-4. Calculated dose from TLD environmental radiation measurements, Tracy and other off-site locations in the vicinity of Site 300, 1999.

\begin{tabular}{|c|c|c|c|c|c|}
\hline \multirow[b]{2}{*}{ Location $^{(a)}$} & \multicolumn{4}{|c|}{ Quarterly dose (mSv) ${ }^{(\mathbf{b})}$} & \multirow{2}{*}{$\begin{array}{c}\text { Annual dose }{ }^{(\mathrm{c})} \\
(\mathrm{mSv})\end{array}$} \\
\hline & Jan-Mar & Apr-Jun & Jul-Sep & Oct-Dec & \\
\hline 3-092-TD & $0.153 \pm 0.008$ & $0.157 \pm 0.014$ & $0.154 \pm 0.000$ & $0.161 \pm 0.004$ & $0.625 \pm 0.017$ \\
\hline 3-093-TD & $0.143 \pm 0.008$ & $0.132 \pm 0.002$ & $0.140 \pm 0.005$ & $0.143 \pm 0.007$ & $0.558 \pm 0.012$ \\
\hline $\operatorname{Mean}^{(d)}$ & $0.148 \pm 0.010$ & $0.144 \pm 0.024$ & $0.147 \pm 0.013$ & $0.152 \pm 0.017$ & $0.591 \pm 0.066$ \\
\hline 3-090-TD & $0.177 \pm 0.003$ & $0.181 \pm 0.009$ & $0.189 \pm 0.006$ & $0.181 \pm 0.011$ & $0.728 \pm 0.016$ \\
\hline 3-094-TD & $0.214 \pm 0.028$ & $0.230 \pm 0.005$ & $0.203 \pm 0.002$ & $0.202 \pm 0.010$ & $0.849 \pm 0.030$ \\
\hline 3-096-TD & $0.202 \pm 0.011$ & $0.198 \pm 0.012$ & $0.238 \pm 0.016$ & $0.231 \pm 0.008$ & $0.869 \pm 0.024$ \\
\hline 3-099-TD & $0.157 \pm 0.008$ & $0.161 \pm 0.003$ & $0.174 \pm 0.014$ & $0.144 \pm 0.002$ & $0.636 \pm 0.017$ \\
\hline 3-120-TD & -(e) $^{(\mathrm{e}}$ & $0.177 \pm 0.014$ & -(f) $^{(\mathrm{n}}$ & -(f) $^{(1)}$ & - (g) \\
\hline Mean & $0.187 \pm 0.025$ & $0.189 \pm 0.023$ & $0.201 \pm 0.027$ & $0.189 \pm 0.036$ & $0.770 \pm 0.109$ \\
\hline
\end{tabular}

Note: Measurement represents the TLD absorbed dose in $\mathrm{mR}$ converted to $\mathrm{mSv}$.

a See Figure 12-2 in the main volume for locations.

b Measurement uncertainty is reported as $\pm 2 \sigma$ of the data.

c Uncertainty is reported as twice the propagated error of the quarterly mean.

d Uncertainty associated with the quarterly mean is reported as two standard errors of the location data.

e Data are not available due to a missing or damaged TLD.

$f$ Location removed.

g Not applicable. 
There are no supplemental data in this chapter. Please see the main volume for details about Radiological Dose Assessment. 



\section{$14 \mathrm{Q}$}

\section{Quality Assurance}

Lucinda M. Garcia

\section{Participation in Laboratory Intercomparison Studies}

Two laboratories at Lawrence Livermore National Laboratory participated in the annual Environmental Monitoring Laboratory (EML) intercomparison studies program sponsored by the U.S. Department of Energy (DOE). The two LLNL laboratories are the Chemistry and Materials Science Environmental Services (CES) Environmental Monitoring Radiation Laboratory (EMRL) and the Hazards Control Department's Analytical Laboratory (HCAL).

The results of CES EMRL's participation in the EML studies are presented in Table 14-1. According to the results, 55 of 58 analyses fell within established acceptance control limits.

The CES result for plutonium-238 in vegetation for the Semi-Annual Report for the Department of Energy's Office of Environmental Management Quality Assurance Program (QAP-51) (Greenlaw 1999) study fell below the lower acceptance control limit. CES attributed this unacceptable result to poor counting statistics for plutonium-238, which was present at a relatively low activity. The count time for this sample was shortened (16 hours as opposed to 72 hours typically used for standard samples) to prevent detector and counting chamber contamination by long-lived decay daughter recoil nuclides from plutonium-239, which was present at an activity ten times higher than that of plutonium-238. If the standard deviation of the CES reported activity is taken into account, the CES result is not statistically different from the EML value.

CES results for cobalt- 60 and cesium-137 in water for the QAP-51 study fell above the upper acceptance control limits. CES attributed these unacceptable results to inattention to detail. Specifically, the efficiency calibration standards for the gamma spectrometer unit were not prepared correctly, leading to an erroneous estimation of the minimum detectable concentration (MDC) and unacceptable results. When CES analysts discovered this problem, they recounted all affected client samples and reissued corrected analytical reports. In the future, CES will count a second source standard that is independent of the standard used for calibration after they have performed efficiency calibration of the gamma spectrometer units. 
The results of HCAL's participation in the 1999 EML studies (see Table 14-2) indicate that ten of ten sample results fell within the $3 \sigma$ acceptance control limits.

CES EMRL participated in two DOE Mixed Analyte Performance Evaluation Program (MAPEP) studies in 1999. The results of these study are presented in Tables 14-3 and 14-4. Sixteen of 16 analytes reported by CES in the first study, and 23 of 23 analytes reported by CES in the second study fell within acceptable limits.

Although contract laboratories are also required to participate in laboratory intercomparison programs, permission to publish their results for comparison purposes was not granted for 1999. 
Table 14-1. LLNL's CES EMRL results from the DOE EML Quality Assurance Program, 1999.

\begin{tabular}{|c|c|c|c|c|c|c|c|}
\hline Analyte & EML study & CES value & EML value & CES/EML & $\begin{array}{l}\text { Control limits } \\
(3 \sigma)^{(a)}\end{array}$ & $\begin{array}{l}\text { Warning limits } \\
(2 \sigma)\end{array}$ & Performance $^{(b)}$ \\
\hline \multicolumn{8}{|c|}{ Air filter (Bq/filter) } \\
\hline \multirow[t]{2}{*}{ Am-241 } & QAP-50 & 0.145 & 0.134 & 1.09 & $0.73-2.58$ & $0.88-1.46$ & Acceptable \\
\hline & QAP-51 & 0.124 & 0.127 & 0.976 & $0.73-2.58$ & $0.88-1.46$ & Acceptable \\
\hline \multirow[t]{2}{*}{ Co-57 } & QAP-50 & 3.24 & 3.01 & 1.08 & $0.65-1.39$ & $0.72-1.13$ & Acceptable \\
\hline & QAP-51 & 8.56 & 7.73 & 1.11 & $0.65-1.39$ & $0.72-1.13$ & Acceptable \\
\hline \multirow[t]{2}{*}{ Co-60 } & QAP-50 & 5.24 & 4.96 & 1.06 & $0.75-1.32$ & $0.83-1.10$ & Acceptable \\
\hline & QAP-51 & 6.83 & 6.35 & 1.08 & $0.75-1.32$ & $0.83-1.10$ & Acceptable \\
\hline \multirow[t]{2}{*}{ Cs-137 } & QAP-50 & 6.59 & 6.05 & 1.09 & $0.73-1.37$ & $0.82-1.14$ & Acceptable \\
\hline & QAP-51 & 7.36 & 6.43 & 1.15 & $0.73-1.37$ & $0.82-1.14$ & Warning \\
\hline$M n-54$ & QAP-51 & 8.70 & 7.91 & 1.10 & $0.76-1.42$ & $0.84-1.18$ & Acceptable \\
\hline \multirow[t]{2}{*}{ Pu-238 } & QAP-50 & 0.274 & 0.272 & 1.01 & $0.74-1.40$ & $0.89-1.15$ & Acceptable \\
\hline & QAP-51 & 0.108 & 0.097 & 1.12 & $0.74-1.40$ & $0.89-1.15$ & Acceptable \\
\hline \multirow[t]{2}{*}{ Pu-239 } & QAP-50 & 0.128 & 0.124 & 1.03 & $0.76-1.44$ & $0.90-1.19$ & Acceptable \\
\hline & QAP-51 & 0.129 & 0.136 & 0.949 & $0.76-1.44$ & $0.90-1.19$ & Acceptable \\
\hline Sb-125 & QAP-50 & 4.19 & 3.59 & 1.17 & $0.61-1.43$ & $0.83-1.19$ & Acceptable \\
\hline $\mathrm{U}(\mathrm{Bq})$ & QAP-51 & 0.127 & 0.133 & 0.955 & $0.80-3.35$ & $0.90-1.53$ & Acceptable \\
\hline U-234 & QAP-50 & 0.073 & 0.060 & 1.21 & $0.83-1.92$ & $0.90-1.40$ & Acceptable \\
\hline U-238 & QAP-50 & 0.064 & 0.061 & 1.05 & $0.84-2.61$ & $0.90-1.31$ & Acceptable \\
\hline \multicolumn{8}{|c|}{ Soil (Bq/kg) } \\
\hline Ac-228 & QAP-50 & 49.9 & 47.2 & 1.06 & $0.79-1.75$ & $0.87-1.31$ & Acceptable \\
\hline Am-241 & QAP-50 & 7.23 & 4.89 & 1.48 & $0.63-2.31$ & $0.79-1.48$ & Acceptable \\
\hline $\mathrm{Bi}-214$ & QAP-50 & 77.6 & 69.9 & 1.11 & $0.75-1.42$ & $0.83-1.18$ & Acceptable \\
\hline $\mathrm{U}(\mathrm{Bq})$ & QAP-51 & 383 & 401 & 0.955 & $0.42-1.39$ & $0.61-1.16$ & Acceptable \\
\hline \multirow[t]{2}{*}{ Cs-137 } & QAP-50 & 759 & 660 & 1.15 & $0.83-1.32$ & $0.90-1.21$ & Acceptable \\
\hline & QAP-51 & 187 & 204 & 0.917 & $0.83-1.32$ & $0.90-1.21$ & Acceptable \\
\hline \multirow[t]{2}{*}{$\mathrm{K}-40$} & QAP-50 & 374 & 363 & 1.03 & $0.78-1.53$ & $0.90-1.25$ & Acceptable \\
\hline & QAP-51 & 715 & 780 & 0.917 & $0.78-1.53$ & $0.90-1.25$ & Acceptable \\
\hline $\mathrm{Pb}-212$ & QAP-50 & 50.7 & 47.9 & 1.06 & $0.74-1.33$ & $0.91-1.21$ & Acceptable \\
\hline $\mathrm{Pb}-214$ & QAP-50 & 87.1 & 71.0 & 1.23 & $0.65-1.45$ & $0.89-1.25$ & Acceptable \\
\hline Pu-238 & QAP-51 & 0.193 & 0.320 & 0.603 & $0.52-2.84$ & $0.74-1.37$ & Warning \\
\hline \multirow[t]{2}{*}{ Pu-239 } & QAP-50 & 8.09 & 8.11 & 0.997 & $0.69-1.74$ & $0.89-1.24$ & Acceptable \\
\hline & QAP-51 & 2.90 & 3.20 & 0.906 & $0.69-1.74$ & $0.89-1.24$ & Acceptable \\
\hline Th-234 & QAP-50 & 146 & 138 & 1.06 & $0.59-1.85$ & $.082-1.48$ & Acceptable \\
\hline $\mathrm{U}(\mu \mathrm{g})$ & QAP-50 & 11.8 & 11.8 & 1.00 & $0.46-1.22$ & $0.67-1.10$ & Acceptable \\
\hline
\end{tabular}




\section{Quality Assurance}

Table 14-1. LLNL's CES EMRL results from the DOE EML Quality Assurance Program, 1999 (concluded).

\begin{tabular}{|c|c|c|c|c|c|c|c|}
\hline Analyte & EML study & CES value & EML value & CES/EML & $\begin{array}{l}\text { Control limits } \\
(3 \sigma)^{(a)}\end{array}$ & $\begin{array}{c}\text { Warning limits } \\
(2 \sigma)\end{array}$ & Performance ${ }^{(b)}$ \\
\hline \multicolumn{8}{|c|}{ Vegetation (Bq/kg) } \\
\hline Am-241 & QAP-51 & 4.24 & 2.88 & 1.47 & $0.68-2.70$ & $0.89-1.60$ & Acceptable \\
\hline $\mathrm{Cm}-244$ & QAP-51 & 2.34 & 1.61 & 1.45 & $0.47-1.74$ & $0.81-1.35$ & Warning \\
\hline Co-60 & QAP-51 & 15.6 & 17.6 & 0.886 & $0.69-1.46$ & $0.86-1.24$ & Acceptable \\
\hline \multirow[t]{2}{*}{ Cs-137 } & QAP-50 & 500 & 467 & 1.07 & $0.80-1.40$ & $0.90-1.25$ & Acceptable \\
\hline & QAP-51 & 378 & 440 & 0.859 & $0.80-1.40$ & $0.90-1.25$ & Warning \\
\hline $\mathrm{K}-40$ & QAP-50 & 643 & 657 & 0.979 & $0.79-1.42$ & $0.90-1.24$ & Acceptable \\
\hline Pu-239 & QAP-51 & 4.66 & 4.30 & 1.08 & $0.68-1.59$ & $0.86-1.23$ & Acceptable \\
\hline \multicolumn{8}{|c|}{ Water (Bq/L) } \\
\hline Am-241 & QAP-50 & 1.21 & 1.15 & 1.06 & $0.75-1.49$ & $0.90-1.24$ & Acceptable \\
\hline \multirow[t]{2}{*}{ Co-60 } & QAP-50 & 54.4 & 51.1 & 1.065 & $0.80-1.20$ & $0.90-1.14$ & Acceptable \\
\hline & QAP-51 & 109 & 52.4 & 2.08 & $0.80-1.20$ & $0.90-1.14$ & Not acceptable \\
\hline \multirow[t]{2}{*}{ Cs-137 } & QAP-50 & 39.4 & 39.4 & 1.00 & $0.80-1.26$ & $0.90-1.18$ & Acceptable \\
\hline & QAP-51 & 163 & 76.0 & 2.15 & $0.80-1.26$ & $0.90-1.18$ & Not acceptable \\
\hline $\mathrm{H}-3$ & QAP-50 & 141 & 121 & 1.17 & $0.71-1.79$ & $0.82-1.22$ & Acceptable \\
\hline U-234 & QAP-50 & 0.256 & 0.269 & 0.953 & $0.80-1.40$ & $0.90-1.22$ & Acceptable \\
\hline U-238 & QAP-50 & 0.277 & 0.262 & 1.06 & $0.80-1.26$ & $0.90-1.17$ & Acceptable \\
\hline
\end{tabular}

a Control limits are established from historical QAP data and reported as the ratio of reported value to EML value. Limits were not applied where historical data were insufficient.

b Data are considered acceptable when they fall within the $2 \sigma$ warning limits. Data should be checked for error when they are between the $2 \sigma$ warning limits and the $3 \sigma$ control limits. Data are considered unacceptable when they are outside the $3 \sigma$ control limits. 
Table 14-2. LLNL's HCAL results from the DOE EML Quality Assurance Program, 1999.

\begin{tabular}{|c|c|c|c|c|c|c|c|}
\hline Analyte & $\begin{array}{l}\text { EML } \\
\text { study }\end{array}$ & CES value & EML value & CES/EML & $\begin{array}{l}\text { Control } \\
\text { limits } \\
(3 \sigma)\end{array}$ & $\begin{array}{c}\text { Warning } \\
\text { limits } \\
(2 \sigma)\end{array}$ & Performance ${ }^{(a)}$ \\
\hline \multicolumn{8}{|c|}{ Air filter (Bq/filter) } \\
\hline Gross alpha & QAP-50 & 1.38 & 1.61 & 0.854 & $0.50-1.55$ & $0.81-1.32$ & Acceptable \\
\hline Gross alpha & QAP-51 & 2.44 & 2.77 & 0.881 & $0.50-1.55$ & $0.81-1.32$ & Acceptable \\
\hline Gross beta & QAP-50 & 1.20 & 1.56 & 0.771 & $0.72-1.67$ & $0.89-1.39$ & Warning \\
\hline Gross beta & QAP-51 & 2.26 & 2.66 & 0.850 & $0.72-1.67$ & $0.89-1.39$ & Warning \\
\hline \multicolumn{8}{|c|}{ Water (Bq/L) } \\
\hline Gross alpha & QAP-50 & 1100 & 1090 & 1.00 & $0.61-1.32$ & $0.83-1.17$ & Acceptable \\
\hline Gross alpha & QAP-51 & 1500 & 1580 & 0.946 & $0.61-1.32$ & $0.83-1.17$ & Acceptable \\
\hline Gross beta & QAP-50 & 1190 & 1100 & 1.08 & $0.55-1.54$ & $0.71-1.32$ & Acceptable \\
\hline Gross beta & QAP-51 & 951 & 740 & 1.29 & $0.55-1.54$ & $0.71-1.32$ & Acceptable \\
\hline Tritium & QAP-50 & 111 & 121 & 0.917 & $0.71-1.79$ & $0.82-1.22$ & Acceptable \\
\hline Tritium & QAP-51 & 82.3 & 80.7 & 1.02 & $0.71-1.79$ & $0.82-1.22$ & Acceptable \\
\hline
\end{tabular}

a Data are considered acceptable when they fall within the $2 \sigma$ warning limits. Data should be checked for error when they are between the $2 \sigma$ warning limits and the $3 \sigma$ control limits. Data are considered unacceptable when they are outside the $3 \sigma$ control limits. 


\section{Quality Assurance}

Table 14-3. LLNL CES EMRL performance in the MAPEP-98-W6 Intercomparison Program for Water.

\begin{tabular}{|l|c|c|c|c|c|l|}
\hline \multicolumn{1}{|c|}{ Analyte } & $\begin{array}{c}\text { CES } \\
\text { value }\end{array}$ & Units & $\begin{array}{c}\text { Reference } \\
\text { value }\end{array}$ & Bias (\%) & $\begin{array}{c}\text { Acceptance } \\
\text { range }\end{array}$ & Performance $^{(a)}$ \\
\hline Antimony & 0.543 & $\mathrm{mg} / \mathrm{L}$ & 0.50 & 9.48 & $0.35-0.64$ & Acceptable \\
Barium & 80.8 & $\mathrm{mg} / \mathrm{L}$ & 79.4 & 1.76 & $55.6-103$ & Acceptable \\
Beryllium & 2.04 & $\mathrm{mg} / \mathrm{L}$ & 1.99 & 2.51 & $1.39-2.59$ & Acceptable \\
Chromium & 0.5 & $\mathrm{mg} / \mathrm{L}$ & 0.50 & 0.81 & $0.35-0.65$ & Acceptable \\
Copper & 0.976 & $\mathrm{mg} / \mathrm{L}$ & 0.99 & -1.71 & $0.69-1.29$ & Acceptable \\
Lead & 2.99 & $\mathrm{mg} / \mathrm{L}$ & 2.98 & 0.34 & $2.09-3.87$ & Acceptable \\
Thallium & 0.509 & $\mathrm{mg} / \mathrm{L}$ & 0.50 & 2.62 & $0.35-0.65$ & Acceptable \\
Zinc & 0.761 & $\mathrm{mg} / \mathrm{L}$ & 0.75 & 1.33 & $0.53-0.98$ & Acceptable \\
Cesium-137 & 621 & $\mathrm{~Bq} / \mathrm{L}$ & 637 & -2.51 & $446-828$ & Acceptable \\
Cobalt-57 & 343 & $\mathrm{~Bq} / \mathrm{L}$ & 358 & -4.19 & $251-465$ & Acceptable \\
Manganese-54 & 222 & $\mathrm{~Bq} / \mathrm{L}$ & 229 & -3.06 & $160-298$ & Acceptable \\
Plutonium-238 & 1.42 & $\mathrm{~Bq} / \mathrm{L}$ & 1.45 & -2.07 & $1.02-1.89$ & Acceptable \\
Plutonium-239+240 & 3.68 & $\mathrm{~Bq} / \mathrm{L}$ & 4.04 & -8.91 & $2.83-5.25$ & Acceptable \\
Uranium-234+233 & 2.62 & $\mathrm{~Bq} / \mathrm{L}$ & 2.67 & -1.87 & $1.87-3.47$ & Acceptable \\
Uranium-238 & 20 & $\mathrm{~Bq} / \mathrm{L}$ & 21.2 & -5.66 & $14.8-27.6$ & Acceptable \\
Zinc-65 & 1480 & $\mathrm{~Bq} / \mathrm{L}$ & 1560 & -5.13 & $1090-2030$ & Acceptable \\
\hline
\end{tabular}

a Acceptable results have $\mid$ bias $\mid \leq 20 \%$. Results with warning have $20 \%<\mid$ bias $\mid \leq 30 \%$. 
Table 14-4. LLNL CES EMRL performance in the MAPEP-99-S6 Intercomparison Program for Soil.

\begin{tabular}{|l|c|c|c|c|c|l|}
\hline \multicolumn{1}{|c|}{ Analyte } & $\begin{array}{c}\text { CES } \\
\text { value }\end{array}$ & Units & $\begin{array}{c}\text { Reference } \\
\text { value }\end{array}$ & Bias (\%) & $\begin{array}{c}\text { Acceptance } \\
\text { range }\end{array}$ & Performance(a) \\
\hline Arsenic & 27 & $\mathrm{mg} / \mathrm{kg}$ & 26.7 & 1.12 & $18.7-34.7$ & Acceptable \\
Barium & 390 & $\mathrm{mg} / \mathrm{kg}$ & 400 & -2.52 & $280-520$ & Acceptable \\
Beryllium & 48 & $\mathrm{mg} / \mathrm{kg}$ & 48.0 & -0.06 & $33.6-62.4$ & Acceptable \\
Cadmium & 14 & $\mathrm{mg} / \mathrm{kg}$ & 14.4 & -2.85 & $10.1-18.7$ & Acceptable \\
Chromium & 79 & $\mathrm{mg} / \mathrm{kg}$ & 79.0 & 0.00 & $55.3-103$ & Acceptable \\
Lead & 78 & $\mathrm{mg} / \mathrm{kg}$ & 77.8 & 0.22 & $54.5-101$ & Acceptable \\
Nickel & 44 & $\mathrm{mg} / \mathrm{kg}$ & 49.6 & -11.3 & $34.7-64.5$ & Acceptable \\
Selenium & 8.7 & $\mathrm{mg} / \mathrm{kg}$ & 9.61 & -9.47 & $6.73-12.5$ & Acceptable \\
Thallium & 79 & $\mathrm{mg} / \mathrm{kg}$ & 96.1 & -17.8 & $67.2-125$ & Acceptable \\
Vanadium & 200 & $\mathrm{mg} / \mathrm{kg}$ & 194 & 2.88 & $136-253$ & Acceptable \\
Zinc & 85 & $\mathrm{mg} / \mathrm{kg}$ & 102 & -16.3 & $71.1-132$ & Acceptable \\
Americium-241 & 6.79 & $\mathrm{~Bq} / \mathrm{kg}$ & 6.55 & 3.66 & $4.59-8.52$ & Acceptable \\
Cesium-134 & 693 & $\mathrm{~Bq} / \mathrm{kg}$ & 752 & -7.85 & $526-978$ & Acceptable \\
Cesium-137 & 312 & $\mathrm{~Bq} / \mathrm{kg}$ & 331 & -5.74 & $232-430$ & Acceptable \\
Cobalt-57 & 354 & $\mathrm{~Bq} / \mathrm{kg}$ & 360 & -1.67 & $252-468$ & Acceptable \\
Cobalt-60 & 134 & $\mathrm{~Bq} / \mathrm{kg}$ & 131 & 2.29 & $91.7-170$ & Acceptable \\
Manganese-54 & 349 & $\mathrm{~Bq} / \mathrm{kg}$ & 345 & 1.16 & $242-449$ & Acceptable \\
Plutonium-238 & 27.2 & $\mathrm{~Bq} / \mathrm{kg}$ & 27.5 & -1.09 & $19.3-35.8$ & Acceptable \\
Plutonium-239+240 & 46.8 & $\mathrm{~Bq} / \mathrm{kg}$ & 48.1 & -2.70 & $33.7-62.5$ & Acceptable \\
Potassium-40 & 636 & $\mathrm{~Bq} / \mathrm{kg}$ & 652 & -2.45 & $456-848$ & Acceptable \\
Uranium-234+233 & 139 & $\mathrm{~Bq} / \mathrm{kg}$ & 157 & -11.45 & $110-204$ & Acceptable \\
Uranium-238 & 31.5 & $\mathrm{~Bq} / \mathrm{kg}$ & 40.7 & -22.6 & $28.5-52.9$ & Warning \\
Zinc-65 & 2880 & $\mathrm{~Bq} / \mathrm{kg}$ & 2840 & 1.41 & $1990-3690$ & Acceptable \\
\hline
\end{tabular}

a Acceptable results have | bias $\mid \leq 20 \%$. Results acceptable with warning have $\mid$ bias $\mid>20 \%$ less but $\leq 30 \%$. 




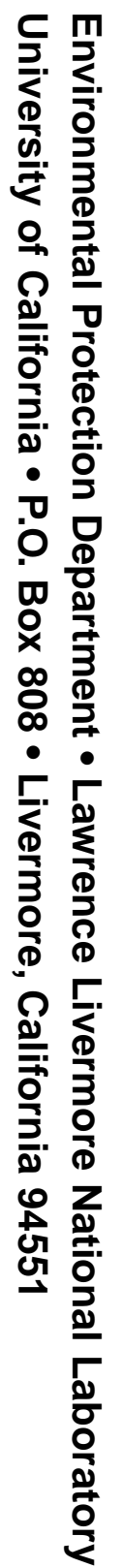

